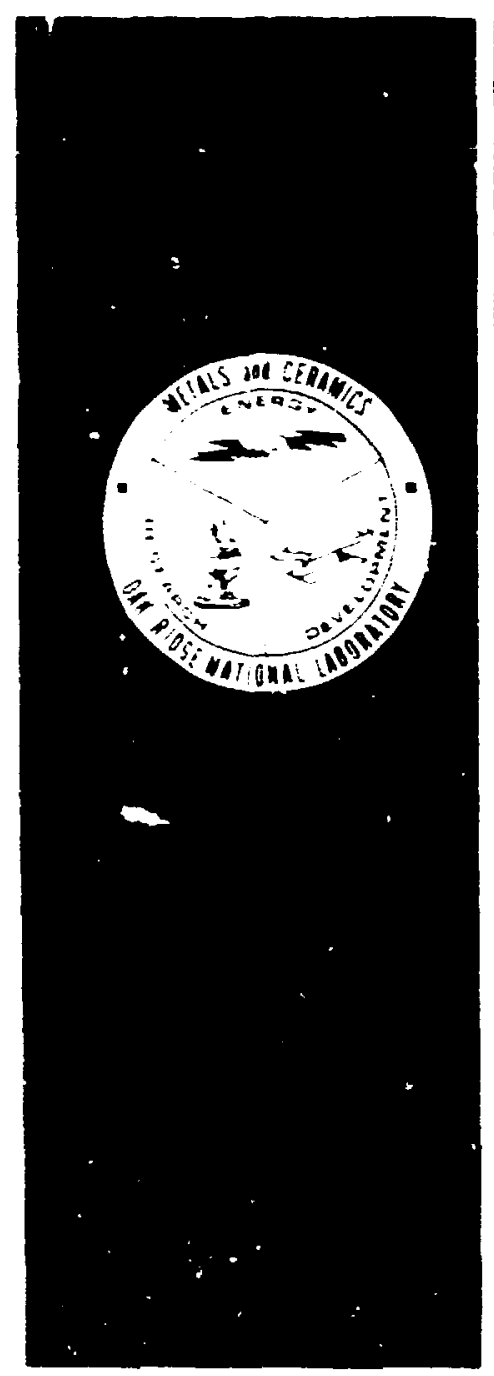

ORNL NUREG-10

\title{
Stress Factors for the Deformation Systems of Zirconium under Multiaxia! Stress
}

D. O. Hobson

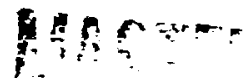

\section{Prepared for the}

U.S. Nuclear Regulatory Commission

Office of Nuclea! Reactor Regulation

Under Interagency Agreements ERDA 40-55i-75 and 40-552-75

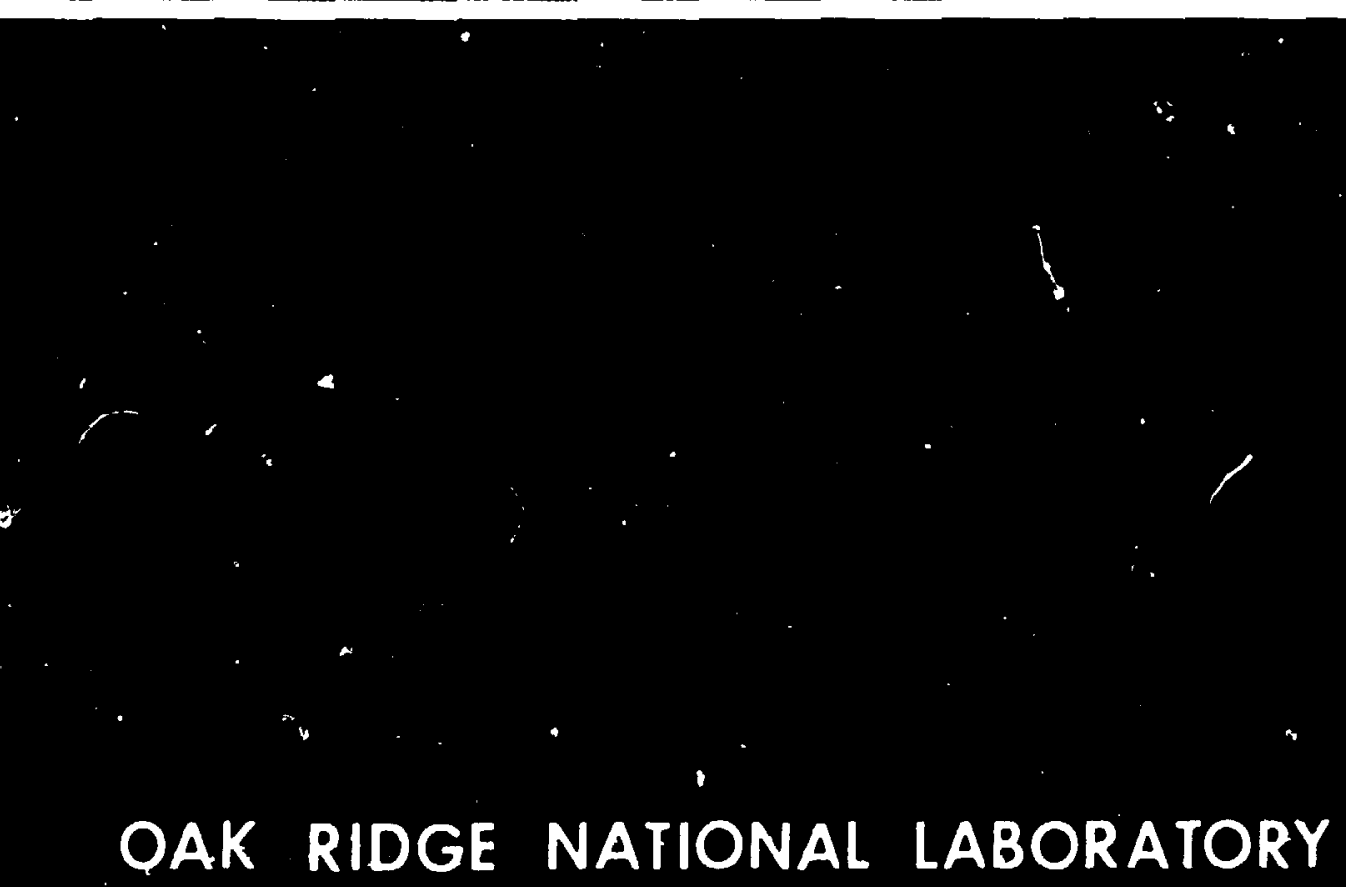

OPERATED BY UMION CARBIOE CORPORATION FOR IHE ENERGY RESEAFK AMO DEYELOPMENT ADMINISTRAIION 


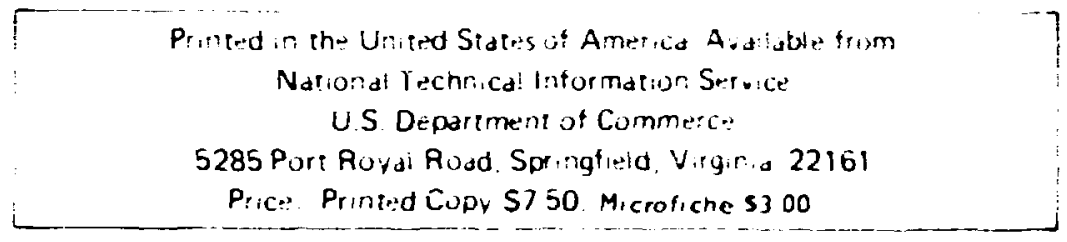

This report was orepared as an account of work sponsared by the United States Government. Neither the United States nor the Energ, Research and Development Administration: Un:ted States Nuc'ear Aegulatory Commission, nor iny of ineir employees, rot any of their cor-sactors. subcontraztors, or their employees. makes any warraney. express or imiplied. or assumes any legal hablity or responsibilify for the accuracy. completeness or usefuiness of any information. apparatus. product or process disclosed, or represents that is use would not infernge privateiv owned ights 
ORNL/MTREG-10

Distribution

Category SRC-3

Contract lio. h-7405-eng-26

METALS AVD CERAMICS DIVISION

STRESS FACTORS FOR THE DEFORYATION SYSTEYS DF
ZIRCONILY LODER MITIAUIAL STRESS

D. O. Hobson

Manuscript Completed - . Wovember 12, 1976

Date Published - December 1976

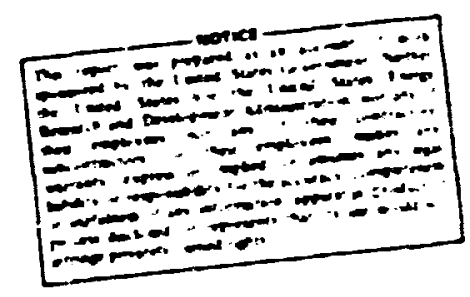

\author{
Prepared for the \\ U.S. Nuclear Regulatory Comission \\ of fice of Nuclear Reactor Regulation \\ Under Interagency Agreements ERDA 40-551-75 and 40-552-75
}

\author{
wotke this document contarm intormation of a deliminapy natwe \\ It is subpect to revision or correction and theretore dee not represents \\ linat repoet \\ Prepared by the \\ OAK RIDGE NATIONAL LABORATORY \\ Dak Ridge, Tennessee 37830 \\ operated by \\ UNION CARBIDE CORPORATION \\ for the \\ ENERGY RESEARCH AND DEVELOPMENT ADMINISTRATION
}




\section{comrenrs}

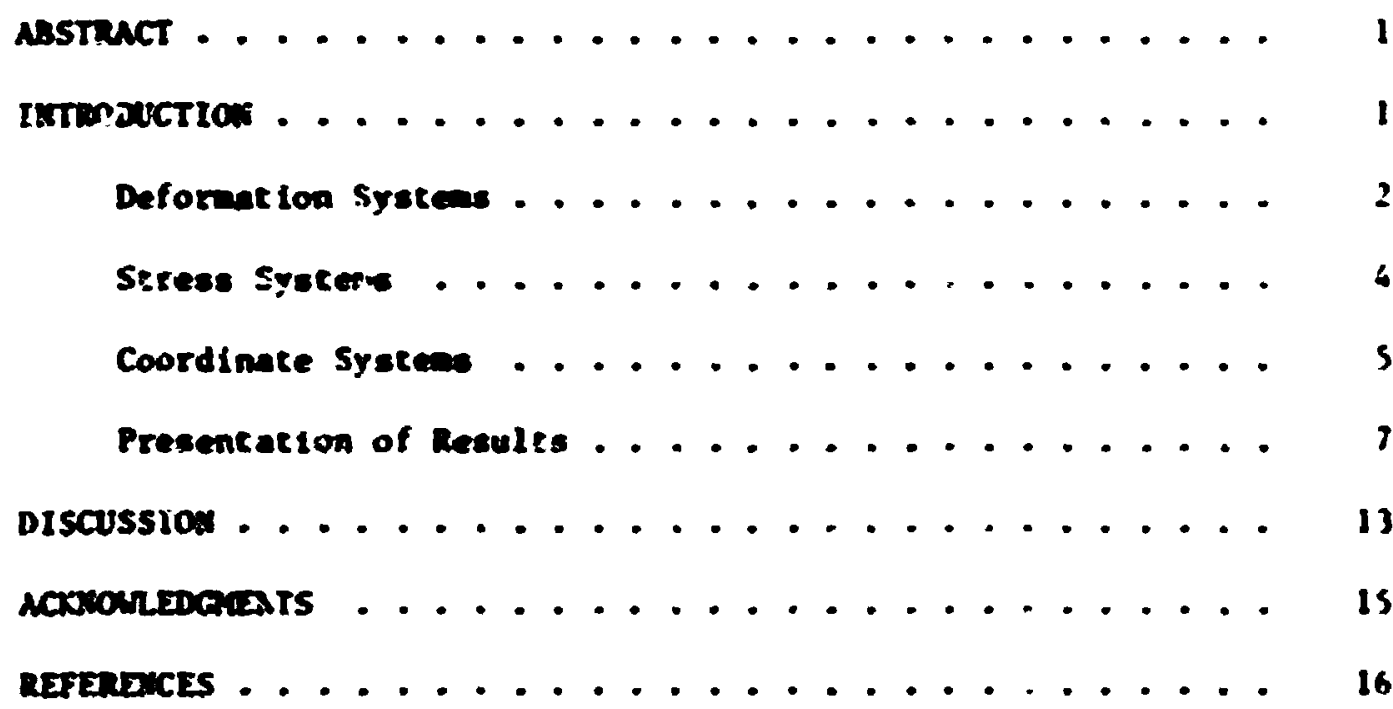




\section{STRESS FACTOES FOR THE DEFOMMTIOA STSTELS OF}

\section{ZIRCOSTAM LADER YULTIAXUL STRESS}

D. O. Hobson

\section{ASTRACT}

Calculation of the resolved shear stresses (rss) that act on various deformation systens in metals and, in particdlar. the deternination of those system subjected to the highest rss by a piven set of multiaxial stresses is of inportance in the study of texture development, rielding and plastic flou. This study exanines the geometrical influences of any stress state on the deformation nodes of ifrconiu. One slip mode and three tuimine modes, comprising twenty-one deformation systens, are considered. Seress factors conputed for these systems are shown on a coordinate system that allows specimen ortentation, Dost highly stressed deformation system, and stress factor to be show without anbigulty.

The information in this report allows the deteraination of the rss that results from any mulciaxial stress state: this information also allows the prediction of the deforation modes that ight operate for any specimen orientation in that stress state.

\section{IMTRODUCT ION}

In bcty-centered cublc metals, with their aultiple silp systens, the applifatio. of stress in any arbitrary direceion will result in accomodating strain, alsci in that direction. For the hexagonal setals (in this case zircon un, and, by extension 2ircaloy) live roon teaperature deformation wodes include tuinning as well as slip. A twinning systea, unlike slip, is operable in only one directior. This nonreversing cheracter of the twimniag ades causes complications when the stress orientations are described under which particular defornation systens operate. In addition, the single node avaslable for alip in zirconiun is confined to shear directions that lie parallel to the basal plane. This requires that twinning be initially the predoninant sechanis for nonbasal deformation, at least at room temperature. 
The author's previous study of the textures developed by $z^{2}$ rcoaiu single crystals cold rolled from vartous spectfied starting orientations showed that the crystais responded co the rolling operacion as if they had been subjected to orthogonal stresses, one censile and one coapresstre. I It was posstble to Ideatify the cuinoing nodes and to follow the coplex tuining sequences that produced the final textures. Biaxia: stress factor calculat lons were ade as an aid to analysis of this texture formation.

The concept of a critical resolved shear stress (crss) for tuinning is debatable, wth purists clafnim thrt there is no such thing and others observing a cendency for a fairly constant resolved stress to inftate turning in polycrystaline caterial. It is not the purpose of this study to enter the crss arena. Rather, the subject wil be approached from a geoners;-cryotaliography standpoint that will tdentify for zirconium the wost hignly stressed deformation systen that results from any arbitrary stress state.

Deformation Systens

Zircontu deforms at roo temprature with one slip wode and chree twinning nodes. These vere sumarized in a review paper by Reed-Hi112 and are shown in Table 1 . It should be eaphasized that the regults presented in chis report are valid for a particular material wich a particular c/a ratio.

Since this study exalnes the resolved shear stress on individual deformation systeas, it is necessery to consider each of those systens (Table 2) and to calculate the shear stresses on the as a function of the ariftrary stress directions.

Twins I through XII are activated by net tensile forces along the [0001] direction and twins XIII through XVIII by net compressits forces In that direction. As staced earilcr, the twinning shear is not reversible. The three slip systems, on the other hand, are fully reversible in the shear directions. 
Table 1. Deformation Elesents in Zirconiu

\begin{tabular}{|c|c|c|c|c|}
\hline \multicolumn{5}{|c|}{ Ivinere } \\
\hline $\begin{array}{c}\text { Mingies or First } \\
\text { Undistorted } \\
\text { Place } \\
\ell_{3}\end{array}$ & $\begin{array}{l}\text { Tuinis } \\
\text { Stheer } \\
\text { Direction } \\
n_{1}\end{array}$ & 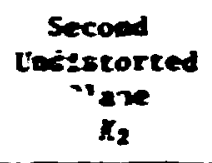 & $\eta_{2}$ & $\begin{array}{l}\text { Mroitende } \\
\text { of } \\
\text { Shear } \\
5\end{array}$ \\
\hline$\{10 \overline{1} 2\}$ & (10ī) & $\{10 \overline{2} 2\}$ & (10īi $)$ & 0.167 \\
\hline$\{11 \overline{2} 1\}$ & $(11 \overline{26})$ & $\{0001\}$ & $(11 \overline{20})$ & 0.63 \\
\hline$\{11 \overline{2} 2\}$ & $(11 \overline{23})$ & $\{11 \overline{26}\}$ & $(22 \overline{6} 3)$ & 0.225 \\
\hline \multicolumn{5}{|c|}{$\underline{\text { sifp }}$} \\
\hline \multicolumn{2}{|c|}{ Slip Pleme } & \multicolumn{3}{|c|}{ Slip Direction } \\
\hline \multicolumn{2}{|l|}{ \{10īo\} } & \multicolumn{3}{|c|}{.11201} \\
\hline
\end{tabular}

Table 2. Deformation Systems Cons:dered in This Study

\begin{tabular}{|c|c|c|c|}
\hline \multicolumn{2}{|c|}{ Deforectoe } & \multirow{2}{*}{$\begin{array}{l}\text { Active } \\
\text { Plase }\end{array}$} & \multirow{2}{*}{$\begin{array}{c}\text { Active } \\
\text { Direction }\end{array}$} \\
\hline Naber & mode & & \\
\hline $\begin{array}{l}\text { I } \\
\text { II } \\
\text { III } \\
\text { vV } \\
\text { V } \\
\text { VI }\end{array}$ & $\begin{array}{l}a \\
a \\
a \\
a\end{array}$ & $\begin{array}{l}(10 \overline{2}) \\
(01 \overline{1}) \\
(\overline{1} 102) \\
(1012) \\
(0 \overline{1} 12) \\
(1102)\end{array}$ & 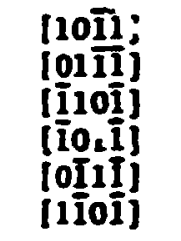 \\
\hline $\begin{array}{l}\text { VII } \\
\text { VIII } \\
\text { IX } \\
X \\
X I \\
X I I\end{array}$ & $\begin{array}{l}: \\
: \\
:\end{array}$ & $\begin{array}{l}(1121) \\
(I 2 I 1) \\
(2111) \\
\left(I_{21}\right) \\
(1211) \\
(2 \overline{I I})\end{array}$ & 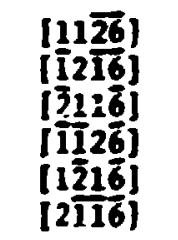 \\
\hline $\begin{array}{l}\text { XIII } \\
\text { XIV } \\
\text { XV } \\
\text { IVI } \\
\text { XVII } \\
\text { XVIII }\end{array}$ & : & $\begin{array}{l}(1132) \\
(1212) \\
(\overline{2112)} \\
(\overline{1122}) \\
(1 \overline{1} 12) \\
(2 \overline{11})\end{array}$ & 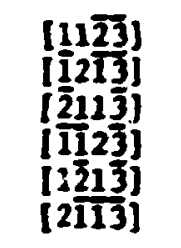 \\
\hline 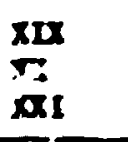 & $\begin{array}{l}b \\
b \\
b\end{array}$ & $\begin{array}{l}(10 \overline{1} 0) \\
(01 \overline{1} 0) \\
(1 \overline{100})\end{array}$ & 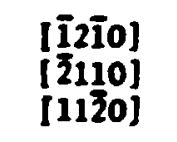 \\
\hline
\end{tabular}




\section{Stress Systens}

According to Barrett and Massalski: ${ }^{3}$

If a force $Z$ is applied to a single-crystai bar having a cross section $h$, as in Figs. If-16, and a slip pliane lies at angle to the cross-section plane (1.e., 90- to the direction of $:)$. the area of the slip plane vil! be $: / \cos$; and the stress on the plane will be $I /(A \cos A)$; the component of this stress in the slip direction $w_{11}$ be this stress miliplifed by $\cos \lambda$, where $\lambda$ is the angle between the direction of $\xi$ and the direction of slip. Thus, the resolved shear stress cending to produce slip ill be

$$
I=E \cos : \cos \lambda \text {. }
$$

The figure referted tc in the quotation is siailar to Fig. 1.

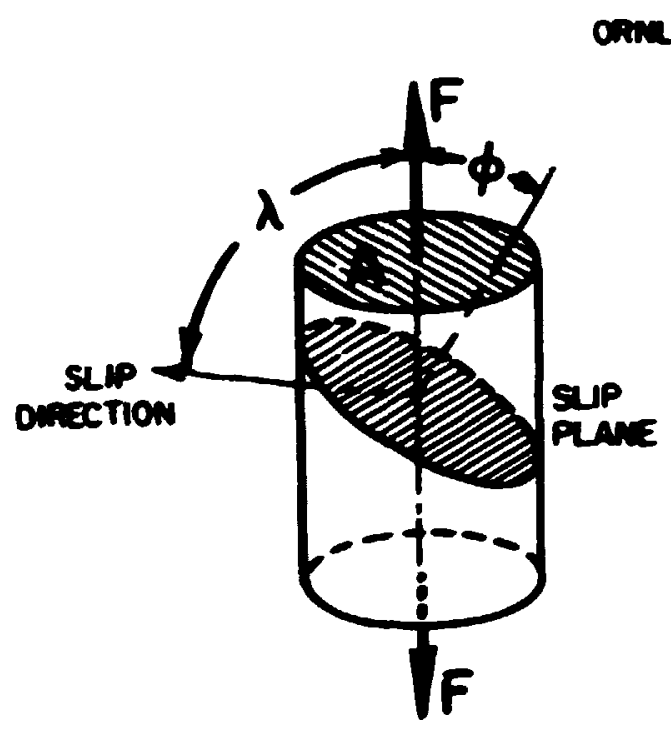

Fig. 1. Coordinates for Calculating Uniaxial Resolved Shear Stresses. 
For this study, the principal force $=$ is complexented by two additional principal stresses and the resulting comined siress factors are calculated by superposition, that is, $T=T_{1}+T_{2}+\tau_{3}$. For this study, the principal stress system is noralized as follows: Let $z i$. $z_{2}$, and $; i$ be the principal stresses, so chosen that $\tau_{i}<z ;<z_{2}$.

Then let

$$
\begin{aligned}
& z_{1}=-i-\frac{j i+j i}{2} \\
& z_{2}=j_{2}-\frac{j i+9 i}{2} \\
& z_{3}=z_{3}-\frac{5 i+j_{i}}{2}
\end{aligned}
$$

and

$$
\because=\frac{J_{1}}{\sigma_{2}} \text { such that }-1<Z<+1 \text {. }
$$

The transformation from $c_{i}^{\prime}$ to $\sigma_{i}$ results in a stress system in which the largest stresses are equal and opposite in sign. This manipulation simplifies the presentation of the results since now only the magnitude of $:$ need be specified along with the orientation angles.

\section{Coorcinate Systeas}

The data were computer-calrilated from a program written for the author by $R$. D. Willians." The data are plotted using a coordinate system (Fig. 2) developed by Hilliams for the presentation of biaxial pole figure datas and consists of a stereographic triangle bounded by

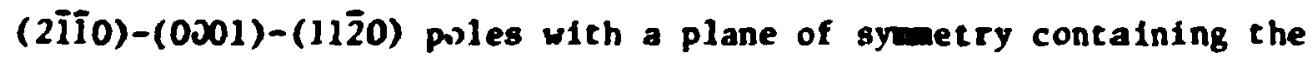
$[0001]$ and the $[10 \overline{1} 0]$ directions.

In this study, the compressive stress direction, $\sigma_{1}$, is located in the stereographic triangle by the angles $a$ and $\rho$. The tensile stress direction, $\sigma_{2}$ is in the plane orthogonal to $\sigma_{2}$ and at an angle 6 from 


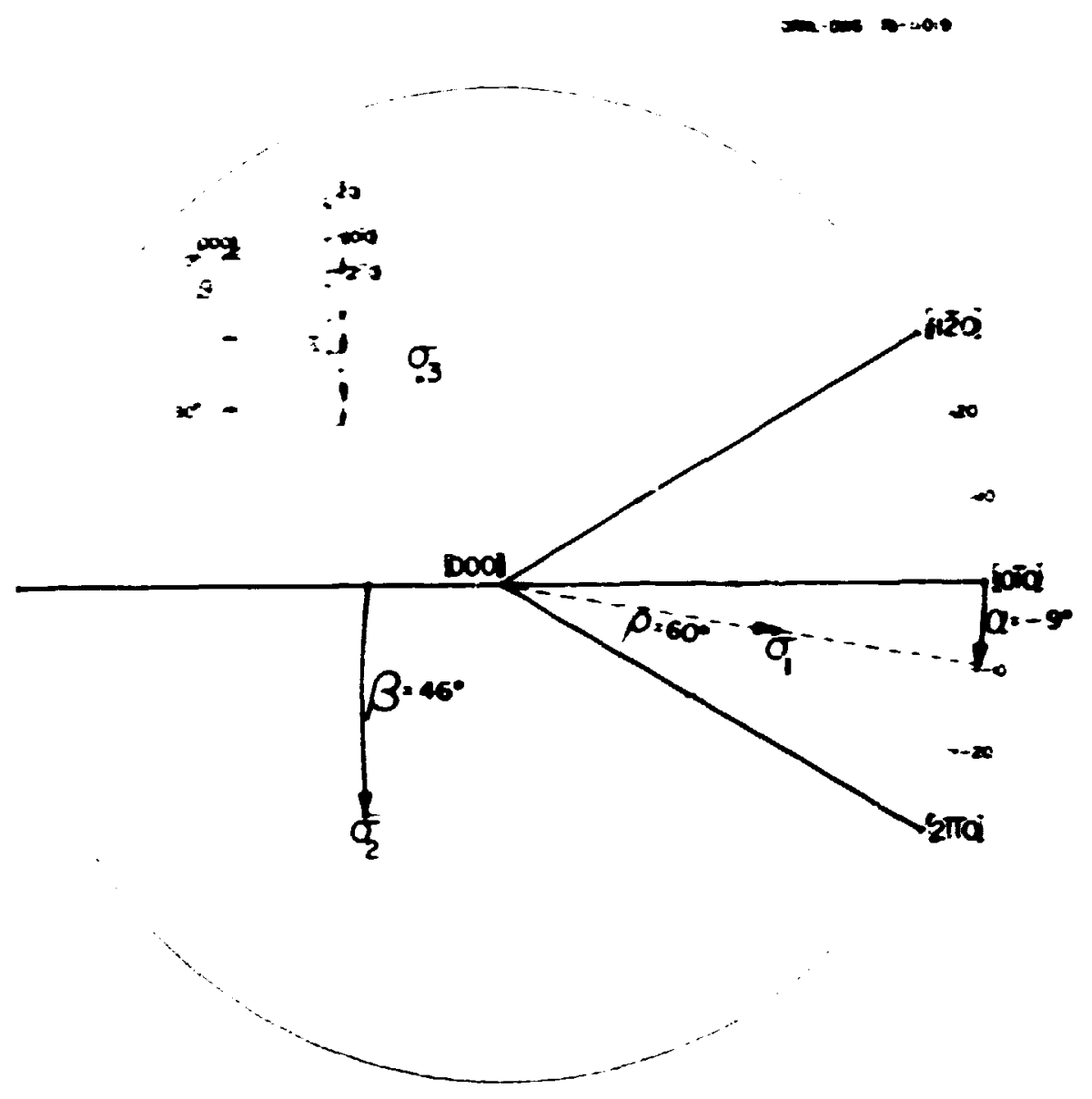

Fig. 2. Coordinate System Used for Plotting Stress Factor Data Showing Relationships Arong Angles $a, 0$, and $E$.

the trace of the symetry plane through [0001] and [10īo]. The three angular coordinates generate the solid shown in the upper left portion of the figure. This coordinate system is one in which the multaxiai stress system is seemingly rotated within a fixed crystallographic framework. It is perhaps easier to visualize the physical meaning of the coordinate system, the angle $B$ in particular, if one visualizes the rotation of the crystallographic system (a single crystal, for instance) zithin a fixed stress system (Fig. 3). It should be pointed out that these two projecticns are, in themselves, related by a set of simple rotations. 


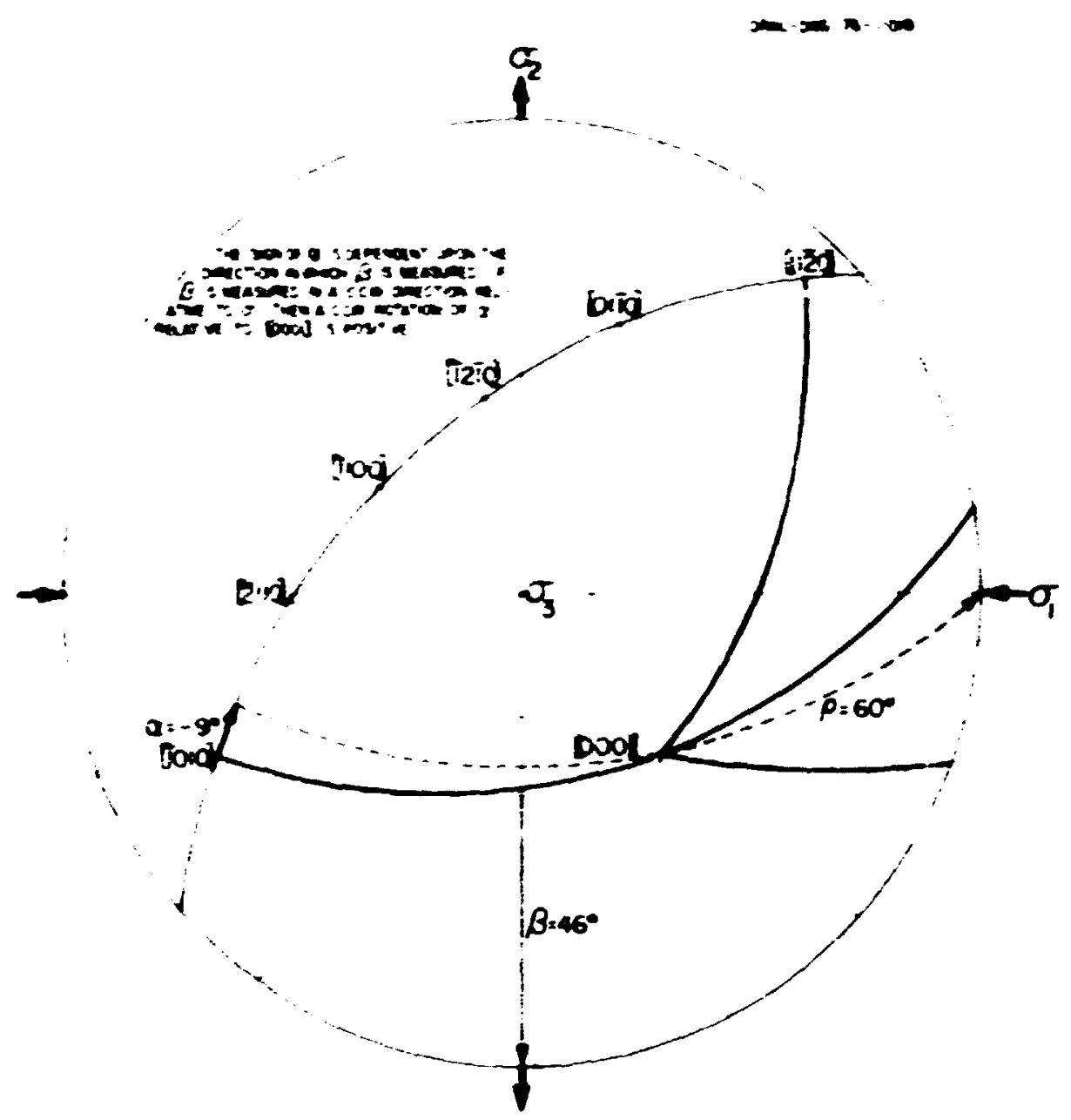

Fig. 3. Rotated Coordinate System for Plotting Stress Factor Data.

\section{Presentation of Results}

With the stress and coordinate systems described above, it is possible to unambiguously present the results of calculations for any arbftrary stress system that acts un any crystallographic orientation. The next problem is that of choosing which results to present from the infinite awount avallable. The computer calculations for this study produced a large printout of information that covered the following ranges and increments: 


$$
\begin{aligned}
& c \text { - from }+30 \text { to }-30^{\circ} \text { in } 5^{\circ} \text { increments } \\
& \beta \text { - from } 0 \text { to } 90^{\circ} \text { in } 5^{\circ} \text { increments } \\
& \rho \text { - from } 0 \text { to } 90^{\circ} \text { in } 5^{\circ} \text { increments }
\end{aligned}
$$

$\sigma_{3}$ frow to to $\sigma_{1}$ in $0.1\left|\sigma_{1}\right|$ increments

The printout consisted of 399 tabulated sets of deformation systemstress factor information, each of which contains the entire specified ranges of $c$ and $\rho$ variables. Each set represented a single combination of the $\sigma_{3}$ and $B$ variables. These tabular data ars in a format that is directly transferable to the stereographic triangle sisown in Fig. 2 . Figure 4 is a more detailed drawing of the triangle. Eact. grid intersection is analogous to a data pair in one of the tabulated sets. The resolved shear stresses were calculated for all systems but only the maximum values, along with their applicable systems, are listed in the tables.

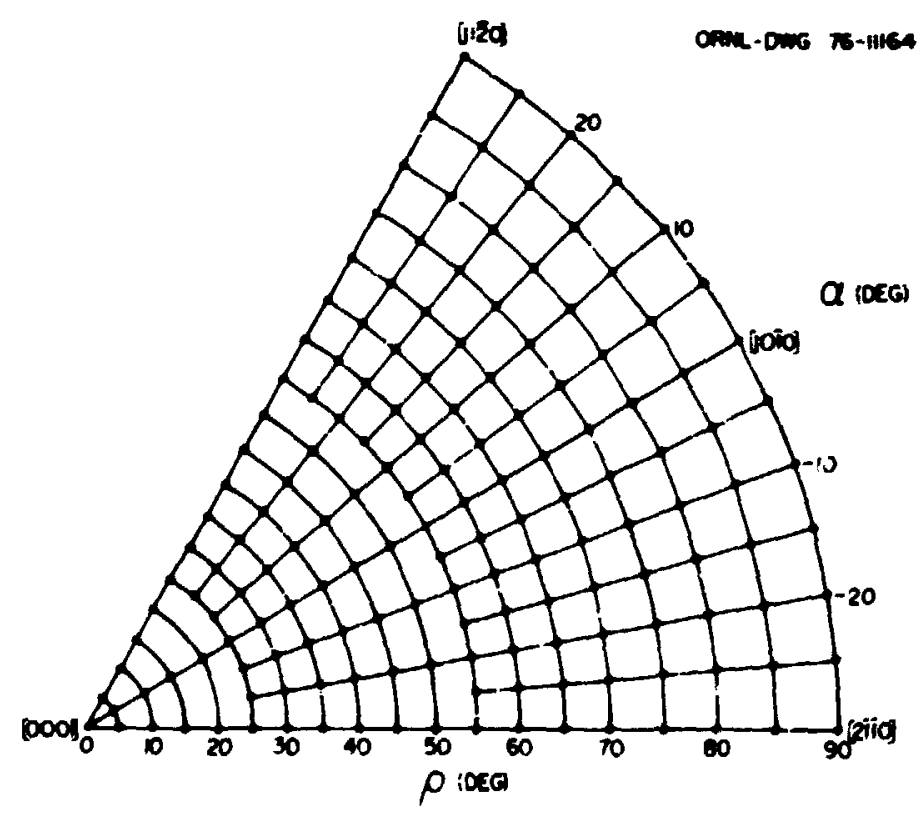

Fig. 4. Data Point Positions Within the Stereographic Triangle. 
When the tabulated data are transferred to the triangular grids, a mapping of the regions in wich each deformation system has the highest rss can be obtained. Within each region contours of constant rss can be drawn. Examples of such mapping are shown (Fig. 5) for $10^{\circ}$ increments of $B$ and for $\sigma=0$.

To compare the calculated results (Fig. 5) with reality, consider the following examples. Reference to Fig. 2 should aid the comparison.

1. $\beta=0^{\circ}, \rho=0^{\circ}, \alpha$ undefined. The compressive stress $\sigma_{1}$, is parallel vo [0001]. Systems XIII or XVIII are operative (Fig. 5). These are the $\{11 \overline{2} 2\}(11 \overline{2} \overline{3})$ compression twinning systems, and it has been found expertmentally ${ }^{2}$ that they do indeed operate under these conditions.

2. $\beta=0^{\circ}, \rho=90^{\circ}, \alpha=0^{\circ}$. The tensile stress, $\sigma_{2}$, is parallel

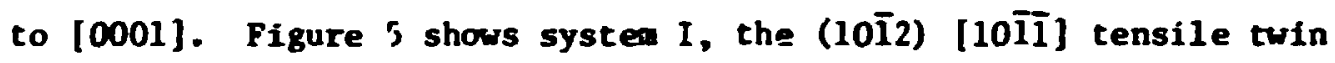
operating. This is also found experimentally.

3. $B=40^{\circ}, \rho=60^{\circ}, Q=-20^{\circ}$. Here, $\sigma_{1}$ lies within the boundaries of system IX, the $(\overline{2} 111)[\overline{2} 11 \overline{6}]$ tension twin. No direct experimental evidence is available to show that this is indeed the system that would operate at this stress state. Note that a slight increase in the value of $p$ (to between 65 and $70^{\circ}$ ), would put $\sigma_{1}$ in a slip region (system XXI). Likewise, an a closer to $-30^{\circ}$ would also call for slip (system XIX). A decrease in $\rho$ (to between 50 and $55^{\circ}$ ) would call for the compression-

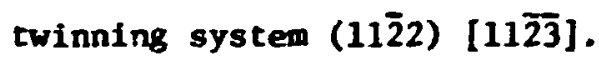

It should be emphasized that the deformation regions maped in the figure are simply interpenetrating convex surfaces and only the systems with the highest stress factors show on the surface of the stereographic triangle. There is no easy way to tell from the information presented in this report how closely the next subjacent system approaches the dominant system for a given combination of $\alpha, \rho$, and $\beta$. A uifferent output format for the calculations could show that information. Indeed, the successful use of the tabulated values to predict which system should operate deperis upon how nearly equal are the stresses required to operate the different systems. 


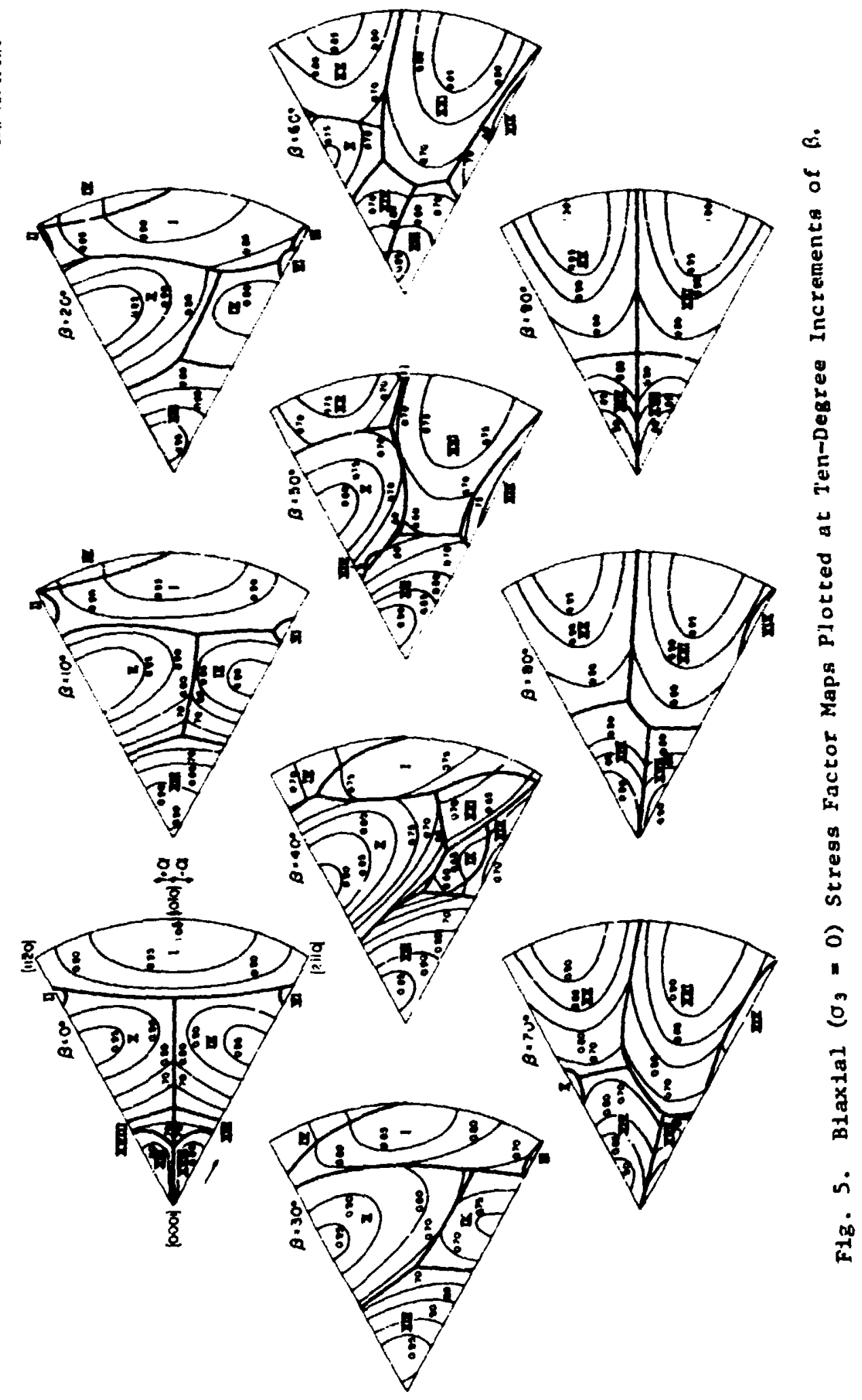


The three examples given above were for a balanced biaxial stress system, $\sigma_{1}=-\sigma_{2}$ and $\sigma_{3}=0$. The effect of adding the tinird stress can be illustrated with two additional exaples in which $\sigma_{3}$ is equal to $0.5\left|\sigma_{1}\right|$ and $-0.5\left|\sigma_{1}\right|$. Figure 6 is a map of the deformation systens for $B=40^{\circ}$ and $\sigma_{3}=0.5 \mid \sigma_{1} i$. It is seen that several changes are ade in the locations of the deformation systens relative to the $B=40^{\circ}$ triangle in Fig. 5. The tension twin, syster I, has shifted to a relatively salil area at high $p$ values near the [10īo] direction. It is flanked on each side by the slip systens $X X$ and XXI. Twinning systens of the fanily $\{11 \overline{2} 1\}(11 \overline{26})$ occupy the interediate $\rho$ positions, including those vacated by the slip systens. Low $p$ values continue to be occupied by syster XIII, the compression twin.

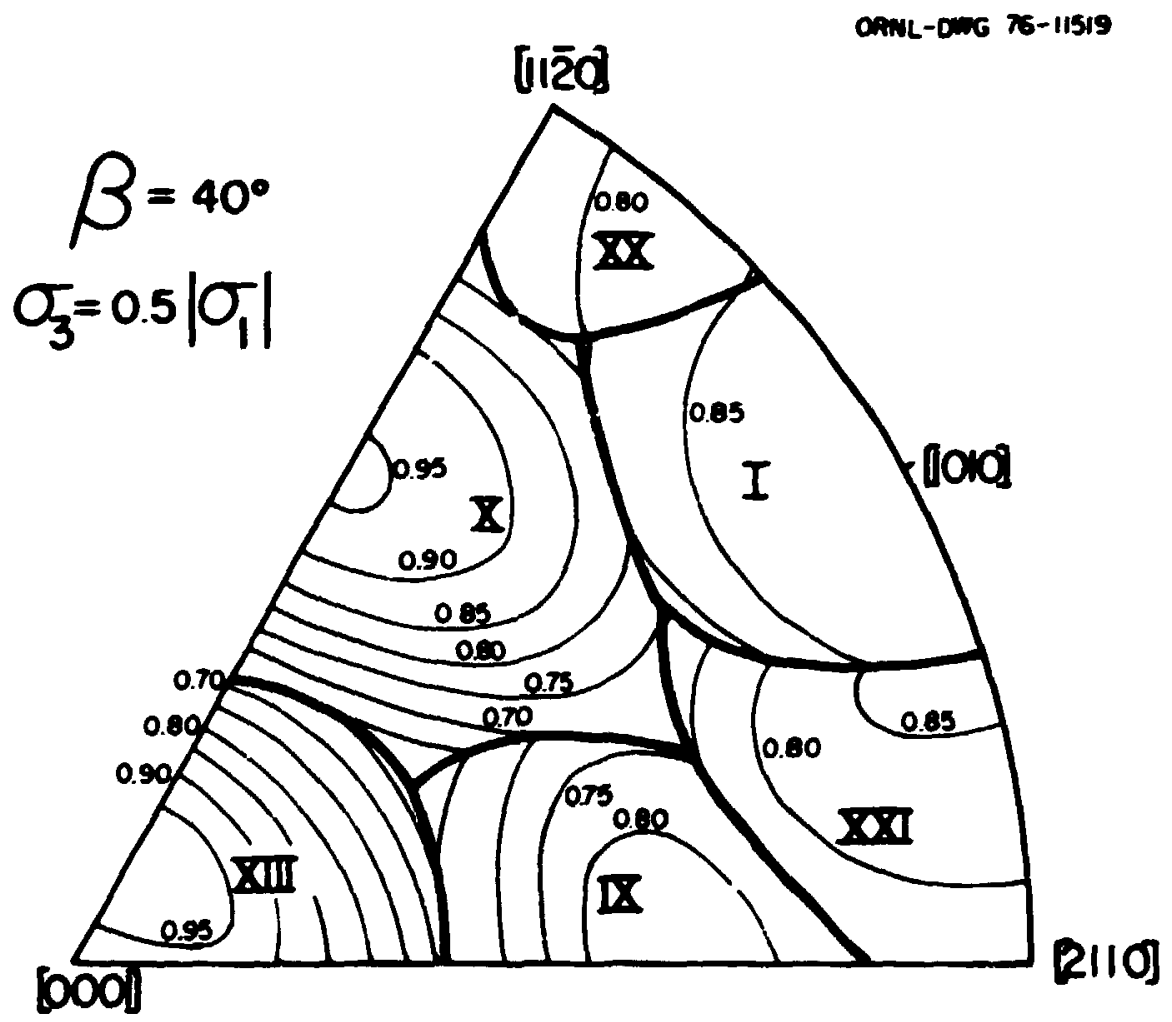

F18. 6. Stress Factor Data Map for $B=40^{\circ}$ and the Intermediate Stress $\sigma_{3}=0.5\left|\sigma_{1}\right|$. 
Figure 7 is a map of the deformation systens for $Q=40^{\circ}$ and $\sigma_{3}$ $=-0.5\left|\sigma_{1}\right|$. The intenediate compressive stress has caused large changes relative to the results given for the stress states with $\sigma_{3}=0$ and $\sigma_{3}=0.5\left|\sigma_{1}\right|$. The tension twinning region (system $I$ ) is still in the sane position, but is greatly reduced by the expanded syster. $X$,

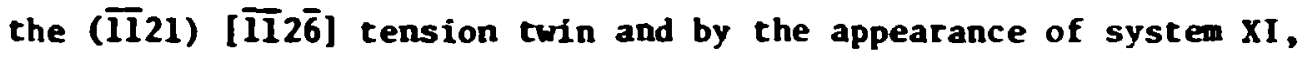
another $\{1 \overline{2} 1\}$ tension twin. The slip systems $X X$ and $X X I$, present in the stress state $\sigma_{3}=0.5\left|\sigma_{1}\right|$ are eliminated fic: $\sigma_{3}=-0.5\left|\sigma_{1}\right|$. Only a small slip region is left at internediate $\rho$ and negative $a$ values. The compressive twin syste (XIII) renains dominant at 100 values - that is, where the maximu compressive stress is near the basal pole orientation - for all three stress states.

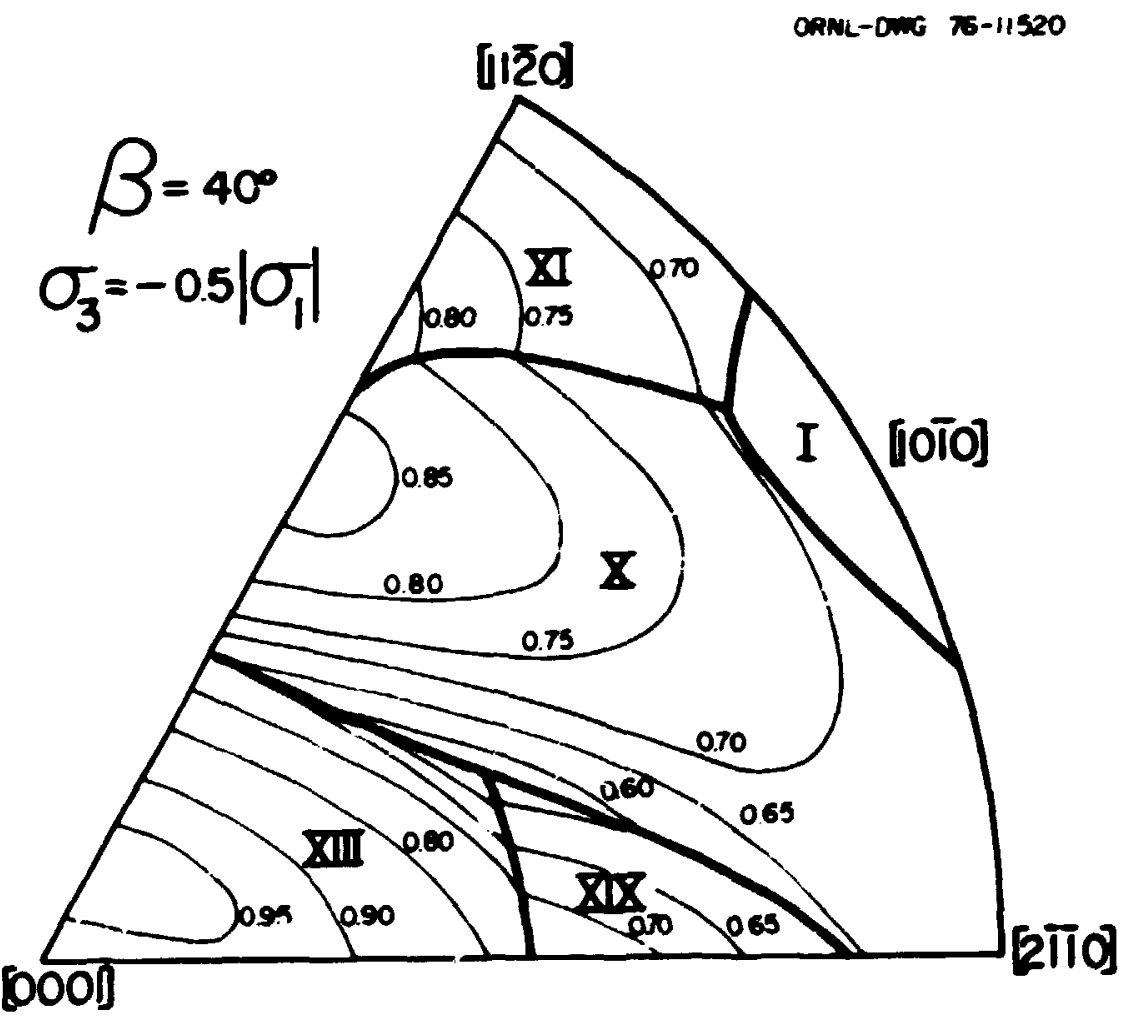

Fig. 7. Stress Factor Map Plotted for $B=40^{\circ}$ and the Intermediate Stress $\sigma_{3}=0.5\left|\sigma_{1}\right|$. 
Because there is such a large anount of information contained in the computi: printout and since it is iapossible to predict which stress states $\mathrm{n}^{-} \mathrm{t}$ be of incerest to the reader, the entire printout is included in the appendix of this report. The choice of increaents for $a, b, 0$, and $\sigma_{3}$ allow the user to approxinate the values of the orientation factors for any arbftrary stress state. Two conventions are used in the printout: (1) The factors are multiplied by $1000-937$ is actually 0.937 - and (2) Values equal to unity are denoted by asterisks.

\section{DISCUSSION}

It was stated in the incroduction that the subject of stress factors would be approached from a geonetry-crystallography standpoint. The results presented in this report follow that approach strictly. The principal stresses are resolved onto the shear planes and in shear directions of the slip and twinning systems of zirconium as a function of the orientation of the zirconium crystal lattice in stress space.

A very valid question that should be asked is: How do the results presented in this report comare with the real behavior of zirconiu? This question can be divided into three other questions: (1) How real is the posicioning of the system boundaries, especially between slip and tuiming systems? (2) How does a real single crystal react compared to predictions? and (3) How does polycrystalline zirconium react compared to predictions?

The answer to Question 1 is that, with the exception of boundaries between systems of the sane fantly, the systes boundartes are probshly not quant ltatively correct in their placement, especially those chat separate the cutming system restons from the silp reglons. One assuption that is Inherent in these calculations is that the syste with the highest stress factor is the one that operates. In realicy, IIp is probably operative at lower values of shear stress than is twinning, and, therefore, the ilp regionn should effectively be larger than show. The relative ease of operation of une tuinning node compared to another is also unknown, and differences is ease of operation could also change the relative olzes of the cuinning regions. 
A partial answer to Question 2 was given in Ref. 1, in wh:ch zirconiu single crystals were cold rolled from various orientations relative to the rolling direction and the operating deformation systens were identified. It can be stated that, to a first approxination, the systems predicted to operate are the ones that do operate. It is probable that crystal orfentations close to a boundary will not be accurately predicted due to specinen size effects, restraints, or the lack of quanticative correctness of the boundary placenent discussed in Question 1 above.

Question 3 is the one cost closely related to reality. The analogy is often made that a highly-textured zirconium specimen will deform under stress as if it were a single crystal subjected to that sane stress. I : is recognized that deforwation of any polycrystalline material is restrained by intergranular interactions and that an external stress state may not be uniformly transmitted throughout the specimen, especinlly at, and after, yielding. The single crystal analogy does seem to work with zirconium specimens that have a strong texture. The shape changes that occur during testing can be related to the texture present in the metal.

Finally, one further conjecture can be made which, if valid, could significantly add to an understanding of the yield behavior of zirconium and Zircaloy. This confecture will be presented following a brief background discussion. It is known ${ }^{6}$ that the room-temperature yield strength of $2 i r c a l o y$ can vary by a factor of 2 under uniaxial stress conditions, depending upon specimen orientation. Differences in crystallographic structure affect the yield strengths obtained by either uniaxial tension or uniaxial compression cesting of highly-textured, polycrystalline Zircaloy (Ref. 6 and Table 3 ). The orientation factors were calculated on the basis of single-crystal analogues related to the textures found in the rolled sheets (Schedule 8 had basal poles concentrated to a very high degree about the sheet normal; Schedule 62 was not as highly developed as schedule 8 and had the basal poles perpendicular to the rolling direction and almost rqually divided with respect to rotation about that direction; Schedule $J$ has basal poles concentrated parallel 
Table 3. Room-Tenperature Yield Strengths of Zircalov-2 in Tension and Compression and Orientation Factors for $\{10 \overline{10}\}(\overline{1} z \overline{1} 0)$ slip

\begin{tabular}{|c|c|c|c|c|}
\hline Schedule & $\begin{array}{l}\text { Specimen } \\
\text { Orientation }\end{array}$ & $\frac{\text { Yield }}{\text { Tension }}$ & $\frac{\text { Strength, ksi }}{\text { Compression }}$ & $\begin{array}{l}\text { Orientation Faztor } \\
\cos x \cos \lambda\end{array}$ \\
\hline-6 & $\begin{array}{l}\mathbf{R D} \\
\mathbf{T D} \\
\mathbf{N D}\end{array}$ & $\begin{array}{l}54.9 \\
58.3\end{array}$ & $\begin{array}{r}66.7 \\
62.7 \\
122.0\end{array}$ & $\begin{array}{l}0.485 \\
0.497 \\
0\end{array}$ \\
\hline-62 & $\begin{array}{l}\text { RD } \\
\text { TD } \\
\text { ND }\end{array}$ & $\begin{array}{l}55.8 \\
63.2\end{array}$ & & $\begin{array}{l}0.433 \\
0.344 \\
0.139\end{array}$ \\
\hline$-J$ & $\begin{array}{l}\text { RD } \\
\text { TD } \\
\text { RD }\end{array}$ & $\begin{array}{l}52.0 \\
70.0\end{array}$ & $\begin{array}{r}65.6 \\
101.3 \\
72.5\end{array}$ & $\begin{array}{l}0.433 \\
0.002 \\
0.433\end{array}$ \\
\hline
\end{tabular}

2RD, rolling direction; TD, transverse direction; and $\mathrm{ND}$, normal direction.

to the transverse direction). It is seen in Table 3 that high orientation factors (0.5 maximu) for prisi slip correspond to the lover yleld strengths. Low factors indicate that twinning had to be the donfnant deformation mechanisa and higher yield strengths are observed.

Now, it is conjectured that, since the yield behavior of zirconium (and Zircaloy) is a function of the crystallographic texture, the yield locus for a material with a given texture should show features related to the orientation factors described and apped in this report. Whether these features might occur as facets in the yield surface, as shape changes, or as some other feature is not known at this tine.

\section{ACKNOWLEDGTEMTS}

The author acknowledges the very large contribution of Robin 0. Willians to this report. He is especially indebted to Williams for the coordinate systen and the computer program. Gratitude is also expressed to R. A. Vandeweer and $M$. H. Yoo for reviewing the manuscript, to George Griffith for editing, and to Denise Jackson for the final report preparation. 
1. D. O. Hobson, "Textures in Deformed Zirconiu Single Crystals," Trans. Met. Soc. ADE, 242(6): 1105-1110 (1968).

2. R. E. Recd-Hill "Role of Deformation Twinging in the Plastic Deformation of a Polycrystailine Anisotropic Metal," pp. 295-320 in Deformation Twinning, Vol. 25, Gordon and Breach, New York, 1964.

3. C. S. Barrett and T. B. Massalski, Structure of Metals, 3rd ed., McGran-H111, New York, 1966, Pp. 404-405.

4. R. O. Willians, Metallurgist, Metals and Ceramics Division, Oak Ridge National Laboratory, private commication.

5. R. O. Willians, "Represent-i.tion of the Textures of Rollad Copper, Brass, and Aluminu by Biaxial Pole figures," Trans. Met. Soc. ARE, $242(1)$ : $105-115$ (1968).

6. P. L. Rittenhouse and M. L. Picklesimer, "Research on the Mechanical Anisotropy of Zircaloy-2," Electrochem. Technol. 4(7-8): 322-329 (1966). 
TABLE OF

STRESS FACTORS FOR THE DEFORYATION SYSTEMS OF ZIRCONIUM UNDER WULTIAXIAL STRESS 


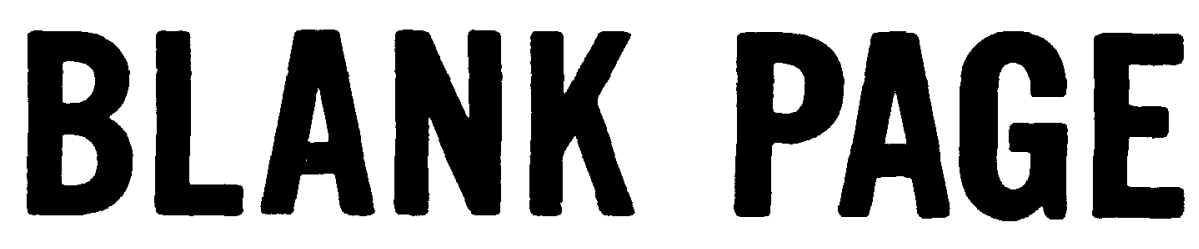




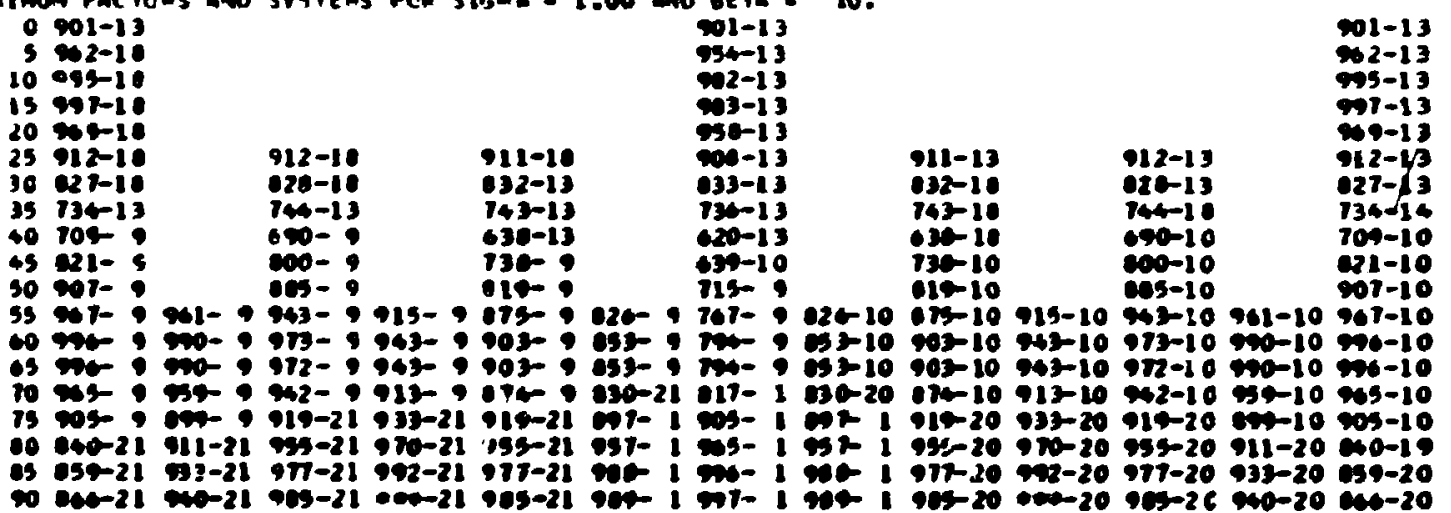




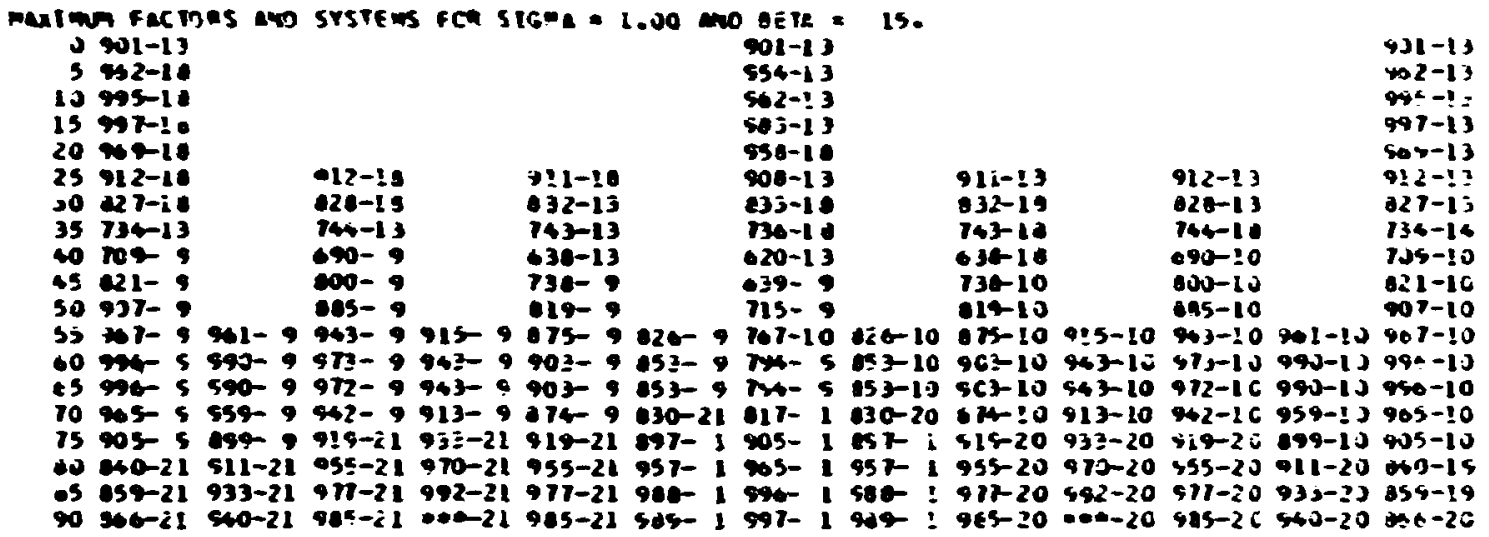

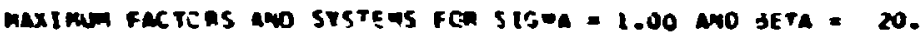

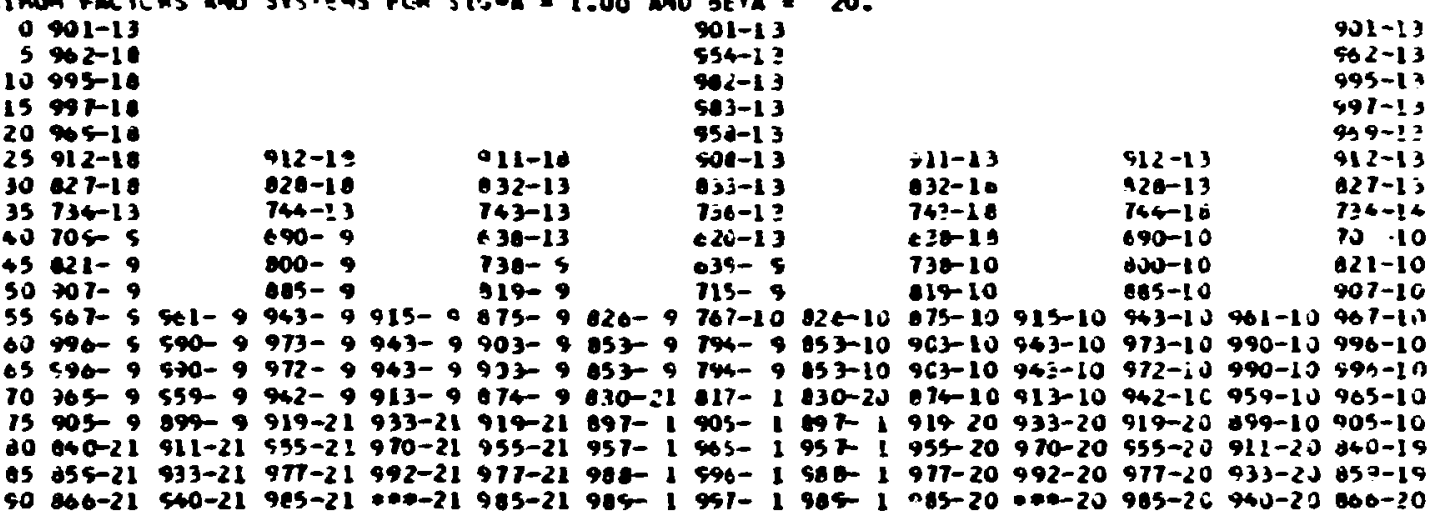

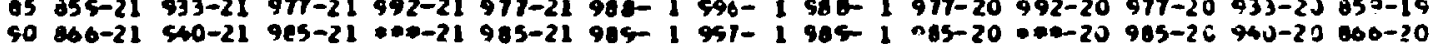

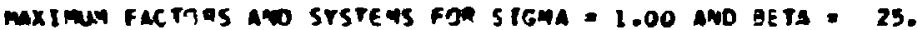

$001-13$

$5962-10$

$13595-10$

15 597-18

$2096-10$

$25912-18$

30 127-18

$35 \quad 734-13$

$40700-9$

$45021-9$

$50907-9$ 60 594-990-9 973-9 943-9 903-9 853-9 794-10 953-10 903-10 943-10 973-10 990-10 996-10 65 59- $9990-9972-9$ 943-9 903-9 853-9 794-9 85 3-10 903-10 943-10 972-10 990-10 996-10

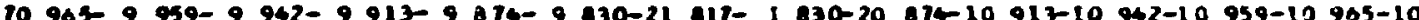
73 905- 9 99- $9919-21933-21919-21$ 997- $1905-19971919-20933-20$ 919-20 $699-10905-10$ 60 940-21 $511-21955-21970-21955-21$ 957- 1 95- $1957-1955-20970-20955-20911-20940-19$ 85 859-21 933-21 977-21 942-21 977-21 9r - 1 96- 1 ser $1977-20992-20977-20933-20$ 959-20

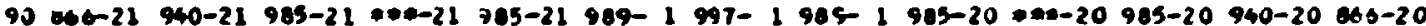

$901-13$ $902-13$ $995-13$ $309-13$ $912-15$ $327-13$ $\begin{array}{lll}743-18 & 744-18 & 734-14 \\ 630-18 & 690-10 & 709-10\end{array}$ $\begin{array}{lll}743-18 & 744-18 & 734-14 \\ 630-18 & 690-10 & 709-10\end{array}$ $730-10 \quad 821-10$ $19-10 \quad 085-10 \quad 907-10$ 630-13

620-13

$982-13$

903-13

9se -13 


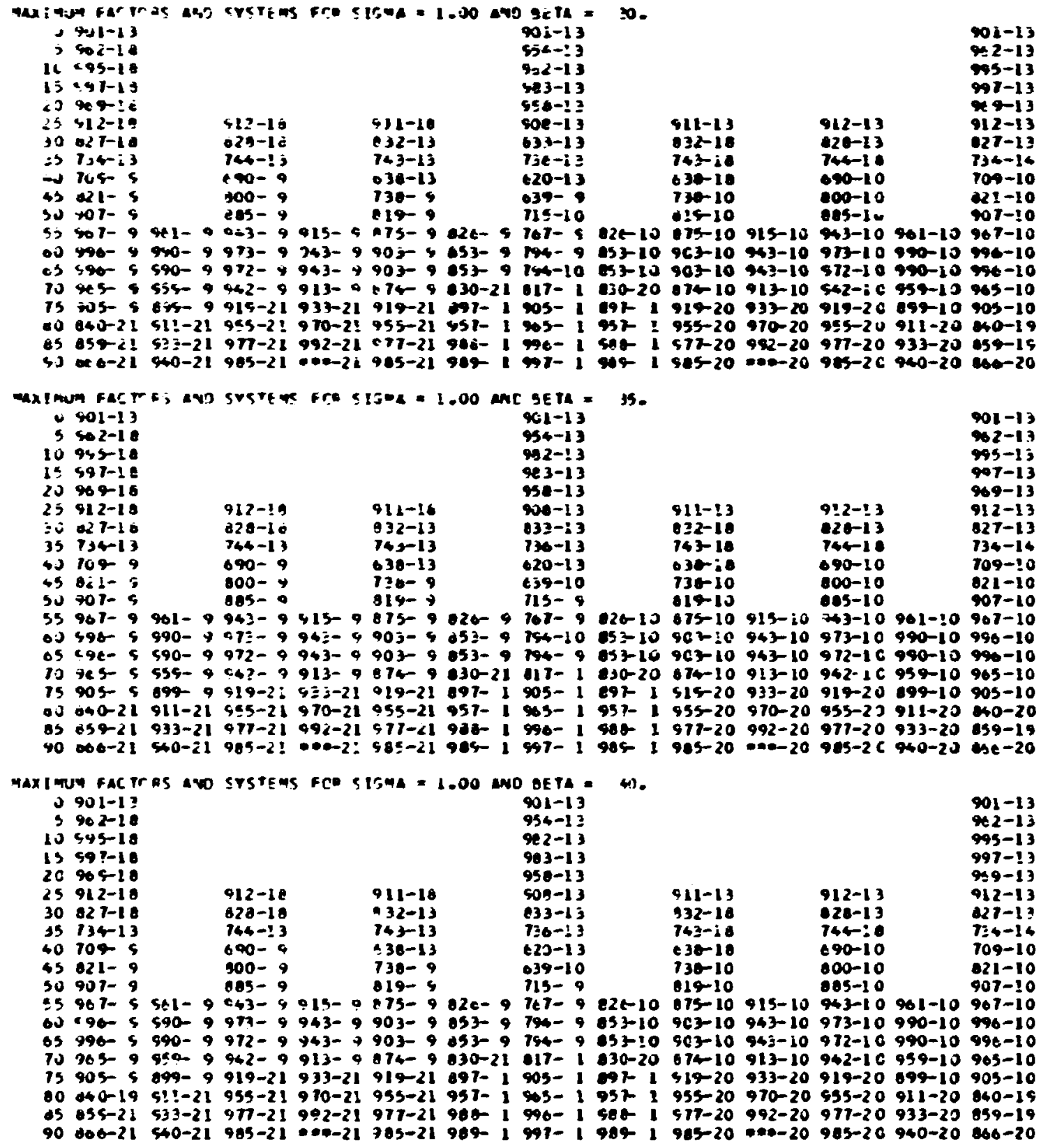




\begin{tabular}{|c|c|c|c|c|c|c|c|c|c|c|c|c|c|}
\hline $\begin{array}{l}x 10 \\
0 \\
5 \\
10 \\
15 \\
20 \\
25 \\
30 \\
35 \\
40 \\
45 \\
50 \\
55 \\
60 \\
65 \\
70 \\
75 \\
80 \\
85 \\
90\end{array}$ & $\begin{array}{l}\text { FAC } \\
901-13 \\
962-18 \\
995-18 \\
557-18 \\
969-18 \\
912-18 \\
627-18 \\
734-13 \\
709-9 \\
61-9 \\
907-9 \\
967-9 \\
996-9 \\
596-9 \\
965-9 \\
905-9 \\
940-19 \\
959-21 \\
864-21\end{array}$ & $\begin{array}{r}91-9 \\
900-9 \\
990-9 \\
99-9 \\
99-9 \\
911-21 \\
933-21 \\
940-21\end{array}$ & $\begin{array}{l}912-18 \\
928-18 \\
746-13 \\
690-9 \\
800-9 \\
905-9 \\
943-9 \\
975-9 \\
972-9 \\
942-9 \\
919-21 \\
955-21 \\
977-21 \\
905-21\end{array}$ & $\begin{array}{l}963-9 \\
943-9 \\
913-9 \\
933-21 \\
970-21 \\
992-21 \\
90-21\end{array}$ & $\begin{array}{l}911-13 \\
932-13 \\
743-13 \\
630-13 \\
730-9 \\
119-9 \\
975-9 \\
909-9 \\
905-9 \\
903-9 \\
874-9 \\
1919-21 \\
9955-21 \\
1977-21 \\
1905-21\end{array}$ & $\begin{array}{l}926- \\
053- \\
853- \\
830-2 \\
697- \\
957- \\
902- \\
969-\end{array}$ & 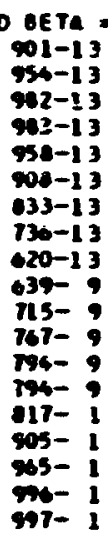 & $\begin{array}{l}026-10 \\
653-10 \\
653-10 \\
630-20 \\
697-1 \\
572 \\
968-1 \\
908-1\end{array}$ & $\begin{array}{l}911-13 \\
832-18 \\
743-10 \\
630-10 \\
739-10 \\
919-10 \\
875-10 \\
903-10 \\
903-10 \\
624-20 \\
919-20 \\
955-20 \\
977-20 \\
945-20\end{array}$ & & $\begin{array}{l}912-13 \\
820-13 \\
764-18 \\
690-10 \\
909-10 \\
625-10 \\
943-10 \\
9 ? 5-10 \\
972-10 \\
942-10 \\
917-2 C \\
955-20 \\
977-20 \\
965-2 C\end{array}$ & $\begin{array}{l}96 \\
99 \\
9 \\
69 \\
91 \\
93 \\
94\end{array}$ & $\begin{array}{l}01-13 \\
22-13 \\
45-13 \\
17-13 \\
69-13 \\
12-13 \\
127-13 \\
36-16 \\
09-10 \\
121-10 \\
07-10 \\
167-10 \\
96-10 \\
96-10 \\
105-10 \\
105-10 \\
40-19 \\
69-20 \\
100-20\end{array}$ \\
\hline $\begin{array}{l}0 \\
5 \\
10 \\
15 \\
20 \\
25 \\
30 \\
35 \\
40 \\
45 \\
50 \\
55 \\
60 \\
65 \\
20 \\
75 \\
10 \\
25 \\
90\end{array}$ & $\begin{array}{l}\text { FAC? } \\
901-13 \\
962-10 \\
995-19 \\
997-11 \\
99-19 \\
912-19 \\
027-19 \\
736-13 \\
704-5 \\
021-9 \\
907-9 \\
967-9 \\
996-9 \\
996-9 \\
965-9 \\
905-9 \\
840-19 \\
959-19 \\
606-21\end{array}$ & $\begin{array}{l}7 \text { MS } \triangle 400 \\
\\
961-9 \\
990-9 \\
990-9 \\
959-9 \\
999-9 \\
911-21 \\
933-21 \\
940-21\end{array}$ & $\begin{array}{l}912-10 \\
920-10 \\
744-13 \\
690-9 \\
900-9 \\
805-9 \\
943-9 \\
973-9 \\
972-9 \\
942-9 \\
919-21 \\
935-21 \\
977-21 \\
985-21\end{array}$ & $\begin{array}{l}915-9 \\
943-9 \\
943-9 \\
913-9 \\
933-21 \\
970-21 \\
992-21 \\
910-21\end{array}$ & 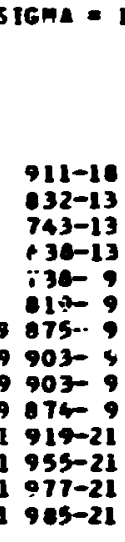 & $\begin{array}{l}926-9 \\
053-9 \\
053- \\
030-2 \\
997- \\
957- \\
908- \\
969-\end{array}$ & 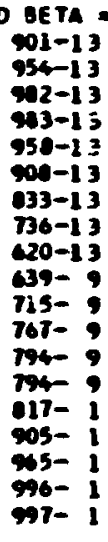 & $\begin{array}{l}\mid \\
026-10 \\
053-10 \\
053-10 \\
050-20 \\
992-1 \\
952-1 \\
96-1 \\
904-1\end{array}$ & $\begin{array}{l}911-13 \\
632-10 \\
743-10 \\
630-10 \\
730-10 \\
19-10 \\
675-10 \\
903-10 \\
903-10 \\
874-10 \\
919-20 \\
955-20 \\
977-20 \\
905-20\end{array}$ & $\begin{array}{r} \\
\\
915-10 \\
94-10 \\
943-10 \\
913-10 \\
933-20 \\
970-20 \\
992-20 \\
900-20\end{array}$ & $\begin{array}{l}912-13 \\
220-13 \\
744-10 \\
490-10 \\
900-10 \\
915-10 \\
943-10 \\
973-10 \\
972-10 \\
942-10 \\
919-20 \\
935-20 \\
977-20 \\
985-20\end{array}$ & $\begin{array}{l}961 \\
990 \\
990 \\
939 \\
999 \\
911 \\
933\end{array}$ & $\begin{array}{l}201-13 \\
962-13 \\
995-13 \\
997-13 \\
99-13 \\
912-13 \\
927-13 \\
734-14 \\
709-10 \\
621-10 \\
907-10 \\
967-10 \\
996-10 \\
996-10 \\
965-10 \\
905-10 \\
960-19 \\
959-19 \\
060-20\end{array}$ \\
\hline $\begin{array}{l}\times 110 \\
0 \\
5 \\
10 \\
15 \\
20 \\
25 \\
30 \\
35 \\
40 \\
45 \\
50 \\
55 \\
60 \\
65 \\
10 \\
75 \\
10 \\
15 \\
90\end{array}$ & $\begin{array}{l}4171 \\
901- \\
962 \\
995 \\
997 \\
969 \\
912 \\
627 \\
734 \\
909 \\
821 \\
937- \\
967 \\
996- \\
996 \\
965 \\
905 \\
940 \\
859\end{array}$ & $\begin{array}{l}990- \\
590- \\
959- \\
999- \\
911-2 \\
933-2 \\
940-2\end{array}$ & $\begin{array}{l}912-18 \\
820-10 \\
744-13 \\
690-9 \\
800-9 \\
905-9 \\
943-9 \\
973-9 \\
972-9 \\
942-9 \\
919-21 \\
955-21 \\
977-21 \\
995-21\end{array}$ & $?$ & 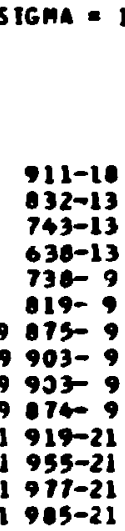 & 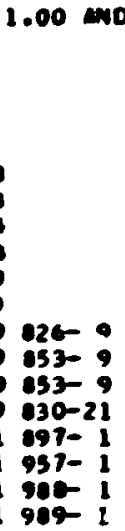 & 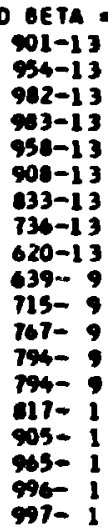 & 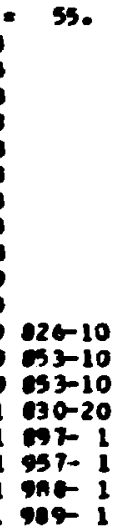 & $\begin{array}{l}911-13 \\
632-18 \\
763-10 \\
630-11 \\
736-10 \\
919-10 \\
675-10 \\
903-10 \\
903-10 \\
974-10 \\
919-20 \\
955-20 \\
978-20 \\
905-20\end{array}$ & $\begin{array}{l} \\
\\
915-10 \\
943-10 \\
943-10 \\
913-10 \\
933-20 \\
970-20 \\
992-20 \\
910-20\end{array}$ & $\begin{array}{l}912-13 \\
920-13 \\
764-10 \\
690-10 \\
900-10 \\
105-10 \\
943-10 \\
973-10 \\
972-10 \\
942-10 \\
919-20 \\
935-20 \\
977-20 \\
965-26\end{array}$ & 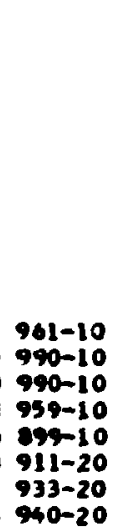 & $\begin{array}{l}901-13 \\
962-13 \\
995-13 \\
997-13 \\
969-13 \\
912-13 \\
927-13 \\
736-14 \\
709-10 \\
921-10 \\
907-10 \\
967-10 \\
996-10 \\
996-10 \\
965-10 \\
905-10 \\
960-20 \\
959-20 \\
666-20\end{array}$ \\
\hline
\end{tabular}




\begin{tabular}{|c|c|c|c|c|c|c|c|c|c|c|c|c|c|}
\hline $\begin{array}{l}0 \\
5 \\
13 \\
15 \\
20 \\
25 \\
30 \\
35 \\
40 \\
45 \\
50 \\
45 \\
00 \\
65 \\
70 \\
75 \\
20 \\
95 \\
90\end{array}$ & $\begin{array}{l}931-13 \\
502-18 \\
595-18 \\
697-18 \\
969-18 \\
912-18 \\
927-18 \\
73-13 \\
709-9 \\
921-9 \\
307-9 \\
967-9 \\
990-5 \\
490-9 \\
945-9 \\
905-9 \\
440-19 \\
354-19 \\
66-21\end{array}$ & $\begin{array}{l}561-9 \\
590-9 \\
590-9 \\
959-9 \\
690-9 \\
911-21 \\
533-21 \\
540-21\end{array}$ & $\begin{array}{l}912-18 \\
828-10 \\
744-13 \\
690-9 \\
800-9 \\
985-9 \\
943-9 \\
973-9 \\
972-9 \\
942-9 \\
919-21 \\
955-21 \\
977-21\end{array}$ & $\begin{array}{l}9 \\
915-9 \\
943-9 \\
943-9 \\
913-9 \\
935-21 \\
970-21 \\
992-21\end{array}$ & $\begin{array}{l}911-10 \\
932-13 \\
743-13 \\
938-13 \\
738-9 \\
81 \cdot 2-9 \\
115-9 \\
903-9 \\
903-9 \\
974-9 \\
919-21 \\
955-21 \\
977-21 \\
905-21\end{array}$ & $\begin{array}{l} \\
620-9 \\
653-9 \\
655-9 \\
630-21 \\
697-1 \\
957-1 \\
900-1 \\
909-1\end{array}$ & $\begin{array}{l}901-13 \\
954-13 \\
902-13 \\
903-13 \\
956-13 \\
908-13 \\
933-13 \\
755-12 \\
620-13 \\
639-9 \\
715-9 \\
767-9 \\
796-9 \\
756-9 \\
917-1 \\
905-1 \\
965-1 \\
996-1\end{array}$ & $\begin{array}{l}24-10 \\
653-10 \\
65 z-10 \\
830-20 \\
697-1 \\
57-1 \\
96-1 \\
964-1\end{array}$ & $\begin{array}{l}511-13 \\
632-18 \\
745-10 \\
630-18 \\
734-10 \\
619-10 \\
875-10 \\
5 c 3-10 \\
5 c 3-10 \\
674-10 \\
919-20 \\
955-20 \\
977-20 \\
985-20\end{array}$ & $\begin{array}{l}915-10 \\
943-10 \\
543-20 \\
913-10 \\
933-20 \\
970-20 \\
992-20 \\
920-20\end{array}$ & $\begin{array}{r}912-13 \\
820-13 \\
744-110 \\
690-10 \\
200-10 \\
945-10 \\
943-10 \\
973-10 \\
972-10 \\
942-110 \\
919-20 \\
955-20 \\
977-20\end{array}$ & $\begin{array}{l}961-10 \\
990-10 \\
990-10 \\
959-10 \\
99-10 \\
911-20 \\
933-20 \\
940-20\end{array}$ & $\begin{array}{l}90 i-13 \\
902-13 \\
995-13 \\
997-13 \\
99-13 \\
912-13 \\
227-13 \\
75-14 \\
709-10 \\
921-10 \\
907-10 \\
967-10 \\
990-10 \\
996-10 \\
965-10 \\
905-10 \\
940-20 \\
959-20 \\
960-20\end{array}$ \\
\hline
\end{tabular}

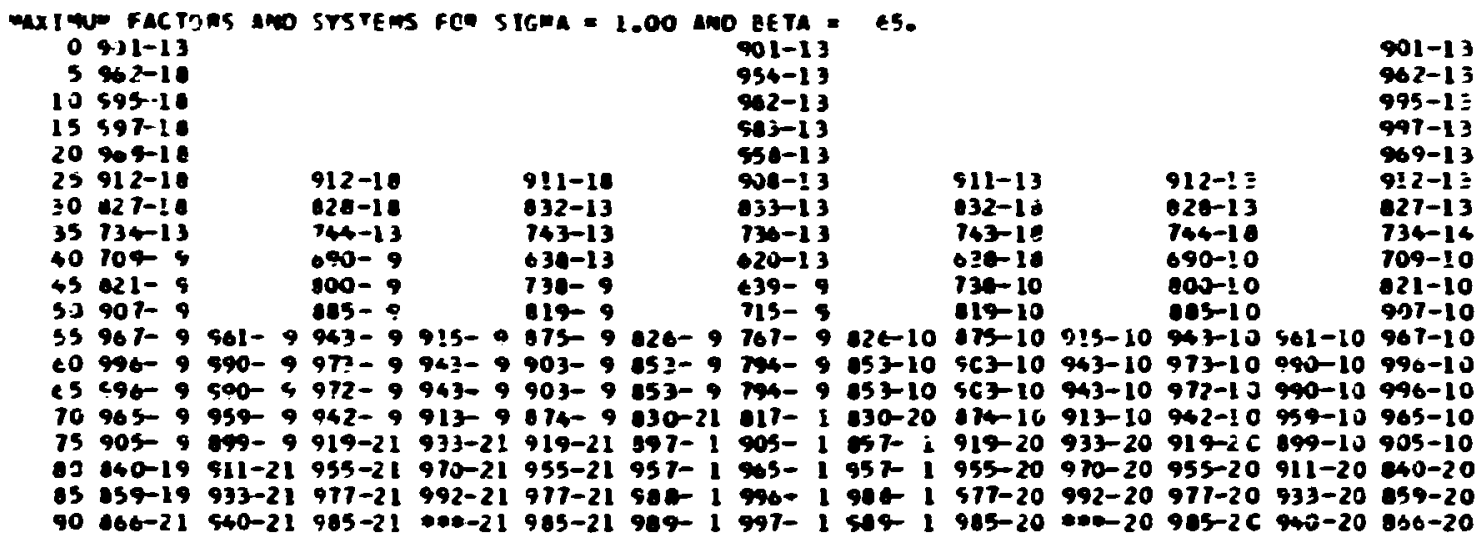

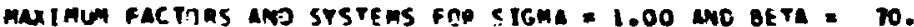

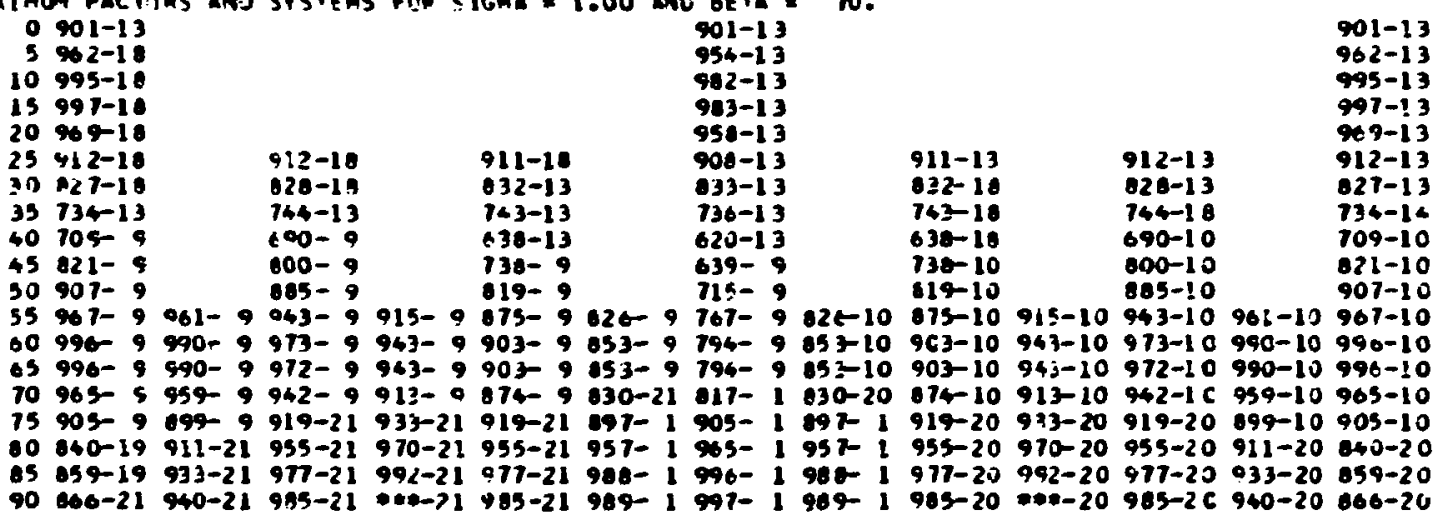




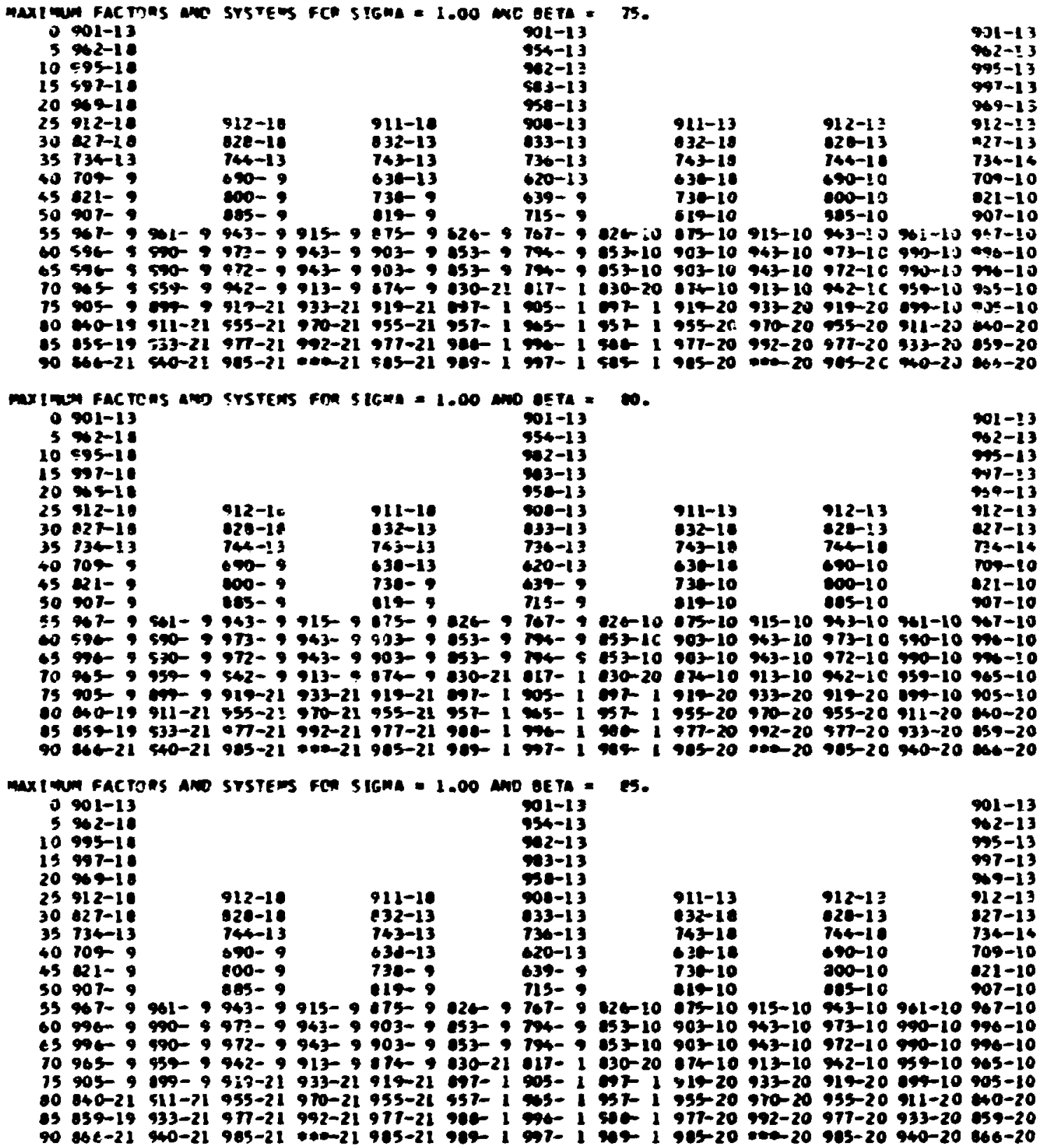




\begin{tabular}{|c|c|c|c|c|c|c|c|c|c|c|c|c|c|}
\hline $\begin{array}{l}10 \\
0 \\
5 \\
1.9 \\
15 \\
20 \\
25 \\
10 \\
35 \\
40 \\
45 \\
50 \\
35 \\
00 \\
85 \\
10 \\
15 \\
10 \\
10 \\
15\end{array}$ & 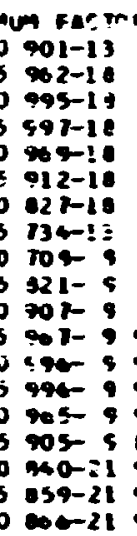 & $\begin{array}{l} \\
\\
951-9 \\
940-9 \\
400-9 \\
595-9 \\
499-9 \\
91:-2 ! \\
933-21 \\
940-21\end{array}$ & 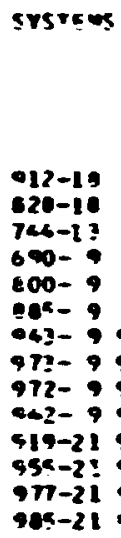 & $\begin{array}{l}915-9 \\
915-9 \\
943-9 \\
945-9 \\
913-9 \\
933-21 \\
970-21 \\
9<2-21 \\
902-21\end{array}$ & $\begin{array}{l}911-18 \\
632-113 \\
743-13 \\
635-11 \\
735-9 \\
919-9 \\
375-9 \\
903-9 \\
903-9 \\
975-9 \\
915-21 \\
955-21 \\
977-21 \\
905-21\end{array}$ & 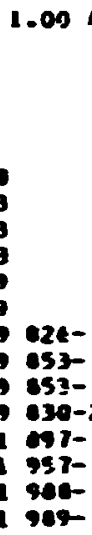 & 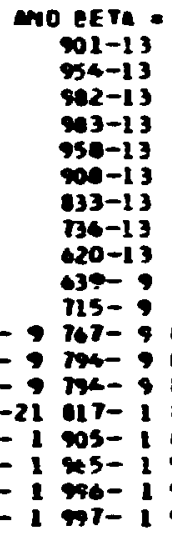 & 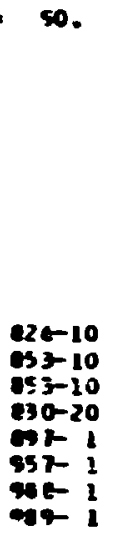 & $\begin{array}{l}911-13 \\
632-10 \\
762-10 \\
630-10 \\
730-10 \\
915-10 \\
175-10 \\
963-10 \\
963-10 \\
614-10 \\
919-20 \\
955-20 \\
577-20 \\
935-20\end{array}$ & $\begin{array}{l}0 \\
915-10 \\
943-10 \\
943-10 \\
913-10 \\
933-20 \\
970-20 \\
992-20 \\
9=2-20\end{array}$ & $\begin{array}{l}912-13 \\
620-13 \\
744-10 \\
690-10 \\
600-10 \\
895-10 \\
943-10 \\
973-10 \\
972-10 \\
942-11 \\
919-20 \\
955-20 \\
971-20 \\
965-20\end{array}$ & 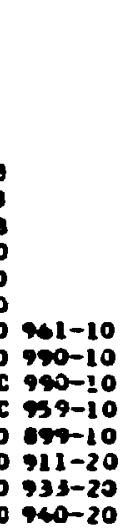 & 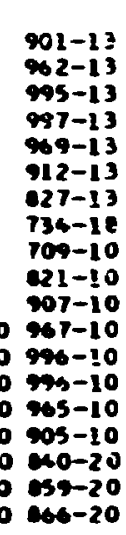 \\
\hline
\end{tabular}




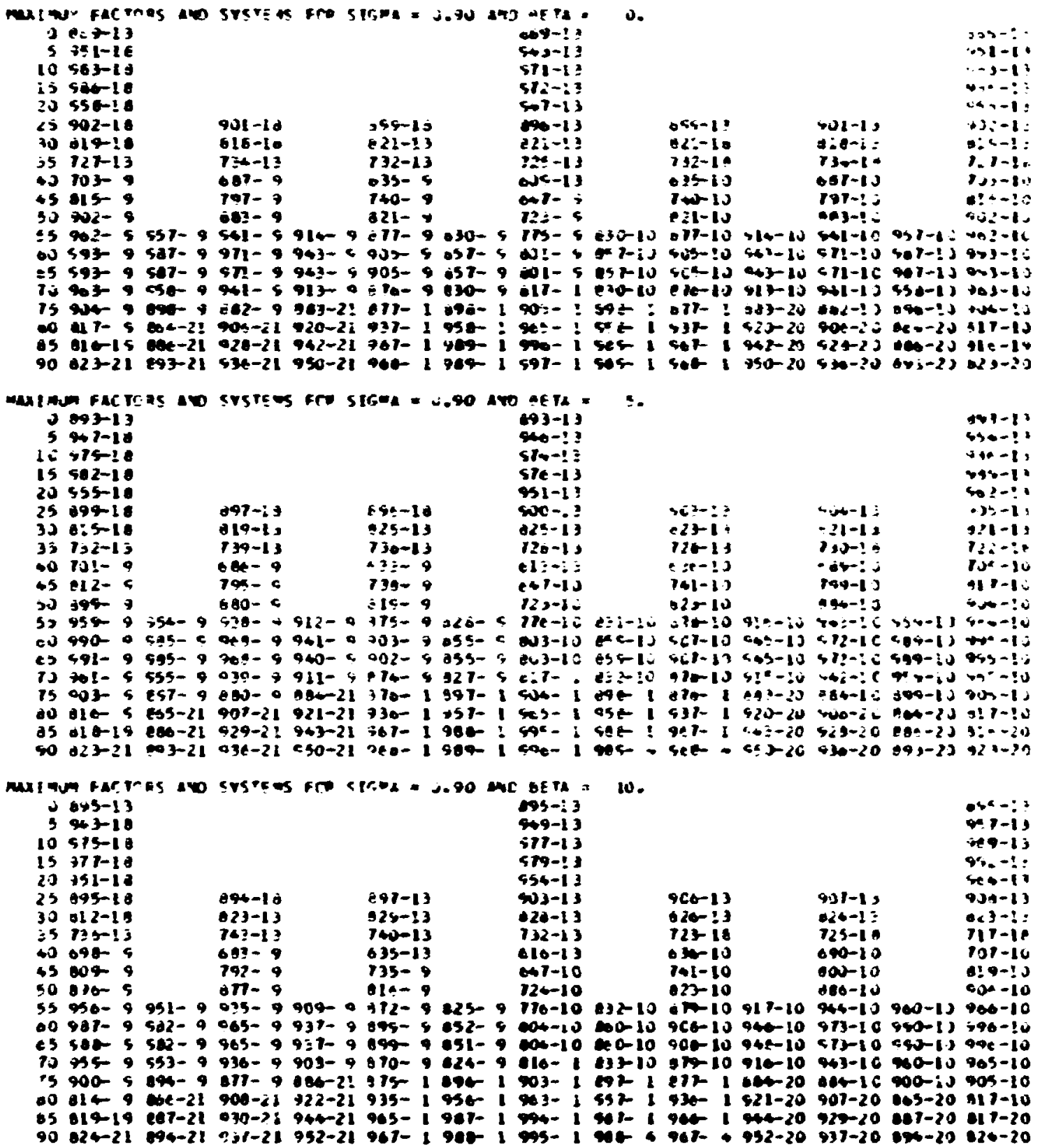




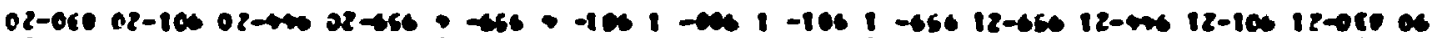

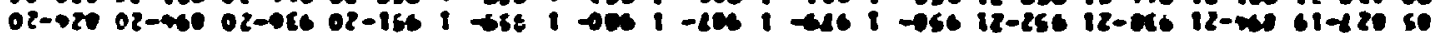

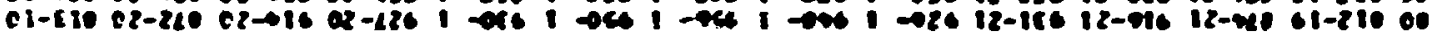

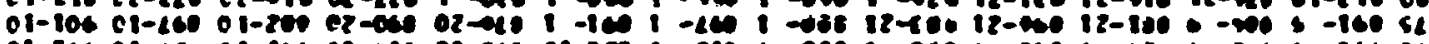

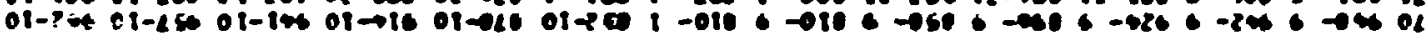

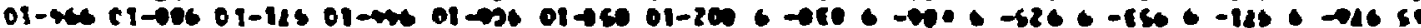

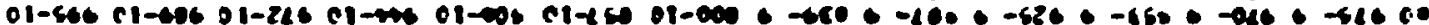

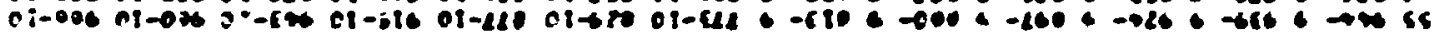

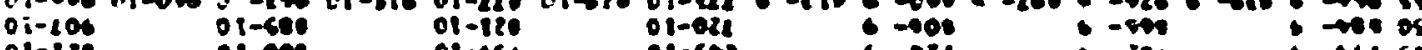
$01-00$

Ci-1: I oit $\rightarrow 00$ ot ot $\rightarrow$ ci-2is pi-21s it-its

: $:-6=4$

ti-100

(1-5es

$(t-2)$

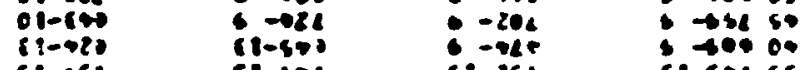

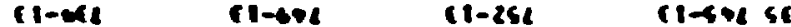

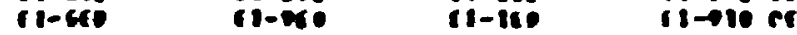
ci-tos iti-los ities 58 ci-es (1-2) ci-ces cton 1- 1- 100 $\lim _{i \rightarrow 0}$

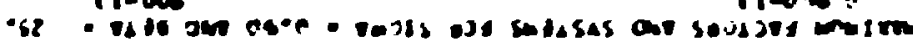

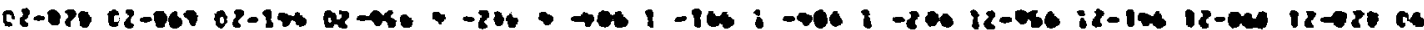

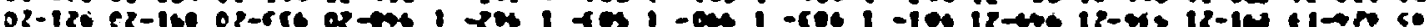

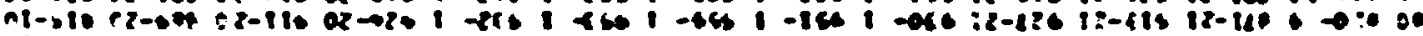

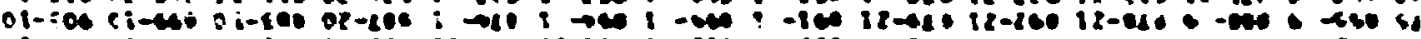

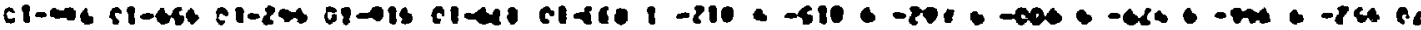

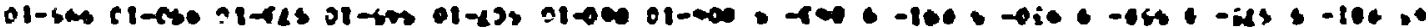

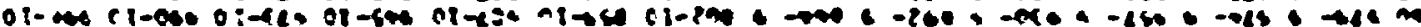

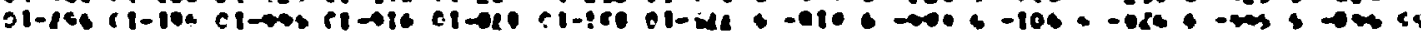
$01-100$

ol-ise

$n 1-4 c^{2}$

11-91

it-9?

$1-116$

ii- oct

ionet

1-1 11

$$
\text { ci-a) }
$$
ot 28 i $-\infty$

$01-0$ ot -91 r!tic ct-18 i-iis ilois

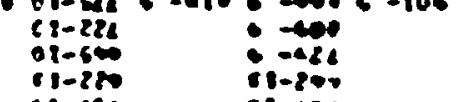
$-48 .-3060$

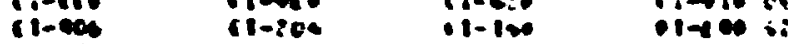
i-es

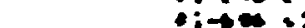
$11-145000$ 11-165

it

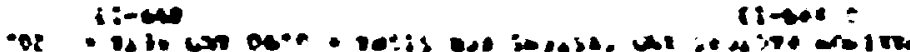

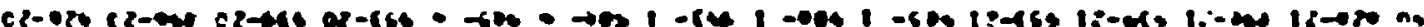

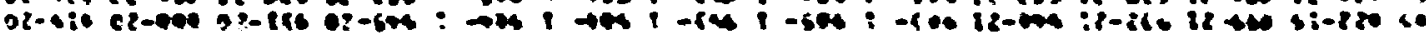

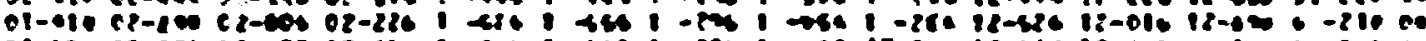

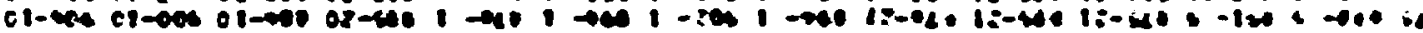

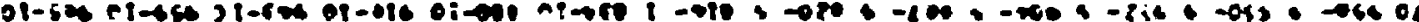

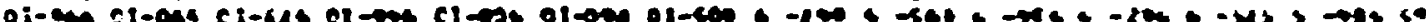

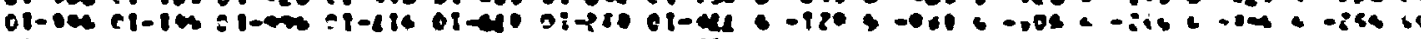
$0 i-40$. $01-0 \pi$

$01-8$

i-ste

$61-56$

c 1-ots

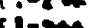

1

c1-8

i $7-64$

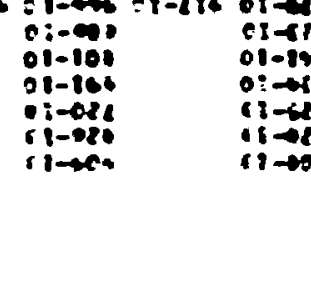

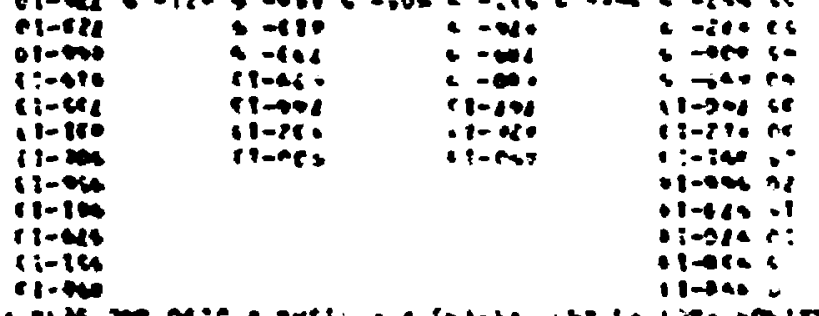




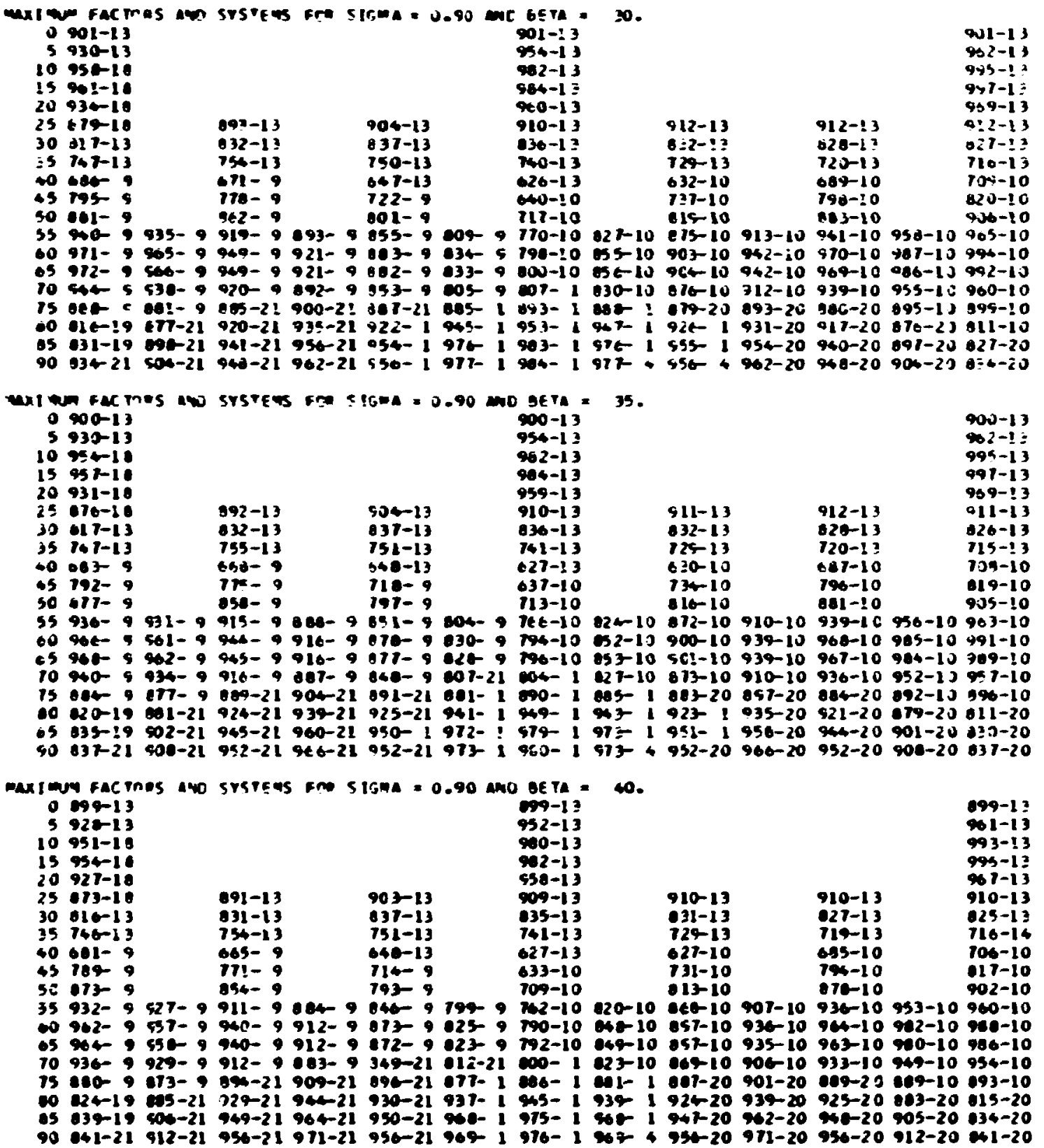




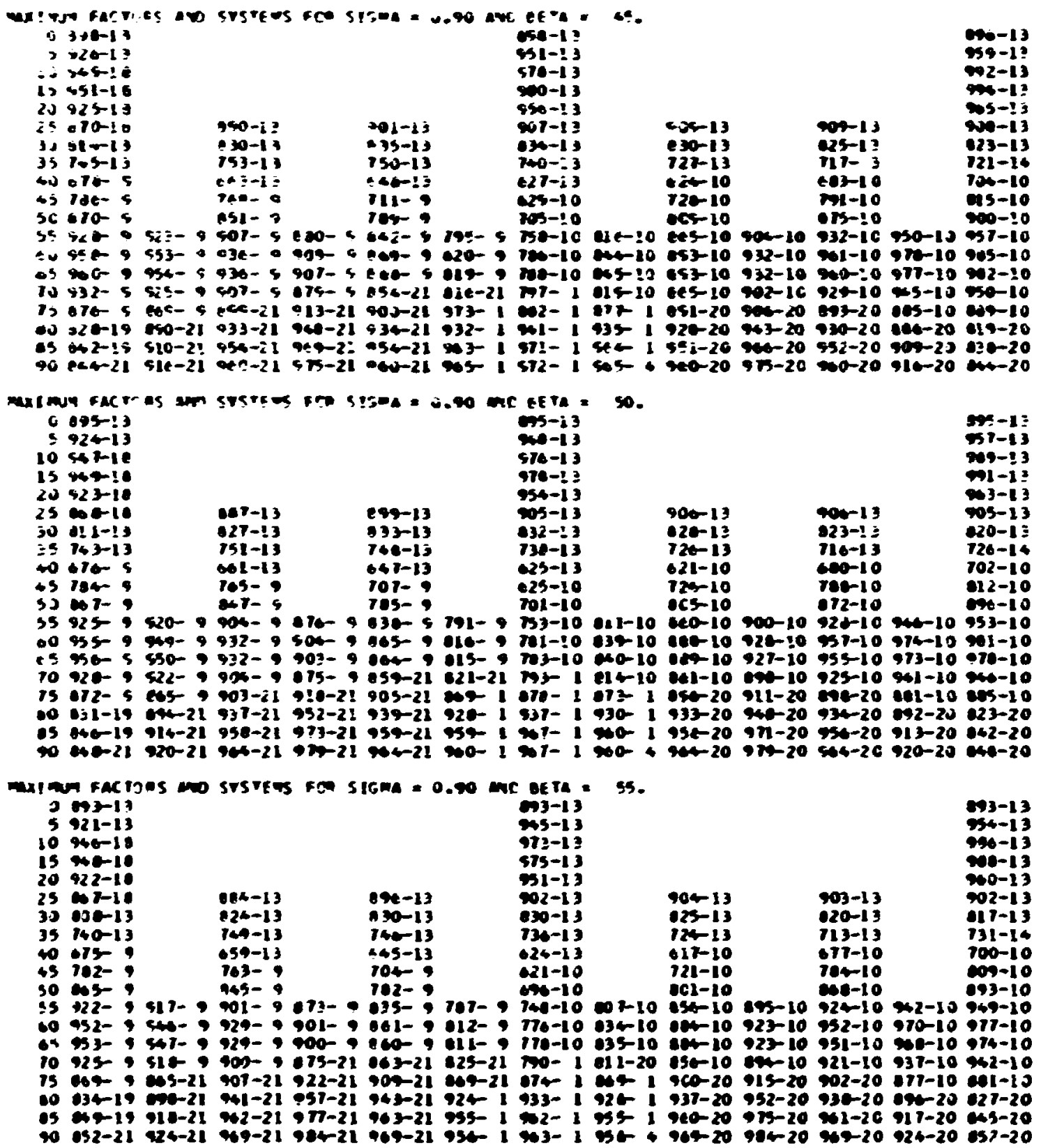




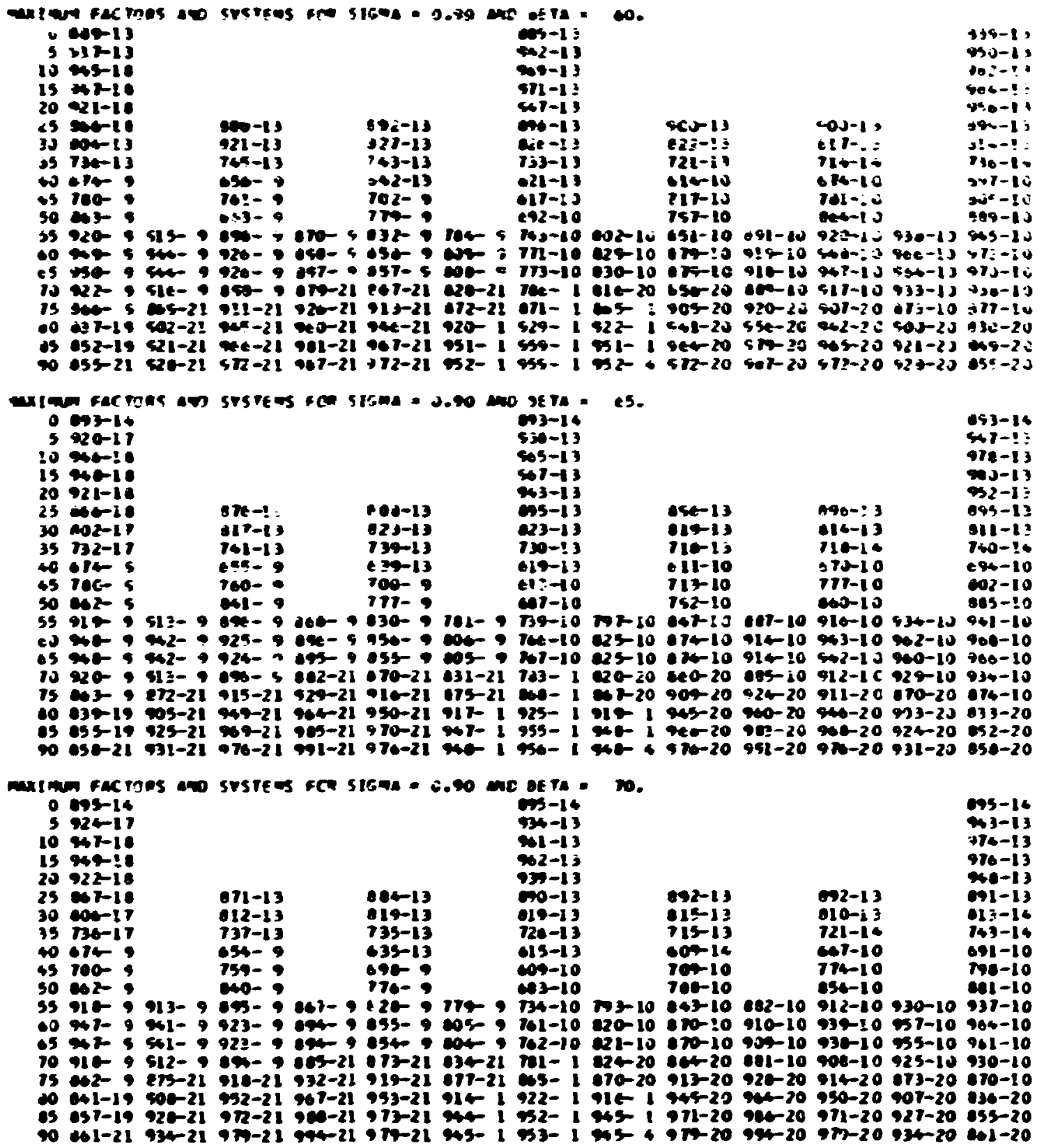




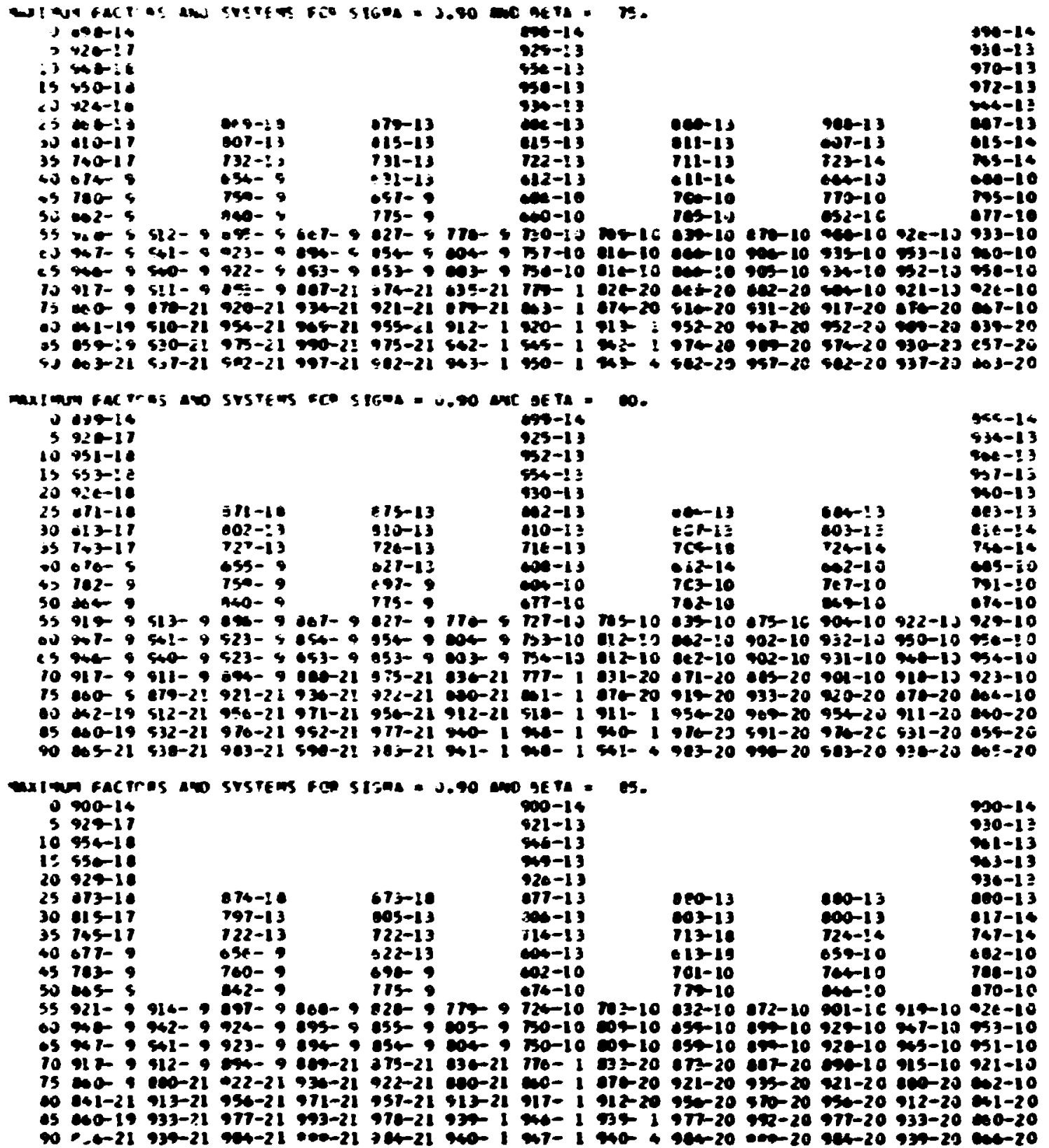




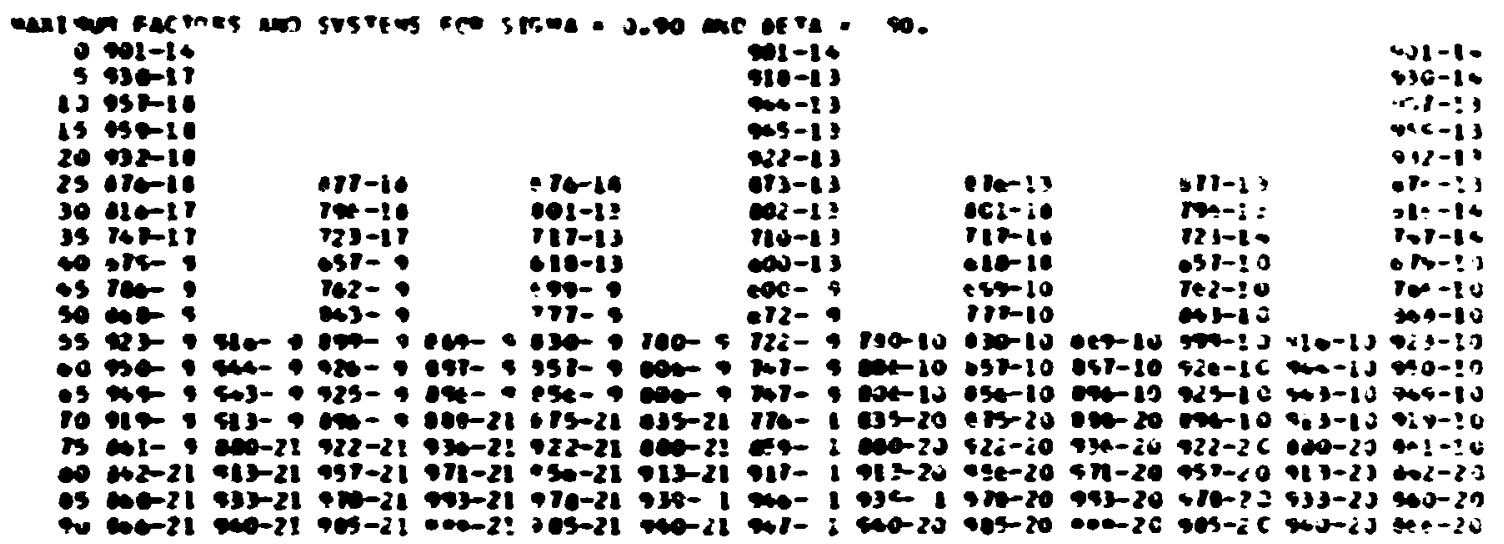




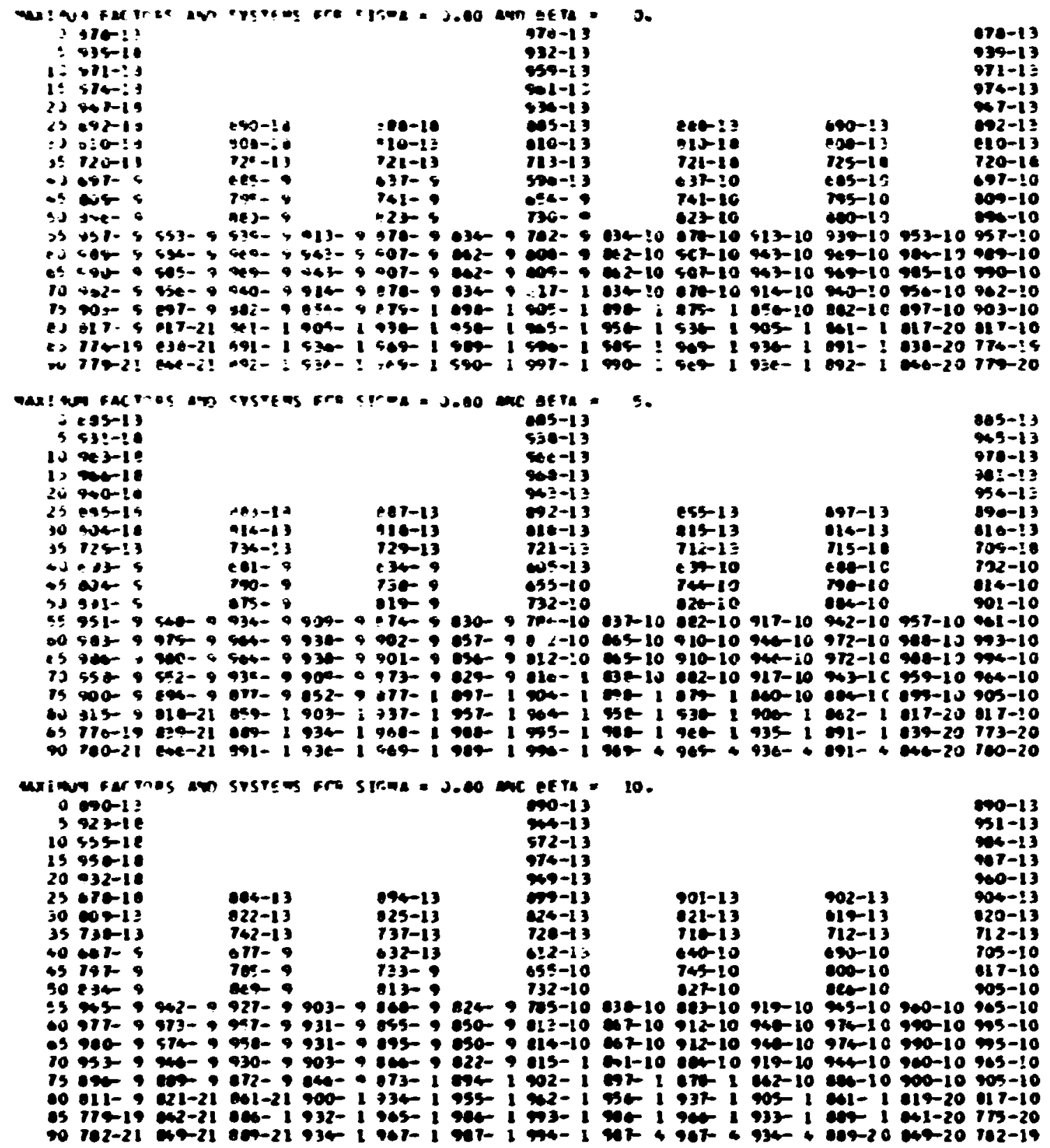




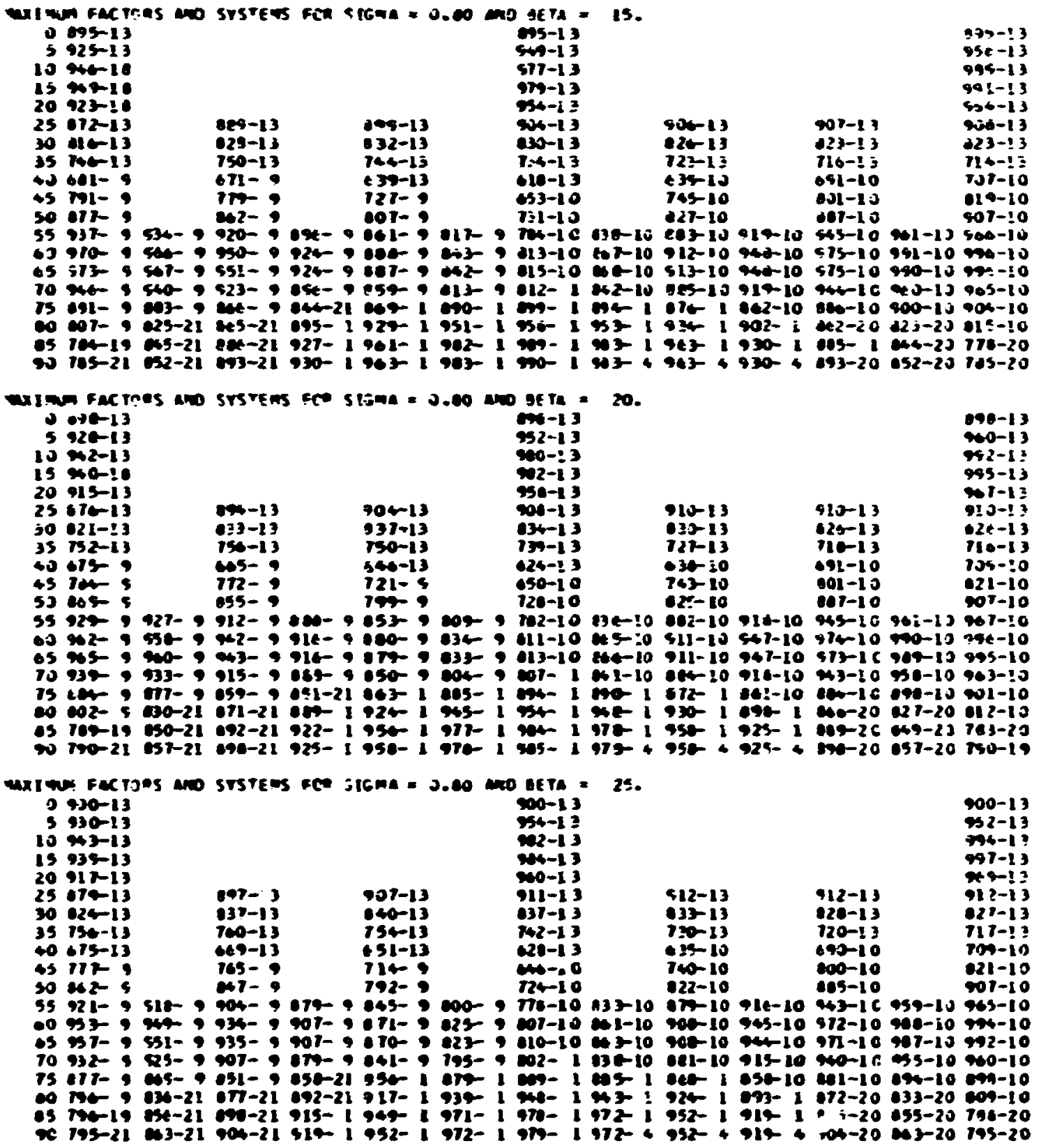




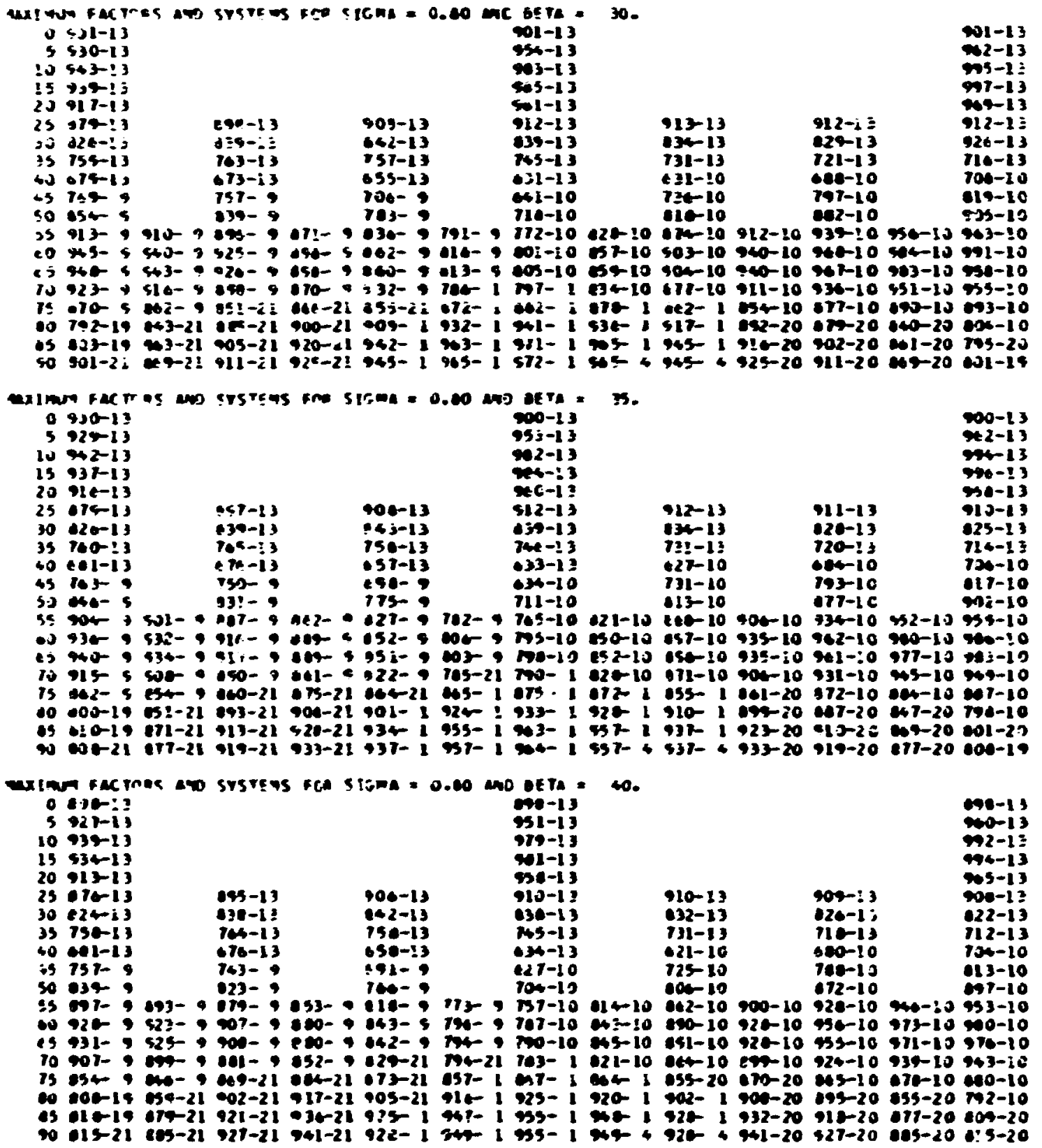




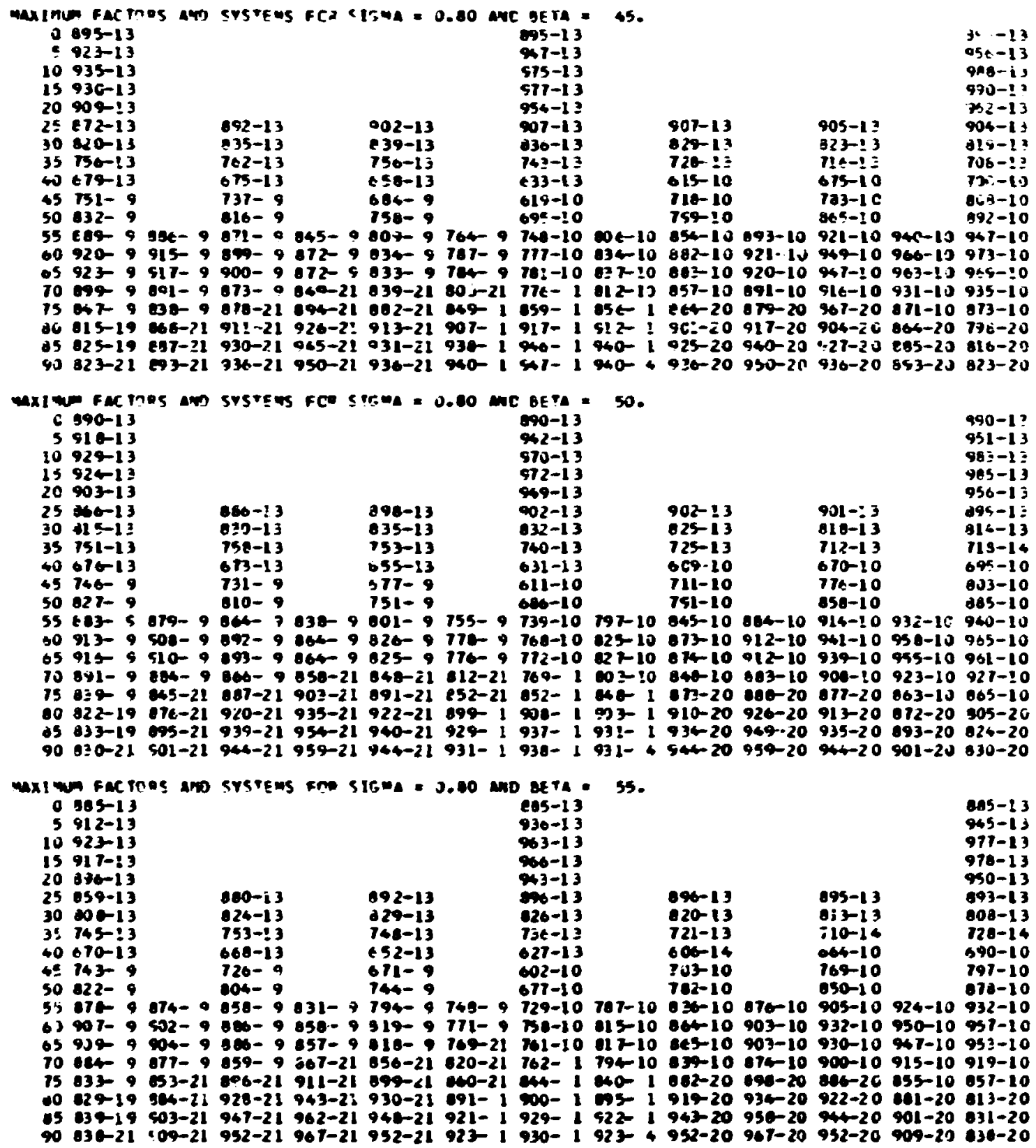




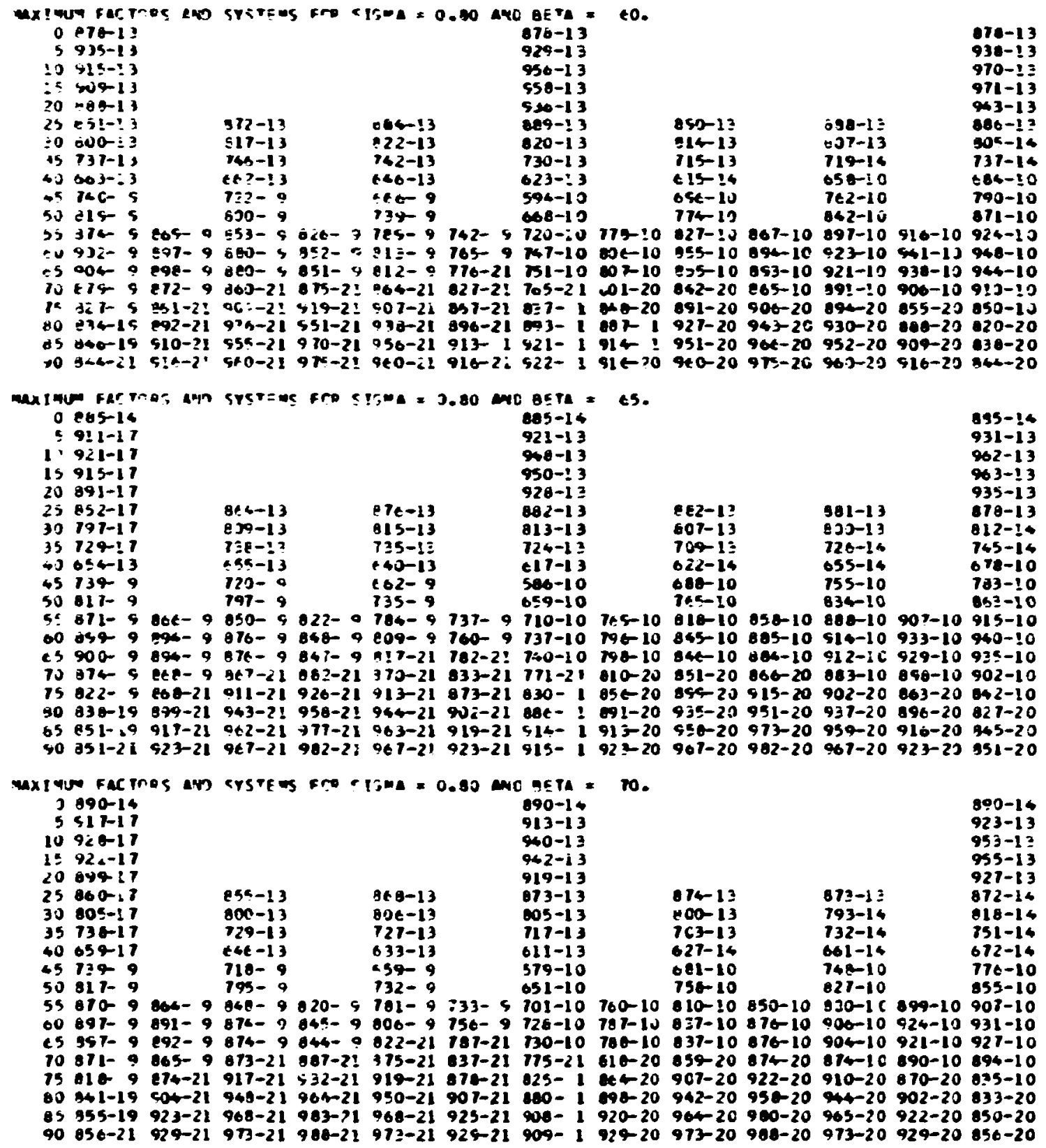




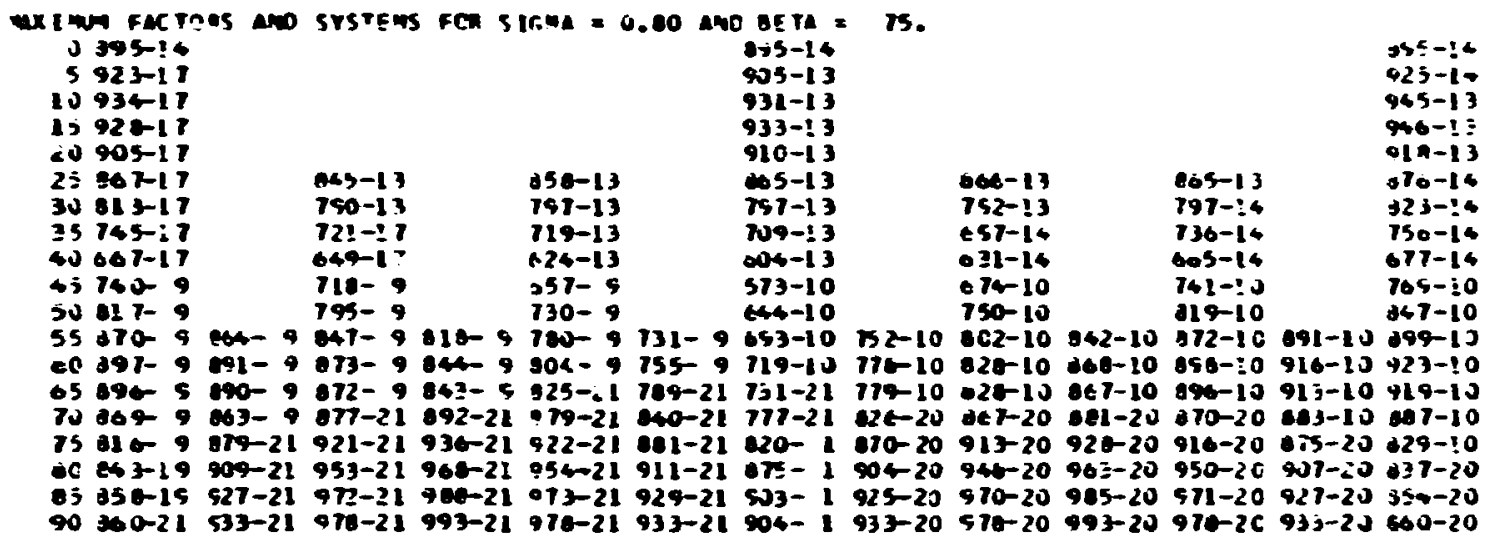

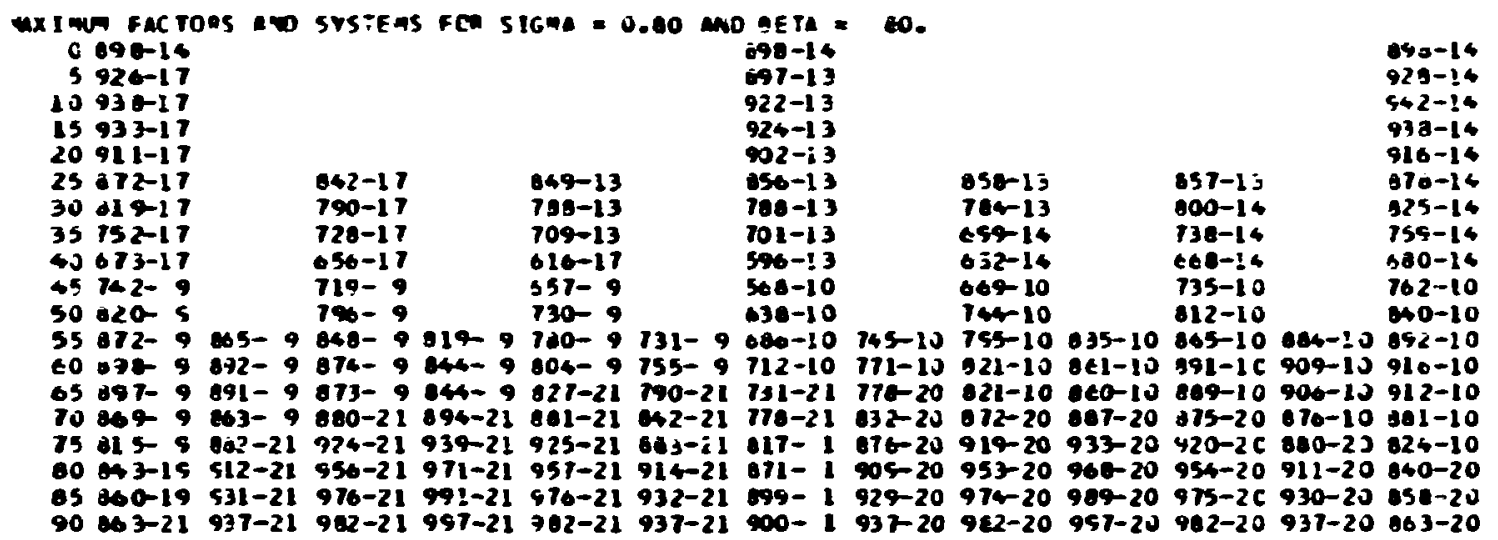

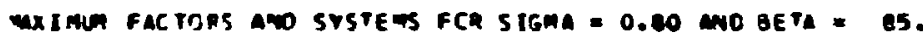

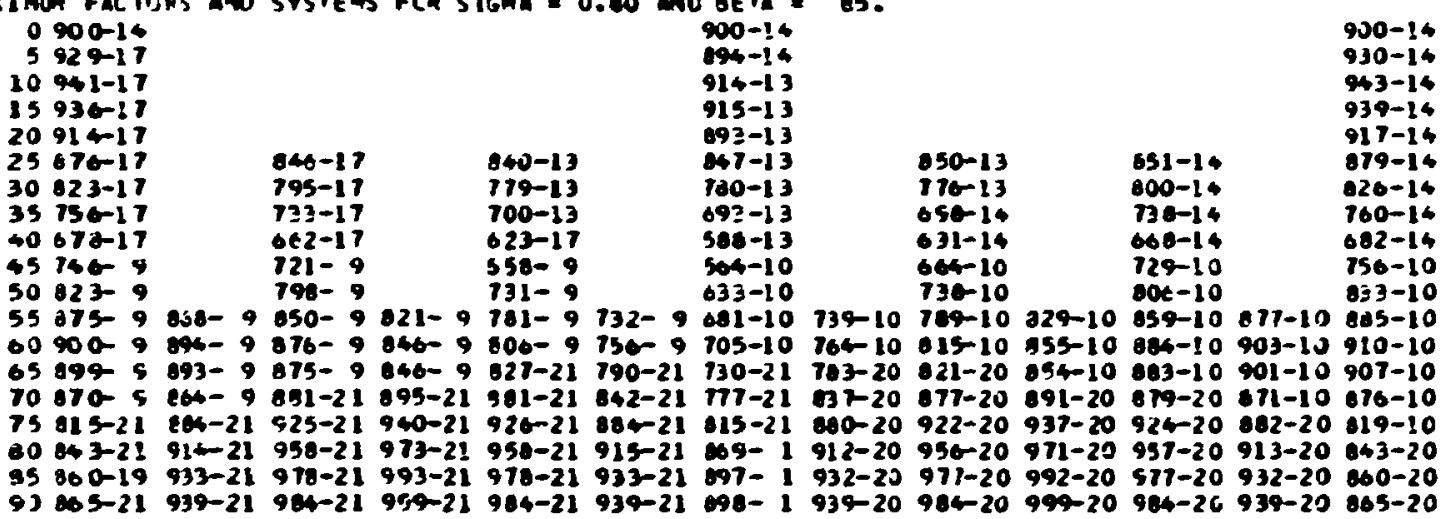




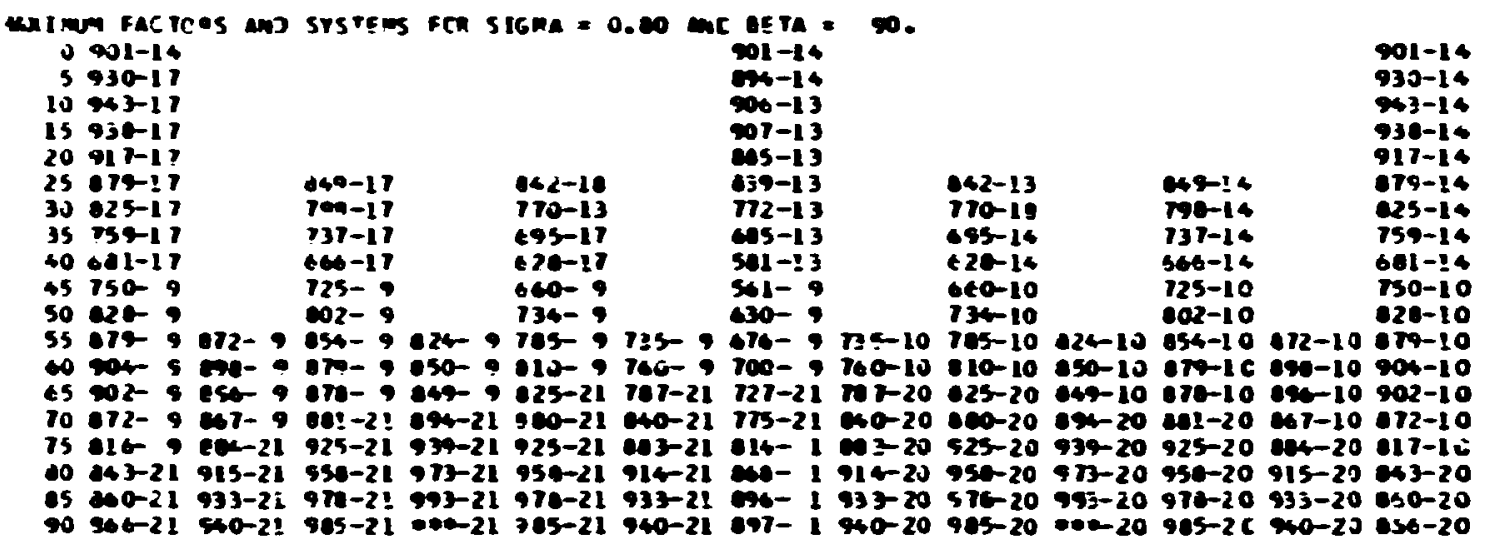




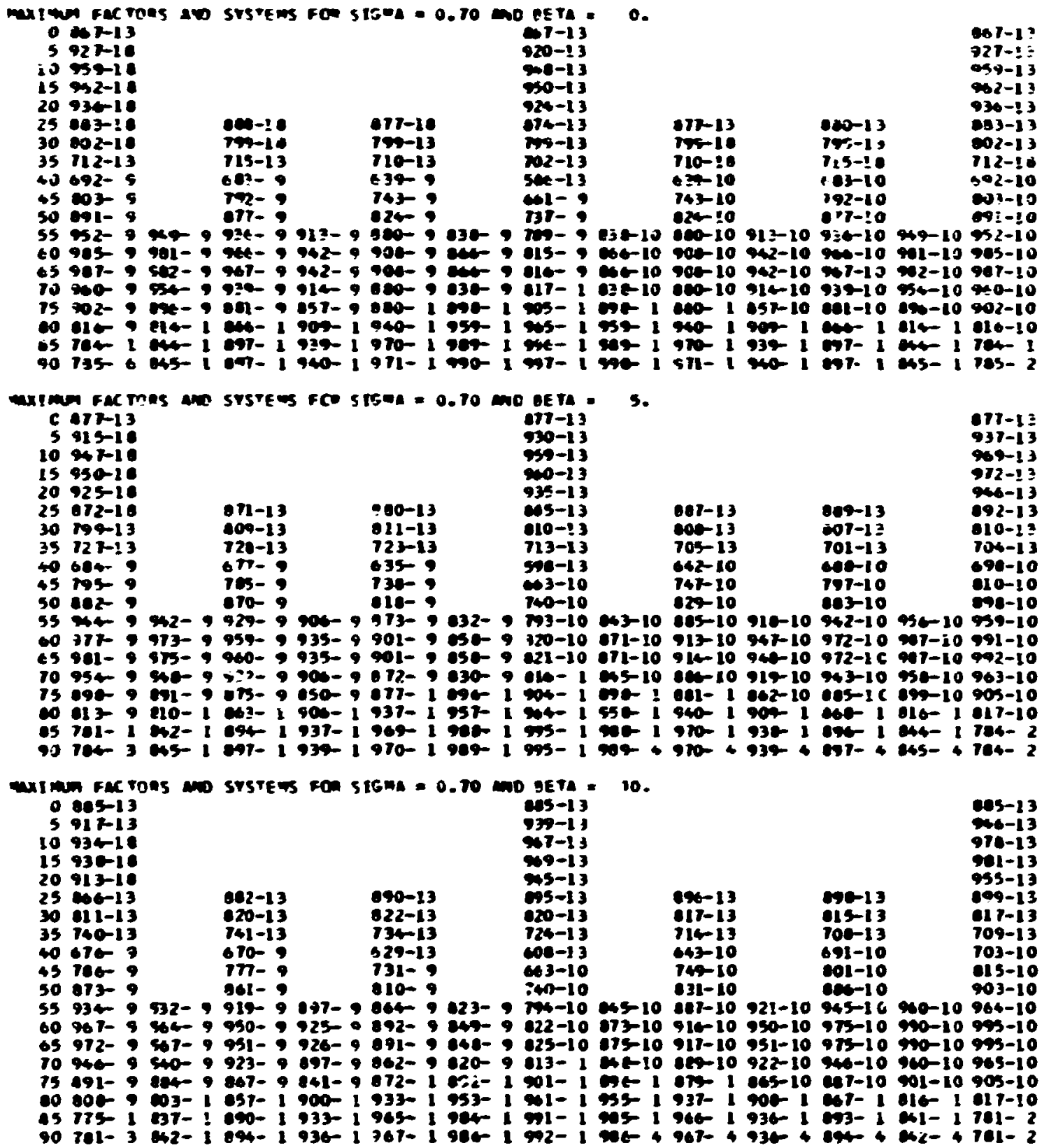




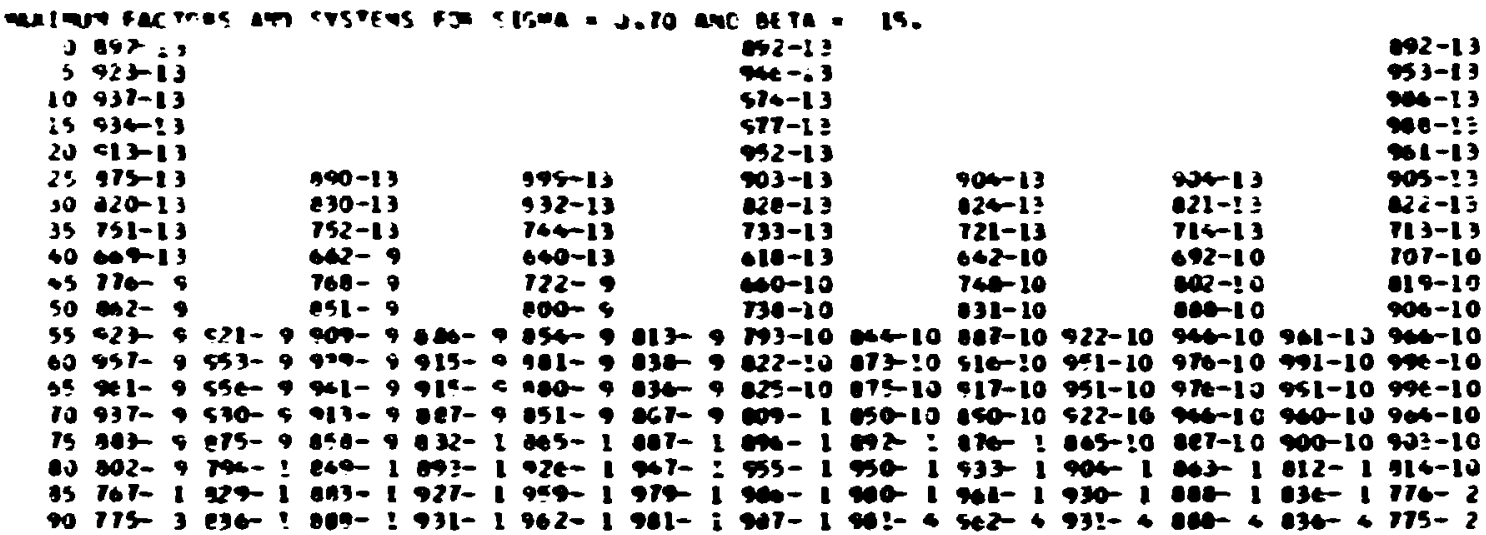

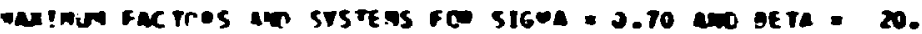

(9) 0 - 13

5 920-1

C) 13

iv $\% 2-13$

951-13

979-13

15 919-13

$20910-13$

25. $1-1$

$30+26-13$

35 700-13

ب2-13

Q50-13

991-13

40 c00-13 $470-12 \quad 40-13 \quad 62-1$

45 TES-9 758-9 712-9

5050 9 $540-9 \quad 700-9 \quad 734-10$

120-10

20-1

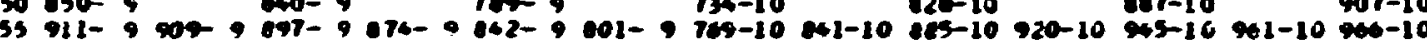

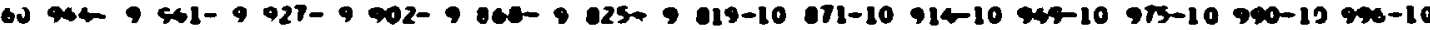

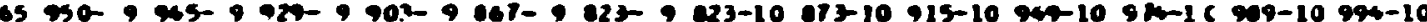

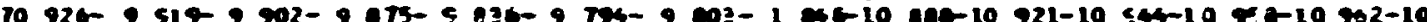

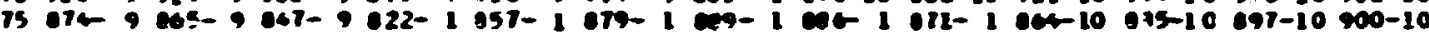

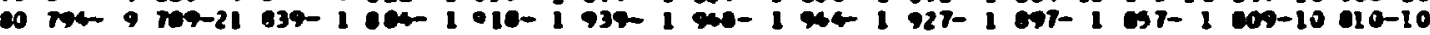

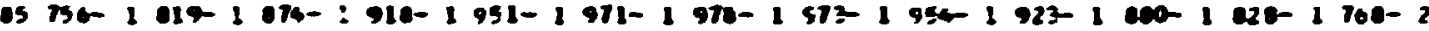

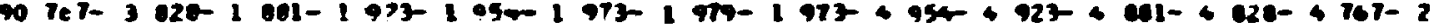

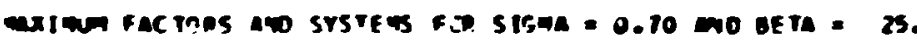

$090-13$
$5930-13$

10 24+13

200-13

3.

15 - 12

$20921-13$

25 exs-13

30 03-13

35 162-13

40 cot-13

45 754

so ase 9

2013-1

$20-13$

910-13

25-13

17-19

21-10

$907-10$

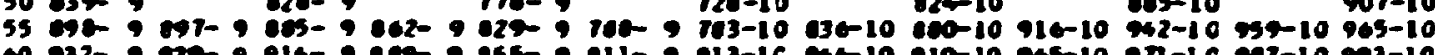

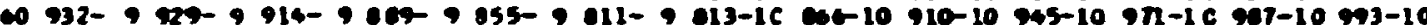

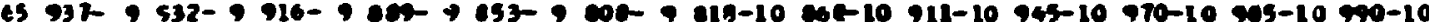

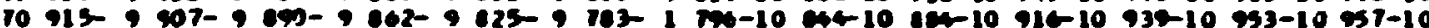

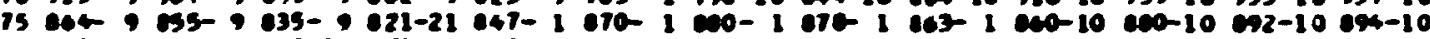

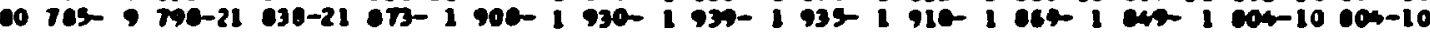

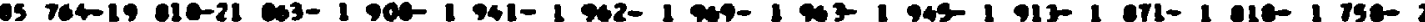

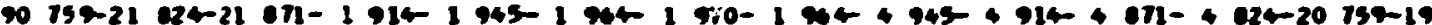




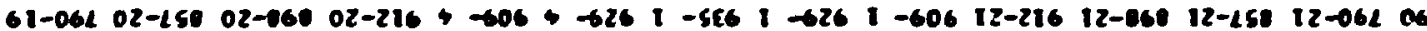

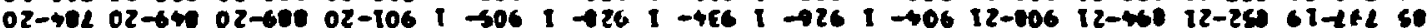

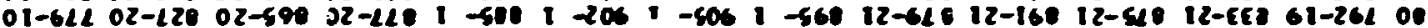

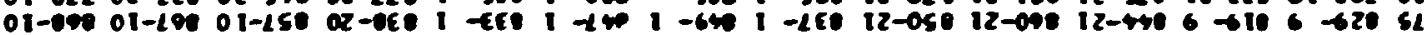

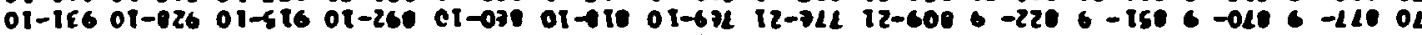

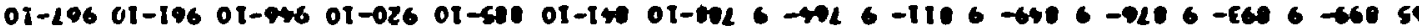

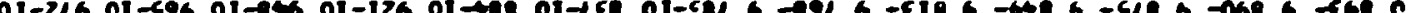

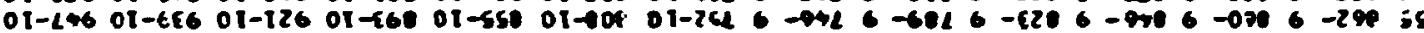

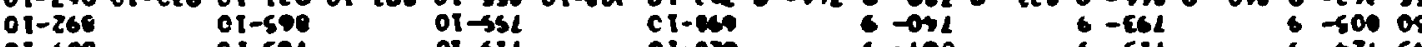

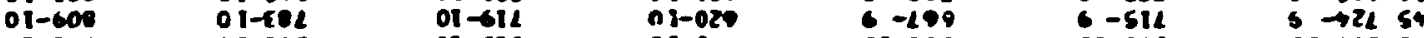

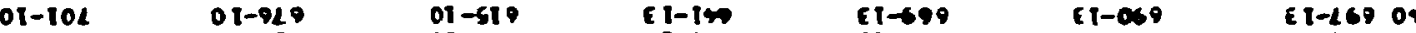

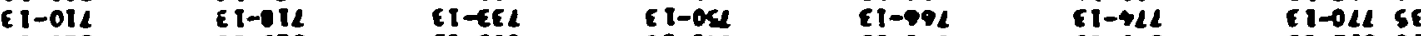

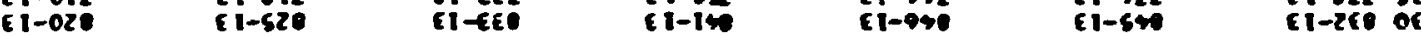
EI-906 EI-006 EI-016 EI-IIS EI-006 EI- ESE EL C I-ise EI-0ess

EI-C6 OI $\begin{array}{ll}E 1-526 & 5 \\ E 1-160 & 0\end{array}$

E i-es6 I-64) E 1-160

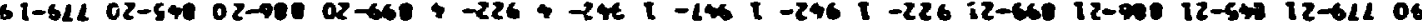

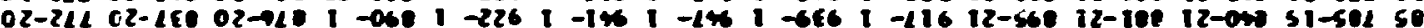

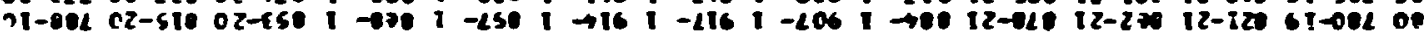

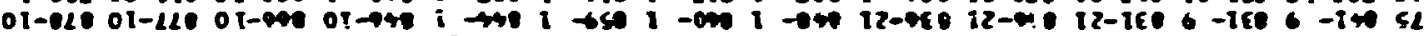

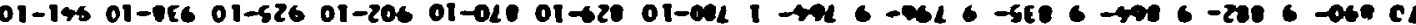

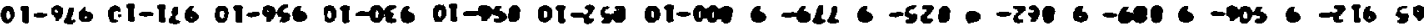

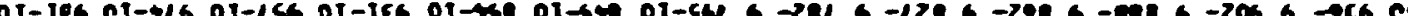

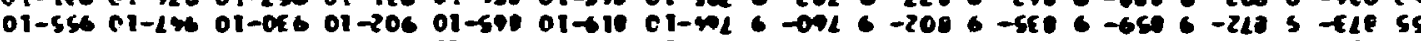

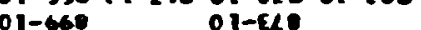
$01-600$

$01-60$ $01-28901-c 28$

$\leq 1-470 \quad$ i $1-120 \quad$ iा

EI-016 II-Tis

C1-1\% E1-14L EI-094 EI-GLL EI-2LL SE CI- E4 EI-040 EI-140 ET EI-416 CI-116 CI-206 EI-50 52 E t-2u

1- 1C I-4. E1-10s E1-246 o1

E $\mathrm{i}-\mathbf{4} \mathbf{4}$

C1-1006 c t-10s e I$=1 \rightarrow 265$

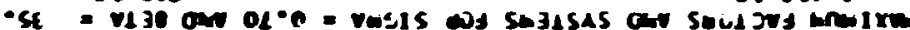

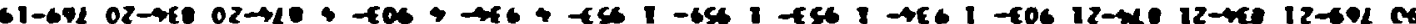

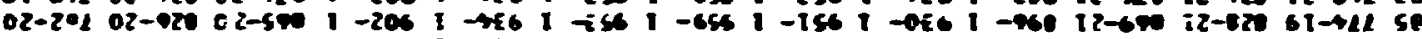

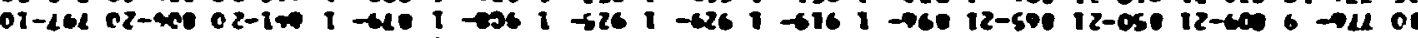

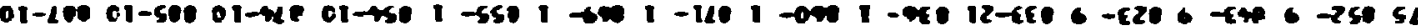

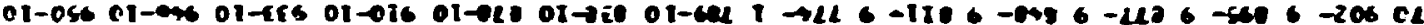

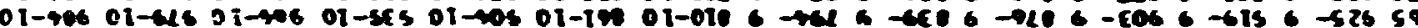

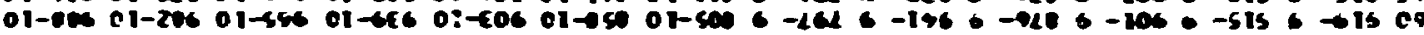

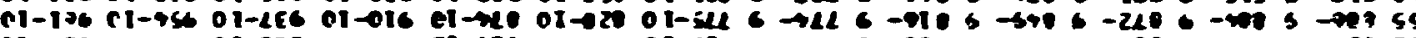

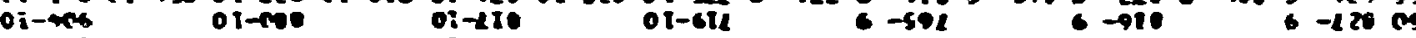
01-010 01-20 01-

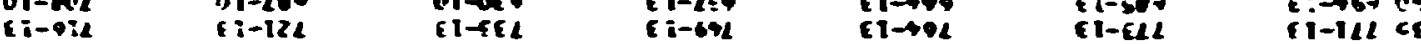

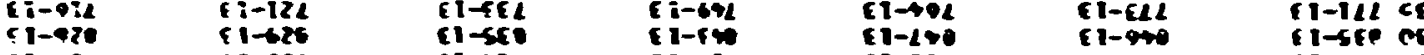

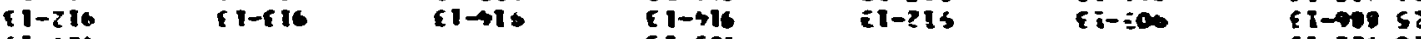
$1-596$ $61-280$ c EI-T) EI-14 51

(1-2)

c I-ch

$+i-29$ c1-6ets $\mathrm{i}_{\mathrm{i} \rightarrow \mathrm{T}} \mathrm{C}$ Ci-ses

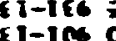

$-\alpha=139$ on or. 


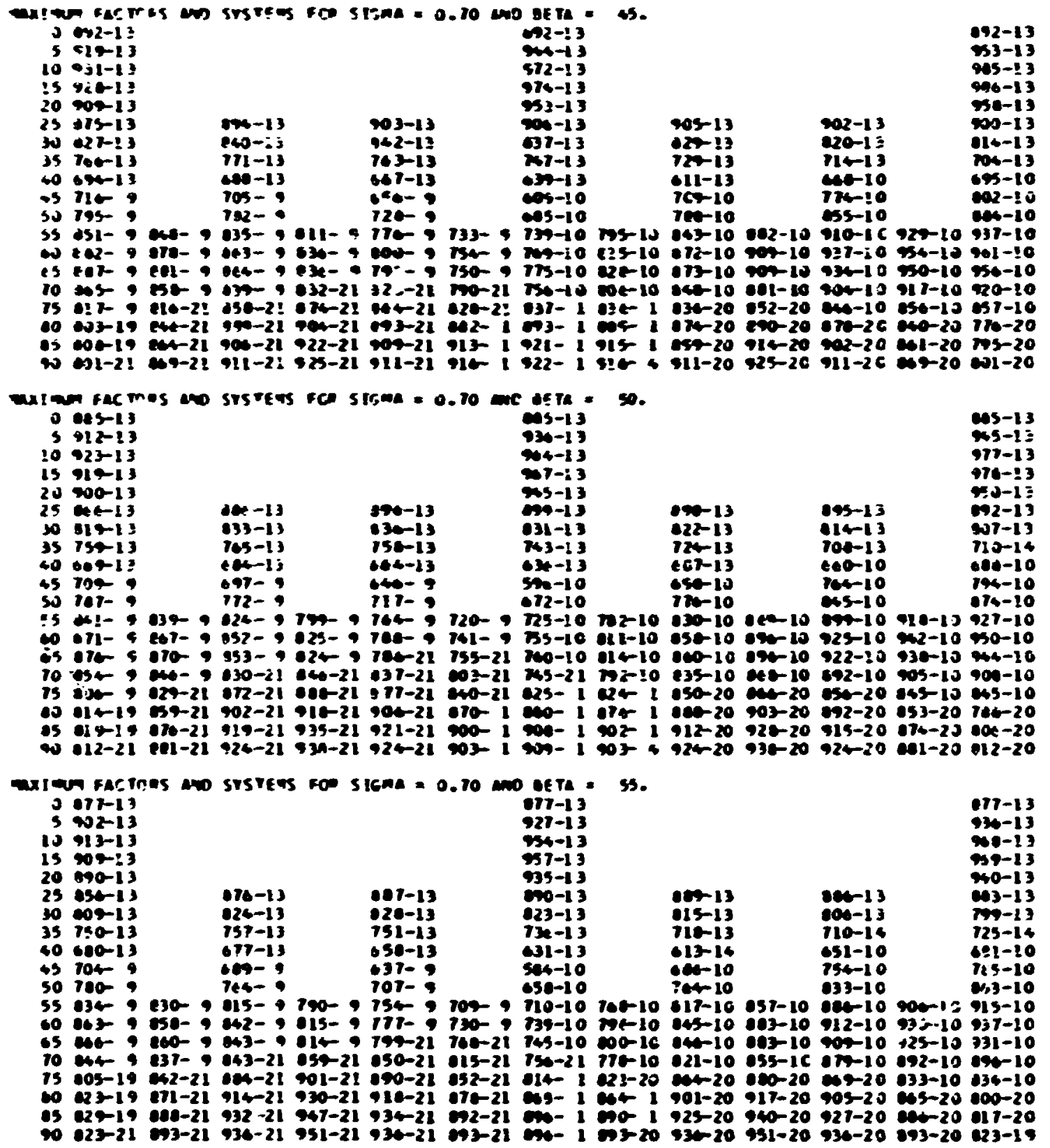




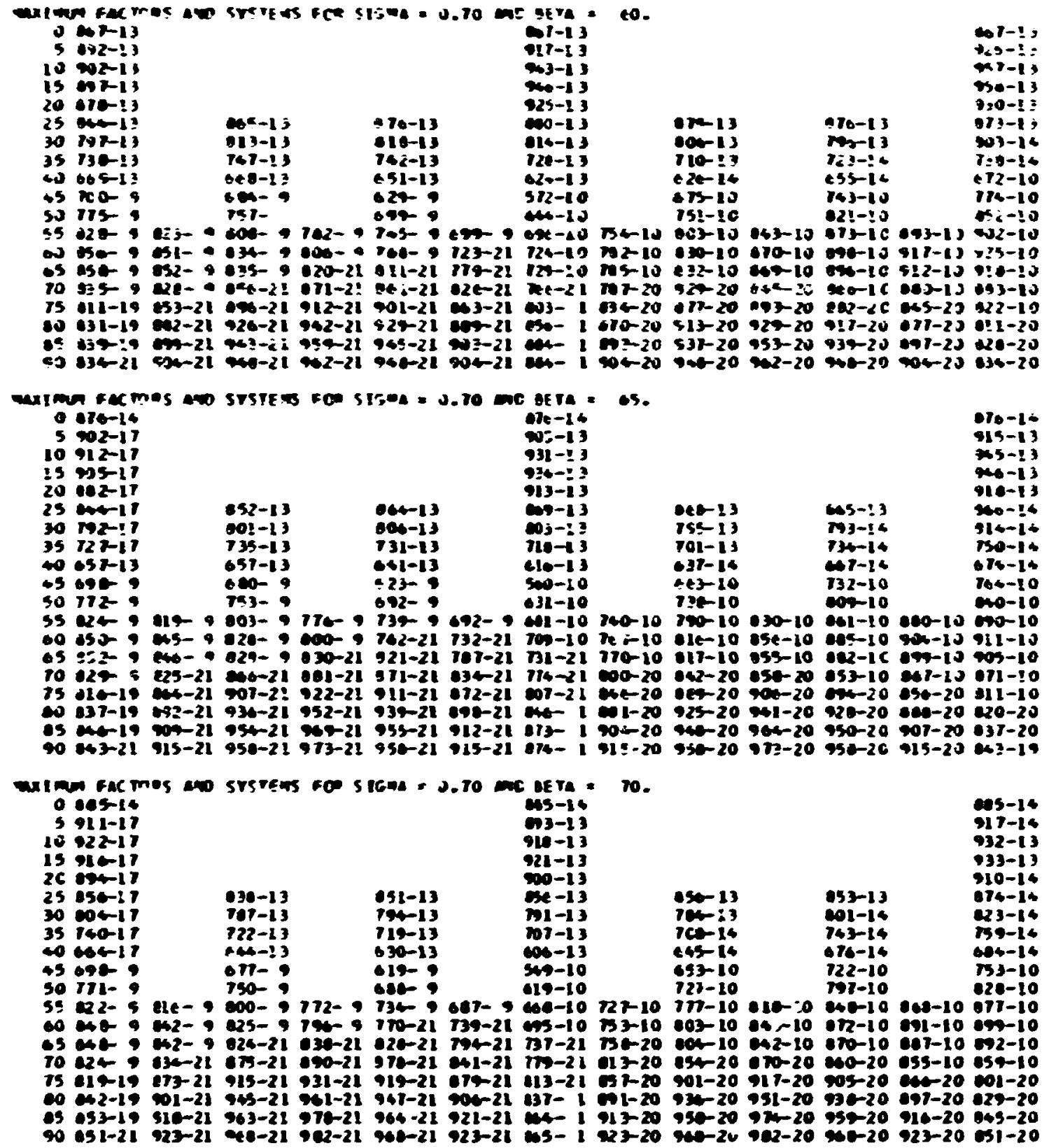




\begin{tabular}{|c|c|c|c|c|c|c|c|c|c|c|c|c|c|}
\hline 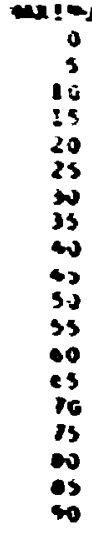 & 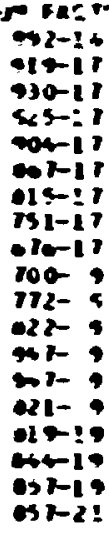 & 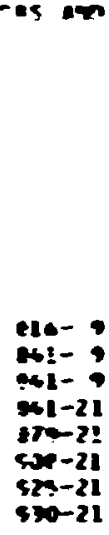 & 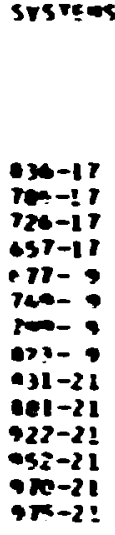 & 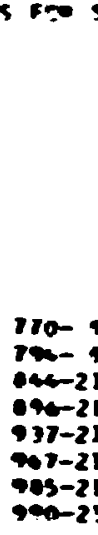 & 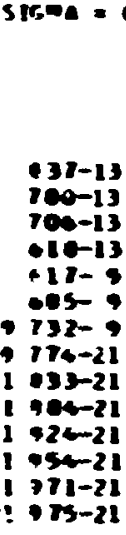 & 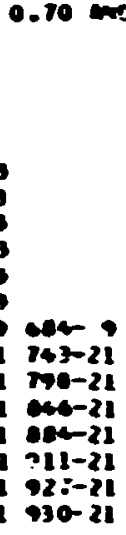 & 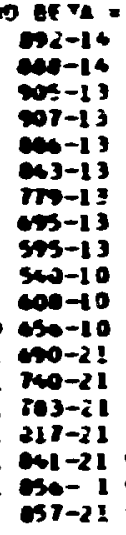 & 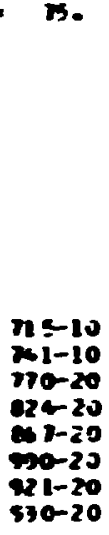 & $\begin{array}{l}66-13 \\
712-13 \\
713-16 \\
650-16 \\
643-10 \\
710-10 \\
745-10 \\
751-10 \\
600-26 \\
045-20 \\
510-20 \\
46-20 \\
45-20 \\
95-26\end{array}$ & 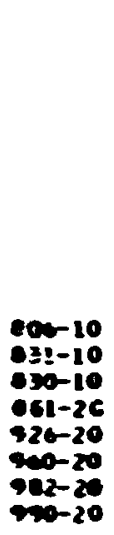 & 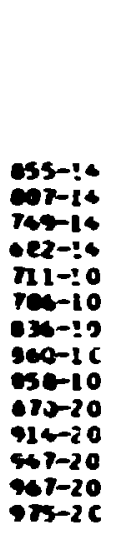 & 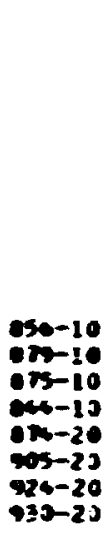 & 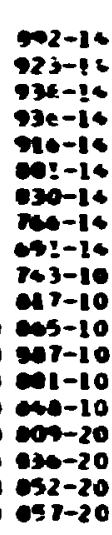 \\
\hline 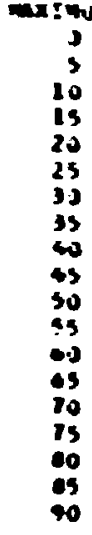 & 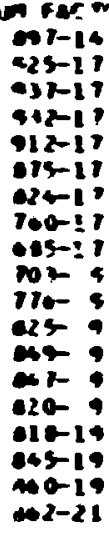 & $\begin{array}{l}10-9 \\
67-9 \\
01-2 \\
60-21 \\
890-21 \\
513-2 ! \\
430-2 ! \\
935-21\end{array}$ & 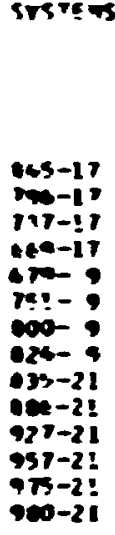 & 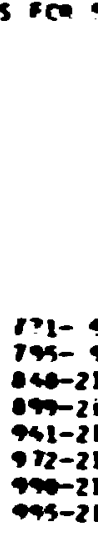 & 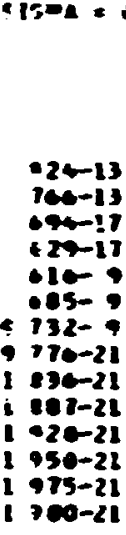 & $\begin{array}{l}3 \\
105-2 \\
74-21 \\
000-21 \\
90-21 \\
601-21 \\
915-22 \\
931-21 \\
935-21 \\
935\end{array}$ & 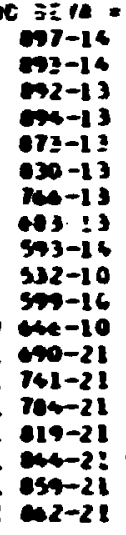 & 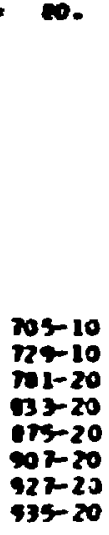 & $\begin{array}{l}931-13 \\
711-14 \\
715-14 \\
652-16 \\
030-10 \\
700-10 \\
7 \leq 5-13 \\
710-10 \\
015-20 \\
974-20 \\
910-20 \\
951-20 \\
972-20 \\
900-20\end{array}$ & $\begin{array}{r}0 \\
795-10 \\
020-10 \\
030-20 \\
109-20 \\
934-20 \\
747-20 \\
900-20 \\
995-20\end{array}$ & $\begin{array}{l}050-16 \\
610-16 \\
752-16 \\
60-16 \\
702-10 \\
775-10 \\
620-10 \\
950-10 \\
67-10 \\
970-26 \\
921-20 \\
959-20 \\
573-20 \\
960-20\end{array}$ & $\begin{array}{r}0 \\
0 \\
045-10 \\
60-10 \\
06-10 \\
940-23 \\
901-20 \\
911-20 \\
929-20 \\
935-20\end{array}$ & 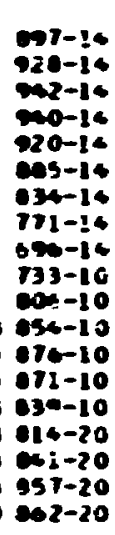 \\
\hline $\begin{array}{l}210 \\
5 \\
10 \\
15 \\
20 \\
25 \\
30 \\
35 \\
40 \\
45 \\
30 \\
35 \\
10 \\
65 \\
70 \\
73 \\
10 \\
65 \\
40\end{array}$ & 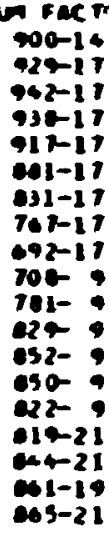 & $\begin{array}{l}222-9 \\
04-9 \\
240-21 \\
915-21 \\
915-21 \\
933-21 \\
939-21\end{array}$ & $\begin{array}{l}052-17 \\
006-17 \\
745-17 \\
677-17 \\
602-9 \\
754-9 \\
003-9 \\
627-9 \\
930-21 \\
907-21 \\
929-21 \\
950-21 \\
970-21 \\
906-21\end{array}$ & $\begin{array}{l}774- \\
790 \\
640- \\
901- \\
943- \\
974- \\
993\end{array}$ & 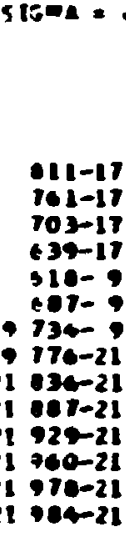 & 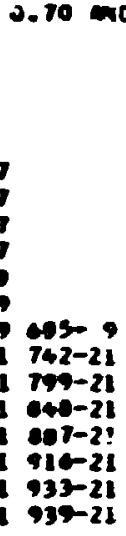 & 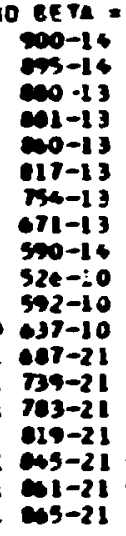 & $\begin{array}{l}0 \\
09-10 \\
690-20 \\
139-20 \\
90-20 \\
61-20 \\
112-20 \\
932-20 \\
99-20\end{array}$ & $\begin{array}{l}19-13 \\
770-14 \\
714-14 \\
052-16 \\
627-10 \\
950-10 \\
74-10 \\
770-10 \\
127-20 \\
901-20 \\
924-20 \\
950-20 \\
974-20 \\
94-20\end{array}$ & $\begin{array}{r}r \\
70-10 \\
11-10 \\
041-20 \\
095-20 \\
939-20 \\
972-20 \\
992-20 \\
949-20\end{array}$ & $\begin{array}{l}059-14 \\
611-16 \\
153-16 \\
64-14 \\
694-10 \\
767-10 \\
910-10 \\
640-16 \\
036-10 \\
003-20 \\
920-20 \\
937-20 \\
911-20 \\
964-20\end{array}$ & $\begin{array}{l} \\
036-10 \\
63-10 \\
59-10 \\
65-10 \\
645-20 \\
609-20 \\
914-20 \\
932-20 \\
939-20\end{array}$ & $\begin{array}{r}900-14 \\
930-14 \\
96-14 \\
961-16 \\
922-14 \\
600-14 \\
636-14 \\
772-14 \\
697-14 \\
123-10 \\
796-10 \\
64-10 \\
60-10 \\
602-10 \\
632-10 \\
010-20 \\
64-20 \\
60-20 \\
65-20\end{array}$ \\
\hline
\end{tabular}




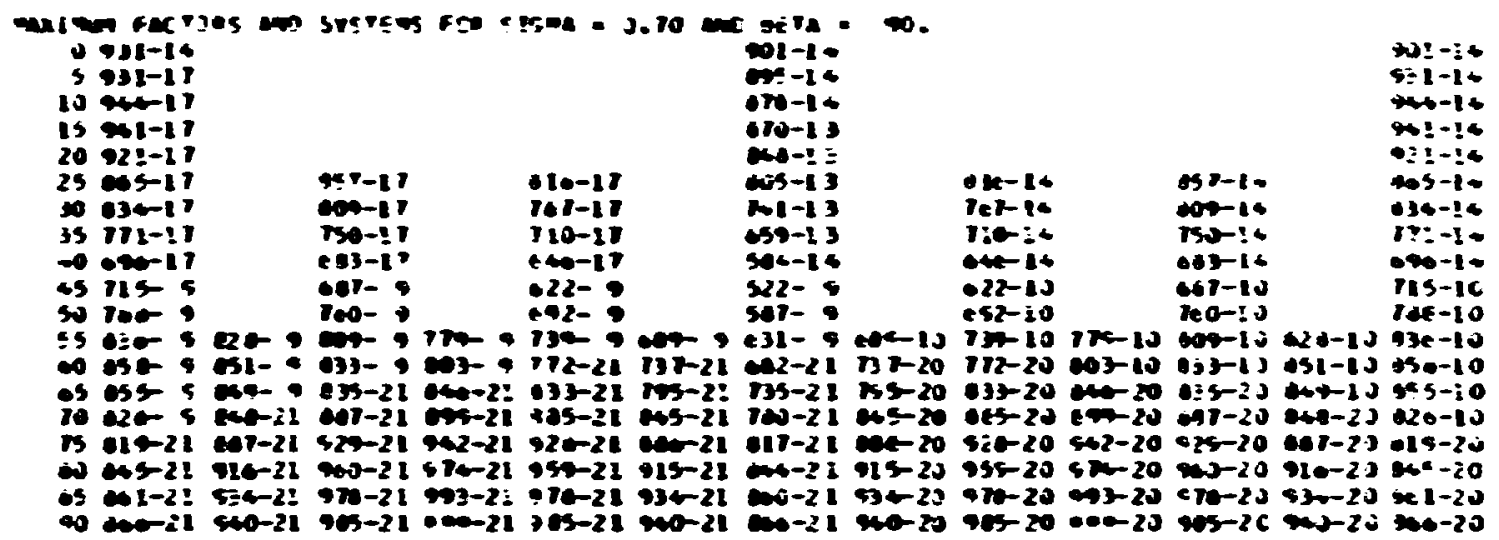




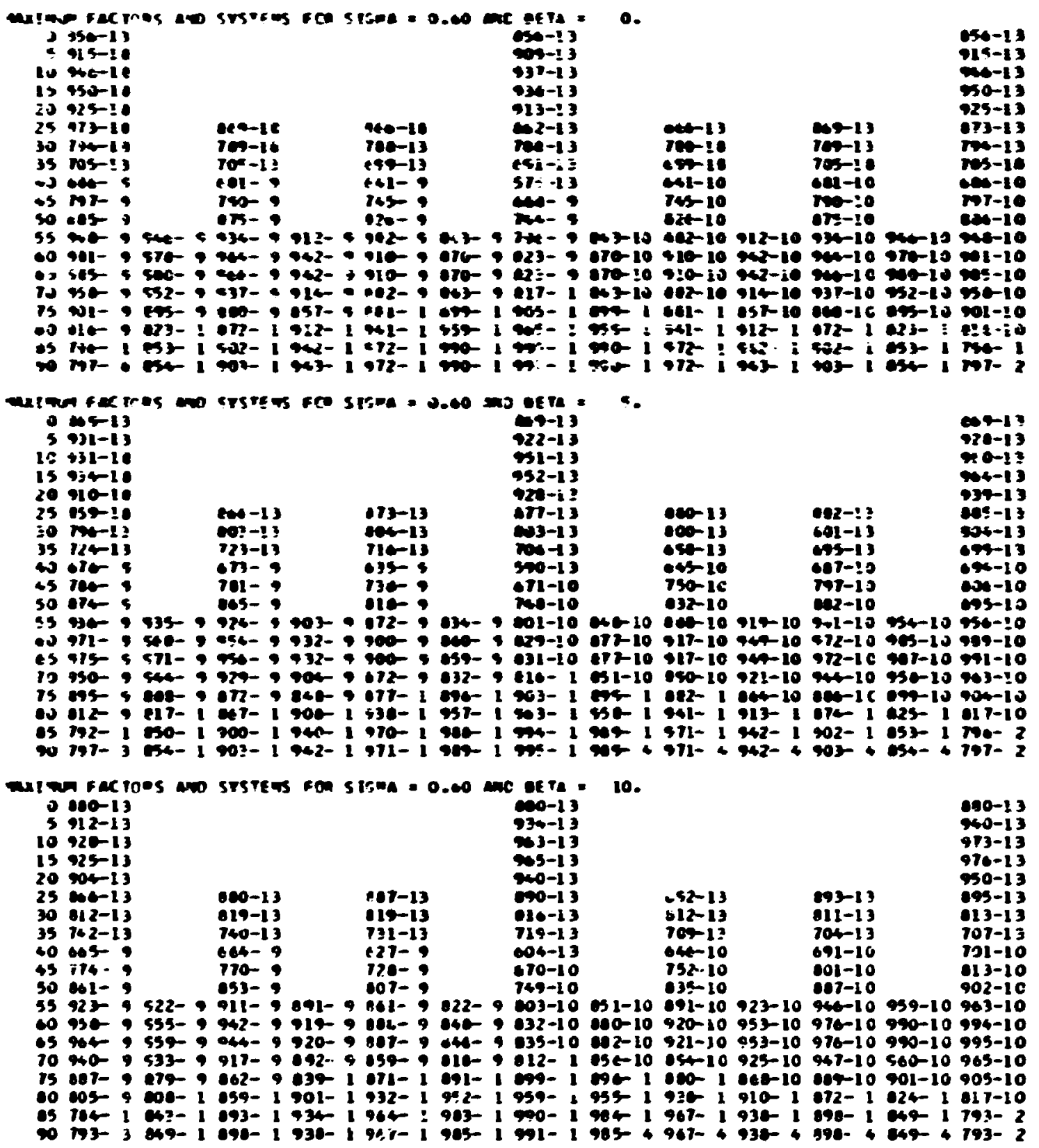




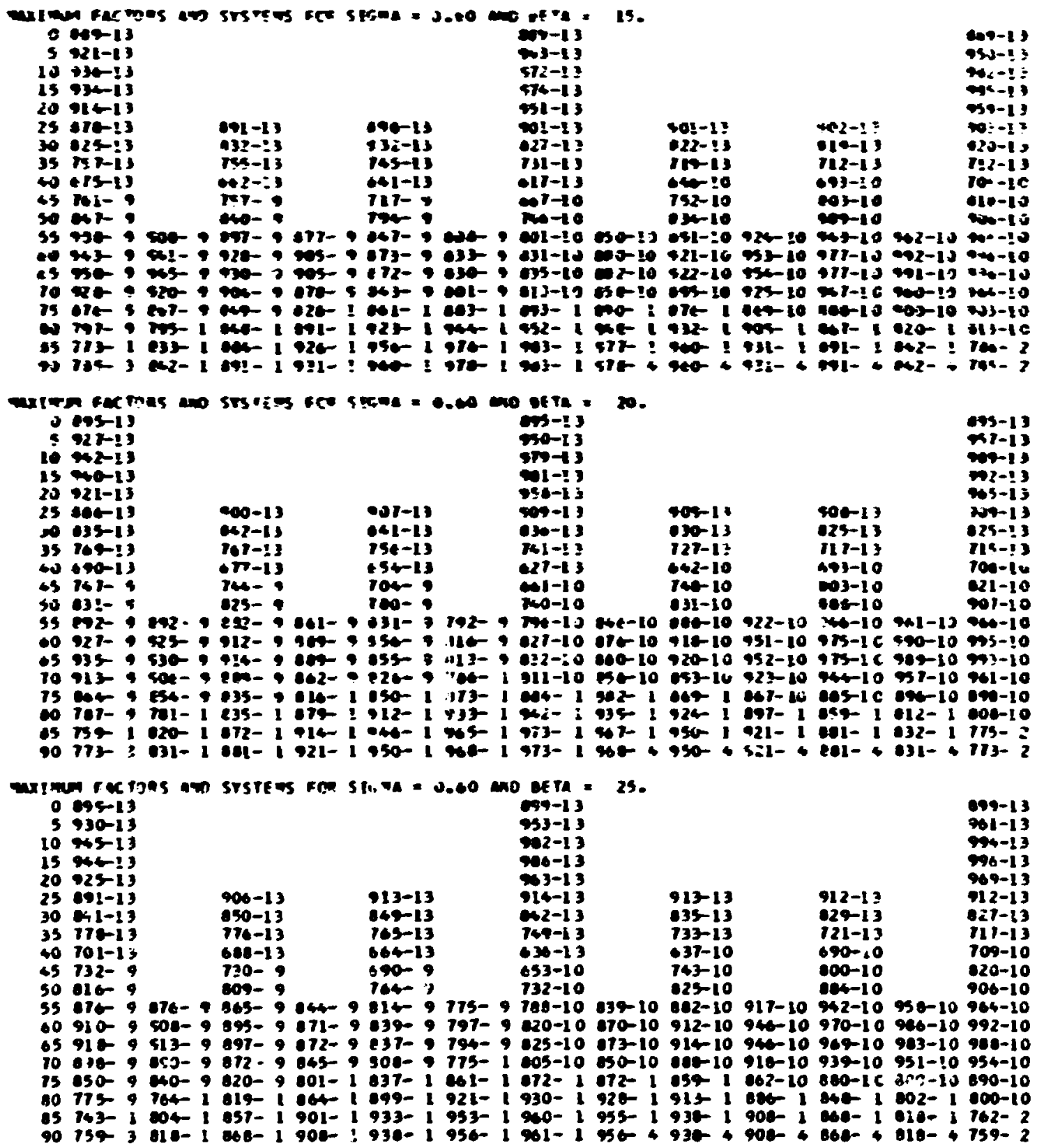




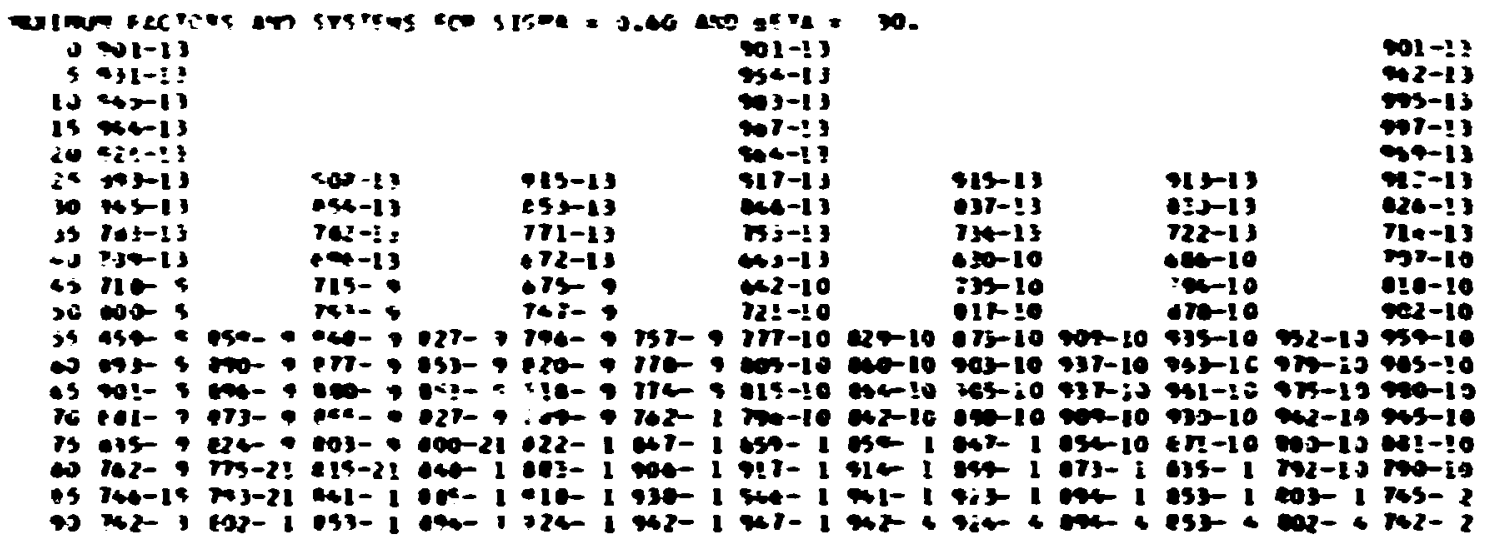

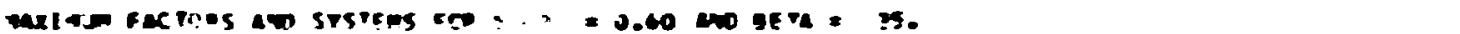

$399-1$

929-1

$4-13$

952-13

1.1 1 :

$205-13$
$23-13$

(5) $201-13$

$29+24-1$

$2591-13$

30 acti:

40 in

$65165-9$

so pos -

$910-13$

$24-13$

155-13

$054-13 \quad$ is $4-1$

$54-23$
$774-13$

$\begin{array}{lll}701-13 & 677-13 & 56-13 \\ 701-9 & \div 55-9 & 29-10\end{array}$

5)-

$701-9$

$\cos -1$

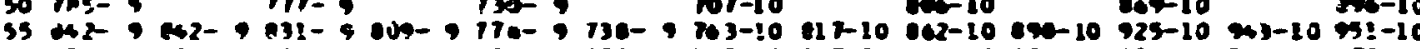

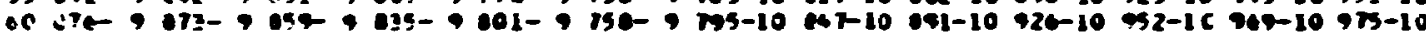

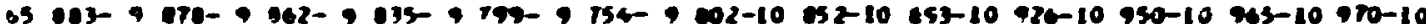

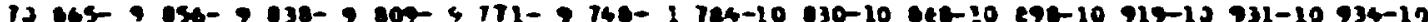

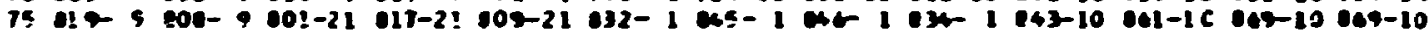

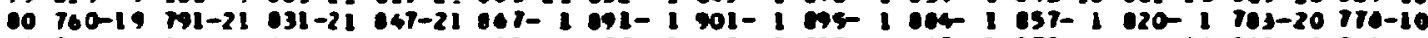

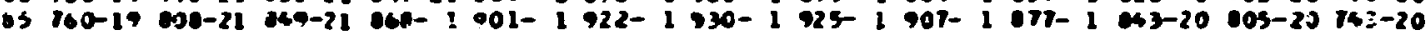

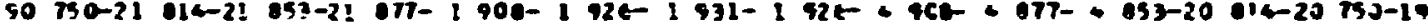

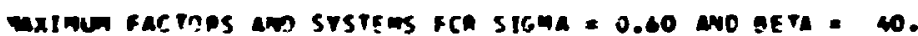

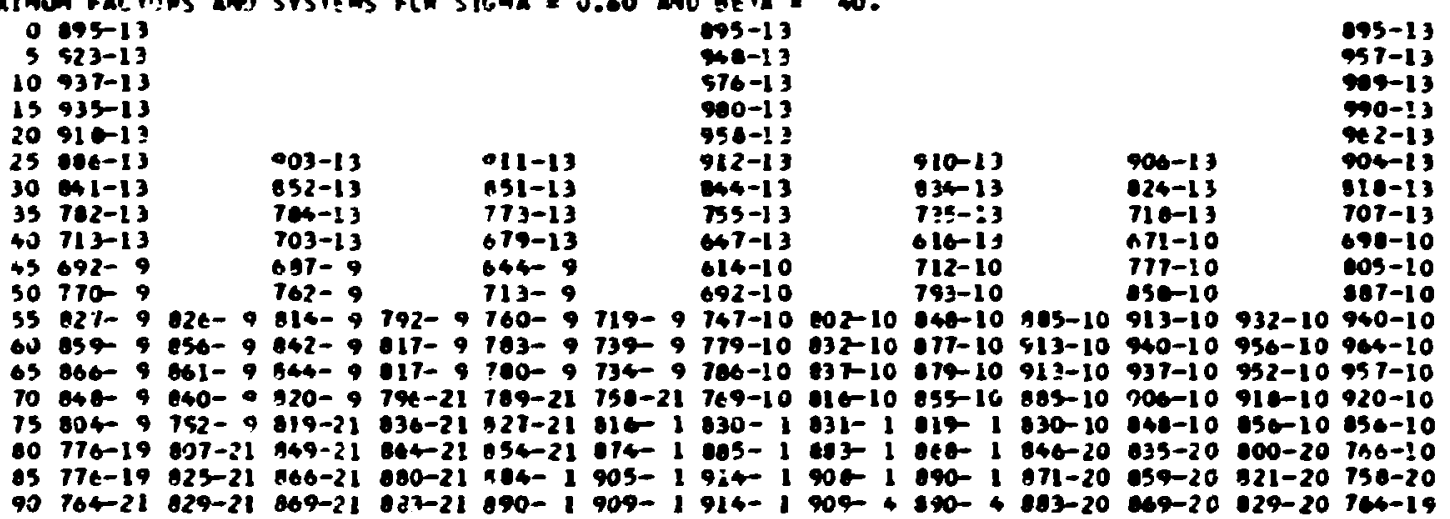




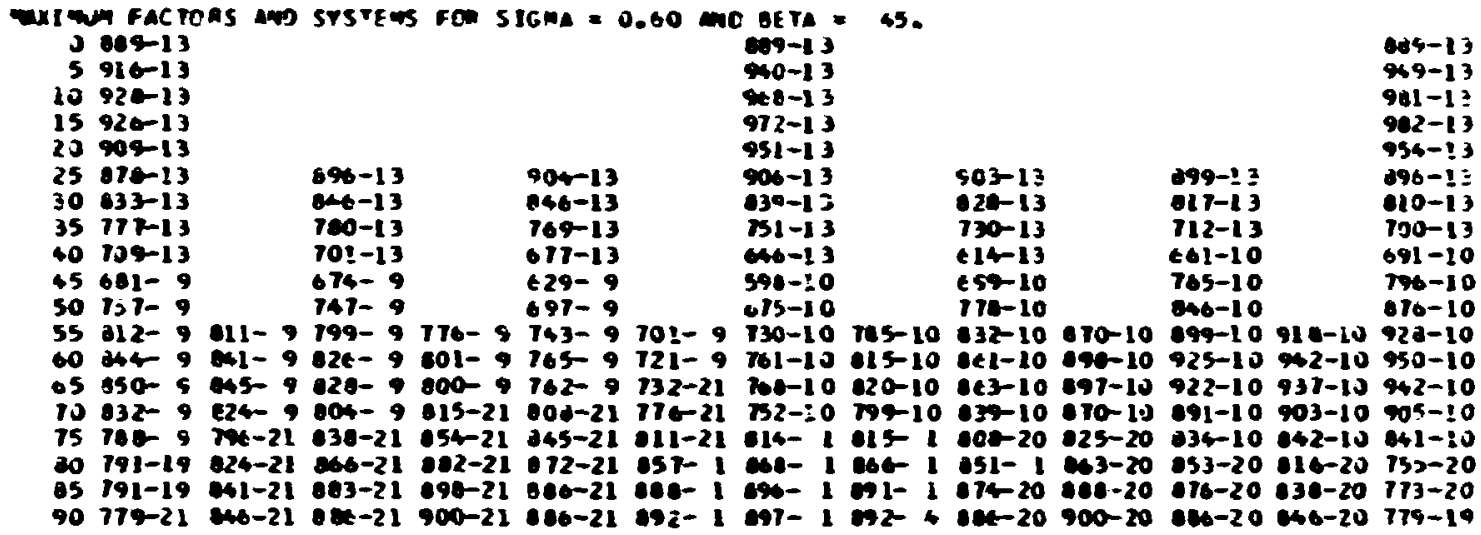

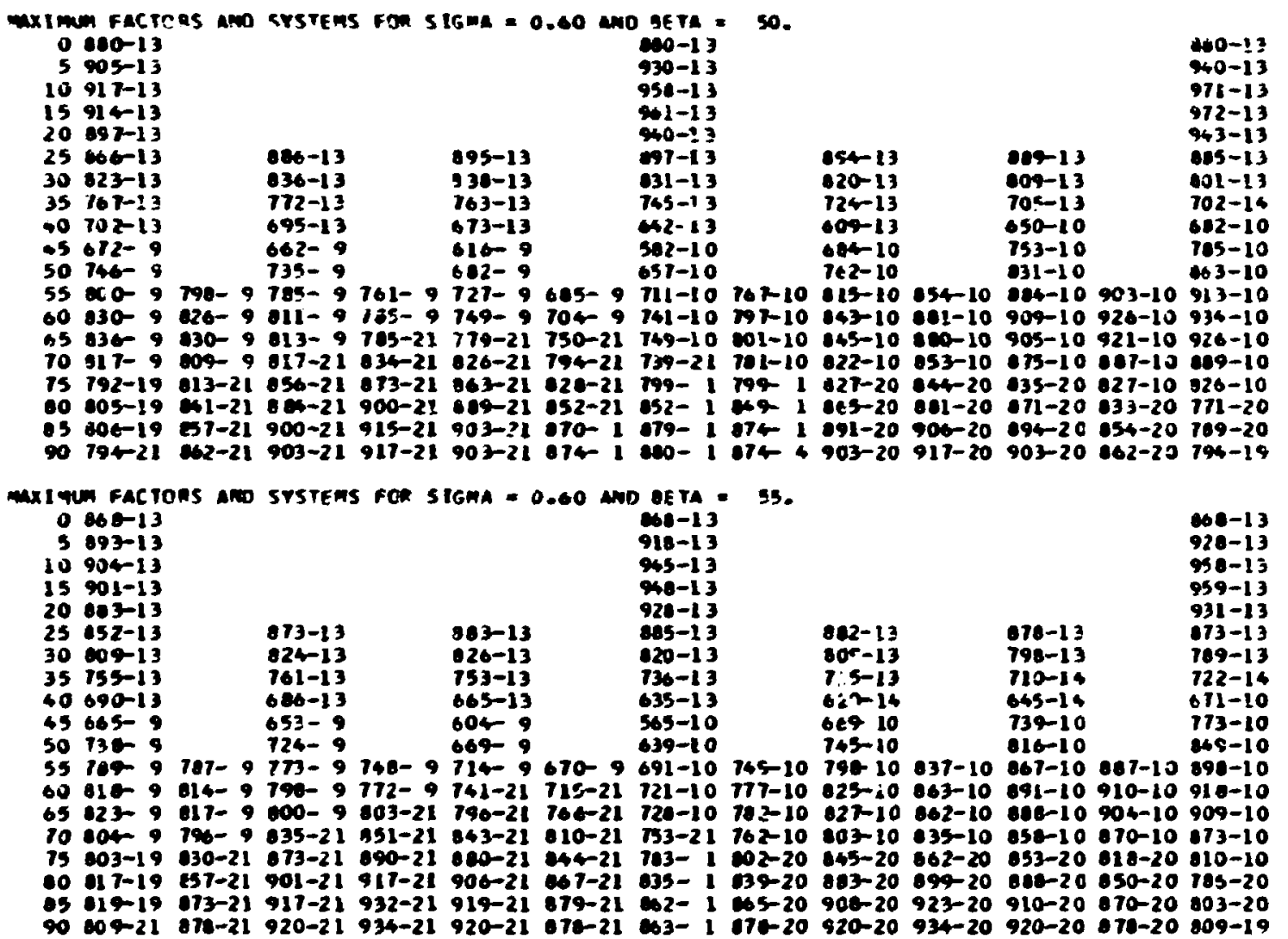




\begin{tabular}{|c|c|c|c|c|c|c|c|c|c|c|c|c|c|}
\hline $\begin{array}{l}0 \\
5 \\
10 \\
15 \\
20 \\
25 \\
10 \\
35 \\
45 \\
15 \\
50 \\
55 \\
03 \\
05 \\
10 \\
75 \\
10 \\
65 \\
90\end{array}$ & $\begin{array}{l}056-13 \\
675-13 \\
605-13 \\
605-13 \\
067-12 \\
030-13 \\
753-13 \\
739-13 \\
670-13 \\
000-9 \\
731-9 \\
701-9 \\
009-9 \\
012-9 \\
192-9 \\
012-19 \\
026-19 \\
032-19 \\
123-21\end{array}$ & 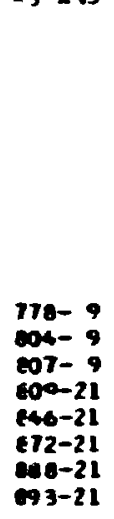 & $\begin{array}{l}657-13 \\
610-15 \\
746-12 \\
676-13 \\
645-9 \\
715-9 \\
765-9 \\
780-9 \\
604-21 \\
651-21 \\
609-21 \\
916-21 \\
632-21 \\
936-21\end{array}$ & $\begin{array}{l} \\
130-0 \\
764-21 \\
620-21 \\
607-21 \\
905-21 \\
932-21 \\
940-21 \\
950-21\end{array}$ & 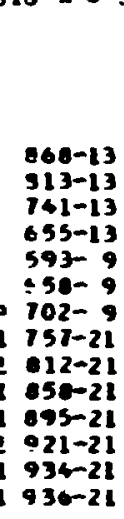 & $\begin{array}{l}\mid \\
012-21 \\
130-21 \\
701-21 \\
024-21 \\
050-21 \\
001-21 \\
093-21 \\
093-21\end{array}$ & $\begin{array}{l}950-13 \\
904-13 \\
930-13 \\
933-13 \\
913-13 \\
671-13 \\
608-13 \\
725-13 \\
626-13 \\
569-10 \\
621-10 \\
672-10 \\
701-10 \\
726-21 \\
766-21 \\
996-21 \\
920-1 \\
646-1 \\
647-1\end{array}$ & $\begin{array}{l}x \\
130-10 \\
750-10 \\
762-10 \\
173-20 \\
620-20 \\
65-20 \\
000-20 \\
61-20\end{array}$ & $\begin{array}{l}969-13 \\
757-13 \\
704-13 \\
630-16 \\
654-10 \\
720-10 \\
76-10 \\
605-10 \\
600-10 \\
615-20 \\
963-20 \\
699-20 \\
924-20 \\
934-20\end{array}$ & $\begin{array}{l} \\
020-10 \\
845-10 \\
446-10 \\
332-20 \\
600-20 \\
916-20 \\
939-20 \\
950-20\end{array}$ & $\begin{array}{l}604-13 \\
760-13 \\
120-14 \\
663-14 \\
724-10 \\
600-10 \\
650-10 \\
74-10 \\
970-10 \\
640-10 \\
670-20 \\
904-20 \\
926-20 \\
936-20\end{array}$ & $\begin{array}{l} \\
871-10 \\
892-10 \\
006-10 \\
853-10 \\
034-20 \\
666-20 \\
005-20 \\
693-20\end{array}$ & $\begin{array}{l}954-1 \equiv \\
914-13 \\
94-13 \\
95-13 \\
914-13 \\
660-13 \\
902-14 \\
740-14 \\
66-14 \\
739-19 \\
934-10 \\
961-10 \\
901-10 \\
992-10 \\
650-111 \\
794-11 \\
601-20 \\
617-20 \\
923-20\end{array}$ \\
\hline $\begin{array}{l}x ! m u \\
0 \\
5 \\
10 \\
15 \\
20 \\
25 \\
35 \\
35 \\
40 \\
45 \\
50 \\
55 \\
60 \\
65 \\
70 \\
75 \\
60 \\
45 \\
90\end{array}$ & $\begin{array}{l}\text { FAC TI } \\
968-14 \\
693-17 \\
902-17 \\
695-17 \\
674-17 \\
637-17 \\
787-17 \\
724-17 \\
657-13 \\
657-9 \\
727-9 \\
176-9 \\
602-9 \\
004-99 \\
790-19 \\
619-15 \\
837-19 \\
642-19 \\
035-21\end{array}$ & $\begin{array}{l}\text { nes am } \\
\\
772-9 \\
797-9 \\
790-9 \\
024-21 \\
059-21 \\
806-21 \\
902-21 \\
506-21\end{array}$ & $\begin{array}{l}940-13 \\
703-13 \\
732-13 \\
659-13 \\
640-9 \\
709-9 \\
750-9 \\
780-9 \\
918-21 \\
865-2 ! \\
903-21 \\
930-21 \\
946-21 \\
950-21\end{array}$ & $\begin{array}{l} \\
\\
730-9 \\
776-21 \\
63-21 \\
801-21 \\
919-21 \\
946-21 \\
961-21 \\
964-21\end{array}$ & $\begin{array}{l}652-13 \\
790-13 \\
727-13 \\
642-13 \\
585-9 \\
650-9 \\
670-21 \\
770-21 \\
770-21 \\
1025-21 \\
671-21 \\
1900-21 \\
1934-21 \\
1940-21 \\
1950-21\end{array}$ & $\begin{array}{l} \\
\\
404-21 \\
742-21 \\
793-21 \\
636-21 \\
870-21 \\
894-21 \\
906-21 \\
906-21\end{array}$ & $\begin{array}{l}65-14 \\
699-13 \\
916-13 \\
917-13 \\
697-13 \\
656-13 \\
793-13 \\
712-13 \\
614-13 \\
539-14 \\
603-10 \\
653-10 \\
692-21 \\
736-21 \\
777-21 \\
606-21 \\
927-21 \\
836-21 \\
935-21\end{array}$ & $\begin{array}{l} \\
71-10 \\
71-10 \\
739-10 \\
742-10 \\
991-20 \\
63-20 \\
611-20 \\
69-20 \\
90-20\end{array}$ & $\begin{array}{l}654-13 \\
703-13 \\
711-16 \\
652-14 \\
630-10 \\
711-10 \\
762-10 \\
707-10 \\
709-10 \\
633-20 \\
679-20 \\
915-20 \\
939-20 \\
950-20\end{array}$ & $\begin{array}{l} \\
002-10 \\
27-10 \\
22-10 \\
950-20 \\
896-20 \\
931-20 \\
954-20 \\
964-20\end{array}$ & $\begin{array}{l}950-13 \\
798-14 \\
742-14 \\
679-14 \\
710-10 \\
786-10 \\
233-10 \\
250-10 \\
853-10 \\
841-20 \\
896-20 \\
920-20 \\
941-20 \\
950-20\end{array}$ & $\begin{array}{l} \\
\\
954-10 \\
975-10 \\
869-10 \\
837-10 \\
849-20 \\
800-20 \\
999-20 \\
906-20\end{array}$ & 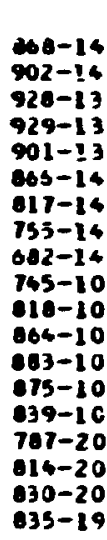 \\
\hline $\begin{array}{l}x 190 \\
0 \\
5 \\
10 \\
15 \\
20 \\
25 \\
30 \\
35 \\
40 \\
45 \\
50 \\
55 \\
60 \\
65 \\
70 \\
75 \\
60 \\
85 \\
90\end{array}$ & $\begin{array}{l}\text { FACT } \\
000-11 \\
905-17 \\
915-17 \\
910-17 \\
609-17 \\
853-17 \\
603-17 \\
741-17 \\
669-17 \\
657-9 \\
726-99 \\
773-9 \\
798-9 \\
799-9 \\
791-19 \\
022-19 \\
843-19 \\
251-19 \\
964-21\end{array}$ & $\begin{array}{l}760-9 \\
793-9 \\
793-9 \\
935-21 \\
071-21 \\
097-21 \\
913-21 \\
510-21\end{array}$ & $\begin{array}{l}822-13 \\
775-13 \\
715-17 \\
640-17 \\
637-9 \\
705-9 \\
752-9 \\
775-9 \\
630-21 \\
971-21 \\
914-21 \\
942-21 \\
950-21 \\
962-21\end{array}$ & $\begin{array}{l} \\
\\
727-21 \\
709-21 \\
044-21 \\
092-21 \\
930-21 \\
958-21 \\
973-21 \\
977-21\end{array}$ & 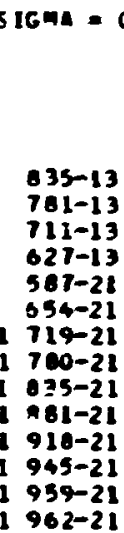 & $\begin{array}{l} \\
093-21 \\
750-21 \\
801-21 \\
045-21 \\
079-21 \\
904-21 \\
917-21 \\
910-21\end{array}$ & $\begin{array}{l}000-14 \\
079-14 \\
97-13 \\
00-13 \\
000-13 \\
639-13 \\
177-13 \\
697-13 \\
009-14 \\
540-14 \\
592-21 \\
648-21 \\
700-21 \\
766-21 \\
704-21 \\
615-21 \\
035-21 \\
066-21 \\
860-20\end{array}$ & $\begin{array}{l}894-10 \\
120-10 \\
754-20 \\
607-20 \\
051-20 \\
65-20 \\
907-20 \\
918-20\end{array}$ & $\begin{array}{l}830-13 \\
774-14 \\
721-14 \\
663-14 \\
624-10 \\
696-10 \\
744-10 \\
770-10 \\
754-20 \\
949-20 \\
995-20 \\
929-20 \\
932-210 \\
962-20\end{array}$ & $\begin{array}{l}7 \\
785-10 \\
109-10 \\
910-20 \\
966-20 \\
911-20 \\
745-20 \\
767-20 \\
971-20\end{array}$ & $\begin{array}{l}954-14 \\
809-14 \\
754-14 \\
691-14 \\
095-10 \\
760-10 \\
616-10 \\
639-10 \\
636-10 \\
951-20 \\
900-20 \\
933-20 \\
954-20 \\
962-20\end{array}$ & $\begin{array}{l} \\
037-10 \\
050-10 \\
852-10 \\
022-20 \\
003-20 \\
092-20 \\
911-20 \\
918-20\end{array}$ & $\begin{array}{l}800-14 \\
913-14 \\
929-14 \\
920-14 \\
911-14 \\
871-14 \\
029-14 \\
707-14 \\
695-14 \\
731-10 \\
802-10 \\
840-10 \\
806-10 \\
858-10 \\
024-10 \\
799-20 \\
025-20 \\
041-20 \\
846-20\end{array}$ \\
\hline
\end{tabular}




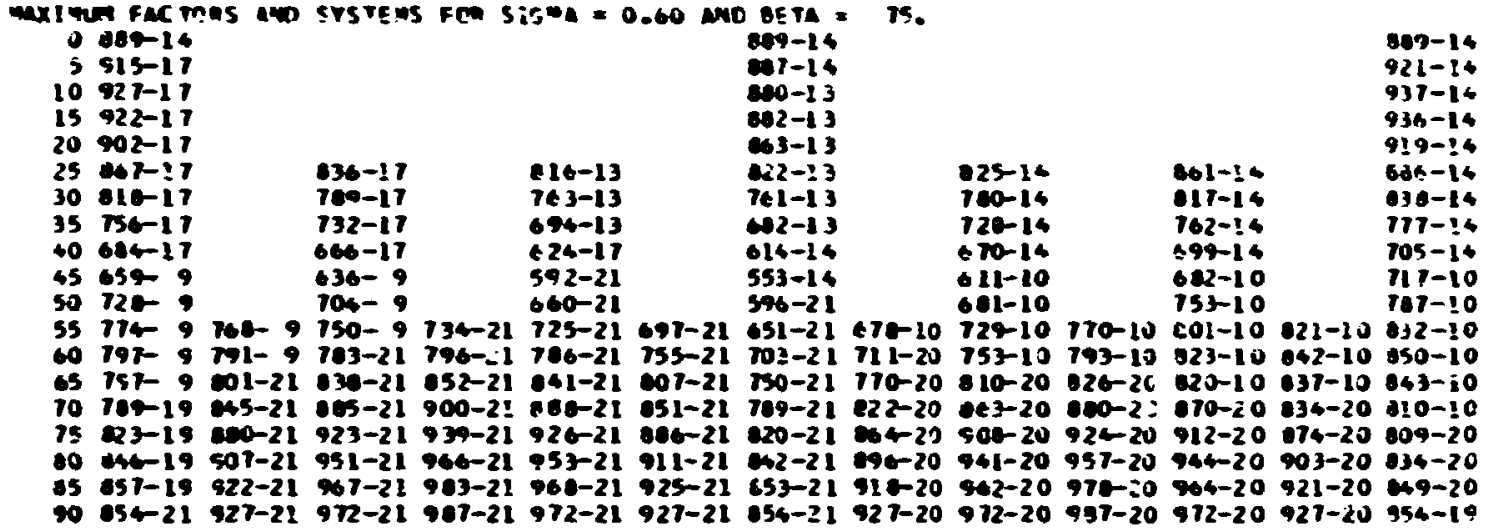

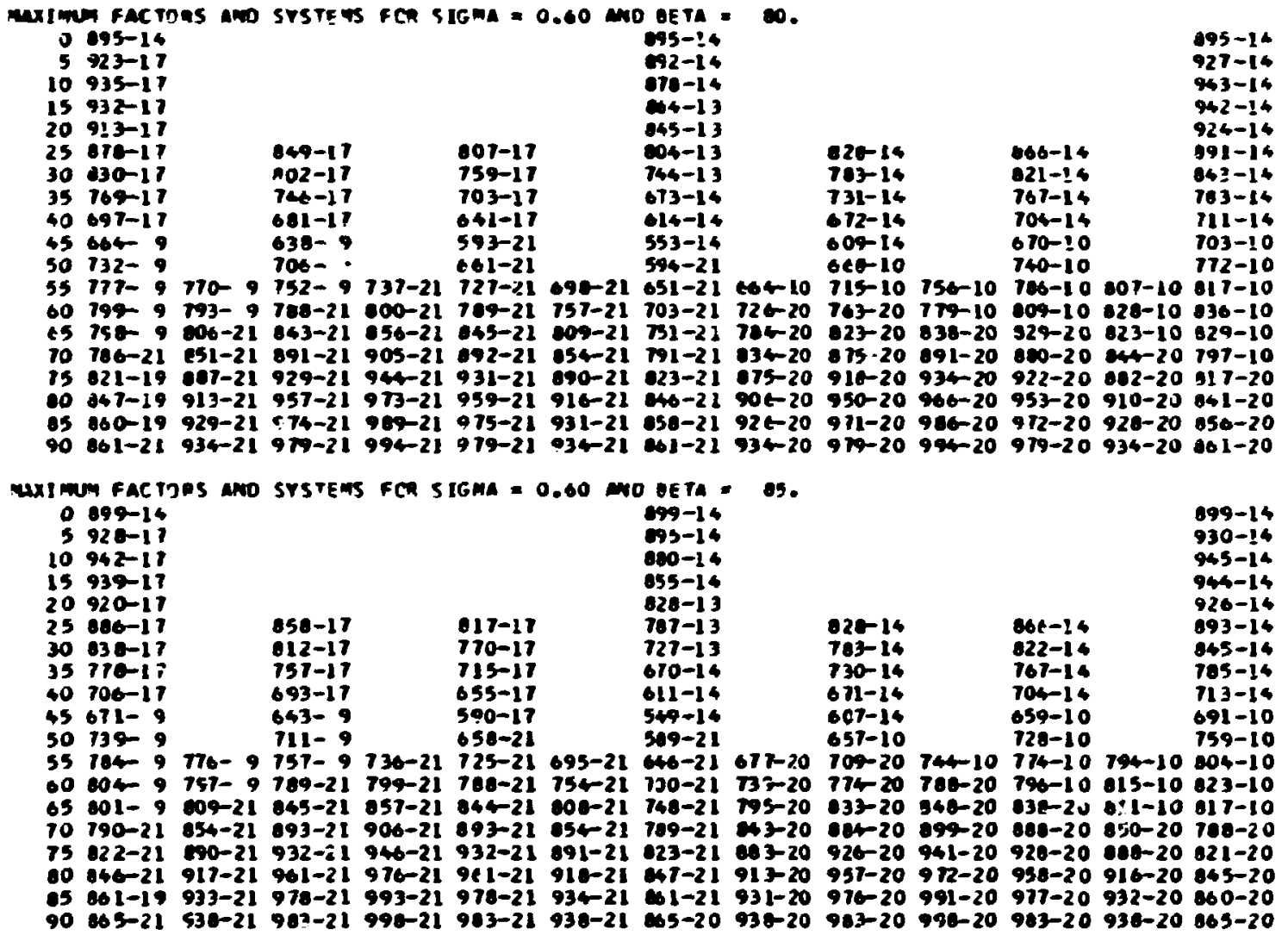




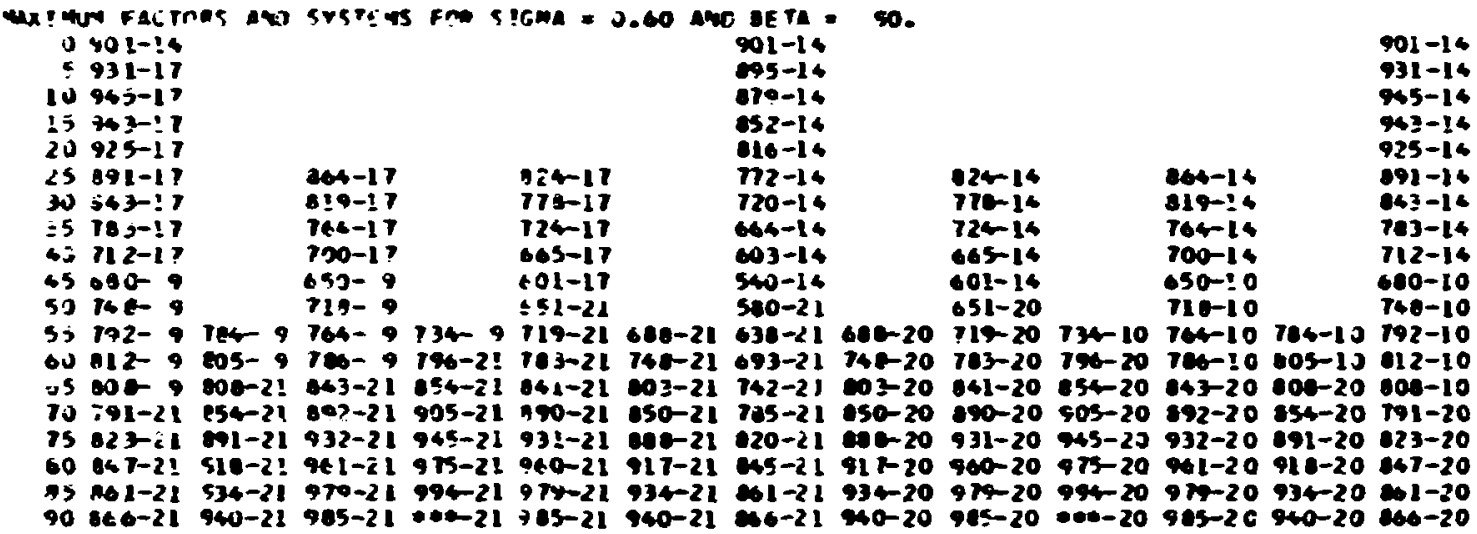




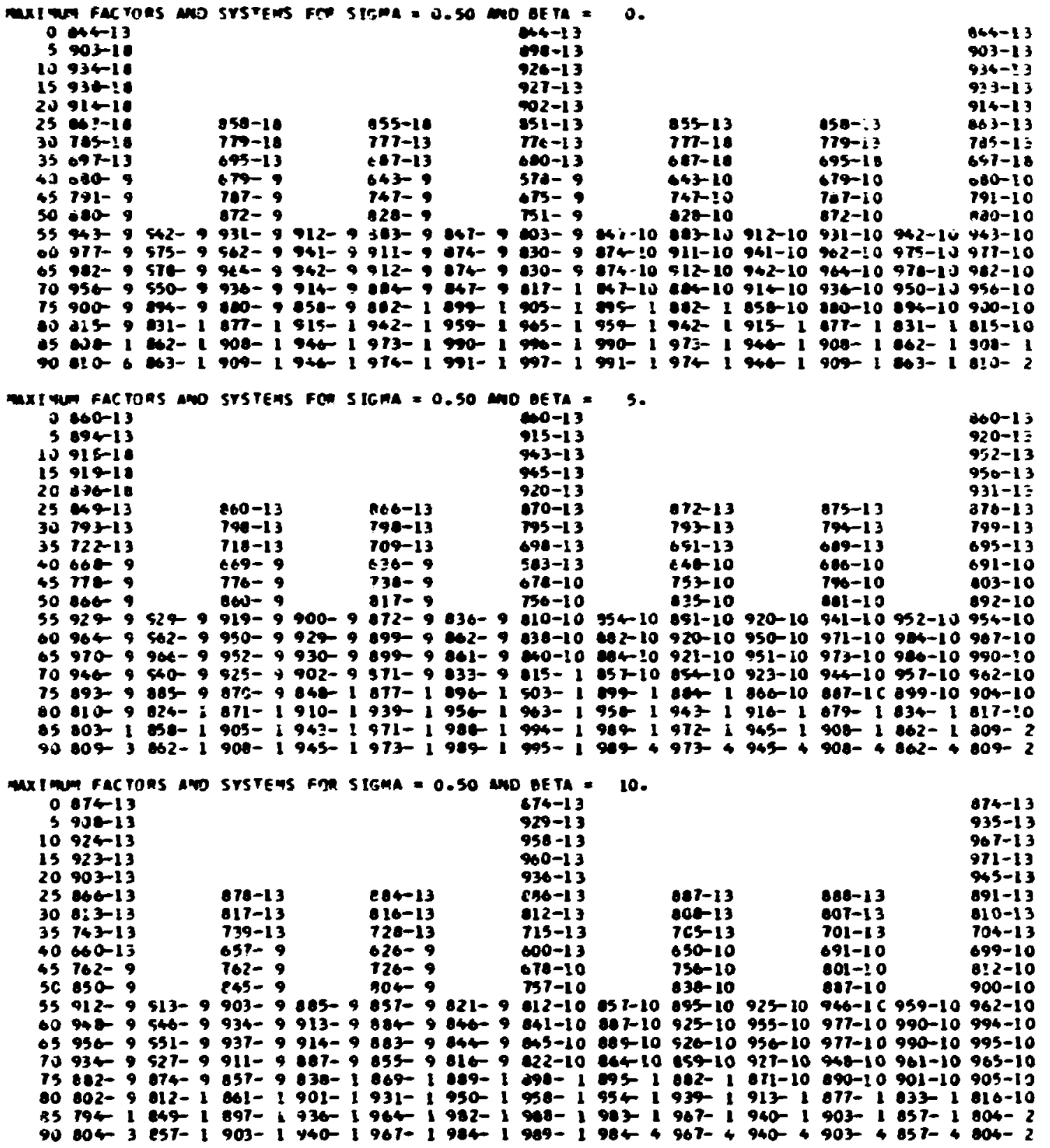




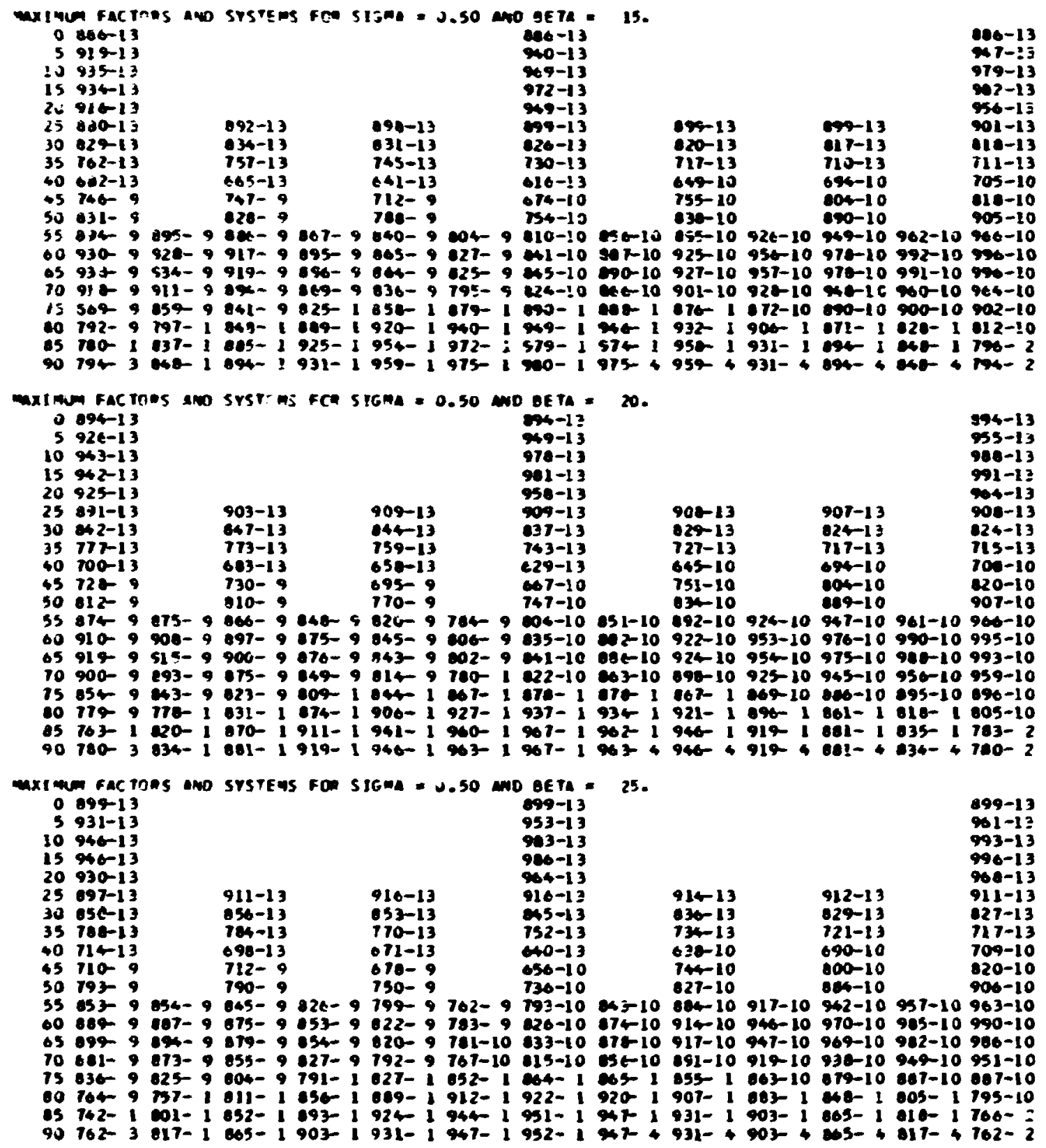




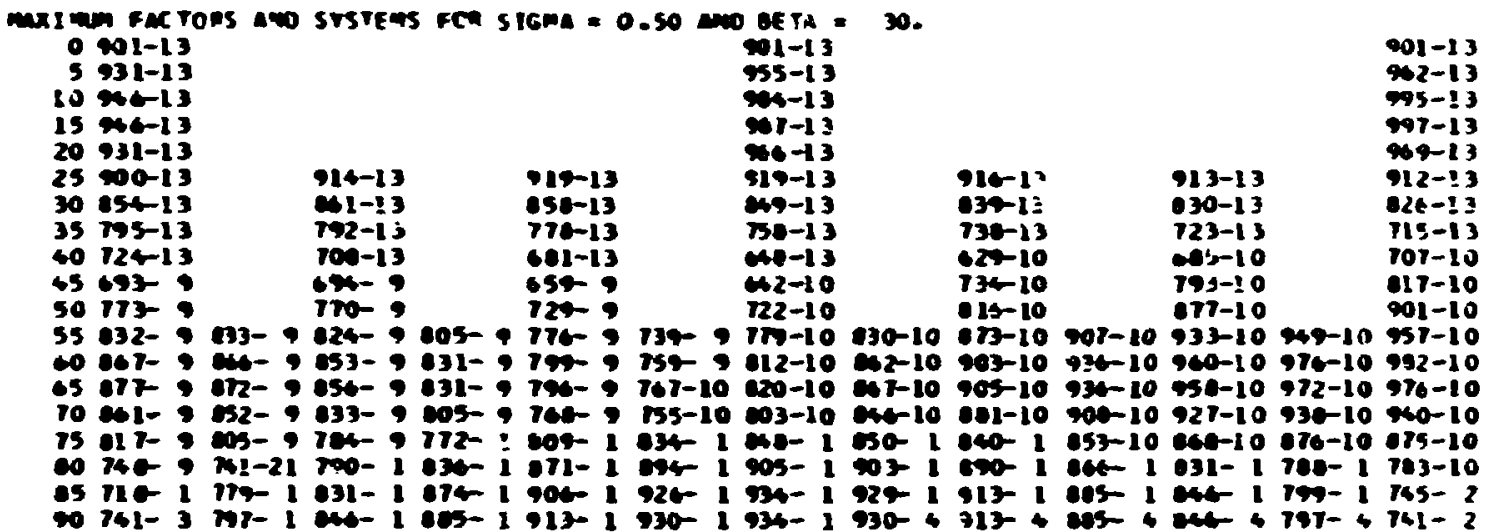

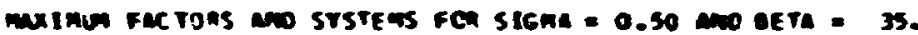

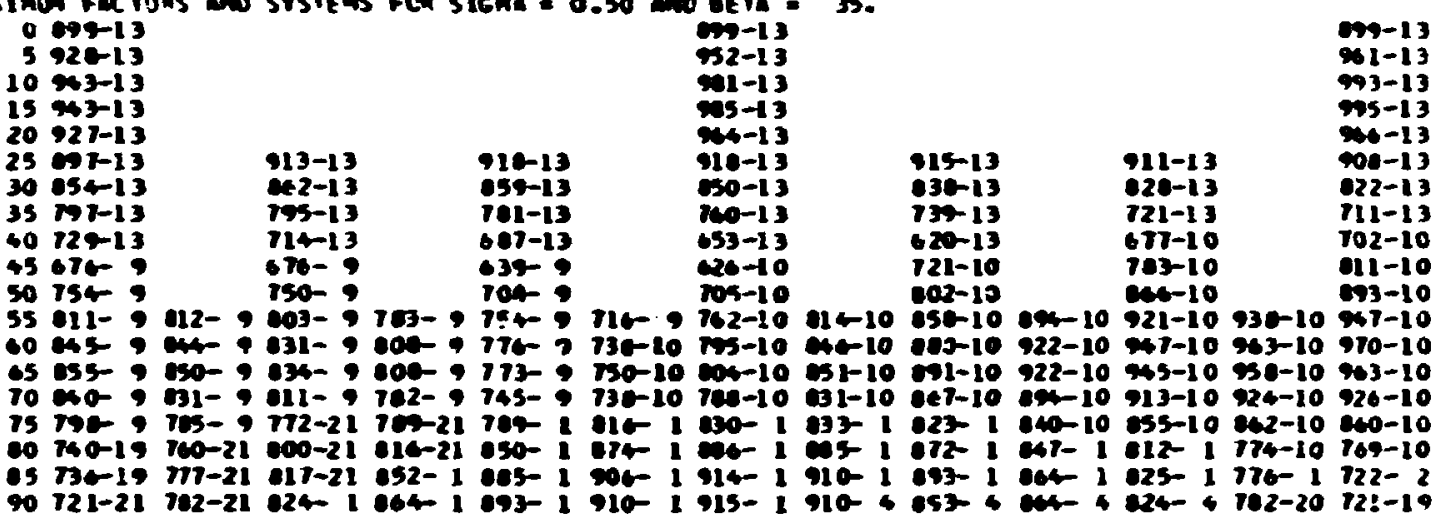

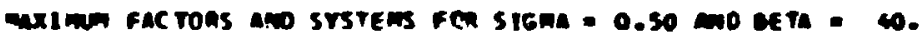

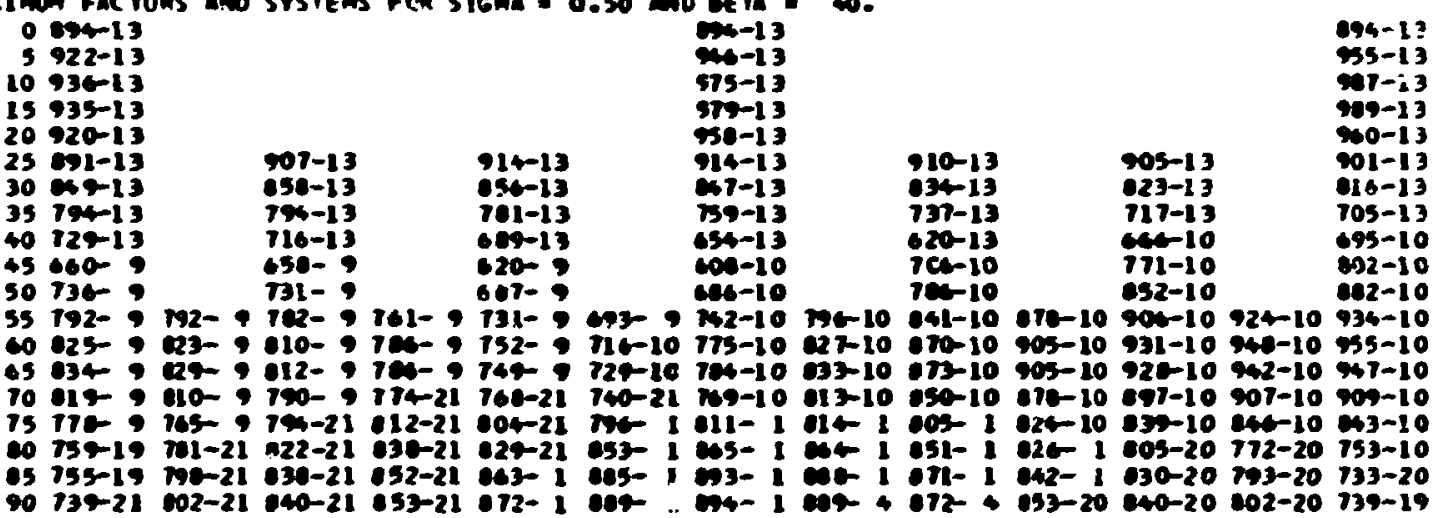




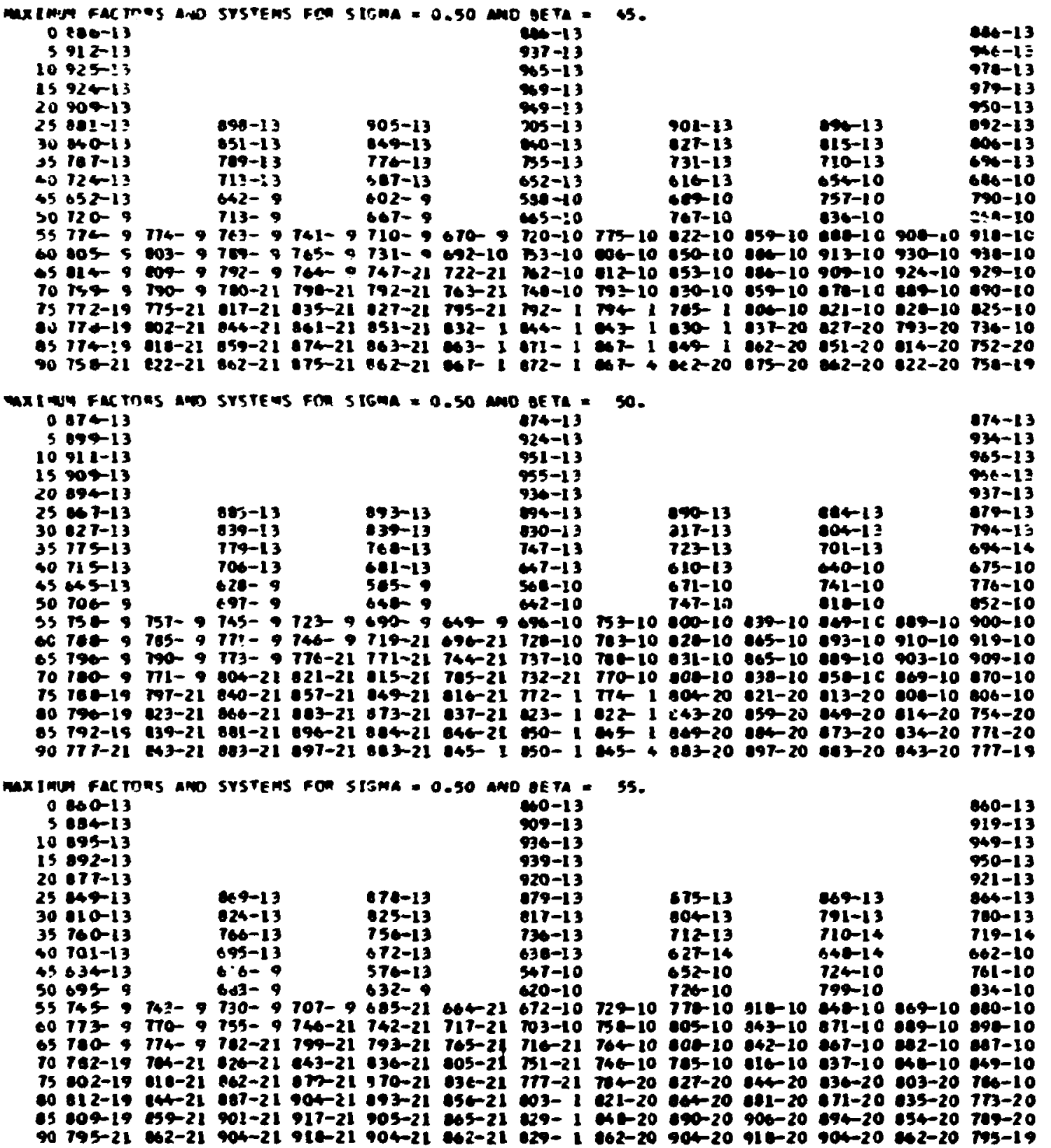




\begin{tabular}{|c|c|c|c|c|c|c|c|c|c|c|c|c|c|}
\hline $\begin{array}{l}0 \\
5 \\
10 \\
15 \\
20 \\
25 \\
30 \\
35 \\
40 \\
45 \\
50 \\
55 \\
60 \\
65 \\
70 \\
15 \\
10 \\
105 \\
90\end{array}$ & $\begin{array}{l}46-13 \\
607-13 \\
376-13 \\
173-13 \\
057-13 \\
025-13 \\
790-13 \\
761-13 \\
603-13 \\
619-9 \\
607-9 \\
735-9 \\
702-9 \\
760-9 \\
791-19 \\
114-19 \\
025-19 \\
025-19 \\
012-21\end{array}$ & $\begin{array}{l}7 \\
\\
732-9 \\
750-9 \\
763-21 \\
604-21 \\
830-21 \\
063-21 \\
077-21 \\
001-21\end{array}$ & $\begin{array}{l}650-13 \\
06-13 \\
740-13 \\
600-13 \\
606-9 \\
672-9 \\
710-9 \\
751-21 \\
002-21 \\
940-21 \\
901-21 \\
906-21 \\
921-21 \\
923-21\end{array}$ & $\begin{array}{l}l \\
710-21 \\
767-21 \\
19-21 \\
903-21 \\
998-21 \\
923-21 \\
930-21 \\
937-21\end{array}$ & $\begin{array}{l}600-13 \\
600-13 \\
761-13 \\
659-13 \\
502-21 \\
645-21 \\
705-21 \\
762-21 \\
613-21 \\
055-21 \\
069-21 \\
012-21 \\
924-21 \\
923-21\end{array}$ & $\begin{array}{l}\text { o3-21 } \\
73-21 \\
703-21 \\
022-21 \\
053-21 \\
074-21 \\
063-21 \\
061-21\end{array}$ & $\begin{array}{l}06-12 \\
m 2-13 \\
917-13 \\
921-13 \\
02-13 \\
202-13 \\
61-13 \\
722-13 \\
627-13 \\
545-16 \\
597-10 \\
646-10 \\
690-21 \\
732-21 \\
707-21 \\
793-21 \\
609-21 \\
010-21 \\
112-21\end{array}$ & & $\begin{array}{l}050-13 \\
709-13 \\
705-14 \\
644-14 \\
632-10 \\
7 c 5-10 \\
755-10 \\
702-10 \\
704-16 \\
001-20 \\
649-20 \\
104-20 \\
911-20 \\
923-20\end{array}$ & & $\begin{array}{l}652-13 \\
705-16 \\
732-14 \\
671-14 \\
702-10 \\
779-10 \\
627-10 \\
69-10 \\
65-10 \\
615-10 \\
650-20 \\
992-20 \\
914-20 \\
923-20\end{array}$ & & 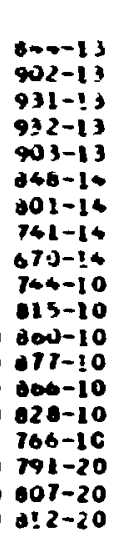 \\
\hline $\begin{array}{l}x 170 \\
0 \\
5 \\
10 \\
15 \\
20 \\
25 \\
30 \\
35 \\
40 \\
45 \\
50 \\
55 \\
60 \\
65 \\
70 \\
75 \\
80 \\
05 \\
90\end{array}$ & $\begin{array}{l}10 \text { FaC In } \\
060-14 \\
603-17 \\
-32-17 \\
060-17 \\
665-17 \\
630-17 \\
701-17 \\
721-17 \\
661-13 \\
616-9 \\
002-9 \\
720-9 \\
753-99 \\
701-19 \\
796-19 \\
021-19 \\
036-19 \\
036-19 \\
027-21\end{array}$ & $\begin{array}{l}7 \\
74-9 \\
769-9 \\
781-21 \\
222-21 \\
055-21 \\
679-21 \\
894-21 \\
090-21\end{array}$ & $\begin{array}{l}829-13 \\
785-13 \\
729-13 \\
662-13 \\
600-9 \\
664-9 \\
714-21 \\
770-21 \\
820-21 \\
664-21 \\
899-21 \\
924-21 \\
938-21 \\
941-21\end{array}$ & $\begin{array}{l} \\
727-21 \\
105-21 \\
330-21 \\
800-21 \\
915-21 \\
940-21 \\
954-21 \\
955-21\end{array}$ & $\begin{array}{l}640-13 \\
709-13 \\
723-13 \\
643-13 \\
599-21 \\
661-21 \\
722-21 \\
770-21 \\
629-21 \\
97-21 \\
905-21 \\
928-21 \\
941-21 \\
941-21\end{array}$ & $\begin{array}{l} \\
\\
690-21 \\
751-21 \\
790-21 \\
037-21 \\
060-21 \\
009-21 \\
099-21 \\
090-21\end{array}$ & $\begin{array}{l}60-14 \\
62-13 \\
97-13 \\
900-13 \\
602-13 \\
643-13 \\
703-13 \\
700-13 \\
610-14 \\
501-14 \\
605-21 \\
650-21 \\
703-21 \\
745-21 \\
700-21 \\
600-21 \\
823-21 \\
030-21 \\
027-21\end{array}$ & 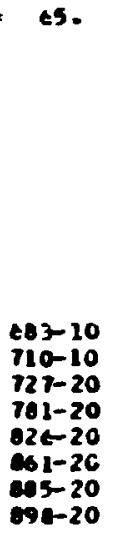 & $\begin{array}{l}840 \\
172 \\
722 \\
667 \\
614 \\
684 \\
723 \\
759 \\
760 \\
624 \\
870 \\
905 \\
929 \\
941\end{array}$ & $\begin{array}{l}7 \\
774-10 \\
796-10 \\
796-10 \\
941-20 \\
897-20 \\
922-20 \\
945-20 \\
995-20\end{array}$ & $\begin{array}{l}045-14 \\
603-14 \\
751-16 \\
691-16 \\
607-10 \\
758-10 \\
805-10 \\
627-16 \\
923-10 \\
834-20 \\
678-20 \\
911-20 \\
932-20 \\
941-20\end{array}$ & $\begin{array}{l} \\
\\
\\
-10 \\
-10 \\
-10 \\
-10 \\
-20 \\
-20 \\
-20 \\
-20\end{array}$ & $\begin{array}{l}-16 \\
-14 \\
-16 \\
-16 \\
-16 \\
-16 \\
-16 \\
-16 \\
-16 \\
-10 \\
-10 \\
-10 \\
-10 \\
-10 \\
-10 \\
-20 \\
-20 \\
-20 \\
-19\end{array}$ \\
\hline $\begin{array}{l}1100 \\
0 \\
5 \\
10 \\
15 \\
20 \\
25 \\
30 \\
35 \\
40 \\
45 \\
50 \\
55 \\
60 \\
05 \\
70 \\
15 \\
10 \\
05 \\
90\end{array}$ & 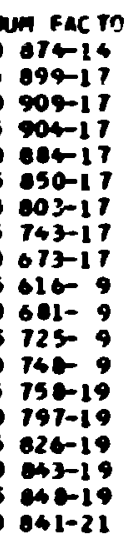 & $\begin{array}{l}0 \\
120-9 \\
769-21 \\
790-21 \\
637-21 \\
670-21 \\
696-21 \\
508-21 \\
512-21\end{array}$ & $\begin{array}{l}918-17 \\
772-17 \\
716-17 \\
652-17 \\
606-21 \\
668-21 \\
728-21 \\
760-21 \\
635-21 \\
676-21 \\
913-21 \\
936-21 \\
953-21 \\
996-21\end{array}$ & $\begin{array}{l} \\
\\
941-21 \\
190-21 \\
950-21 \\
994-21 \\
929-21 \\
955-21 \\
969-21 \\
911-21\end{array}$ & $\begin{array}{l}010-13 \\
760-13 \\
703-13 \\
624-13 \\
610-21 \\
073-21 \\
73-21 \\
790-21 \\
641-21 \\
96-21 \\
918-21 \\
942-21 \\
955-21 \\
950-21\end{array}$ & $\begin{array}{r}\text { } \\
700-21 \\
701-21 \\
009-21 \\
049-21 \\
000-21 \\
902-21 \\
913-21 \\
012-21\end{array}$ & 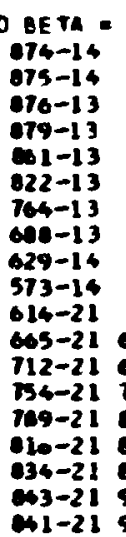 & $\begin{array}{r}0 \\
61-10 \\
60-20 \\
749-20 \\
01-20 \\
61-20 \\
17-20 \\
901-20 \\
912-20\end{array}$ & $\begin{array}{l}625-14 \\
764-14 \\
735-14 \\
681-14 \\
622-14 \\
665-10 \\
712-10 \\
736-10 \\
750-20 \\
844-20 \\
694-20 \\
923-20 \\
945-20 \\
954-20\end{array}$ & $\begin{array}{l} \\
\\
753-10 \\
770-10 \\
800-20 \\
962-20 \\
900-20 \\
939-20 \\
961-20 \\
970\end{array}$ & $\begin{array}{l}850-14 \\
817-14 \\
765-14 \\
706-14 \\
669-10 \\
739-10 \\
705-10 \\
805-10 \\
801-10 \\
853-20 \\
996-20 \\
927-20 \\
940-20 \\
901-20\end{array}$ & $\begin{array}{l}0 \\
\\
\\
000-10 \\
025-10 \\
018-10 \\
020-20 \\
659-20 \\
688-20 \\
905-20 \\
01<-20\end{array}$ & $\begin{array}{l}974-14 \\
908-14 \\
926-: 4 \\
927-14 \\
911-14 \\
800-14 \\
634-14 \\
776-14 \\
707-14 \\
708-10 \\
776-10 \\
910-10 \\
034-10 \\
824-10 \\
180-10 \\
797-20 \\
021-20 \\
836-20 \\
941-20\end{array}$ \\
\hline
\end{tabular}




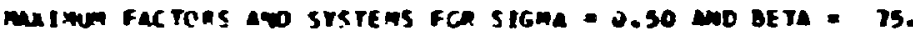

- oce-14

5 912-1?

$10823-18$

15 919-11

20 sodit

$25607-17$

$30621-17$

35 162-17

$40093-17$

45 ol 99

50 $603-9$

$671-2 !$

930-21 760-21 741-21

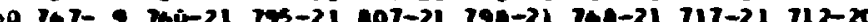

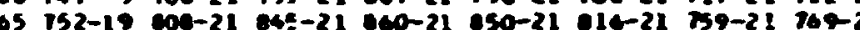

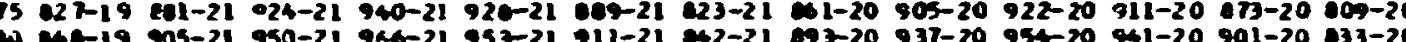

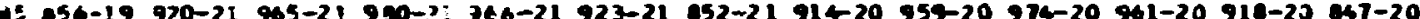

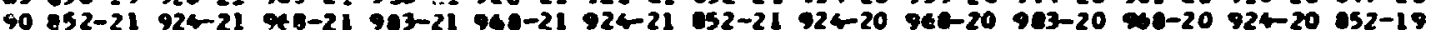

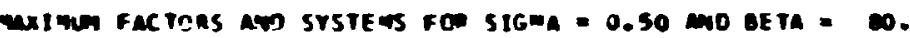

$0 \rightarrow 4-14$

$5921-17$

12 934-17

15 931-17

20 914-17

25 at-11

30 835-17

35 i77-17

40 roti?

$45632-17$

30 o.6- 9

052-11

$000-17$

755-1]

694-11

(26-1

795-13

693-17

(3)-17

6
$600-21$

690-1

(35-1 4

579-1

$617-2$

743-14

$609-14$

631-16
900-10

$936-16$

937-14

$091-10$

045-14

707-14

$719-16$

$\begin{array}{ll}716-14 & 797-16 \\ 653-10 & 691-19\end{array}$

157-20
B.9-14

92-14

(50)

27-16

$700-14$

$765-17 \quad 762-14$

$\begin{array}{lll}112-17 & 091-14 \\ 034-11 & 630-14\end{array}$

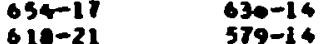

$750-14$

$692-14$

$122-14$

657-14

$928-16$

944-14

697-14

$852-16$

$795-16$
$727-14$

$674-10$

$38-10$ 753-21 744-21 715-21 660-21 671-20 705-20 719-20 747-10 760-10 779-10 $\begin{array}{lllllllllll} & \end{array}$

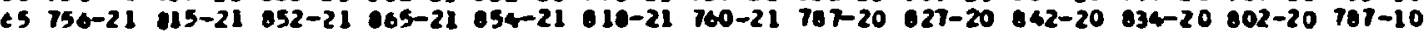

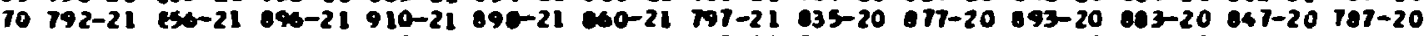
75 024-19 $969-21 \quad 932-21 \quad 947-21 \quad 934-21 \quad 99-21 \quad 927-21 \quad 974-20 \quad 516-20 \quad 934-20 \quad 923-20 \quad 884-20 \quad 819-20$ 10 - 49 -19 914-21 958-21 $973-21$ 959-21 $917-21 \quad 47-21$ 904-20 945-20 965-20 952-20 910-20 841-20

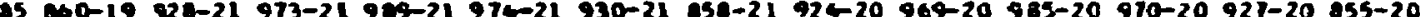
90 959-21 933-21 971-21 992-21 971-21 933-21 959-21 933-20 971-20 992-20 977-20 935-20 959-20

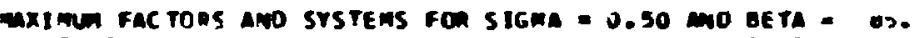

0 099-14

592018

10 942-17

15 940-11

$20923-17$

25 091-: ?

30 840-1?

35 ite-1 7

$40720-17$

45 64-11

$50 \div 90-9$

55 73-9 79-9 763-21 752-21 741-21 711-21 663-21 649-20 721-20 735-20 732-10 753-10 763-10

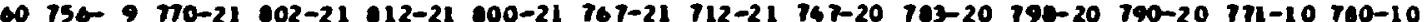

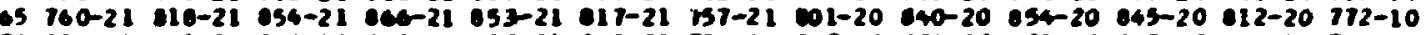

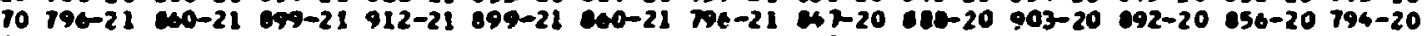
$75 \quad 920-21 \quad 94-21 \quad 935-21$ 950-21 936-2: 694-21 $126-21$ 10s-20 $920-20$ 943-20 $931-20 \quad 091-20 \quad 025-20$ 00 1-21 910-21 962-21 917-21 963-21 919-21 949-21 913-20 957-20 913-20 959-20 917-20 $447-20$

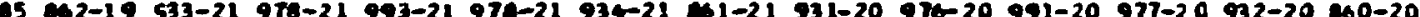

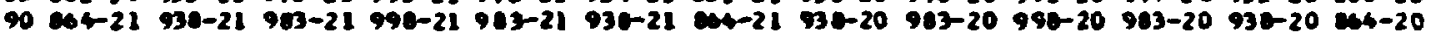




\begin{tabular}{|c|c|c|c|c|c|c|c|c|c|c|c|c|c|}
\hline $\begin{array}{l}3 \\
5 \\
10 \\
15 \\
20 \\
25 \\
30 \\
35 \\
40 \\
45 \\
50 \\
55 \\
60 \\
65 \\
70 \\
75 \\
60 \\
65 \\
90\end{array}$ & 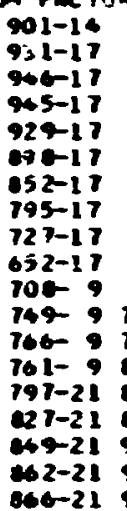 & $\begin{array}{l}739-9 \\
767-21 \\
117-21 \\
60-21 \\
66-21 \\
919-21 \\
535-21 \\
260-21\end{array}$ & $\begin{array}{l}671-17 \\
629-17 \\
771-17 \\
710-17 \\
652-17 \\
676--9 \\
730-21 \\
795-21 \\
652-21 \\
990-21 \\
935-21 \\
962-21 \\
979-21\end{array}$ & $\begin{array}{l}1 \\
746-21 \\
007-21 \\
662-21 \\
910-21 \\
940-21 \\
977-21 \\
990-21 \\
0 * 0-21\end{array}$ & $\begin{array}{l}632-17 \\
709-17 \\
739-17 \\
603-17 \\
623-17 \\
689-21 \\
734-21 \\
794-21 \\
649-21 \\
694-21 \\
934-21 \\
942-21 \\
979-21 \\
705-21\end{array}$ & $\begin{array}{l}7 \\
702-21 \\
759-21 \\
11-21 \\
955-21 \\
991-21 \\
910-21 \\
934-21 \\
940-21\end{array}$ & $\begin{array}{l}901-14 \\
695-14 \\
800-14 \\
955-24 \\
022-14 \\
760-14 \\
732-14 \\
620-14 \\
022-14 \\
563-14 \\
590-21 \\
652-21 \\
704-21 \\
750-21 \\
790-21 \\
923-21 \\
66-21 \\
61-21 \\
606-21\end{array}$ & $\begin{array}{l}02-20 \\
102-20 \\
759-20 \\
011-20 \\
055-20 \\
911-20 \\
918-20 \\
934-20 \\
940-20\end{array}$ & $\begin{array}{l}632-14 \\
749-14 \\
799-14 \\
603-14 \\
623-16 \\
669-20 \\
734-20 \\
794-20 \\
049-20 \\
694-20 \\
924-20 \\
962-20 \\
979-20 \\
905-20\end{array}$ & $\begin{array}{l} \\
746-20 \\
907-20 \\
902-20 \\
910-20 \\
946-20 \\
977-20 \\
994-20 \\
91-20\end{array}$ & $\begin{array}{l}811-14 \\
829-14 \\
717-16 \\
710-14 \\
652-14 \\
670-10 \\
130-20 \\
790-20 \\
852-20 \\
990-20 \\
935-20 \\
962-20 \\
979-20\end{array}$ & $\begin{array}{l}739-13 \\
767-20 \\
117-20 \\
860-20 \\
494-20 \\
919-20 \\
935-20 \\
940-20\end{array}$ & $\begin{array}{l}901-16 \\
93114 \\
940-14 \\
9<5-14 \\
929-14 \\
998-14 \\
952-14 \\
795-14 \\
727-14 \\
652-14 \\
708-10 \\
769-10 \\
764-10 \\
761-10 \\
797-20 \\
927-20 \\
149-20 \\
862-20 \\
962-20\end{array}$ \\
\hline
\end{tabular}




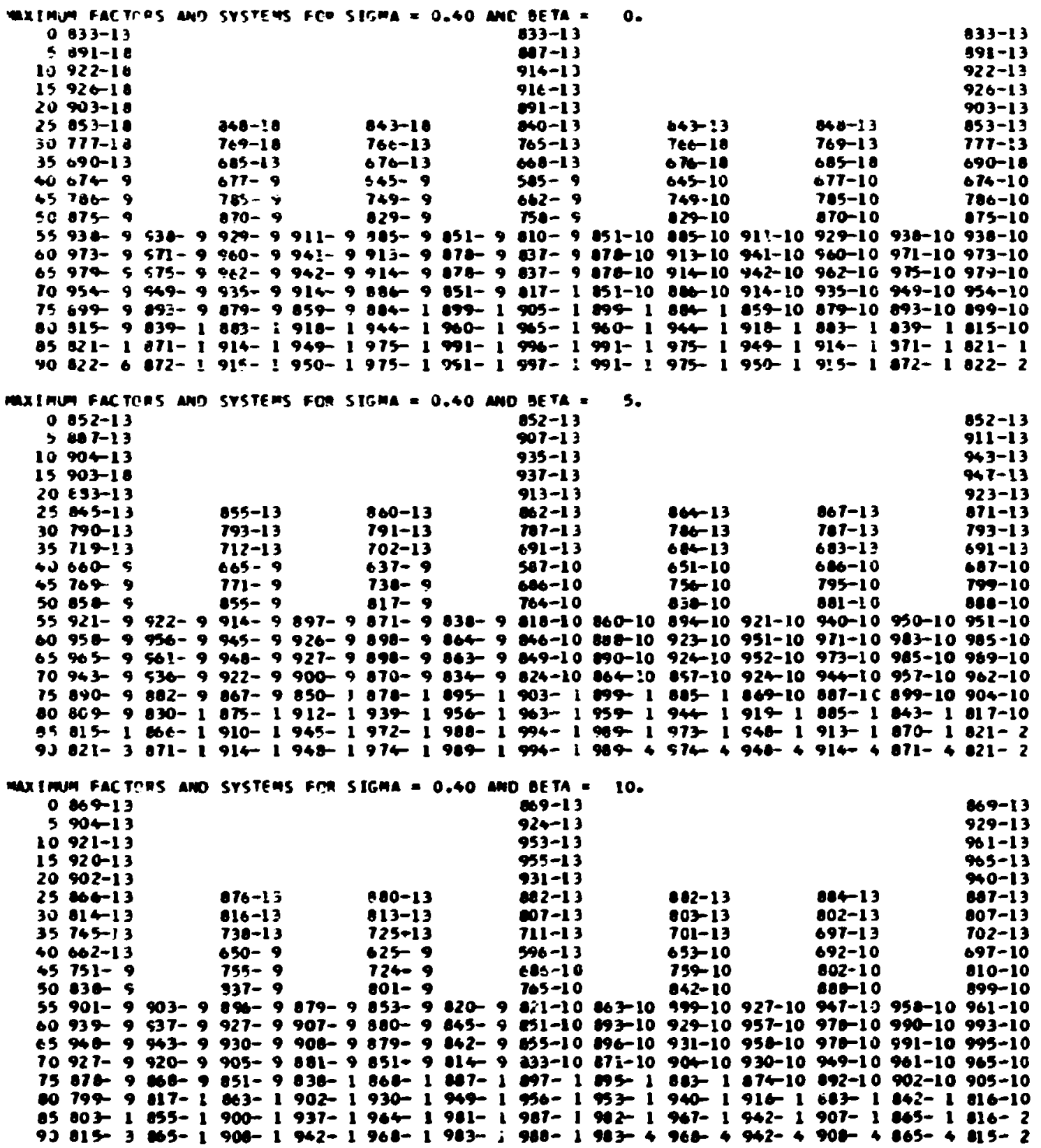




\begin{tabular}{|c|c|c|c|c|c|c|c|c|c|c|c|c|c|}
\hline $\begin{array}{l}13 \\
15 \\
15 \\
20 \\
25 \\
30 \\
35 \\
40 \\
45 \\
50 \\
55 \\
60 \\
85 \\
70 \\
75 \\
80 \\
85 \\
90\end{array}$ & 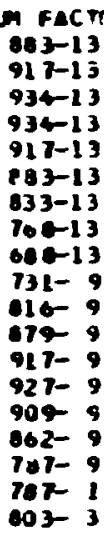 & $\begin{array}{l}3 \\
3 \\
3 \\
3 \\
3 \\
3 \\
9 \\
902-9 \\
910-9 \\
523-9 \\
901-9 \\
651-9 \\
290-1 \\
150-1 \\
054-1\end{array}$ & $\begin{array}{l}893-13 \\
836-13 \\
760-13 \\
668-13 \\
736-9 \\
817-9 \\
874-9 \\
905-9 \\
909-9 \\
805-9 \\
833-9 \\
867-1 \\
896-1 \\
897-1\end{array}$ & $\begin{array}{l}858- \\
680- \\
687- \\
860- \\
822- \\
607- \\
924- \\
932- \\
932-\end{array}$ & $\begin{array}{r}897-13 \\
631-13 \\
746-13 \\
642-13 \\
106-9 \\
7101-9 \\
9832-9 \\
9858-9 \\
9850-9 \\
982-9 \\
1854-1 \\
1917-1 \\
1252-1 \\
1957-1\end{array}$ & $\begin{array}{ll}3 \\
3 \\
3 \\
3 \\
9 \\
9 \\
9 \\
9 & 000-9 \\
9 & 022-9 \\
9 & 19-9 \\
9 & 91-10 \\
1 & 676-1 \\
1 & 937-1 \\
1 & 96+1 \\
1 & 912-1\end{array}$ & $\begin{array}{l}803-13 \\
938-13 \\
267-13 \\
970-13 \\
947-13 \\
690-13 \\
624-13 \\
729-13 \\
615-13 \\
601-10 \\
761-10 \\
818-10 \\
950-10 \\
956-10 \\
635-10 \\
687-1 \\
196-1 \\
1976-1 \\
976-1\end{array}$ & 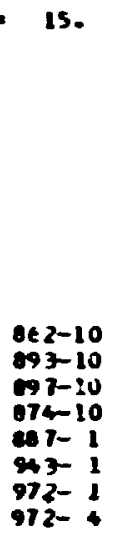 & $\begin{array}{l}857-13 \\
612-13 \\
715-13 \\
652-10 \\
759-10 \\
642-10 \\
659-10 \\
930-10 \\
932-10 \\
906-10 \\
670-1 \\
931-1 \\
957-1 \\
957-4\end{array}$ & $\begin{array}{l} \\
\\
929-10 \\
950-10 \\
959-10 \\
931-10 \\
970-10 \\
900-1 \\
931-1 \\
932-4\end{array}$ & $\begin{array}{l}997-13 \\
015-13 \\
800-13 \\
095-10 \\
695-10 \\
691-10 \\
950-10 \\
919-10 \\
919-10 \\
950-10 \\
091-10 \\
870-1 \\
897-1 \\
091-4\end{array}$ & $\begin{array}{l}2-10 \\
22-10 \\
1-10 \\
0-10 \\
5-1 \\
5-1 \\
6-1\end{array}$ & 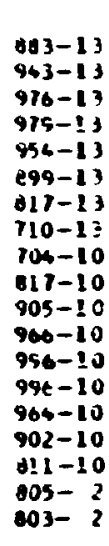 \\
\hline $\begin{array}{l}x 170 \\
0 \\
5 \\
10 \\
15 \\
20 \\
25 \\
30 \\
35 \\
40 \\
45 \\
50 \\
55 \\
60 \\
45 \\
10 \\
75 \\
80 \\
85 \\
90\end{array}$ & $\begin{array}{l}\text { FAC T } \\
693-13 \\
926-13 \\
943-13 \\
944-13 \\
920-13 \\
076-13 \\
240-13 \\
786-13 \\
710-13 \\
710-99 \\
793-9 \\
855-9 \\
893-9 \\
964-9 \\
800-9 \\
943-9 \\
771-9 \\
766-1 \\
786-3\end{array}$ & $\begin{array}{l}3 \\
3 \\
3 \\
3 \\
3 \\
3 \\
9 \\
9058-9 \\
892-9 \\
899-9 \\
9079-9 \\
961-9 \\
776-1 \\
021-1 \\
3030-1\end{array}$ & $\begin{array}{l}907-13 \\
851-13 \\
770-13 \\
690-13 \\
716-9 \\
755-9 \\
851-9 \\
881-9 \\
885-9 \\
862-9 \\
811-9 \\
827-1 \\
860-1 \\
801-1\end{array}$ & $\begin{array}{l}834- \\
861- \\
862- \\
836- \\
803- \\
669- \\
901- \\
911-\end{array}$ & $\begin{array}{r}910-13 \\
846-13 \\
163-13 \\
662-13 \\
607-9 \\
760-9 \\
9009-9 \\
9833-9 \\
9831-9 \\
9002-9 \\
1037-1 \\
1000-1 \\
1936-1 \\
1942-1\end{array}$ & $\begin{array}{ll}3 \\
3 \\
3 \\
3 \\
9 \\
9 \\
9 & 716-9 \\
9 & 797-9 \\
9 & 803-10 \\
9 & 100-10 \\
1 & 861-1 \\
1 & 921-1 \\
1 & 954-1 \\
1 & 957-1\end{array}$ & $\begin{array}{l}0 E 1 A= \\
993-13 \\
947-13 \\
917-13 \\
901-13 \\
950-13 \\
910-13 \\
038-13 \\
746-13 \\
631-13 \\
672-10 \\
753-10 \\
911-10 \\
964-10 \\
651-10 \\
032-10 \\
973-1 \\
931-1 \\
961-1 \\
962-1\end{array}$ & 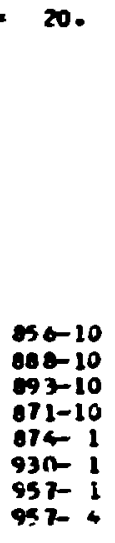 & $\begin{array}{l}900-13 \\
829-13 \\
727-13 \\
047-10 \\
754-10 \\
937-10 \\
895-10 \\
925-10 \\
920-10 \\
903-10 \\
863-1 \\
910-1 \\
942-1 \\
942-4\end{array}$ & $\begin{array}{l} \\
925-10 \\
925-10 \\
955-10 \\
950-10 \\
928-10 \\
972-10 \\
996-1 \\
917-1 \\
911-4\end{array}$ & $\begin{array}{rl}-1 & 3 \\
-1 & 3 \\
-1 & 3 \\
-1 & 0 \\
-1 & 0 \\
-10 & 0 \\
-1 & 0 \\
-1 & 0 \\
-1 & 0 \\
-1 & 0 \\
-1 & 0 \\
-1 \\
-1 \\
-1\end{array}$ & $\begin{array}{l}961-10 \\
900-10 \\
900-10 \\
956-10 \\
894-10 \\
824-1 \\
638-1 \\
038-1\end{array}$ & $\begin{array}{l}993-13 \\
954-13 \\
907-13 \\
990-13 \\
963-13 \\
907-13 \\
926-13 \\
715-13 \\
700-10 \\
820-10 \\
907-10 \\
906-10 \\
995-10 \\
992-10 \\
950-10 \\
896-10 \\
803-10 \\
790-2 \\
706-2\end{array}$ \\
\hline $\begin{array}{l}110 \\
0 \\
5 \\
10 \\
15 \\
20 \\
25 \\
30 \\
35 \\
40 \\
45 \\
50 \\
55 \\
60 \\
65 \\
70 \\
75 \\
80 \\
85 \\
90\end{array}$ & $\begin{array}{l}9460 \\
899-13 \\
931-13 \\
947-13 \\
949-13 \\
934-13 \\
904-13 \\
850-13 \\
799-13 \\
727-13 \\
686-9 \\
370-9 \\
630-9 \\
867-9 \\
679-9 \\
644-9 \\
622-9 \\
753-9\end{array}$ & 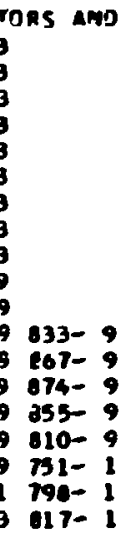 & $\begin{array}{l}915-13 \\
862-13 \\
792-13 \\
707-13 \\
695-9 \\
771-9 \\
826-9 \\
856-9 \\
860-9 \\
637-9 \\
780-9 \\
604-9 \\
646-1 \\
862-1\end{array}$ & $\begin{array}{l}835- \\
836- \\
610- \\
781- \\
847- \\
806- \\
890-\end{array}$ & $\begin{array}{r}919-13 \\
857-13 \\
776-13 \\
677-13 \\
665-9 \\
736-9 \\
9793-9 \\
9006-9 \\
9804-9 \\
9775-9 \\
1017-1 \\
10130-1 \\
1980-1 \\
1916-1 \\
1924-1\end{array}$ & 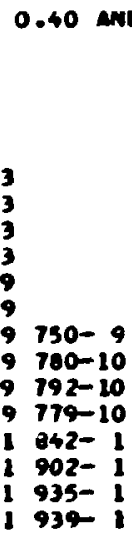 & $\begin{array}{l}999-13 \\
953-13 \\
903-13 \\
207-13 \\
965-13 \\
918-13 \\
67-13 \\
755-13 \\
646-13 \\
659-10 \\
740-10 \\
799-10 \\
932-10 \\
961-10 \\
824-10 \\
956-1 \\
913-1 \\
943-1 \\
943-1\end{array}$ & $\begin{array}{l} \\
\\
046-10 \\
410-10 \\
98-10 \\
063-10 \\
859-1 \\
913-1 \\
939-1 \\
939-4\end{array}$ & $\begin{array}{l}915-13 \\
837-19 \\
735-13 \\
639-10 \\
745-10 \\
620-10 \\
886-10 \\
916-10 \\
919-10 \\
895-10 \\
851-1 \\
901-1 \\
924-1 \\
924-4\end{array}$ & $\begin{array}{l} \\
918-10 \\
967-10 \\
947-10 \\
920-10 \\
865-10 \\
879-1 \\
890-1 \\
990-4\end{array}$ & $\begin{array}{l}912-13 \\
829-13 \\
722-13 \\
691-10 \\
800-10 \\
804-10 \\
941-10 \\
969-10 \\
960-10 \\
937-10 \\
870-10 \\
840-1 \\
862-1 \\
862-4\end{array}$ & $\begin{array}{l}2 \\
\\
956-10 \\
964-10 \\
960-10 \\
947-10 \\
804-10 \\
800-1 \\
019-1 \\
617-4\end{array}$ & $\begin{array}{l}999-13 \\
90-13 \\
993-17 \\
996-13 \\
960-12 \\
911-13 \\
627-13 \\
117-13 \\
797-10 \\
620-10 \\
906-10 \\
963-10 \\
909-10 \\
964-10 \\
969-10 \\
863-10 \\
791-10 \\
769-2 \\
765-2\end{array}$ \\
\hline
\end{tabular}




\begin{tabular}{|c|c|c|c|c|c|c|c|c|c|c|c|c|c|}
\hline $\begin{array}{l}0 \\
5 \\
12 \\
15 \\
20 \\
23 \\
33 \\
35 \\
40 \\
65 \\
50 \\
55 \\
60 \\
85 \\
70 \\
75 \\
80 \\
85 \\
90\end{array}$ & 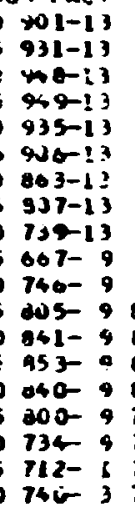 & $\begin{array}{l} \\
808-9 \\
41-9 \\
840-9 \\
631-9 \\
784-9 \\
723-1 \\
71-1 \\
793-1\end{array}$ & $\begin{array}{l}919-13 \\
600-13 \\
601-13 \\
720-13 \\
673-9 \\
767-9 \\
200-9 \\
620-9 \\
233-9 \\
811-9 \\
706-9 \\
778-9 \\
622-1 \\
039-1\end{array}$ & $\begin{array}{l} \\
\\
703-9 \\
000-9 \\
009-9 \\
103-9 \\
752-1 \\
623-1 \\
863-1 \\
074-1\end{array}$ & 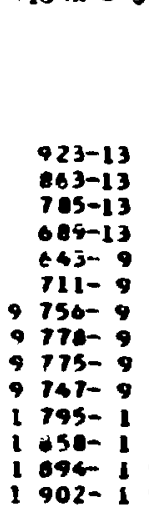 & $\begin{array}{l}2 \\
726-10 \\
7 \in 2-10 \\
715-10 \\
765-10 \\
022-1 \\
901-1 \\
913-1 \\
910-1\end{array}$ & $\begin{array}{l}901-13 \\
955-13 \\
904-13 \\
908-13 \\
967-13 \\
921-13 \\
952-13 \\
762-13 \\
654-13 \\
643-10 \\
723-10 \\
702-10 \\
916-10 \\
926-10 \\
910-10 \\
257-1 \\
993-1 \\
921-1 \\
922-1\end{array}$ & 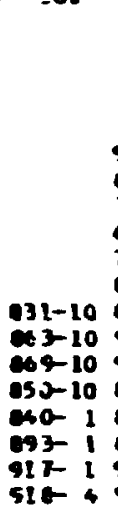 & $\begin{array}{l}917-13 \\
640-13 \\
740-13 \\
620-10 \\
733-10 \\
015-10 \\
672-10 \\
903-10 \\
904-10 \\
102-10 \\
932-1 \\
981-1 \\
902-1 \\
962-4\end{array}$ & $\begin{array}{l}2 \\
900-10 \\
934-10 \\
935-10 \\
907-10 \\
253-10 \\
159-1 \\
076-1 \\
875-4\end{array}$ & $\begin{array}{l}913-13 \\
931-13 \\
123-13 \\
624-10 \\
792-10 \\
675-10 \\
931-10 \\
950-10 \\
955-10 \\
926-10 \\
966-10 \\
920-1 \\
840-1 \\
929-4\end{array}$ & $\begin{array}{l} \\
\\
94:-10 \\
973-10 \\
969-10 \\
934-10 \\
671-10 \\
707-1 \\
795-1 \\
793-4\end{array}$ & $\begin{array}{l}901-13 \\
962-13 \\
993-12 \\
997-13 \\
969-13 \\
911-12 \\
920-11 \\
715-13 \\
707-10 \\
916-10 \\
900-10 \\
955-10 \\
979-10 \\
972-10 \\
935-10 \\
869-10 \\
710-10 \\
763-2 \\
740-2\end{array}$ \\
\hline $\begin{array}{l}1 \mathrm{m0} \\
0 \\
5 \\
10 \\
15 \\
20 \\
25 \\
30 \\
35 \\
40 \\
45 \\
50 \\
55 \\
60 \\
85 \\
70 \\
75 \\
80 \\
85 \\
90\end{array}$ & $\begin{array}{l}\text { Un FAC TMI } \\
994-13 \\
920-13 \\
943-13 \\
944-13 \\
531-13 \\
904-13 \\
003-13 \\
810-13 \\
745-13 \\
609-13 \\
723-9 \\
700-9 \\
115-9 \\
227-9 \\
014-9 \\
170-9 \\
720-19 \\
711-19 \\
711-3\end{array}$ & $\begin{array}{l} \\
703-9 \\
814-9 \\
622-9 \\
205-9 \\
763-9 \\
730-21 \\
746-21 \\
306-1\end{array}$ & $\begin{array}{l}916-13 \\
669-13 \\
805-13 \\
727-13 \\
651-9 \\
723-9 \\
776-9 \\
603-9 \\
807-9 \\
705-2 \\
742-21 \\
769-21 \\
795-1 \\
613-1\end{array}$ & $\begin{array}{l} \\
750-9 \\
701-9 \\
761-9 \\
156-9 \\
160-21 \\
197-1 \\
037-1 \\
851-1\end{array}$ & $\begin{array}{l}922-13 \\
365-13 \\
789-13 \\
696-13 \\
620-9 \\
605-9 \\
9729-9 \\
9750-9 \\
9747-9 \\
9719-21 \\
1772-1 \\
1133-1 \\
1869-1 \\
1676-1\end{array}$ & $\begin{array}{l} \\
703-10 \\
740-10 \\
754-10 \\
745-12 \\
799-1 \\
057-1 \\
009-1 \\
894-1\end{array}$ & $\begin{array}{l}999-13 \\
45 i-13 \\
921-13 \\
995-11 \\
965-113 \\
920-11 \\
053-11 \\
705-13 \\
659-13 \\
623-10 \\
703-10 \\
761-10 \\
796-10 \\
600-10 \\
792-10 \\
215-1 \\
970-1 \\
698-1 \\
898-1\end{array}$ & $\begin{array}{l} \\
012-10 \\
045-10 \\
051-10 \\
032-10 \\
020-1 \\
670-1 \\
995-1 \\
095-4\end{array}$ & $\begin{array}{l}910-13 \\
640-13 \\
74-13 \\
623-13 \\
710-10 \\
759-10 \\
655-10 \\
68-10 \\
158-10 \\
645-10 \\
117-10 \\
659-1 \\
678-1 \\
870-4\end{array}$ & $\begin{array}{l} \\
\\
990-10 \\
310-10 \\
918-10 \\
891-10 \\
9: 7-10 \\
837-1 \\
851-1 \\
651-4\end{array}$ & $\begin{array}{l}911-13 \\
620-13 \\
722-12 \\
674-10 \\
760-10 \\
862-10 \\
916-10 \\
242-10 \\
939-10 \\
906-10 \\
850-10 \\
624-1 \\
814-1 \\
113-4\end{array}$ & $\begin{array}{l}i-10 \\
7-10 \\
-10 \\
-10 \\
-10 \\
-1 \\
b-1\end{array}$ & 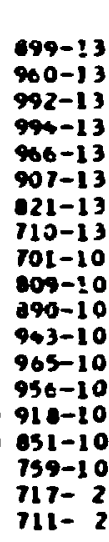 \\
\hline $\begin{array}{l}\times 140 \\
0 \\
5 \\
10 \\
15 \\
20 \\
25 \\
30 \\
35 \\
40 \\
45 \\
50 \\
35 \\
60 \\
65 \\
70 \\
75 \\
80 \\
85 \\
90\end{array}$ & $\begin{array}{l}\text { for fac ror } \\
893-13 \\
920-13 \\
920-13 \\
934-13 \\
935-13 \\
922-13 \\
690-13 \\
857-13 \\
806-13 \\
765-13 \\
673-13 \\
702-9 \\
757-9 \\
790-9 \\
802-9 \\
769-9 \\
753-9 \\
773-19 \\
734-19 \\
714-21\end{array}$ & $\begin{array}{l}759-9 \\
759-9 \\
789-9 \\
797-9 \\
780-9 \\
739-9 \\
755-21 \\
711-21 \\
774-21\end{array}$ & $\begin{array}{l}911-13 \\
865-13 \\
806-13 \\
729-13 \\
643-13 \\
700-9 \\
750-9 \\
777-9 \\
700-9 \\
760-9 \\
770-21 \\
795-21 \\
610-21 \\
011-21\end{array}$ & $\begin{array}{l}7 \\
731-9 \\
736-9 \\
754-9 \\
752-21 \\
707-21 \\
8122-21 \\
824-21 \\
024-1\end{array}$ & $\begin{array}{l}916-13 \\
861-13 \\
700-13 \\
699-13 \\
597-9 \\
660-9 \\
9702-9 \\
9722-9 \\
9719-9 \\
1740-21 \\
1701-21 \\
11007-1 \\
11043-1 \\
1653-1\end{array}$ & $\begin{array}{l} \\
671-10 \\
716-10 \\
729-10 \\
722-21 \\
776-1 \\
632-1 \\
664-1 \\
604-1\end{array}$ & $\begin{array}{l}693-13 \\
964-13 \\
973-13 \\
978-13 \\
958-13 \\
915-13 \\
850-13 \\
764-13 \\
661-13 \\
602-10 \\
600-10 \\
737-10 \\
775-10 \\
702-10 \\
769-10 \\
793-1 \\
645-1 \\
972-1 \\
673-1\end{array}$ & 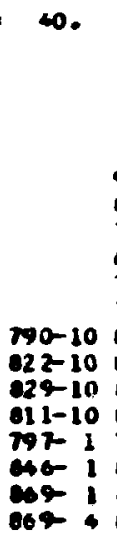 & $\begin{array}{l}910-13 \\
635-13 \\
739-13 \\
623-13 \\
700-10 \\
739-10 \\
634-10 \\
804-10 \\
867-10 \\
643-10 \\
797-10 \\
636-1 \\
852-1 \\
053-\end{array}$ & $\begin{array}{l} \\
\\
071-10 \\
898-10 \\
890-10 \\
071-10 \\
010-10 \\
912-1 \\
023-1 \\
024-4\end{array}$ & $\begin{array}{l}904-13 \\
022-13 \\
111-13 \\
061-10 \\
766-10 \\
245-10 \\
091-10 \\
923-10 \\
919-10 \\
600-10 \\
931-10 \\
779-10 \\
800-20 \\
811-20\end{array}$ & $\begin{array}{l}0 \\
911-10 \\
939-10 \\
933-10 \\
897-10 \\
935-10 \\
740-10 \\
765-20 \\
774-20\end{array}$ & $\begin{array}{l}693-13 \\
954-13 \\
915-13 \\
987-13 \\
950-13 \\
899-13 \\
813-13 \\
793-13 \\
693-10 \\
794-10 \\
877-10 \\
927-10 \\
967-10 \\
937-10 \\
690-10 \\
931-10 \\
740-10 \\
700-20 \\
714-19\end{array}$ \\
\hline
\end{tabular}




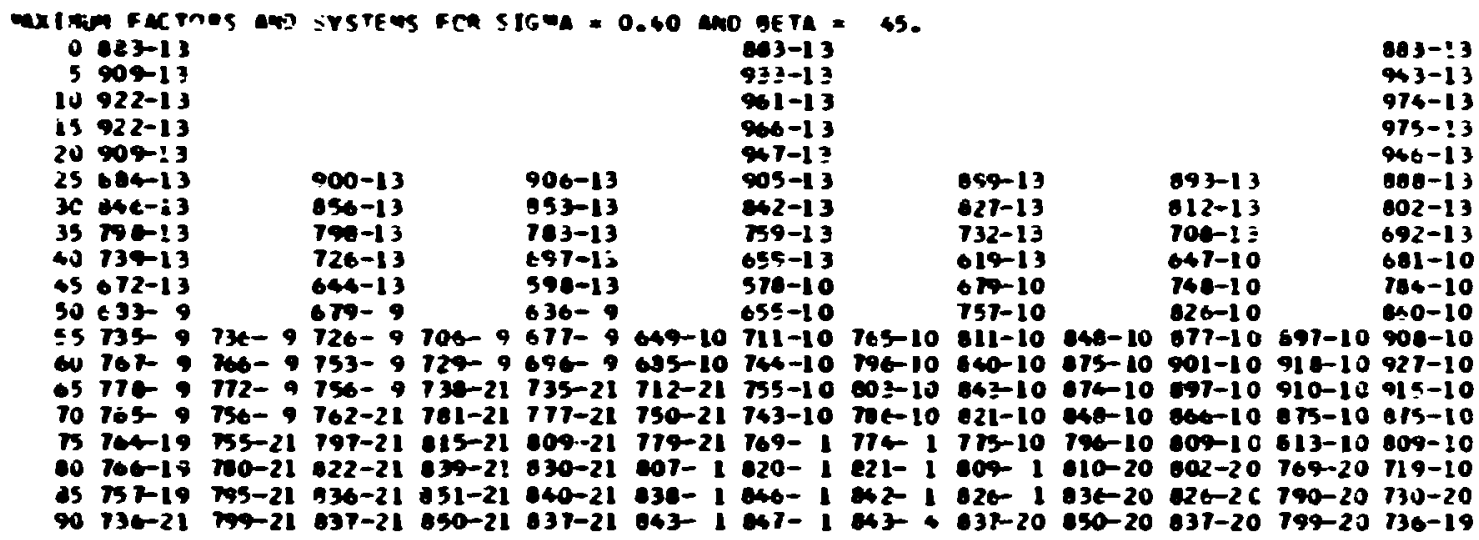

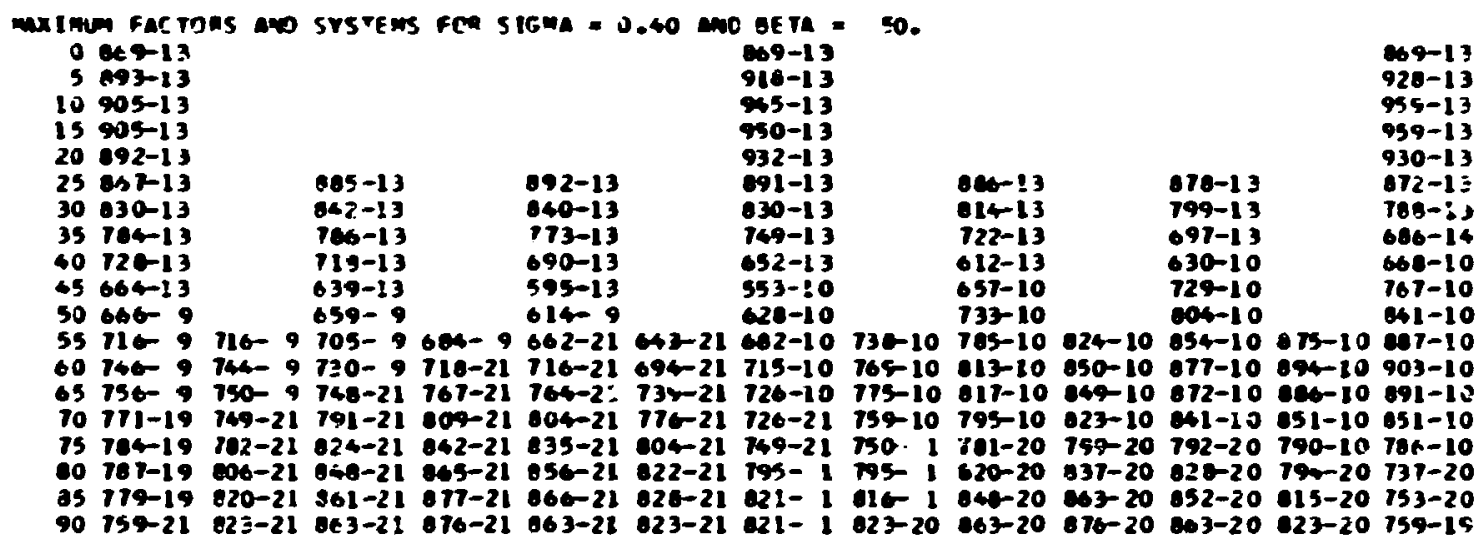

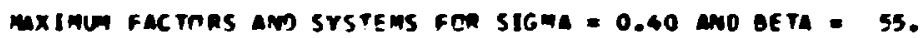

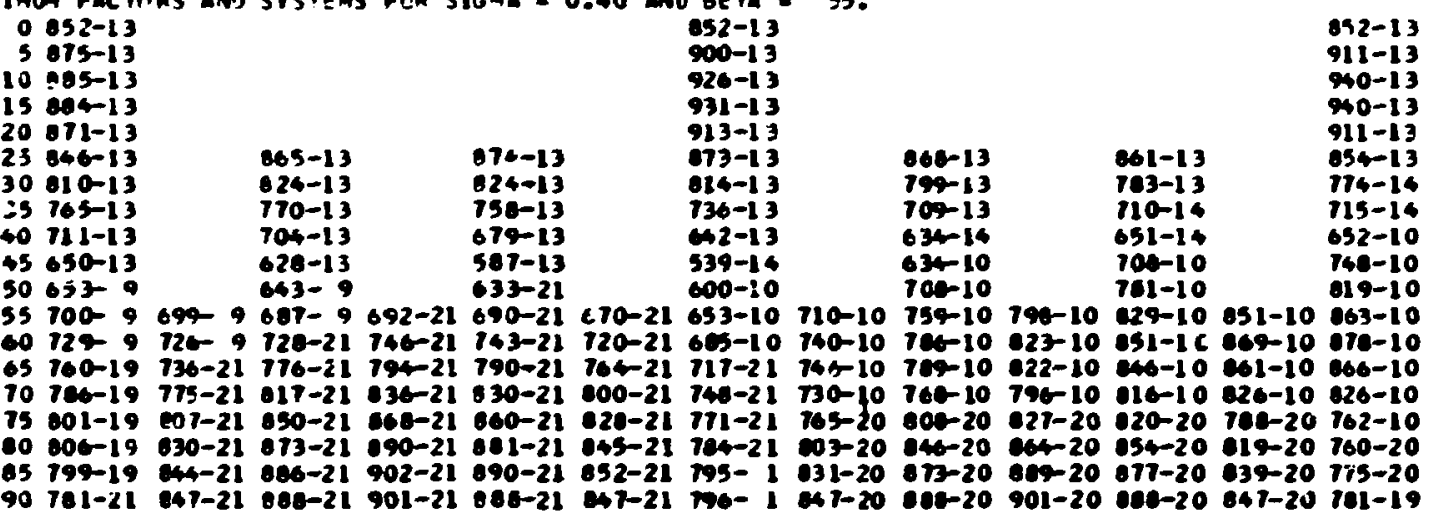




\begin{tabular}{|c|c|c|c|c|c|c|c|c|c|c|c|c|c|}
\hline $\begin{array}{l}0 \\
5 \\
10 \\
15 \\
26 \\
25 \\
30 \\
35 \\
40 \\
45 \\
50 \\
55 \\
60 \\
65 \\
70 \\
75 \\
60 \\
85 \\
90\end{array}$ & $\begin{array}{l}833-13 \\
854-13 \\
603-13 \\
861-13 \\
846-13 \\
021-13 \\
705-19 \\
742-13 \\
609-13 \\
620-15 \\
643-19 \\
606-99 \\
731-19 \\
706-19 \\
796-19 \\
015-19 \\
822-19 \\
010-19 \\
001-21\end{array}$ & $\begin{array}{l}0 \\
\\
606-9 \\
117-21 \\
761-21 \\
690-21 \\
630-21 \\
63-21 \\
66-21 \\
69-21\end{array}$ & $\begin{array}{l}842-17 \\
802-13 \\
150-13 \\
606-13 \\
612-13 \\
646-21 \\
702-21 \\
754-21 \\
901-21 \\
941-21 \\
674-21 \\
897-21 \\
909-21 \\
911-21\end{array}$ & $\begin{array}{l} \\
717-21 \\
711-2 ! \\
19-21 \\
959-21 \\
991-21 \\
914-21 \\
925-2 ! \\
925-21\end{array}$ & $\begin{array}{l}852-13 \\
604-13 \\
740-13 \\
663-13 \\
599-21 \\
658-21 \\
714-21 \\
767-21 \\
813-21 \\
132-21 \\
652-21 \\
903-21 \\
903-21 \\
913-21 \\
911-21\end{array}$ & $\begin{array}{l}093-21 \\
742-21 \\
705-21 \\
021-21 \\
46-21 \\
646-21 \\
613-21 \\
64-21\end{array}$ & $\begin{array}{l}633-13 \\
679-13 \\
904-13 \\
900-13 \\
691-13 \\
653-13 \\
795-13 \\
719-13 \\
620-13 \\
564-14 \\
607-21 \\
655-21 \\
690-21 \\
736-21 \\
767-21 \\
790-21 \\
603-21 \\
607-21 \\
601-21\end{array}$ & $\begin{array}{l}0 \\
42-10 \\
110-10 \\
11-10 \\
74-20 \\
71-20 \\
920-20 \\
054-20 \\
64-20\end{array}$ & $\begin{array}{l}646-13 \\
731-13 \\
713-14 \\
601-14 \\
611-10 \\
612-10 \\
731-10 \\
757-10 \\
760-10 \\
760-20 \\
635-20 \\
672-20 \\
697-20 \\
911-20\end{array}$ & & $\begin{array}{l}641-13 \\
767-16 \\
737-14 \\
679-14 \\
697-10 \\
757-10 \\
603-10 \\
624-10 \\
619-10 \\
601-2 C \\
646-20 \\
679-20 \\
901-20 \\
911-2 C\end{array}$ & $\begin{array}{l}10 \\
10 \\
10 \\
10 \\
10 \\
20 \\
20 \\
20 \\
23\end{array}$ & $\begin{array}{l}833-13 \\
890-13 \\
919-13 \\
919-13 \\
990-13 \\
944-14 \\
799-14 \\
742-14 \\
675-14 \\
720-10 \\
797-10 \\
050-10 \\
953-10 \\
940-10 \\
201-10 \\
750-20 \\
701-20 \\
796-20 \\
001-20\end{array}$ \\
\hline $\begin{array}{l}\times 100 \\
0 \\
5 \\
10 \\
15 \\
20 \\
25 \\
30 \\
35 \\
40 \\
45 \\
50 \\
55 \\
60 \\
65 \\
70 \\
75 \\
10 \\
85 \\
90\end{array}$ & 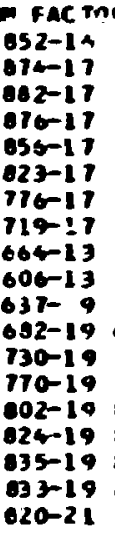 & 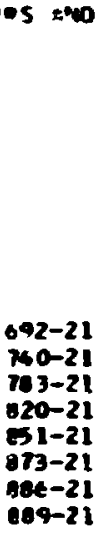 & $\begin{array}{l}917-13 \\
717-13 \\
726-12 \\
664-13 \\
6 i 2-21 \\
669-21 \\
724-21 \\
716-21 \\
623-21 \\
963-21 \\
895-21 \\
917-21 \\
930-21 \\
932-21\end{array}$ & $\begin{array}{l}7 \\
730-21 \\
792-21 \\
799-21 \\
890-21 \\
912-21 \\
934-21 \\
946-21 \\
946-21\end{array}$ & $\begin{array}{l}920-13 \\
701-13 \\
719-13 \\
646-13 \\
622-21 \\
670-21 \\
734-21 \\
766-21 \\
633-21 \\
672-21 \\
902-21 \\
923-21 \\
933-21 \\
932-21\end{array}$ & $\begin{array}{l}711-21 \\
760-21 \\
603-21 \\
639-21 \\
667-21 \\
605-21 \\
692-21 \\
609-21\end{array}$ & $\begin{array}{l}652-14 \\
656-13 \\
60-13 \\
640-13 \\
667-13 \\
630-13 \\
773-13 \\
700-13 \\
636-14 \\
504-14 \\
624-21 \\
671-21 \\
714-21 \\
752-21 \\
703-21 \\
606-21 \\
620-21 \\
025-21 \\
020-21\end{array}$ & $\begin{array}{l}0 \\
654-10 \\
61-10 \\
110-20 \\
311-20 \\
115-20 \\
651-20 \\
675-20 \\
009-20\end{array}$ & $\begin{array}{l}220 \\
79 \\
723 \\
622 \\
621 \\
651 \\
705 \\
730 \\
7 \in 0 \\
114 \\
600 \\
895 \\
919 \\
932\end{array}$ & $\begin{array}{l}70 \\
746-10 \\
769-10 \\
779-20 \\
633-20 \\
078-20 \\
912-20 \\
935-20 \\
946-20\end{array}$ & $\begin{array}{l}14 \\
14 \\
14 \\
14 \\
10 \\
10 \\
10 \\
10 \\
10 \\
20 \\
20 \\
20 \\
20 \\
20\end{array}$ & $\begin{array}{l}000-10 \\
017-10 \\
000-10 \\
197-20 \\
636-20 \\
866-20 \\
962-20 \\
089-20\end{array}$ & $\begin{array}{l}932-14 \\
909-16 \\
907-14 \\
909-14 \\
695-16 \\
605-14 \\
621-14 \\
765-14 \\
699-16 \\
797-10 \\
773-10 \\
913-10 \\
627-10 \\
614-10 \\
776-10 \\
777-20 \\
801-20 \\
015-20 \\
020-19\end{array}$ \\
\hline $\begin{array}{l}110 \\
2 \\
5 \\
10 \\
15 \\
20 \\
25 \\
30 \\
35 \\
40 \\
45 \\
50 \\
55 \\
60 \\
65 \\
10 \\
75 \\
60 \\
15 \\
90\end{array}$ & $\begin{array}{l}\text { FAC TO } \\
869-14 \\
693-17 \\
902-17 \\
690-17 \\
679-17 \\
647-17 \\
602-17 \\
745-17 \\
676-17 \\
603-17 \\
635-9 \\
677-19 \\
723-19 \\
700-19 \\
804-19 \\
830-19 \\
844-19 \\
846-19 \\
834-21\end{array}$ & $\begin{array}{l}710-21 \\
758-21 \\
01-21 \\
030-21 \\
368-21 \\
690-21 \\
903-21 \\
507-21\end{array}$ & $\begin{array}{l}15-17 \\
771-17 \\
717-17 \\
657-17 \\
629-21 \\
686-21 \\
742-21 \\
793-21 \\
64 c-21 \\
600-21 \\
912-21 \\
935-21 \\
946-21 \\
950-21\end{array}$ & $\begin{array}{l} \\
754-21 \\
900-21 \\
950-21 \\
996-21 \\
929-21 \\
951-21 \\
904-21 \\
965-21\end{array}$ & $\begin{array}{l}902-13 \\
755-13 \\
695-13 \\
622-13 \\
634-21 \\
692-21 \\
769-21 \\
601-21 \\
940-21 \\
907-21 \\
910-21 \\
940-21 \\
950-21 \\
990-21\end{array}$ & $\begin{array}{r}21 \\
3-21 \\
6-21 \\
3-21 \\
1-21 \\
10-21\end{array}$ & $\begin{array}{l}609-14 \\
671-14 \\
64-14 \\
658-11 \\
641-11 \\
05-11 \\
750-13 \\
699-14 \\
649-14 \\
597-14 \\
655-21 \\
601-21 \\
725-21 \\
763-21 \\
194-21 \\
918-21 \\
633-21 \\
639-21 \\
94-21\end{array}$ & 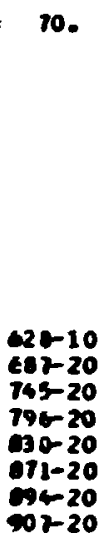 & $\begin{array}{l}631-14 \\
754-14 \\
769-14 \\
690-14 \\
644-14 \\
634-10 \\
67 \%-10 \\
726-20 \\
164-20 \\
639-20 \\
962-20 \\
916-20 \\
939-20 \\
950-20\end{array}$ & $\begin{array}{l} \\
721-10 \\
74-20 \\
805-20 \\
657-20 \\
900-20 \\
933-20 \\
955-20\end{array}$ & $\begin{array}{l}663-14 \\
825-14 \\
777-14 \\
721-14 \\
659-14 \\
710-10 \\
753-10 \\
772-10 \\
200-20 \\
850-20 \\
691-20 \\
922-20 \\
942-20 \\
990-20\end{array}$ & $\begin{array}{l}775-10 \\
792-10 \\
703-10 \\
10-20 \\
1056-20 \\
603-20 \\
900-20 \\
907-20\end{array}$ & $\begin{array}{l}269-14 \\
904-14 \\
923-14 \\
926-14 \\
912-14 \\
963-14 \\
639-14 \\
784-14 \\
718-14 \\
606-10 \\
769-10 \\
708-10 \\
901-10 \\
709-10 \\
763-20 \\
795-20 \\
910-20 \\
931-20 \\
930-20\end{array}$ \\
\hline
\end{tabular}




\begin{tabular}{|c|c|c|c|c|c|c|c|c|c|c|c|c|c|}
\hline $\begin{array}{l}x 17 \\
0 \\
5 \\
10 \\
15 \\
20 \\
25 \\
30 \\
35 \\
40 \\
45 \\
50 \\
55 \\
10 \\
05 \\
70 \\
75 \\
40 \\
45 \\
90\end{array}$ & 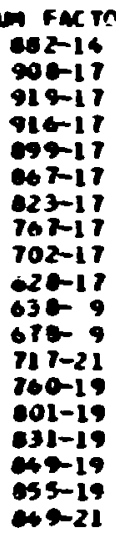 & $\begin{array}{l}723-21 \\
72-21 \\
015-21 \\
52-21 \\
202-21 \\
02-21 \\
917-21 \\
521-21\end{array}$ & $\begin{array}{l}637-17 \\
79-17 \\
743-17 \\
693-17 \\
639-21 \\
697-21 \\
753-21 \\
606-21 \\
653-21 \\
693-21 \\
925-21 \\
949-21 \\
962-21 \\
965-21\end{array}$ & $\begin{array}{l}0 \\
\\
965-21 \\
769-21 \\
667-21 \\
900-21 \\
941-21 \\
965-21 \\
970-21 \\
960-21\end{array}$ & $\begin{array}{l}794-17 \\
749-17 \\
696-17 \\
641-17 \\
642-21 \\
701-21 \\
757-21 \\
610-21 \\
650-21 \\
996-21 \\
930-21 \\
952-21 \\
964-21 \\
945-21\end{array}$ & $\begin{array}{l}0 \\
\\
731-21 \\
100-21 \\
225-21 \\
202-21 \\
91-21 \\
911-21 \\
921-21 \\
921-21\end{array}$ & 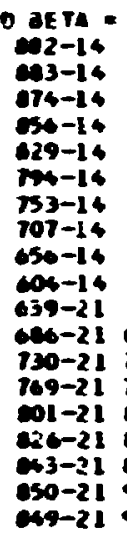 & $\begin{array}{r} \\
654-20 \\
11-20 \\
1+5-20 \\
11-20 \\
150-20 \\
\cos -20 \\
110-20 \\
921-20\end{array}$ & $\begin{array}{l}941-14 \\
004-14 \\
759-14 \\
705-14 \\
054-14 \\
621-20 \\
615-20 \\
752-20 \\
910-20 \\
60-20 \\
902-20 \\
934-20 \\
955-20 \\
905-20\end{array}$ & & $\begin{array}{l}174-14 \\
630-14 \\
769-14 \\
733-14 \\
672-14 \\
607-10 \\
729-10 \\
765-20 \\
621-20 \\
670-20 \\
909-20 \\
938-20 \\
957-20 \\
45-20\end{array}$ & $\begin{array}{l}52-10 \\
0-10 \\
92-20 \\
37-20 \\
12-20 \\
9-20 \\
15-20 \\
21-20\end{array}$ & $\begin{array}{l}202-14 \\
917-14 \\
935-14 \\
938-14 \\
924-14 \\
995-14 \\
853-14 \\
790-14 \\
133-14 \\
004-10 \\
726-10 \\
764-10 \\
777-10 \\
766-10 \\
779-20 \\
010-20 \\
9 \equiv 1-20 \\
964-20 \\
069-19\end{array}$ \\
\hline $\begin{array}{l}0 \\
5 \\
10 \\
15 \\
20 \\
25 \\
30 \\
15 \\
40 \\
45 \\
50 \\
35 \\
60 \\
65 \\
10 \\
75 \\
10 \\
65 \\
90\end{array}$ & $\begin{array}{l}6 \text { Fac } 10 \\
692-14 \\
920-17 \\
932-17 \\
931-17 \\
915-17 \\
694-17 \\
641-17 \\
720-17 \\
721-17 \\
640-17 \\
44-9 \\
604-21 \\
727-21 \\
765-21 \\
797-21 \\
227-19 \\
950-19 \\
601-19 \\
450-21\end{array}$ & $\begin{array}{l}0 \text { No } \\
\\
731-21 \\
730-21 \\
64-21 \\
62-21 \\
992-21 \\
916-21 \\
927-21 \\
931-21\end{array}$ & $\begin{array}{l}955-17 \\
616-17 \\
74-17 \\
706-17 \\
643-21 \\
702-21 \\
759-21 \\
913-21 \\
661-21 \\
902-21 \\
934-21 \\
950-21 \\
972-21 \\
976-21\end{array}$ & $\begin{array}{l}l \\
\\
770-21 \\
924-21 \\
974-21 \\
916-21 \\
950-21 \\
974-21 \\
900-21 \\
991-21\end{array}$ & $\begin{array}{l}914-17 \\
771-17 \\
721-17 \\
666-17 \\
643-21 \\
703-21 \\
760-21 \\
114-21 \\
863-21 \\
904-21 \\
937-21 \\
960-21 \\
974-21 \\
976-21\end{array}$ & $\begin{array}{l}0 \\
732-21 \\
703-21 \\
62-21 \\
60-21 \\
697-21 \\
916-21 \\
930-21 \\
931-21\end{array}$ & 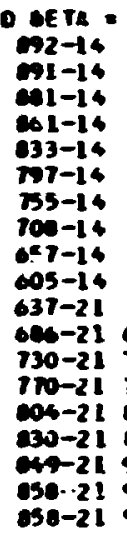 & $\begin{array}{r}67-20 \\
675-20 \\
3720 \\
790-20 \\
63-20 \\
17-20 \\
903-20 \\
922-20 \\
931-20\end{array}$ & $\begin{array}{l}16 \\
14 \\
14 \\
14 \\
14 \\
20 \\
20 \\
20 \\
20 \\
20 \\
20 \\
20 \\
20 \\
20\end{array}$ & $\begin{array}{r}-20 \\
-20 \\
-20 \\
-20 \\
-20 \\
-20 \\
20 \\
20 \\
20\end{array}$ & $\begin{array}{l}61-14 \\
43-14 \\
795-14 \\
760-14 \\
679-14 \\
647-10 \\
724-20 \\
765-20 \\
939-20 \\
646-20 \\
923-20 \\
931-26 \\
* 69-20 \\
976-20\end{array}$ & $\begin{array}{l}-10 \\
-20 \\
-20 \\
-20 \\
-20 \\
-20 \\
-20 \\
-20\end{array}$ & $\begin{array}{l}-14 \\
-14 \\
-14 \\
-14 \\
-14 \\
-14 \\
-14 \\
-14 \\
-14 \\
-14 \\
-10 \\
-10 \\
-10 \\
-20 \\
-20 \\
-20 \\
-20 \\
-20 \\
-20\end{array}$ \\
\hline $\begin{array}{l}0 \\
5 \\
10 \\
15 \\
20 \\
25 \\
30 \\
35 \\
40 \\
45 \\
50 \\
55 \\
60 \\
65 \\
10 \\
75 \\
60 \\
85 \\
90\end{array}$ & 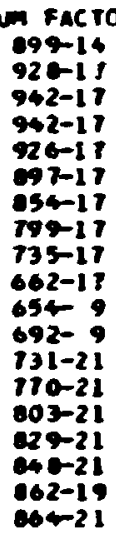 & $\begin{array}{l} \\
73-21 \\
703-21 \\
70-21 \\
864-21 \\
697-21 \\
920-21 \\
533-21 \\
530-21\end{array}$ & $\begin{array}{l}969-17 \\
629-17 \\
760-17 \\
723-17 \\
660-17 \\
701-21 \\
760-21 \\
214-21 \\
263-21 \\
905-21 \\
939-21 \\
963-21 \\
978-21 \\
903-21\end{array}$ & $\begin{array}{l}7 \\
7 \\
7 \in 8-21 \\
024-21 \\
075-21 \\
910-21 \\
953-21 \\
970-21 \\
993-21 \\
990-21\end{array}$ & $\begin{array}{l}929-17 \\
700-17 \\
740-17 \\
696-17 \\
637-21 \\
690-21 \\
757-21 \\
912-21 \\
662-21 \\
905-21 \\
939-21 \\
964-21 \\
979-21 \\
903-21\end{array}$ & $\begin{array}{l}727-21 \\
779-21 \\
226-21 \\
60-21 \\
994-21 \\
921-21 \\
934-21 \\
930-21\end{array}$ & 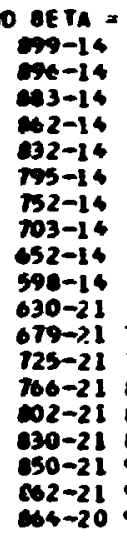 & $\begin{array}{l}0 . \\
100-20 \\
75<-20 \\
00-20 \\
50-20 \\
06-20 \\
913-20 \\
330-20 \\
930-20\end{array}$ & $\begin{array}{l}945-14 \\
667-14 \\
7 \in 1-14 \\
710-14 \\
655-14 \\
670-20 \\
733-20 \\
792-20 \\
94-20 \\
692-20 \\
930-20 \\
950-20 \\
976-20 \\
989-20\end{array}$ & $\begin{array}{l} \\
7+7-20 \\
107-20 \\
961-20 \\
900-20 \\
965-20 \\
973-20 \\
991-20 \\
990-20\end{array}$ & $\begin{array}{l}002-14 \\
796-14 \\
741-14 \\
679-14 \\
619-20 \\
742-20 \\
900-20 \\
522-20 \\
697-20 \\
933-20 \\
960-20 \\
976-20 \\
983-20\end{array}$ & $\begin{array}{l}717-20 \\
771-20 \\
020-20 \\
061-20 \\
994-20 \\
910-20 \\
932-20 \\
930-20\end{array}$ & $\begin{array}{l}999-14 \\
931-14 \\
960-14 \\
969-14 \\
935-14 \\
900-14 \\
264-14 \\
010-14 \\
745-14 \\
674-14 \\
685-10 \\
122-10 \\
736-10 \\
765-20 \\
600-20 \\
020-20 \\
648-20 \\
960-20 \\
664-20\end{array}$ \\
\hline
\end{tabular}




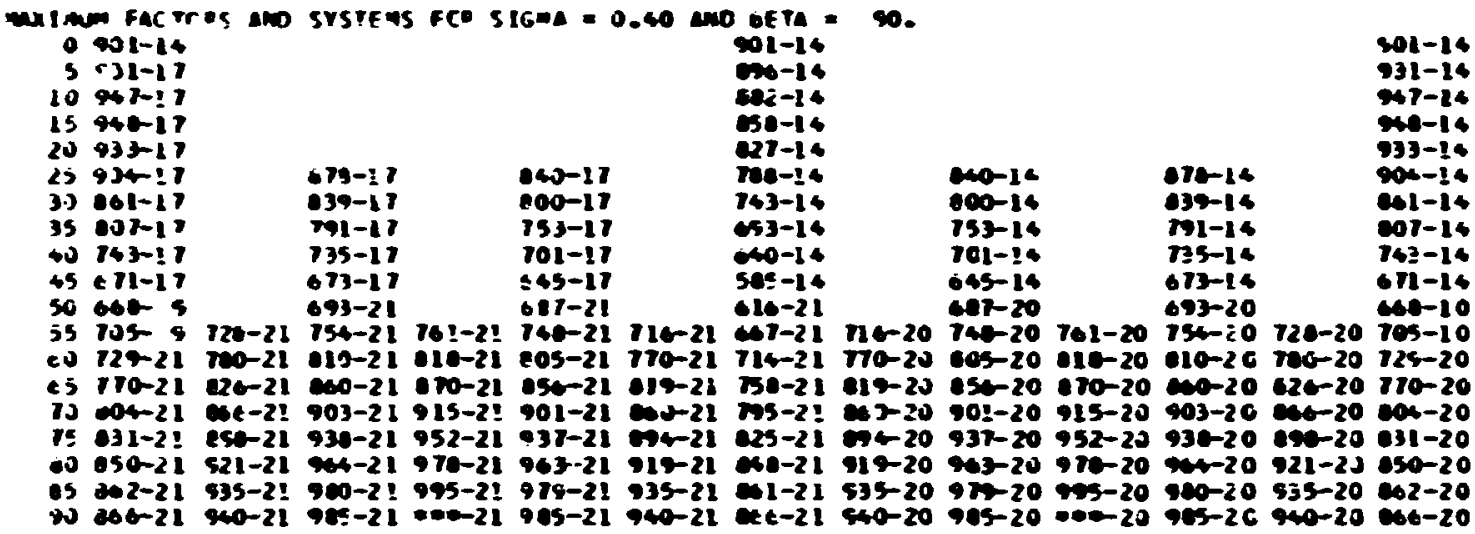




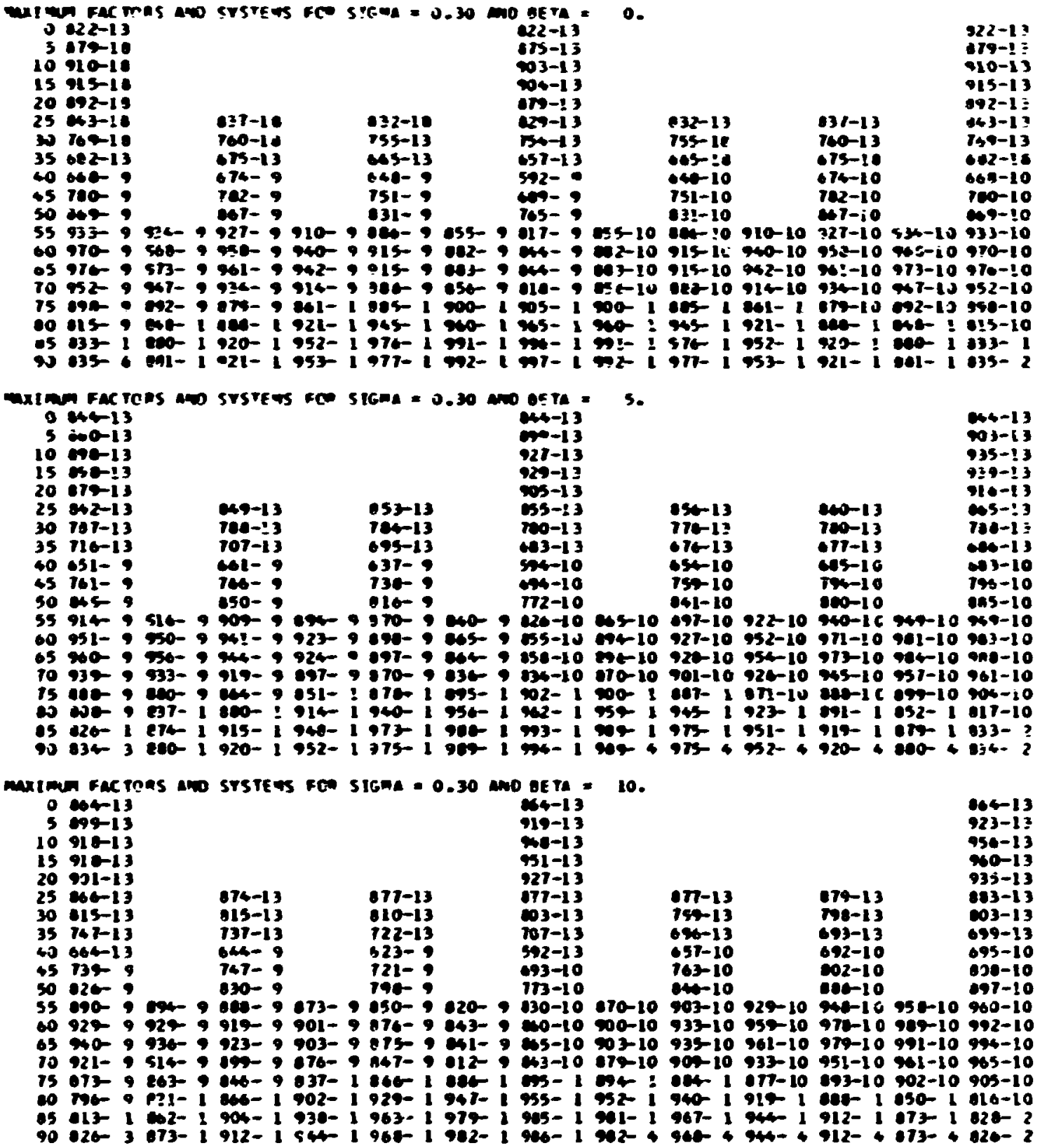




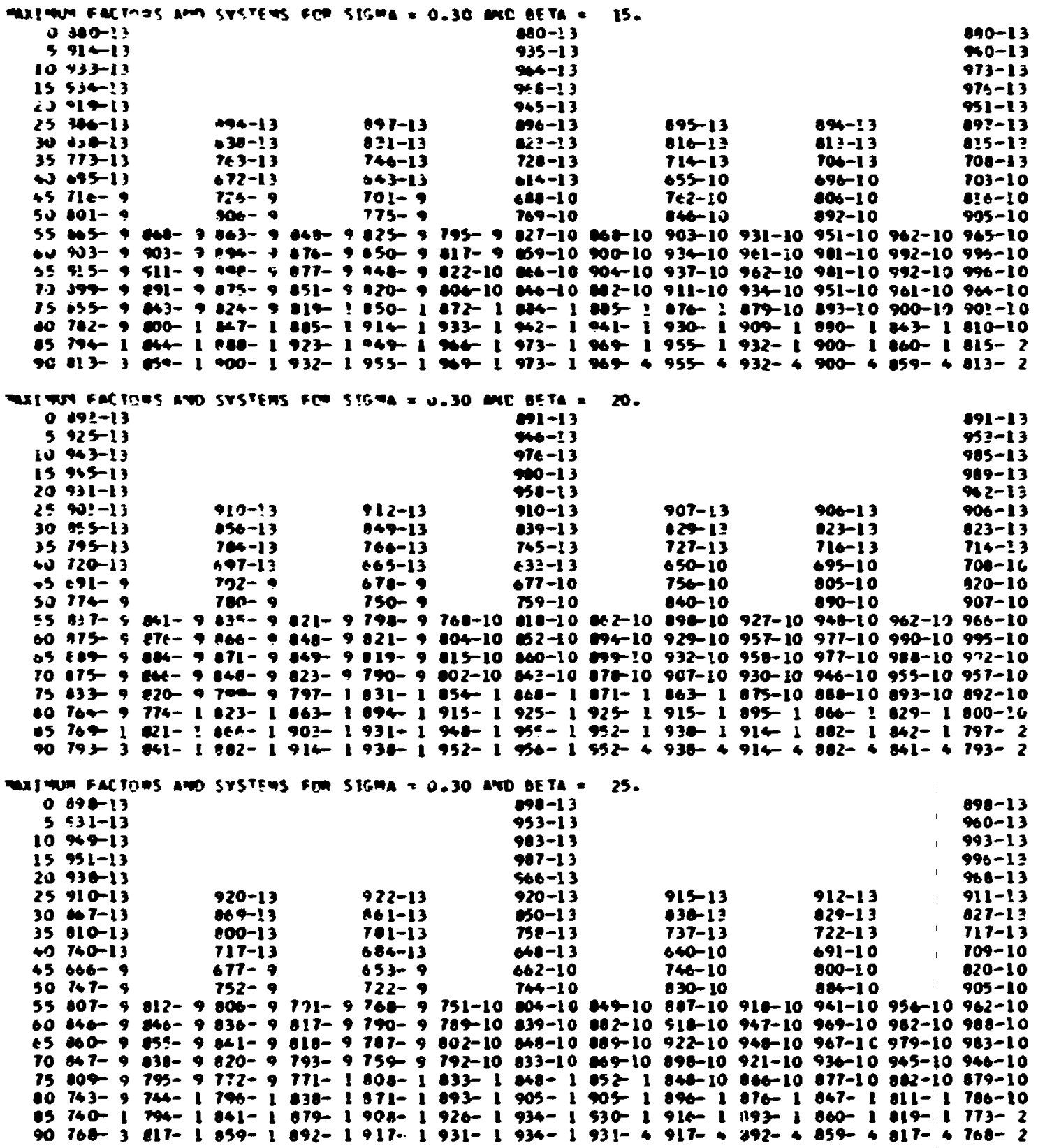




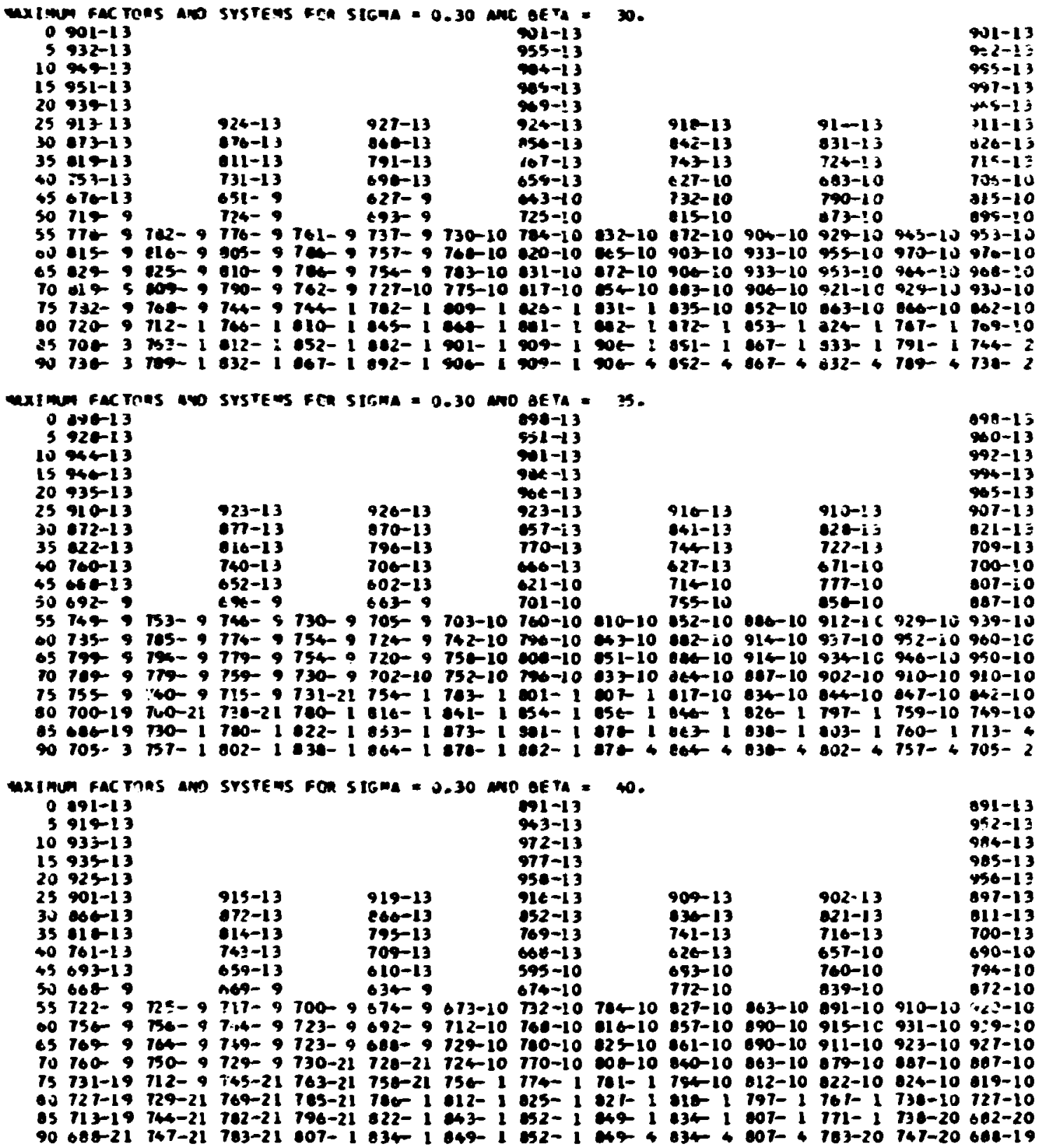




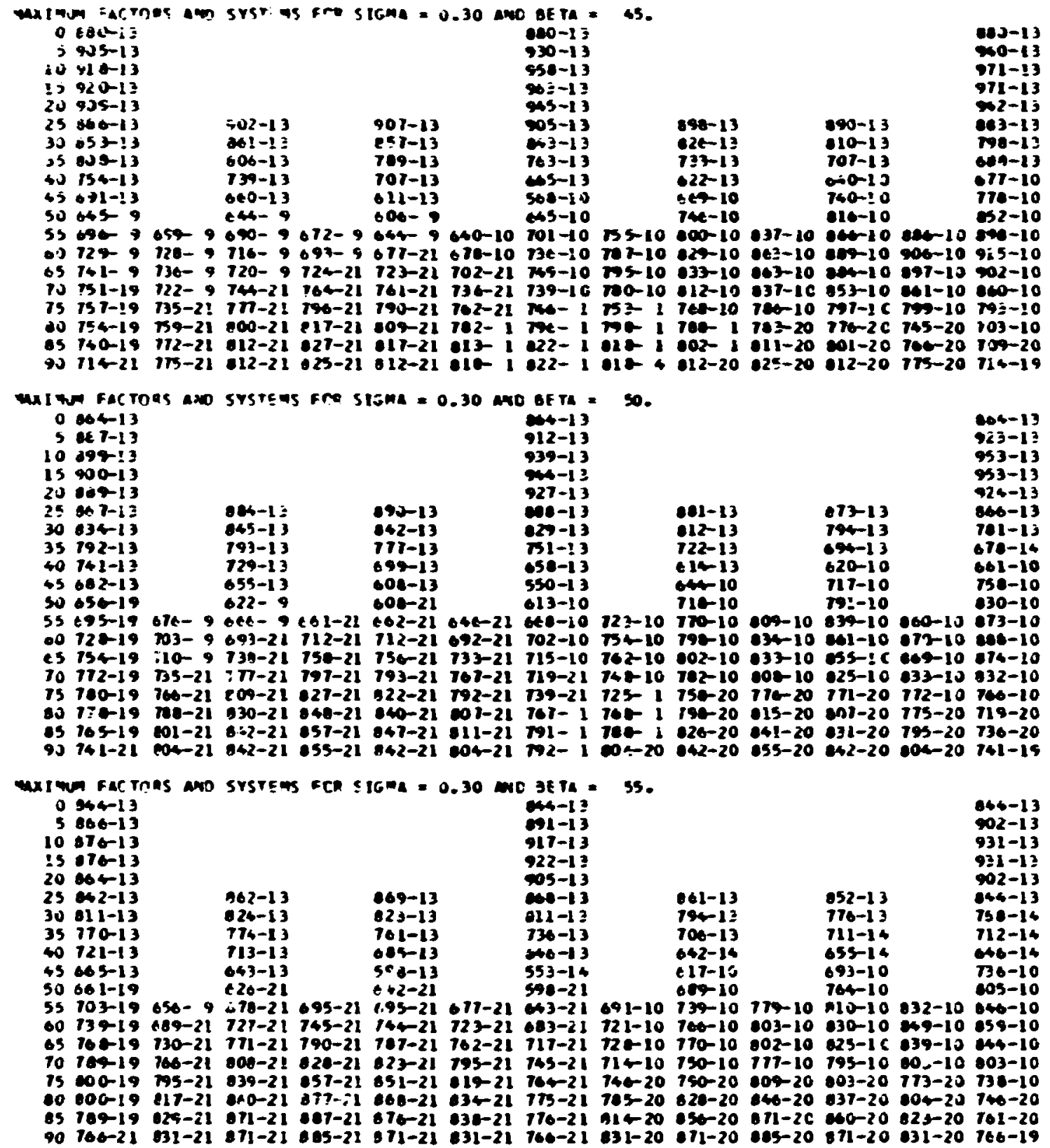




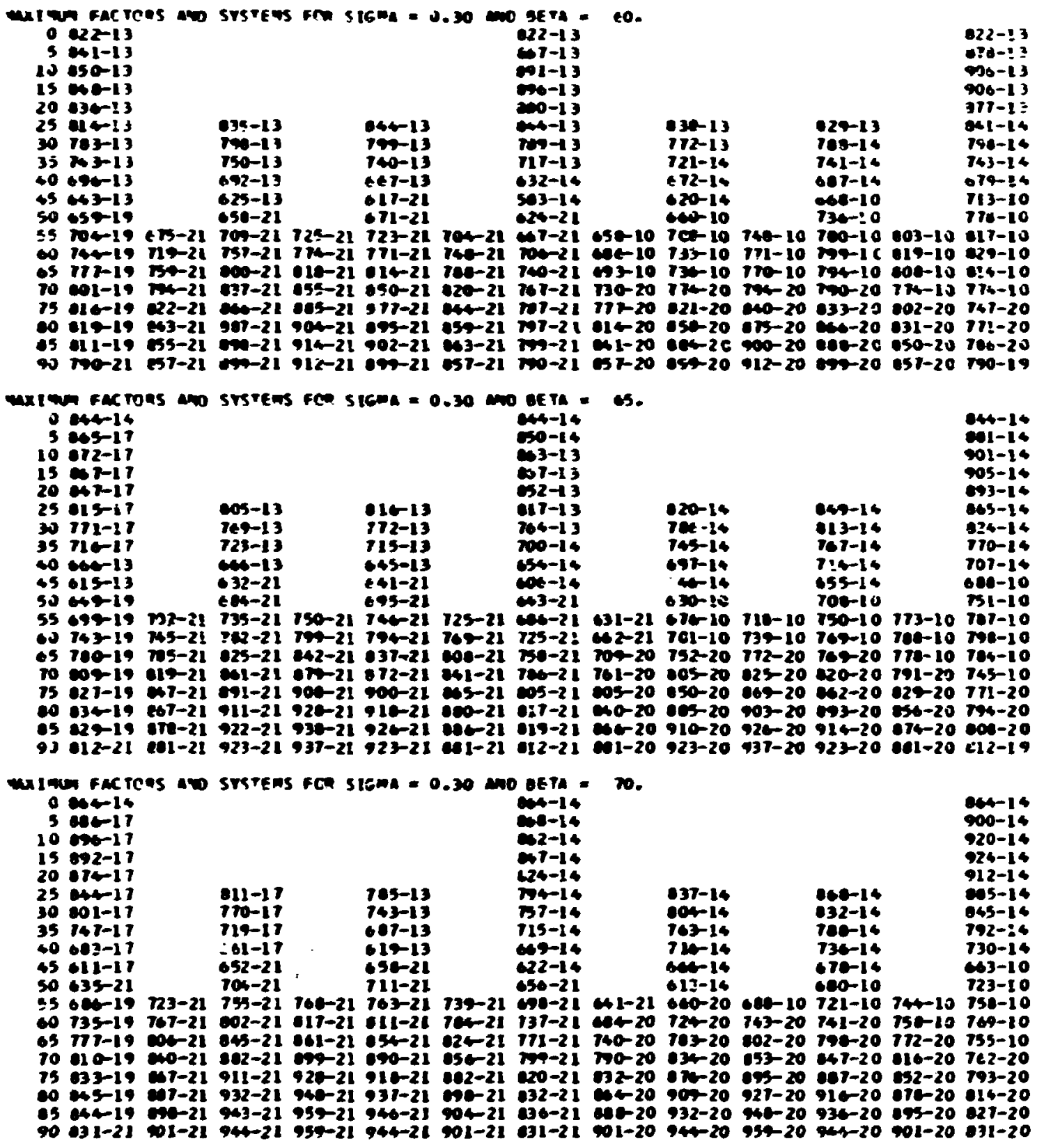




\begin{tabular}{|c|c|c|c|c|c|c|c|c|c|c|c|c|c|}
\hline $\begin{array}{l}150 \\
0 \\
5 \\
10 \\
15 \\
20 \\
25 \\
30 \\
35 \\
40 \\
25 \\
50 \\
30 \\
03 \\
45 \\
10 \\
15 \\
10 \\
05 \\
90\end{array}$ & 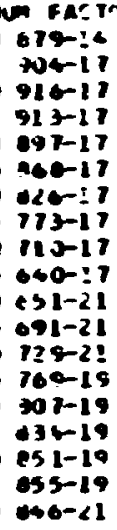 & $\begin{array}{l}1 \\
\\
134-21 \\
703-2 ! \\
022-21 \\
25 e-21 \\
293-21 \\
503-21 \\
515-21 \\
510-21\end{array}$ & $\begin{array}{l}931-11 \\
797-17 \\
740-17 \\
692-17 \\
06+-21 \\
717-21 \\
709-21 \\
9 ! 7-21 \\
960-21 \\
947-21 \\
920-2 ! \\
963-21 \\
960-21 \\
962-21\end{array}$ & $\begin{array}{l} \\
\\
\\
701-21 \\
930-21 \\
075-21 \\
913-21 \\
943-21 \\
9 e+21 \\
975-21 \\
9 n-21\end{array}$ & $\begin{array}{l}196-17 \\
752-: 8 \\
76,-17 \\
049-17 \\
967-21 \\
721-21 \\
173-21 \\
922-21 \\
906-21 \\
903-21 \\
932-21 \\
952-21 \\
962-21 \\
962-21\end{array}$ & 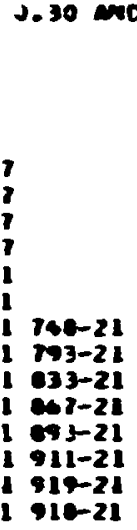 & 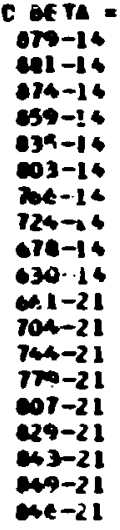 & $\begin{array}{l}0 \\
051-20 \\
115-20 \\
769-20 \\
015-20 \\
65<-20 \\
005-20 \\
900-20 \\
116-20\end{array}$ & $\begin{array}{l}645-14 \\
615-14 \\
774-14 \\
720-14 \\
670-14 \\
620-20 \\
693-20 \\
755-20 \\
610-20 \\
059-20 \\
659-20 \\
530-20 \\
951-20 \\
962-20\end{array}$ & $\begin{array}{l}711-20 \\
773-20 \\
129-20 \\
17-20 \\
917-20 \\
947-20 \\
967-20 \\
977-20\end{array}$ & $\begin{array}{l}00:-14 \\
66-14 \\
602-14 \\
150-14 \\
693-14 \\
654-10 \\
709-20 \\
769-20 \\
826-20 \\
670-20 \\
904-20 \\
936-20 \\
954-20 \\
962-20\end{array}$ & $\begin{array}{l}7 \\
\\
75-10 \\
745-20 \\
75-20 \\
030-20 \\
071-20 \\
096-20 \\
912-20 \\
910-20\end{array}$ & $\begin{array}{l}679-14 \\
915-14 \\
935-14 \\
930-14 \\
927-14 \\
900-14 \\
660-14 \\
608-14 \\
747-14 \\
670-14 \\
600-10 \\
730-10 \\
761-10 \\
765-20 \\
702-20 \\
030-20 \\
030-20 \\
042-20 \\
060-19\end{array}$ \\
\hline $\begin{array}{l}10 \\
0 \\
3 \\
10 \\
15 \\
20 \\
25 \\
30 \\
35 \\
65 \\
43 \\
50 \\
35 \\
60 \\
85 \\
70 \\
75 \\
80 \\
15 \\
90\end{array}$ & 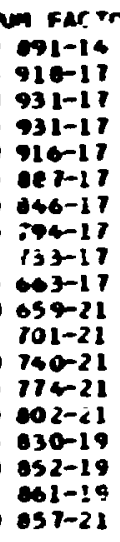 & $\begin{array}{l}\cos 4+00 \\
\\
747-21 \\
792-21 \\
232-21 \\
267-21 \\
994-21 \\
914-21 \\
52 E-21 \\
530-21\end{array}$ & $\begin{array}{l}959-17 \\
620-17 \\
773-17 \\
710-17 \\
669-21 \\
723-21 \\
778-21 \\
925-21 \\
209-21 \\
907-21 \\
937-21 \\
959-21 \\
97:-21 \\
974-21\end{array}$ & $\begin{array}{l} \\
\\
796-21 \\
937-21 \\
902-21 \\
921-21 \\
932-21 \\
975-21 \\
907-21 \\
909-21\end{array}$ & $\begin{array}{l}917-17 \\
777-17 \\
730-17 \\
379-17 \\
660-21 \\
723-21 \\
777-21 \\
627-21 \\
872-21 \\
910-21 \\
940-21 \\
961-21 \\
973-21 \\
974-21\end{array}$ & $\begin{array}{ll} & \\
\\
7 \\
7 \\
1 \\
1 \\
1 \\
1 \\
1 & 799-21 \\
1 & 93-21 \\
1 & 972-21 \\
1 & 900-21 \\
1 & 919-21 \\
1 & 929-21 \\
1 & 930-21\end{array}$ & 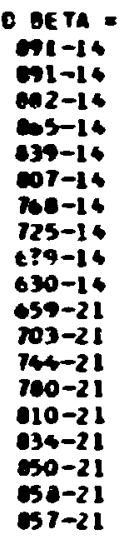 & $\begin{array}{l}0 \\
07-20 \\
742-20 \\
993-20 \\
637-20 \\
973-20 \\
902-20 \\
920-20 \\
930-20\end{array}$ & $\begin{array}{l}155-14 \\
620-14 \\
779-14 \\
733-14 \\
622-14 \\
600-20 \\
722-20 \\
701-20 \\
934-20 \\
600-20 \\
916-20 \\
947-20 \\
964-20 \\
974-20\end{array}$ & $\begin{array}{l}739-20 \\
797-20 \\
951-20 \\
857-20 \\
935-20 \\
963-20 \\
901-20 \\
909-20\end{array}$ & $\begin{array}{l}109 \\
654 \\
010 \\
752 \\
701 \\
673 \\
735 \\
793 \\
644 \\
600 \\
624 \\
951 \\
968 \\
974\end{array}$ & $\begin{array}{l}0 \\
713-20 \\
767-20 \\
914-20 \\
954-20 \\
906-20 \\
910-20 \\
924-20 \\
930-20\end{array}$ & $\begin{array}{l}991-14 \\
925-14 \\
94-14 \\
960-14 \\
936-14 \\
910-14 \\
970-14 \\
919-14 \\
750-14 \\
699-14 \\
671-10 \\
704-10 \\
121-20 \\
762-20 \\
794-20 \\
823-20 \\
042-20 \\
053-20 \\
057-20\end{array}$ \\
\hline $\begin{array}{l}100 \\
0 \\
5 \\
10 \\
15 \\
20 \\
25 \\
30 \\
35 \\
40 \\
45 \\
50 \\
55 \\
60 \\
65 \\
70 \\
75 \\
10 \\
15 \\
90\end{array}$ & $\begin{array}{l}\text { FAC Th } \\
990-14 \\
927-17 \\
942-17 \\
243-17 \\
929-17 \\
902-17 \\
601-17 \\
110-17 \\
769-17 \\
660-17 \\
661-21 \\
704-2 \\
744-21 \\
779-21 \\
004-21 \\
833-21 \\
950-21 \\
802-19 \\
604-21\end{array}$ & $\begin{array}{l}0 \\
190-21 \\
740-21 \\
937-21 \\
912-21 \\
900-21 \\
921-21 \\
533-21 \\
937-21\end{array}$ & $\begin{array}{l}315-17 \\
938-17 \\
792-17 \\
739-17 \\
679-17 \\
721-21 \\
776-21 \\
927-21 \\
972-21 \\
911-21 \\
942-21 \\
965-21 \\
970-21 \\
902-21\end{array}$ & $\begin{array}{l}0 \\
104-21 \\
037-21 \\
004-21 \\
924-21 \\
954-21 \\
900-21 \\
994-21 \\
997-21\end{array}$ & $\begin{array}{l}935-17 \\
797-17 \\
152-17 \\
102-11 \\
661-21 \\
710-21 \\
773-21 \\
825-21 \\
971-21 \\
911 \\
911-21 \\
943-21 \\
960-21 \\
979-21 \\
902-21\end{array}$ & 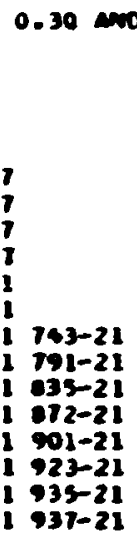 & 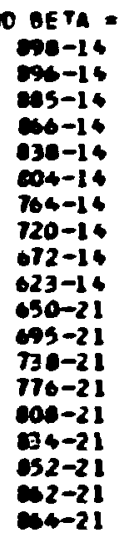 & $\begin{array}{l}112-20 \\
764-20 \\
12-20 \\
034-20 \\
60-20 \\
913-20 \\
930-20 \\
937-20\end{array}$ & $\begin{array}{l}654-14 \\
619-14 \\
777-14 \\
730-14 \\
675-14 \\
66-20 \\
745-20 \\
601-20 \\
652-20 \\
690-20 \\
931-20 \\
950-20 \\
975-20 \\
902-20\end{array}$ & $\begin{array}{l}160-20 \\
117-20 \\
601-20 \\
912-20 \\
947-20 \\
971-20 \\
991-20 \\
997-20\end{array}$ & $\begin{array}{l}690-14 \\
855-14 \\
111-14 \\
759-14 \\
701-14 \\
696-20 \\
135-20 \\
910-20 \\
959-20 \\
902-20 \\
935-20 \\
961-20 \\
97-20\end{array}$ & 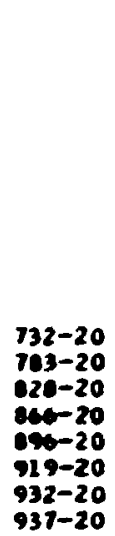 & $\begin{array}{l}992-14 \\
931-14 \\
949-14 \\
952-14 \\
939-14 \\
913-16 \\
973-14 \\
022-14 \\
161-14 \\
693-14 \\
640-10 \\
691-20 \\
735-20 \\
174-20 \\
906-20 \\
031-20 \\
049-20 \\
600-20 \\
604-20\end{array}$ \\
\hline
\end{tabular}




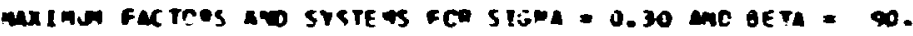

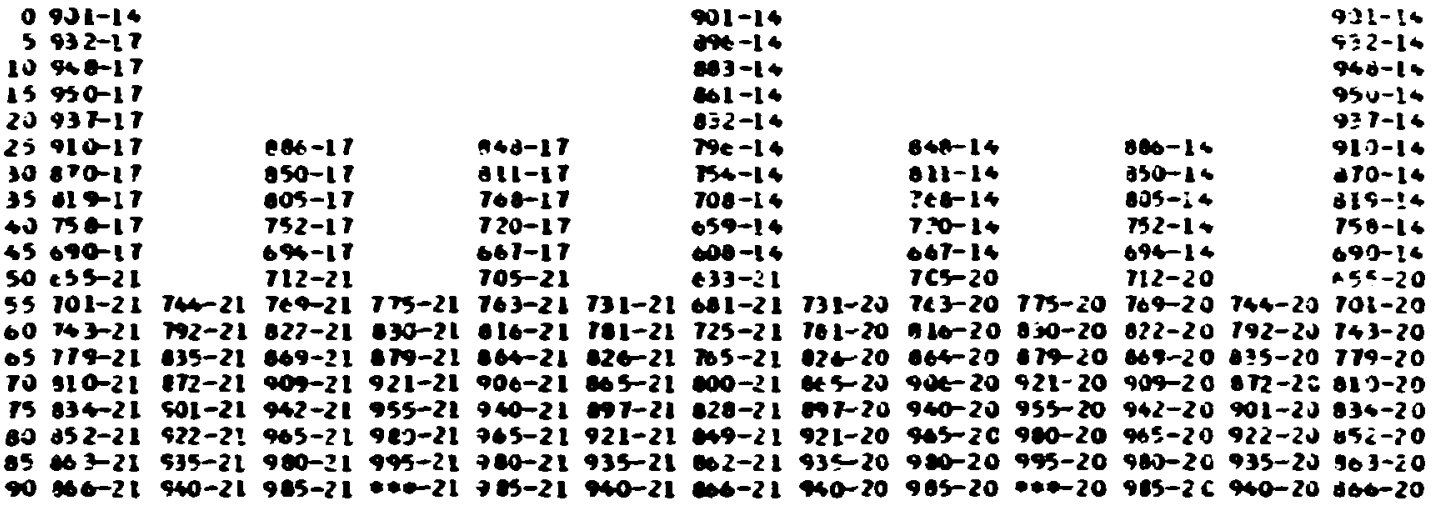




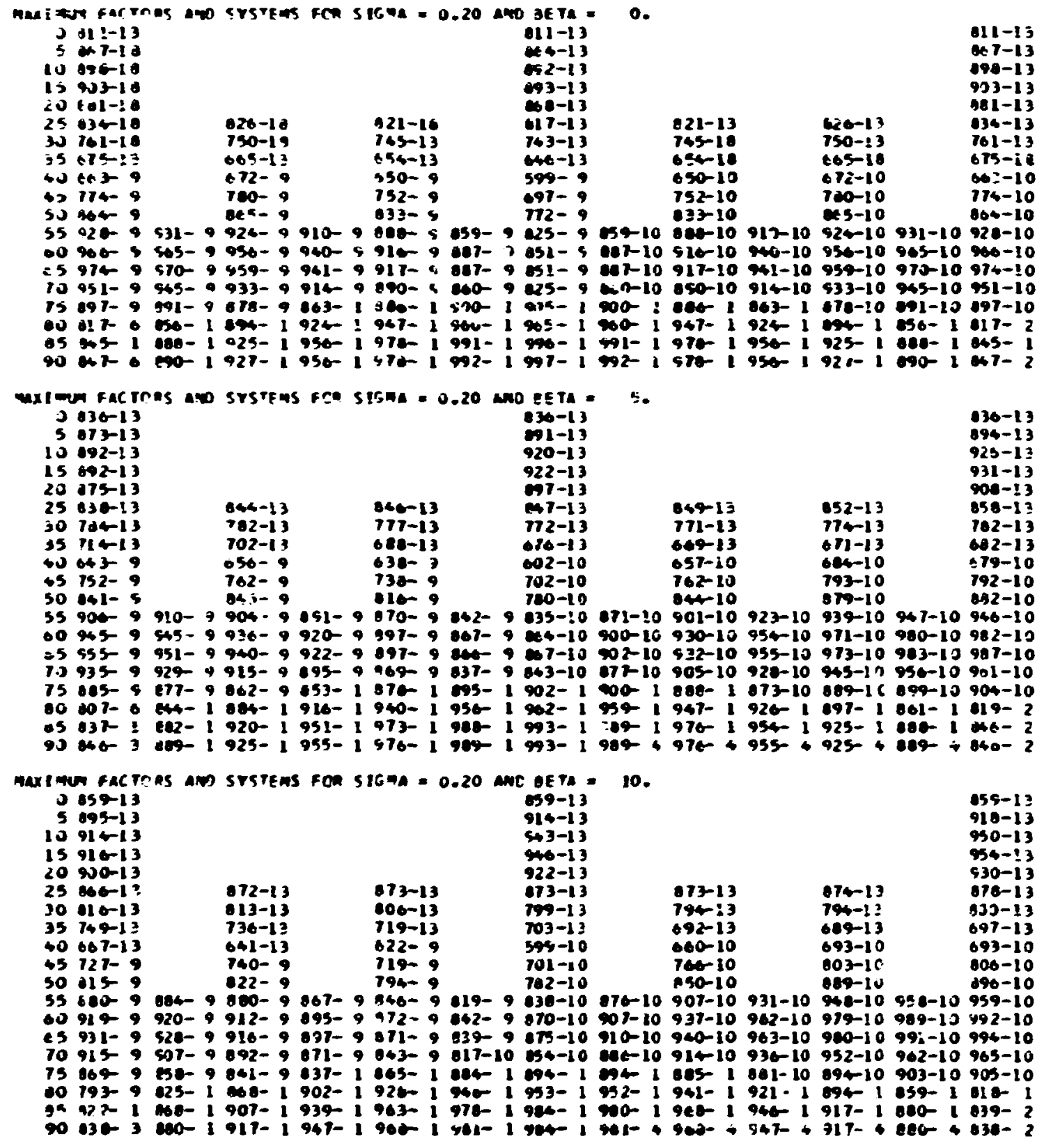




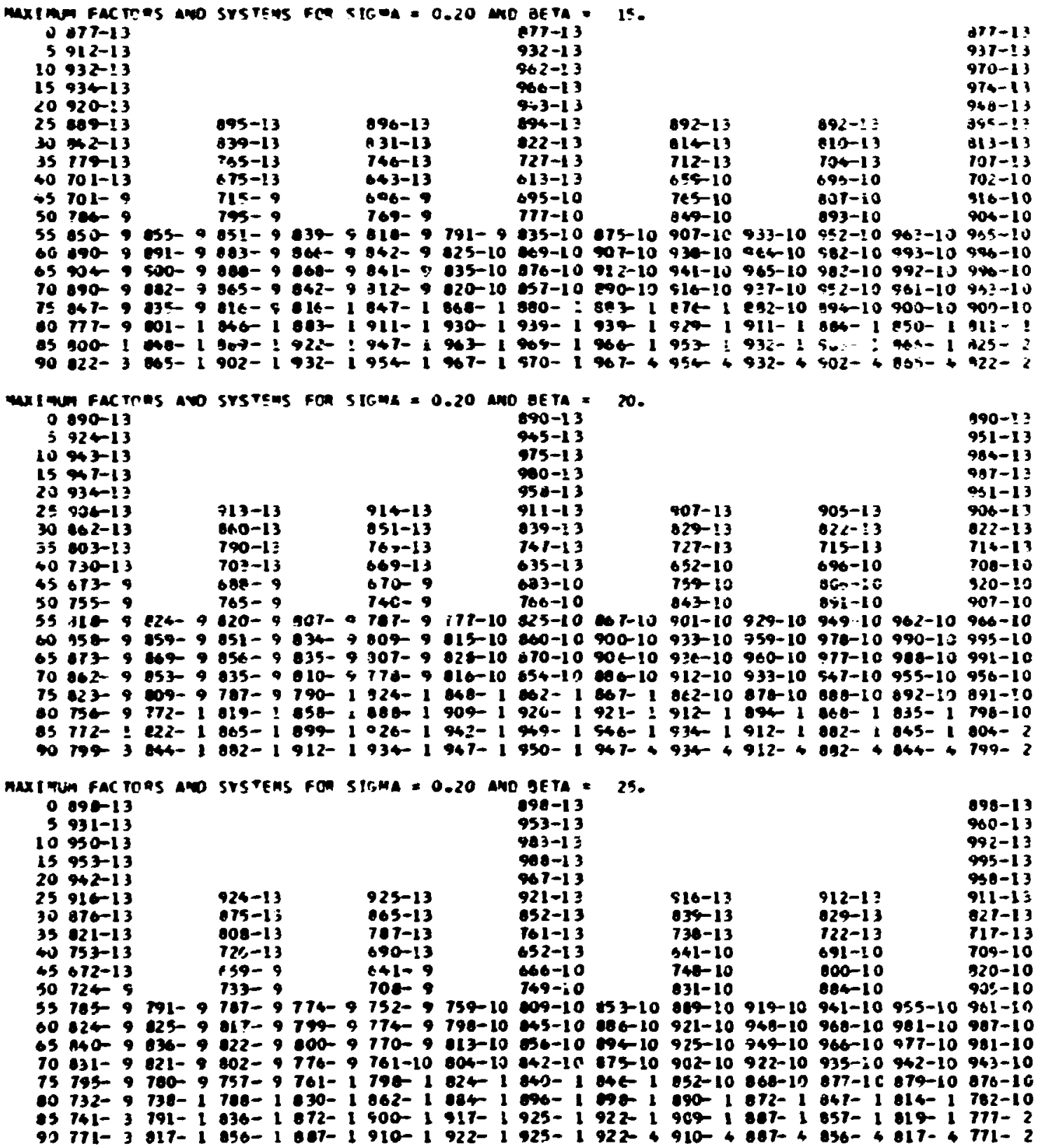




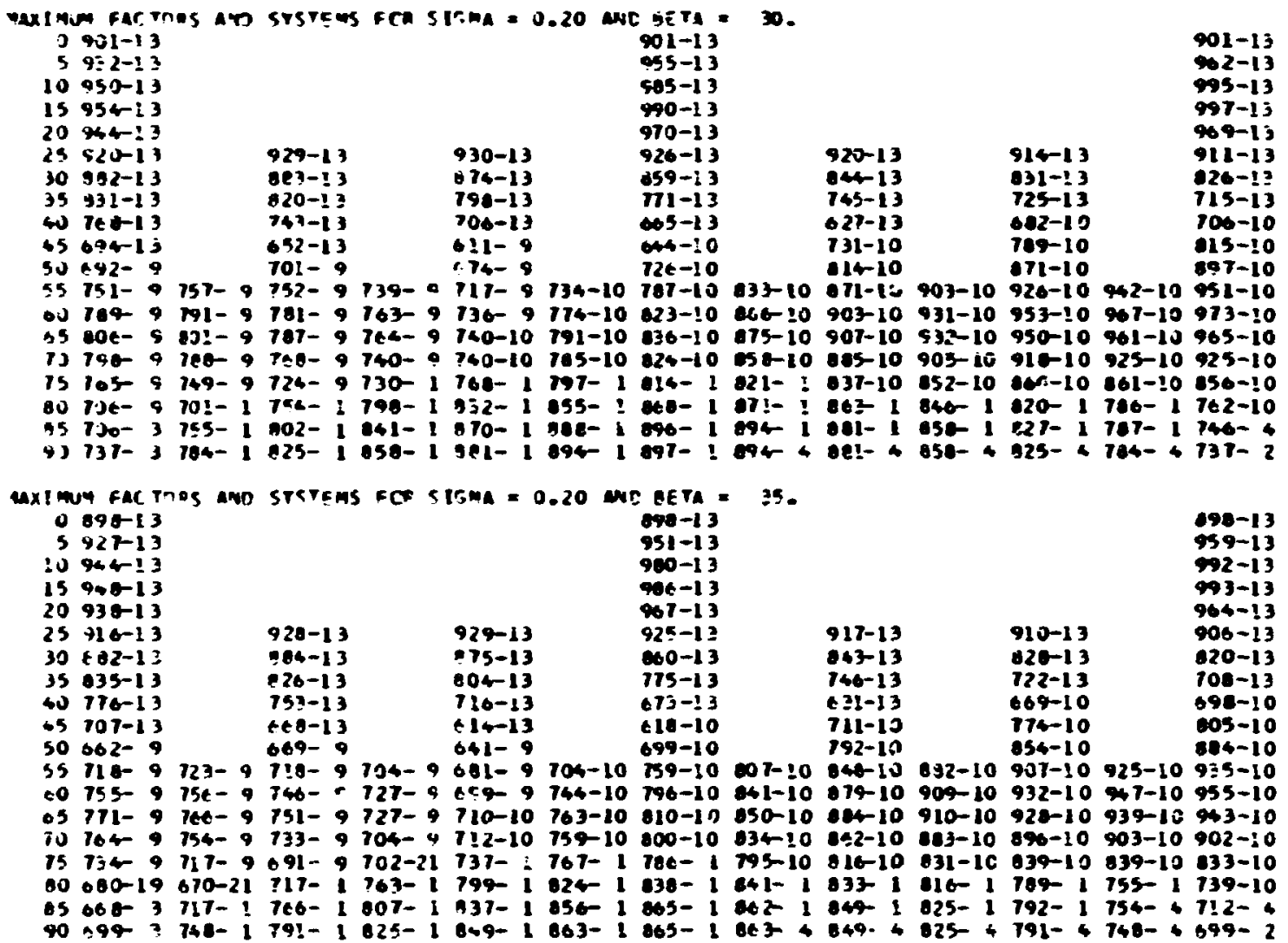

WAXIRUM FACTIAS AN SYSTEAS FLQ SIG

$060-13$

5 91 ? 13

$10932-13$

$15936-13$

$20927-13$

25 900-13

$30.74-13$

$39330-13$

40 777-13

$45113-13$

So $042-19$ $55687-9691-938-9 \quad 647-9 \quad 60-10$

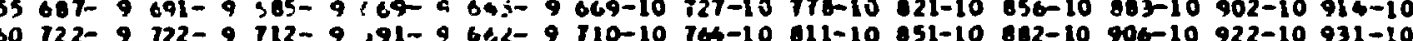

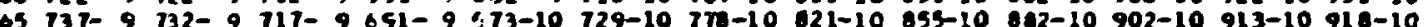
$70731-9720-9699-0708-21$ 708-21 727-10 770-10 06-10 835-10 85s-10 870-10 876-10 875-10 $15720-19$ 685-9 720-: $739-21735-21735-1755-2708-10$ 791-10 606-10 $13-10$ 813-10 806-10

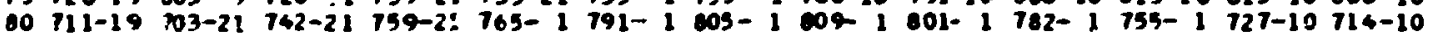

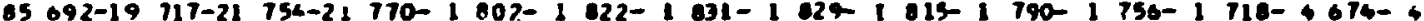

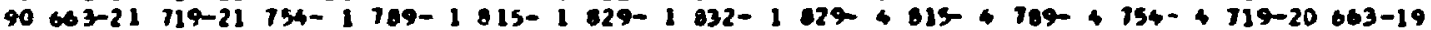




\begin{tabular}{|c|c|c|c|c|c|c|c|c|c|c|c|c|c|}
\hline $\begin{array}{l}0 \\
6 \\
5 \\
10 \\
15 \\
20 \\
25 \\
30 \\
35 \\
40 \\
45 \\
50 \\
55 \\
65 \\
65 \\
70 \\
75 \\
10 \\
85 \\
90\end{array}$ & $\begin{array}{l}\text { FACT0 } \\
877-13 \\
901-13 \\
915-13 \\
910-13 \\
999-13 \\
309-13 \\
659-13 \\
919-13 \\
769-13 \\
110-13 \\
604-19 \\
694-19 \\
120-19 \\
730-19 \\
769-19 \\
750-19 \\
741-19 \\
722-19 \\
693-21\end{array}$ & $\begin{array}{l}7 \\
7 \\
601-9 \\
691-9 \\
700-9 \\
610-9 \\
715-21 \\
737-21 \\
750-21 \\
752-21\end{array}$ & $\begin{array}{l}905-13 \\
607-13 \\
3: 9-12 \\
752-13 \\
677-13 \\
610-9 \\
654-9 \\
679-9 \\
659-21 \\
727-21 \\
757-21 \\
770-21 \\
780-21 \\
780-21\end{array}$ & $\begin{array}{l}7 \\
837-9 \\
636-21 \\
610-21 \\
767-2 ! \\
776-21 \\
795-21 \\
803-21 \\
800-21\end{array}$ & $\begin{array}{l}900-13 \\
860-13 \\
796-13 \\
716-13 \\
624-13 \\
575-9 \\
620-21 \\
600-21 \\
711-21 \\
740-21 \\
772-21 \\
709-21 \\
794-21 \\
780-21\end{array}$ & $\begin{array}{l}7 \\
\\
631-10 \\
671-10 \\
692-21 \\
723-21 \\
746-21 \\
759-21 \\
787-1 \\
794-1\end{array}$ & $\begin{array}{l}671-13 \\
926-13 \\
956-13 \\
960-13 \\
963-13 \\
904-13 \\
645-13 \\
76 c-13 \\
671-13 \\
563-13 \\
636-10 \\
692-10 \\
720-10 \\
742-10 \\
735-10 \\
726-1 \\
772-1 \\
797-1 \\
797-1\end{array}$ & 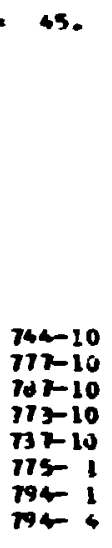 & $\begin{array}{l}890-5 j \\
825-13 \\
734-13 \\
425-19 \\
660-10 \\
736-10 \\
769-10 \\
819-10 \\
823-10 \\
863-10 \\
761-10 \\
767-1 \\
779-1 \\
786-20\end{array}$ & $\begin{array}{l} \\
62 t-10 \\
852-10 \\
651-10 \\
326-10 \\
777-10 \\
157-20 \\
765-20 \\
600-20\end{array}$ & $\begin{array}{l}607-! 3 \\
807-13 \\
705-13 \\
632-10 \\
731-10 \\
600-13 \\
855-10 \\
677-10 \\
672-19 \\
640-10 \\
765-10 \\
751-20 \\
775-20 \\
708-20\end{array}$ & $\begin{array}{l}0 \\
8 \\
7 \\
7 \\
7 \\
7\end{array}$ & $\begin{array}{l}677-13 \\
930-13 \\
981-13 \\
968-13 \\
936-13 \\
079-13 \\
794-13 \\
604-13 \\
372-10 \\
771-10 \\
64-10 \\
885-10 \\
903-10 \\
886-10 \\
345-10 \\
777-10 \\
686-10 \\
587-20 \\
693-19\end{array}$ \\
\hline $\begin{array}{r}1 \text { 1 } 0 \\
0 \\
5 \\
10 \\
15 \\
20 \\
25 \\
30 \\
35 \\
40 \\
45 \\
50 \\
25 \\
60 \\
65 \\
70 \\
15 \\
80 \\
65 \\
90\end{array}$ & $\begin{array}{l}\text { W FAC T } \\
\text { d55-13 } \\
091-13 \\
093-13 \\
695-13 \\
680-13 \\
607-13 \\
630-13 \\
000-13 \\
754-13 \\
700-13 \\
677-19 \\
711-19 \\
739-19 \\
760-19 \\
773-19 \\
770-19 \\
770-19 \\
752-19 \\
723-21\end{array}$ & $\begin{array}{l}7 \\
75-19 \\
655-19 \\
677-19 \\
692-19 \\
72:-21 \\
750-21 \\
771-2 i \\
702-21 \\
784-21\end{array}$ & $\begin{array}{l}804-13 \\
840-13 \\
800-12 \\
740-13 \\
670-13 \\
592-13 \\
640-21 \\
686-21 \\
728-21 \\
764-21 \\
793-21 \\
813-21 \\
823-21 \\
822-21\end{array}$ & $\begin{array}{l}l \\
\\
\\
659-21 \\
707-21 \\
749-21 \\
705-21 \\
012-21 \\
830-21 \\
030-21 \\
035-21\end{array}$ & $\begin{array}{l}689-13 \\
84 j-13 \\
182-13 \\
707-13 \\
220-13 \\
316-21 \\
662-21 \\
100-21 \\
109-21 \\
102-21 \\
800-21 \\
82=-21 \\
620-21 \\
822-21\end{array}$ & $\begin{array}{l} \\
\\
640-21 \\
691-21 \\
720-21 \\
750-21 \\
760-21 \\
792-21 \\
793-21 \\
704-21\end{array}$ & $\begin{array}{l}959-13 \\
900-13 \\
933-13 \\
939-13 \\
423-13 \\
696-13 \\
029-13 \\
754-13 \\
603-13 \\
559-13 \\
539-10 \\
054-10 \\
699-10 \\
703-10 \\
713-21 \\
729-21 \\
738-1 \\
762-1 \\
703-1\end{array}$ & $\begin{array}{l}0 \\
709-10 \\
740-10 \\
750-10 \\
737-10 \\
102-10 \\
741-1 \\
764-20 \\
104-20\end{array}$ & $\begin{array}{l}617-13 \\
309-13 \\
721-13 \\
615-13 \\
630-10 \\
704-10 \\
755-10 \\
783-10 \\
768-10 \\
769-10 \\
735-20 \\
775-20 \\
604-20 \\
822-20\end{array}$ & $\begin{array}{l} \\
794-10 \\
118-10 \\
110-10 \\
193-10 \\
154-20 \\
793-20 \\
620-20 \\
035-20\end{array}$ & $\begin{array}{l}-113 \\
-13 \\
-11 \\
-11 \\
-110 \\
-10 \\
-10 \\
-10 \\
-10 \\
-10 \\
-10 \\
-10 \\
-10 \\
-20 \\
-20 \\
-20\end{array}$ & & $\begin{array}{l}0<9-13 \\
917-13 \\
967-13 \\
947-13 \\
917-13 \\
8: 9-13 \\
774-13 \\
670-14 \\
654-10 \\
769-10 \\
819-10 \\
860-10 \\
972-10 \\
850-10 \\
013-10 \\
740-10 \\
702-20 \\
710-20\end{array}$ \\
\hline $\begin{array}{l}5 \\
10 \\
15 \\
20 \\
25 \\
30 \\
35 \\
40 \\
45 \\
50 \\
55 \\
60 \\
65 \\
70 \\
75 \\
80 \\
95 \\
90\end{array}$ & $\begin{array}{l}\text { In FAC TC } \\
836-13 \\
857-13 \\
867-13 \\
867-13 \\
850-13 \\
839-13 \\
811-13 \\
775-13 \\
731-13 \\
621-13 \\
603-19 \\
720-19 \\
752-19 \\
717-19 \\
793-19 \\
799-19 \\
795-19 \\
719-19 \\
752-11\end{array}$ & $\begin{array}{l}0 \\
0 \\
\\
662-19 \\
687-19 \\
124-21 \\
757-21 \\
763-21 \\
603-21 \\
814-21 \\
61 t-21\end{array}$ & $\begin{array}{l}858-13 \\
824-12 \\
778-13 \\
722-13 \\
657-13 \\
673-21 \\
680-21 \\
725-21 \\
765-21 \\
800-21 \\
627-21 \\
946-21 \\
954-21 \\
855-21\end{array}$ & $\begin{array}{l} \\
\\
694-21 \\
764-21 \\
785-21 \\
220-21 \\
646-21 \\
66-21 \\
872-21 \\
60-21\end{array}$ & $\begin{array}{l}665-13 \\
621-13 \\
763-13 \\
692-13 \\
610-13 \\
651-21 \\
700-21 \\
764-21 \\
704-21 \\
816-21 \\
641-21 \\
656-21 \\
861-21 \\
855-21\end{array}$ & $\begin{array}{l}0 \\
614-21 \\
125-21 \\
71-21 \\
790-21 \\
011-21 \\
123-21 \\
025-21 \\
016-21\end{array}$ & 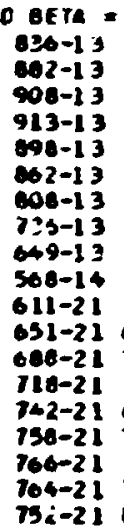 & $\begin{array}{l}0 \\
671-10 \\
102-10 \\
710-10 \\
690-10 \\
128-20 \\
767-20 \\
79720 \\
916-20\end{array}$ & $\begin{array}{l}854-13 \\
760-13 \\
704-11 \\
649-14 \\
600-10 \\
671-10 \\
720-10 \\
767-10 \\
750-10 \\
732-10 \\
771-20 \\
610-20 \\
630-20 \\
855-20\end{array}$ & $\begin{array}{l}7 \\
760-10 \\
753-10 \\
782-10 \\
757-10 \\
791-20 \\
820-20 \\
854-20 \\
860-20\end{array}$ & $\begin{array}{l}844-1= \\
760-13 \\
711-14 \\
658-14 \\
670-10 \\
747-10 \\
791-10 \\
610-1 c \\
604-10 \\
774-10 \\
767-20 \\
621-20 \\
644-20 \\
655-20\end{array}$ & $\begin{array}{l}0 \\
814-10 \\
829-10 \\
617-10 \\
701-10 \\
750-20 \\
719-20 \\
001-20 \\
016-20\end{array}$ & $\begin{array}{l}630-13 \\
993-13 \\
922-13 \\
921-13 \\
392-13 \\
625-13 \\
763-11 \\
709-14 \\
646-14 \\
724-10 \\
790-10 \\
629-10 \\
639-10 \\
922-10 \\
740-10 \\
714-10 \\
733-20 \\
747-20 \\
752-19\end{array}$ \\
\hline
\end{tabular}




\begin{tabular}{|c|c|c|c|c|c|c|c|c|c|c|c|c|c|}
\hline $\begin{array}{l}1 \\
5 \\
10 \\
53 \\
20 \\
25 \\
30 \\
35 \\
40 \\
45 \\
50 \\
35 \\
00 \\
65 \\
70 \\
15 \\
40 \\
05 \\
93\end{array}$ & 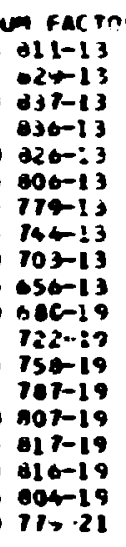 & $\begin{array}{l}0 \\
\\
281-21 \\
12:-21 \\
357-21 \\
709-21 \\
e 15-21 \\
63-21 \\
3:-21 \\
266-21\end{array}$ & 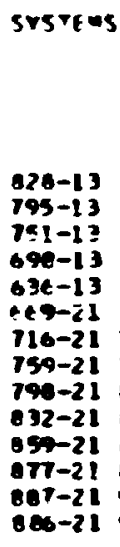 & $\begin{array}{l}0 \\
133-21 \\
710-21 \\
010-21 \\
031-21 \\
070-21 \\
0 \%-21 \\
903-21 \\
900-21\end{array}$ & $\begin{array}{l}630-13 \\
794-11 \\
739-11 \\
611-13 \\
635-21 \\
605-21 \\
632-21 \\
110-21 \\
815-21 \\
367-21 \\
871-21 \\
306-21 \\
891-21 \\
696-21\end{array}$ & $\begin{array}{l}0 \\
714-21 \\
755-21 \\
790-21 \\
019-21 \\
034-21 \\
451-21 \\
453-21 \\
1046-21\end{array}$ & 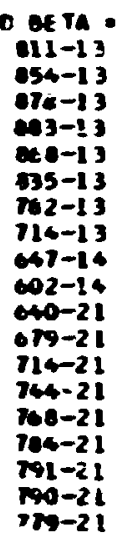 & $\begin{array}{l}0 \\
63-10 \\
662-10 \\
600-21 \\
116-20 \\
762-20 \\
000-20 \\
220-20 \\
06-20\end{array}$ & $\begin{array}{l}027-13 \\
767-16 \\
120-14 \\
604-16 \\
635-14 \\
637-10 \\
604-10 \\
709-10 \\
112-10 \\
700-20 \\
607-20 \\
64-20 \\
60-20 \\
06-20\end{array}$ & $\begin{array}{l}725-10 \\
740-10 \\
745-10 \\
701-20 \\
227-20 \\
662-20 \\
600-20 \\
900-20\end{array}$ & $\begin{array}{l}624-14 \\
76-114 \\
746-14 \\
695-14 \\
649-10 \\
715-10 \\
757-10 \\
75-10 \\
76-10 \\
770-20 \\
621-20 \\
656-20 \\
675-20 \\
86-20\end{array}$ & $\begin{array}{l}700-10 \\
794-10 \\
762-10 \\
753-20 \\
791-20 \\
20-20 \\
30-20 \\
060-20\end{array}$ & $\begin{array}{l}61-13 \\
66-23 \\
693-13 \\
692-13 \\
63-13 \\
637-14 \\
797-14 \\
745-14 \\
603-14 \\
697-10 \\
760-10 \\
796-10 \\
605-10 \\
780-10 \\
746-10 \\
730-20 \\
762-20 \\
315-20 \\
179-19\end{array}$ \\
\hline $\begin{array}{l}x 10 \\
0 \\
5 \\
20 \\
15 \\
20 \\
25 \\
30 \\
35 \\
40 \\
45 \\
50 \\
55 \\
60 \\
85 \\
10 \\
75 \\
80 \\
65 \\
90\end{array}$ & 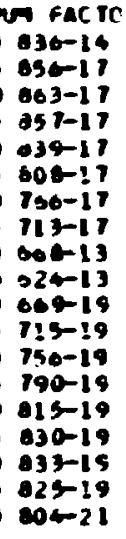 & $\begin{array}{l}712-21 \\
751-21 \\
707-21 \\
117-21 \\
112-21 \\
660-21 \\
171-21 \\
273-21\end{array}$ & $\begin{array}{l}193-13 \\
7 \in 1-13 \\
719-13 \\
669-13 \\
652-21 \\
699-21 \\
745-21 \\
790-21 \\
327-21 \\
660-21 \\
966-21 \\
905-21 \\
916-21 \\
914-21\end{array}$ & $\begin{array}{l}0 \\
9 \\
7 \leq 1-21 \\
805-21 \\
965-21 \\
679-2 ! \\
905-21 \\
922-21 \\
931-2 ! \\
929-21\end{array}$ & $\begin{array}{l}104-13 \\
764-13 \\
111-13 \\
646-13 \\
6 \in 2-21 \\
711-21 \\
750-21 \\
002-21 \\
940-21 \\
873-21 \\
897-21 \\
913-21 \\
919-21 \\
914-21\end{array}$ & $\begin{array}{l}3 \\
3 \\
730-21 \\
776-21 \\
013-21 \\
042-21 \\
063-21 \\
1076-21 \\
1074-21 \\
1873-21\end{array}$ & $\begin{array}{l}10 \text { EE TA }= \\
636-14 \\
643-14 \\
66-13 \\
651-13 \\
630-13 \\
604-12 \\
754-13 \\
114-14 \\
672-14 \\
620-14 \\
602-21 \\
701-21 \\
735-21 \\
165-21 \\
769-21 \\
605-21 \\
613-21 \\
613-21 \\
604-21\end{array}$ & $\begin{array}{l}0 \\
0 \\
44-21 \\
475-21 \\
700-20 \\
75:-20 \\
79-20 \\
030-20 \\
054-20 \\
073-20\end{array}$ & $\begin{array}{l}124-14 \\
193-14 \\
754-14 \\
713-16 \\
665-14 \\
610-16 \\
646-10 \\
604-20 \\
163-20 \\
796-20 \\
640-20 \\
675-20 \\
900-20 \\
924-20\end{array}$ & $\begin{array}{l}090-10 \\
710-10 \\
106-20 \\
117-20 \\
060-20 \\
993-20 \\
910-20 \\
929-20\end{array}$ & $\begin{array}{l}851-14 \\
610-14 \\
770-14 \\
720-14 \\
671-14 \\
603-10 \\
725-10 \\
739-10 \\
763-20 \\
613-20 \\
053-20 \\
806-20 \\
504-20 \\
914-20\end{array}$ & $\begin{array}{l}167-10 \\
159-10 \\
760-10 \\
705-20 \\
622-20 \\
048-20 \\
665-20 \\
673-20\end{array}$ & $\begin{array}{l}836-14 \\
676-16 \\
695-14 \\
901-14 \\
691-14 \\
605-14 \\
026-14 \\
775-14 \\
715-14 \\
669-10 \\
720-10 \\
752-10 \\
770-10 \\
733-10 \\
736-20 \\
706-20 \\
738-20 \\
600-20 \\
004-19\end{array}$ \\
\hline $\begin{array}{l}x 19 \\
0 \\
5 \\
10 \\
15 \\
20 \\
25 \\
30 \\
35 \\
40 \\
45 \\
50 \\
55 \\
00 \\
65 \\
10 \\
15 \\
00 \\
05 \\
90\end{array}$ & 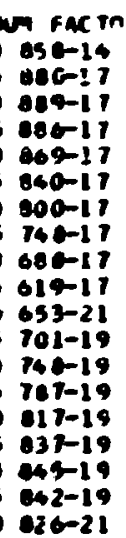 & $\begin{array}{l}131-21 \\
776-21 \\
011-21 \\
261-21 \\
066-21 \\
903-21 \\
904-21 \\
96-21\end{array}$ & $\begin{array}{l}806-17 \\
760-17 \\
720-17 \\
665-17 \\
674-21 \\
722-21 \\
768-21 \\
912-21 \\
650-21 \\
683-21 \\
910-21 \\
926-21 \\
930-21 \\
939-21\end{array}$ & $\begin{array}{l}102-21 \\
1021-21 \\
267-21 \\
901-21 \\
927-21 \\
949-21 \\
994-21 \\
953-21\end{array}$ & $\begin{array}{l}769-13 \\
130-23 \\
679-13 \\
632-21 \\
601-21 \\
730-21 \\
776-21 \\
922-21 \\
600-21 \\
693-21 \\
910-21 \\
934-21 \\
941-21 \\
934-21\end{array}$ & $\begin{array}{l}3 \\
3 \\
1 \\
1 \\
7 s 9-21 \\
79-21 \\
141-21 \\
100-21 \\
1002-21 \\
090-21 \\
900-21 \\
090-21\end{array}$ & $\begin{array}{l}64-14 \\
60-14 \\
640-14 \\
629-14 \\
02-14 \\
709-14 \\
711-14 \\
490-14 \\
64-14 \\
677-21 \\
715-21 \\
750-21 \\
700-21 \\
64-21 \\
021-21 \\
031-21 \\
632-21 \\
02-21\end{array}$ & $\begin{array}{l} \\
654 \\
600 \\
796 \\
704 \\
025 \\
601\end{array}$ & $\begin{array}{l}14 \\
014 \\
170 \\
13 \\
600 \\
639 \\
160 \\
722 \\
179 \\
129 \\
170 \\
903\end{array}$ & $\begin{array}{l}190-20 \\
742-20 \\
794-20 \\
949-20 \\
969-20 \\
921-20 \\
942-20 \\
953-20\end{array}$ & $\begin{array}{l}672-14 \\
640-14 \\
799-14 \\
750-14 \\
690-14 \\
651-10 \\
669-10 \\
742-20 \\
797-20 \\
64-20 \\
602-20 \\
911-20 \\
930-20 \\
934-20\end{array}$ & $\begin{array}{l}713-10 \\
725-10 \\
712-20 \\
014-20 \\
146-20 \\
173-20 \\
109-20 \\
096-20\end{array}$ & $\begin{array}{l}8510-14 \\
696-14 \\
917-14 \\
923-14 \\
913-14 \\
800-14 \\
050-14 \\
000-14 \\
741-14 \\
615-14 \\
691-10 \\
729-10 \\
730-10 \\
725-20 \\
762-20 \\
790-20 \\
210-20 \\
622-20 \\
926-20\end{array}$ \\
\hline
\end{tabular}




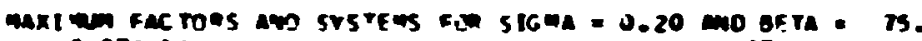
- 876-14 $5901-17$

$10912-17$

15 910-17

$2090-17$

25 cin

30 24-17

35 TTE-17

$40719-7$

$45 \div 52-17$

So $671-21$

(s) $1-14$

936-14

60-16

838-17 795-17

12:-14

794-17

Tra-16

T01-17

$6 e 8-21 \quad+92-21$

$7 \$ 2-21$

141-14

699-! 6

$43-21$

\section{7}

790-21 $7-21717-20757-20770-20774-20 \quad 751-20 \quad 709-20$

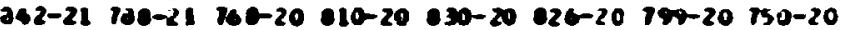

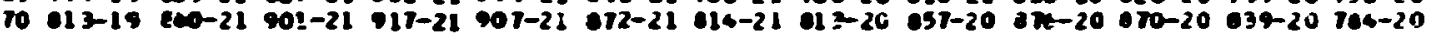

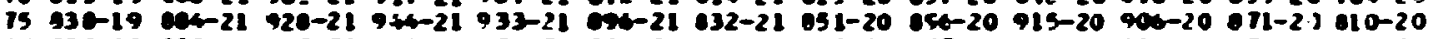

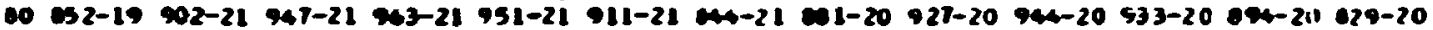

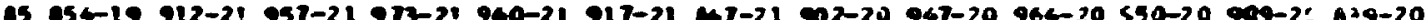

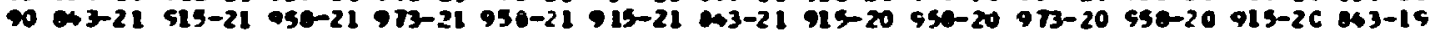

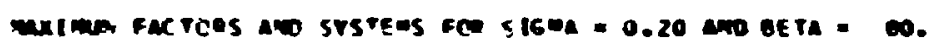

0 ope-14

$5916-17$

$10930-17$

15 930-17

20 917-17

25 ea-17

30 es -17

35 cos-17

$4074-17$

$4567+17$

so 6e1-21

(No-14

191-14

93-14

ase-14

$\begin{array}{rr}82-17 & 021-17 \\ 626-17 & 703-11\end{array}$

626-11

$21-17 \quad 016-14$

$702-14$

$731-17 \quad 091-17 \quad 700-14$

$60-14$

41-21

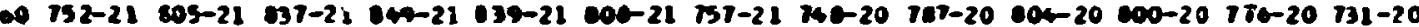

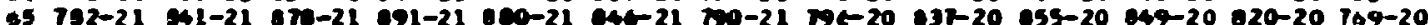

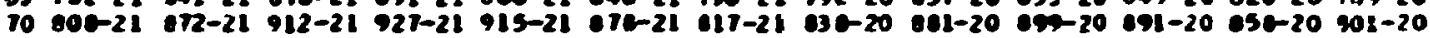

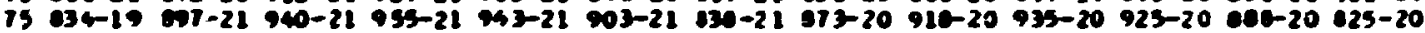

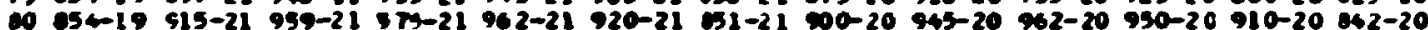

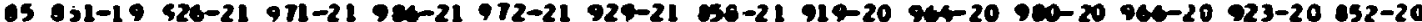

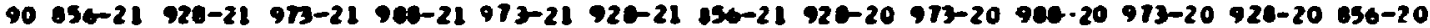

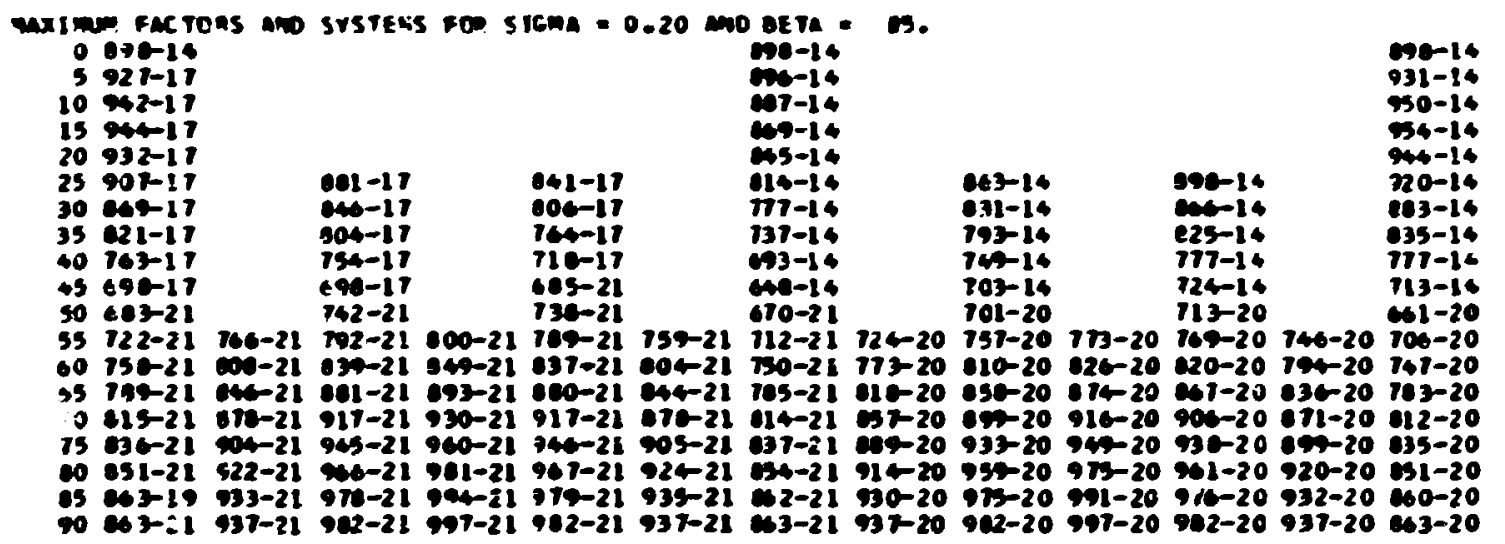




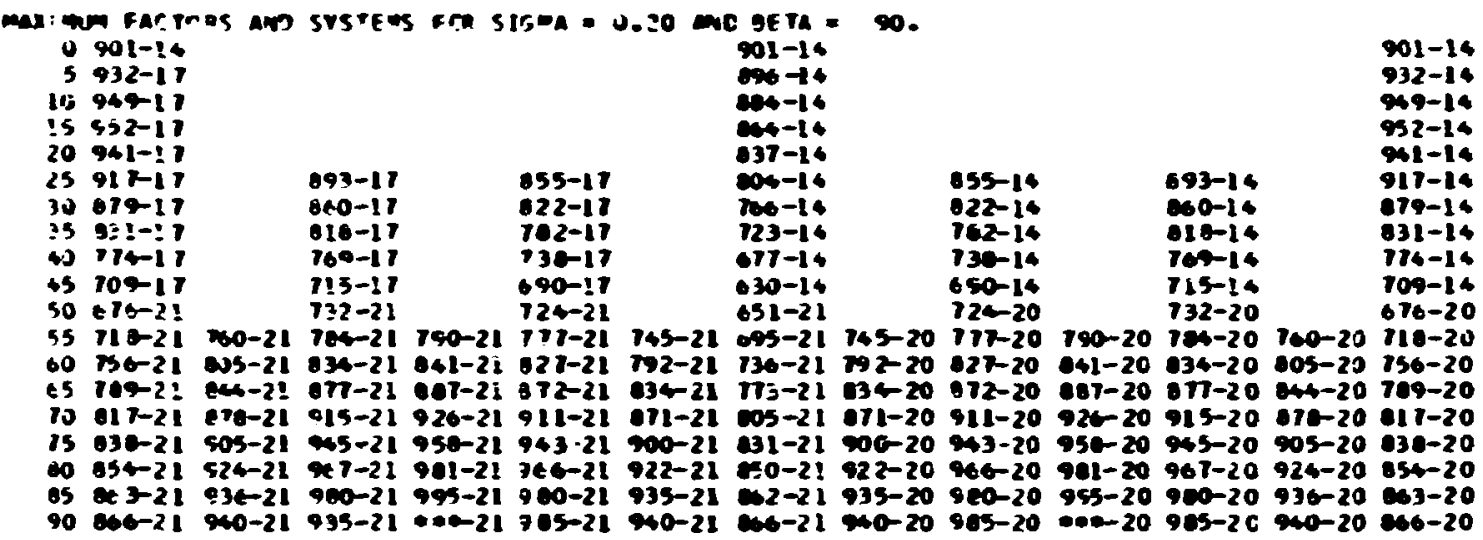




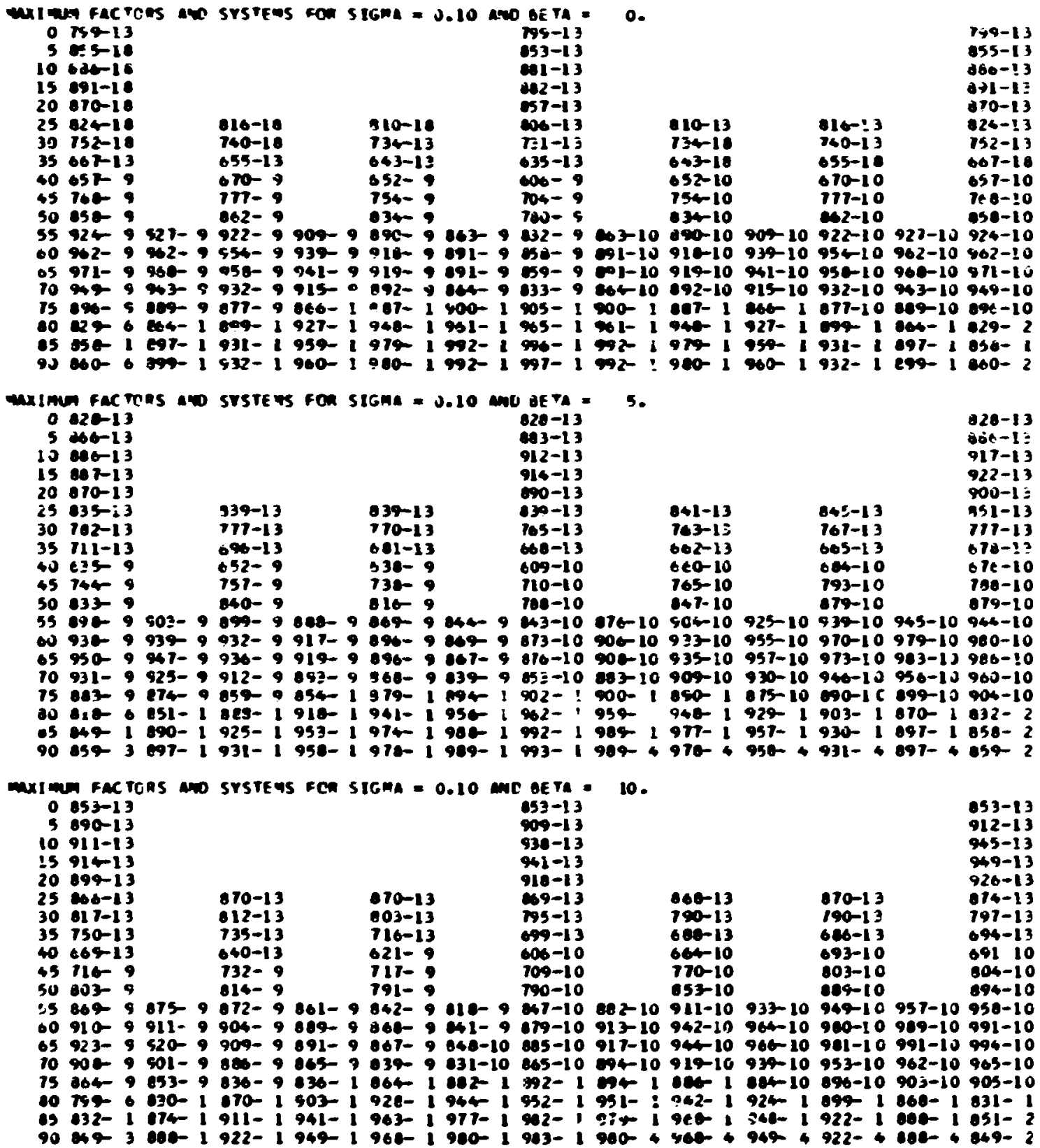




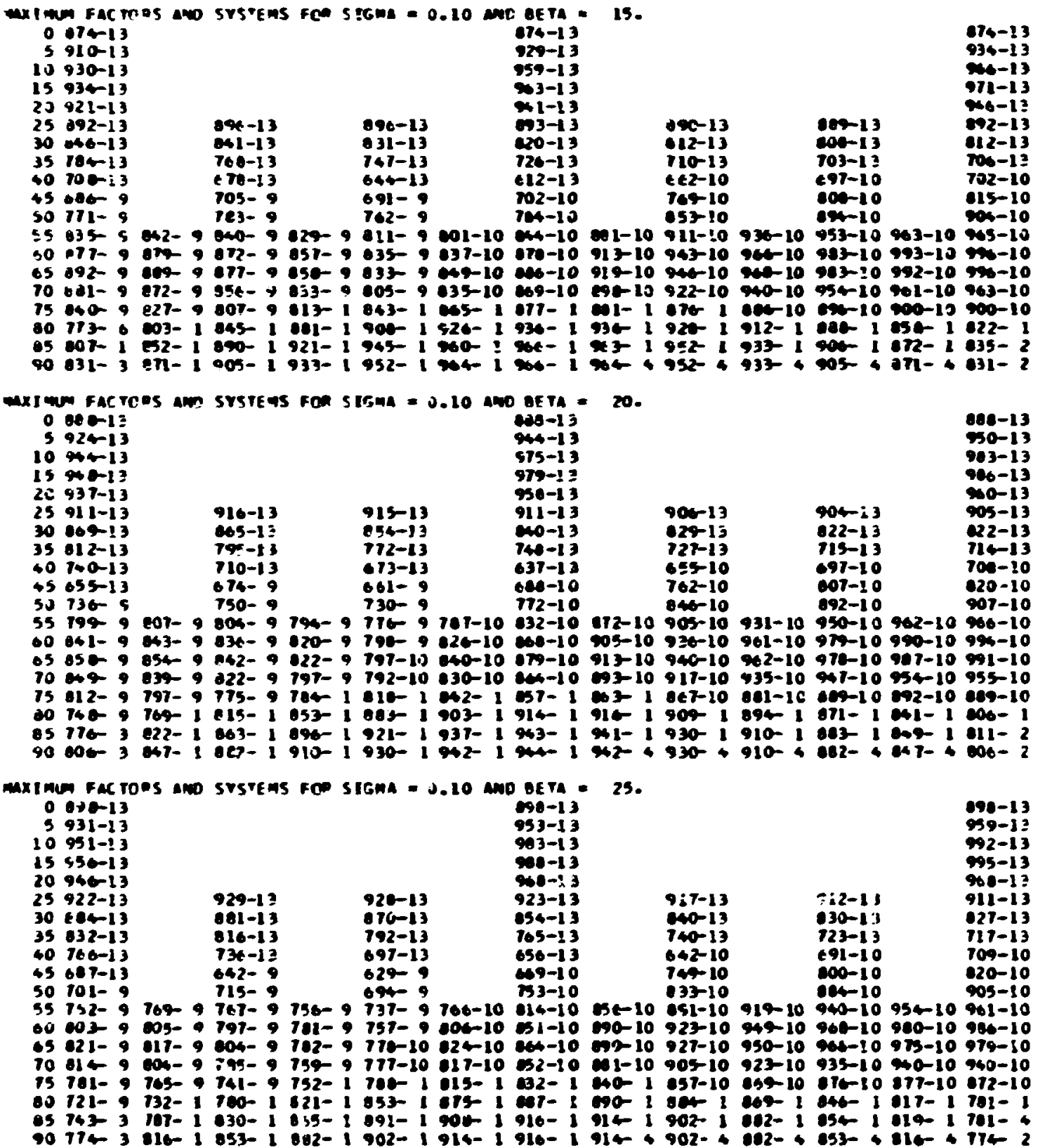




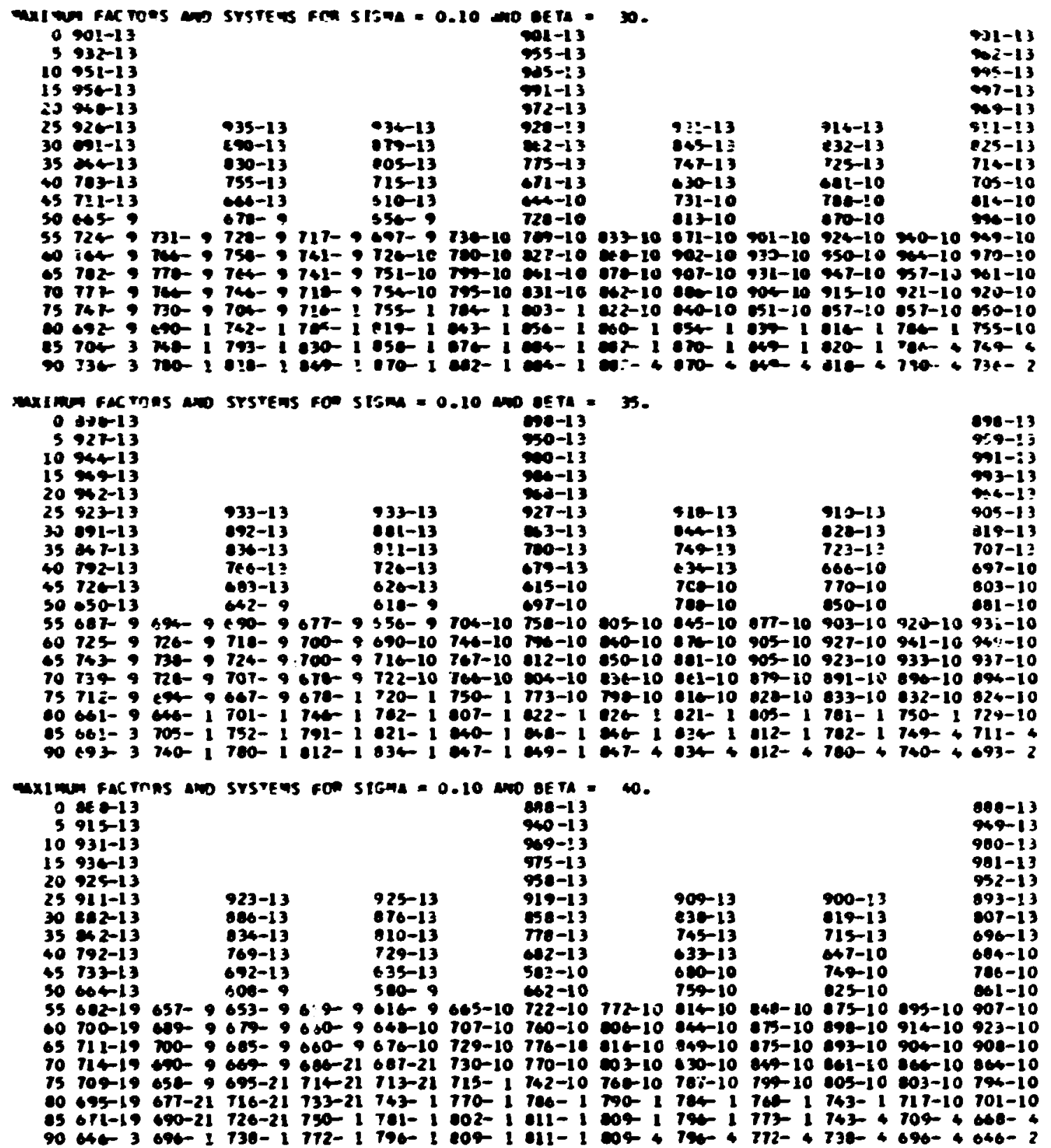




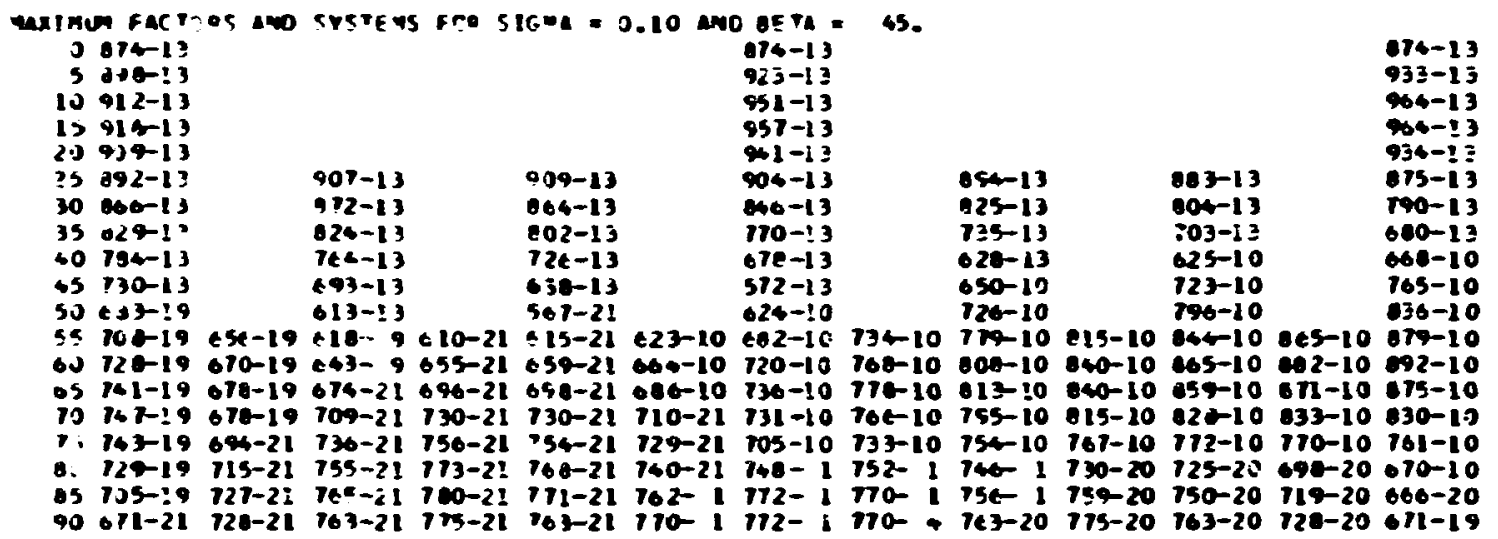

\begin{tabular}{|c|c|c|c|c|c|c|c|c|c|c|c|c|c|}
\hline $\begin{array}{l}0 \\
5 \\
10 \\
15 \\
20 \\
25 \\
30 \\
15 \\
43 \\
45 \\
50 \\
55 \\
00 \\
05 \\
70 \\
15 \\
20 \\
85 \\
90\end{array}$ & $\begin{array}{l}053-13 \\
575-13 \\
01-13 \\
090-13 \\
083-13 \\
607-13 \\
6 \$ 2-13 \\
008-13 \\
767-13 \\
718-13 \\
199-19 \\
727-19 \\
750-19 \\
7 \in t-19 \\
774-19 \\
772-19 \\
761-19 \\
730-19 \\
705-21\end{array}$ & $\begin{array}{l}673-19 \\
609-19 \\
700-19 \\
700-21 \\
736-21 \\
753-21 \\
764-21 \\
765-21\end{array}$ & $\begin{array}{l}903-13 \\
8 \times 1-12 \\
907-13 \\
152-13 \\
686-13 \\
412-13 \\
639-21 \\
680-21 \\
713-21 \\
751-21 \\
777-2 ! \\
709-21 \\
003-21 \\
902-21\end{array}$ & $\begin{array}{l}658-21 \\
701-21 \\
740-21 \\
772-21 \\
797-21 \\
813-21 \\
819-21 \\
014-21\end{array}$ & $\begin{array}{l}900-13 \\
845-13 \\
787-13 \\
710-13 \\
3 j-13 \\
616-21 \\
062-21 \\
704-21 \\
7+1-21 \\
711-21 \\
794-21 \\
007-21 \\
810-21 \\
002-21\end{array}$ & $\begin{array}{l}\mid \\
650-21 \\
689-21 \\
723-21 \\
769-21 \\
768-21 \\
777-21 \\
776-21 \\
765-21\end{array}$ & $\begin{array}{l}13-13 \\
900-13 \\
927-13 \\
922-13 \\
918-13 \\
833-13 \\
825-13 \\
756-13 \\
68-13 \\
500-13 \\
504-21 \\
640-10 \\
676-10 \\
692-10 \\
700-21 \\
720-21 \\
724-21 \\
733-1 \\
733-1\end{array}$ & $\begin{array}{l}694-10 \\
724-10 \\
73-10 \\
725-10 \\
69-10 \\
714-1 \\
76-20 \\
765-20\end{array}$ & $\begin{array}{l}873-13 \\
800-13 \\
720-13 \\
617-13 \\
617-10 \\
650-10 \\
760-10 \\
7 \in 6-10 \\
773-10 \\
756-10 \\
716-10 \\
753-20 \\
723-20 \\
802-20\end{array}$ & $\begin{array}{l}77-10 \\
779-10 \\
603-10 \\
002-10 \\
777-10 \\
732-20 \\
771-20 \\
790-20 \\
914-20\end{array}$ & $\begin{array}{l}062-1 \\
105-1 \\
600-1 \\
615-1 \\
694-1 \\
764-1 \\
009-1 \\
629-1 \\
022-1 \\
791-1 \\
737-1 \\
765-2 \\
709-2 \\
602-2\end{array}$ & $\begin{array}{r}932-10 \\
86-10 \\
734-10 \\
797-10 \\
730-10 \\
736-20 \\
756-20 \\
765-20\end{array}$ & $\begin{array}{r}953-13 \\
911-13 \\
71-13 \\
940-13 \\
911-13 \\
652-13 \\
768-13 \\
662-14 \\
647-10 \\
740-10 \\
908-10 \\
647-10 \\
957-10 \\
639-10 \\
796-10 \\
724-10 \\
685-20 \\
700-20 \\
705-19\end{array}$ \\
\hline
\end{tabular}




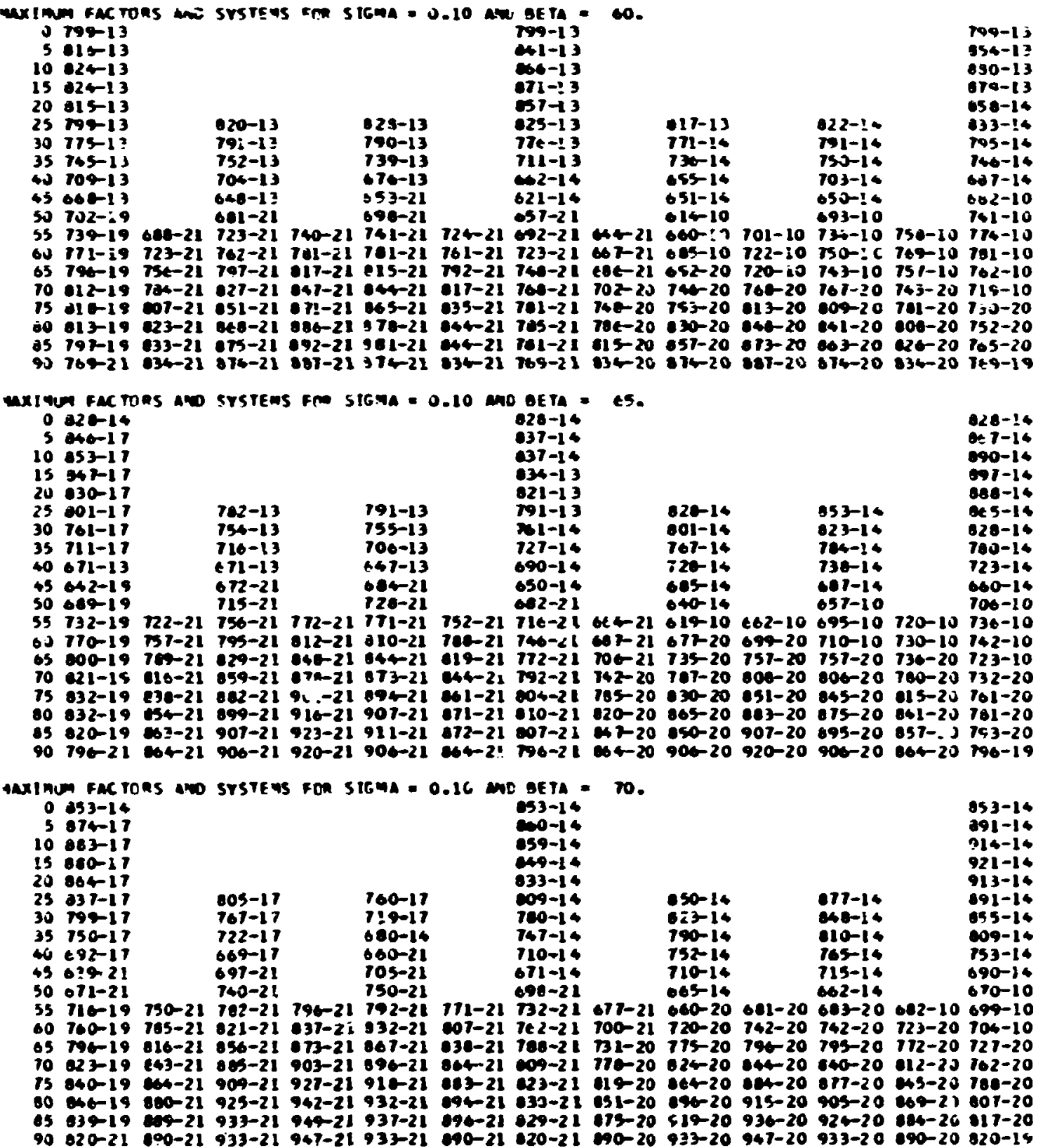




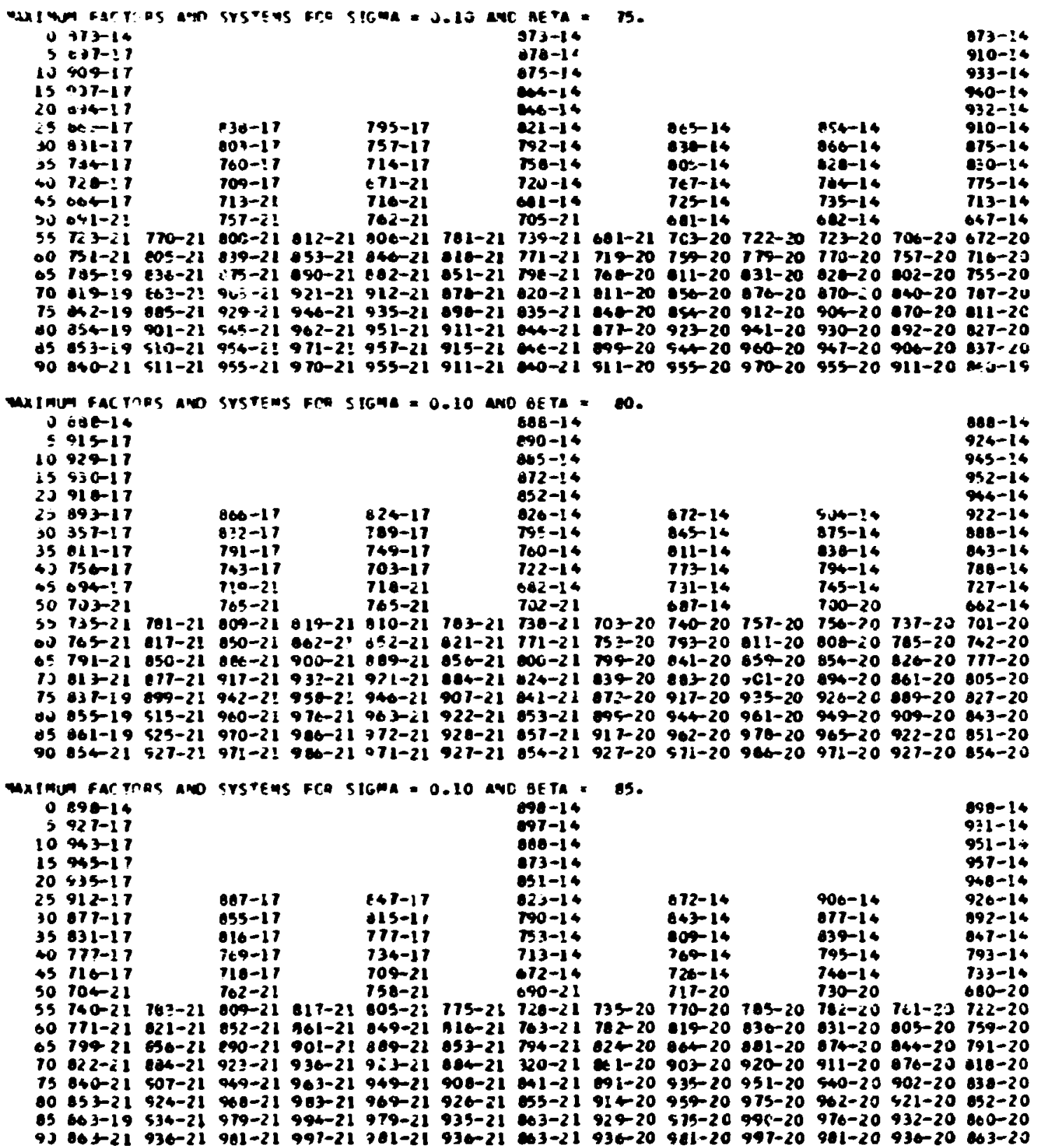




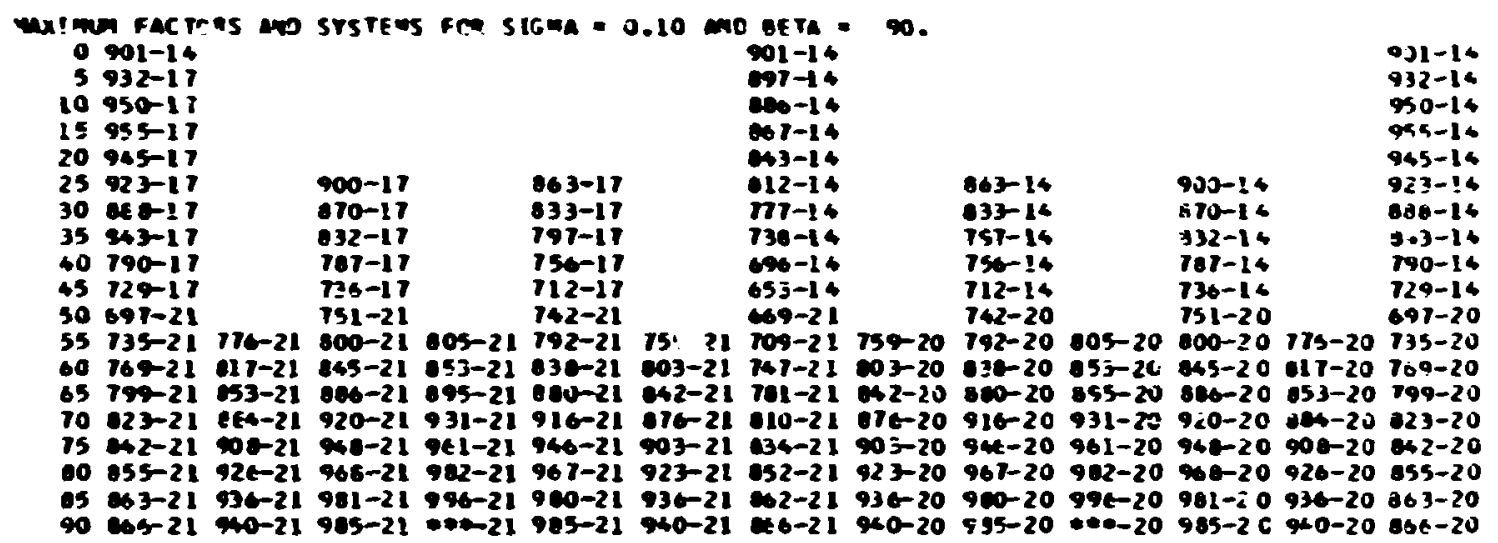




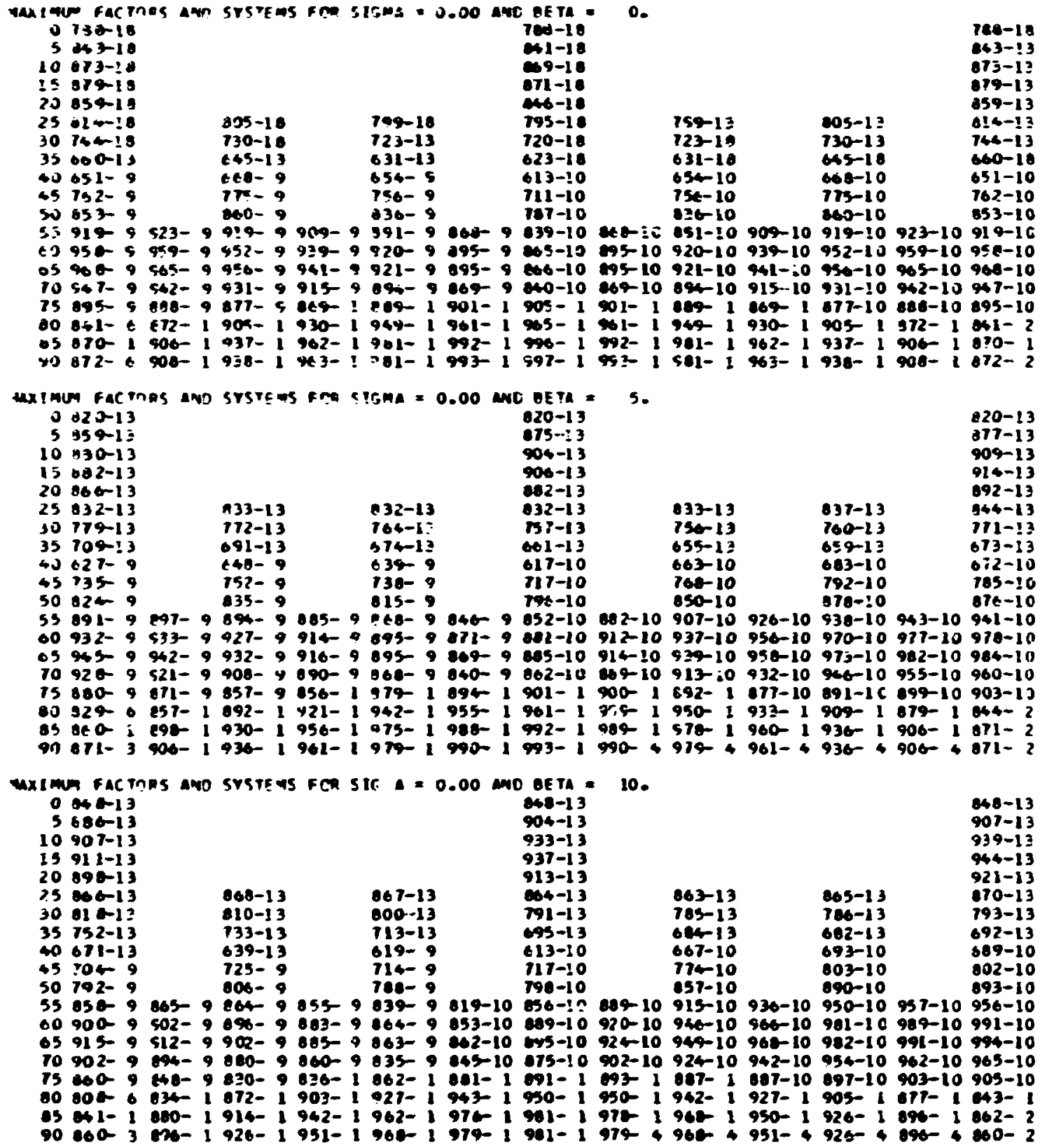




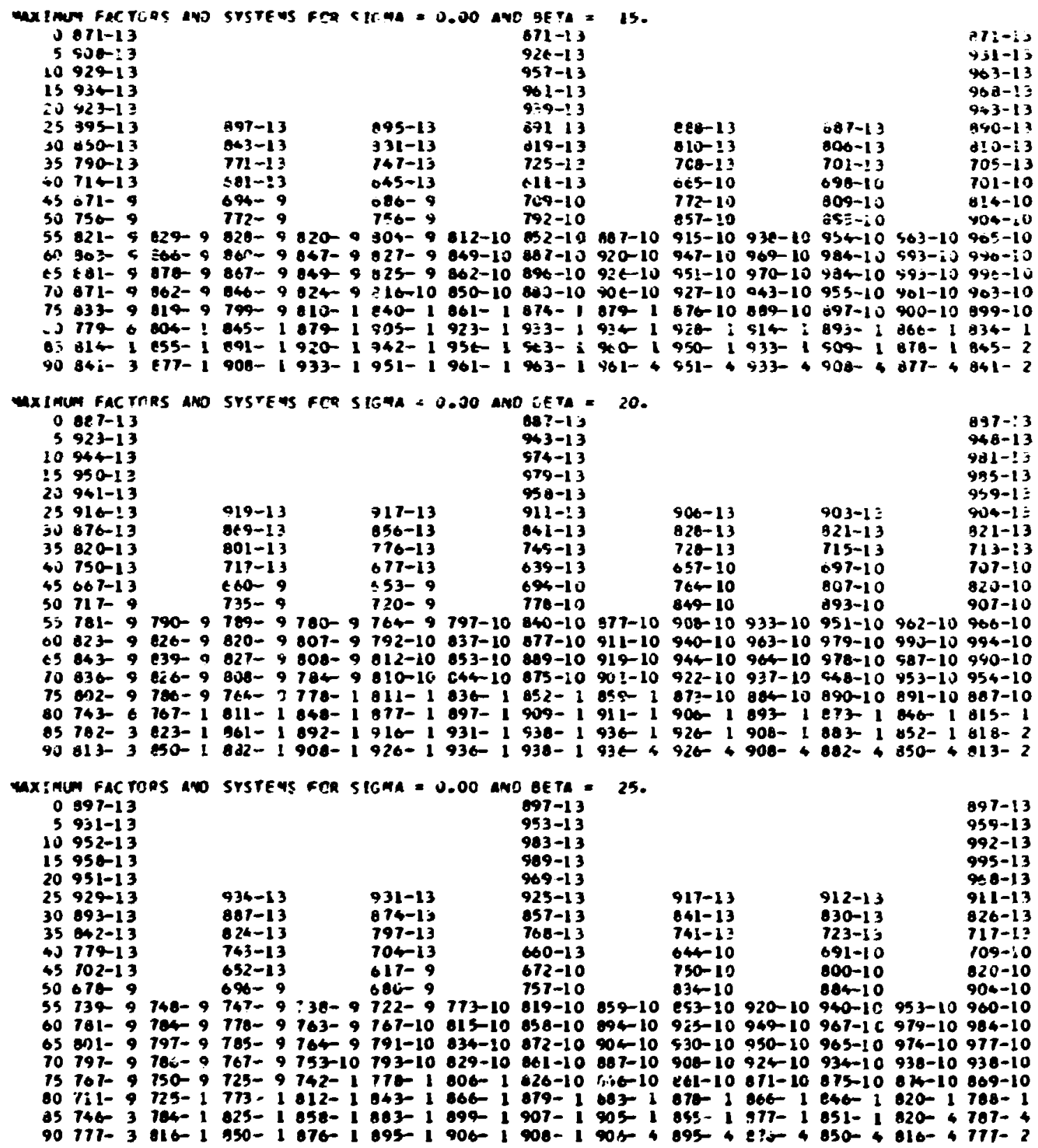




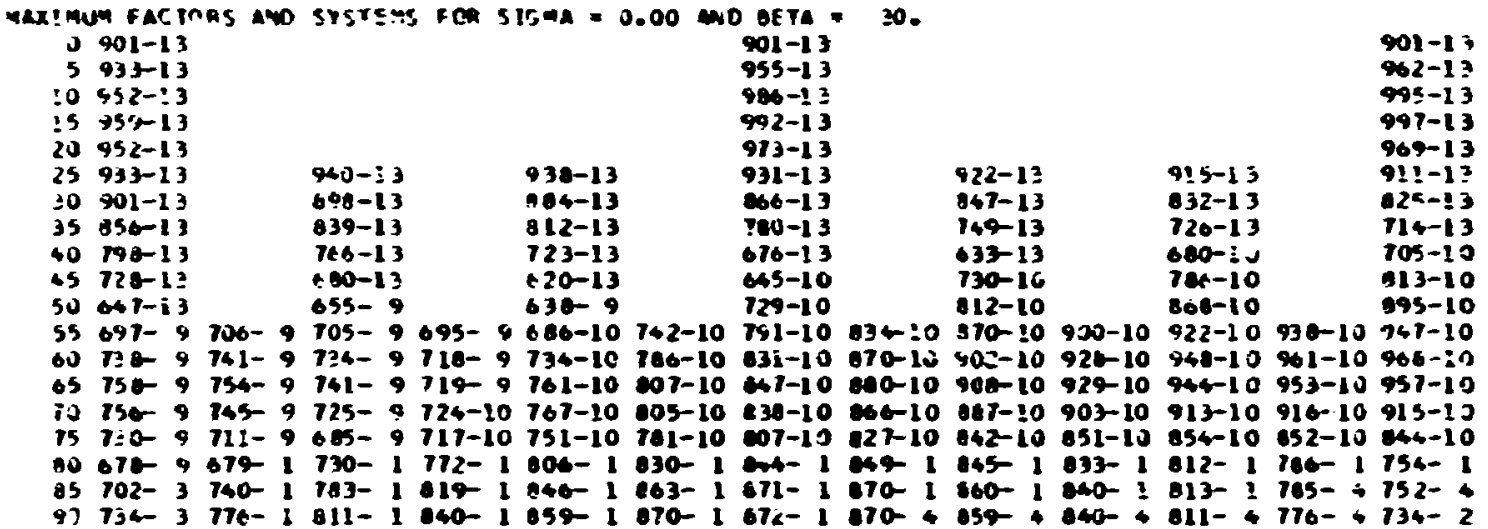

Wximun factinas awo srstets ene sigma $=0.00$ mo Bete $=35$

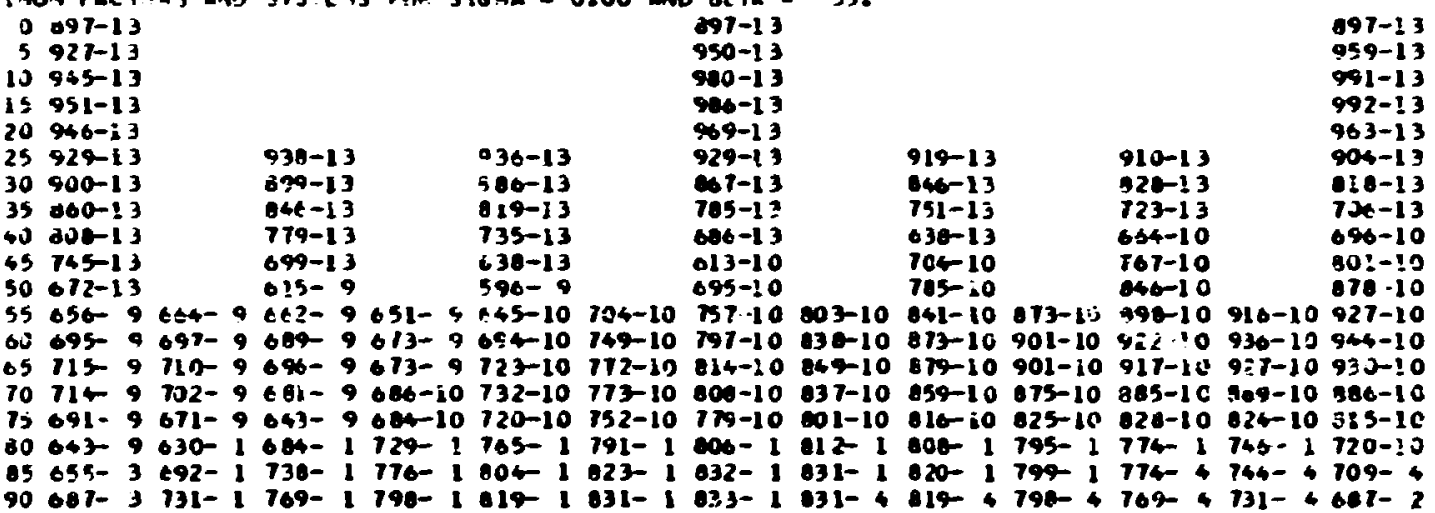

MXIMUM FACTCRS AND SYSTEMJ FCR SIGMA $=0.00$ AND EETA $=40$.

o e $7-13$

$3914-13$

ox $7-13$

$939-13$

947-13

$974-13$

$15936-13$

$974-13$

$20931-1$

$25916-1$

31 90-13

35 as -13

40 cos- 13

$45752-13$

50 6. 7-13

$927-13 \quad 927-13 \quad 920-13$

892-13 $821-13 \quad 061-13$

$61-13$
$703-13$

$618-13$
$740-13$

$608-13$
500-13

709-13

$64-13$

$55693-19066-19621-9600-$ .

59-15 661-10 117-10 760-10 807-10 841-10 868-10 886-10 901-10

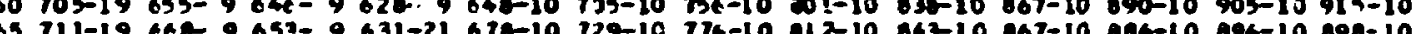

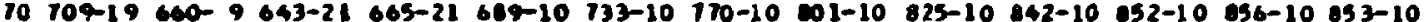
$75690-19632-19670-21690-21650-21716-10$ 745-10 76-10 764-10 $193-10796-10792-10761-10$ $00679-19651-21699-21706-21722-1749-1706-1771-1767-1753-1731-1704-10680-10$ $05650-19663-21690-21731-1761-1701-1790-1$ 189- $1711-1755-1730-4699-4662-4$ $90635-3682-1722-1754-1777-1799-1791-1795-4777-4754-4722-4682-4639-2$ 


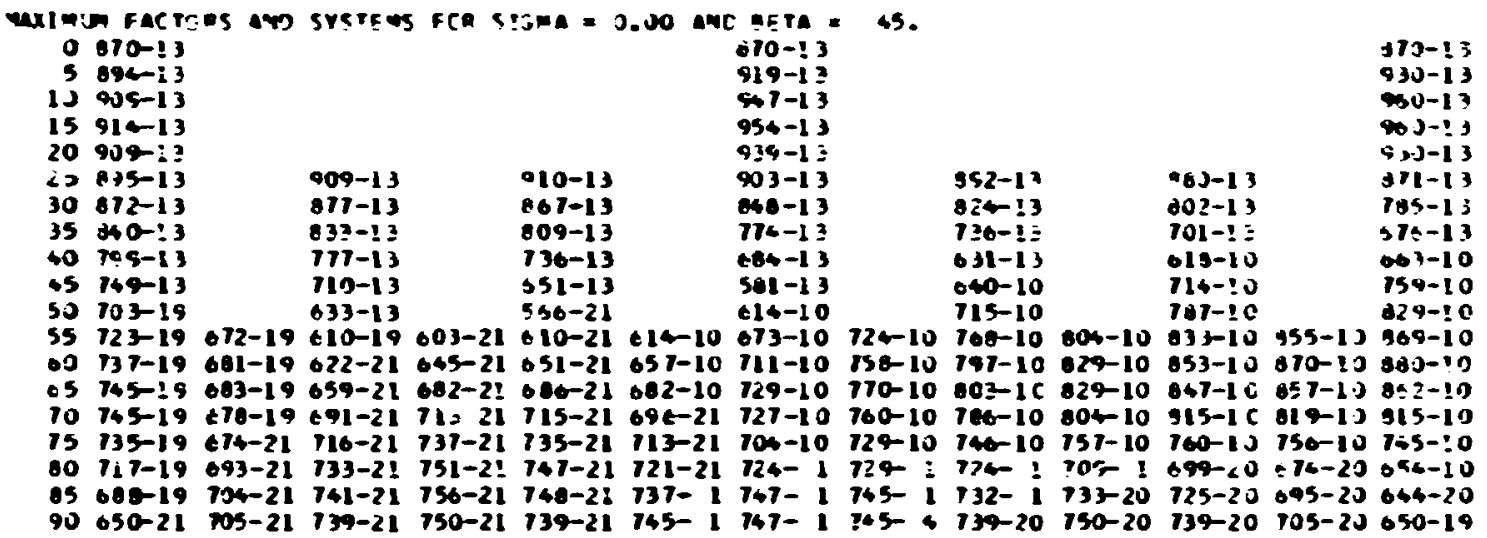

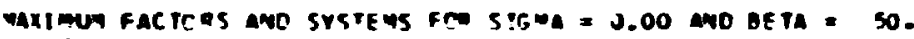

- ont-13

5 869-13

ex $3-13$

10 $801-13$

64t-13

921-13

is 005 : 3

20 800-i3

928-13

$914-13$

aco

25 \% 7-13

30 6 $6-1$ ?

35 016-13

40 780-13

45 136-13

so 120-19

\section{3-13}

\section{$0.6-13$}

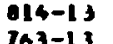

$763-13$
$702-13$

$632-13$

800-1 3

028-1 3

$\begin{array}{ll}846-19 & 020-13 \\ 792-13 & 758-13 \\ 725-13 & 674-13\end{array}$

$\begin{array}{ll}725-13 & 674-13 \\ 645-13 & 577-13\end{array}$

$02 \mathrm{C}-21$

$577-13$

657-21 $662-21$ 632-21 $627-21$ 679-10 725-10 704-10 794-10 $817-10$ :33-10 60 762-i9 702-19 t73-2i e9t-2i 700-21 607-21 $363-10$ 71 2-10 753-10 767-10 013-10 030-10 941-10 $65772-19737-10708-21$ 731-21 734-21 717-21 6b2 21 724-10 759-10 786-10 80:-10 61 7-10 821-10 ro 775-19 705-19 739-21 760-21 761-21 740-21 700-21 714-10 742-19 762-10 774-10 773-10 773-10 75 769-19 710-21 761-21 702-21 780-21 755-21 710-21 045-10 7C5-10 717-10 721-10 717-10 70e-10 00 752-19 735-21 777-21 795-21 790-21 162-21 711-2i $\quad 609-20 \quad 730-20 \quad 740-20$ 746-20 110-20 608-20 os 125-19 745-21 784-21 $100-21 \quad 791-21 \quad 759-21 \quad 704-21 \quad 722-20 \quad 161-20 \quad 716-20 \quad 768-20 \quad 736-20 \quad 602-20$ $90607-21$ 746-21 781-21 793-21 761-21 74t-21 704-1 74t-20 791-20 793-20 761-20 746-20 s67-19

WXIMUM FACTOES RUD SYSTEAS FOR SIGMA $=0.00$ MUC BETA $=35$. 0 820-13

5 838-13

10 of $0-13$

020-13

64-13

$089-13$

15 o51-13

20 o4 $5-13$

25 632-13

30 012-13

3) 135-13

to 752-13

45 $113-13$

50 727-19

$095-13$
$802-13$

$351-13 \quad 956-13 \quad 051-13$

$\begin{array}{lll}824-13 & 819-13 & 002-13 \\ 707-13 & 760-13 & 736-13\end{array}$

$\begin{array}{ll}760-13 & 136-13 \\ 106-13 & 657-13\end{array}$

$\begin{array}{lll}760-13 & 706-13 & 657-13 \\ 605-13 & 632-13 & 602-21\end{array}$ $62-21$
$637-21$

969-13

$304-13$

120-13

e15-13

cor-10

669-21

(19 60 110-19 715-19 121-21 143-21 746-21 130-21 697-21 664-10 707-10 743-10 769-10 700-10 800-10 65 793-19 724-19 754-21 776-21 777-21 758-21 720-21 675-10 712-10 741-10 762-10 774-10 779-10 70 000-19 736-21 702-21 004-21 $003-21 \quad 700-21$ 737-21 $674-21$ 696-10 710-10 732-10 737-10 734-10 75 197-19 760-21 $004-21$ :25-21 $021-21$ 795-21 746-21 090-20 134-20 156-20 753-20 729-20 603-20

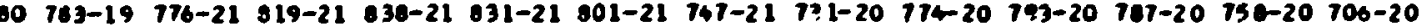

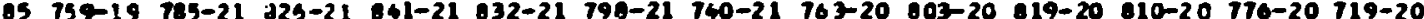

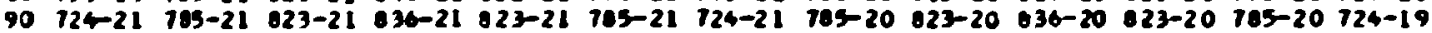




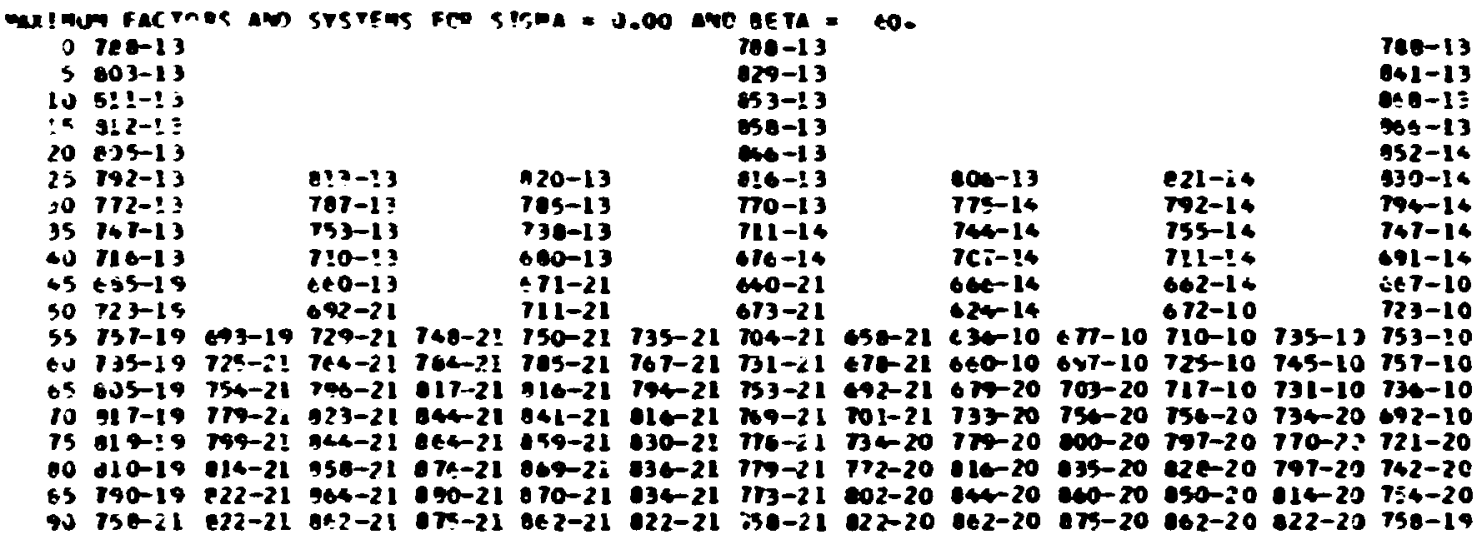

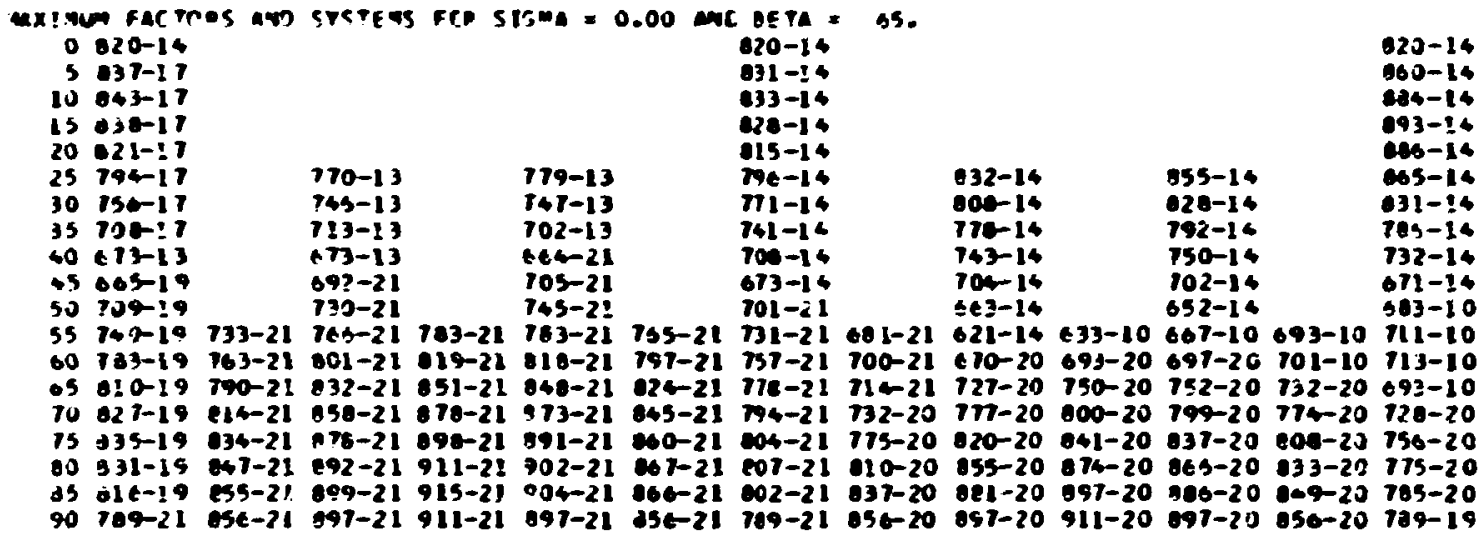

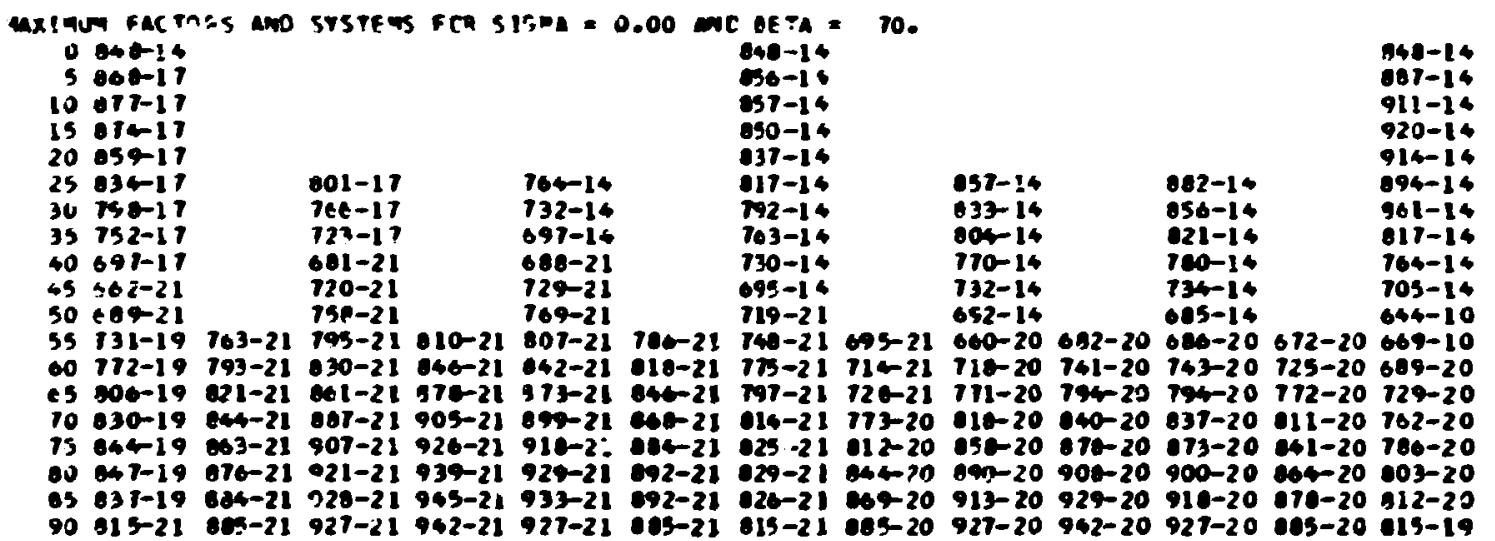




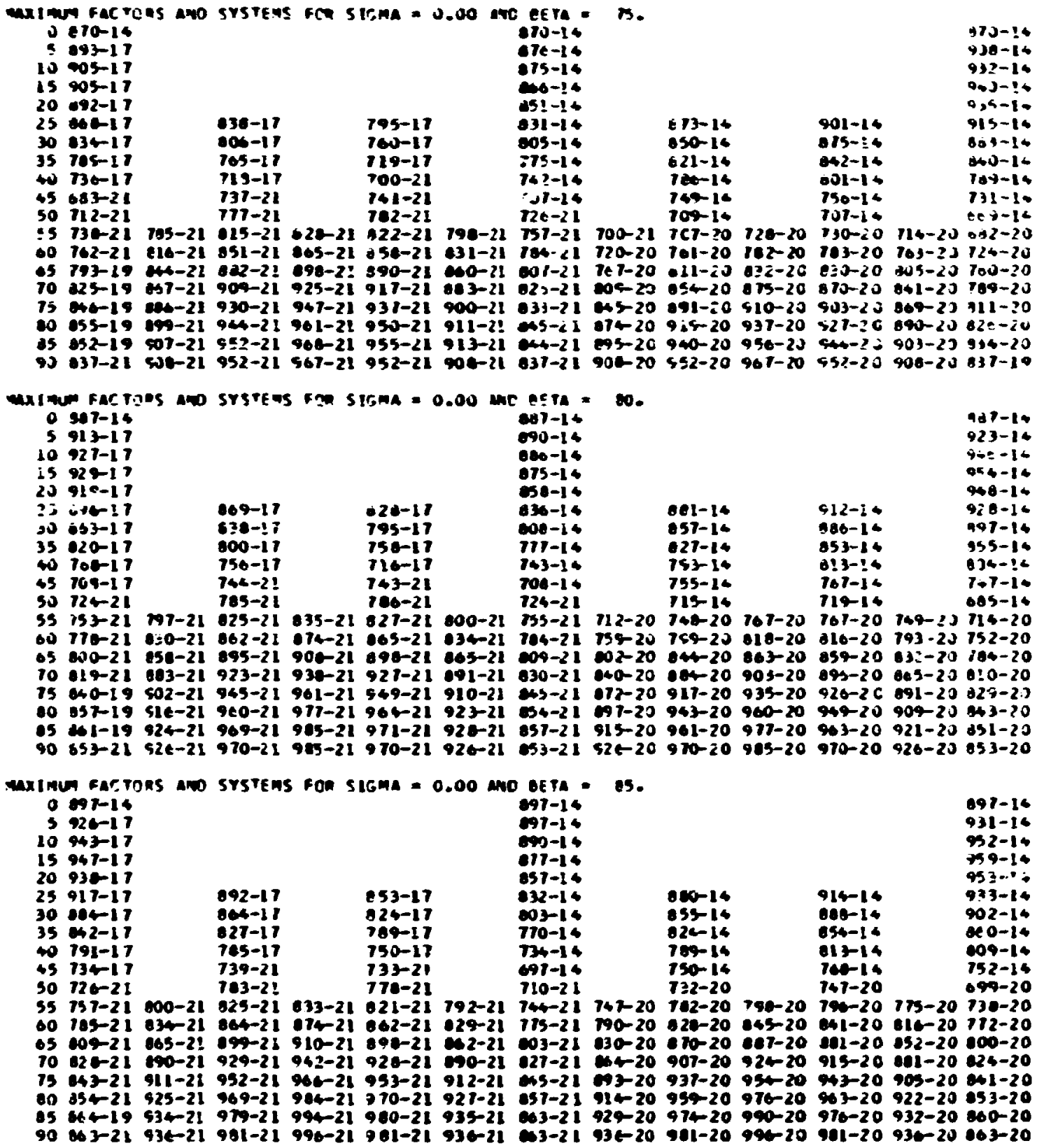




\begin{tabular}{|c|c|c|c|c|c|c|c|c|c|c|c|c|c|}
\hline $\begin{array}{l}1 \\
5 \\
12 \\
15 \\
23 \\
25 \\
35 \\
35 \\
40 \\
45 \\
53 \\
35 \\
10 \\
63 \\
10 \\
15 \\
65 \\
05\end{array}$ & 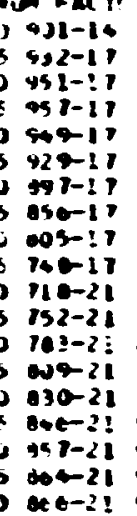 & $\begin{array}{l}97-21 \\
690-21 \\
662-21 \\
690-21 \\
512-21 \\
527-21 \\
531-21 \\
560-21\end{array}$ & 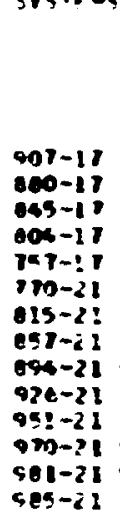 & $\begin{array}{l} \\
\\
920-2: \\
0+2-21 \\
903-21 \\
937-21 \\
904-2 ! \\
904-21 \\
99 t-21 \\
0.0-21\end{array}$ & 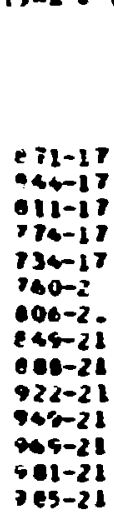 & $\begin{array}{l}10-21 \\
14-21 \\
050-21 \\
201-21 \\
900-21 \\
925-21 \\
930-21 \\
940-21\end{array}$ & 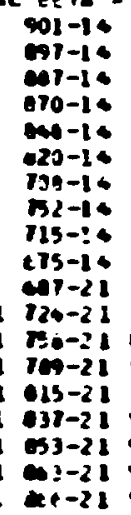 & $\begin{array}{l}114-20 \\
014-20 \\
50-20 \\
01-20 \\
50 k-20 \\
925-20 \\
0.20\end{array}$ & 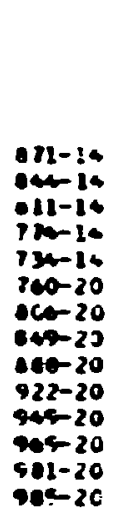 & $\begin{array}{l}120-20 \\
964-20 \\
903-20 \\
937-20 \\
904-20 \\
964-20 \\
59-20\end{array}$ & 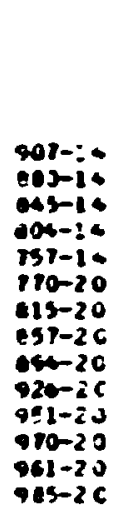 & $\begin{array}{l}1 \\
792-23 \\
030-20 \\
002-20 \\
090-23 \\
12-20 \\
528-20 \\
911-20\end{array}$ & 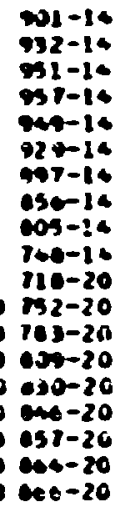 \\
\hline
\end{tabular}




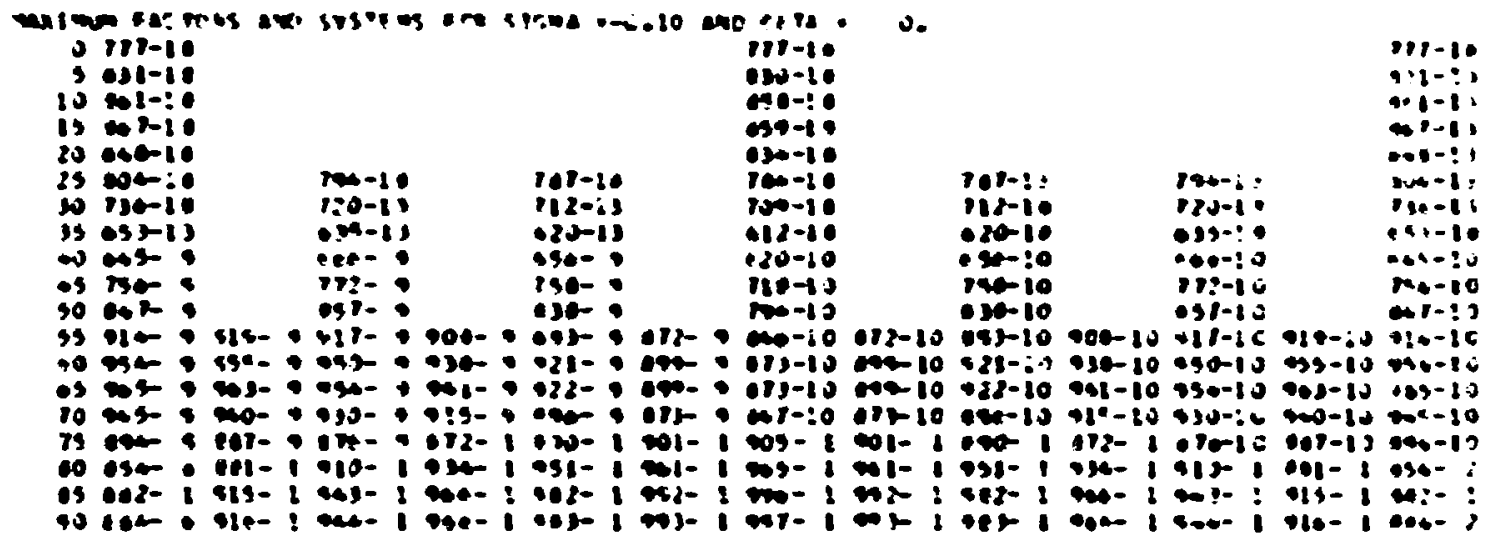

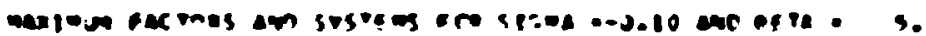

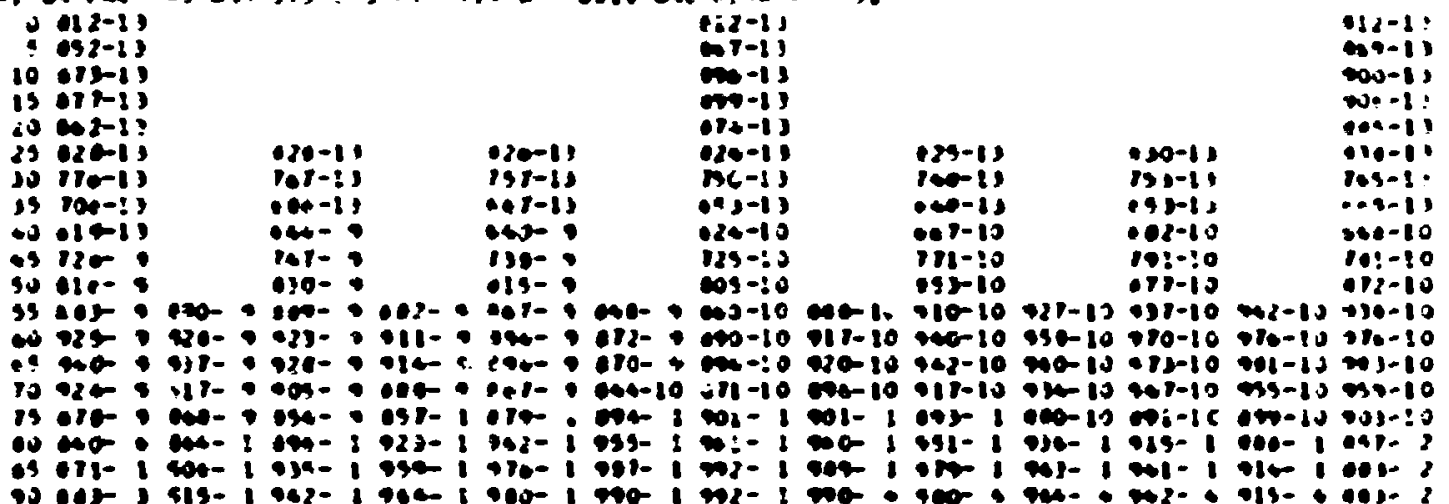

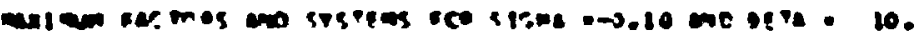

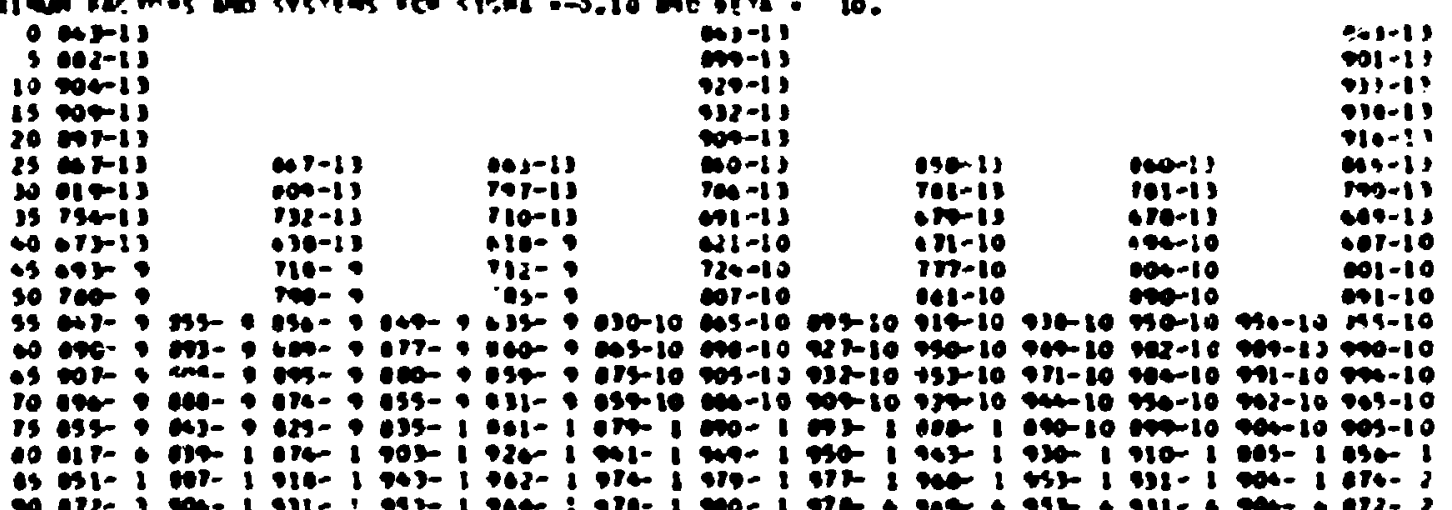




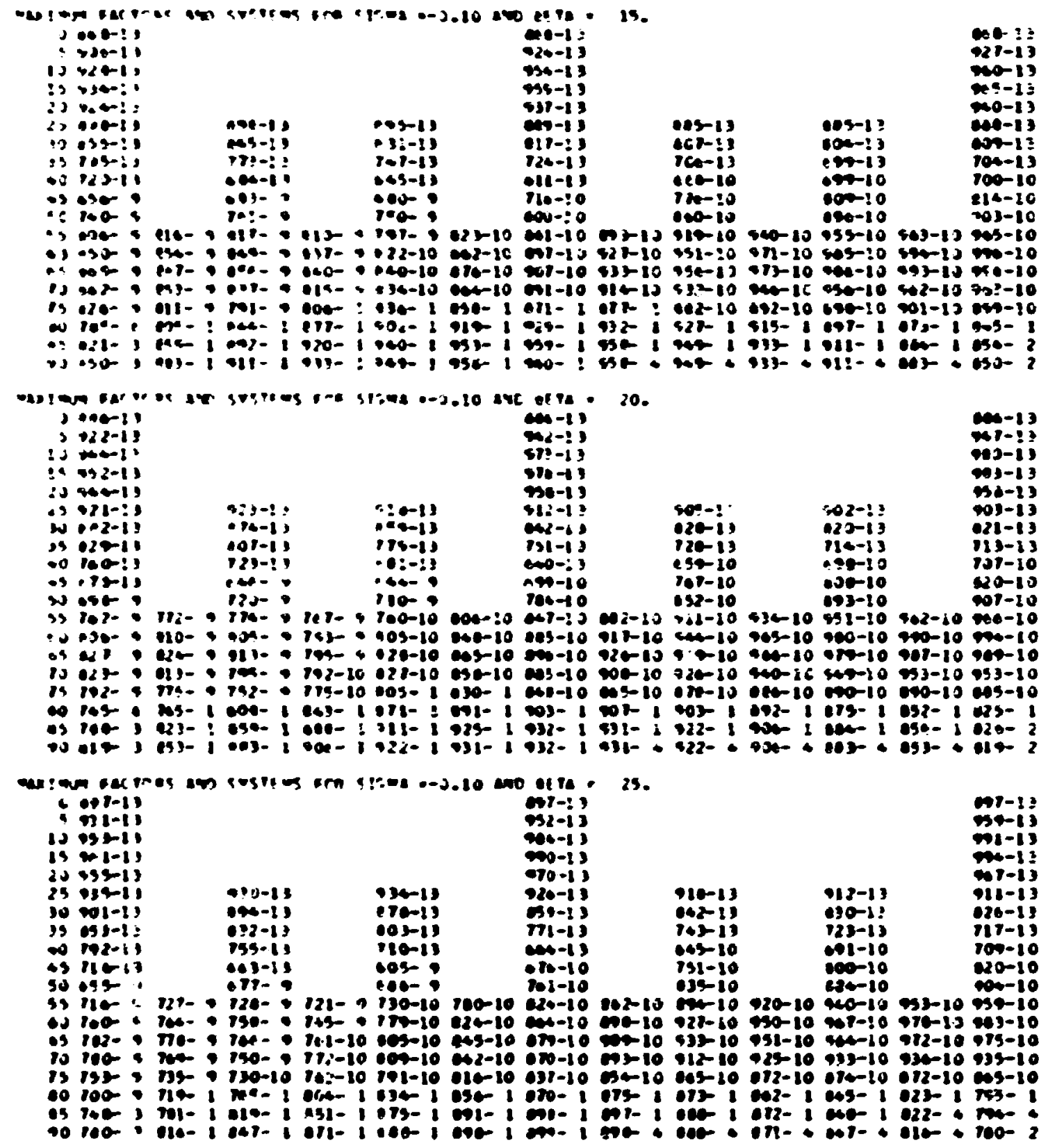




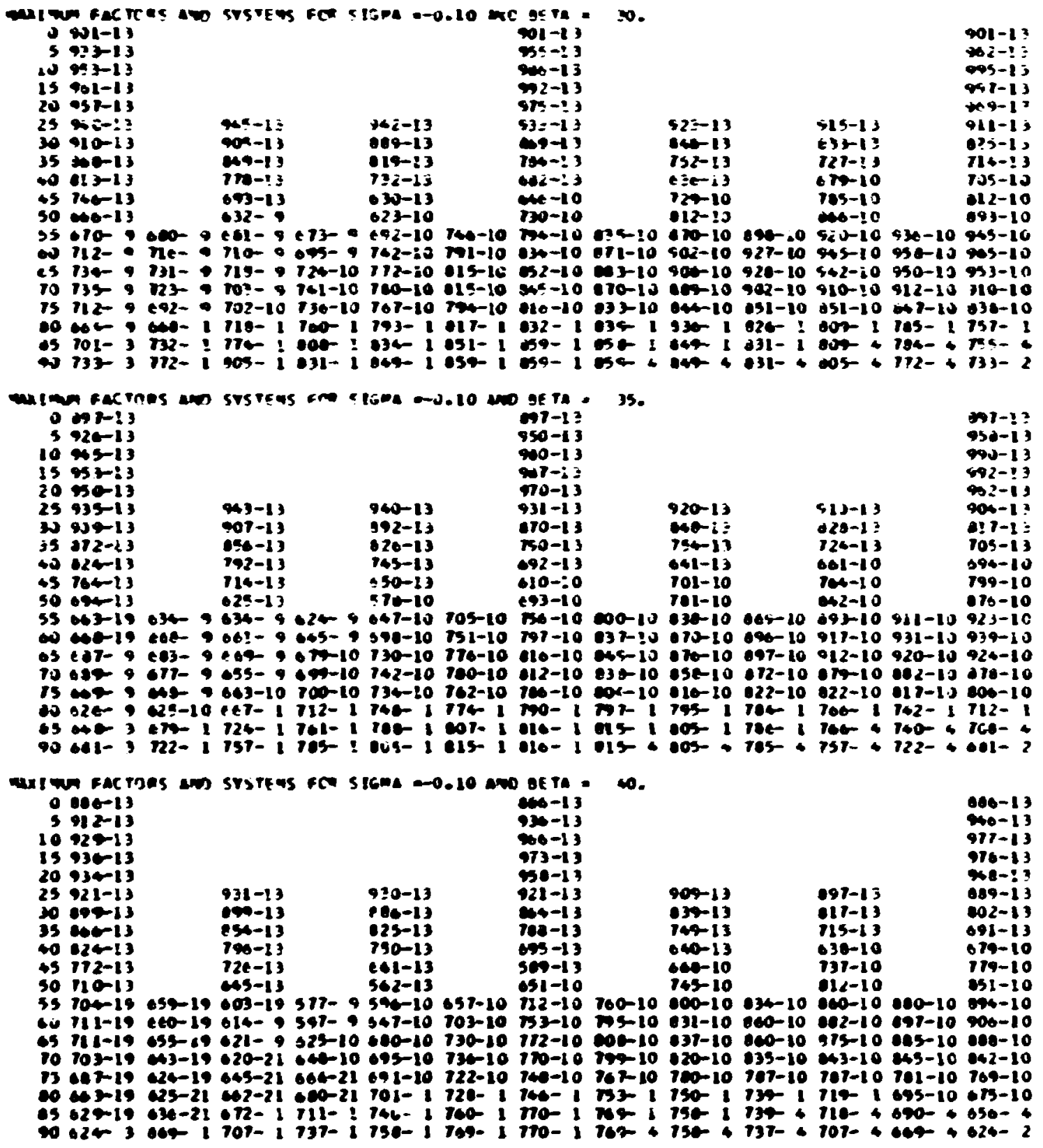




\begin{tabular}{|c|c|c|c|c|c|c|c|c|c|c|c|c|c|}
\hline $\begin{array}{l}0 \\
5 \\
10 \\
15 \\
43 \\
25 \\
30 \\
55 \\
00 \\
05 \\
50 \\
55 \\
73 \\
05 \\
10 \\
12 \\
10 \\
65 \\
90\end{array}$ & 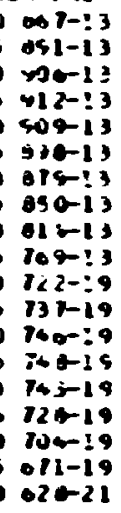 & $\begin{array}{r}05-19 \\
652-19 \\
699-19 \\
69-19 \\
6 !-19 \\
611-21 \\
681-21 \\
61-21\end{array}$ & $\begin{array}{l}411-13 \\
382-13 \\
642-13 \\
700-13 \\
727-13 \\
64-13 \\
620-19 \\
020-19 \\
646-21 \\
674-21 \\
696-2 ! \\
711-21 \\
71 b-21 \\
114-21\end{array}$ & $\begin{array}{l} \\
\\
590-21 \\
036-2 ! \\
c e 0-21 \\
096-21 \\
717-21 \\
129-2 ! \\
132-21 \\
125-21\end{array}$ & $\begin{array}{l}911-13 \\
871-13 \\
115-13 \\
740-13 \\
644-13 \\
572-13 \\
605-21 \\
642-21 \\
674-21 \\
659-21 \\
717-21 \\
726-21 \\
725-21 \\
714-21\end{array}$ & $\begin{array}{l} \\
605-10 \\
650-10 \\
677-10 \\
605-10 \\
697-21 \\
101-21 \\
112-1 \\
121-1\end{array}$ & $\begin{array}{r}67-13 \\
916-13 \\
24-13 \\
951-1 \\
537-13 \\
503-13 \\
647-12 \\
77=-13 \\
651-13 \\
541-13 \\
604-13 \\
63-10 \\
713-10 \\
723-10 \\
723-10 \\
702-10 \\
700-1 \\
722-11 \\
722-1\end{array}$ & $\begin{array}{l}714-10 \\
749-10 \\
7 \in 2-10 \\
153-10 \\
724-10 \\
707-1 \\
721-1 \\
721-4\end{array}$ & 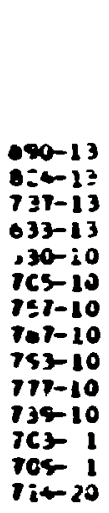 & $\begin{array}{l}793-10 \\
617-10 \\
117-10 \\
793-10 \\
742-10 \\
690-1 \\
707-20 \\
725-20\end{array}$ & $\begin{array}{l}677-13 \\
720-13 \\
703-13 \\
611-10 \\
704-10 \\
777-10 \\
622-10 \\
61-10 \\
834-10 \\
002-10 \\
74-10 \\
674-20 \\
700-20 \\
714-20\end{array}$ & $\begin{array}{r}4-10 \\
65-10 \\
40-13 \\
04-10 \\
741-10 \\
659-10 \\
71-20 \\
61-20\end{array}$ & 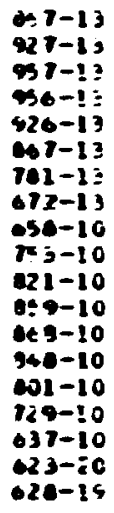 \\
\hline
\end{tabular}




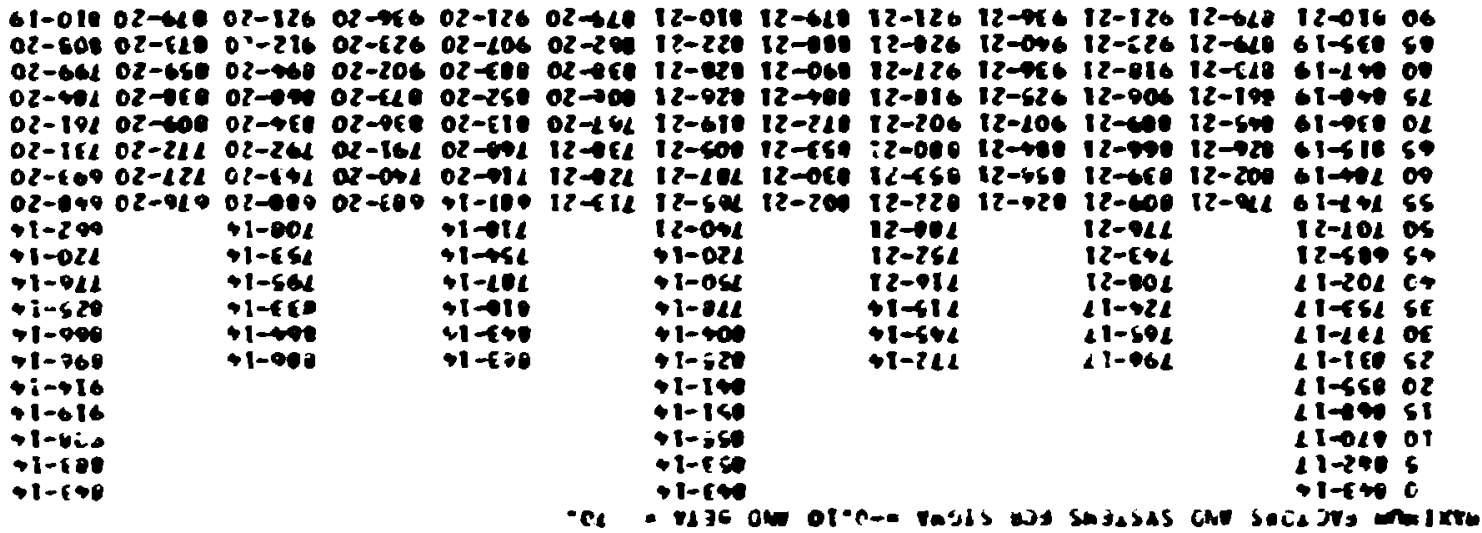

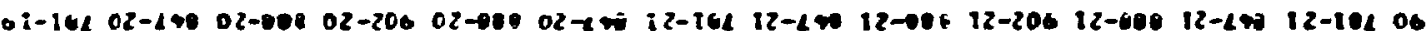

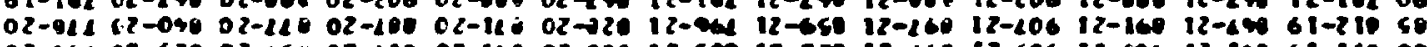

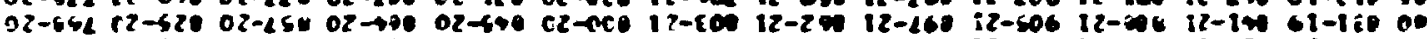

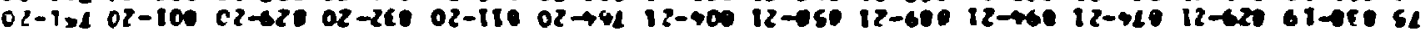

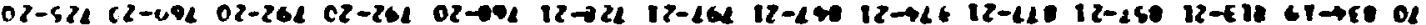

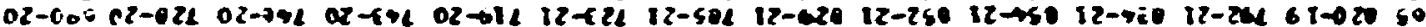

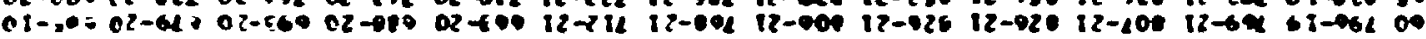

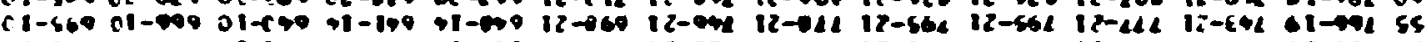

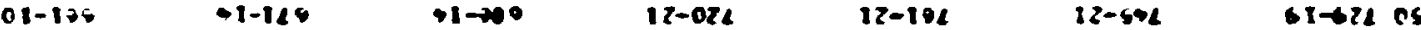

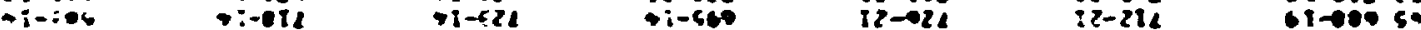

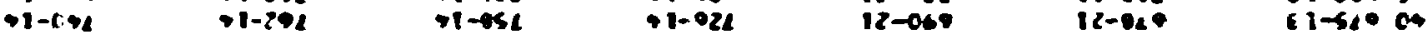

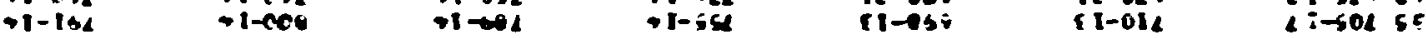

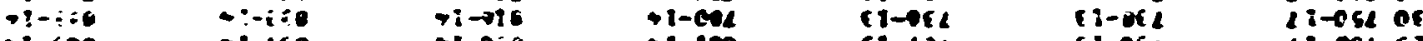

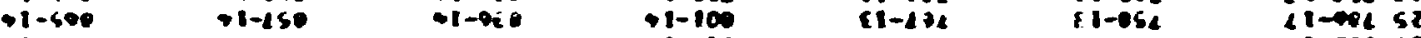

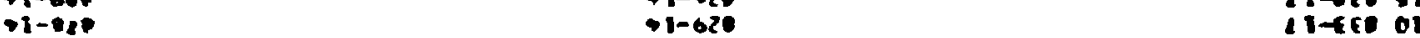

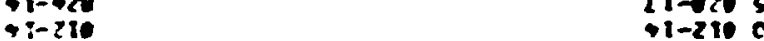

Ti -210

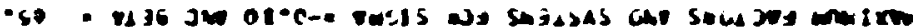

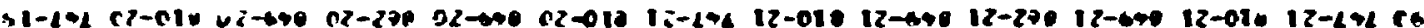

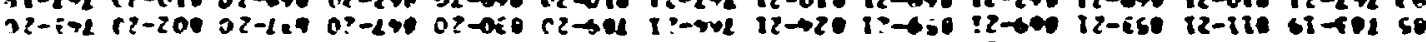

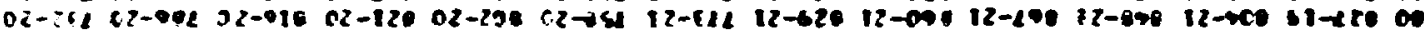

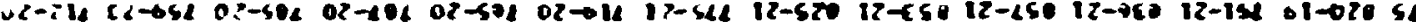

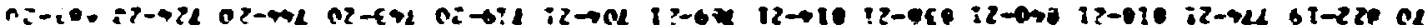

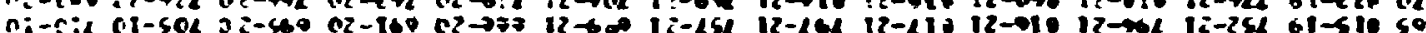

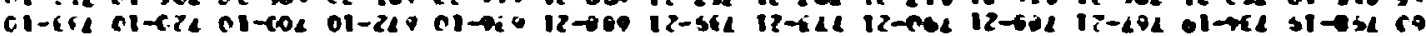

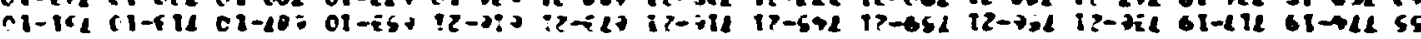

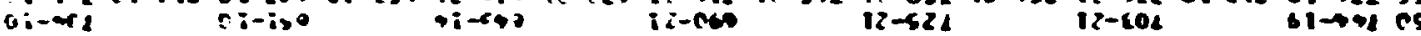
(istion

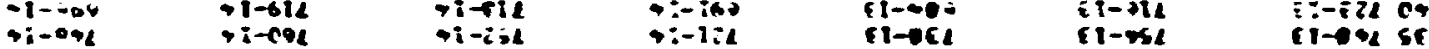

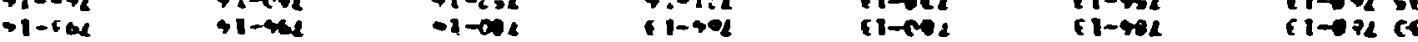

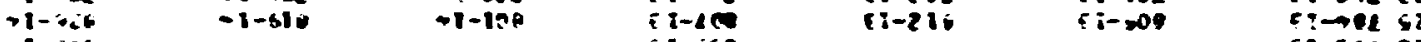

10

$.1-114$

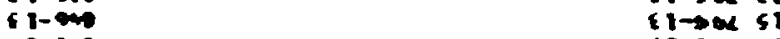
CI- I+ i-are ci-iere Gi-ele $\quad 1-1<2$ 


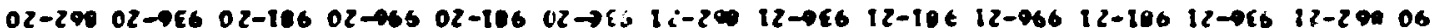

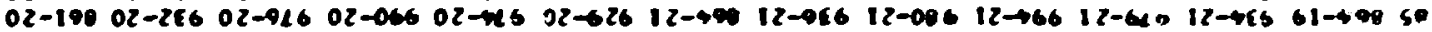

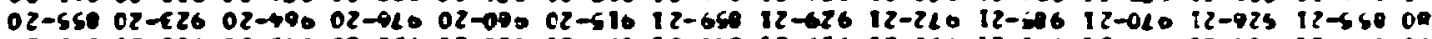

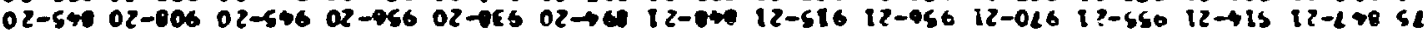

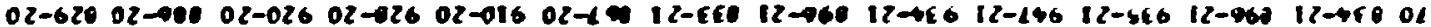

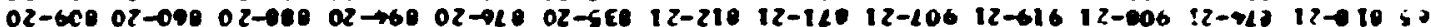

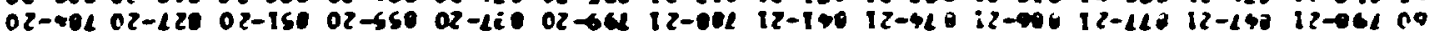

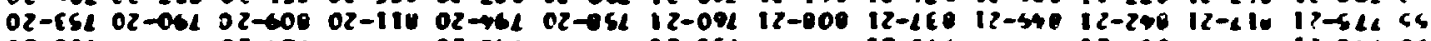
$0 z-811$ oz-99L $02-02$

$+1-520$

$1-06$

$1-250$

$1 \rightarrow 18$

$\rightarrow 1+00$

$1-210$

$1-090$

$\rightarrow 1-00$

1- 120 $12-125$

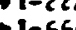

$12-662$

$12-194$

$12-\infty 0$

$12-601$

$11-904 \quad 41-000$

$-1-92 \quad 11-100 \quad 4-010$

$41-24 d$

$\begin{array}{ll}41-666 & 41-21 \\ 11-650 & 11-061\end{array}$

$12-1$ is 11,500 or

$+1-0+6 \quad+1-226 \quad+160$

$+1-150$ $\rightarrow 1-190$

LI-ZeO CE

$11-22652$

i-cose

$1:-965$

$\rightarrow-280$

-1-260

1- 1-40

11-6\$6 C1

$+1-256$

-1-26

i-ied

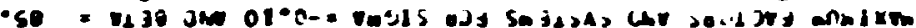

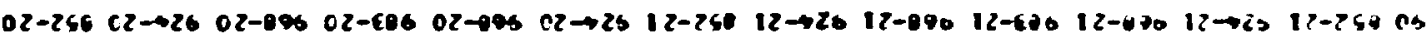

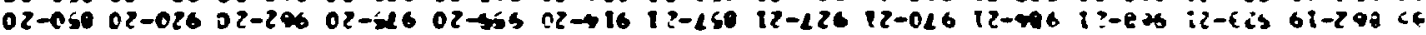

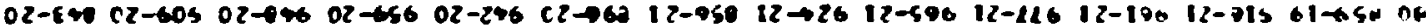

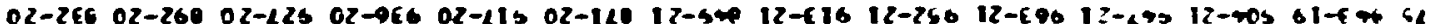

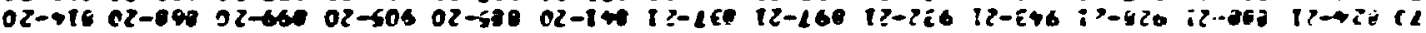

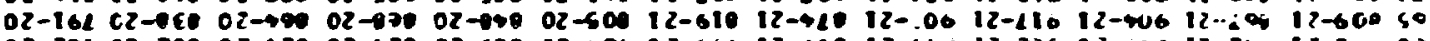

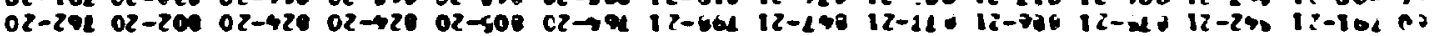

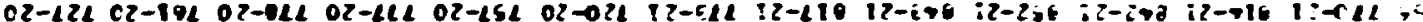

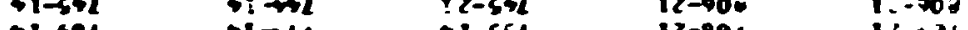

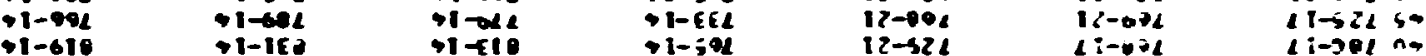
$\checkmark 1-610$ ग1-1ED

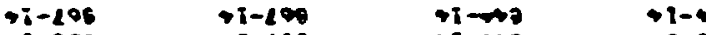

$\rightarrow 1-906 \quad+1-160 \quad+1$ ac $\quad+1-1$

$\rightarrow 1-466+1-615 \quad+1-050$

$+1-968$

$\begin{array}{lll}1 i-182 & 11-600 & 1 \\ 11-200 & 11-540 & 1\end{array}$

$1-25$

$1-95$ $\rightarrow 1-\$ 40$ $-1-40$

-1-E 24 $-i-04$

$1-\infty 0$

$1 i-424$

11-200 litasficis

11 -ces $\mathrm{PC}$

$41+5401$

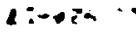

c t-i ?a 6

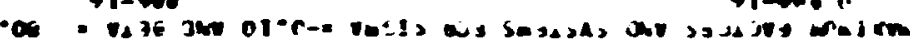

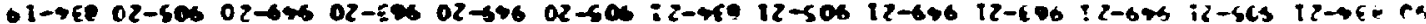

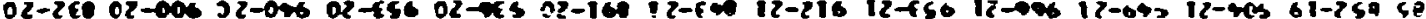

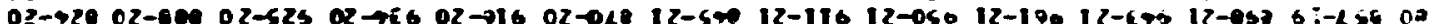

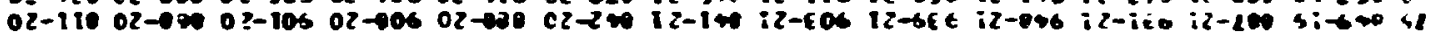

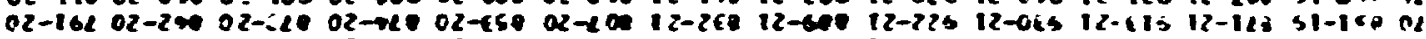

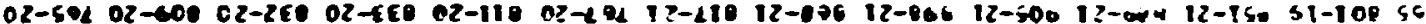

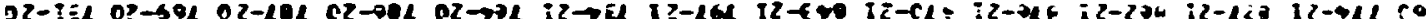

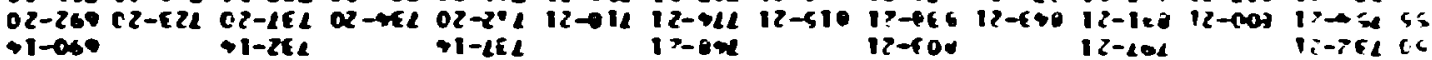

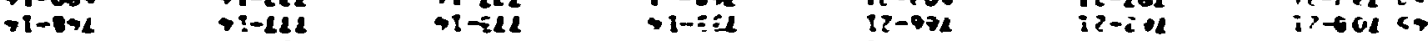

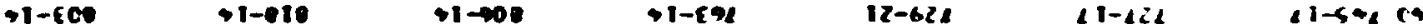

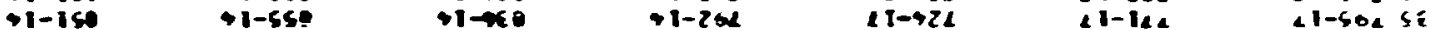

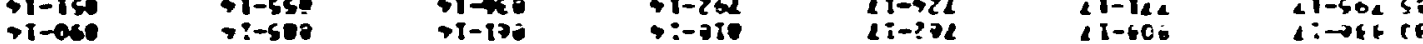

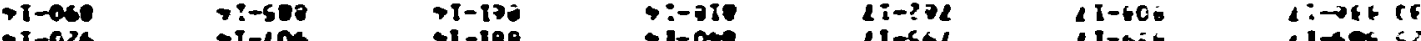

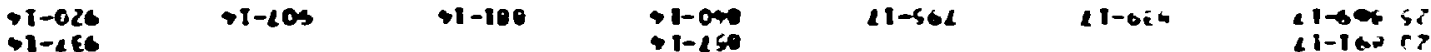
$\rightarrow i-4>0$ 1-610 11-1C? 6

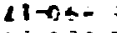
$+1-200$ 


\begin{tabular}{|c|c|c|c|c|c|c|c|c|c|c|c|c|c|}
\hline 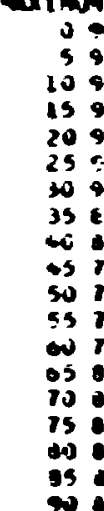 & 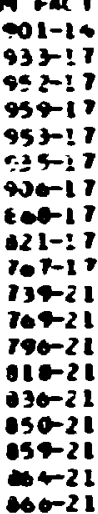 & $\begin{array}{l} \\
09-21 \\
092-21 \\
e 72-21 \\
64-21 \\
515-21 \\
20-21 \\
537-21 \\
540-21\end{array}$ & 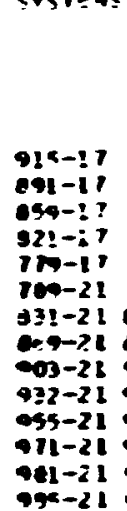 & 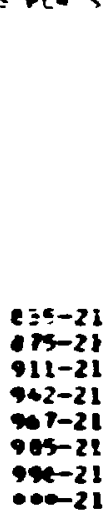 & $\begin{array}{l}079-17 \\
855-17 \\
620-17 \\
793-17 \\
7 ; 0-17 \\
770-21 \\
021-21 \\
960-21 \\
990-21 \\
921-21 \\
952-21 \\
970-21 \\
901-21 \\
705-21\end{array}$ & 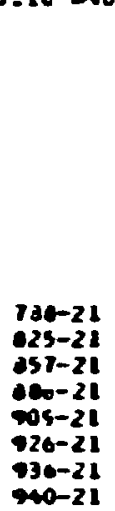 & 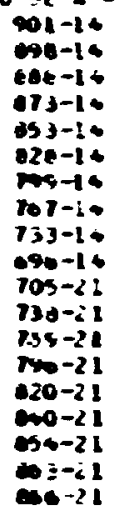 & 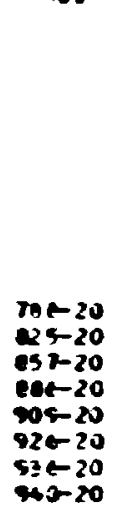 & $\begin{array}{l}679-16 \\
655-16 \\
620-16 \\
153-16 \\
190-16 \\
170-20 \\
621-20 \\
600-20 \\
680-20 \\
527-20 \\
952-20 \\
670-20 \\
501-20 \\
505-20\end{array}$ & $\begin{array}{l}0 \\
035-20 \\
615-20 \\
91:-20 \\
942-20 \\
967-20 \\
905-20 \\
950-20 \\
0.0-20\end{array}$ & 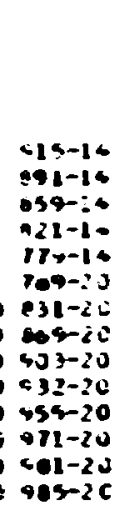 & 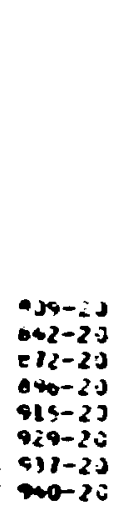 & 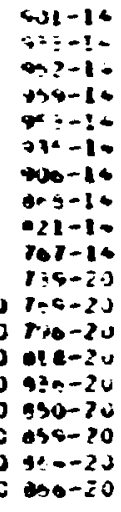 \\
\hline
\end{tabular}




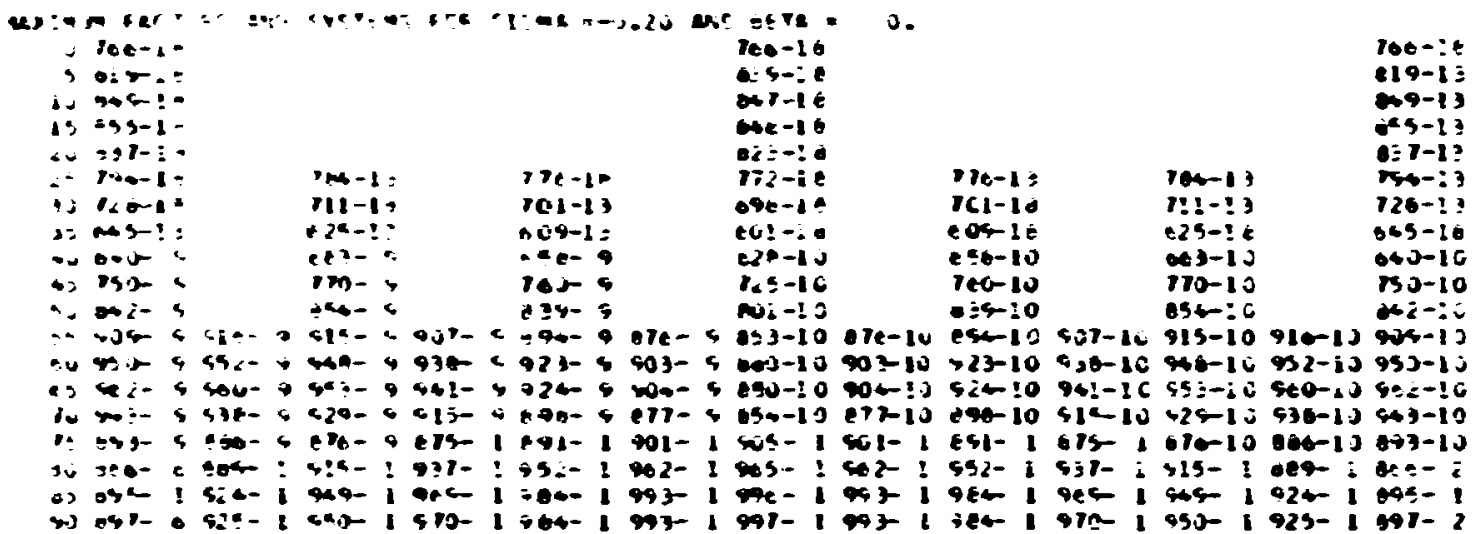

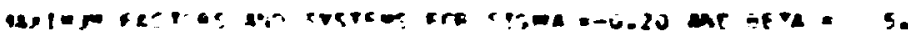

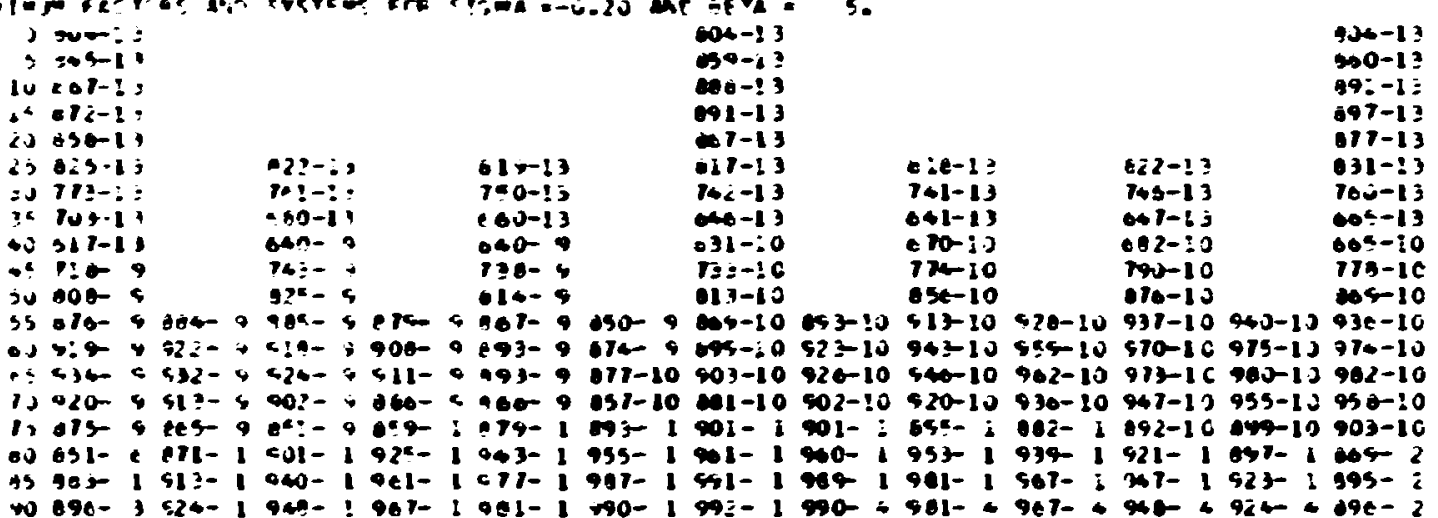

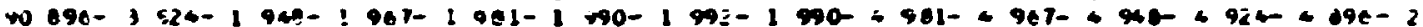

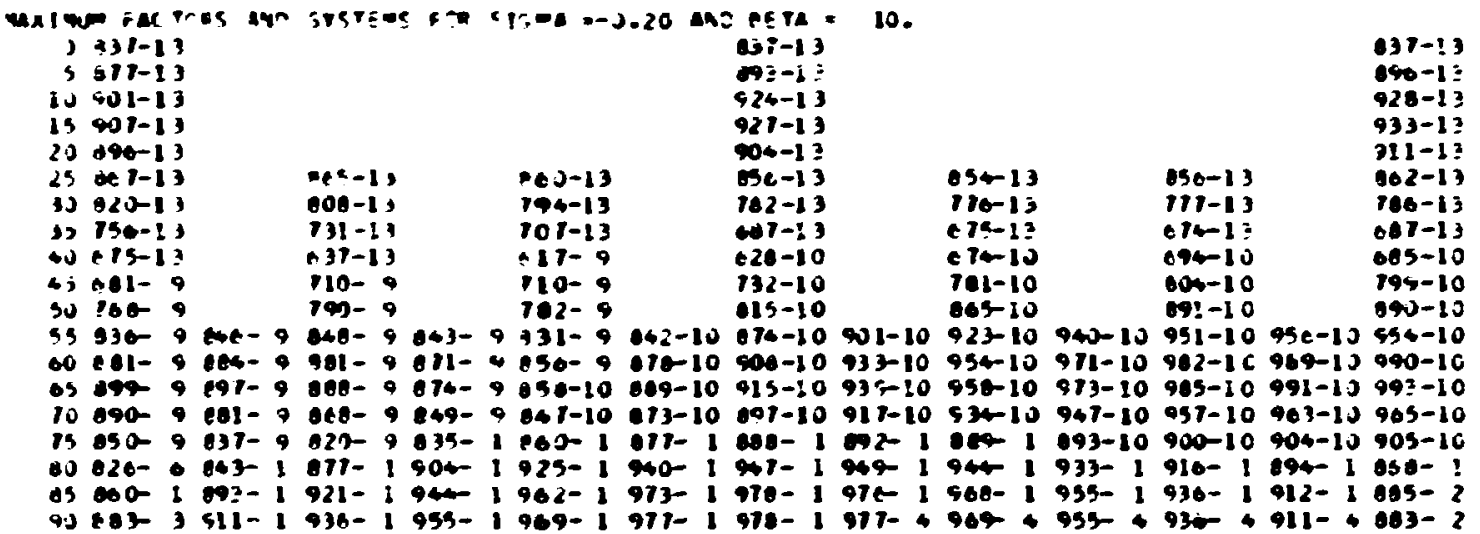




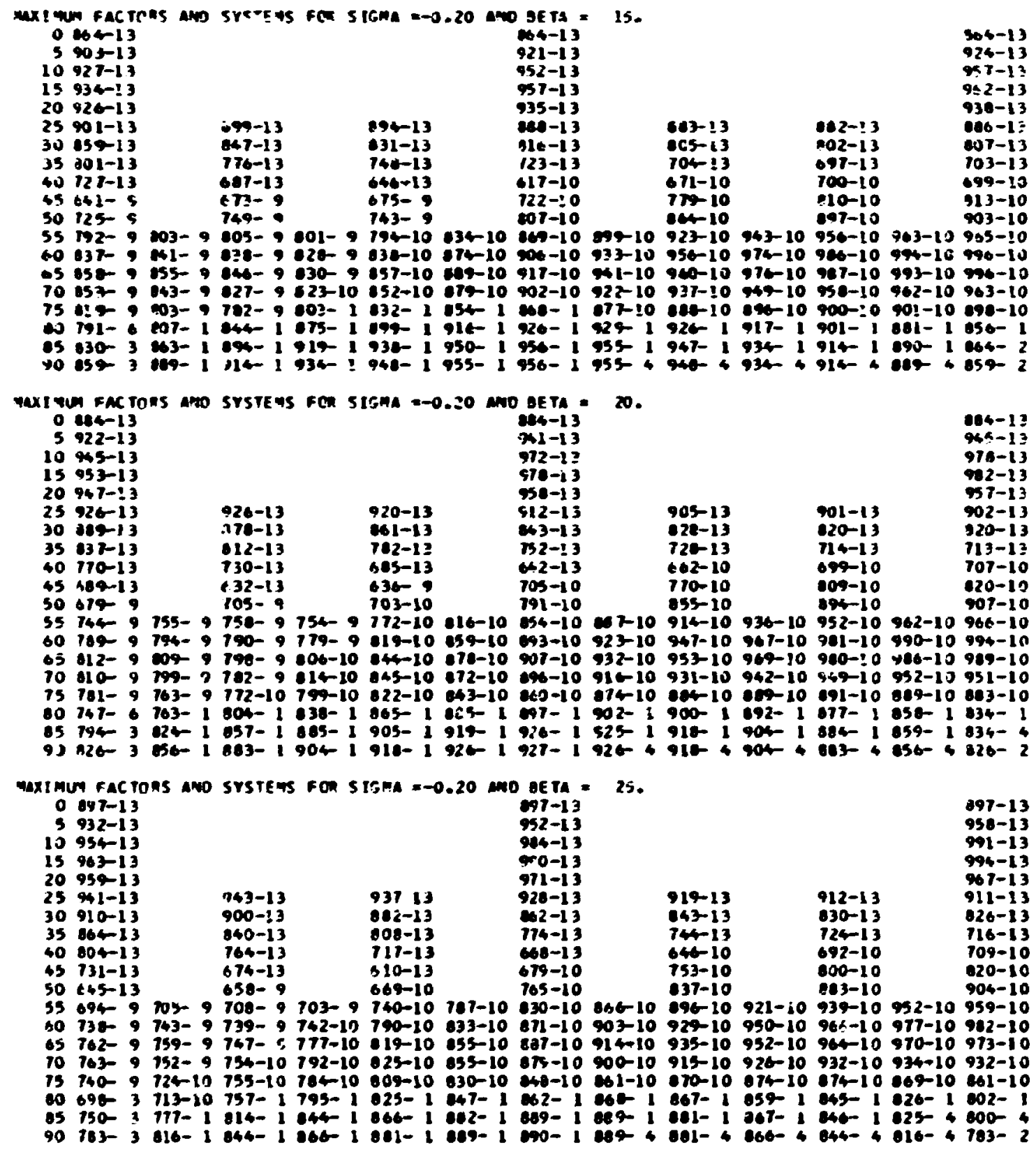




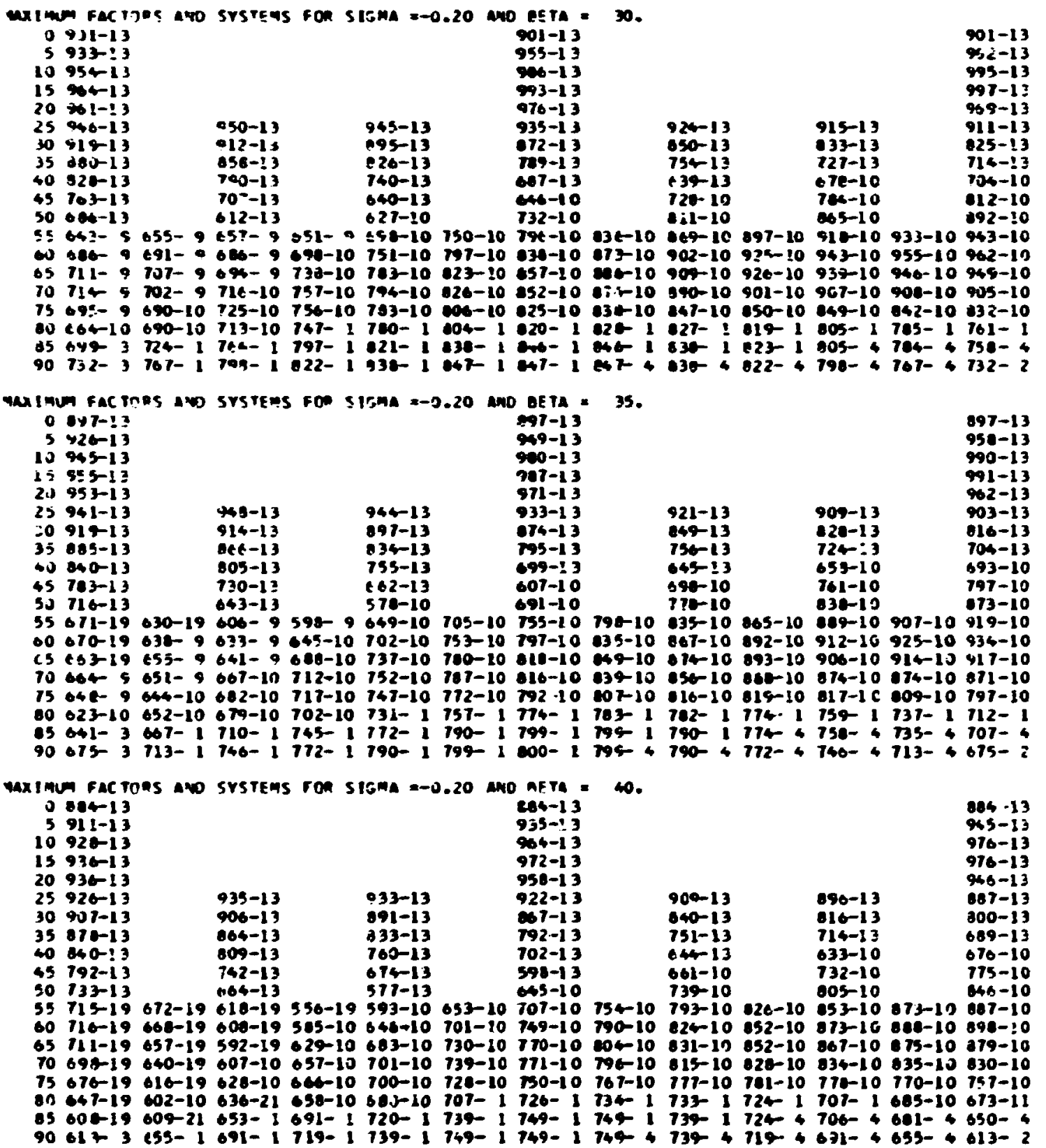




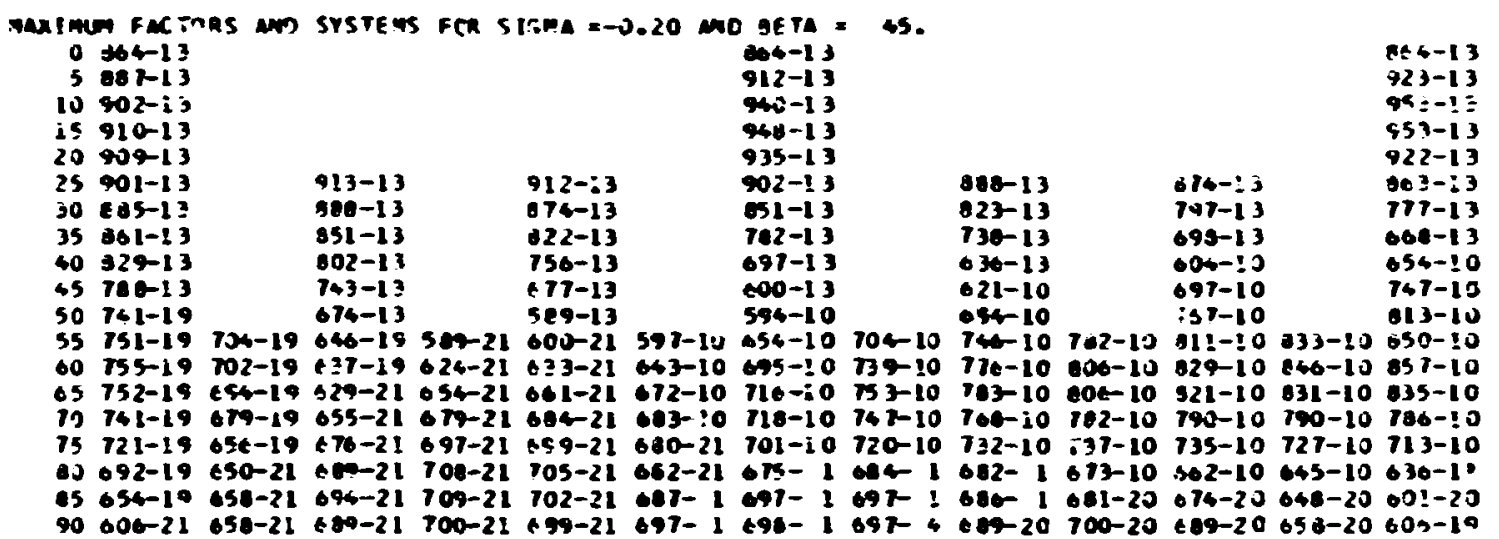

\begin{tabular}{|c|c|c|c|c|c|c|c|c|c|c|c|c|c|}
\hline $\begin{array}{l}5 \\
10 \\
15 \\
20 \\
25 \\
30 \\
35 \\
40 \\
45 \\
50 \\
55 \\
00 \\
05 \\
70 \\
75 \\
10 \\
65 \\
90\end{array}$ & $\begin{array}{r}637-13 \\
657-13 \\
049-13 \\
675-13 \\
674-13 \\
067-13 \\
653-13 \\
633-13 \\
656-13 \\
772-13 \\
762-19 \\
776-19 \\
754-19 \\
705-19 \\
777-19 \\
761-19 \\
734-19 \\
698-19 \\
651-21\end{array}$ & $\begin{array}{l}720-19 \\
728-19 \\
723-17 \\
711-19 \\
690-19 \\
700-21 \\
707-21 \\
707-21\end{array}$ & $\begin{array}{l}802-13 \\
260-13 \\
878-13 \\
785-13 \\
734-13 \\
673-13 \\
6 \times 3-19 \\
660-21 \\
688-21 \\
711-21 \\
730-21 \\
741-21 \\
745-21 \\
741-21\end{array}$ & $\begin{array}{l}7 \\
654-21 \\
685-21 \\
713-21 \\
735-21 \\
752-21 \\
761-2 ! \\
701-21 \\
752-21\end{array}$ & $\begin{array}{l}983-13 \\
649-13 \\
002-13 \\
742-13 \\
671-13 \\
629-21 \\
602-21 \\
643-21 \\
719-21 \\
739-21 \\
752-21 \\
757-21 \\
754-21 \\
741-21\end{array}$ & $\begin{array}{l}0 \\
\\
650-21 \\
684-21 \\
704-21 \\
722-21 \\
731-21 \\
732-21 \\
724-21 \\
707-21\end{array}$ & 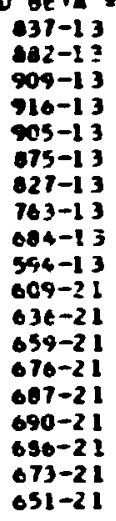 & $\begin{array}{l}650-10 \\
64-10 \\
698-10 \\
692-10 \\
66-10 \\
64-20 \\
680-20 \\
707-20\end{array}$ & $\begin{array}{l}600-13 \\
784-13 \\
710-13 \\
622-13 \\
578-16 \\
64 t-10 \\
650-10 \\
723-10 \\
630-10 \\
716-10 \\
642-10 \\
685-20 \\
710-20 \\
741-20\end{array}$ & $\begin{array}{l} \\
734-10 \\
750-10 \\
755-10 \\
732-10 \\
689-10 \\
704-20 \\
733-20 \\
792-20\end{array}$ & $\begin{array}{l}945-13 \\
771-13 \\
675-13 \\
609-14 \\
659-10 \\
724-10 \\
765-10 \\
760-10 \\
772-10 \\
741-10 \\
687-10 \\
701-20 \\
725-20 \\
741-20\end{array}$ & $\begin{array}{l}789-10 \\
798-10 \\
782-10 \\
742-10 \\
681-10 \\
677-20 \\
657-20 \\
707-20\end{array}$ & $\begin{array}{l}637-13 \\
094-13 \\
923-13 \\
921-13 \\
691-13 \\
533-13 \\
748-13 \\
641-13 \\
026-10 \\
114-10 \\
774-10 \\
607-10 \\
910-10 \\
754-10 \\
737-10 \\
607-10 \\
623-20 \\
547-20 \\
651-19\end{array}$ \\
\hline
\end{tabular}




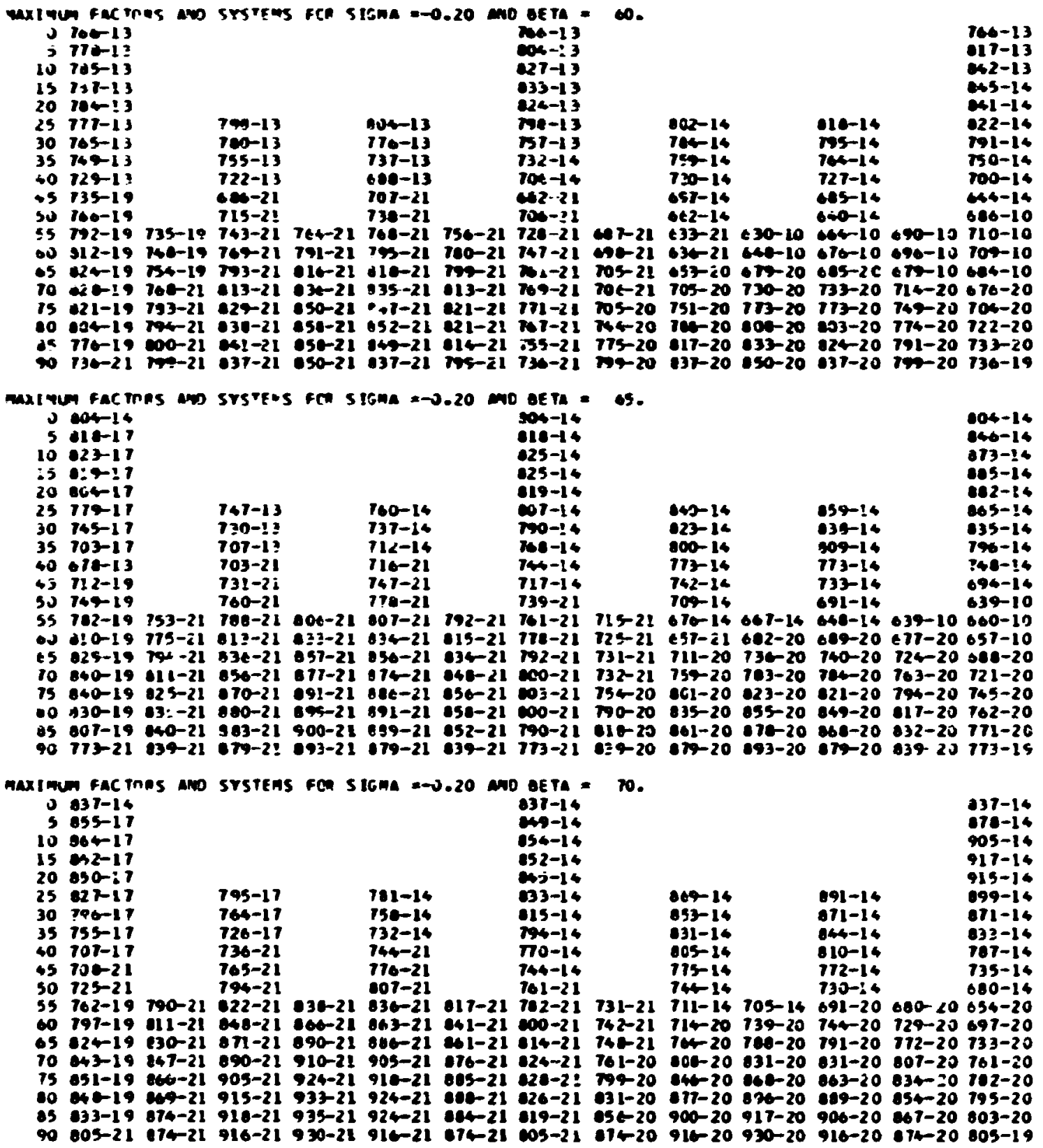




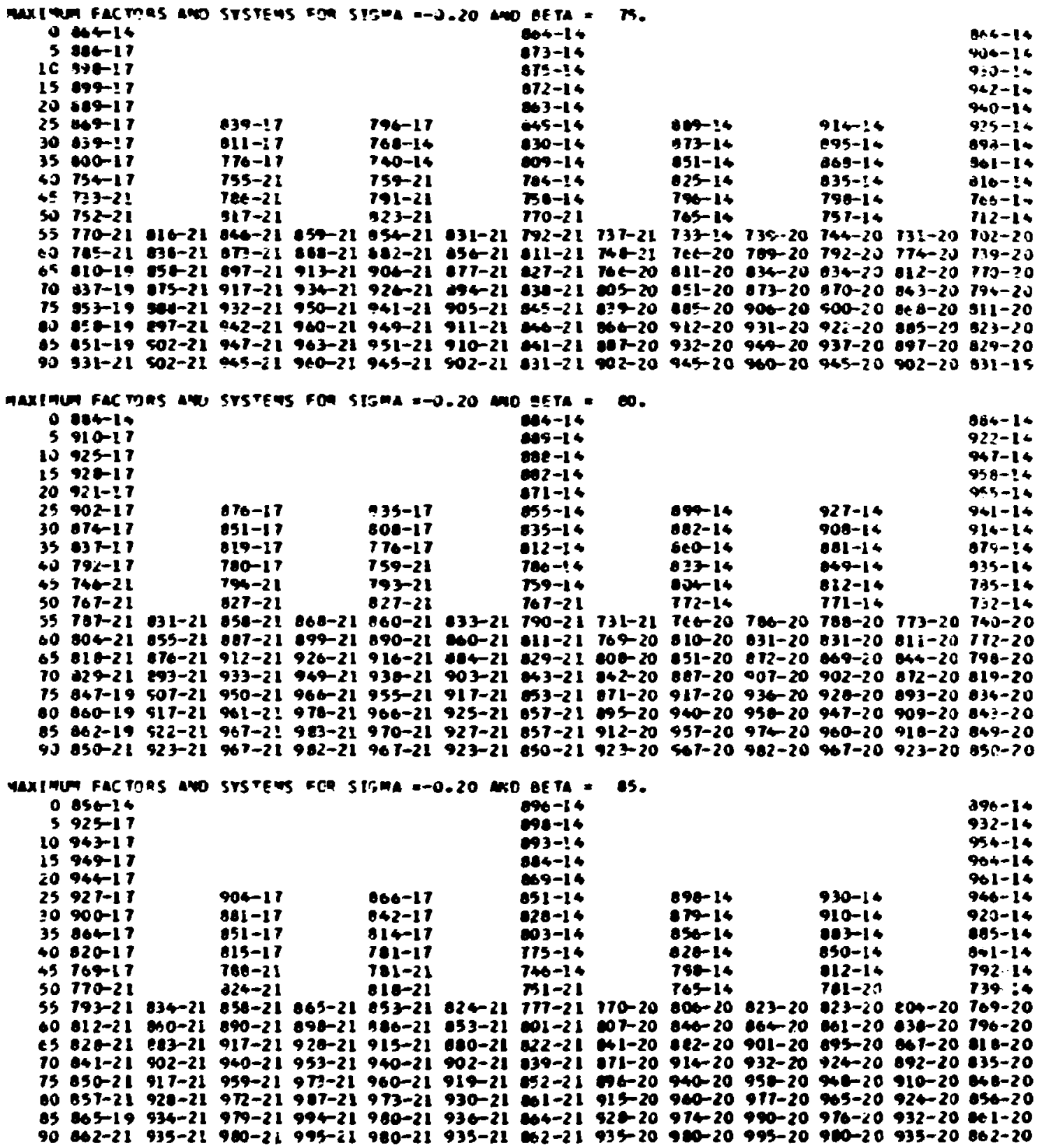




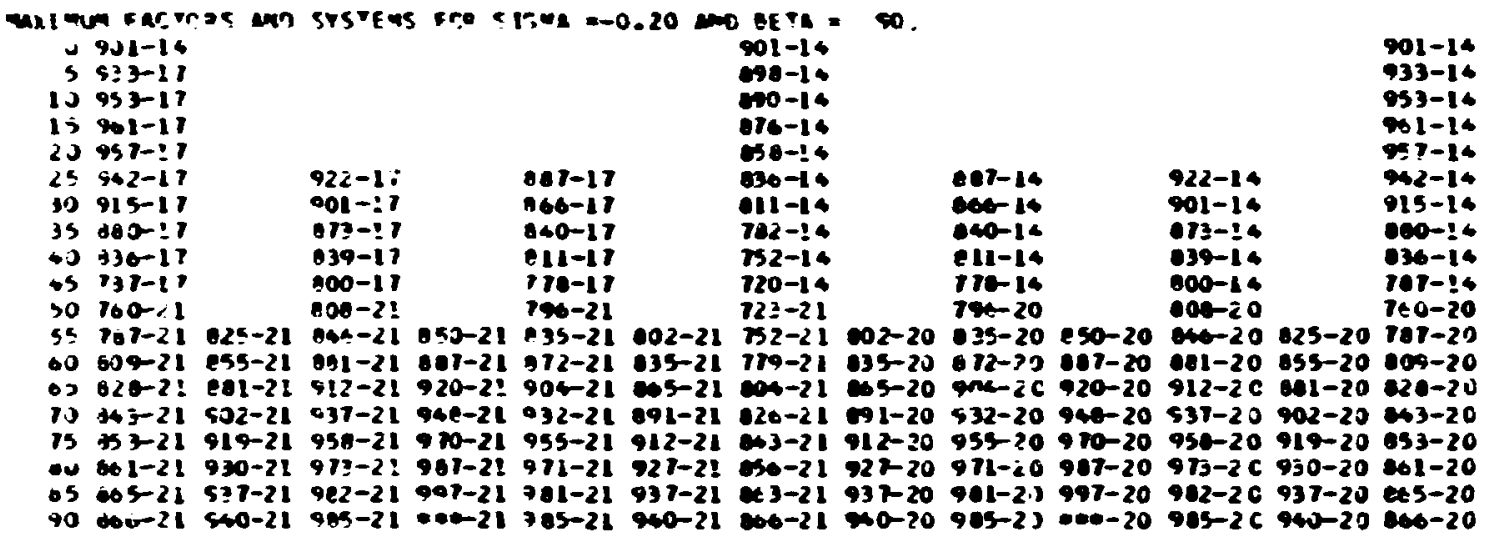




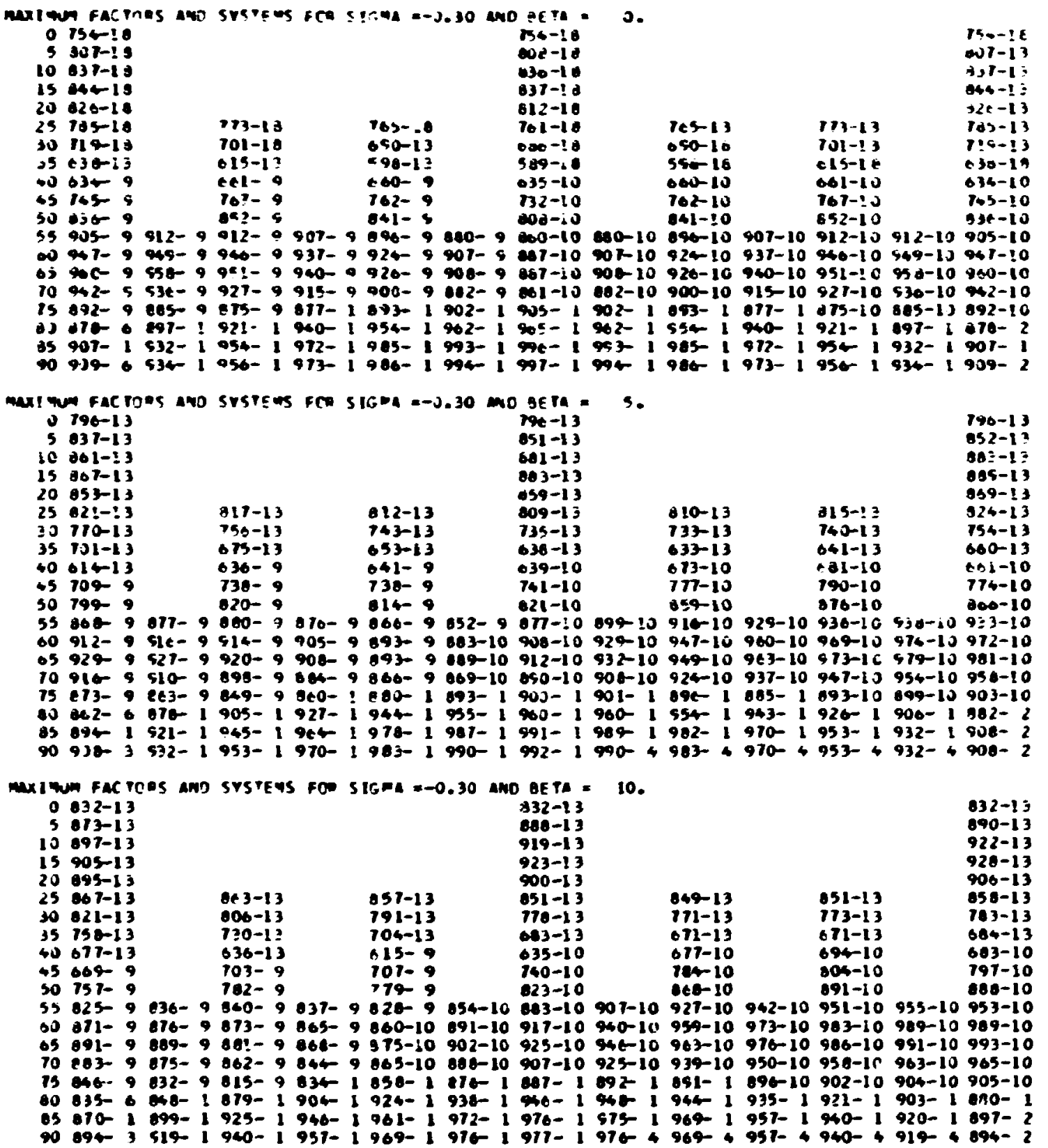




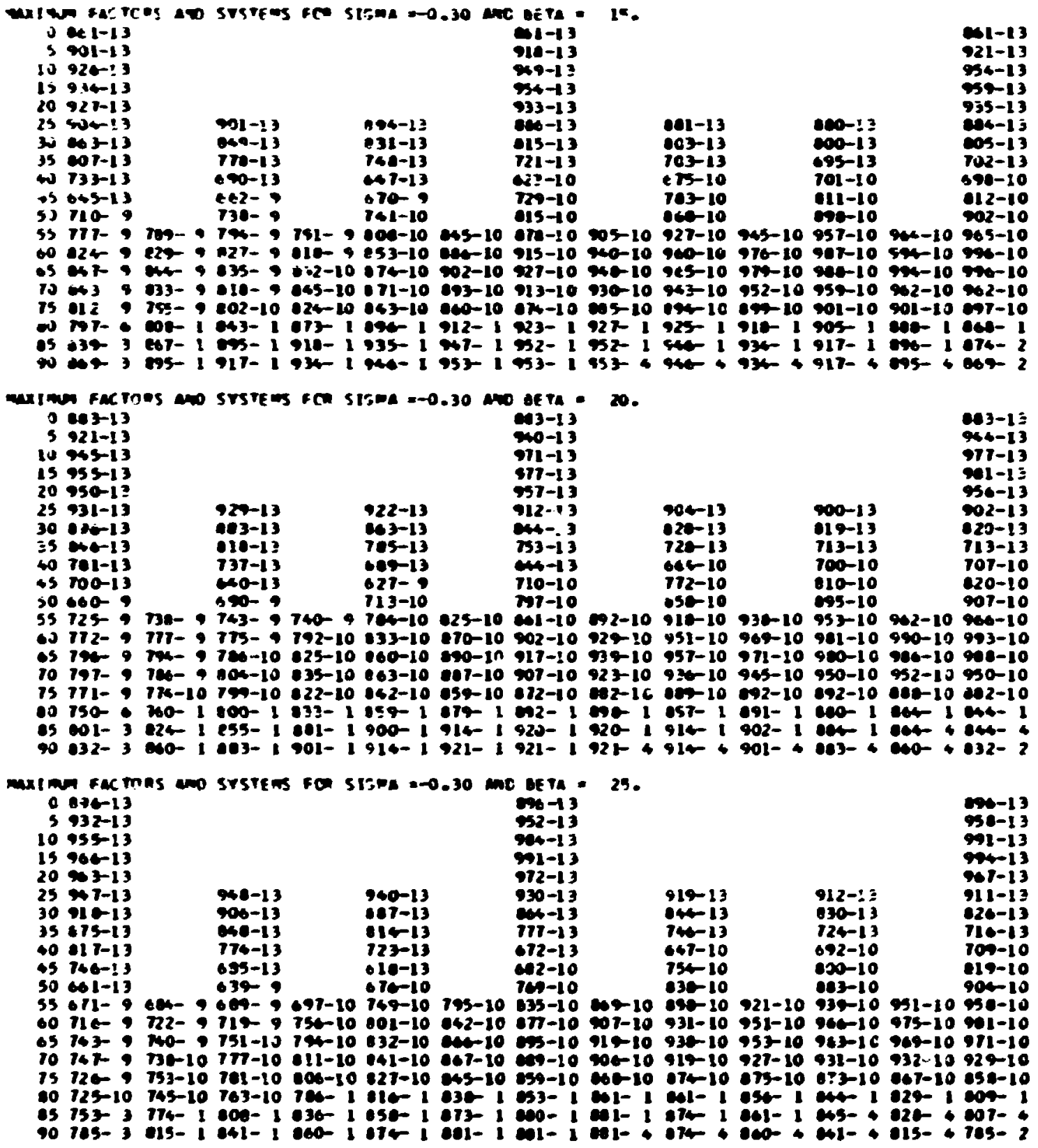




\begin{tabular}{|c|c|c|c|c|c|c|c|c|c|c|c|c|c|}
\hline $\begin{array}{l}10 \\
0 \\
5 \\
10 \\
15 \\
20 \\
25 \\
30 \\
35 \\
40 \\
45 \\
50 \\
55 \\
60 \\
05 \\
73 \\
75 \\
40\end{array}$ & 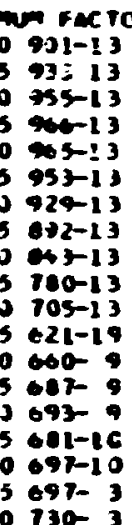 & $\begin{array}{r}0 \\
65-9 \\
666-9 \\
693-9 \\
693-10 \\
117-10 \\
20-10\end{array}$ & $\begin{array}{l}956-13 \\
920-11 \\
660-12 \\
801-13 \\
720-13 \\
727-13 \\
633-9 \\
662-19 \\
702-10 \\
736-10 \\
746-10 \\
740-10 \\
754-1\end{array}$ & $\begin{array}{r}5 \\
647-10 \\
700-10 \\
751-10 \\
776-10 \\
776-10 \\
757-10\end{array}$ & $\begin{array}{l}949-13 \\
900-13 \\
0 \geq 3-13 \\
149-13 \\
650-13 \\
631-10 \\
70+-10 \\
759-10 \\
793-10 \\
807-10 \\
800-10 \\
771-10\end{array}$ & $\begin{array}{l}356-10 \\
753-10 \\
031-10 \\
036-10 \\
0 \\
019-10 \\
091-1 \\
026-1\end{array}$ & 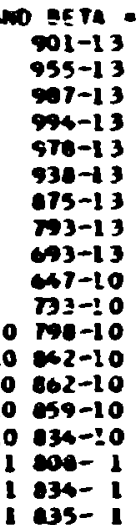 & 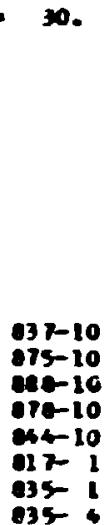 & $\begin{array}{l}925-13 \\
052-13 \\
756-13 \\
642-13 \\
120-10 \\
610-10 \\
869-10 \\
902-10 \\
909-10 \\
691-10 \\
84-10 \\
618-1 \\
620-1 \\
827-4\end{array}$ & $\begin{array}{l}3 \\
3 \\
395-10 \\
924-10 \\
925-10 \\
900-10 \\
950-10 \\
13-1 \\
13-1 \\
014-1 \\
013-4\end{array}$ & 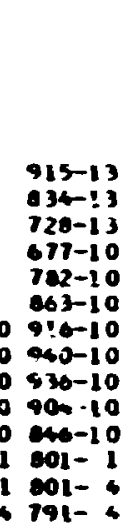 & $\begin{array}{l} \\
931-10 \\
952-10 \\
962-10 \\
904-10 \\
630-10 \\
705-1 \\
703-4 \\
163-4\end{array}$ & 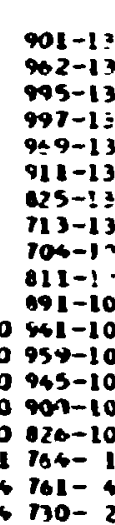 \\
\hline
\end{tabular}

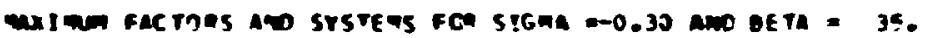

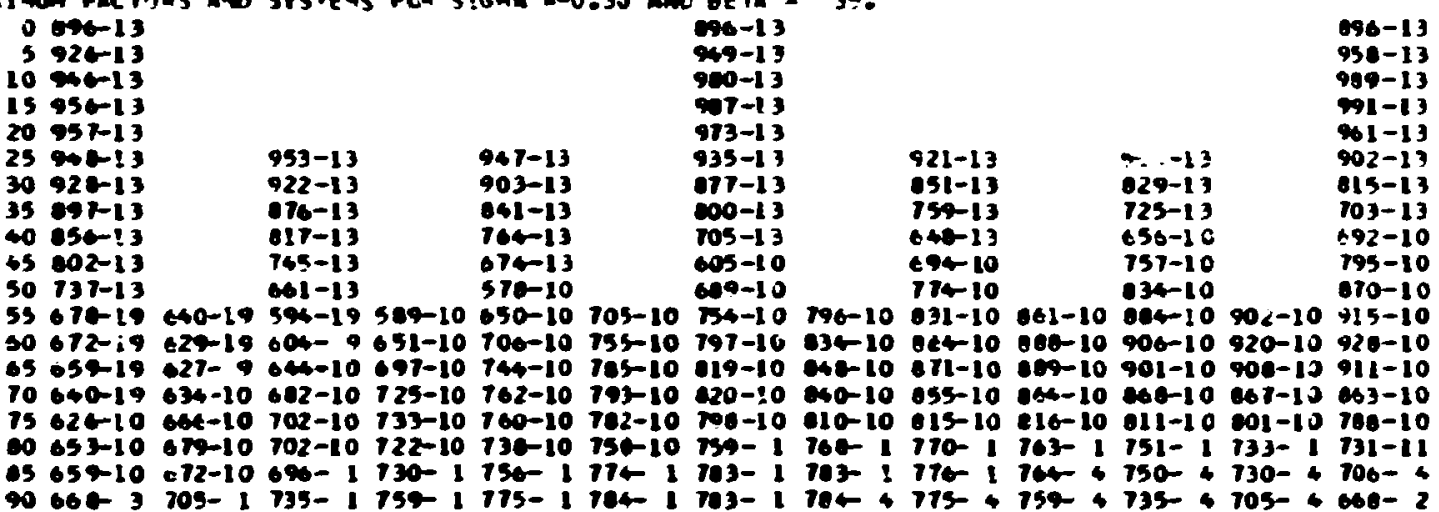

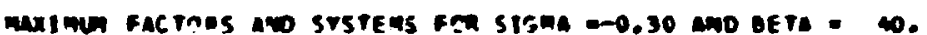

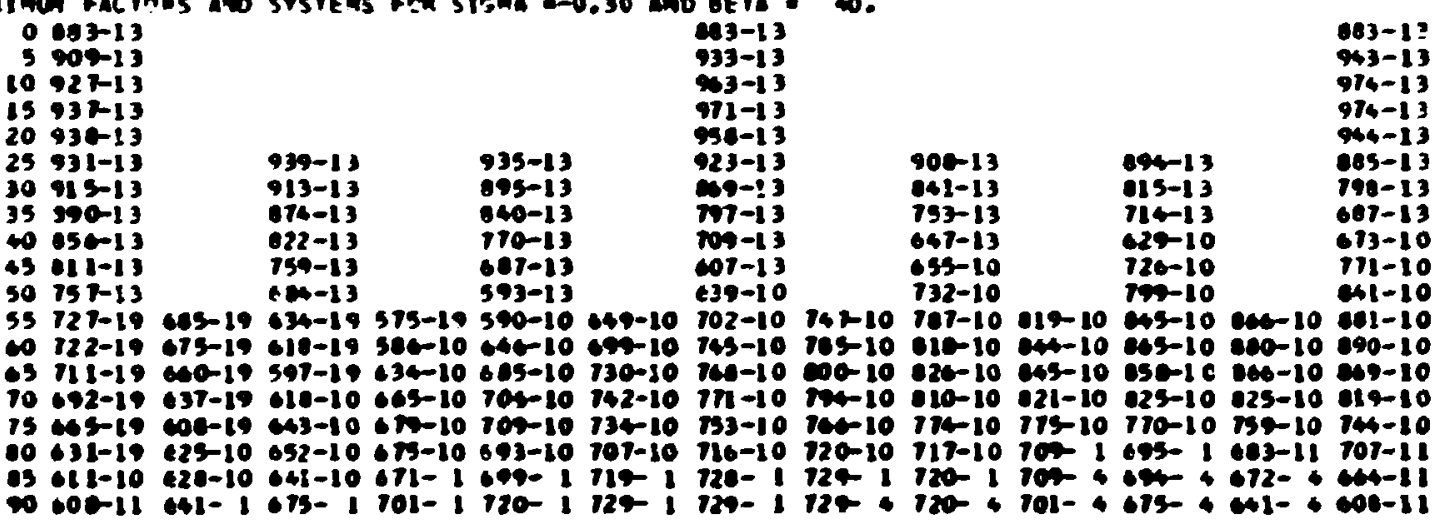




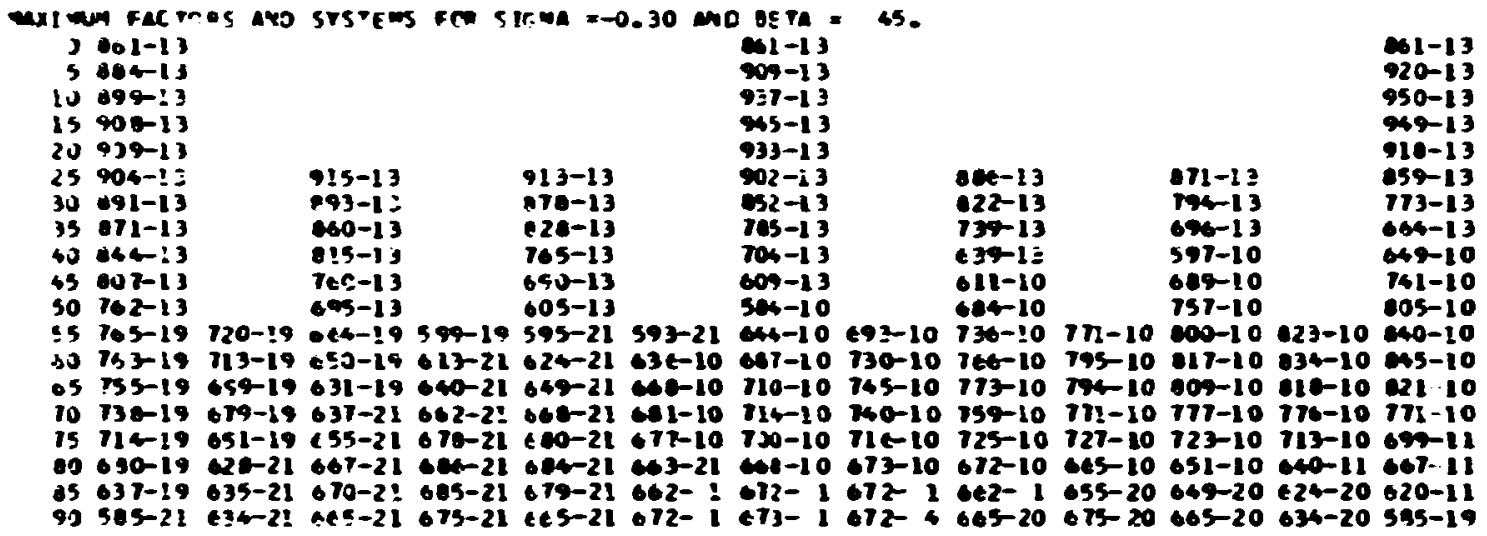

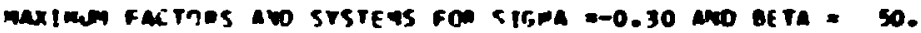

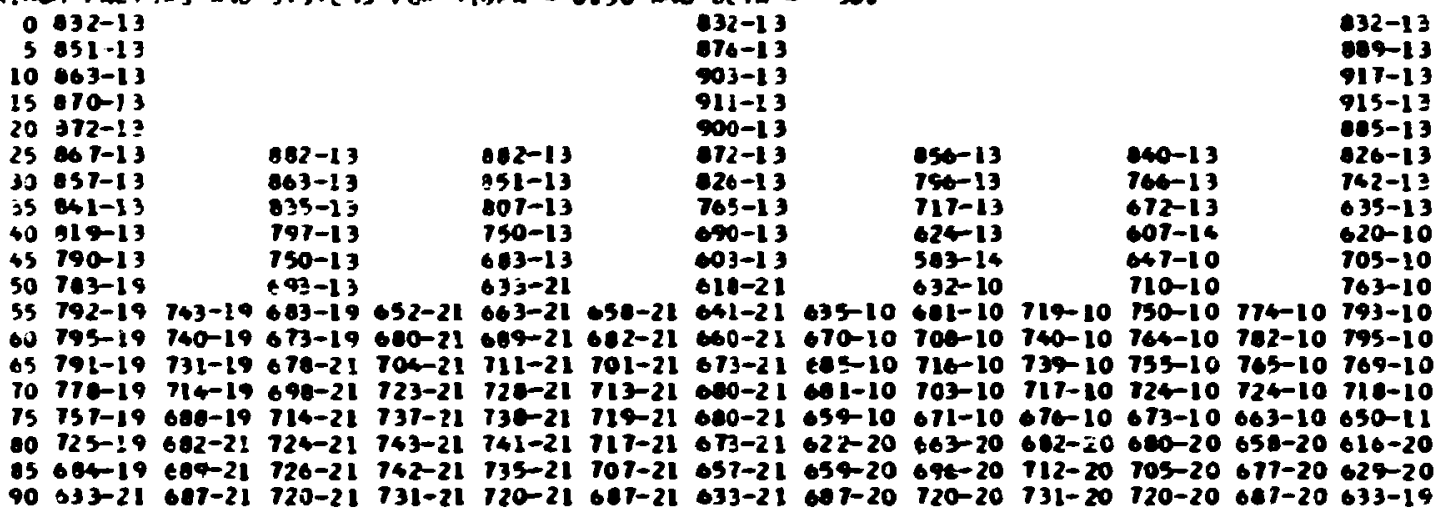

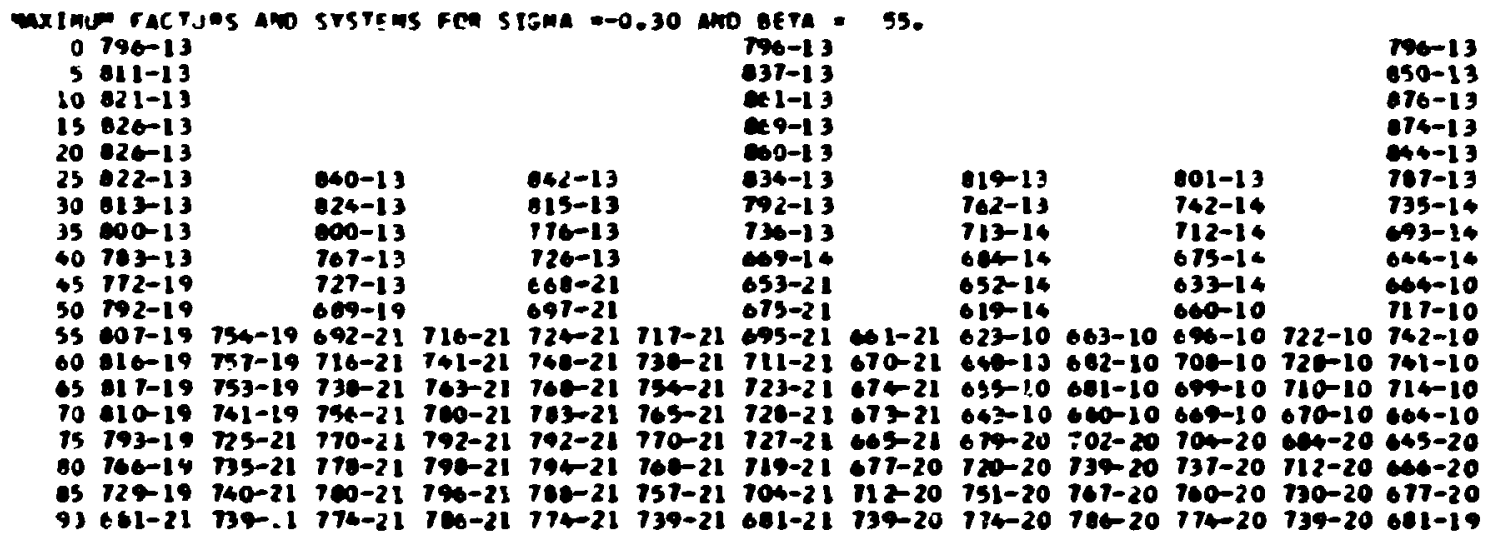




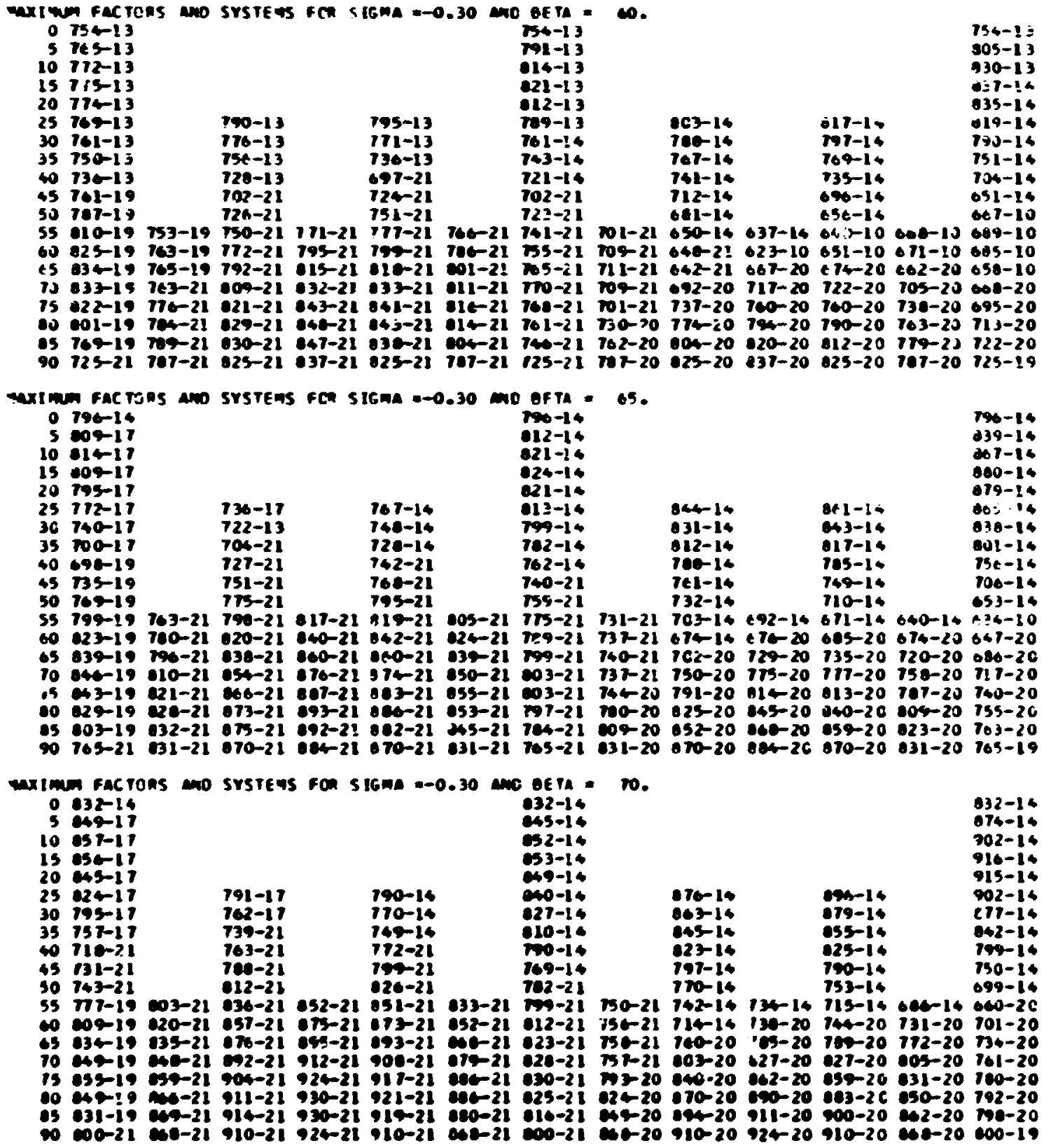




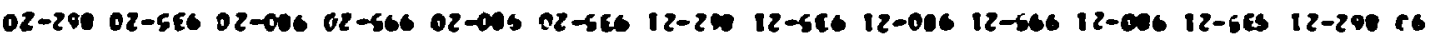

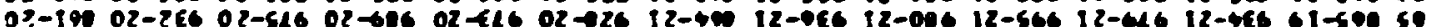

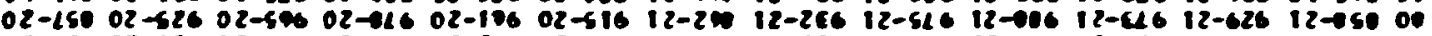

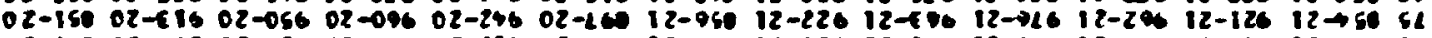

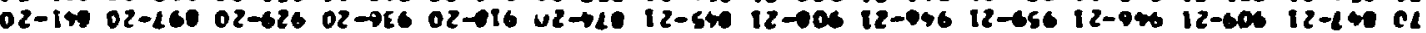

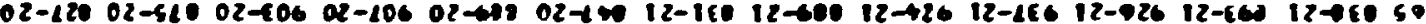

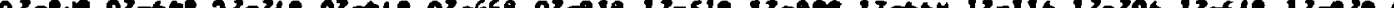

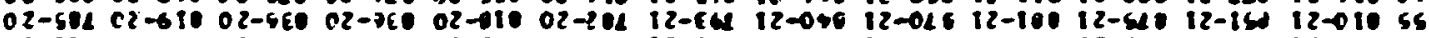

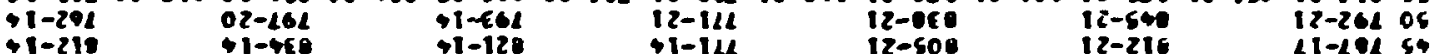

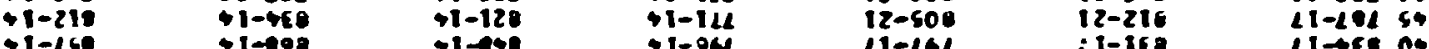

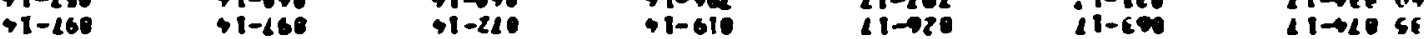

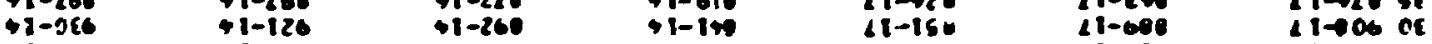

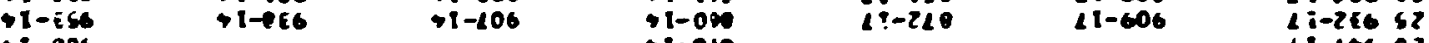
$1-918$ $+1-400$

$1-20^{2}$ $1-66$ 15651 $1: 460$ $+1-960$

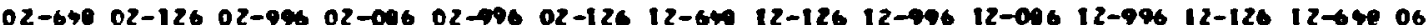

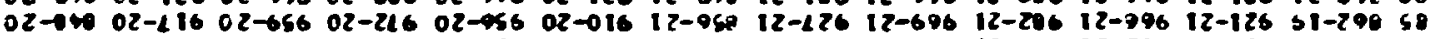

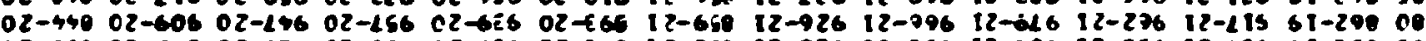

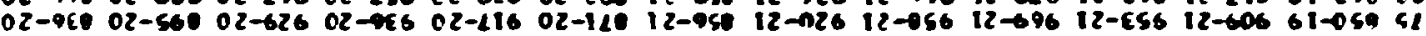

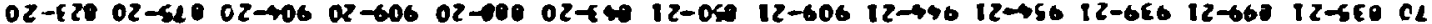

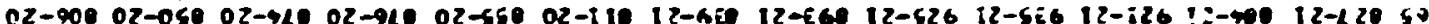

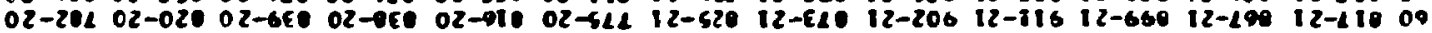

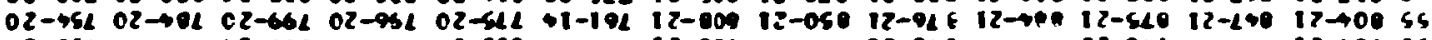

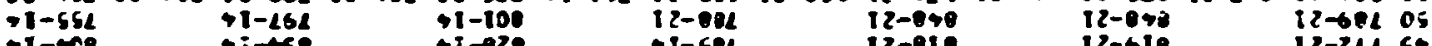

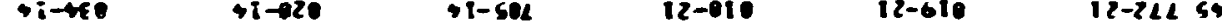

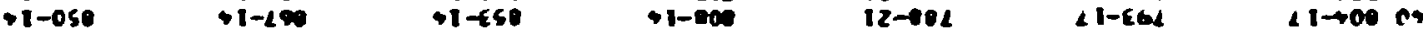

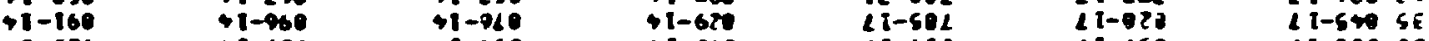

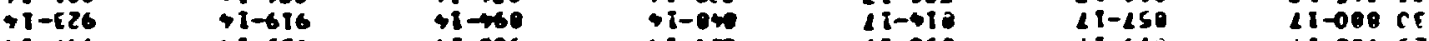

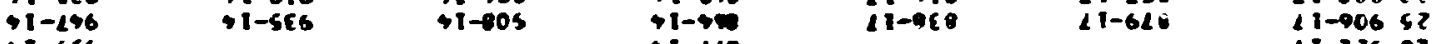

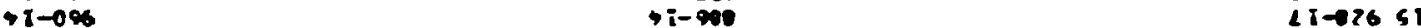
$+1-146 \quad+1-050 \quad 11-62601$ $+1-126$ $+1-500$

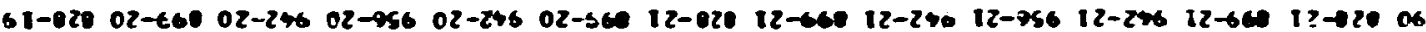

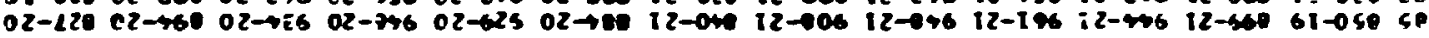

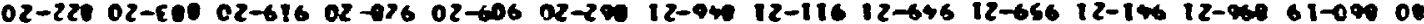

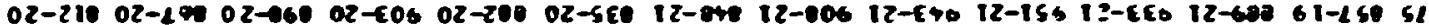

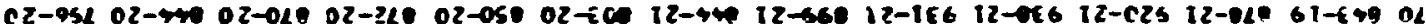

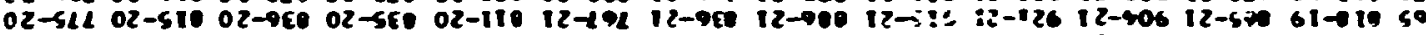

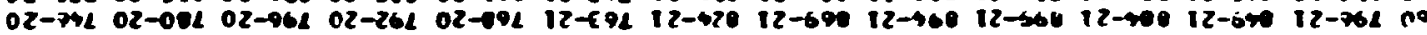

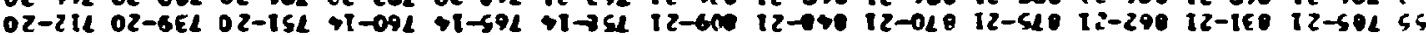

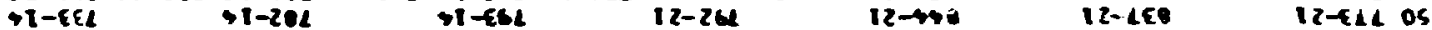
$+1-402 \quad+1-610 \quad+1-020 \quad 12-910 \quad 12-116 \quad 12-95254$ $\begin{array}{lll}+1-40 L & +1-610 & +1-020\end{array}$ $+i-210+1-100+1-190+1-920$ $+8-906+1-506$ $1-0$ is $t-1 \overline{2} \quad+1-25$ $4 \pi-246$ $41-626$

$1-206$ $+1-13$ 12-001 iz-902 $41-69 \mathrm{CH}$ $12-092 \quad 11-28 L \quad C 1-900 \mathrm{SE}$

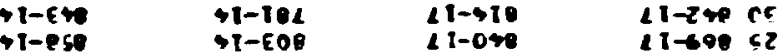
$\begin{array}{lll}91-850 & 11-600 & 11-6952\end{array}$ $\begin{array}{ll}\rightarrow 1-04 & 21-299<2 \\ 1-910 & 41-96051\end{array}$ -i-5L5 li-the o: $41-110 \quad 41-2005$

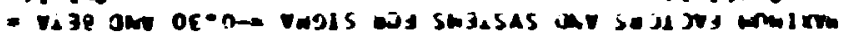




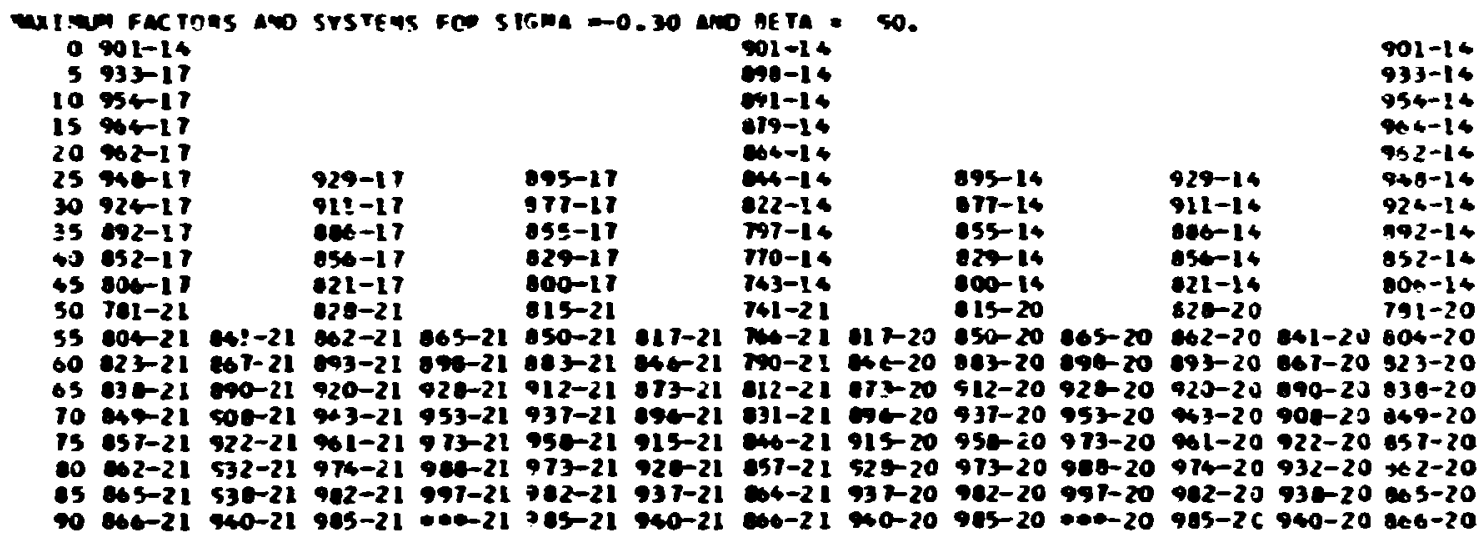




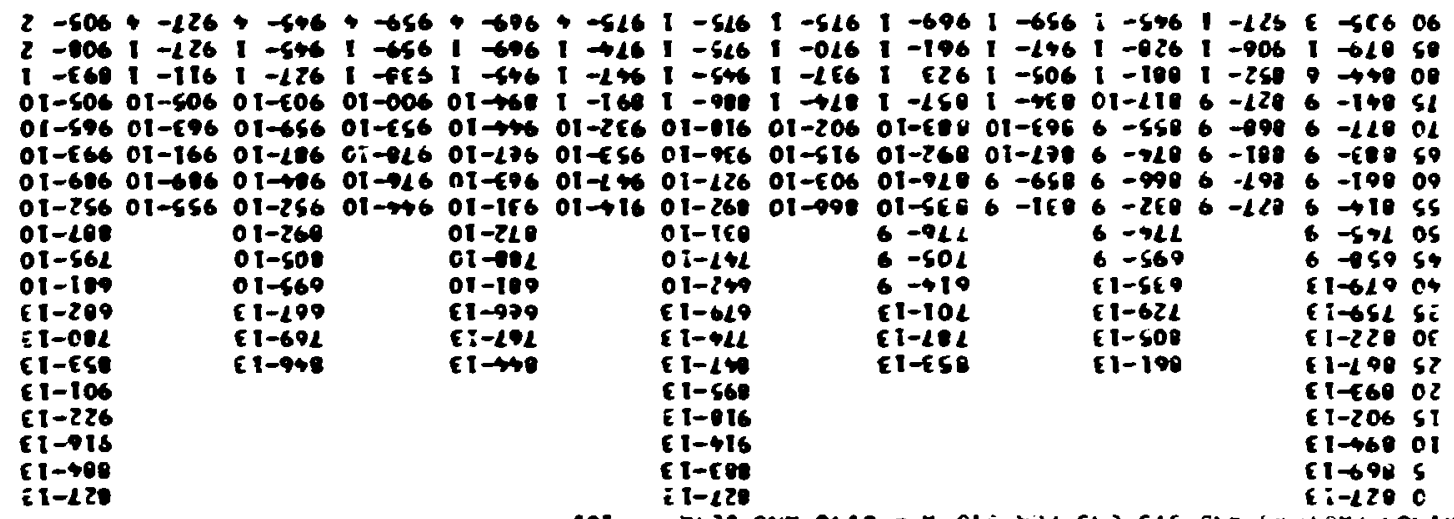

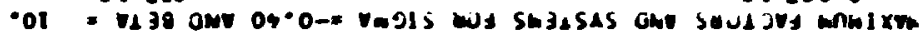

$2-026+-146+-656+-216+-106+-0661-1661-0661 \rightarrow 061-6151-6501-145 E-02606$

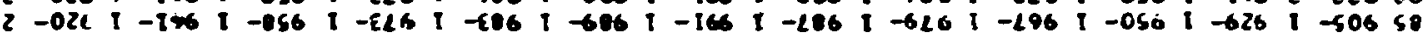

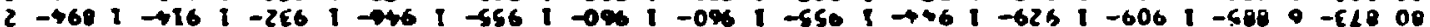

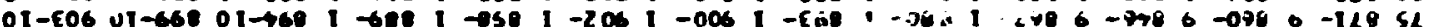

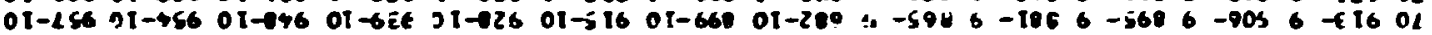

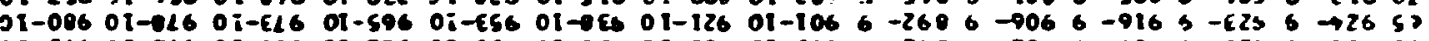
01-116 01-216 $01-696$ 01-296 $01-056$ 01-566 01-916 01-56e $6-2696-206 \quad 6-6066-0156-966$ 09

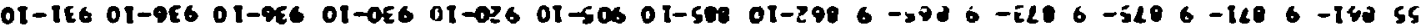

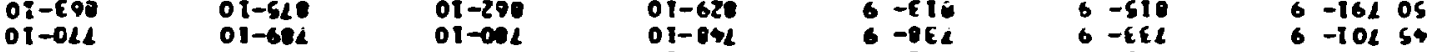

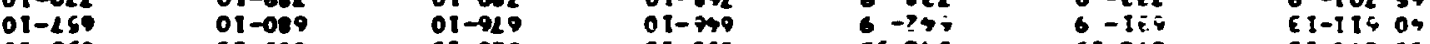

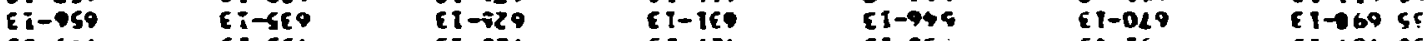
CI-69L EI-EEL EI-92L EI-12L EL EI-9EL EI-TSL EI-LOL OE

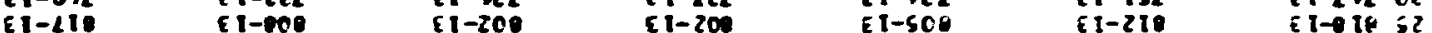
$51-200$ EI-SL $\varepsilon I-428$ $1-190$ si EI 1 - 58 or

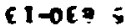
EI-E⿻ CI-9 91 C

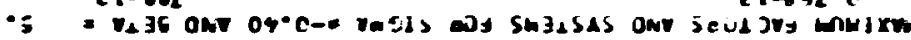

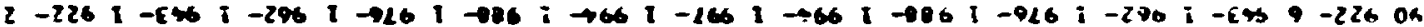

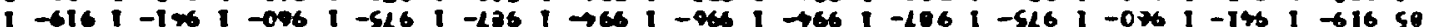

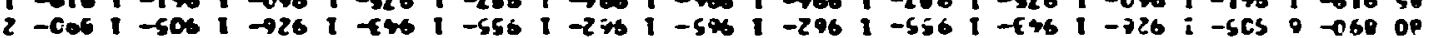

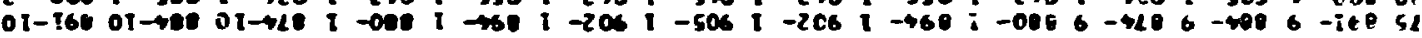

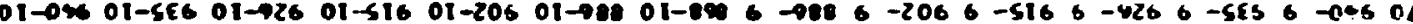

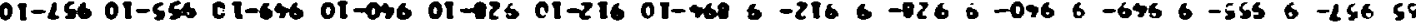

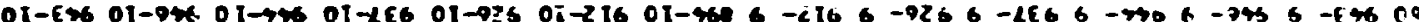

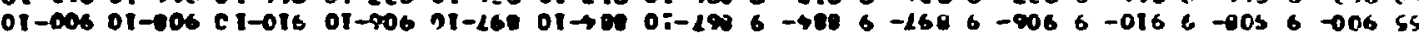
$01-168$ $01-6 \bar{c}$

$01-9 x$ 1069

c1-112 $01-6+9$ $0 \mathrm{i}-59 \mathrm{~s}$

$01-659$

$01-509$

E i-29

E $\mathrm{i}-294 \quad$ it $\rightarrow 5:$ $0 \mathrm{i}-6 \mathrm{EL}$

$01-249$

$6-591 \quad 6-6154$

EI-STO

EI-Z $\mathbf{E S}$

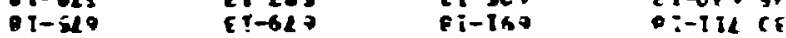

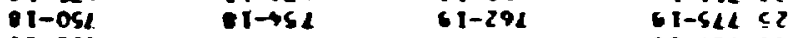
(1-108 $1-920$ ci-2Ef si EI-C20 ET-SQ

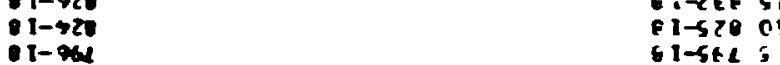

$01-69$

i- $-5 \times 2$ $6 T-E+1 C$ 


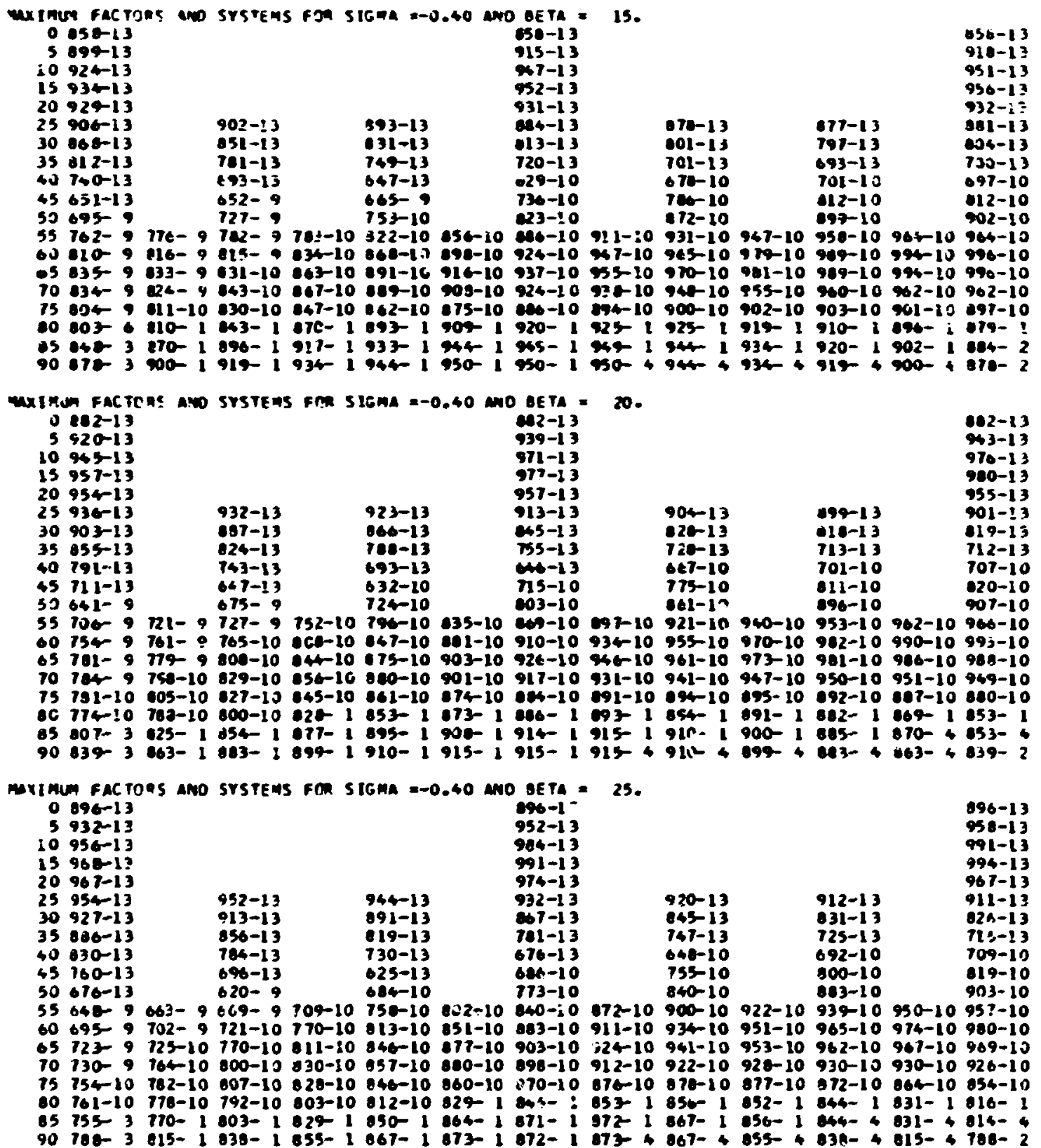




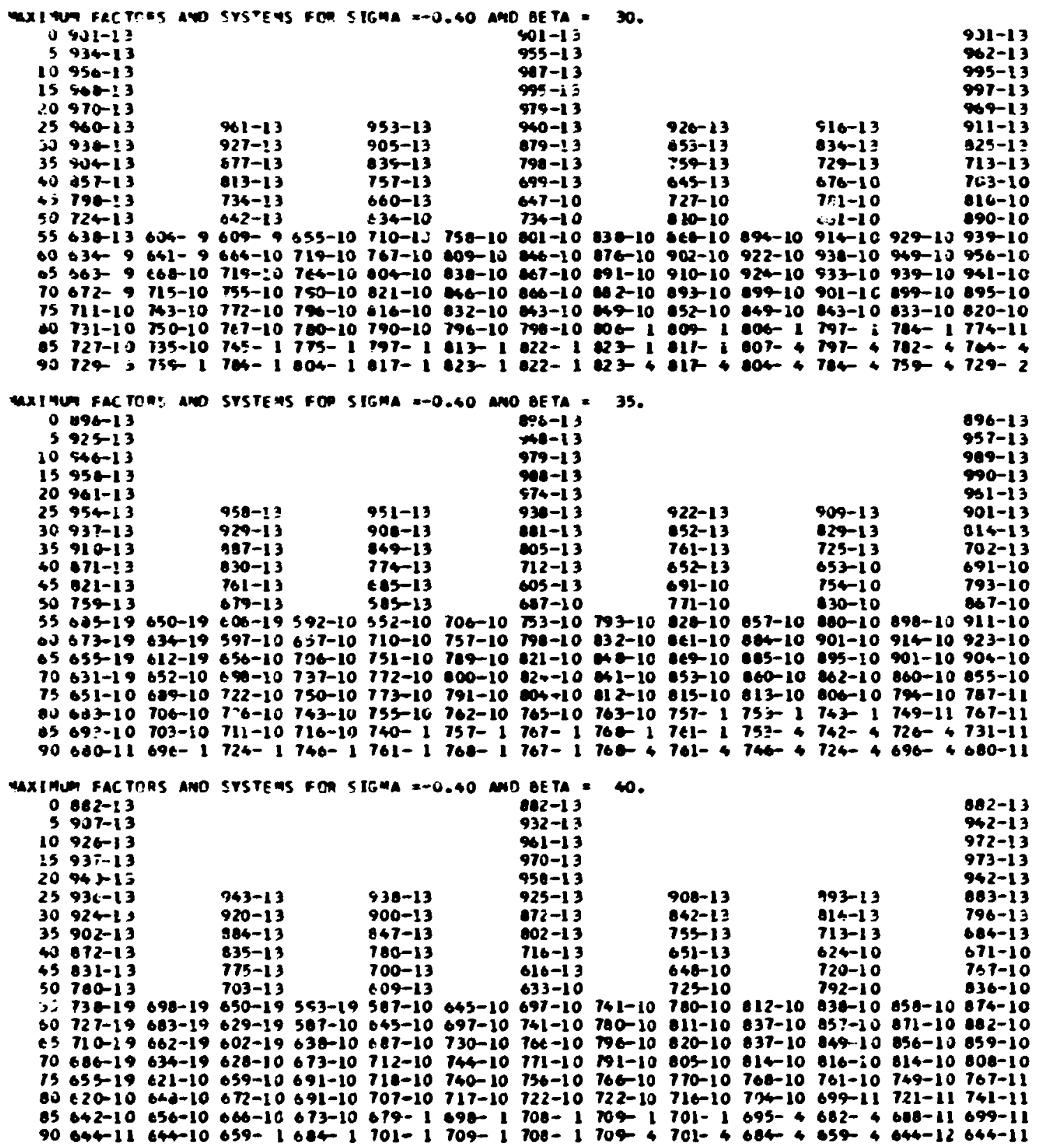




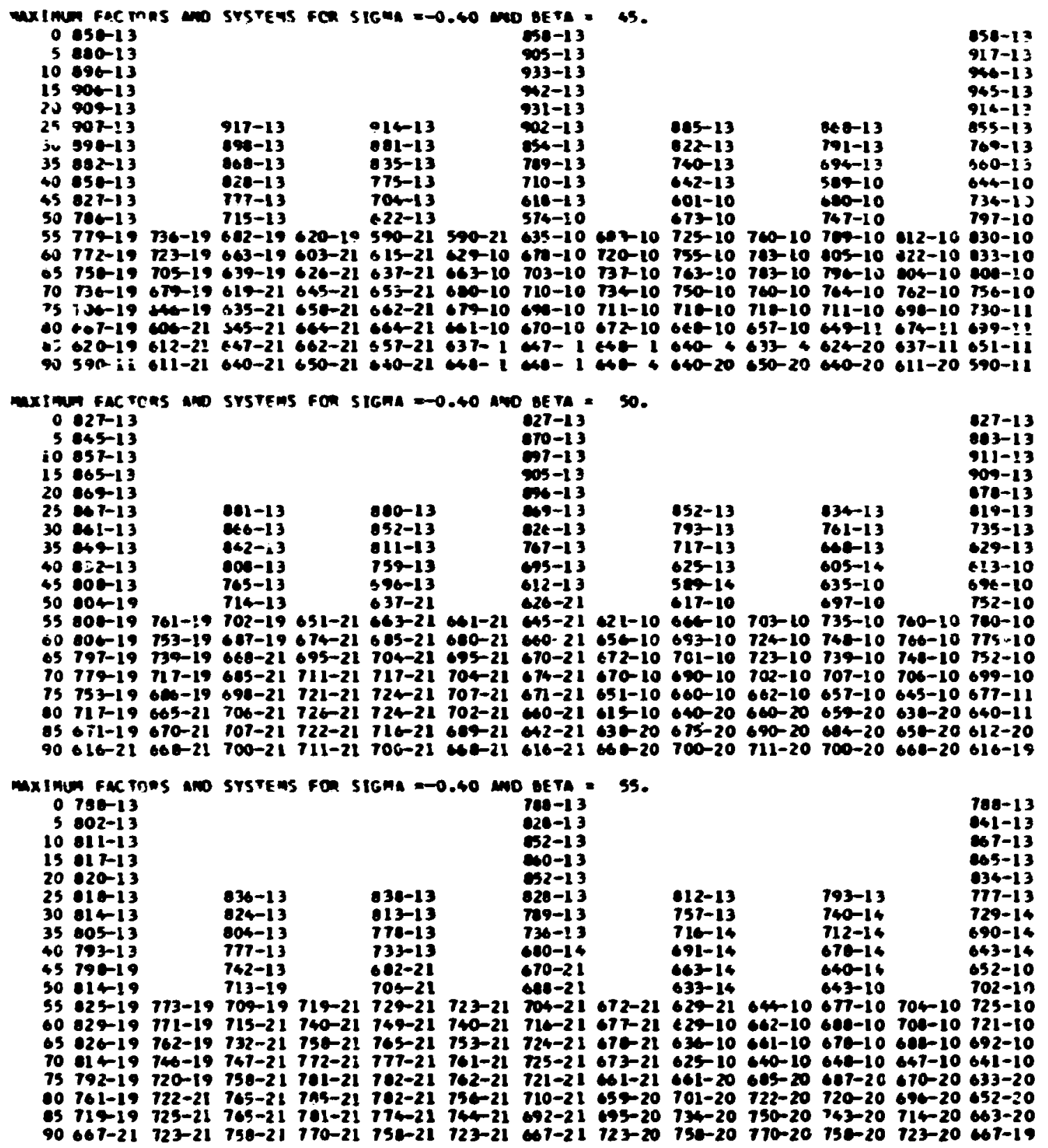




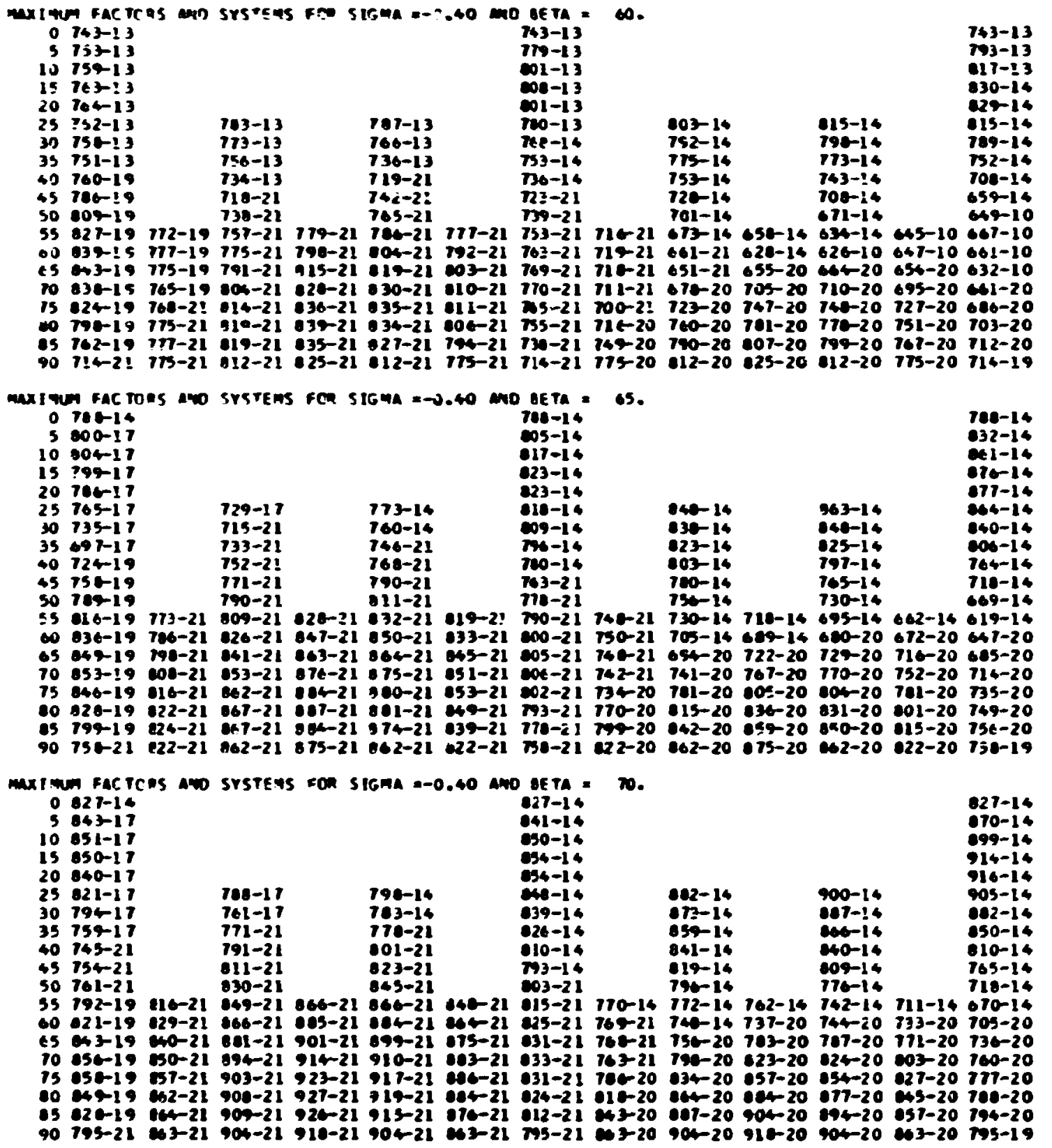




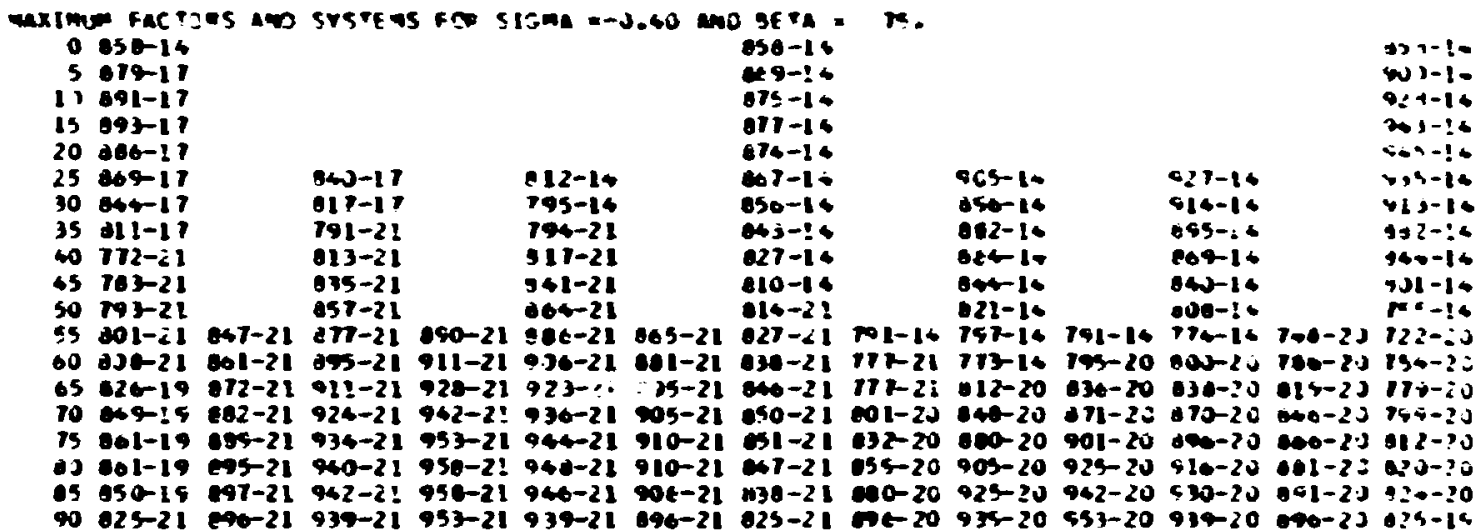

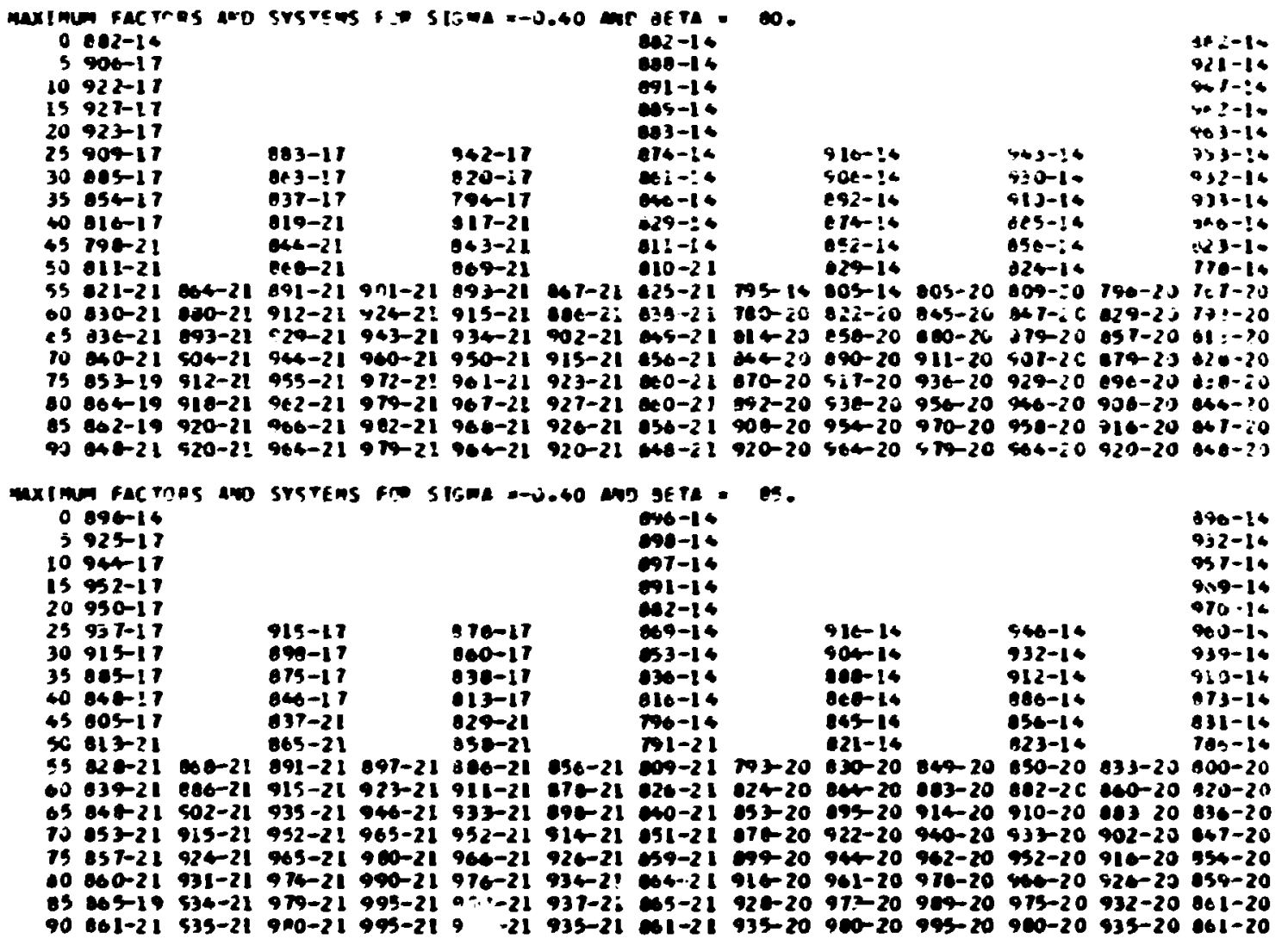




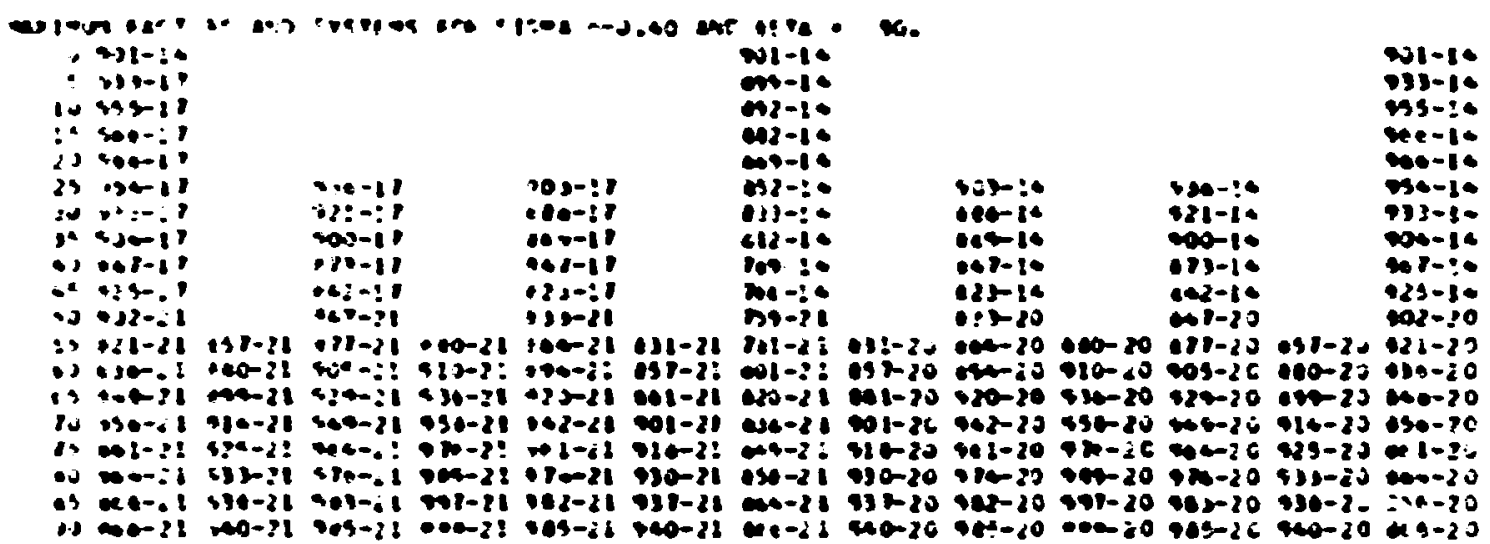




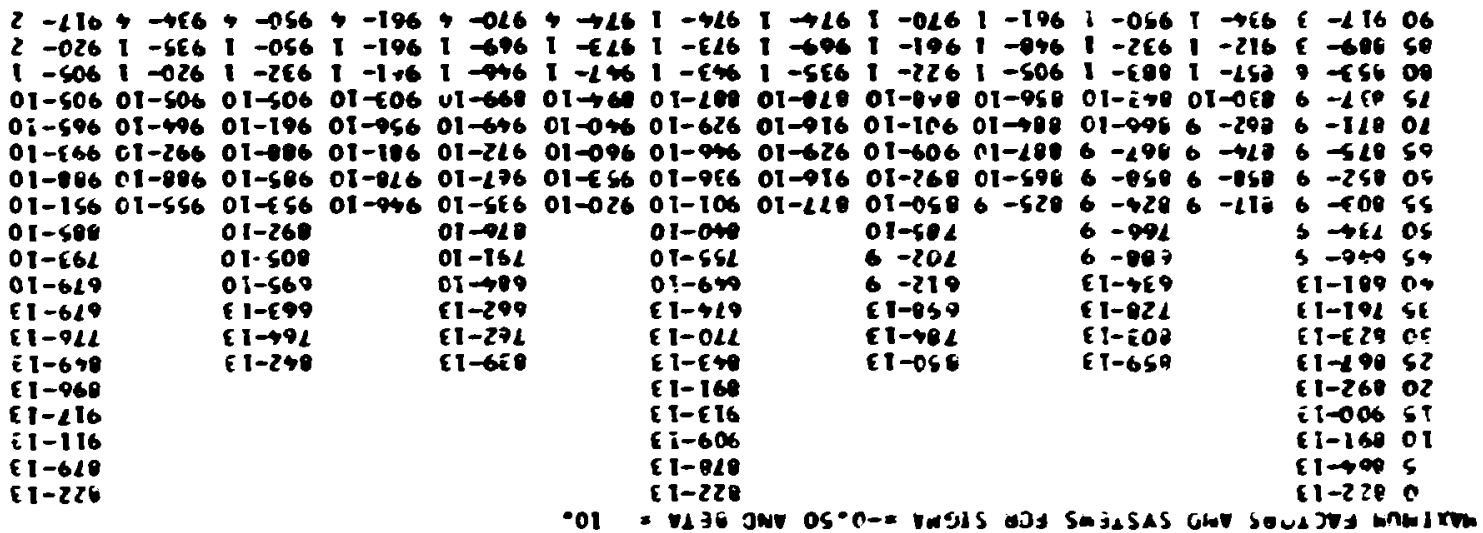

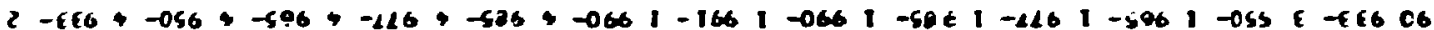

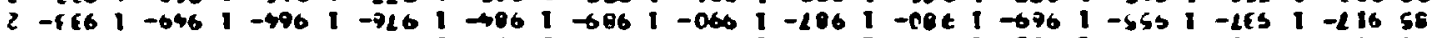

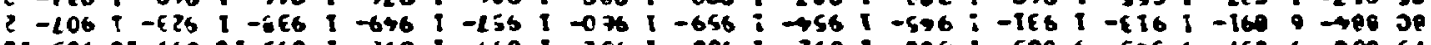

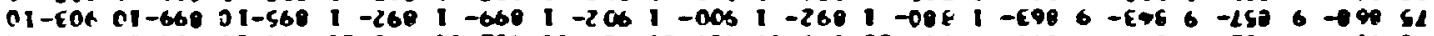

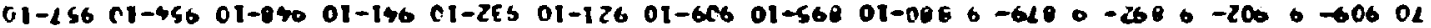

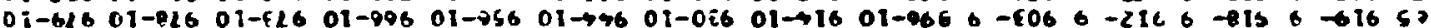

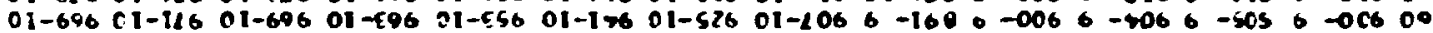

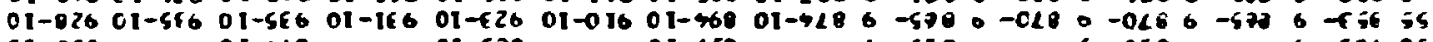

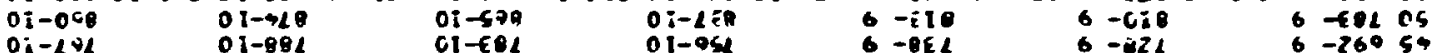

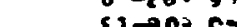

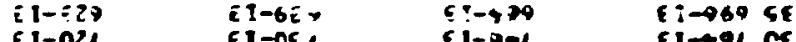

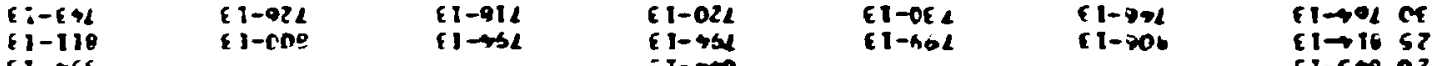
EI 1 - SE

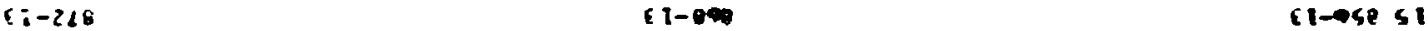

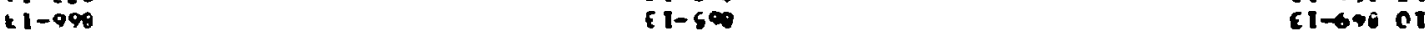

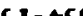

[1-OBL

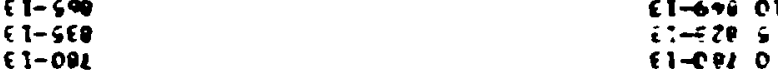

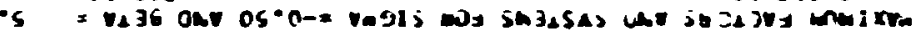

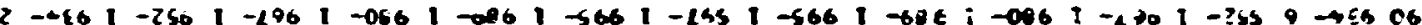

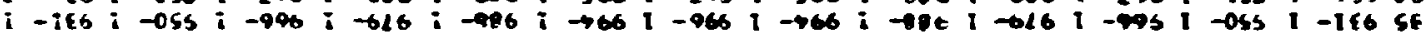

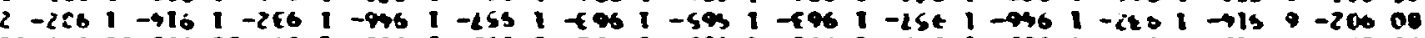

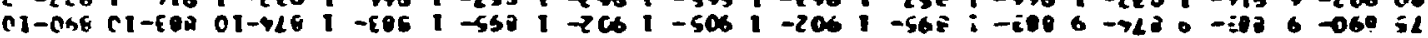

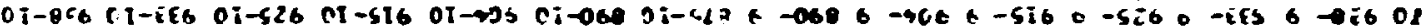

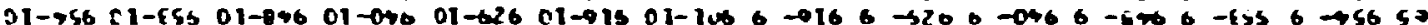

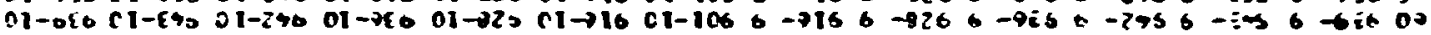

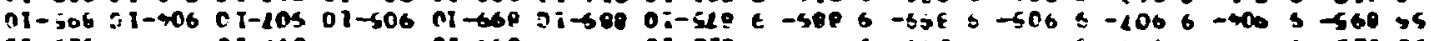

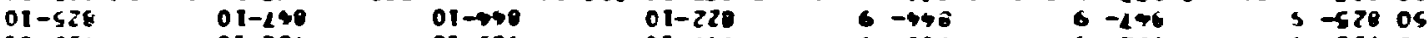

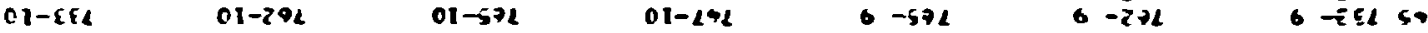

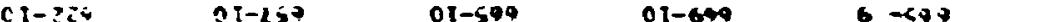
$\begin{array}{llllll}91-629 & 01-565 & 81-515 & 01-205 & 61-545 & 1-655\end{array}$

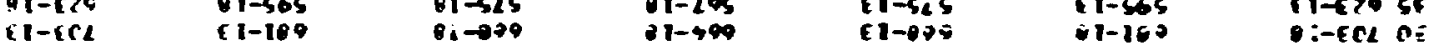

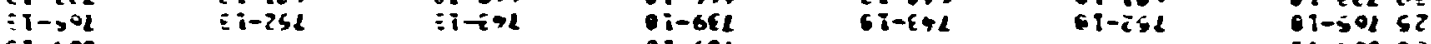
CI-GCF

$1-n>5$

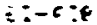

Ei-fFi

ai- $2+1$

\begin{tabular}{|c|c|}
\hline $\begin{array}{l}91-919 \\
01-519 \\
91-594 \\
91-262\end{array}$ & 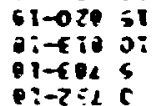 \\
\hline
\end{tabular}

- 


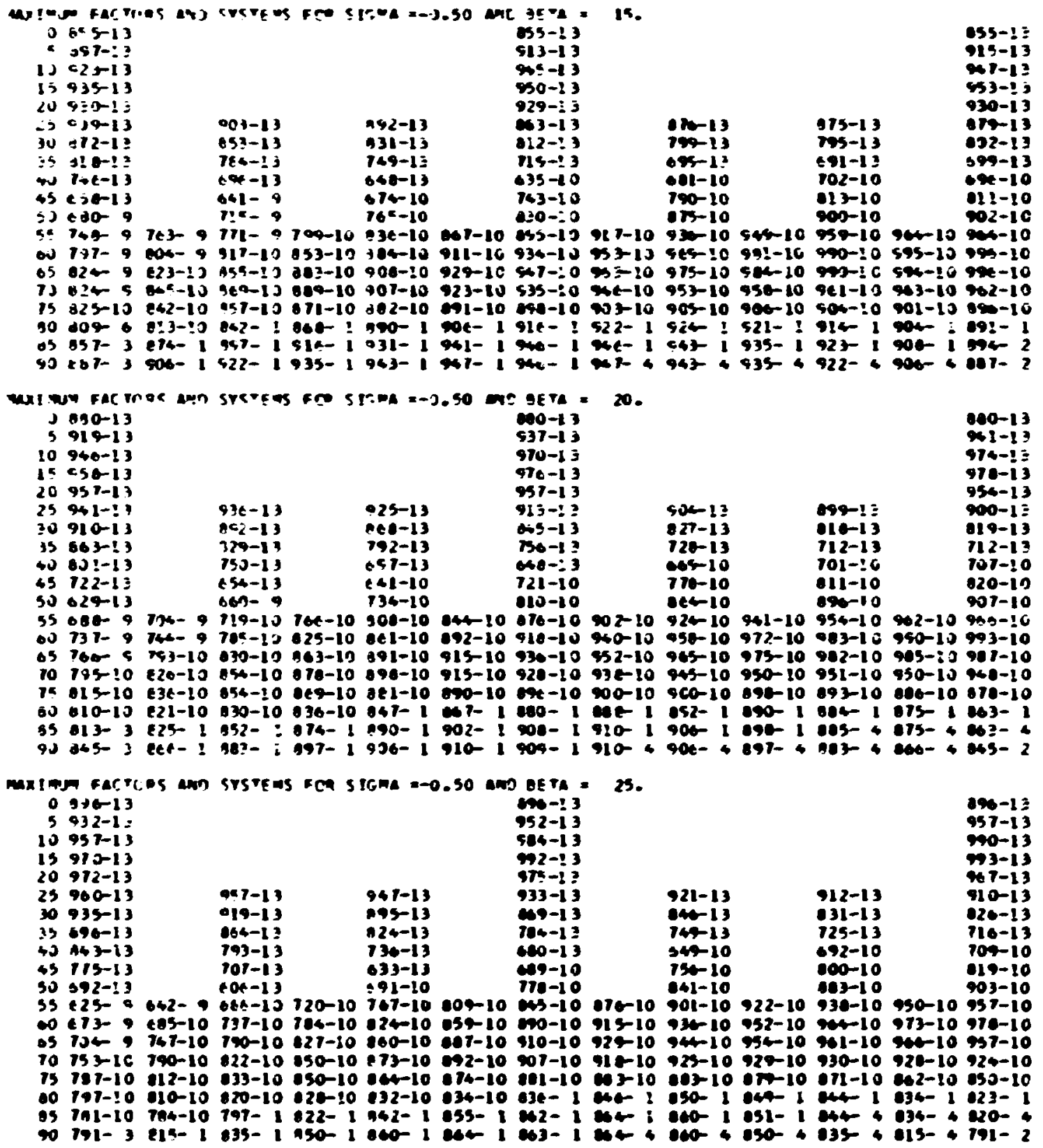




\begin{tabular}{|c|c|c|c|c|c|c|c|c|c|c|c|c|c|}
\hline $\begin{array}{l}0 \\
5 \\
13 \\
15 \\
20 \\
23 \\
35 \\
35 \\
00 \\
05 \\
33 \\
55 \\
00 \\
63 \\
10 \\
15 \\
30 \\
05 \\
95\end{array}$ & 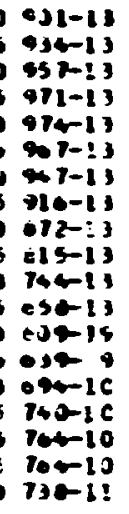 & 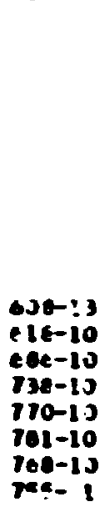 & 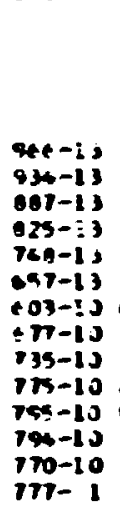 & 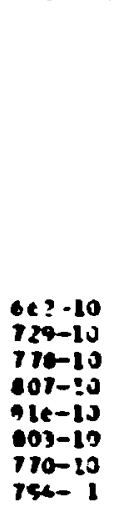 & $\begin{array}{l}757-12 \\
010-13 \\
140-13 \\
700-13 \\
=7 j-13 \\
030-10 \\
110-10 \\
775-10 \\
115-10 \\
934-10 \\
132-10 \\
509-10 \\
705-1 \\
000-1\end{array}$ & $\begin{array}{l}0 \\
\\
162-20 \\
015-10 \\
06-10 \\
050-10 \\
041-10 \\
081-10 \\
001-1 \\
911-1\end{array}$ & 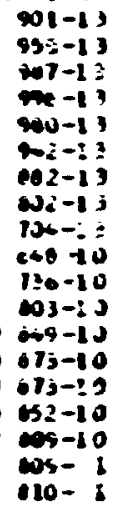 & 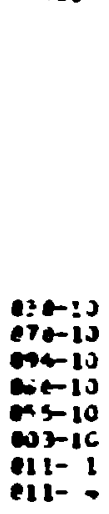 & 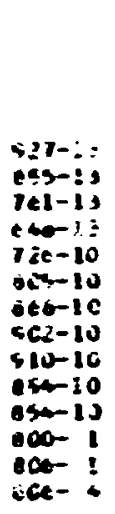 & $\begin{array}{l}0 \\
92-10 \\
921-10 \\
922-10 \\
996-80 \\
640-10 \\
790-1 \\
900-4 \\
190-4\end{array}$ & 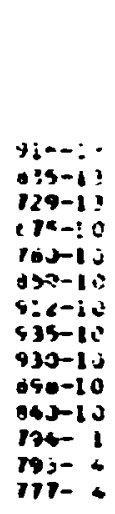 & 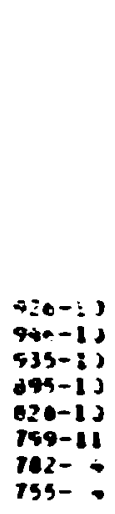 & 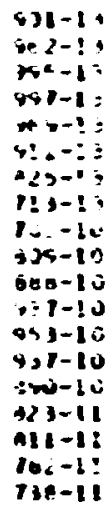 \\
\hline $\begin{array}{l}100 \\
0 \\
5 \\
10 \\
15 \\
20 \\
25 \\
30 \\
35 \\
-6 \\
15 \\
50 \\
56 \\
60 \\
65 \\
70 \\
75 \\
00 \\
15 \\
90\end{array}$ & 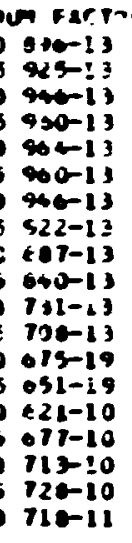 & $\begin{array}{l}\text { cec-10 } \\
63 e-10 \\
612-10 \\
670-13 \\
712-10 \\
73-13 \\
735-13 \\
121-11\end{array}$ & $\begin{array}{l}903-13 \\
957-13 \\
697-13 \\
647-13 \\
176-13 \\
697-13 \\
618-19 \\
605-1.3 \\
047-10 \\
113-13 \\
141-10 \\
150-10 \\
739-10 \\
723-11\end{array}$ & $\begin{array}{l} \\
595-10 \\
593-10 \\
603-10 \\
715-10 \\
750-10 \\
700-10 \\
763-10 \\
760-10 \\
733-1\end{array}$ & $\begin{array}{l}654-13 \\
914-13 \\
950-13 \\
705-13 \\
697-13 \\
595-13 \\
654-10 \\
716-10 \\
757-10 \\
702-10 \\
780-10 \\
771-10 \\
737-10 \\
760-1\end{array}$ & $\begin{array}{l}2 \\
\\
\\
700-10 \\
159-10 \\
792-10 \\
607-10 \\
201-10 \\
775-10 \\
7+1-1 \\
752-1\end{array}$ & 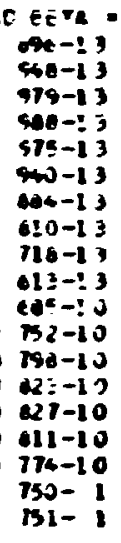 & $\begin{array}{l}\text { in 1-10 } \\
0 \geq 1-10 \\
40-10 \\
0+2-10 \\
115-10 \\
7 \in 9-10 \\
152-1 \\
732\end{array}$ & 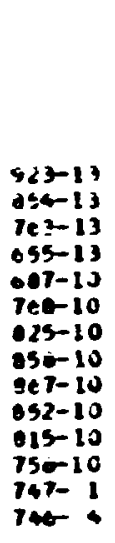 & $\begin{array}{l}05-10 \\
053-10 \\
070-10 \\
000-i 0 \\
050-10 \\
610-10 \\
700-11 \\
740-11 \\
733-1\end{array}$ & $\begin{array}{l}909-13 \\
924-13 \\
125-13 \\
653-10 \\
151-16 \\
827-10 \\
915-16 \\
690-19 \\
690-16 \\
857-10 \\
800-13 \\
115-1 ! \\
754-11 \\
123-1 !\end{array}$ & $\begin{array}{l}0 \\
051-13 \\
904-13 \\
095-13 \\
053-13 \\
000-11 \\
700-11 \\
702-11 \\
721-11\end{array}$ & 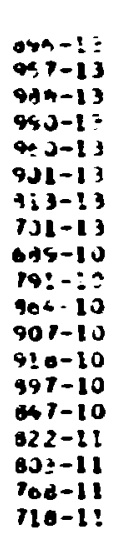 \\
\hline $\begin{array}{l}3 \\
10 \\
15 \\
20 \\
25 \\
30 \\
35 \\
40 \\
45 \\
50 \\
35 \\
60 \\
65 \\
10 \\
15 \\
00 \\
05 \\
01\end{array}$ & $\begin{array}{l}0.46 \mathrm{~m} \\
000-13 \\
906-13 \\
925-13 \\
537-13 \\
943-13 \\
941-13 \\
932-13 \\
914-13 \\
008-13 \\
051-13 \\
003-13 \\
749-19 \\
733-19 \\
710-19 \\
081-19 \\
644-19 \\
645-10 \\
673-10 \\
674-11\end{array}$ & $\begin{array}{r}n 1-19 \\
691-19 \\
64-19 \\
631-19 \\
639-10 \\
671-10 \\
606-10 \\
601-21\end{array}$ & $\begin{array}{l}967-13 \\
92 e-13 \\
896-13 \\
649-13 \\
792-13 \\
723-13 \\
665-19 \\
699-19 \\
609-19 \\
639-10 \\
674-10 \\
691-10 \\
691-10 \\
681-11\end{array}$ & $\begin{array}{l} \\
\\
611-19 \\
309-10 \\
642-10 \\
681-10 \\
703-10 \\
700-10 \\
694-10 \\
601-11\end{array}$ & $\begin{array}{l}941-13 \\
905-13 \\
855-13 \\
790-13 \\
713-13 \\
624-13 \\
504-10 \\
645-10 \\
650-10 \\
117-10 \\
120-10 \\
720-10 \\
694-10 \\
602-1\end{array}$ & $\begin{array}{l}0 \\
\\
41-10 \\
695-10 \\
730-10 \\
747-10 \\
746-10 \\
726-10 \\
690-10 \\
699-1\end{array}$ & $\begin{array}{l}400-13 \\
930-13 \\
900-13 \\
969-13 \\
950-13 \\
920-13 \\
675-13 \\
61-13 \\
722-13 \\
925-13 \\
627-10 \\
992-10 \\
730-10 \\
706-10 \\
171-10 \\
799-10 \\
720-10 \\
607-1\end{array}$ & $\begin{array}{l}735-10 \\
774-10 \\
792-10 \\
78 x-10 \\
704-1 . \\
724-10 \\
64-1\end{array}$ & $\begin{array}{l}906-12 \\
042-13 \\
757-13 \\
654-13 \\
042-10 \\
710-10 \\
773-10 \\
005-10 \\
016-10 \\
000-10 \\
7 \in 7-10 \\
114-10 \\
096-11 \\
002-4\end{array}$ & $\begin{array}{l}0 \\
100-10 \\
029-10 \\
030-10 \\
607-10 \\
702-10 \\
719-11 \\
700-11 \\
601-11\end{array}$ & $\begin{array}{l}892-13 \\
813-13 \\
113-13 \\
619-10 \\
715-10 \\
705-10 \\
050-10 \\
040-10 \\
640-10 \\
607-10 \\
752-10 \\
739-11 \\
716-11 \\
081-11\end{array}$ & $\begin{array}{l}2 \\
\\
251-10 \\
263-10 \\
047-16 \\
606-10 \\
75-11 \\
750-11 \\
726-11 \\
601-11\end{array}$ & $\begin{array}{l}669-13 \\
960-13 \\
971-13 \\
971-13 \\
940-13 \\
600-13 \\
793-17 \\
602-13 \\
000-10 \\
763-10 \\
631-10 \\
260-10 \\
076-10 \\
269-10 \\
609-11 \\
201-11 \\
116-11 \\
734-11 \\
679-11\end{array}$ \\
\hline
\end{tabular}




\begin{tabular}{|c|c|c|c|c|c|c|c|c|c|c|c|c|c|}
\hline $\begin{array}{l}0 \\
5 \\
10 \\
15 \\
20 \\
25 \\
50 \\
35 \\
40 \\
45 \\
50 \\
55 \\
00 \\
15 \\
10 \\
15 \\
63 \\
85 \\
13\end{array}$ & 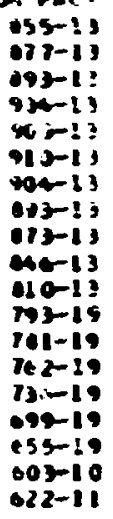 & $\begin{array}{l}51-19 \\
334-19 \\
710-19 \\
674-16 \\
64-19 \\
5 \%-19 \\
\text { c17-10 } \\
\text { c22-11 }\end{array}$ & $\begin{array}{l}920-13 \\
404-11 \\
671-13 \\
441-13 \\
163-11 \\
13 e-13 \\
100-19 \\
615-19 \\
647-19 \\
111-19 \\
615-21 \\
622-21 \\
620-10 \\
62-11\end{array}$ & $\begin{array}{l} \\
\\
640-19 \\
60-19 \\
612-21 \\
620-21 \\
640-21 \\
642-21 \\
630-21 \\
025-21\end{array}$ & 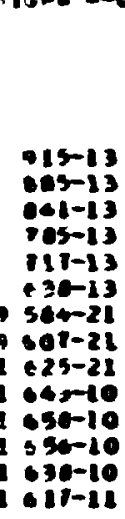 & $\begin{array}{l}0 \\
301-21 \\
622-10 \\
690-10 \\
670-10 \\
641-10 \\
667-10 \\
437-10 \\
424-1\end{array}$ & 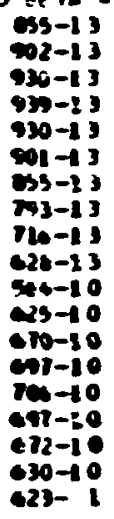 & 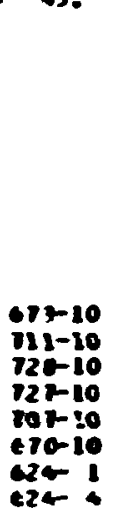 & $\begin{array}{l}103-13 \\
621-13 \\
741-13 \\
645-13 \\
391-20 \\
603-10 \\
114-10 \\
146-10 \\
153-10 \\
741-10 \\
115-10 \\
663-10 \\
634-11 \\
617-11\end{array}$ & 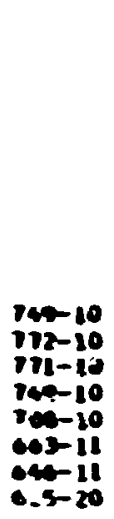 & 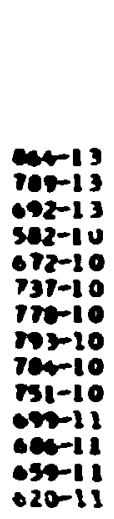 & $\begin{array}{l}0 \\
01-10 \\
010-10 \\
791-10 \\
700-10 \\
710-11 \\
700-11 \\
671-11 \\
022-11\end{array}$ & 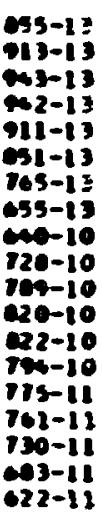 \\
\hline $\begin{array}{l}110 \\
0 \\
5 \\
10 \\
15 \\
20 \\
25 \\
30 \\
35 \\
40 \\
45 \\
50 \\
55 \\
60 \\
65 \\
10 \\
75 \\
60 \\
65 \\
90\end{array}$ & 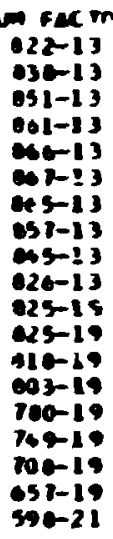 & 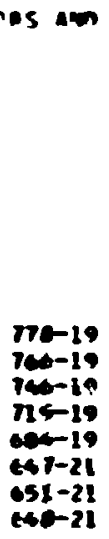 & $\begin{array}{l}601-12 \\
40-13 \\
64-13 \\
619-13 \\
701-13 \\
735-19 \\
721-19 \\
702-19 \\
671-19 \\
672-21 \\
602-21 \\
601921 \\
601-21 \\
600-21\end{array}$ & $\begin{array}{l}0 \\
05-19 \\
669-21 \\
606-21 \\
690-21 \\
700-21 \\
100-21 \\
103-21 \\
60-21\end{array}$ & $\begin{array}{l}970-13 \\
054-13 \\
010-13 \\
760-13 \\
700-13 \\
642-21 \\
603-21 \\
601-21 \\
190-21 \\
100-21 \\
120-21 \\
100-21 \\
650-21 \\
600-21\end{array}$ & $\begin{array}{l}\mid \\
03-21 \\
610-21 \\
60-21 \\
695-21 \\
695-21 \\
41-21 \\
612-21 \\
040-21\end{array}$ & 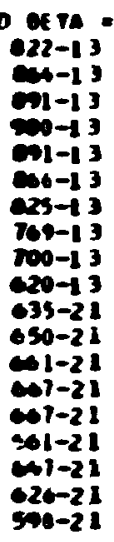 & 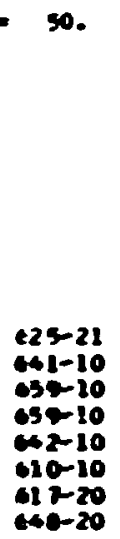 & $\begin{array}{l}64-13 \\
791-13 \\
110-13 \\
627-13 \\
394-14 \\
043-10 \\
691-10 \\
679-10 \\
607-10 \\
67-10 \\
64-10 \\
617-20 \\
693-20 \\
600-20\end{array}$ & $\begin{array}{r} \\
420-10 \\
70-10 \\
100-10 \\
601-10 \\
640-10 \\
630-20 \\
60-20 \\
450-20\end{array}$ & $\begin{array}{l}620-13 \\
75-13 \\
65-1 ? \\
604-10 \\
623-10 \\
605-10 \\
720-10 \\
132-10 \\
722-10 \\
691-10 \\
640-10 \\
630-20 \\
603-20 \\
600-20\end{array}$ & $\begin{array}{l}0 \\
740-10 \\
750-10 \\
730-10 \\
600-10 \\
60-11 \\
64-11 \\
630-20 \\
040-20\end{array}$ & 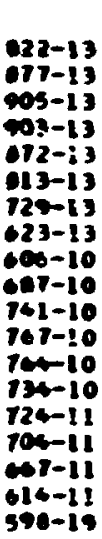 \\
\hline $\begin{array}{l}0 \\
5 \\
10 \\
15 \\
20 \\
25 \\
30 \\
35 \\
40 \\
65 \\
50 \\
55 \\
40 \\
65 \\
70 \\
75 \\
60 \\
65 \\
90\end{array}$ & $\begin{array}{c}0 A C \\
100-13 \\
792-13 \\
002-13 \\
009-13 \\
013-13 \\
015-13 \\
014-13 \\
010-13 \\
059-19 \\
025-19 \\
634-19 \\
042-19 \\
062-19 \\
634-19 \\
017-19 \\
091-19 \\
755-19 \\
704-19 \\
052-21\end{array}$ & $\begin{array}{l}791-19 \\
75-19 \\
m 2-19 \\
731-19 \\
721-19 \\
m 0-21 \\
711-21 \\
m 0-21\end{array}$ & $\begin{array}{l}132-13 \\
624-13 \\
200-13 \\
706-13 \\
750-13 \\
737-19 \\
729-19 \\
710-19 \\
727-21 \\
730-21 \\
747-21 \\
751-21 \\
730-21 \\
742-21\end{array}$ & $\begin{array}{l}0 \\
123-21 \\
123-21 \\
135-21 \\
734-21 \\
711-21 \\
111-21 \\
711-21 \\
152-21 \\
753-21\end{array}$ & 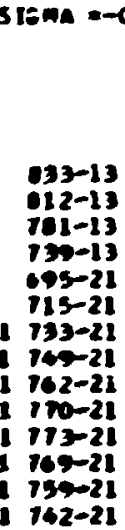 & $\begin{array}{l}7 \\
730-21 \\
743-21 \\
752-21 \\
750-21 \\
754-21 \\
745-21 \\
130-21 \\
700-21\end{array}$ & 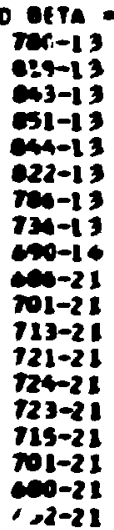 & 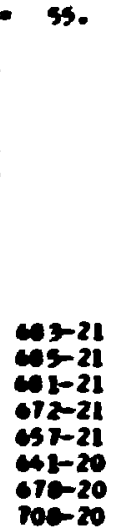 & $\begin{array}{l}604-13 \\
752-13 \\
720-14 \\
64-14 \\
674-16 \\
644-14 \\
643-21 \\
636-21 \\
624-21 \\
607-10 \\
642-20 \\
659-20 \\
717-20 \\
742-20\end{array}$ & $\begin{array}{l}624-11 \\
64210 \\
641-10 \\
621-20 \\
607-20 \\
707-20 \\
733-20 \\
753-20\end{array}$ & 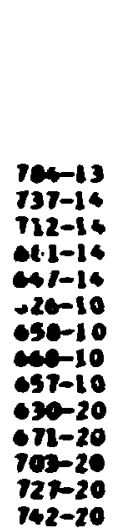 & $\begin{array}{l}60 \\
601 \\
625 \\
65\end{array}$ & $\begin{array}{l}700-13 \\
633-13 \\
650-13 \\
65-13 \\
624-13 \\
107-13 \\
124-14 \\
607-16 \\
63-16 \\
60-10 \\
607-10 \\
100-10 \\
102-10 \\
671-10 \\
657-11 \\
631-11 \\
639-20 \\
640-20 \\
632-19\end{array}$ \\
\hline
\end{tabular}




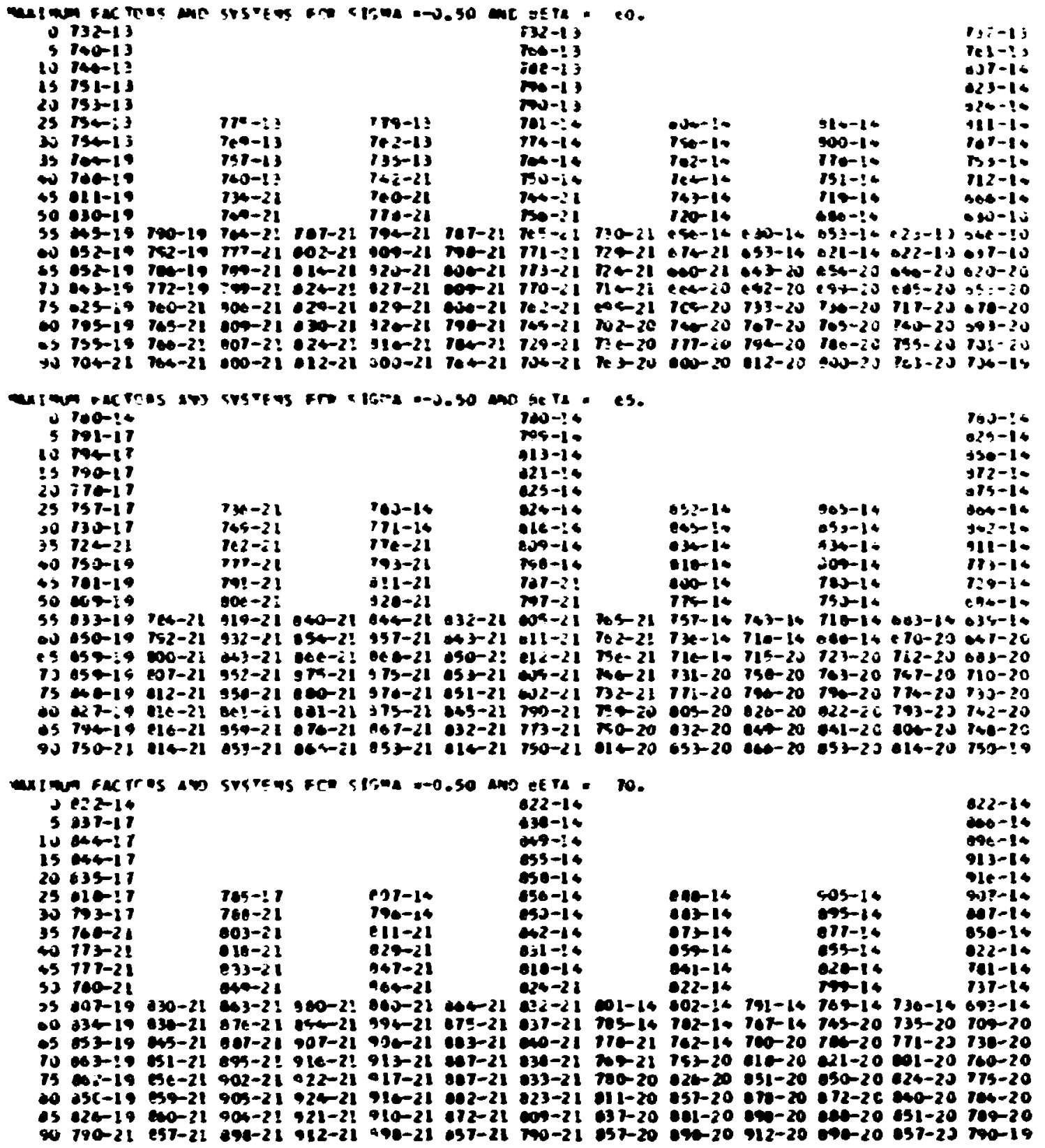




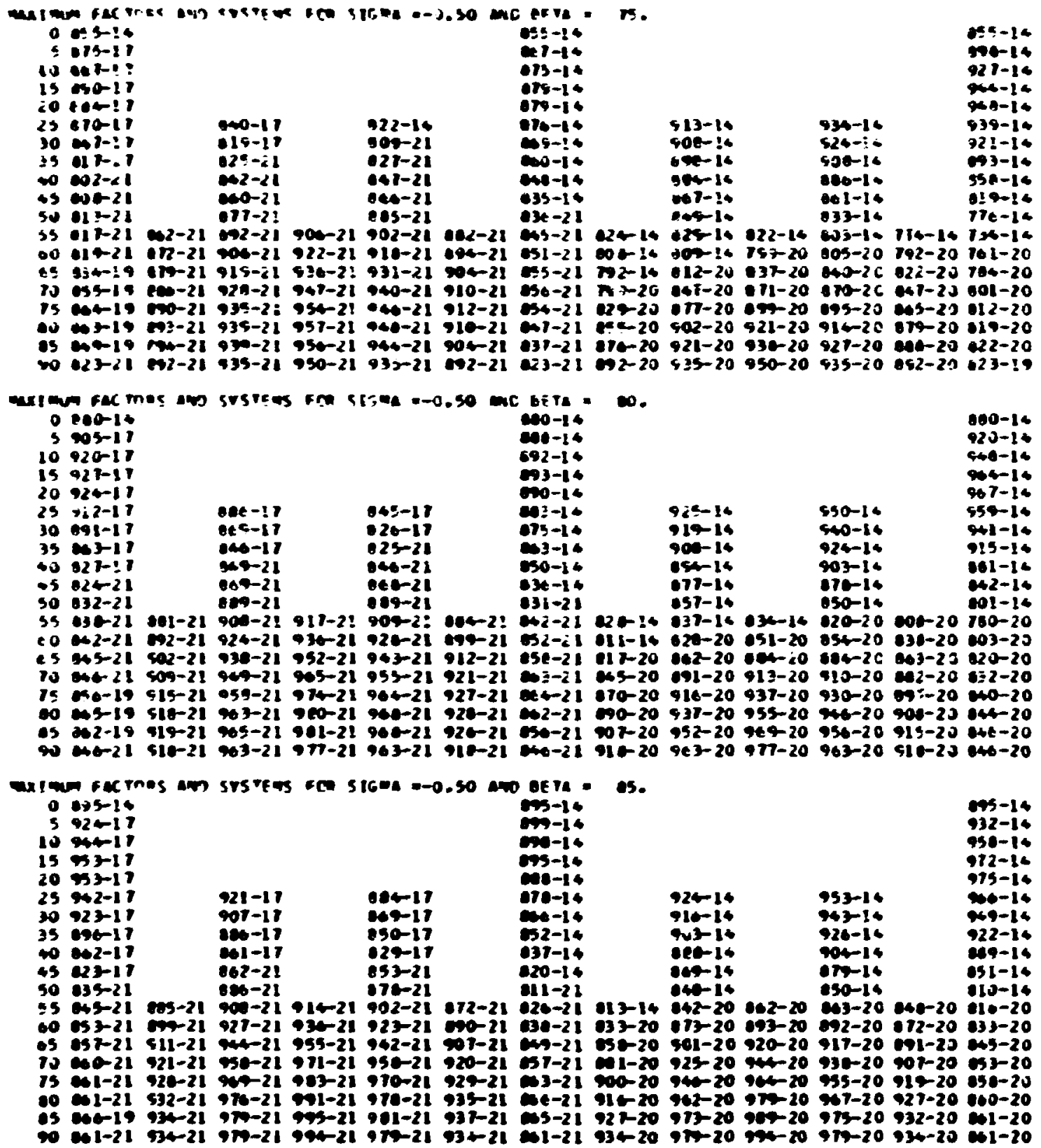




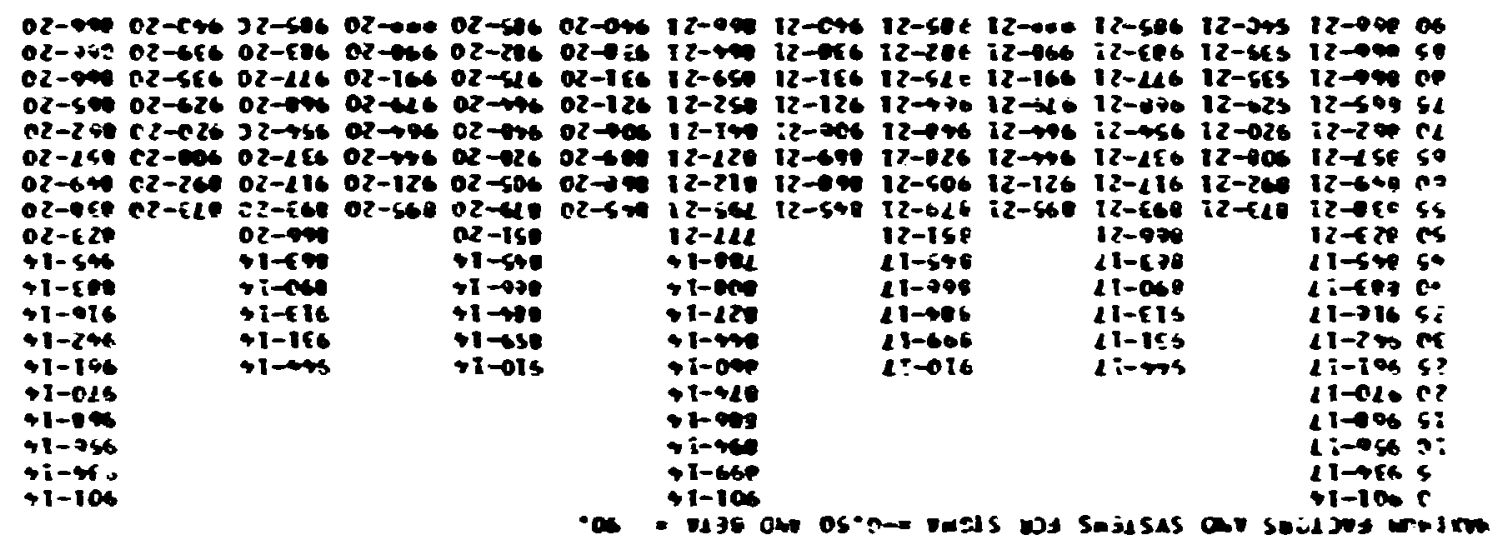




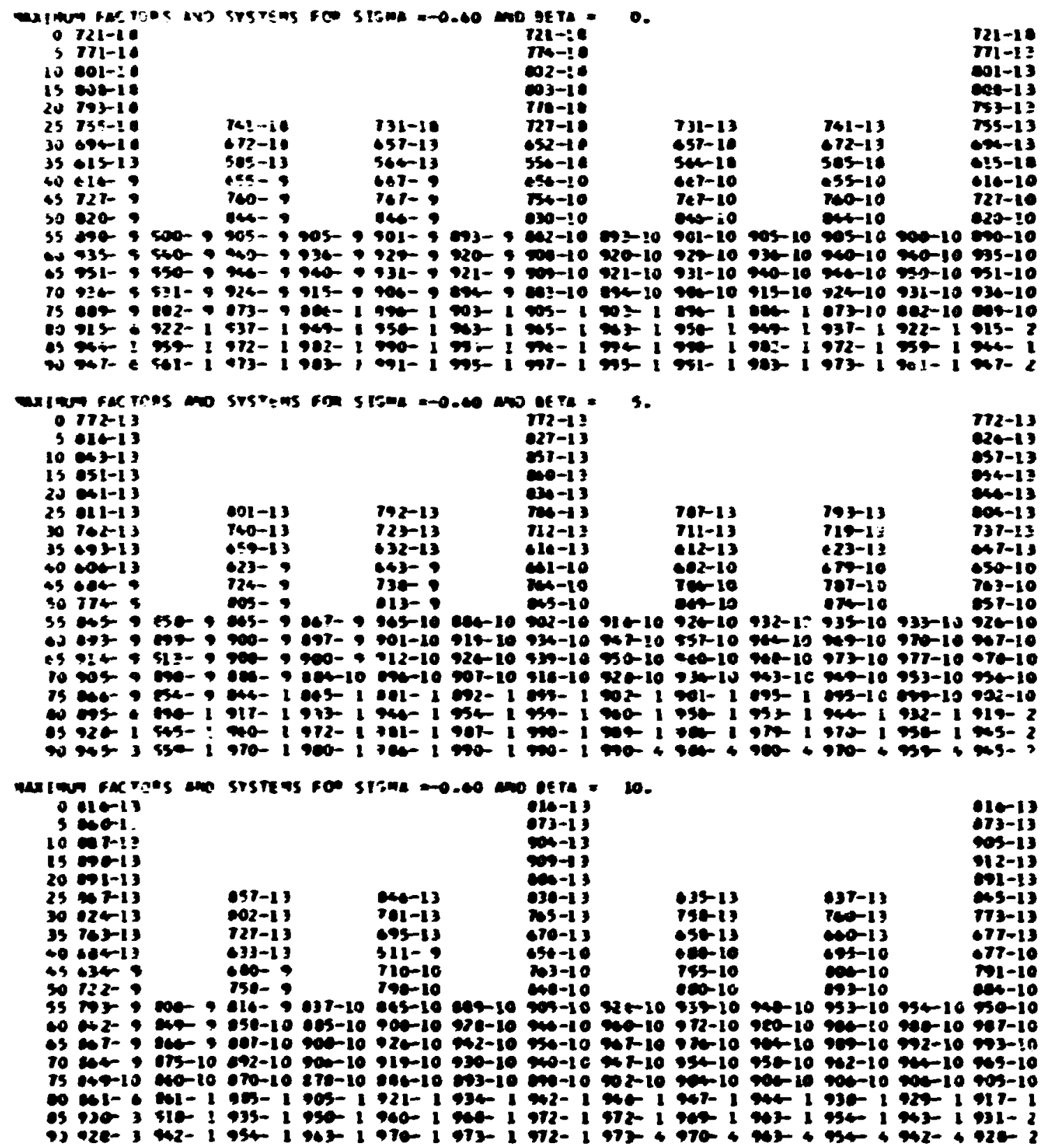




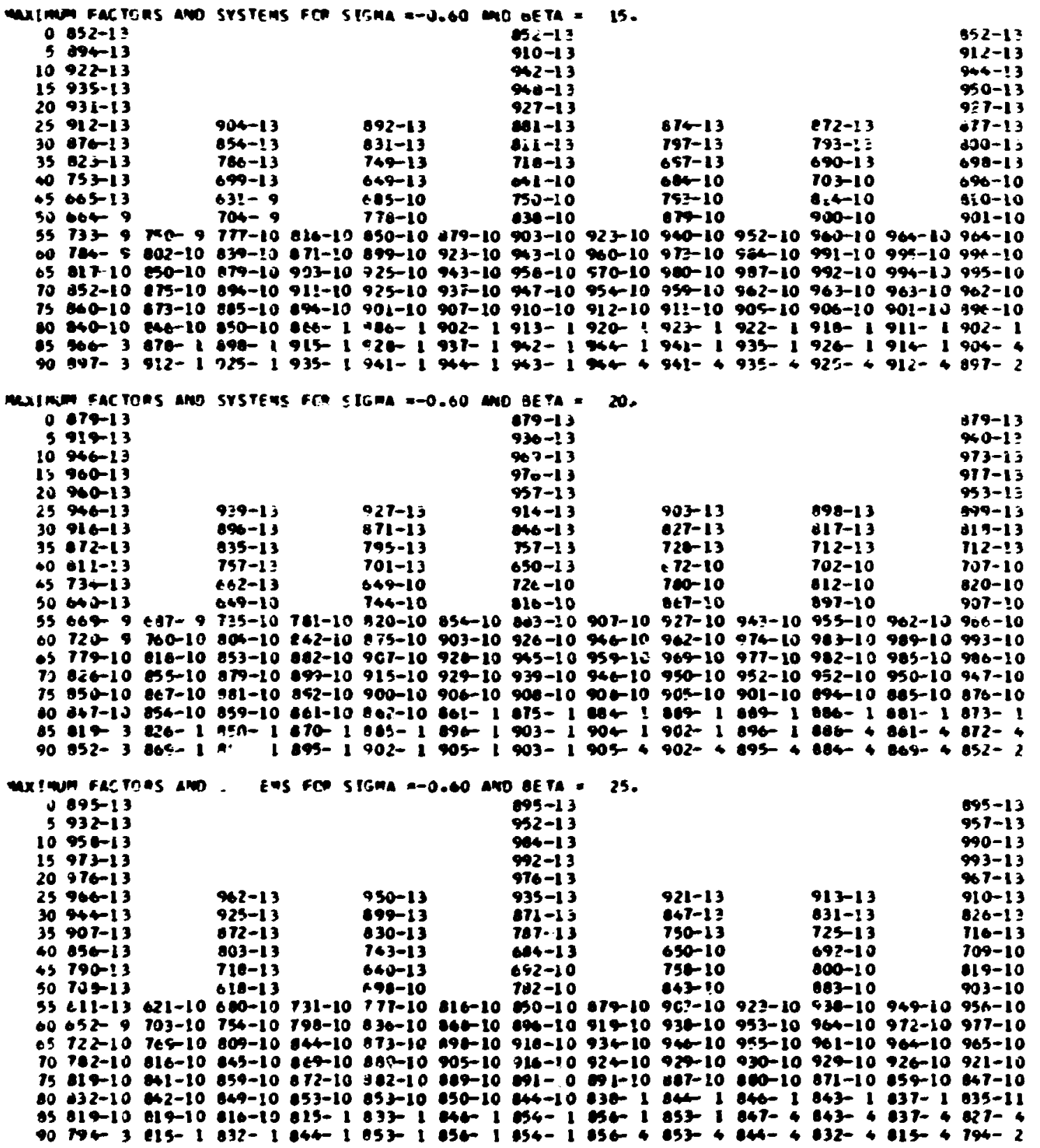




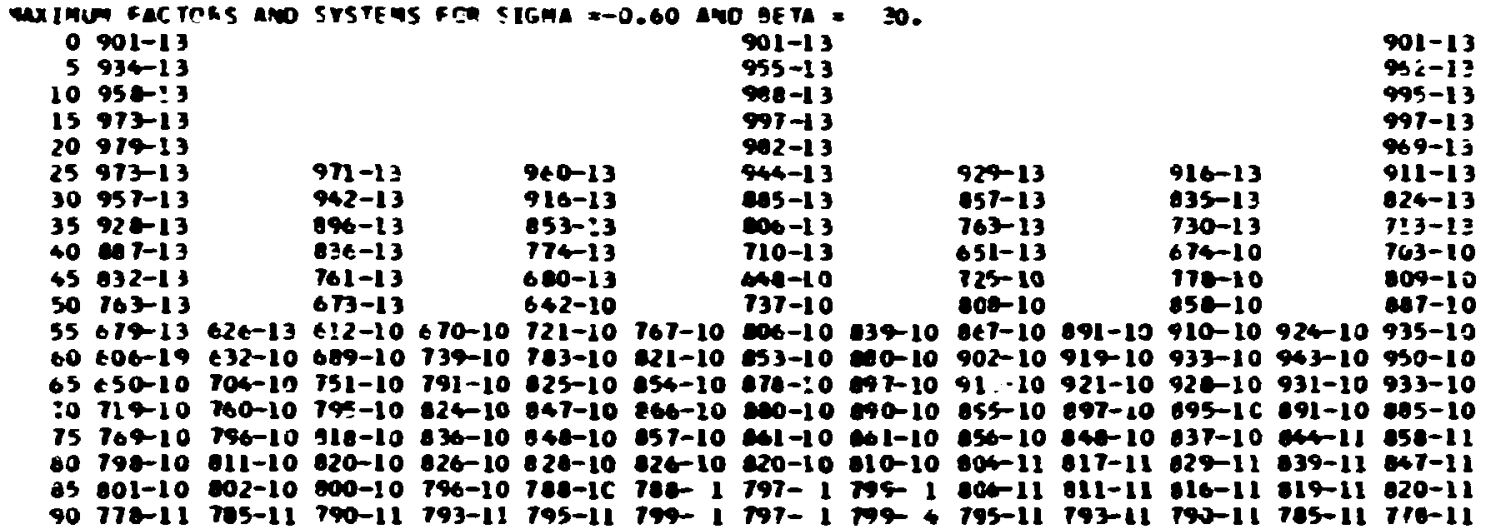

maximu factrins and ststens for sigma =-0.60 Mo ofta = 3.

0 095-13

5 925-13

1094.13

$15961-1$ ?

$20960-13$

25 9.6-1

30 .

$35935-13$

$40903-13$

45 . $059-13$

$50203-13$

53 732-13 091-13 031-19 590-10

$1079-10=29-10$ s5s-10 $97=-10 \quad 091-10903-10913-10$

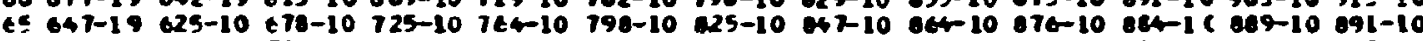
70 441-10 e E0-10 729-10 763-10 791-10 014-10 831-:0 843-10 850-10 053-10 851-15 046-10 E57-1

75 702-10 134-10 76!-15 703-10 799-10 611-10 817-10 e1 -10 815-10 807-10 016-11 830-11 056-11

80 743-10 761-10 776-10 703-10 708-10 733-10 783-10 774-10 705-11 000-11 815-11 820-11 839-11

os 702-10 766-10 767-10 764-10 757-10 750-11 760-11 776-11 704-11 791-11 797-11 002-11 005-11

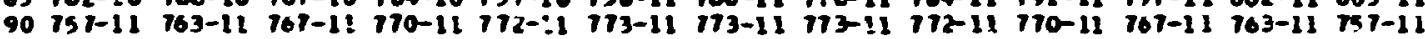

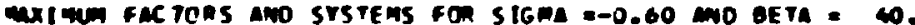

\section{$0.79-13$}

$5904-13$

1092413

$15937-13$

$2096-13$

$25946-13$

$30940-13$

$35926-13$

$40904-13$

$45871-13$

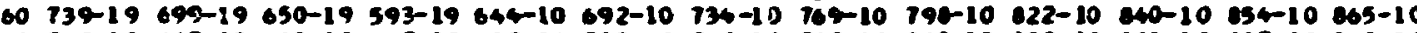
o5 $110-19667-19$ 613-19 6+7-10 692-10 730-10 762-10 760-10 $600-10$ 22-10 $831-10$ 637-10 840-10 70 675-19 e26-19 649-10 669-10 723-10 750-10 771-10 706-10 766-10 799-10 790-10 811-11 841-11 $75633-19656-10699-10$ 716-10 737-10 752-10 761-10 765-10 763-10 761-11 787-11 811-11 634-11 $00671-10$ 69\%-10 T'1-10 725-10 733-10 736-10 734-10 726-10 743-11 761-11 779-11 795-11 310-11 es 704-10 72-10 7.5-10 715-10 711-10 710-11 721-11 730-11 740-11 749-11 757-11 764-11 710-11 $90715-11$ 20-11 122-11 724-11 725-11 726-11 726-11 126-11 125-11 124-11 722-11 120-11 715-11
$019-13$

939-13 $969-13$ $930-13$ 900-13 $890-13$ :78-13 043-13 012-13 791-13 $759-13 \quad 712-13 \quad 680-13$ $050-13$ 014-10 605-10 $636-10 \quad 709-10 \quad 759-10$

$933-13 \quad 943-13 \quad 927-13$

$903-13$

062-13

729-13

$14-13$ 


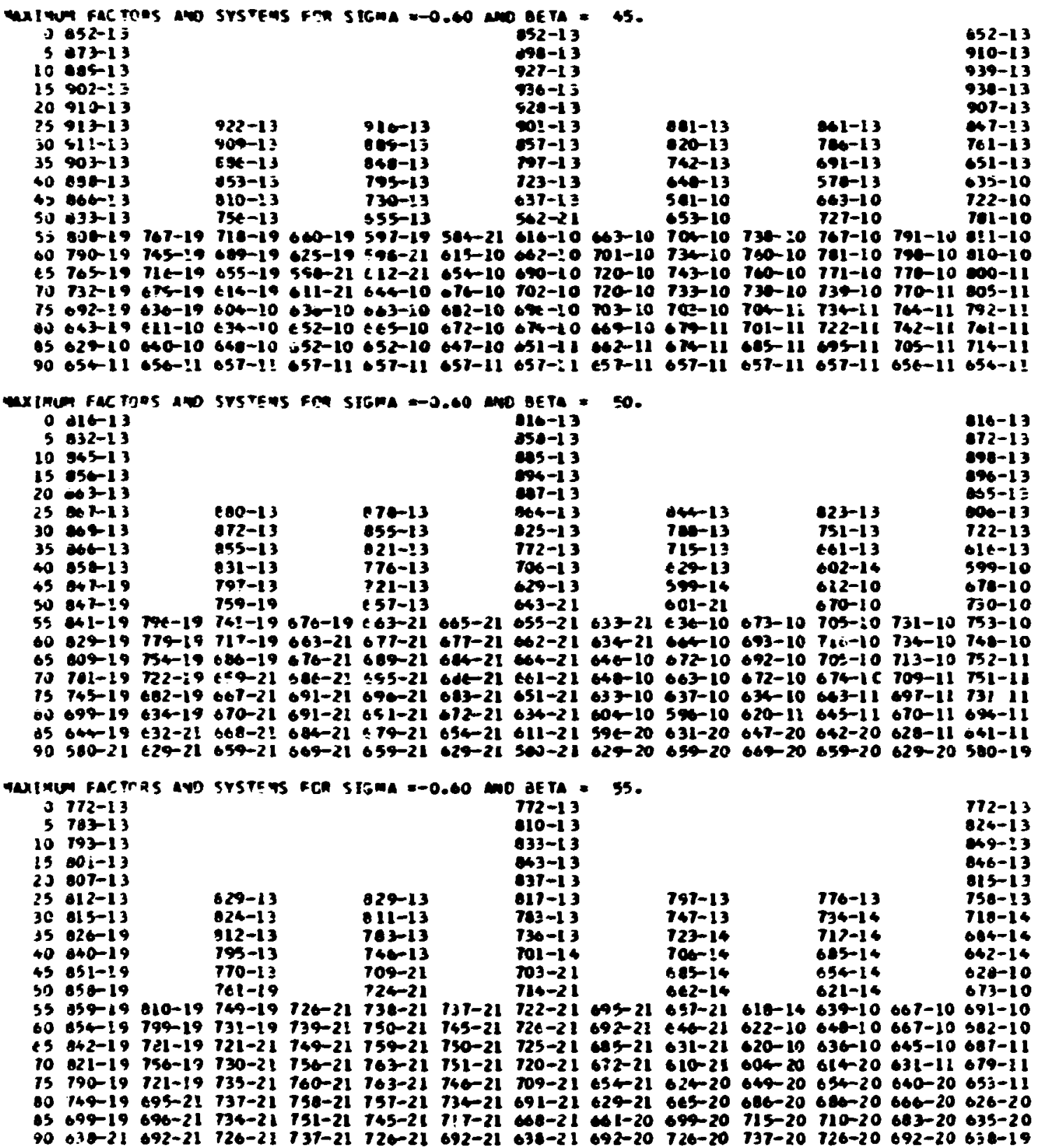




\begin{tabular}{|c|c|c|c|c|c|c|c|c|c|c|c|c|c|}
\hline $\begin{array}{l}0 \\
3 \\
10 \\
15 \\
20 \\
25 \\
30 \\
35 \\
40 \\
45 \\
50 \\
55 \\
60 \\
45 \\
10 \\
15 \\
80 \\
65 \\
90\end{array}$ & 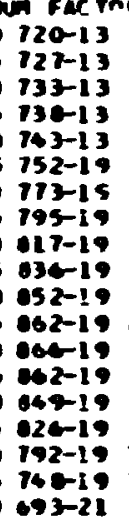 & $\begin{array}{l}0 \\
00-19 \\
60-19 \\
75-19 \\
75-19 \\
752-21 \\
55-21 \\
55-21 \\
752-21\end{array}$ & $\begin{array}{l}7 \in 0-13 \\
765-13 \\
750-13 \\
746-13 \\
750-21 \\
761-21 \\
770-21 \\
760-21 \\
760-21 \\
794-21 \\
797-21 \\
796-21 \\
790-21 \\
700-21\end{array}$ & $\begin{array}{l} \\
194-21 \\
005-21 \\
014-21 \\
020-21 \\
022-21 \\
020-21 \\
013-21 \\
000-21\end{array}$ & $\begin{array}{l}711-13 \\
731-13 \\
750-21 \\
746-21 \\
770-21 \\
791-21 \\
903-21 \\
13-21 \\
130-21 \\
920-21 \\
124-21 \\
923-21 \\
17-21 \\
100-21 \\
100-21\end{array}$ & $\begin{array}{l}0 \\
790-21 \\
904-21 \\
00-21 \\
07-21 \\
002-21 \\
791-21 \\
754-21 \\
752-21\end{array}$ & $\begin{array}{l}m 20-13 \\
754-13 \\
m 5-13 \\
m 3-13 \\
m 1-14 \\
m 3-14 \\
m 1-16 \\
m 5-14 \\
205-14 \\
765-21 \\
m 2-21 \\
7 m-21 \\
m-21 \\
m-21 \\
m 1-21 \\
m 5-21 \\
763-21 \\
m 20-21 \\
623-21\end{array}$ & $\begin{array}{l}0 \\
45-21 \\
40-21 \\
30-21 \\
1 \leq-21 \\
1-21 \\
0-20 \\
23-20 \\
32-20\end{array}$ & $\begin{array}{l}105-14 \\
000-14 \\
790-14 \\
720-14 \\
750-14 \\
730-14 \\
720-16 \\
700-14 \\
60-14 \\
60-14 \\
695-20 \\
732-20 \\
704-20 \\
706-20\end{array}$ & $\begin{array}{l}7 \\
701-16 \\
670-16 \\
657-14 \\
679-20 \\
120-20 \\
756-20 \\
700-20 \\
000-20\end{array}$ & $\begin{array}{l}112-14 \\
601-14 \\
702-14 \\
750-14 \\
731-14 \\
701-14 \\
672-14 \\
64-16 \\
643-20 \\
607-20 \\
726-20 \\
752-20 \\
776-20 \\
700-20\end{array}$ & $\begin{array}{l}0 \\
43-14 \\
600-14 \\
637-20 \\
67-20 \\
704-20 \\
720-20 \\
743-20 \\
75-20\end{array}$ & $\begin{array}{l}-13 \\
-13 \\
-14 \\
-14 \\
-14 \\
-11 \\
-14 \\
-14 \\
-14 \\
-14 \\
-14 \\
-14 \\
-10 \\
-10 \\
-20 \\
-20 \\
-20 \\
-20 \\
-20\end{array}$ \\
\hline $\begin{array}{l}210 \\
0 \\
5 \\
10 \\
15 \\
20 \\
25 \\
30 \\
35 \\
40 \\
45 \\
50 \\
55 \\
40 \\
65 \\
10 \\
75 \\
60 \\
65 \\
90\end{array}$ & 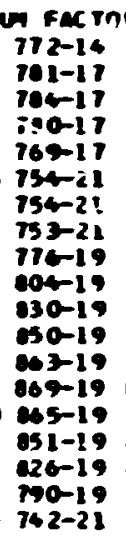 & $\begin{array}{l}21 \\
79-21 \\
79-19 \\
02-21 \\
05-21 \\
00-21 \\
009-21 \\
004-21 \\
005-21\end{array}$ & $\begin{array}{l}714-21 \\
792-21 \\
792-21 \\
001-21 \\
011-21 \\
021-21 \\
030-21 \\
030-21 \\
045-21 \\
051-21 \\
054-21 \\
055-21 \\
052-21 \\
84-21\end{array}$ & 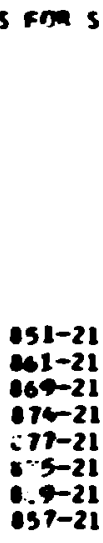 & 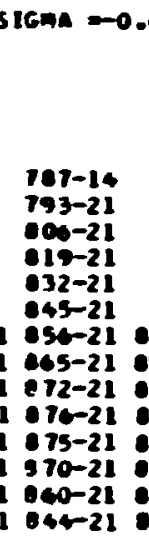 & $\begin{array}{l}.40 \times 0 \\
\\
\\
040-21 \\
052-21 \\
055-21 \\
054-21 \\
050-21 \\
040-21 \\
025-21 \\
005-21\end{array}$ & 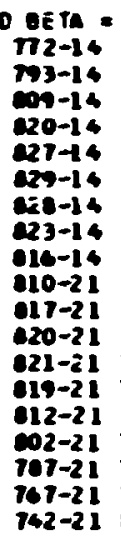 & 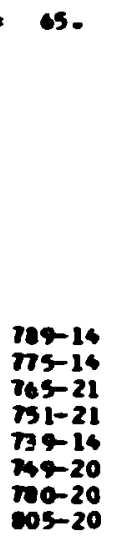 & $\begin{array}{l}14 \\
14 \\
14 \\
14 \\
14 \\
14 \\
14 \\
14 \\
14 \\
14 \\
20 \\
-20 \\
20 \\
-20\end{array}$ & $\begin{array}{l}769-14 \\
769 \\
767-14 \\
727-14 \\
750-20 \\
760-20 \\
016-20 \\
040-20 \\
657-20\end{array}$ & 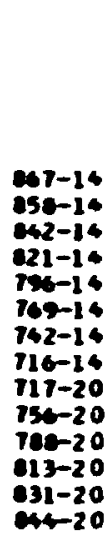 & $\begin{array}{l}105-14 \\
673-12 \\
705-20 \\
741-20 \\
767-20 \\
720-20 \\
79-20 \\
605-20\end{array}$ & $\begin{array}{l}772-14 \\
610-14 \\
650-16 \\
260-14 \\
672-14 \\
64-14 \\
655-14 \\
610-16 \\
761-16 \\
741-14 \\
699-14 \\
650-14 \\
647-20 \\
601-20 \\
707-20 \\
725-20 \\
730-20 \\
741-20 \\
762-19\end{array}$ \\
\hline $\begin{array}{l}1 \\
1 \\
2 \\
2 \\
3 \\
3 \\
5 \\
5 \\
5 \\
6\end{array}$ & $\begin{array}{l}14 \\
17 \\
17 \\
17 \\
17 \\
21 \\
21 \\
21 \\
21 \\
21 \\
19 \\
19 \\
19 \\
19 \\
19 \\
19 \\
19 \\
21\end{array}$ & 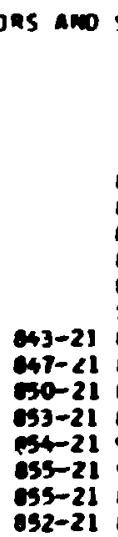 & $\begin{array}{l}914-21 \\
024-21 \\
035-21 \\
045-21 \\
046-21 \\
361-21 \\
976-21 \\
935-21 \\
992-21 \\
997-21 \\
901-21 \\
901-21 \\
99-21 \\
993-21\end{array}$ & $\begin{array}{l} \\
994- \\
904- \\
913- \\
919- \\
921- \\
921- \\
916-\end{array}$ & 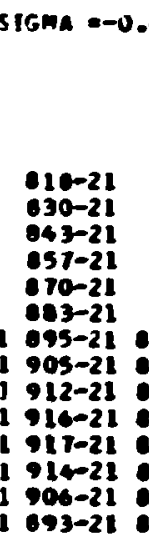 & 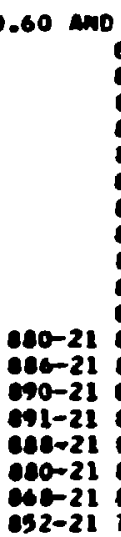 & 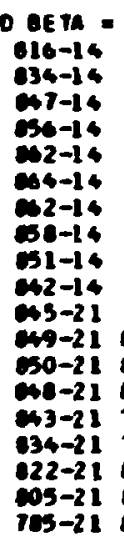 & $\begin{array}{l}033-14 \\
0330-14 \\
200-14 \\
006-14 \\
106-14 \\
004-20 \\
030-20 \\
052-20\end{array}$ & $\begin{array}{l}993-14 \\
693-14 \\
007-14 \\
070-14 \\
063-14 \\
040-14 \\
932-14 \\
014-14 \\
000-14 \\
194-20 \\
622-20 \\
051-20 \\
074-20 \\
093-20\end{array}$ & $\begin{array}{l}0 \\
020-16 \\
790-14 \\
700-16 \\
014-20 \\
040-20 \\
072-20 \\
092-20 \\
90-20\end{array}$ & 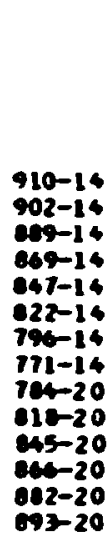 & $\begin{array}{l}761-14 \\
737-20 \\
711-20 \\
794-20 \\
020-20 \\
836-20 \\
846-20 \\
852-20\end{array}$ & $\begin{array}{l}916-14 \\
261-14 \\
693-14 \\
912-14 \\
917-14 \\
910-14 \\
693-14 \\
066-14 \\
033-14 \\
796-14 \\
736-14 \\
716-14 \\
712-20 \\
740-20 \\
760-20 \\
713-20 \\
761-20 \\
704-20 \\
705-19\end{array}$ \\
\hline
\end{tabular}




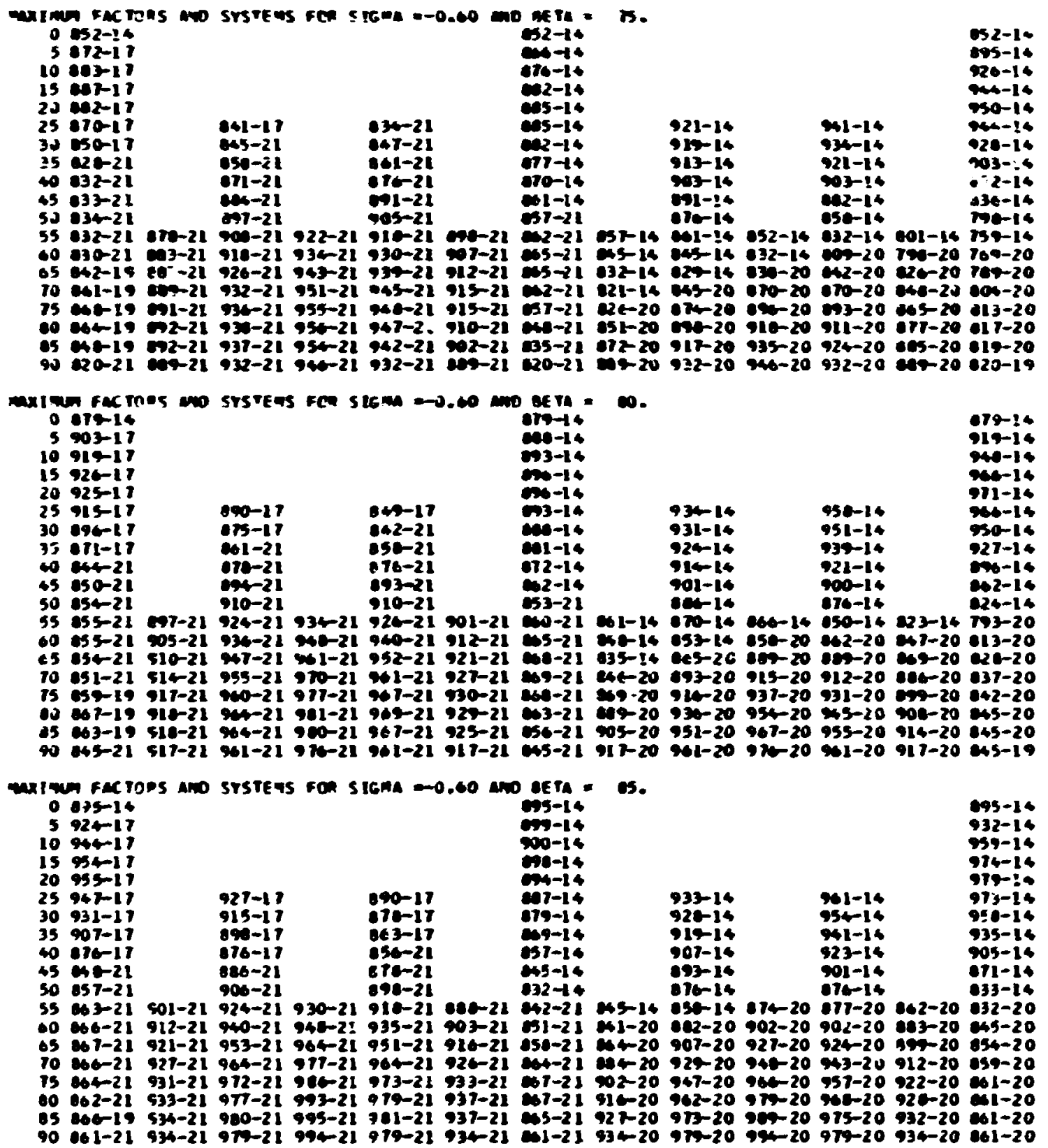




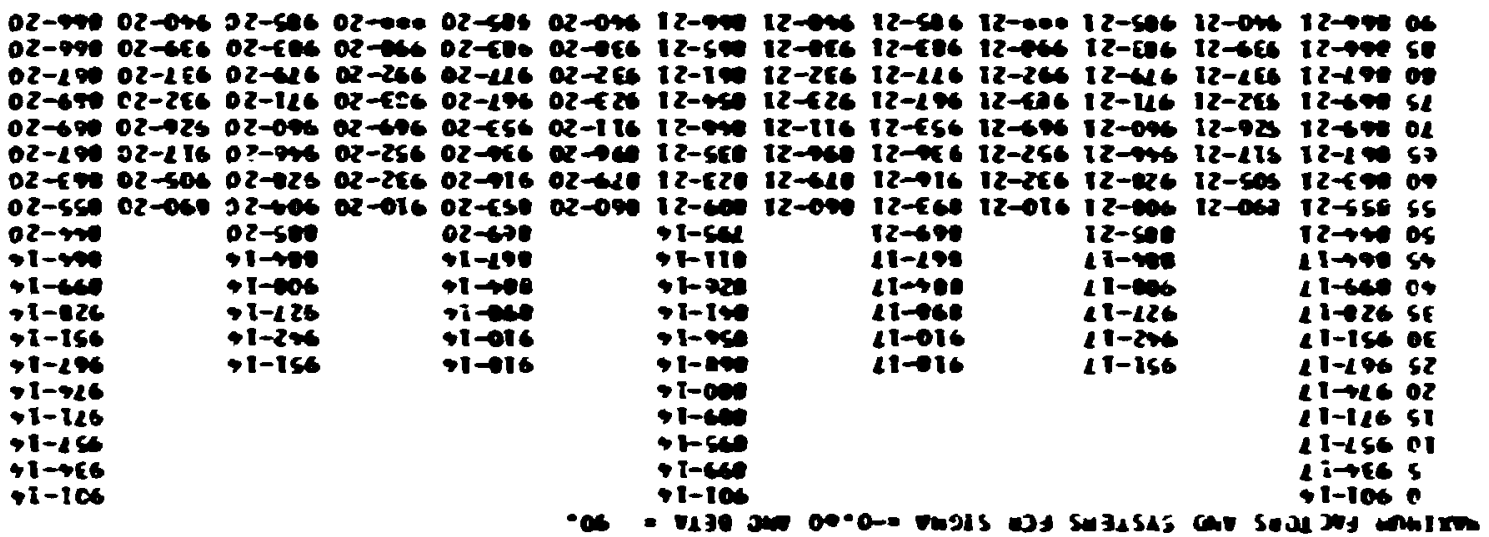




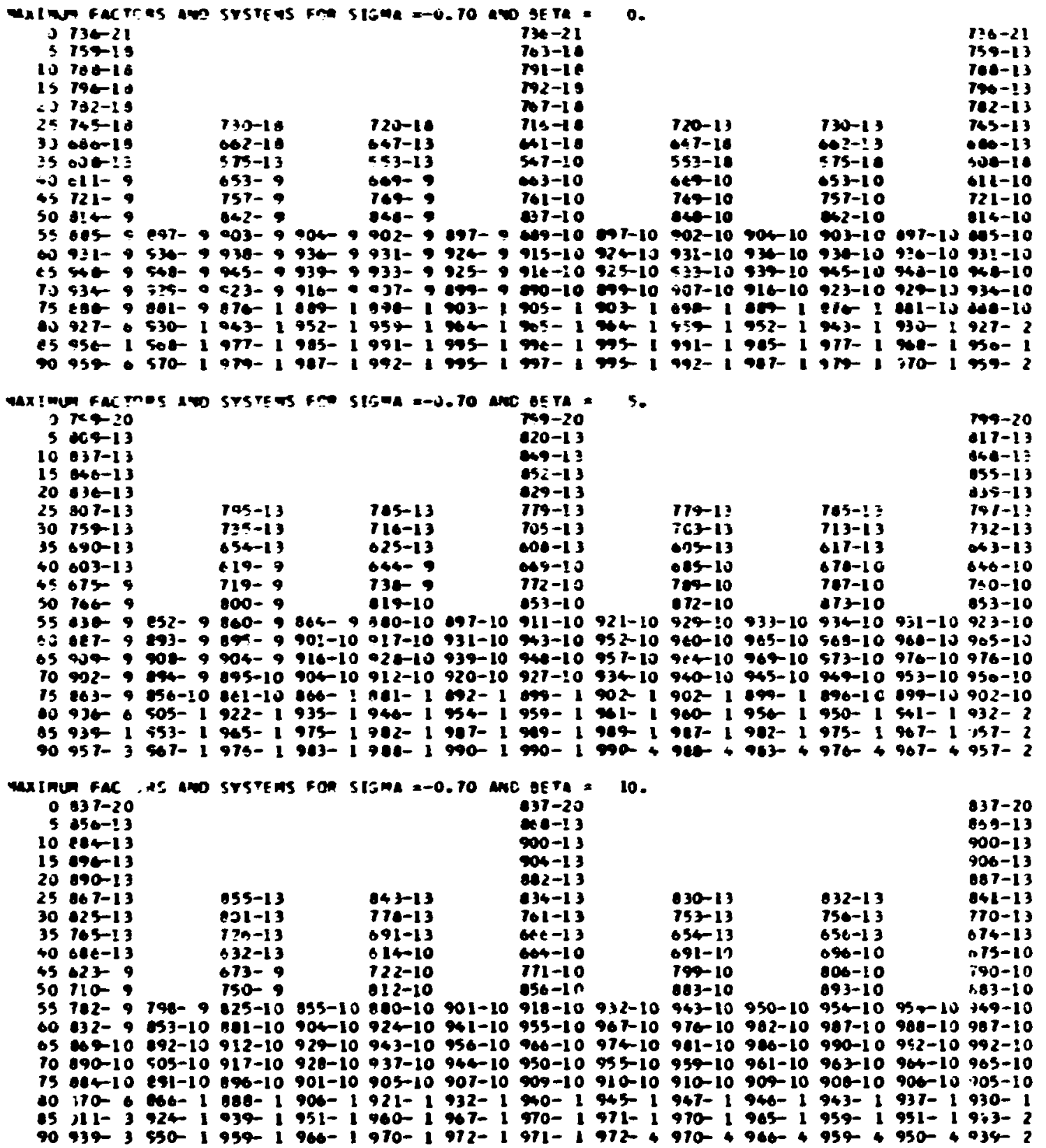




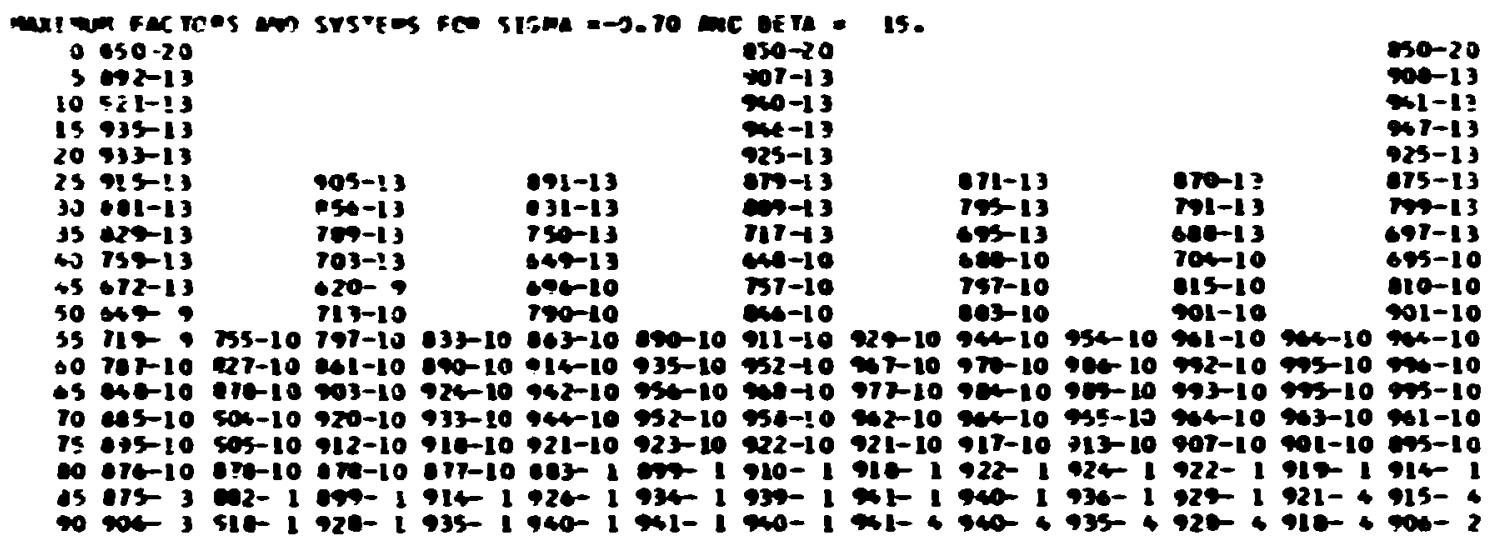

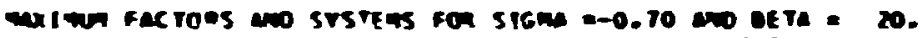

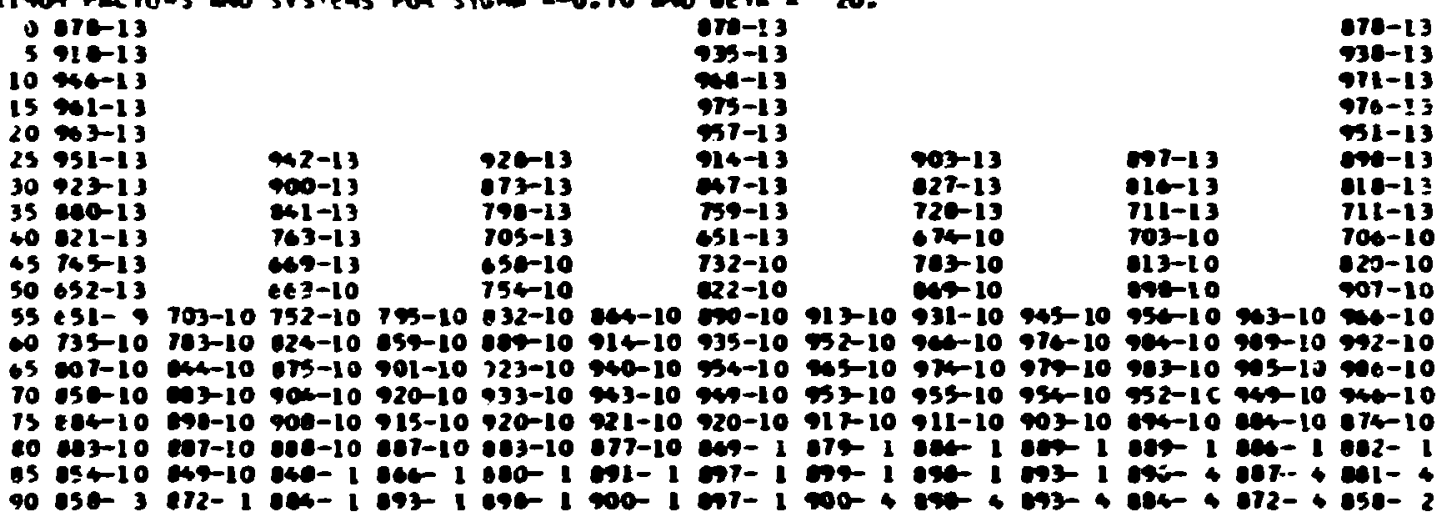

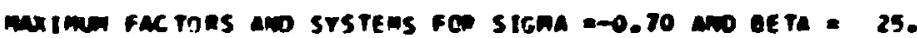

0 os 13

$3932-13$

25

$452-13$

ren -13

$15975-13$

$20900-13$

$2397 \div-13$

30 95 -13

35 9it -13

$40 \mathrm{cos}-13$

45 c05-13

So $724-13$

993-13

206-13 953-13 971-13

$732-13 \quad 72-13$

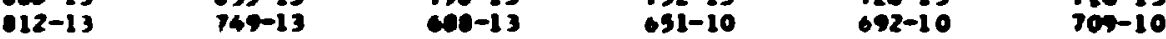

$720-13 \quad 640-13 \quad 65-10 \quad 759-10 \quad 000-10 \quad 019-10$

$630-13 \quad 700-10 \quad 70-10 \quad 003-10 \quad 302-10$

S5 620-13 630-10 693-10 743-10 706-10 e23-10 e55-10 ex2-10 905-10 923-10 936-10 946-10 955-10

40 66-10 72-10 770-10 11-10 967-10 e77-10 902-10 923-10 940-10 953-10 963-10 971-10 976-10

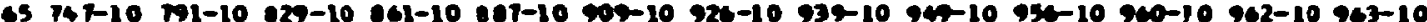

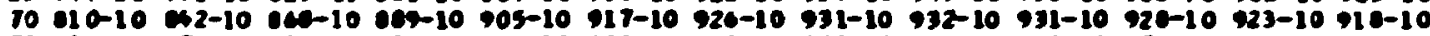

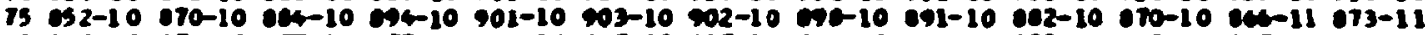

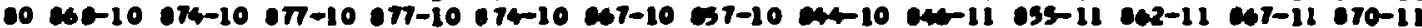

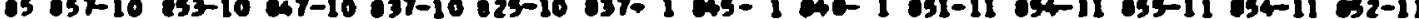

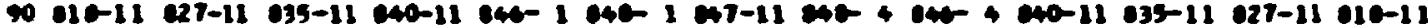




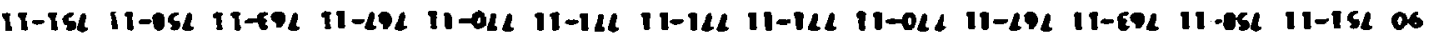

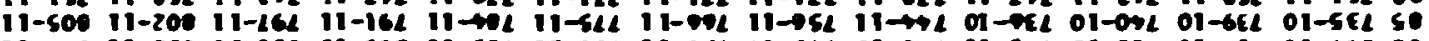

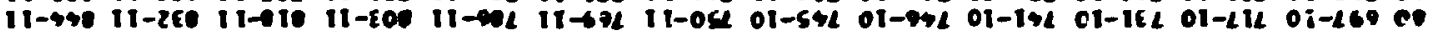

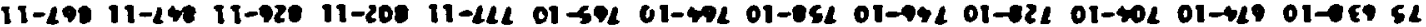

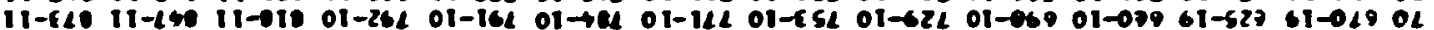

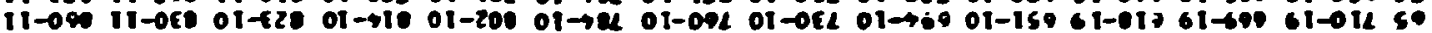

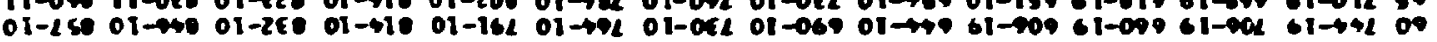

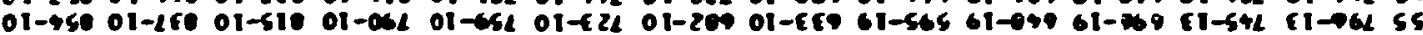
1-070 $01-214$ oitos $01-010$

c1- 249

C1-60 01-01 c: $1-214$ 61-10\%

(1-20 cisee (1-19L 61-192

c1-76 ct $\rightarrow$ os ci-sio c $1-44$

1-106

c1-096

f $1-264$ c I- Ic 1- IN c1-026 c 1-15 CI-OS:

C1-SEL

हा- 110

c1-516 (I-2) 2 E $1-0$ so os C I-OSA 59 E $1-616$ os E I-A66 5E c $1-469$ (I-156 52 EI-2 $w$ o ? c1-2 c $1-25$ $1-25$
$61-226$ CI -1 <6 51 $1-226$
$61-2065$ E $1-210$

c1-020

c $1-100$

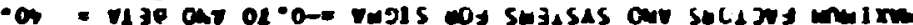

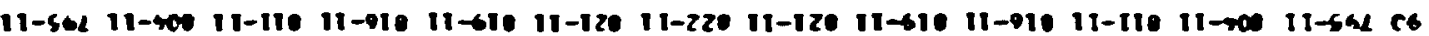

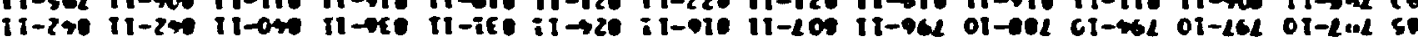

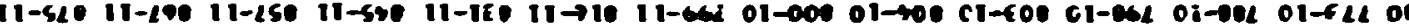

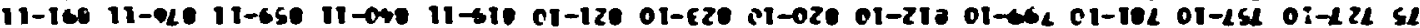

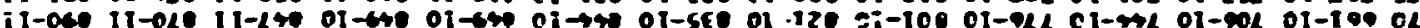

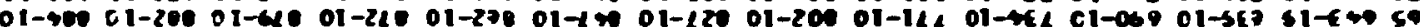

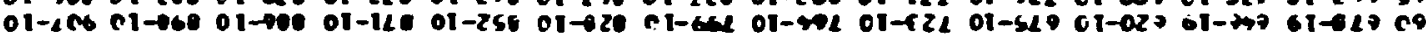

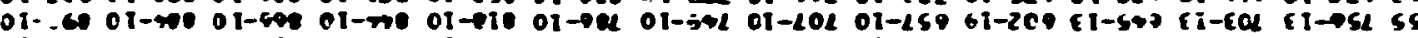
$01-95$

$01-98$ ci-ble ol-19. $01-48$ ol-10

(1-6) C1- CEP ¿I-270 EI-ISL E I-9Z2 $01-290$
$61-02$ t I-620 c1- 150 c $1-152$ c I-16 it-is c 1-126

i i : :-606 $1-6$ E $1-64$ E $!-14$

1-00,

ci-ocs citsen (1-129 EI-12L ET-400 (I)

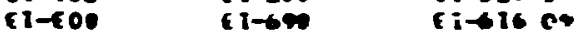

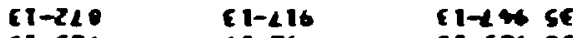
I1-526 (1-250 EI-506 Or

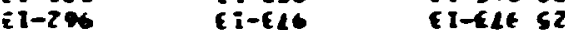
c1-245 02 $c 1-2<6$ o $41-c 965$ cI-14601 $6 \rightarrow 265$

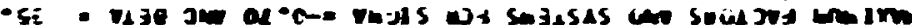

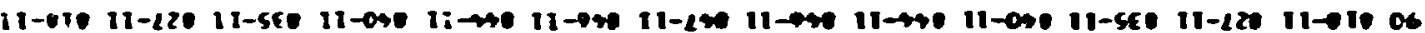

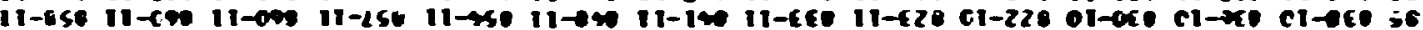

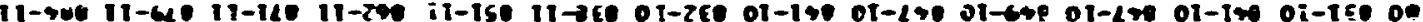

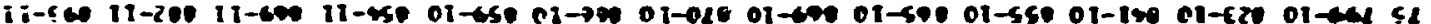

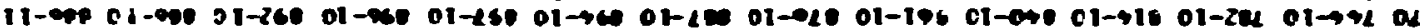

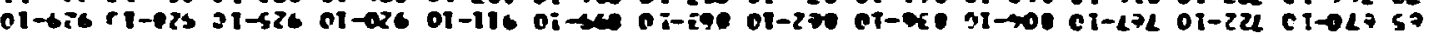

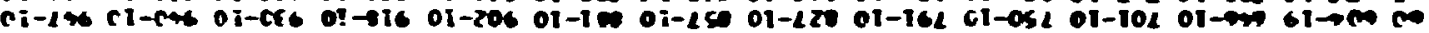

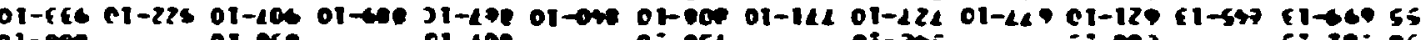
$01-00$ $01-\operatorname{cose}$

$0:-\infty$ $01-2 \mathrm{Cs}$

| $1-212$ $07-114$ $01-210$

i $1-56$

$01-190$ o i-es

oi -421

$61-45$

$61-52$

$=1-214$

ile ci-64 C $1-514$ E $1-906 \quad$ E $1-1465$ E:-6e E1-12s ET-646 $61-146$ (1-4) $1-426$ cI-6es e:$=7-140$ C1-ses c1-ion I- IS4 c I-9es c1-ose

:i-in

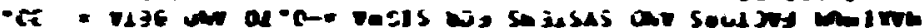




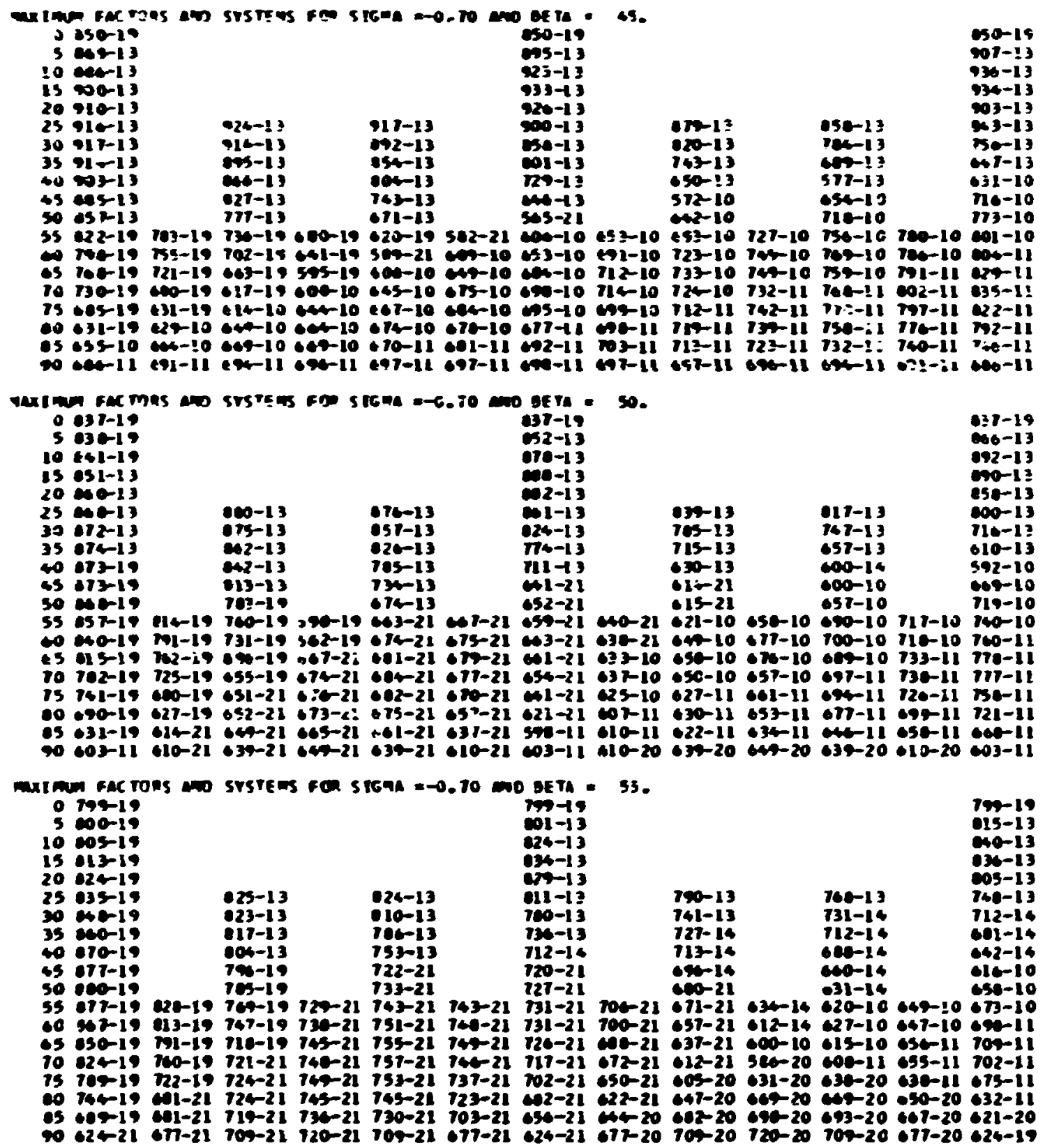




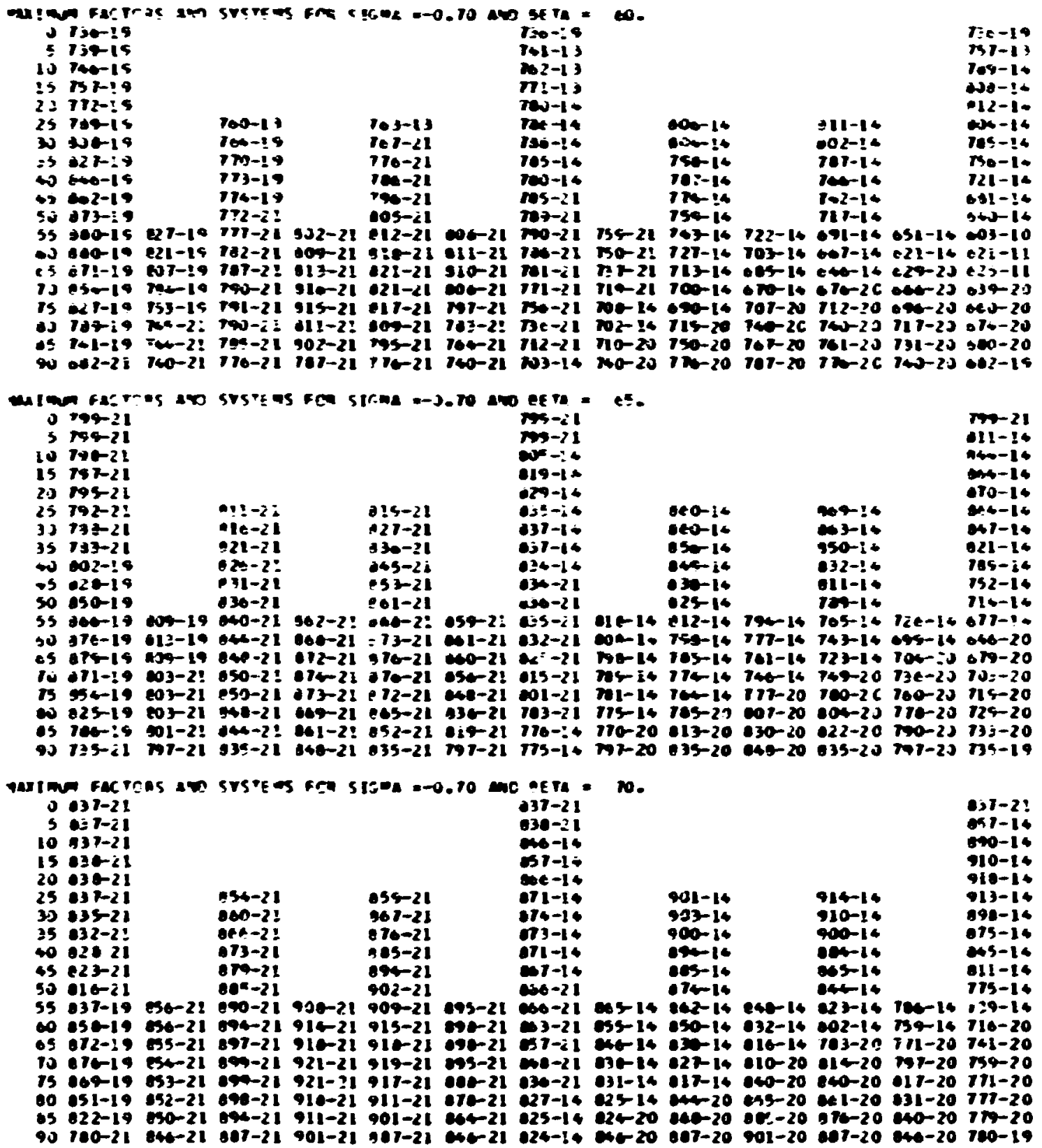




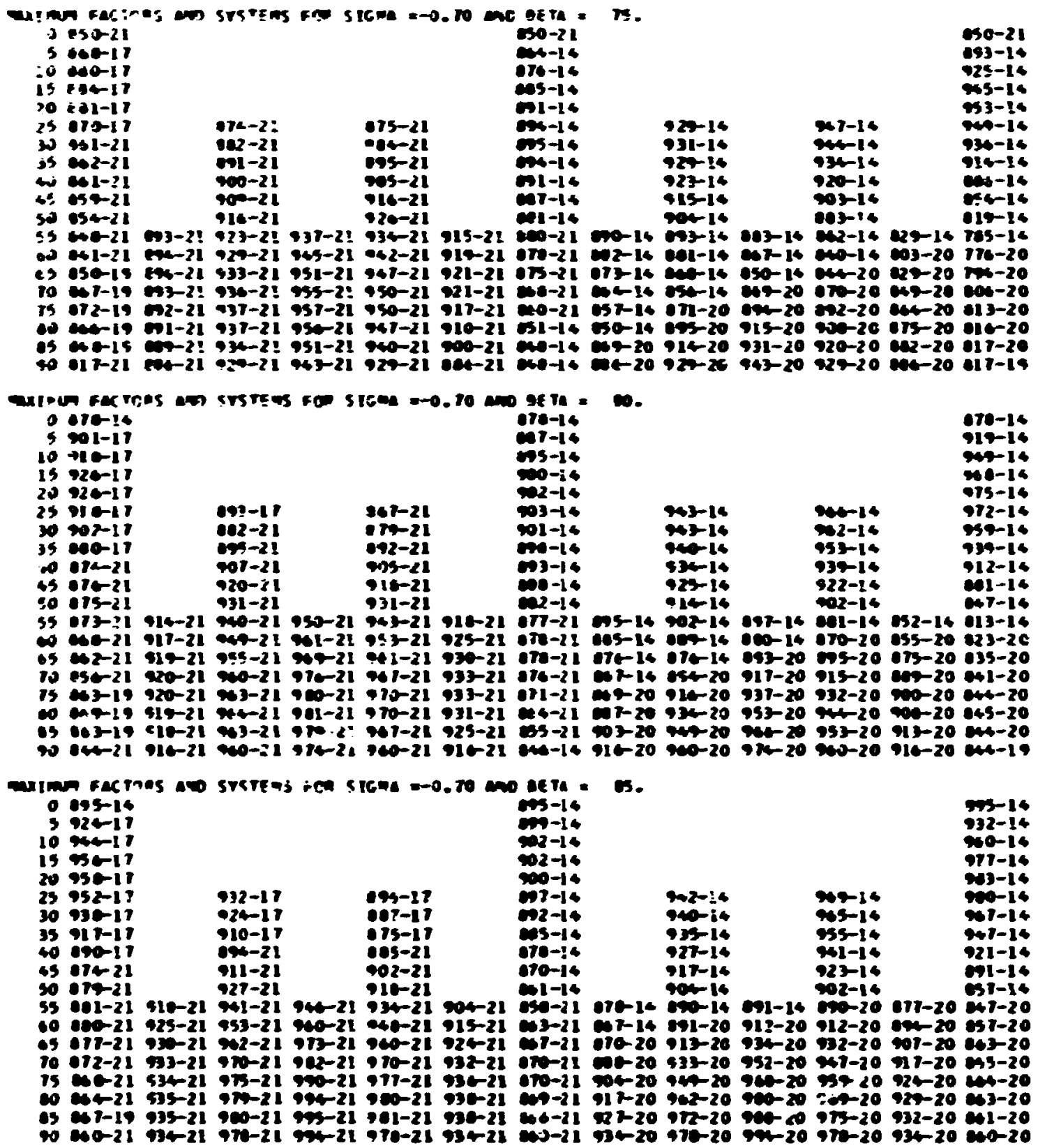




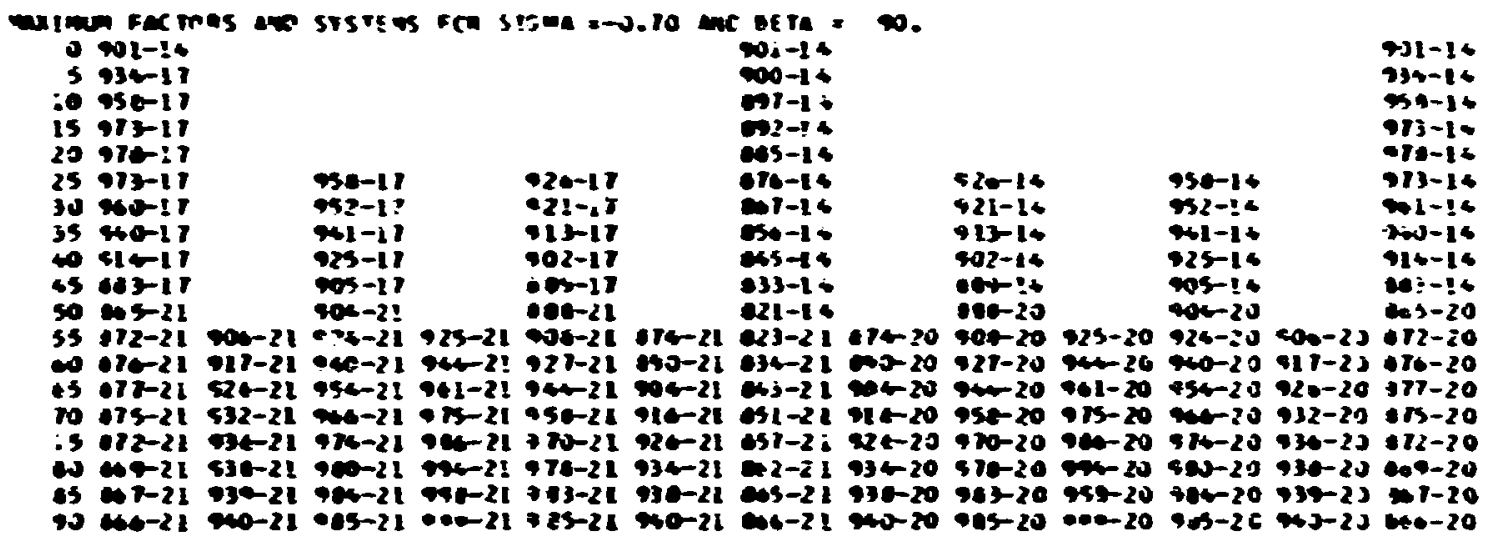




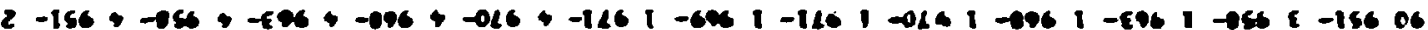

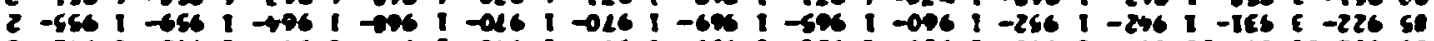

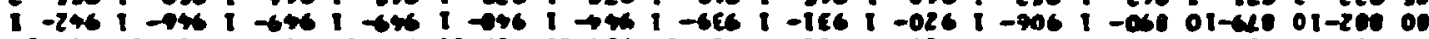

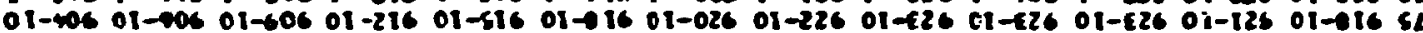

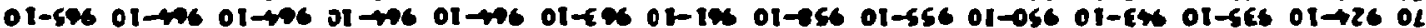

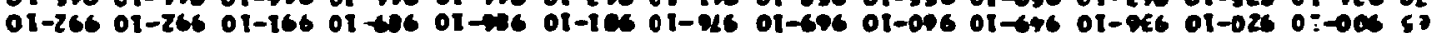

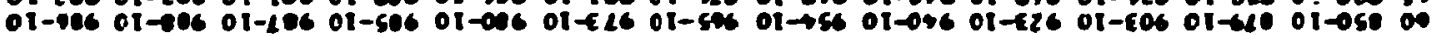

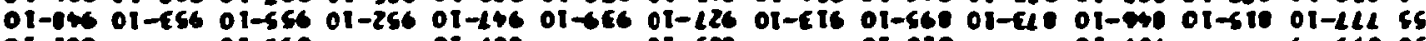

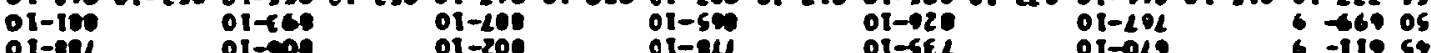

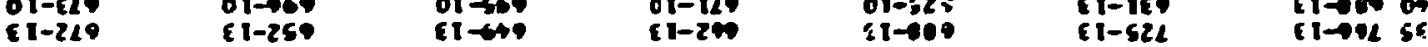

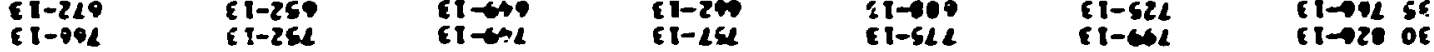

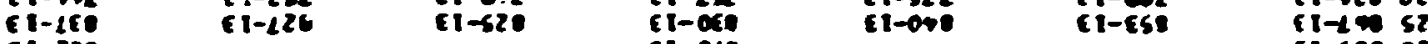
CI- 12e C 1-106 $1=0$ si II$61-605$ SI c 1-su o z-con cI-set $0 z-44$
$0 z-04$ C1-103 01 $0 z-0=0$

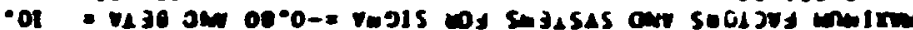

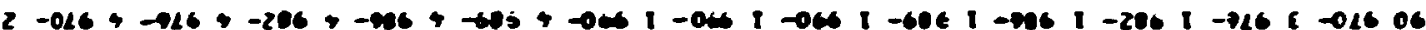

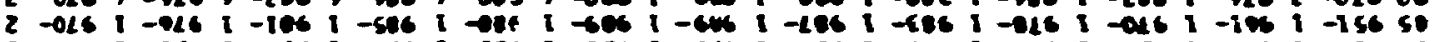

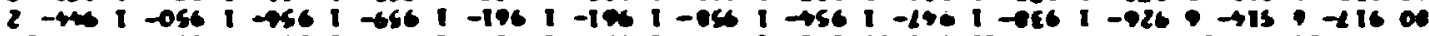

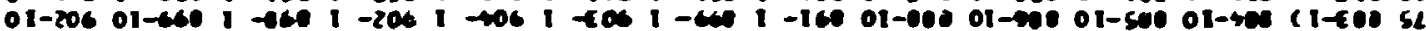

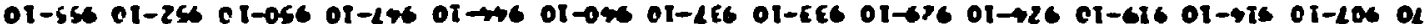

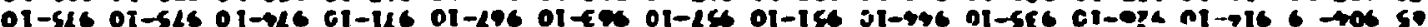

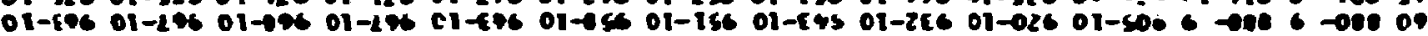

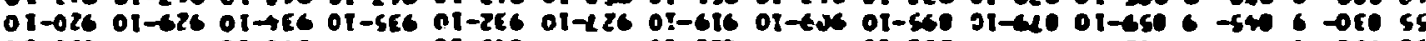

01-2L 01 to

$01-290 \quad 01-019$

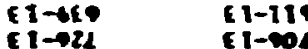

$0-\cos$

$01-692$

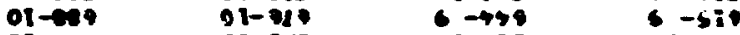

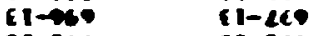

c1-019

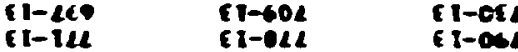

- 0 si os $\rightarrow 99$ E 1-16L EI-QLL CI-TL

E $\mathrm{i}-160$

E1-190

(-120

c1-00 56

ct-ost of $E 1 \rightarrow \infty 52$ ct-2es or $[1-1451$ C1-Oe o : (1- 24

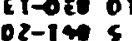
cz-0\% $0<-c 0$ $02 \rightarrow 40$

$0 z-\infty$

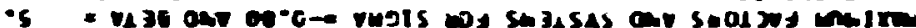

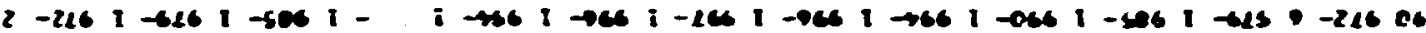

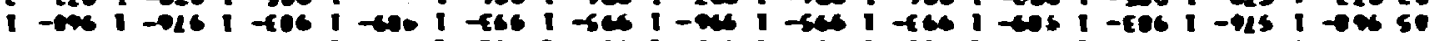

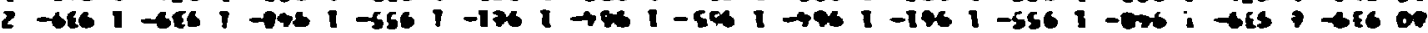

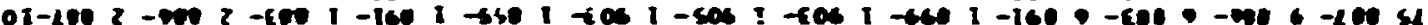

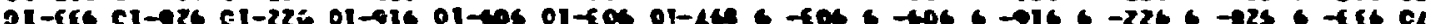

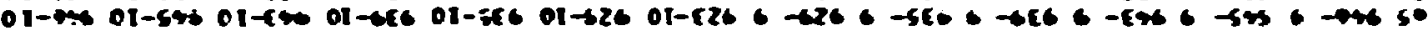

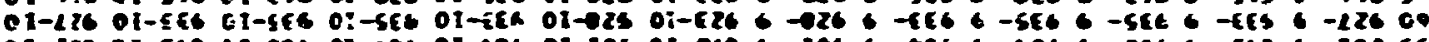

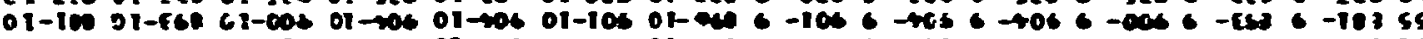

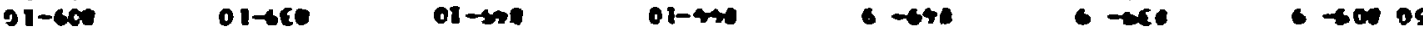

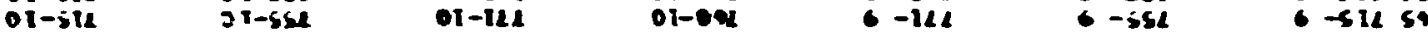

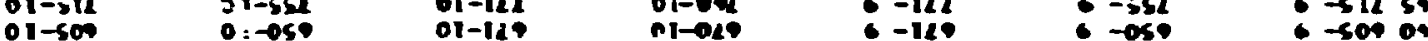

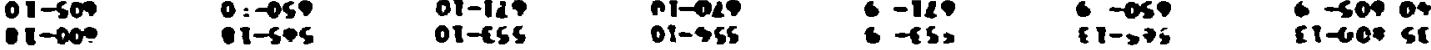

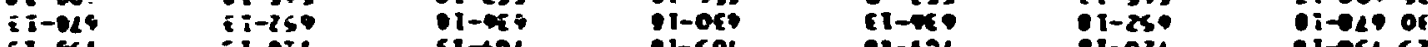

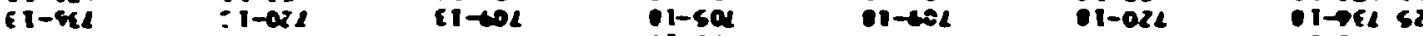
6i-942

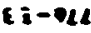

$12-5 i 4$

01-4id

$1-16108$

$1-215$ $1 \rightarrow 24$ C. 17-4u $?-4,18$ 


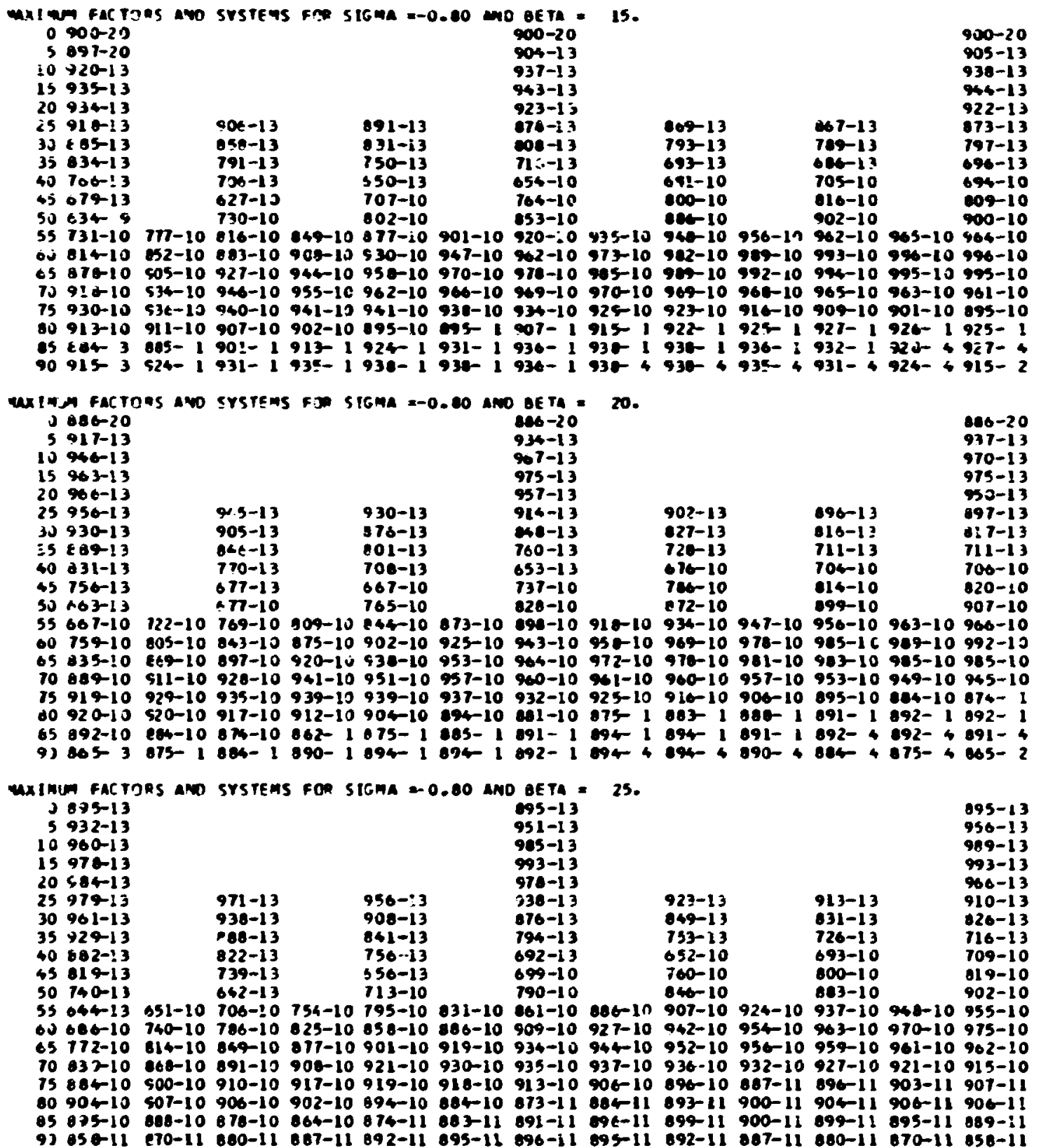




\begin{tabular}{|c|c|c|c|c|c|c|c|c|c|c|c|c|c|}
\hline $\begin{array}{l}140 \\
0 \\
5 \\
10 \\
15 \\
20 \\
25 \\
30 \\
35 \\
40 \\
45 \\
50 \\
55 \\
60 \\
65 \\
10 \\
15 \\
60 \\
45 \\
90\end{array}$ & 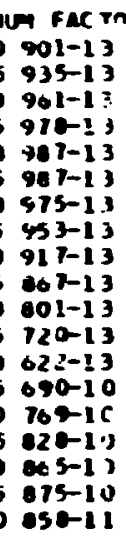 & $\begin{array}{l}0 \\
\\
263-13 \\
660-10 \\
741-10 \\
605-10 \\
669-10 \\
67-10 \\
669-10 \\
670-11\end{array}$ & $\begin{array}{l}902-13 \\
959-13 \\
915-13 \\
659-13 \\
700-13 \\
703-13 \\
630-10 \\
714-10 \\
763-10 \\
936-10 \\
665-10 \\
674-20 \\
660-10 \\
800-11\end{array}$ & $\begin{array}{l} \\
655-10 \\
760-16 \\
910-10 \\
957-23 \\
075-10 \\
872-10 \\
059-11 \\
087-11\end{array}$ & $\begin{array}{l}968-13 \\
926-13 \\
667-13 \\
791-13 \\
701-13 \\
650-10 \\
733-11 \\
799-10 \\
847-10 \\
74-10 \\
891-10 \\
866-10 \\
872-11 \\
095-11\end{array}$ & $\begin{array}{l} \\
\\
77 s-10 \\
732-10 \\
670-10 \\
067-10 \\
962-10 \\
652-11 \\
063-11 \\
096-11\end{array}$ & $\begin{array}{l}901-13 \\
950-13 \\
900-13 \\
996-13 \\
995-13 \\
949-13 \\
992-13 \\
915-13 \\
721-13 \\
650-10 \\
740-10 \\
910-10 \\
960-10 \\
900-10 \\
94-10 \\
979-10 \\
974-11 \\
991-11 \\
997-11\end{array}$ & $\begin{array}{l}0 \\
\\
061-10 \\
603-10 \\
902-10 \\
69-10 \\
672-10 \\
007-11 \\
97-11 \\
09-11\end{array}$ & $\begin{array}{l}931-13 \\
860-13 \\
760-13 \\
657-13 \\
724-10 \\
907-10 \\
96-10 \\
901-10 \\
912-10 \\
990-10 \\
903-11 \\
990-11 \\
902-11 \\
993-11\end{array}$ & $\begin{array}{l} \\
\\
900-10 \\
910-10 \\
910-10 \\
955-10 \\
990-11 \\
907-11 \\
904-11 \\
907-11\end{array}$ & $\begin{array}{l}917-13 \\
636-12 \\
732-13 \\
672-10 \\
770-10 \\
954-10 \\
905-10 \\
920-10 \\
922-10 \\
993-11 \\
911-11 \\
914-11 \\
904-11 \\
900-11\end{array}$ & $\begin{array}{l} \\
920-10 \\
937-10 \\
924-10 \\
907-11 \\
921-11 \\
919-11 \\
91-11 \\
970-11\end{array}$ & $\begin{array}{r}901-13 \\
962-13 \\
995-13 \\
997-13 \\
969-13 \\
911-13 \\
924-13 \\
712-13 \\
792-10 \\
907-10 \\
995-10 \\
932-10 \\
965-10 \\
925-10 \\
919-11 \\
9280-11 \\
920-11 \\
996-11 \\
958-11\end{array}$ \\
\hline $\begin{array}{l}x ! m 0 \\
6 \\
5 \\
10 \\
15 \\
20 \\
25 \\
30 \\
35 \\
40 \\
45 \\
50 \\
55 \\
60 \\
65 \\
10 \\
75 \\
80 \\
15 \\
90\end{array}$ & 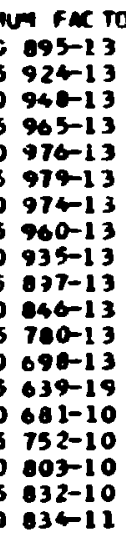 & $\begin{array}{l}724-12 \\
650-19 \\
652-10 \\
72+10 \\
779-10 \\
15-10 \\
6<9-10 \\
245-11\end{array}$ & $\begin{array}{l}570-13 \\
959-13 \\
927-13 \\
602-13 \\
023-13 \\
750-13 \\
605-13 \\
620-10 \\
701-10 \\
760-10 \\
001-10 \\
022-10 \\
822-10 \\
855-11\end{array}$ & $\begin{array}{l} \\
\text { e10-19 } \\
601-10 \\
743-10 \\
769-10 \\
816-10 \\
626-10 \\
830-11 \\
861-11\end{array}$ & $\begin{array}{l}965-13 \\
930-13 \\
879-13 \\
813-13 \\
733-13 \\
641-13 \\
559-10 \\
727-10 \\
770-10 \\
811-10 \\
826-10 \\
821-10 \\
943-11 \\
866-11\end{array}$ & $\begin{array}{l}707-10 \\
760-10 \\
604-10 \\
620-10 \\
630-10 \\
629-11 \\
0295-11 \\
069-11 \\
669-11\end{array}$ & 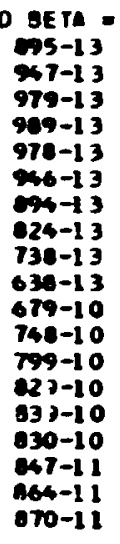 & $\begin{array}{l}35 . \\
\\
704-10 \\
60-10 \\
44-10 \\
46-10 \\
63-11 \\
663-11 \\
072-11 \\
669-11\end{array}$ & $\begin{array}{l}926-13 \\
850-13 \\
771-13 \\
660-13 \\
677-10 \\
757-10 \\
614-10 \\
049-10 \\
659-10 \\
947-10 \\
864-11 \\
677-11 \\
870-11 \\
966-11\end{array}$ & $\begin{array}{l} \\
040-10 \\
067-10 \\
860-10 \\
864-11 \\
003-11 \\
809-11 \\
001-11 \\
661-11\end{array}$ & $\begin{array}{l}900-13 \\
229-13 \\
727-13 \\
643-10 \\
741-10 \\
615-10 \\
862-10 \\
081-10 \\
673-10 \\
206-11 \\
900-11 \\
899-11 \\
903-11 \\
055-11\end{array}$ & $\begin{array}{l} \\
200-10 \\
993-110 \\
802-11 \\
906-11 \\
914-11 \\
906-11 \\
903-11 \\
945-11\end{array}$ & $\begin{array}{l}895-13 \\
950-13 \\
907-13 \\
900-113 \\
958-11 \\
998-13 \\
911-13 \\
690-13 \\
605-10 \\
194-10 \\
955-11 \\
695-10 \\
902-10 \\
903-11 \\
923-11 \\
920-11 \\
911-11 \\
000-11 \\
034-11\end{array}$ \\
\hline $\begin{array}{l}\times 140 \\
0 \\
5 \\
10 \\
15 \\
20 \\
25 \\
30 \\
35 \\
40 \\
45 \\
50 \\
55 \\
80 \\
65 \\
10 \\
75 \\
80 \\
85\end{array}$ & 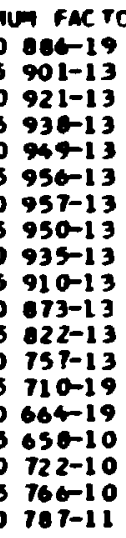 & $\begin{array}{l}769-13 \\
114-19 \\
671-19 \\
628-10 \\
692-10 \\
740-10 \\
767-10 \\
797-11\end{array}$ & $\begin{array}{l}959-13 \\
967-13 \\
923-13 \\
980-13 \\
941-13 \\
782-13 \\
712-19 \\
671-19 \\
624-19 \\
670-10 \\
719-10 \\
751-10 \\
765-10 \\
804-11\end{array}$ & $\begin{array}{l}7 \\
667-19 \\
620-19 \\
655-10 \\
706-10 \\
740-10 \\
750-10 \\
775-11\end{array}$ & $\begin{array}{l}949-13 \\
920-13 \\
677-13 \\
021-13 \\
752-13 \\
671-13 \\
617-19 \\
643-10 \\
696-10 \\
734-10 \\
755-10 \\
759-10 \\
799-11\end{array}$ & $\begin{array}{l}625-10 \\
600-10 \\
731-10 \\
750-10 \\
764-10 \\
775-11 \\
601-11\end{array}$ & $\begin{array}{l}96-19 \\
925-13 \\
955-13 \\
966-13 \\
957-13 \\
930-13 \\
603-13 \\
921-13 \\
763-13 \\
453-13 \\
609-10 \\
677-10 \\
726-10 \\
750-10 \\
772-10 \\
768-11 \\
795-11 \\
111-11 \\
17-11\end{array}$ & $\begin{array}{l} \\
\\
\\
71-10 \\
750-10 \\
70-10 \\
71-10 \\
75-11 \\
013-11 \\
020-11 \\
010-11\end{array}$ & $\begin{array}{l}900-13 \\
865-13 \\
763-13 \\
665-13 \\
623-10 \\
690-10 \\
752-10 \\
705-10 \\
796-10 \\
797-11 \\
620-11 \\
930-11 \\
020-11 \\
814-11\end{array}$ & $\begin{array}{l} \\
\\
702-10 \\
006-10 \\
007-10 \\
028-11 \\
043-11 \\
045-11 \\
034-11 \\
010-11\end{array}$ & $\begin{array}{l}808-13 \\
810-13 \\
712-13 \\
605-10 \\
697-10 \\
766-10 \\
806-11 \\
824-10 \\
833-11 \\
856-11 \\
865-11 \\
858-11 \\
038-11 \\
804-11\end{array}$ & $\begin{array}{l} \\
829-10 \\
837-10 \\
864-11 \\
862-11 \\
204-11 \\
669-11 \\
640-11 \\
797-11\end{array}$ & $\begin{array}{l}886-19 \\
936-13 \\
966-13 \\
906-13 \\
935-113 \\
974-113 \\
707-13 \\
675-13 \\
660-10 \\
752-10 \\
915-10 \\
848-10 \\
859-11 \\
991-11 \\
905-11 \\
901-11 \\
978-11 \\
840-11 \\
707-11\end{array}$ \\
\hline
\end{tabular}




\begin{tabular}{|c|c|c|c|c|c|c|c|c|c|c|c|c|c|}
\hline $\begin{array}{l}0 \\
5 \\
10 \\
15 \\
20 \\
25 \\
30 \\
35 \\
40 \\
45 \\
50 \\
55 \\
60 \\
65 \\
70 \\
75 \\
90 \\
45 \\
90\end{array}$ & 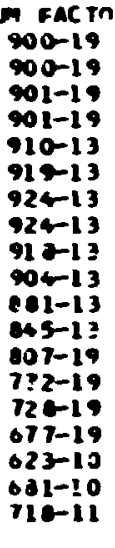 & $\begin{array}{l}7 \\
799-19 \\
760-19 \\
760-19 \\
690-19 \\
626-19 \\
667-10 \\
607-10 \\
726-11\end{array}$ & $\begin{array}{l}925-13 \\
915-13 \\
904-13 \\
979-13 \\
243-13 \\
797-13 \\
753-19 \\
715-19 \\
671-19 \\
620-19 \\
624-10 \\
645-10 \\
699-10 \\
731-11\end{array}$ & $\begin{array}{l} \\
\\
701-19 \\
656-19 \\
606-19 \\
611-10 \\
651-10 \\
677-10 \\
697-11 \\
734-11\end{array}$ & 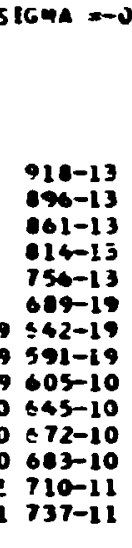 & $\begin{array}{l}000 \\
590-19 \\
602-10 \\
645-10 \\
673-10 \\
696-10 \\
696-11 \\
722-11 \\
738-11\end{array}$ & $\begin{array}{l}900-19 \\
997-19 \\
920-13 \\
930-13 \\
926-13 \\
900-13 \\
60-13 \\
604-13 \\
790-13 \\
455-13 \\
579-19 \\
597-10 \\
465-10 \\
677-10 \\
695-10 \\
693-10 \\
710-11 \\
732-11 \\
739-11\end{array}$ & $\begin{array}{l}0 \\
62-10 \\
62-10 \\
753-10 \\
707-10 \\
722-11 \\
736-11 \\
744-11 \\
738-11\end{array}$ & $\begin{array}{l}777-13 \\
619-13 \\
766-13 \\
652-13 \\
562-10 \\
632-10 \\
602-10 \\
712-10 \\
723-10 \\
733-11 \\
751-11 \\
750-11 \\
753-11 \\
737-11\end{array}$ & $\begin{array}{l}\text { } \\
716-10 \\
737-10 \\
744-11 \\
7 \in 9-11 \\
797-11 \\
777-11 \\
761-11 \\
734-11\end{array}$ & $\begin{array}{l}655-13 \\
781-13 \\
687-13 \\
577-13 \\
646-10 \\
700-10 \\
745-10 \\
757-10 \\
705-11 \\
603-11 \\
606-11 \\
796-11 \\
760-11 \\
731-11\end{array}$ & $\begin{array}{l} \\
\\
770-1 \\
794-11 \\
023-11 \\
035-11 \\
030-11 \\
010-11 \\
774-11 \\
726-11\end{array}$ & $\begin{array}{l}930-15 \\
904-12 \\
932-13 \\
930-13 \\
999-13 \\
639-13 \\
152-13 \\
663-13 \\
025-10 \\
110-10 \\
765-10 \\
7910 \\
633-11 \\
650-11 \\
605-11 \\
253-11 \\
233-11 \\
770-11 \\
710-11\end{array}$ \\
\hline $\begin{array}{l}5 \\
10 \\
15 \\
20 \\
25 \\
30 \\
35 \\
40 \\
45 \\
50 \\
55 \\
80 \\
65 \\
70 \\
75 \\
80 \\
05 \\
90\end{array}$ & $\begin{array}{l}\text { FAC Tn } \\
606-19 \\
907-19 \\
609-19 \\
993-19 \\
997-19 \\
930-19 \\
904-19 \\
905-19 \\
904-19 \\
999-19 \\
909-19 \\
813-19 \\
651-19 \\
621-19 \\
703-19 \\
137-19 \\
681-19 \\
617-19 \\
630-11\end{array}$ & $\begin{array}{l} \\
7 \\
831-19 \\
004-19 \\
770-19 \\
720-19 \\
670-19 \\
621-19 \\
595-21 \\
634-11\end{array}$ & $\begin{array}{l}800-13 \\
678-13 \\
869-13 \\
853-13 \\
629-11 \\
607-19 \\
779-19 \\
746-19 \\
706-19 \\
660-19 \\
635-21 \\
635-21 \\
630-21 \\
636-11\end{array}$ & $\begin{array}{l} \\
719-19 \\
679-19 \\
650-21 \\
661-21 \\
661-21 \\
656-21 \\
645-21 \\
637-11\end{array}$ & $\begin{array}{l}875-13 \\
850-13 \\
831-13 \\
794-13 \\
767-13 \\
696-19 \\
6063-21 \\
9670-21 \\
9673-21 \\
1673-21 \\
1673-21 \\
1669-21 \\
1658-21 \\
1642-21 \\
1638-11\end{array}$ & $\begin{array}{l}0 \\
\\
609-21 \\
673-21 \\
673-21 \\
660-21 \\
650-21 \\
642-21 \\
622-11 \\
638-11\end{array}$ & $\begin{array}{l}D \text { OETA } \\
606-19 \\
603-19 \\
673-19 \\
603-13 \\
670-13 \\
854-13 \\
624-13 \\
776-13 \\
76-13 \\
653-21 \\
660-21 \\
664-21 \\
663-21 \\
658-21 \\
640-21 \\
631-21 \\
619-11 \\
633-11 \\
630-11\end{array}$ & $\begin{array}{l} \\
\\
648-21 \\
642-21 \\
630-21 \\
625-10 \\
628-11 \\
642-11 \\
645-11 \\
630-11\end{array}$ & $\begin{array}{l}835-13 \\
762-13 \\
716-13 \\
632-13 \\
631-21 \\
639-21 \\
622-21 \\
634-10 \\
644-10 \\
647-11 \\
601-11 \\
664-11 \\
056-11 \\
630-11\end{array}$ & $\begin{array}{l} \\
\\
\\
643-10 \\
662-10 \\
669-11 \\
606-11 \\
693-11 \\
686-11 \\
667-11 \\
637-11\end{array}$ & $\begin{array}{l}612-13 \\
762-12 \\
654-13 \\
590-14 \\
560-10 \\
663-10 \\
675-10 \\
680-11 \\
716-11 \\
726-11 \\
725-11 \\
703-11 \\
670-11 \\
636-11\end{array}$ & $\begin{array}{l} \\
\\
\\
705-10 \\
736-11 \\
761-11 \\
767-11 \\
750-11 \\
729-11 \\
667-11 \\
634-11\end{array}$ & $\begin{array}{l}600-19 \\
679-19 \\
900-13 \\
904-13 \\
852-13 \\
103-13 \\
709-13 \\
004-13 \\
505-10 \\
360-10 \\
700-10 \\
768-11 \\
706-11 \\
805-11 \\
206-11 \\
765-11 \\
760-11 \\
695-11 \\
630-11\end{array}$ \\
\hline $\begin{array}{l}5 \\
10 \\
15 \\
20 \\
25 \\
30 \\
35 \\
40 \\
45 \\
50 \\
55 \\
60 \\
65 \\
70 \\
75 \\
80 \\
85\end{array}$ & $\begin{array}{l}\text { m FAC TD } \\
846-19 \\
047-19 \\
051-19 \\
858-19 \\
866-19 \\
075-19 \\
635-19 \\
893-19 \\
930-19 \\
903-19 \\
901-19 \\
094-19 \\
880-19 \\
850-19 \\
028-19 \\
788-19 \\
738-19 \\
679-19 \\
610-21\end{array}$ & $\begin{array}{l}0 \\
\\
\\
647-19 \\
627-19 \\
600-19 \\
765-19 \\
222-19 \\
670-19 \\
660-21 \\
661-21\end{array}$ & $\begin{array}{l}847-19 \\
846-19 \\
842-19 \\
835-19 \\
824-19 \\
809-19 \\
769-19 \\
762-19 \\
729-19 \\
712-21 \\
712-21 \\
710-21 \\
704-21 \\
693-21\end{array}$ & $\begin{array}{l} \\
7 \\
723-21 \\
737-21 \\
740-21 \\
741-21 \\
739-21 \\
732-21 \\
721-21 \\
704-21\end{array}$ & $\begin{array}{l}820-13 \\
808-13 \\
780-13 \\
760-13 \\
736-21 \\
743-21 \\
1748-21 \\
1751-21 \\
1752-21 \\
1750-21 \\
1743-21 \\
1732-21 \\
17321 \\
1716-21 \\
1093-21\end{array}$ & $\begin{array}{l} \\
750-21 \\
751-21 \\
740-21 \\
741-21 \\
729-21 \\
112-21 \\
690-21 \\
661-21\end{array}$ & $\begin{array}{l}046-19 \\
043-19 \\
033-19 \\
625-13 \\
422-13 \\
605-13 \\
777-13 \\
736-13 \\
7=2-21 \\
737-21 \\
740-21 \\
739-21 \\
735-21 \\
727-21 \\
714-21 \\
696-21 \\
673-21 \\
640-14 \\
645-14\end{array}$ & $\begin{array}{l}0 \\
717-21 \\
707-21 \\
692-21 \\
612-21 \\
640-14 \\
642-14 \\
639-14 \\
661-20\end{array}$ & $\begin{array}{l}763-13 \\
737-14 \\
731-14 \\
720-14 \\
700-14 \\
698-21 \\
605-21 \\
667-21 \\
646-14 \\
635-14 \\
627-14 \\
629-20 \\
664-20 \\
693-20\end{array}$ & $\begin{array}{l} \\
650-14 \\
632-14 \\
616-16 \\
603-14 \\
614-20 \\
651-20 \\
661-20 \\
704-20\end{array}$ & $\begin{array}{l}759-13 \\
729-14 \\
713-14 \\
691-14 \\
667-14 \\
641-14 \\
616-14 \\
607-10 \\
628-11 \\
634-11 \\
625-11 \\
653-20 \\
676-20 \\
693-20\end{array}$ & $\begin{array}{l} \\
\\
631-11 \\
665-11 \\
601-11 \\
679-11 \\
662-11 \\
635-20 \\
652-20 \\
601-20\end{array}$ & $\begin{array}{l}246-19 \\
830-19 \\
630-13 \\
627-13 \\
795-13 \\
736-13 \\
707-14 \\
677-14 \\
641-14 \\
604-10 \\
643-10 \\
609-11 \\
720-11 \\
732-11 \\
724-11 \\
697-11 \\
654-11 \\
607-20 \\
610-19\end{array}$ \\
\hline
\end{tabular}




\begin{tabular}{|c|c|c|c|c|c|c|c|c|c|c|c|c|c|}
\hline $\begin{array}{l}0 \\
5 \\
10 \\
15 \\
20 \\
25 \\
30 \\
35 \\
40 \\
45 \\
50 \\
55 \\
50 \\
45 \\
10 \\
75 \\
10 \\
15 \\
90\end{array}$ & 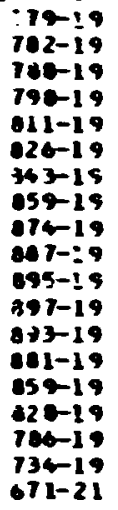 & $\begin{array}{l}75-19 \\
035-19 \\
617-19 \\
791-19 \\
750-19 \\
730-21 \\
733-21 \\
720-21\end{array}$ & $\begin{array}{l}795-19 \\
600-19 \\
002-19 \\
003-19 \\
600-19 \\
793-19 \\
764-21 \\
705-21 \\
705-21 \\
785-21 \\
783-21 \\
700-21 \\
774-21 \\
763-21\end{array}$ & $\begin{array}{l}010-21 \\
012-21 \\
113-21 \\
012-21 \\
908-21 \\
001-21 \\
791-21 \\
775-21\end{array}$ & $\begin{array}{l}793-21 \\
790-21 \\
003-21 \\
000-21 \\
14-21 \\
118-21 \\
1121-21 \\
1023-21 \\
1922-21 \\
1010-21 \\
1011-21 \\
1000-21 \\
1704-21 \\
1703-21\end{array}$ & $\begin{array}{l}110-21 \\
917-21 \\
012-21 \\
004-21 \\
792-21 \\
776-21 \\
755-21 \\
730-14\end{array}$ & $\begin{array}{ll}-1 & 9 \\
-2 & 1 \\
-2 & 1 \\
-2 & 1 \\
-2 & 1 \\
-2 & 1 \\
-2 & 1 \\
-2 & 1 \\
-2 & 1 \\
-2 & 1 \\
-2 & 1 \\
-2 & 1 \\
-2 & 1 \\
-2 & 1 \\
-2 & 1 \\
-2 & 1 \\
-1 & 1 \\
-1 & 4 \\
-1 & 4\end{array}$ & $\begin{array}{l}770-16 \\
759-16 \\
760-16 \\
752-16 \\
76=-16 \\
761-16 \\
730-16 \\
730-16\end{array}$ & $\begin{array}{l}964-14 \\
809-14 \\
964-14 \\
799-14 \\
796-14 \\
776-16 \\
760-14 \\
754-14 \\
763-14 \\
734-16 \\
726-16 \\
721-16 \\
737-20 \\
763-20\end{array}$ & $\begin{array}{l}743-16 \\
720-16 \\
714-16 \\
703-16 \\
684-16 \\
727-20 \\
754-20 \\
175-20\end{array}$ & $\begin{array}{l}810-14 \\
604-14 \\
792-14 \\
774-14 \\
754-14 \\
732-14 \\
710-14 \\
690-16 \\
673-14 \\
665-20 \\
699-20 \\
727-20 \\
746-20 \\
763-20\end{array}$ & $\begin{array}{l}606-14 \\
642-14 \\
621-14 \\
657-20 \\
605-20 \\
806-20 \\
720-20 \\
720-20\end{array}$ & $\begin{array}{l}779-19 \\
770-21 \\
700-14 \\
000-14 \\
607-14 \\
601-14 \\
703-14 \\
757-14 \\
725-14 \\
669-14 \\
551-14 \\
616-14 \\
630-11 \\
642-11 \\
631-20 \\
651-20 \\
604-20 \\
670-20 \\
671-19\end{array}$ \\
\hline
\end{tabular}




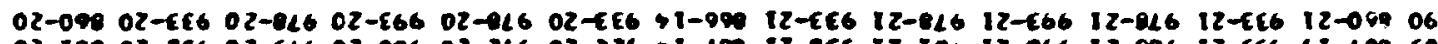

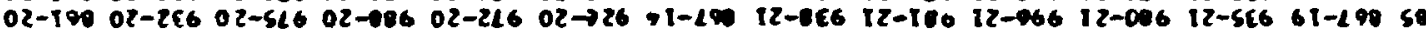

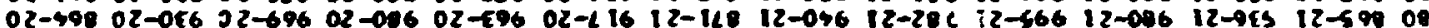

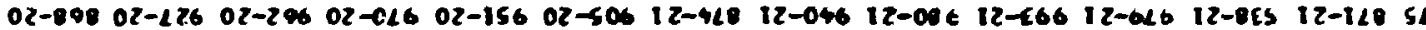

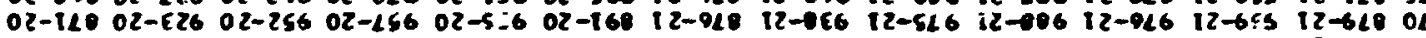

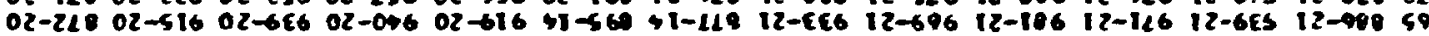

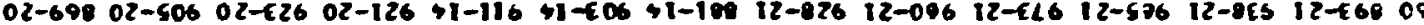

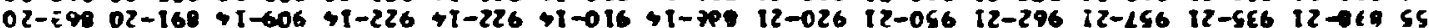
$\$ 1-266 \quad 12-066 \quad 12-069 \quad 12-10605$ $\begin{array}{lllllll}4-016 & \$ 1-546 & +1-046 & 11-469 & 12-926 & 12-966 & 12-00654\end{array}$

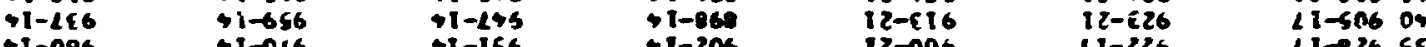
$\begin{array}{lllllll}9-096 & 91-046 & 91-156 & 91-206 & 12-006 & 11-226 & 21-2658\end{array}$

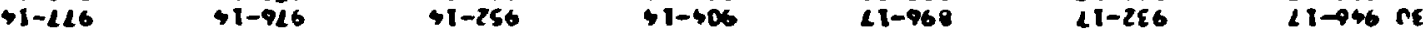

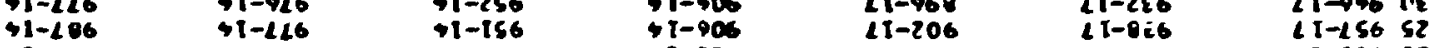
$11-19602$ $41-25 s 51$

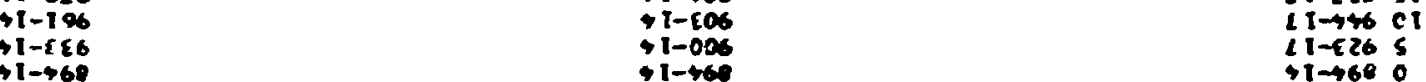

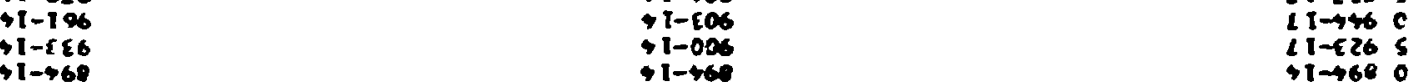

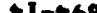

$1-460$

$1-4600$

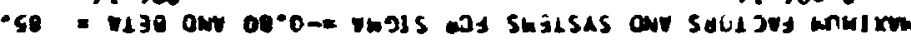

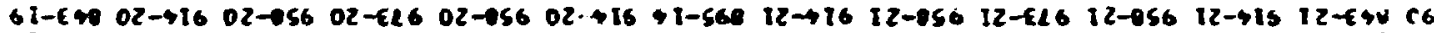

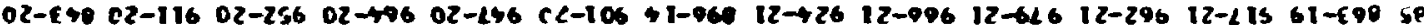

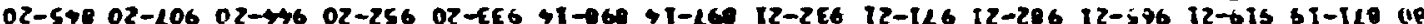

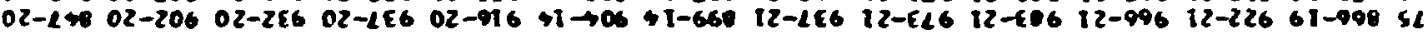
$02-94902-76902-016 \quad 02-616+1-906+1-016+1-106 \quad 12-6 E 6 \quad 12-7<6 \quad 12-196 \quad 12-596 \quad 12-52512-290 \quad 02$

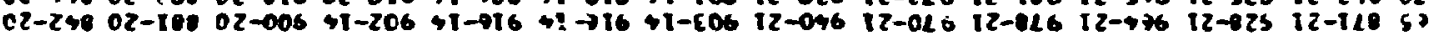

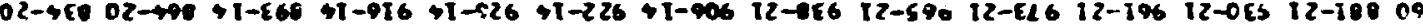

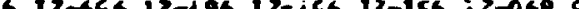

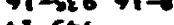

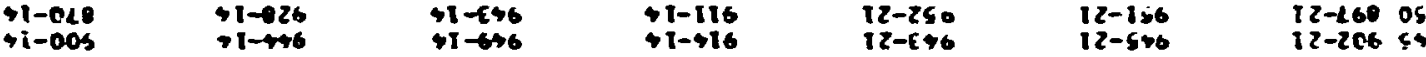
$\begin{array}{lllllll}9-126 & \$ 1-256 & 1-456 & 1-516 & 12-466 & 12-2166 & 12-51604\end{array}$

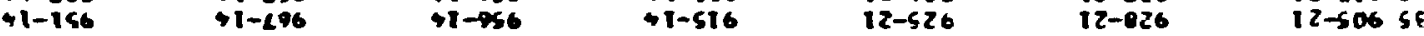
$12-026$

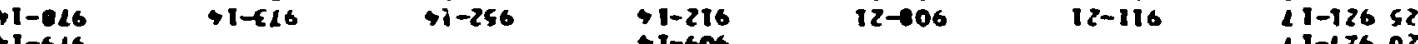
$41-606$ $11-016$ $11-646+1-96$ $21-52651$ $41-016$ c1 210065 11-201 $21-0609$

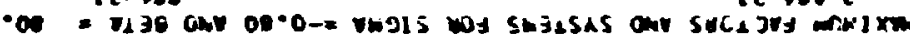

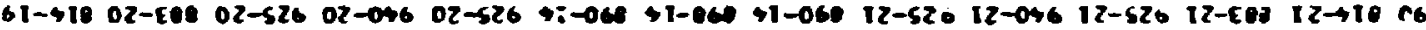

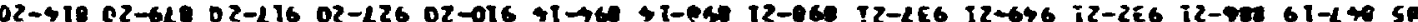

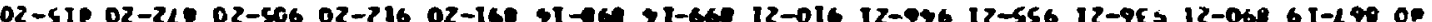

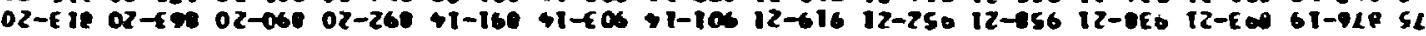

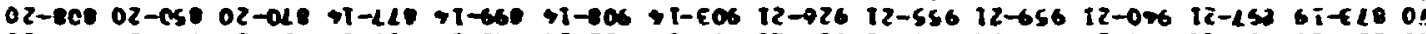

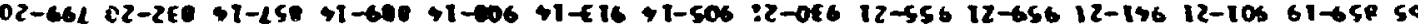

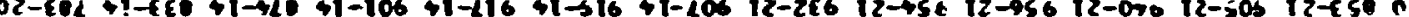

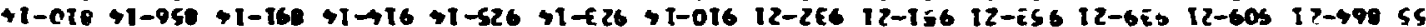

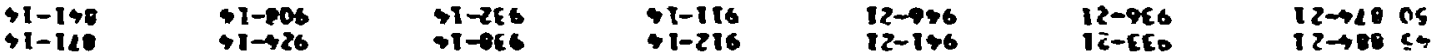
$\$ 1-006+1-466$

$5 i-\$ 26 \quad 4 T-494$

$+1-696 \quad+1-696 \quad 41-296$

$\rightarrow 1-456 \quad+1-450 \quad+1-2 E S$

$4-59$

$41-946$

$+1-926$

$12-160$ $+1-216 \quad 12-466 \quad 12-520$ $-1-116 \quad 12-026 \quad 12-520$ $12-025 \quad 12-669+8$ $\begin{array}{llll}12-406 & 12-916 & 12-51\rangle & 12-1065 ?\end{array}$ $12-\cos$ $12-12051$ $12-105$ 12-1C6 01

$12-006 \quad 12-0065$

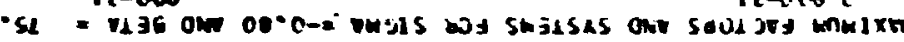




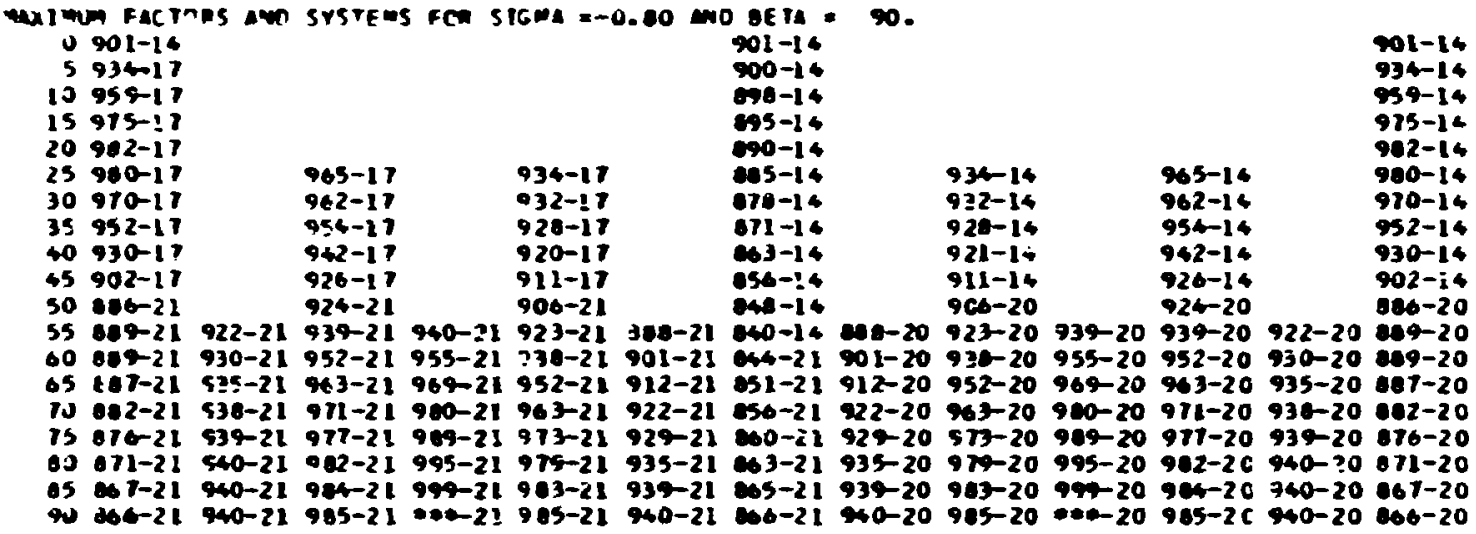




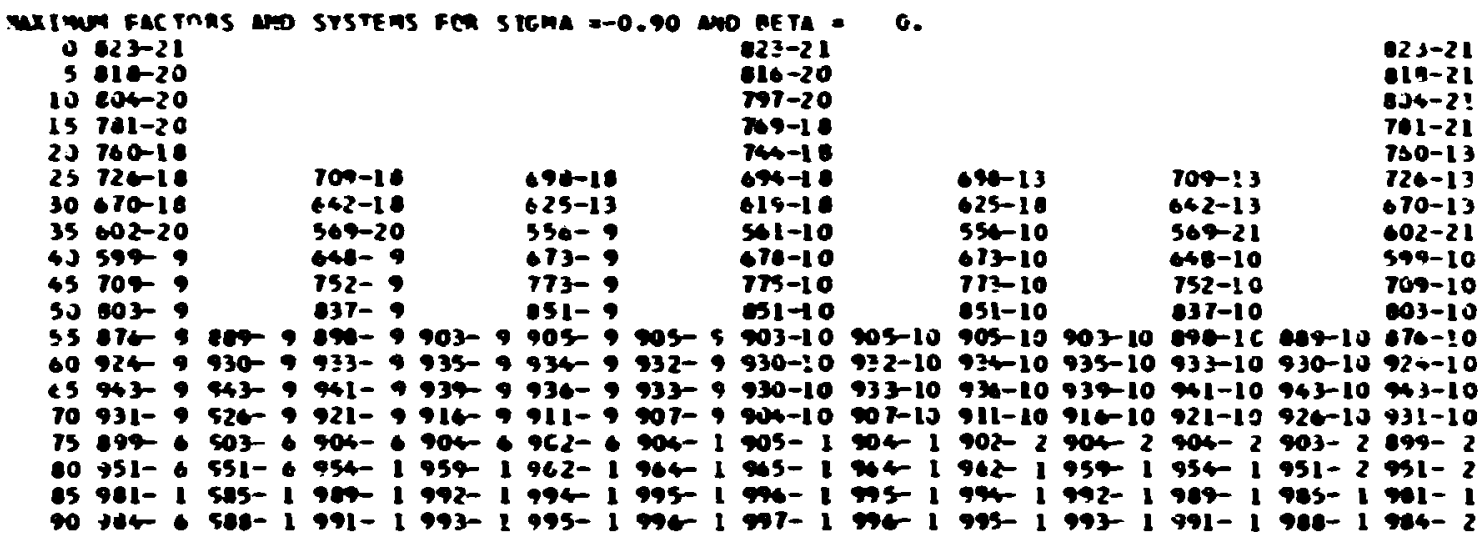

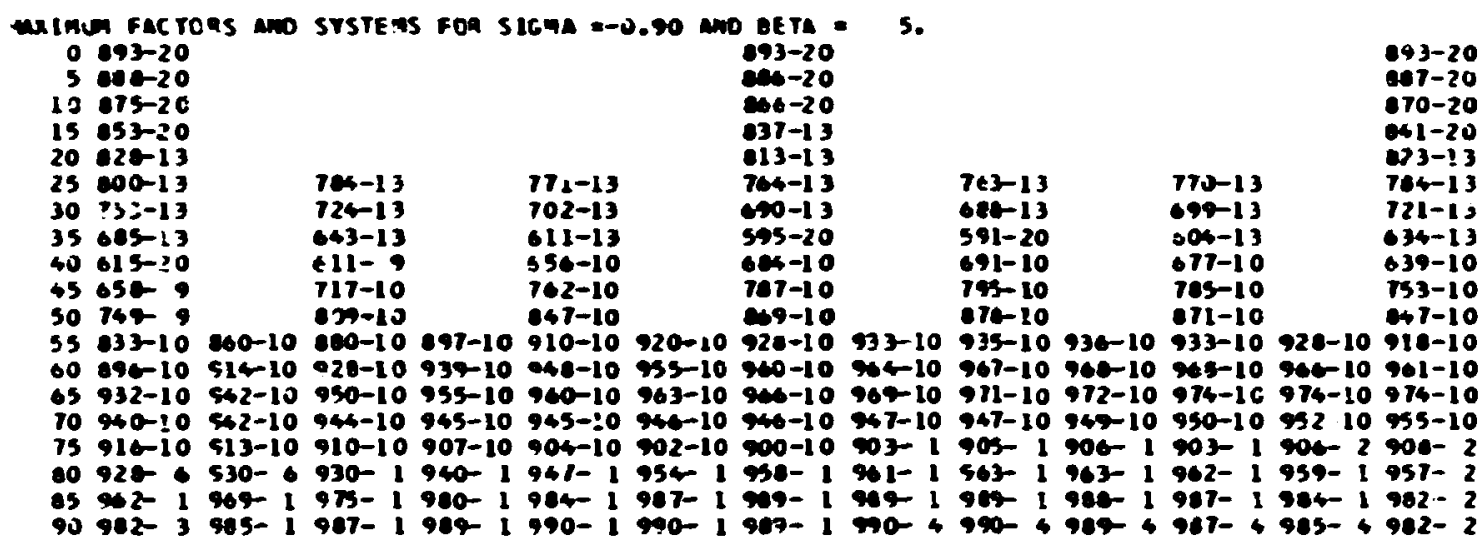

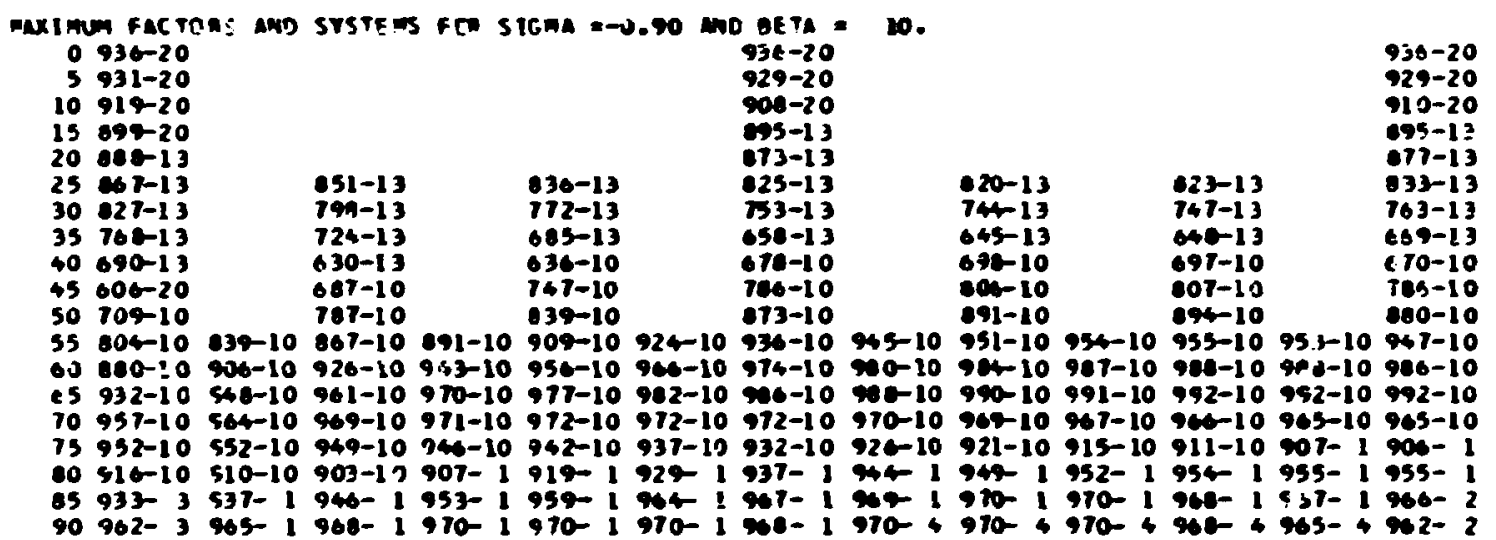




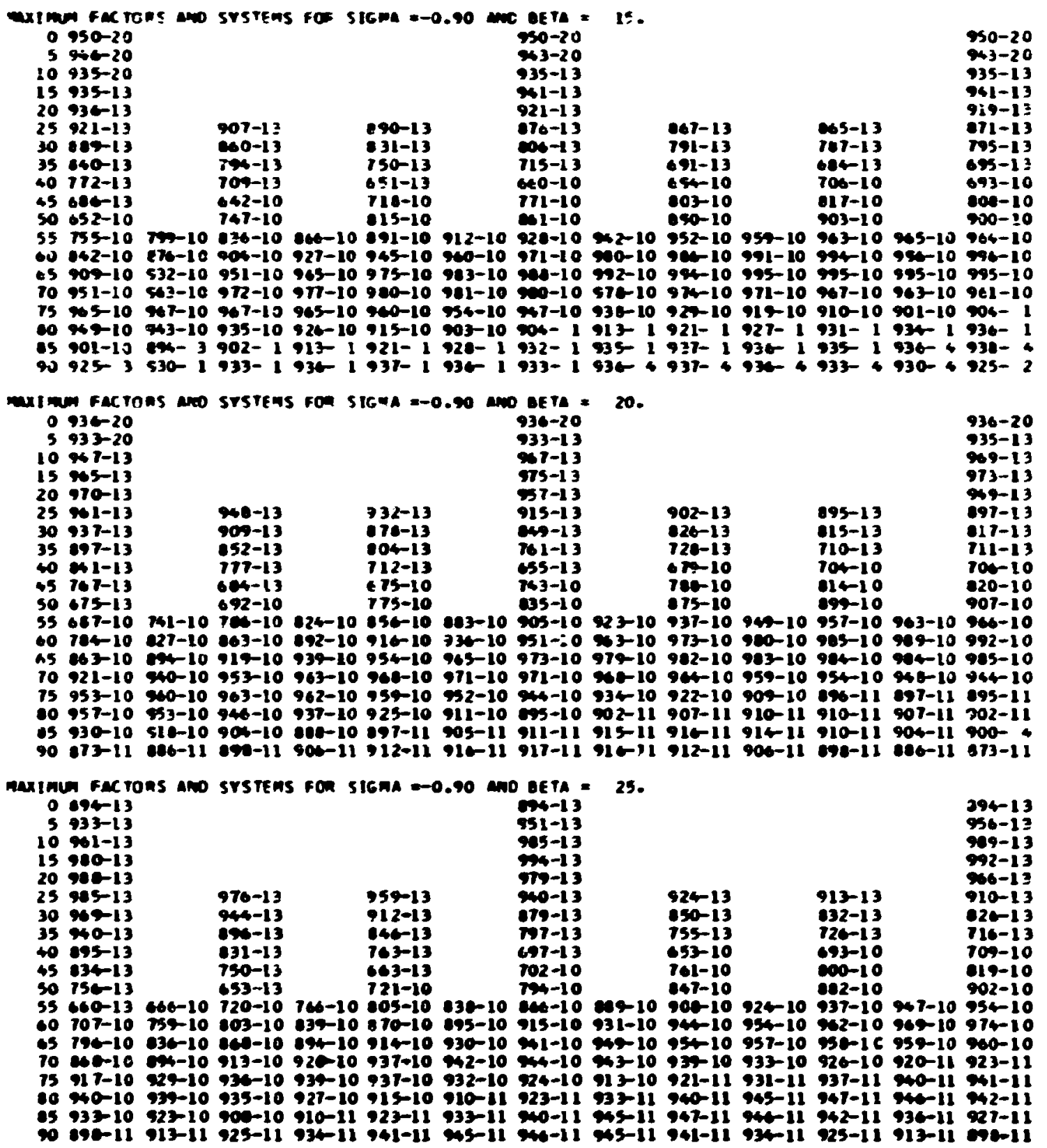




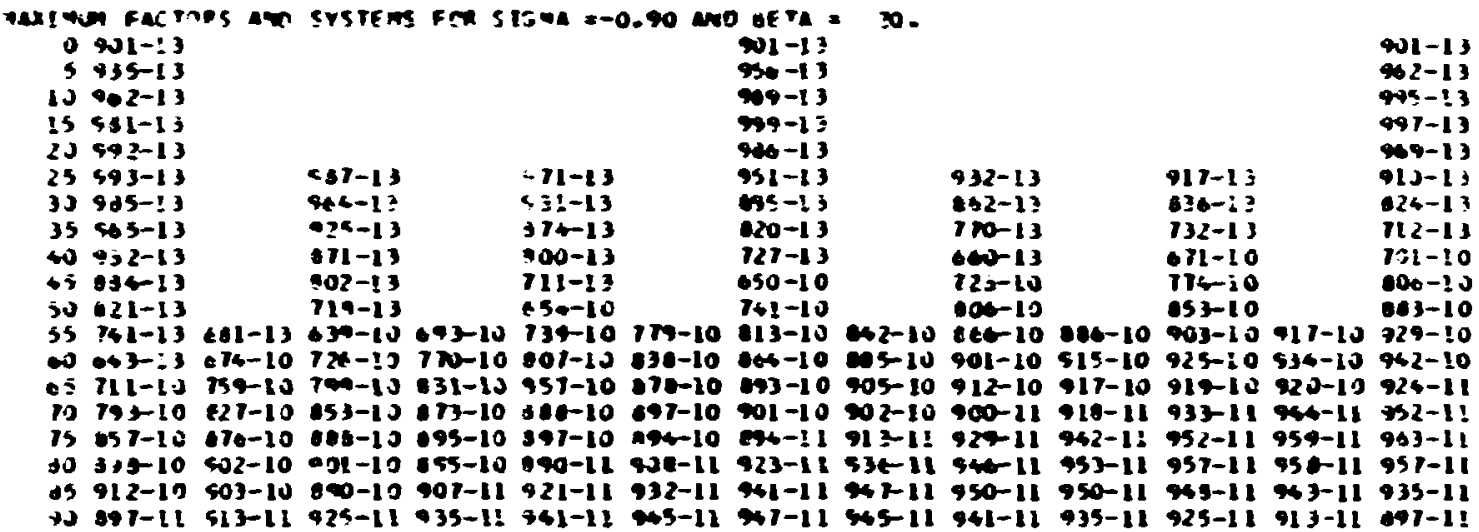

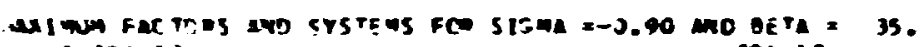

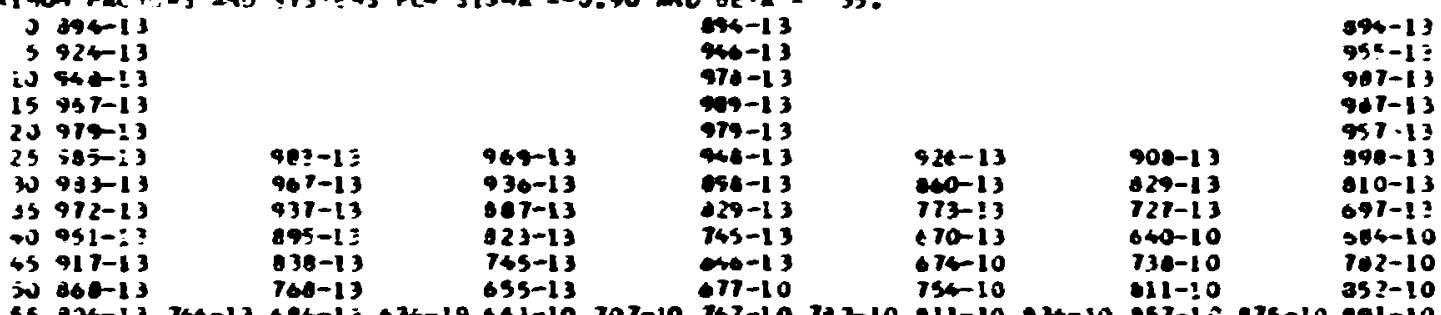

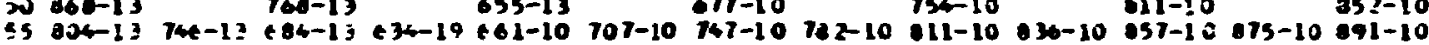

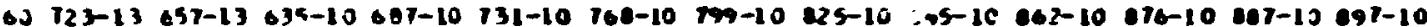

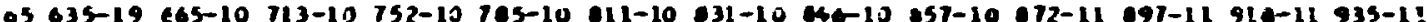
$70700-10 \quad 762-10775-10$ e01-10 $921-10$ e35-10 045-10 69-11 $185-11$ 904-11 920-11 $943-11957-11$

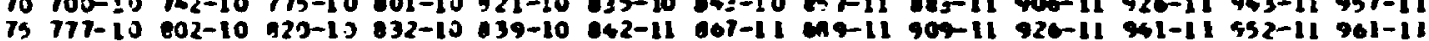

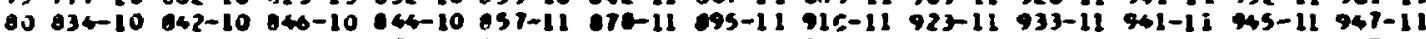

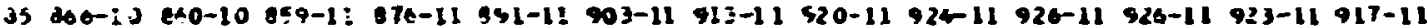

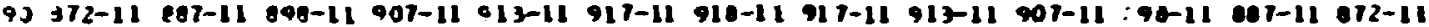

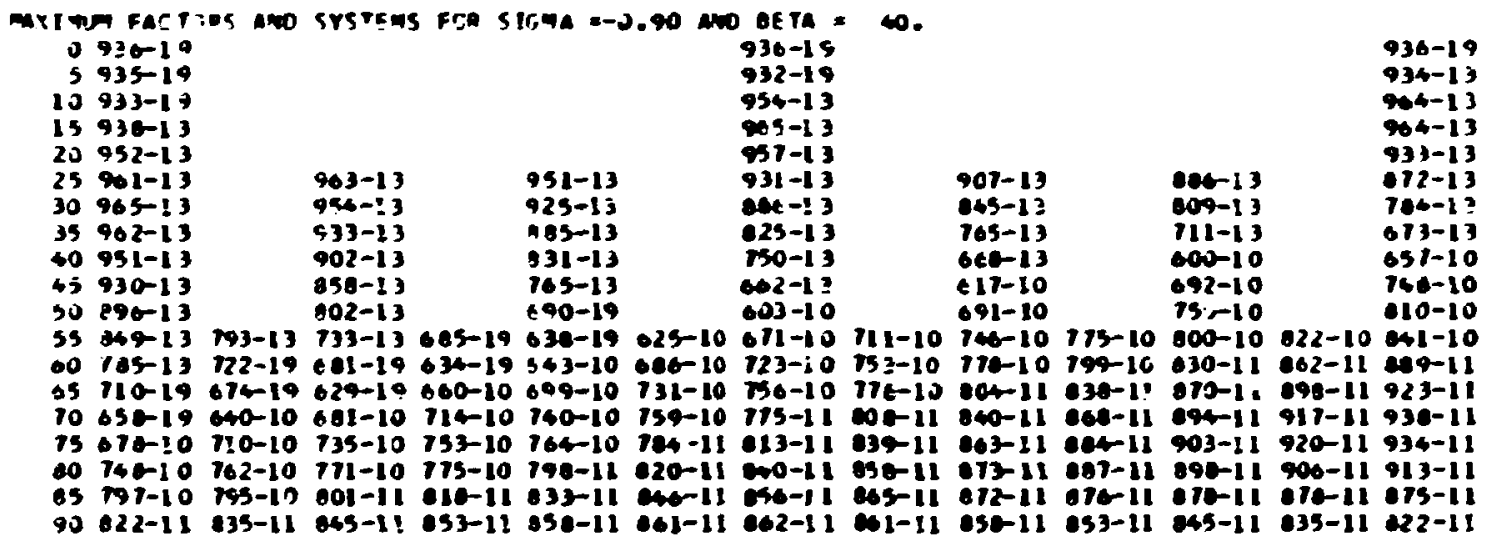




\begin{tabular}{|c|c|c|c|c|c|c|c|c|c|c|c|c|c|}
\hline $\begin{array}{l}3 \\
3 \\
10 \\
15 \\
20 \\
25 \\
30 \\
55 \\
40 \\
45 \\
50 \\
55 \\
05 \\
05 \\
10 \\
15 \\
10 \\
45 \\
90\end{array}$ & $\begin{array}{l}91-19 \\
950-19 \\
969-19 \\
940-19 \\
946-19 \\
92-19 \\
930-19 \\
235-113 \\
933-13 \\
924-13 \\
904-13 \\
073-13 \\
627-113 \\
215-19 \\
726-19 \\
670-19 \\
645-10 \\
700-10 \\
750-11\end{array}$ & $\begin{array}{l}0 \\
\\
622-1 ? \\
117-19 \\
132-19 \\
600-19 \\
621-19 \\
665-10 \\
111-10 \\
300-11\end{array}$ & $\begin{array}{l}920-13 \\
925-13 \\
213-13 \\
851-13 \\
600-11 \\
617-13 \\
771-19 \\
128-19 \\
679-19 \\
623-19 \\
634-10 \\
900-10 \\
720-11 \\
704-11\end{array}$ & $\begin{array}{l} \\
\\
721-19 \\
672-19 \\
617-19 \\
616-10 \\
650-10 \\
699-11 \\
736-11 \\
773-11\end{array}$ & $\begin{array}{l}919-13 \\
699-13 \\
967-13 \\
624-13 \\
769-13 \\
710-19 \\
865-19 \\
610-19 \\
602-10 \\
640-10 \\
670-10 \\
714-11 \\
750-11 \\
771-11\end{array}$ & 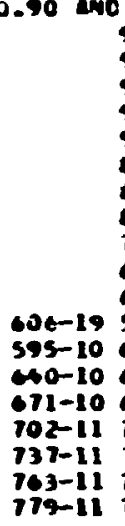 & $\begin{array}{l}950-19 \\
966-19 \\
936-19 \\
928-13 \\
922-13 \\
999-13 \\
61-113 \\
600-13 \\
742-13 \\
672-19 \\
611-19 \\
507-10 \\
637-10 \\
671-10 \\
690-11 \\
733-11 \\
759-1 \\
774-11 \\
700-11\end{array}$ & $\begin{array}{l}0 \\
032-10 \\
672-10 \\
697-11 \\
735-11 \\
762-11 \\
775-11 \\
794-11 \\
779-11\end{array}$ & $\begin{array}{l}875-13 \\
61 e-13 \\
746-13 \\
650-19 \\
582-19 \\
62 !-10 \\
671-10 \\
702-10 \\
760-11 \\
771-11 \\
750-11 \\
790-11 \\
753-11 \\
777-11\end{array}$ & $\begin{array}{l}0 \\
705-10 \\
762-11 \\
701-11 \\
000-11 \\
17-11 \\
015-11 \\
000-11 \\
773-11\end{array}$ & $\begin{array}{l}952-13 \\
770-13 \\
695-12 \\
560-19 \\
637-10 \\
690-10 \\
736-11 \\
785-11 \\
19-11 \\
830-11 \\
861-11 \\
830-11 \\
005-11 \\
760-11\end{array}$ & $\begin{array}{l} \\
\\
\\
\\
m 0-11 \\
625-11 \\
855-11 \\
600-11 \\
606-11 \\
065-11 \\
000-11 \\
760-11\end{array}$ & $\begin{array}{l}950-15 \\
93-19 \\
929-13 \\
921-13 \\
695-13 \\
635-13 \\
760-13 \\
639-12 \\
621-10 \\
704-10 \\
750-10 \\
816-11 \\
661-11 \\
608-11 \\
695-11 \\
904-11 \\
655-11 \\
209-11 \\
750-11\end{array}$ \\
\hline $\begin{array}{l}4100 \\
0 \\
5 \\
10 \\
15 \\
20 \\
25 \\
30 \\
35 \\
40 \\
45 \\
50 \\
55 \\
60 \\
65 \\
70 \\
75 \\
60 \\
95 \\
90\end{array}$ & $\begin{array}{l}\text { W FACM } \\
936-19 \\
930-19 \\
597-19 \\
935-19 \\
941-19 \\
942-15 \\
942-19 \\
939-19 \\
934-19 \\
925-19 \\
910-19 \\
990-19 \\
662-19 \\
920-19 \\
795-19 \\
733-19 \\
673-19 \\
604-19 \\
657-11\end{array}$ & $\begin{array}{l}269-19 \\
11-19 \\
170-19 \\
71-19 \\
676-19 \\
614-19 \\
609-10 \\
64-11\end{array}$ & $\begin{array}{l}919-19 \\
908-19 \\
975-19 \\
678-19 \\
857-19 \\
831-19 \\
799-19 \\
761-19 \\
716-19 \\
665-19 \\
619-21 \\
617-21 \\
617-11 \\
608-11\end{array}$ & $\begin{array}{l} \\
\\
740-19 \\
690-19 \\
649-21 \\
649-21 \\
646-21 \\
639-21 \\
631-11 \\
671-11\end{array}$ & $\begin{array}{l}605-19 \\
802-19 \\
806-13 \\
603-19 \\
765-19 \\
723-19 \\
676-19 \\
666-21 \\
660-21 \\
662-21 \\
655-21 \\
642-21 \\
664-11 \\
672-11\end{array}$ & $\begin{array}{l}0 \\
671-21 \\
671-21 \\
669-21 \\
659-21 \\
646-21 \\
631-11 \\
657-11 \\
673-11\end{array}$ & $\begin{array}{l}\text { D BE T1 } \\
936-19 \\
932-19 \\
921-19 \\
904-19 \\
619-19 \\
655-13 \\
623-13 \\
170-13 \\
122-13 \\
665-21 \\
600-21 \\
664-21 \\
664-21 \\
655-21 \\
641-21 \\
630-11 \\
654-11 \\
669-11 \\
676-11\end{array}$ & $\begin{array}{l}50 . \\
\\
655-21 \\
645-21 \\
630-21 \\
639-11 \\
643-11 \\
677-11 \\
600-11 \\
673-11\end{array}$ & $\begin{array}{l}631-13 \\
760-13 \\
713-13 \\
669-21 \\
640-21 \\
649-21 \\
633-21 \\
619-10 \\
654-11 \\
680-11 \\
655-1 ! \\
690-11 \\
691-11 \\
672-11\end{array}$ & $\begin{array}{l}l \\
0 \\
620-10 \\
660-11 \\
701-11 \\
720-11 \\
726-11 \\
719-11 \\
701-11 \\
671-11\end{array}$ & $\begin{array}{l}800-13 \\
737-13 \\
650-13 \\
619-21 \\
600-21 \\
630-10 \\
675-11 \\
710-11 \\
746-11 \\
759-11 \\
756-11 \\
139-11 \\
709-11 \\
698-11\end{array}$ & $\begin{array}{l}0 \\
720-11 \\
760-11 \\
790-11 \\
796-11 \\
705-11 \\
756-11 \\
711-11 \\
666-11\end{array}$ & $\begin{array}{l}936-19 \\
928-19 \\
905-19 \\
977-13 \\
465-13 \\
780-13 \\
703-13 \\
590-13 \\
578-10 \\
651-19 \\
716-11 \\
773-11 \\
612-11 \\
631-11 \\
631-11 \\
611-11 \\
775-11 \\
125-11 \\
657-11\end{array}$ \\
\hline $\begin{array}{l}0 \\
3 \\
10 \\
15 \\
20 \\
25 \\
30 \\
35 \\
40 \\
45 \\
50 \\
55 \\
60 \\
65 \\
70 \\
75 \\
00 \\
45 \\
00\end{array}$ & $\begin{array}{l}\text { faC IC } \\
693-19 \\
974-19 \\
697-19 \\
902-19 \\
907-19 \\
915-19 \\
922-19 \\
927-19 \\
930-19 \\
929-19 \\
923-19 \\
912-19 \\
693-19 \\
06-19 \\
631-19 \\
797-19 \\
732-19 \\
664-19 \\
595-21\end{array}$ & $\begin{array}{c}05-19 \\
41-19 \\
10-19 \\
70-19 \\
22-19 \\
451-21 \\
440-21\end{array}$ & $\begin{array}{l}660-19 \\
864-19 \\
877-19 \\
666-19 \\
852-19 \\
633-19 \\
609-19 \\
770-19 \\
740-19 \\
706-21 \\
701-21 \\
67-21 \\
67-21 \\
67-21\end{array}$ & $\begin{array}{l}7 \\
733-19 \\
737-21 \\
735-21 \\
733-21 \\
727-21 \\
719-21 \\
706-21 \\
097-21\end{array}$ & $\begin{array}{l}8 \times 1-19 \\
932-19 \\
609-19 \\
711-19 \\
750-21 \\
752-21 \\
753-21 \\
752-21 \\
749-21 \\
763-21 \\
734-21 \\
720-21 \\
701-21 \\
677-21\end{array}$ & $\begin{array}{l}7 \\
757-21 \\
75-21 \\
764-21 \\
736-21 \\
721-21 \\
701-21 \\
677-16 \\
675-16\end{array}$ & $\begin{array}{r}93-19 \\
99-19 \\
879-19 \\
962-19 \\
639-19 \\
609-19 \\
774-13 \\
750-21 \\
733-21 \\
734-21 \\
733-21 \\
740-21 \\
740-21 \\
720-21 \\
711-21 \\
60-21 \\
605-14 \\
402-14 \\
41-16\end{array}$ & 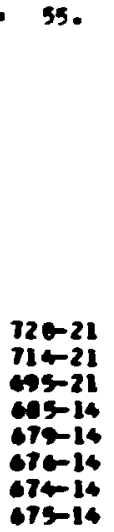 & $\begin{array}{l}776-13 \\
739-21 \\
736-21 \\
734-21 \\
727-21 \\
710-21 \\
699-21 \\
682-14 \\
671-14 \\
663-14 \\
657-14 \\
654-14 \\
694-16 \\
677-20\end{array}$ & $\begin{array}{l}0 \\
660-14 \\
632-14 \\
633-16 \\
630-14 \\
623-14 \\
633-20 \\
664-20 \\
607-20\end{array}$ & $\begin{array}{l}751-13 \\
726-14 \\
713-14 \\
695-21 \\
676-21 \\
651-14 \\
636-14 \\
632-11 \\
653-11 \\
659-11 \\
651-11 \\
630-20 \\
660-20 \\
677-20\end{array}$ & $\begin{array}{l}0 \\
655-11 \\
600-11 \\
705-11 \\
703-11 \\
60-11 \\
633-11 \\
630-20 \\
64-20\end{array}$ & $\begin{array}{l}093-19 \\
005-19 \\
661-19 \\
622-19 \\
196-13 \\
729-13 \\
701-14 \\
674-14 \\
646-21 \\
612-21 \\
661-11 \\
711-11 \\
743-11 \\
734-11 \\
746-11 \\
719-11 \\
676-11 \\
617-11 \\
595-19\end{array}$ \\
\hline
\end{tabular}




\begin{tabular}{|c|c|c|c|c|c|c|c|c|c|c|c|c|c|}
\hline $\begin{array}{l}150 \\
0 \\
5 \\
10 \\
15 \\
25 \\
25 \\
30 \\
35 \\
40 \\
45 \\
30 \\
55 \\
60 \\
65 \\
70 \\
75 \\
00 \\
65 \\
90\end{array}$ & 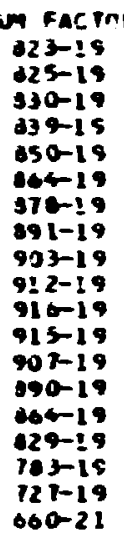 & $\begin{array}{l}7 \\
126-19 \\
649-19 \\
627-19 \\
797-19 \\
750-19 \\
72 t-21 \\
722-21 \\
717-21\end{array}$ & $\begin{array}{l}833-19 \\
835-19 \\
835-19 \\
833-19 \\
827-19 \\
616-19 \\
801-19 \\
787-21 \\
794-21 \\
780-21 \\
776-21 \\
770-21 \\
762-21 \\
751-21\end{array}$ & $\begin{array}{l}0 \\
10-21 \\
10-21 \\
10-21 \\
112-21 \\
100-21 \\
001-21 \\
792-21 \\
779-21 \\
7 \in 2-21\end{array}$ & $\begin{array}{l}827-21 \\
328-21 \\
830-21 \\
331-21 \\
031-21 \\
031-21 \\
030-21 \\
027-21 \\
023-21 \\
015-21 \\
815 \\
805-21 \\
791-21 \\
773-21 \\
755-14\end{array}$ & 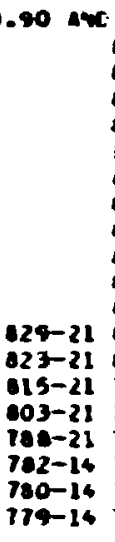 & 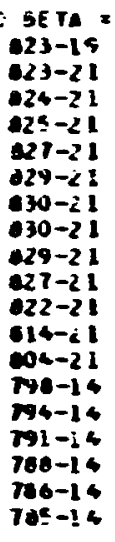 & $\begin{array}{c}00-16 \\
032-16 \\
792-16 \\
792-16 \\
707-14 \\
763-14 \\
780-16 \\
715-14 \\
779-16\end{array}$ & 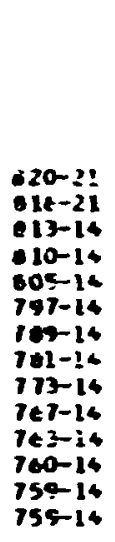 & $\begin{array}{l} \\
766-16 \\
753-16 \\
743-16 \\
735-14 \\
729-16 \\
720-16 \\
741-20 \\
702-20\end{array}$ & $\begin{array}{l}10 d-: 4 \\
905-1\rangle \\
790-14 \\
702-14 \\
765-14 \\
767-14 \\
729-14 \\
713-14 \\
100-14 \\
690-14 \\
601-20 \\
716-20 \\
735-20 \\
751-20\end{array}$ & $\begin{array}{l}\text { cos-14 } \\
003-11 \\
64-14 \\
647-20 \\
67-20 \\
64+-20 \\
100-20 \\
117-20\end{array}$ & 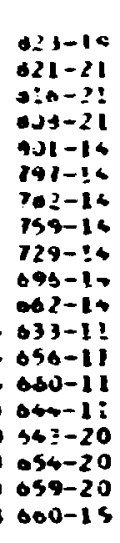 \\
\hline $\begin{array}{r}x 1 m 0 \\
0 \\
5 \\
10 \\
15 \\
20 \\
25 \\
30 \\
35 \\
40 \\
45 \\
50 \\
55 \\
60 \\
65 \\
70 \\
75 \\
80 \\
85 \\
90\end{array}$ & 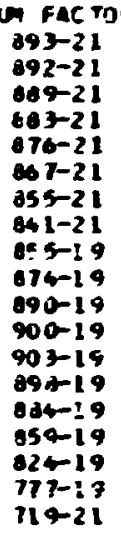 & $\begin{array}{l}0 \\
94-19 \\
641-19 \\
630-19 \\
81:-19 \\
195-21 \\
790-21 \\
700-21 \\
100-21\end{array}$ & $\begin{array}{l}88 \leq-21 \\
803-21 \\
879-21 \\
875-21 \\
871-21 \\
866-2 ! \\
862-21 \\
857-21 \\
852-21 \\
647-21 \\
862-21 \\
836-2 ! \\
820-2 ! \\
818-21\end{array}$ & $\begin{array}{l}l \\
\\
885-21 \\
882-21 \\
878-21 \\
873-21 \\
866-21 \\
857-21 \\
845-2: \\
630-21\end{array}$ & 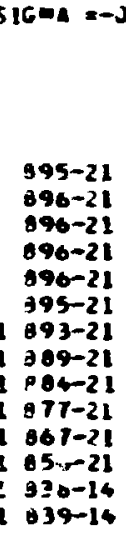 & $\begin{array}{l}.90 \text { MC } \\
\\
806-21 \\
879-21 \\
871-21 \\
061-14 \\
365-16 \\
860-14 \\
859-14 \\
859-14\end{array}$ & 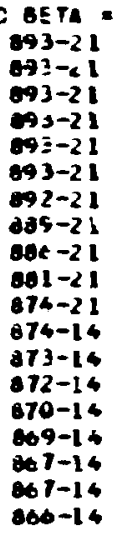 & $\begin{array}{l}07-14 \\
87-14 \\
673-14 \\
070-16 \\
61-14 \\
64-16 \\
802-16 \\
660-14 \\
659-14\end{array}$ & $\begin{array}{l}875-21 \\
875-14 \\
879-14 \\
879-14 \\
87 t-14 \\
872-14 \\
66 e-14 \\
860-14 \\
855-14 \\
850-14 \\
646-14 \\
862-18 \\
940-14 \\
839-14\end{array}$ & $\begin{array}{l} \\
\\
045-16 \\
835-14 \\
A \geq ?-16 \\
819-14 \\
616-14 \\
010-14 \\
811-20 \\
830-20\end{array}$ & $\begin{array}{l}273-14 \\
873-14 \\
06 i-14 \\
05 t-14 \\
063-14 \\
020-14 \\
012-16 \\
798-14 \\
700-14 \\
776-14 \\
709-14 \\
706-20 \\
604-20 \\
010-20\end{array}$ & $\begin{array}{l}0 \\
769-16 \\
750-14 \\
733-14 \\
725-20 \\
740-20 \\
702-23 \\
713-20 \\
700-20\end{array}$ & 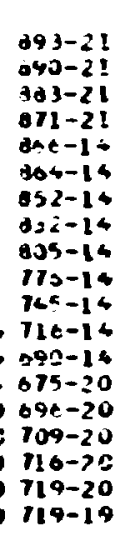 \\
\hline $\begin{array}{l}0 \\
5 \\
10 \\
15 \\
20 \\
25 \\
30 \\
35 \\
40 \\
45 \\
50 \\
55 \\
60 \\
65 \\
10 \\
75 \\
60 \\
85 \\
90\end{array}$ & $\begin{array}{l}\text { FACro } \\
936-21 \\
535-21 \\
933-21 \\
929-21 \\
926-21 \\
917-21 \\
900-21 \\
997-21 \\
804-21 \\
865-21 \\
052-21 \\
807-19 \\
693-19 \\
090-19 \\
009-19 \\
876-19 \\
053-19 \\
017-19 \\
770-21\end{array}$ & $\begin{array}{l}683-21 \\
873-21 \\
805-21 \\
657-21 \\
850-21 \\
845-21 \\
60-21 \\
035-21\end{array}$ & $\begin{array}{l}934-21 \\
932-21 \\
930-21 \\
928-21 \\
924-21 \\
921-21 \\
917-21 \\
992-21 \\
907-21 \\
902-21 \\
997-21 \\
991-21 \\
994-21 \\
975-21\end{array}$ & $\begin{array}{l} \\
\\
\\
9 \\
935-21 \\
933-21 \\
930-21 \\
925-21 \\
919-21 \\
912-21 \\
902-21 \\
989-21\end{array}$ & $\begin{array}{l}939-21 \\
940-21 \\
941-21 \\
941-21 \\
941-21 \\
940-21 \\
939-21 \\
1936-21 \\
1931-21 \\
1923-21 \\
1917-21 \\
1906-21 \\
1992-21 \\
1993-14\end{array}$ & $\begin{array}{l}0.90 \text { MC } \\
926-21 \\
920-21 \\
912-21 \\
900-14 \\
909-14 \\
911-14 \\
912-14 \\
914-14\end{array}$ & $\begin{array}{l}\text { C BE TA } \\
936-21 \\
935-21 \\
935-21 \\
935-61 \\
933-<2 \\
932-21 \\
930-21 \\
926-21 \\
922-21 \\
910-21 \\
919-14 \\
921-14 \\
922-14 \\
922-14 \\
922-14 \\
922-14 \\
921-14 \\
921-14 \\
921-14\end{array}$ & $\begin{array}{l} \\
920-14 \\
926-14 \\
924-14 \\
922-14 \\
920-14 \\
910-14 \\
916-14 \\
914-14\end{array}$ & $\begin{array}{l}914-2 ! \\
923-14 \\
928-14 \\
930-14 \\
929-14 \\
927-14 \\
923-14 \\
918-14 \\
913-14 \\
909-14 \\
904-14 \\
900-14 \\
896-14 \\
993-14\end{array}$ & $\begin{array}{l}7 \\
\\
906-14 \\
991-14 \\
889-14 \\
801-14 \\
874-14 \\
860-14 \\
873-20 \\
689-20\end{array}$ & $\begin{array}{l}924-14 \\
920-14 \\
922-14 \\
914-14 \\
903-14 \\
990-14 \\
917-14 \\
664-14 \\
851-14 \\
661-14 \\
632-14 \\
850-20 \\
864-20 \\
675-20\end{array}$ & $\begin{array}{l}0 \\
930-16 \\
010-14 \\
001-16 \\
793-20 \\
10-20 \\
021-20 \\
29-20 \\
935-20\end{array}$ & $\begin{array}{l}93 c-21 \\
933-21 \\
925-21 \\
900-21 \\
919-14 \\
919-14 \\
939-14 \\
991-14 \\
900-14 \\
841-14 \\
812-14 \\
785-14 \\
760-14 \\
745-20 \\
759-20 \\
760-20 \\
770-20 \\
770-20 \\
970-19\end{array}$ \\
\hline
\end{tabular}




\begin{tabular}{|c|c|c|c|c|c|c|c|c|c|c|c|c|c|}
\hline $\begin{array}{l}0 \\
5 \\
13 \\
15 \\
20 \\
25 \\
30 \\
35 \\
40 \\
45 \\
50 \\
55 \\
00 \\
15 \\
10 \\
75 \\
00 \\
25 \\
90\end{array}$ & 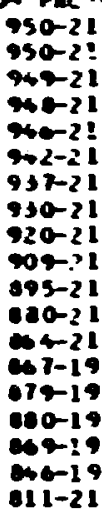 & $\begin{array}{l}5 \\
\\
524-21 \\
510-21 \\
500-21 \\
90 !-21 \\
69-21 \\
60-21 \\
004-21 \\
260-21\end{array}$ & $\begin{array}{l}954-21 \\
957-21 \\
950-2 ! \\
950-21 \\
958-21 \\
954-21 \\
956-21 \\
951-21 \\
948-21 \\
964-21 \\
940-21 \\
935-21 \\
929-21 \\
922-21\end{array}$ & $\begin{array}{l}966-21 \\
26-21 \\
966-21 \\
966-21 \\
960-21 \\
954-21 \\
966-21 \\
936-21\end{array}$ & $\begin{array}{l}957-21 \\
959-21 \\
962-21 \\
964-21 \\
365-21 \\
966-21 \\
1967-21 \\
996-21 \\
1963-21 \\
1959-21 \\
1953-21 \\
19 \\
1945-21 \\
1935-21 \\
1922-21\end{array}$ & $\begin{array}{l}9 \\
940-21 \\
964-21 \\
939-22 \\
931-21 \\
931-16 \\
934-14 \\
937-16 \\
940-14\end{array}$ & 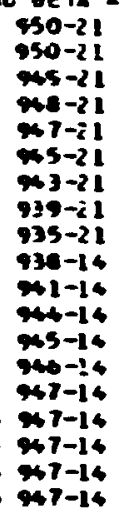 & $\begin{array}{l}x \\
95-14 \\
95-14 \\
54-24 \\
931-16 \\
249-16 \\
46-14 \\
96-14 \\
40-16\end{array}$ & $\begin{array}{l}945-14 \\
95-14 \\
959-14 \\
9 \in 2-14 \\
9 \in 2-14 \\
960-14 \\
957-14 \\
952-14 \\
947-14 \\
942-14 \\
930-14 \\
930-14 \\
925-14 \\
922-20\end{array}$ & $\begin{array}{l}0 \\
945-14 \\
636-14 \\
527-14 \\
910-14 \\
910-14 \\
900-20 \\
924-20 \\
936-20\end{array}$ & $\begin{array}{l}960-14 \\
963-14 \\
961-14 \\
954-14 \\
965-14 \\
933-14 \\
920-14 \\
907-14 \\
996-14 \\
901-14 \\
900-20 \\
902-2 c \\
913-20 \\
922-20\end{array}$ & $\begin{array}{l}0 \\
\\
\\
06-1 t \\
005-14 \\
047-10 \\
851-20 \\
062-20 \\
610-20 \\
676-20 \\
100-20\end{array}$ & $\begin{array}{l}950-21 \\
946-21 \\
935-21 \\
965-14 \\
959-14 \\
959-14 \\
95 !-14 \\
935-14 \\
914-14 \\
839-14 \\
062-16 \\
936-14 \\
911-14 \\
604-20 \\
811-20 \\
613-20 \\
8: 3-20 \\
312-20 \\
811-19\end{array}$ \\
\hline $\begin{array}{l}x I m \\
0 \\
5 \\
10 \\
15 \\
20 \\
25 \\
30 \\
35 \\
40 \\
45 \\
50 \\
55 \\
60 \\
65 \\
10 \\
75 \\
80 \\
05 \\
90\end{array}$ & $\begin{array}{l}9 \text { FAC } \\
936-21 \\
936-21 \\
937-21 \\
934-21 \\
941-21 \\
942-21 \\
941-21 \\
939-21 \\
935-21 \\
928-21 \\
919-21 \\
907-21 \\
674-21 \\
880-21 \\
667-21 \\
669-19 \\
672-19 \\
963-19\end{array}$ & $\begin{array}{l}\text { oos ans } \\
\\
\\
548-21 \\
542-21 \\
934-21 \\
930-21 \\
925-21 \\
520-21 \\
516-21\end{array}$ & $\begin{array}{l}952-21 \\
957-21 \\
9 \in 2-21 \\
966-21 \\
970-21 \\
972-21 \\
973-21 \\
973-21 \\
972-21 \\
971-21 \\
968-21 \\
9 \times 5-21 \\
961-21\end{array}$ & $\begin{array}{l}913-21 \\
985-21 \\
987-21 \\
987-21 \\
905-21 \\
903-21 \\
978-21\end{array}$ & 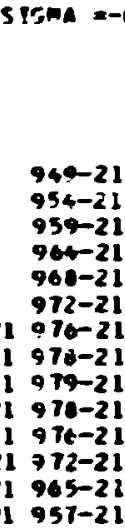 & $\begin{array}{l}952-21 \\
951-21 \\
949-21 \\
945-21 \\
990-21 \\
933-21 \\
934-14\end{array}$ & $\begin{array}{l}\text { CEE T1 } \\
936-21 \\
935-21 \\
935-21 \\
935-21 \\
934-21 \\
933-21 \\
931-21 \\
933-14 \\
936-14 \\
939-14 \\
961-14 \\
943-14 \\
964-14 \\
945-14 \\
965-14 \\
945-14 \\
965-14 \\
965-14\end{array}$ & $\begin{array}{l}0 \\
91-16 \\
90-14 \\
952-14 \\
95-14 \\
930-16 \\
946-14 \\
942-16\end{array}$ & $\begin{array}{l}961-14 \\
964-14 \\
772-14 \\
974-14 \\
974-14 \\
971-14 \\
967-14 \\
962-14 \\
936-14 \\
949-14 \\
961-14 \\
939-14 \\
946-20\end{array}$ & $\begin{array}{l}960-16 \\
951-14 \\
941-16 \\
930-16 \\
939-20 \\
951-20 \\
963-20\end{array}$ & $\begin{array}{l}991-14 \\
904-14 \\
982-14 \\
976-14 \\
966-14 \\
955-14 \\
941-14 \\
927-14 \\
912-14 \\
920-20 \\
933-20 \\
943-20 \\
951-20 \\
957-20\end{array}$ & $\begin{array}{l}7 \\
7 \\
910-14 \\
890-14 \\
987-20 \\
890-20 \\
903-20 \\
907-20 \\
910-20 \\
912-20\end{array}$ & $\begin{array}{l}935-21 \\
931-21 \\
950-14 \\
972-14 \\
963-14 \\
964-14 \\
977-14 \\
963-14 \\
943-14 \\
919-14 \\
893-14 \\
067-16 \\
846-20 \\
849-20 \\
651-20 \\
849-20 \\
846-20 \\
842-20 \\
841-19\end{array}$ \\
\hline
\end{tabular}

maximUn faC TOUS ANO SYSTEMS FER SIGMA $=-0.90$ ANO BETA = 85 .

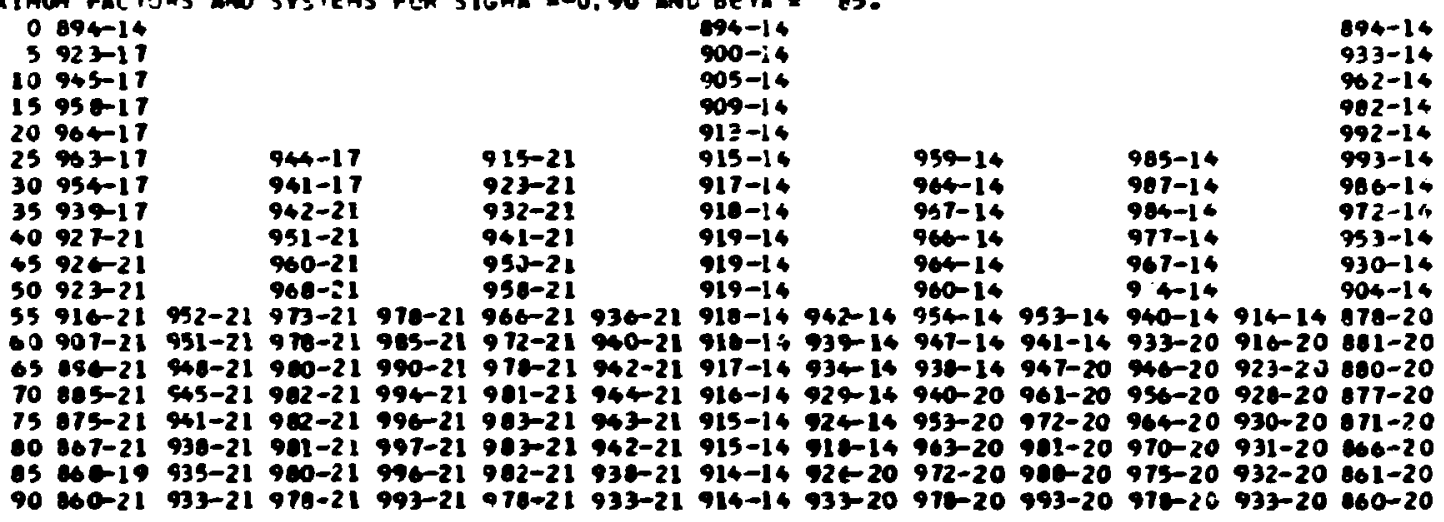




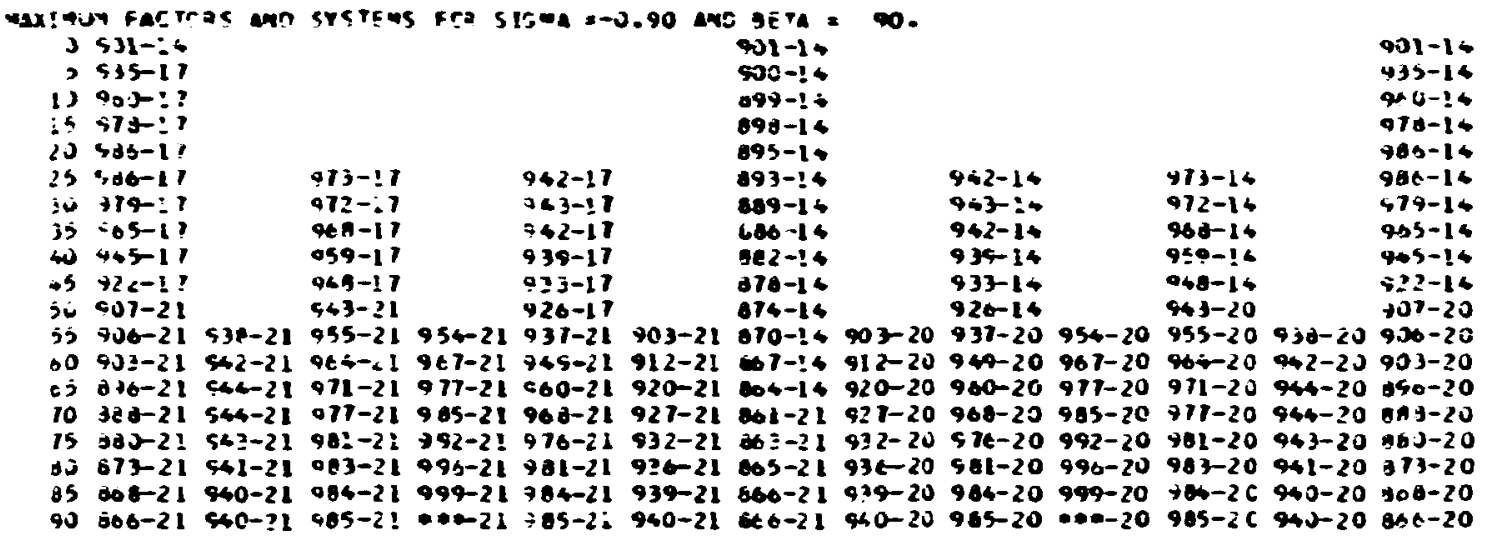




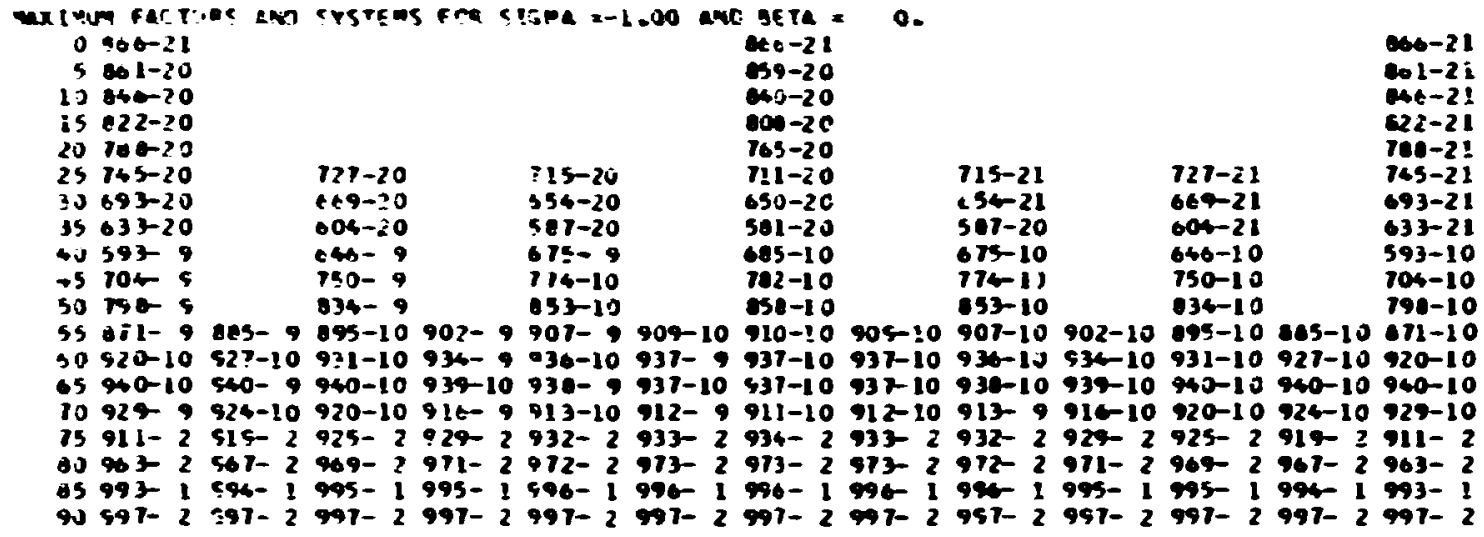

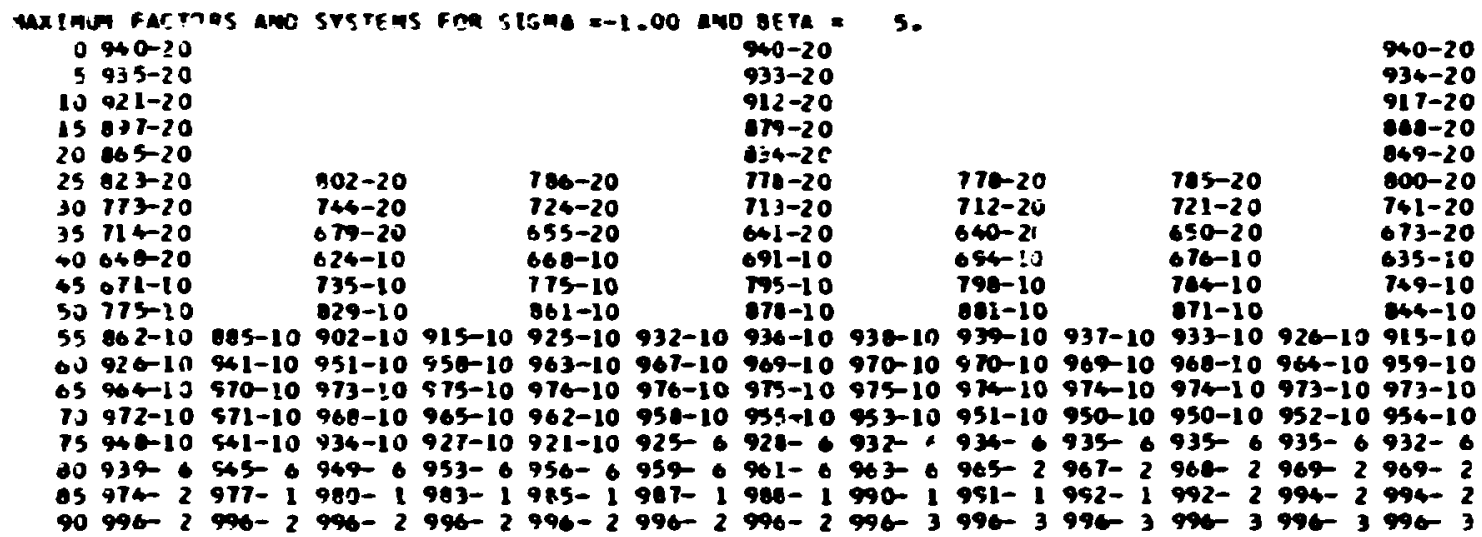

\begin{tabular}{|c|c|c|c|c|c|c|c|c|c|c|c|c|c|}
\hline $\begin{array}{l}0 \\
5 \\
10 \\
15 \\
20 \\
25 \\
30 \\
35 \\
40 \\
45 \\
50 \\
55 \\
60 \\
65 \\
70 \\
75 \\
80 \\
85\end{array}$ & $\begin{array}{l}985-20 \\
980-20 \\
968-20 \\
946-20 \\
915-20 \\
676-20 \\
129-20 \\
173-20 \\
109-20 \\
639-20 \\
733-10 \\
931-10 \\
909-10 \\
964-10 \\
990-10 \\
207-10 \\
951-10 \\
945-3 \\
973-3\end{array}$ & $\begin{array}{l} \\
963-10 \\
932-10 \\
576-10 \\
994-10 \\
902-10 \\
940-10 \\
950-13\end{array}$ & $\begin{array}{l}952-20 \\
796-13 \\
731-20 \\
660-20 \\
704-10 \\
906-10 \\
969-10 \\
949-10 \\
905-10 \\
994-10 \\
976-10 \\
929-10 \\
954-3 \\
973-1\end{array}$ & $\begin{array}{l}909-10 \\
962-10 \\
991-10 \\
993-10 \\
960-10 \\
924-3 \\
957-3 \\
972-1\end{array}$ & $\begin{array}{l}833-13 \\
769-20 \\
699-20 \\
648-10 \\
760-10 \\
953-10 \\
924-10 \\
972-10 \\
99-10 \\
990-10 \\
960-10 \\
932-3 \\
960-3 \\
971-3\end{array}$ & $\begin{array}{l}9 \\
936-10 \\
979-10 \\
996-10 \\
917-10 \\
9 \pm 2-10 \\
930-1 \\
963- \\
970-\end{array}$ & $\begin{array}{r}995-20 \\
970-20 \\
951-20 \\
923-20 \\
976-20 \\
921-13 \\
732-20 \\
670-20 \\
695-10 \\
794-10 \\
991-10 \\
10945-10 \\
10 \\
10 \\
104-10 \\
10 \\
992-10 \\
10 \\
943-10 \\
3\end{array}$ & $\begin{array}{l}0 \\
\\
\\
951-10 \\
97-10 \\
99-10 \\
978-10 \\
934-10 \\
949-3 \\
96-1\end{array}$ & $\begin{array}{l}16-13 \\
745-20 \\
600-20 \\
702-10 \\
909-10 \\
695-10 \\
955-10 \\
984-10 \\
995-10 \\
974-10 \\
920-10 \\
953-3 \\
970-1 \\
971-2\end{array}$ & $\begin{array}{l}9 \\
957-10 \\
989-10 \\
999-10 \\
970-10 \\
924-3 \\
957-1 \\
972-1 \\
972-4\end{array}$ & $\begin{array}{l}916-1 ? \\
740-20 \\
672-20 \\
697-10 \\
907-10 \\
694-10 \\
956-10 \\
999-10 \\
993-10 \\
967-10 \\
928-1 \\
961-3 \\
974-3 \\
973-4\end{array}$ & $\begin{array}{l}953-10 \\
910-10 \\
992-10 \\
965-10 \\
931-3 \\
965-3 \\
976-3 \\
973-4\end{array}$ & $\begin{array}{r}985-20 \\
970-20 \\
959-20 \\
927-20 \\
903-20 \\
120-13 \\
763-20 \\
609-20 \\
668-10 \\
704-10 \\
978-10 \\
10969-10 \\
10965-10 \\
10992-10 \\
10965-10 \\
3933-3 \\
396-3 \\
3979-3 \\
4973-2\end{array}$ \\
\hline
\end{tabular}




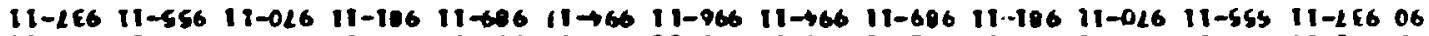

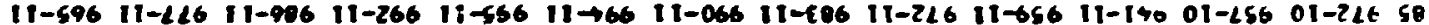

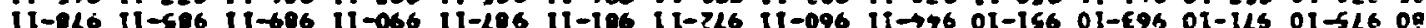

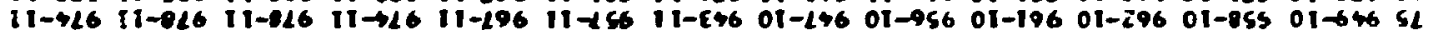

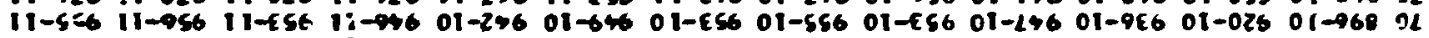

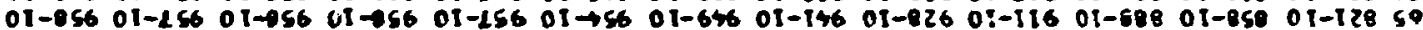

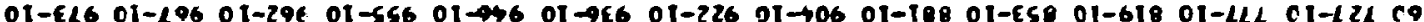

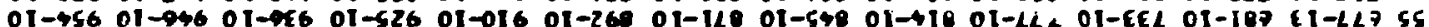

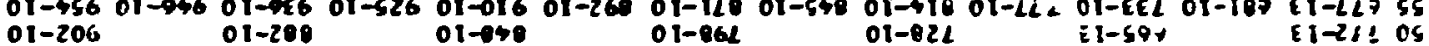

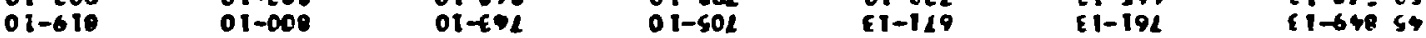
$01-602 \quad 01-669 \quad 01 \rightarrow 59 \quad$ EI-102 EI-69L EI-14LEC6 EI-0IL EI-LZL ET-XSL EI-000 EI-ISB E EI-406 SE

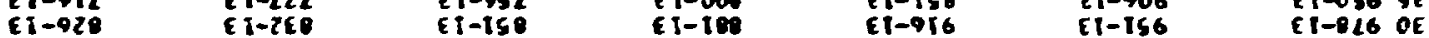

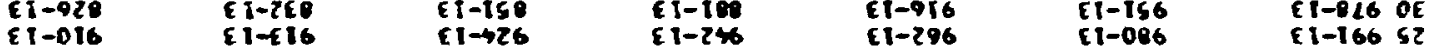
E $1-165 ; 02$

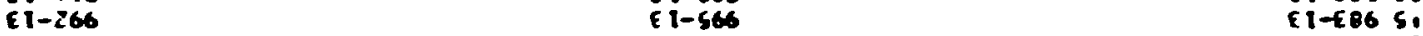

E1-686 EI-506 ET-296 OI Et-556 $02-046$ $\begin{array}{ll}\text { E } 1-156 & 0 z-1 \in 6 \\ 0 z-046 & 0 z-046\end{array}$

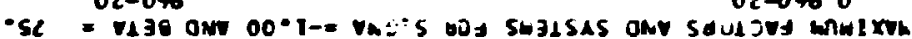

11-116 11-026 II-146 II-246 II-656 II-96 II-596 II-996 II-656 iI-256 II-146 II-825 II-i16 06

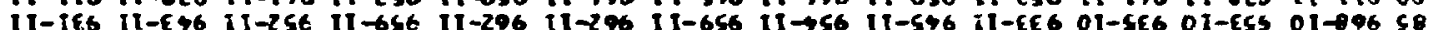

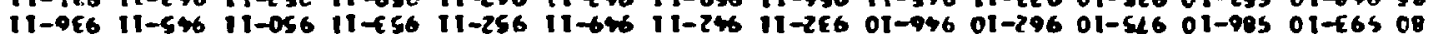

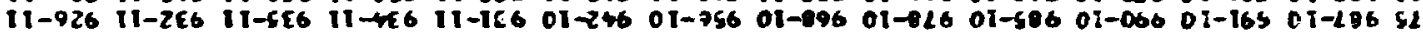

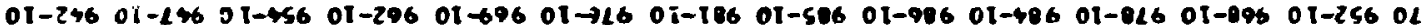

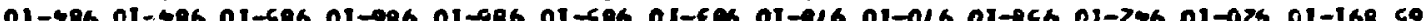

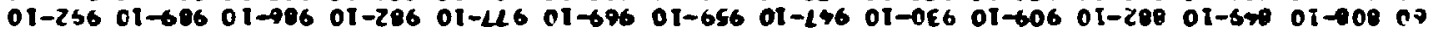

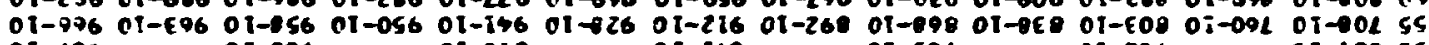
$01-006 \quad 01-918$ $01-14$ $01-028 \quad 01-510 \quad 01-162$ c1 $01-150$ $01-901 \quad 01-50 L \quad 01-189 \quad$ EI-L59 E1-0IL EI-0IL EI-62L EI-910 EI $\rightarrow 10$ EI $\rightarrow 270$ EI-468 $\quad \equiv 1-460$ CI-0S9 EI-188 EI-416 EI-4X OE EI-516 EI-EE6 EI-256 EI-946 S2 E I-1S5 ह1-416 C1-995 51

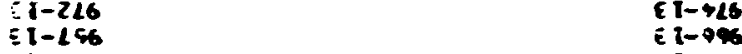

$02-216$ 02-026 $02-2<6$ or $0<-206 \mathrm{~s}$ $02-506$

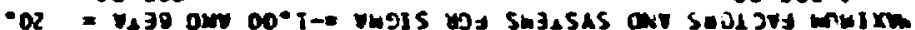

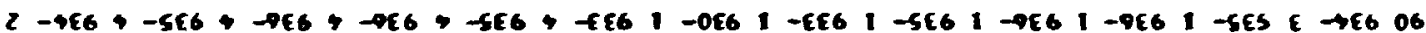

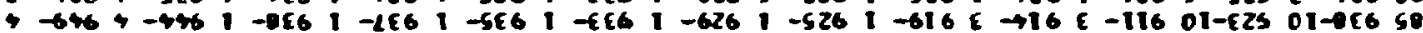

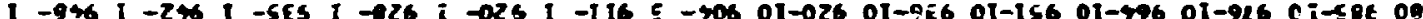

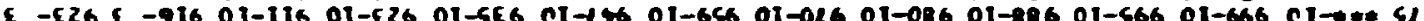
01-196 01-496 01-996 01-916 01-056 01-986 01-166 01-966 01-066 01-666 01-866 01-655 01-786 CL

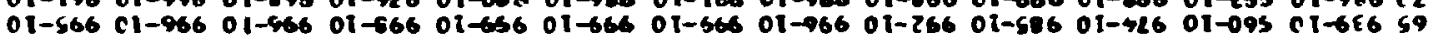

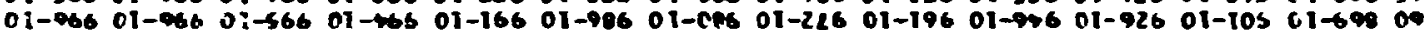

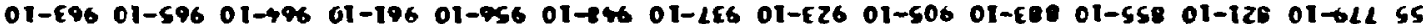
$0 \mathrm{i}-6 \mathrm{7} \quad \mathrm{CI}-220 \quad 0 \mathrm{i}-7 \mathrm{JL} \quad 0 !-517 \mathrm{CS}$

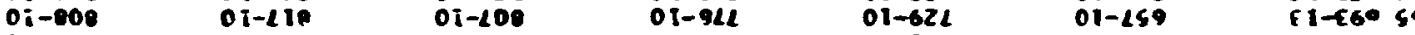
$01-260 \quad 01-101 \quad 01-259 \quad 01-99$ C1-469 E1-209 EI-0S? ET-4T

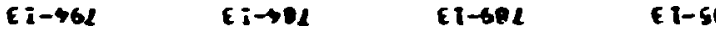

C 1-990 CI-290 CI-590 EI-26L ET T-4 SE

(1-210 CI-4LO EI-068 ET-606 E $1-26$ S2 Q Z $\rightarrow$ ES O2 $02-50651$

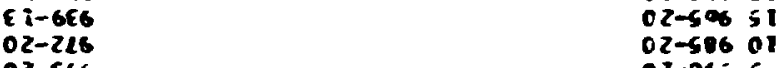

$02-166$

$0 Z \rightarrow \infty$

c2- 


\begin{tabular}{|c|c|c|c|c|c|c|c|c|c|c|c|c|c|}
\hline $\begin{array}{l}0 \\
5 \\
10 \\
15 \\
20 \\
25 \\
50 \\
35 \\
40 \\
45 \\
50 \\
55 \\
60 \\
65 \\
70 \\
75 \\
80 \\
85 \\
90\end{array}$ & $\begin{array}{l}901-13 \\
935-13 \\
963-13 \\
983-13 \\
596-13 \\
90-13 \\
994-13 \\
971-13 \\
967-13 \\
932-13 \\
960-13 \\
761-13 \\
694-13 \\
731-10 \\
910-10 \\
987-10 \\
932-10 \\
949-10 \\
937-11\end{array}$ & $\begin{array}{l}0 \\
\\
700-13 \\
608-10 \\
777-10 \\
949-10 \\
902-10 \\
932-10 \\
537-10 \\
555-11\end{array}$ & $\begin{array}{l}992-13 \\
971-13 \\
934-13 \\
083-13 \\
816-13 \\
733-13 \\
646-10 \\
739-10 \\
915-10 \\
973-10 \\
911-10 \\
927-10 \\
930-11 \\
970-11\end{array}$ & $\begin{array}{l}700-10 \\
701-10 \\
945-10 \\
890-10 \\
915-10 \\
910-10 \\
955-11 \\
902-11\end{array}$ & $\begin{array}{l}575-13 \\
937-13 \\
881-13 \\
900-13 \\
721-13 \\
658-10 \\
745-10 \\
916-10 \\
968-10 \\
901-10 \\
913-10 \\
939-11 \\
970-11 \\
990-11\end{array}$ & $\begin{array}{l}5 \\
703-16 \\
94-10 \\
904-10 \\
907-10 \\
922-11 \\
950-11 \\
992-11 \\
995-11\end{array}$ & 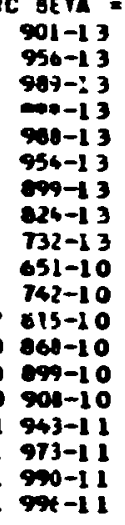 & $\begin{array}{l}\text { } \\
\\
42-10 \\
06-10 \\
907-10 \\
926-11 \\
961-11 \\
96-11 \\
990-11 \\
995-11\end{array}$ & $\begin{array}{l}933-13 \\
863-13 \\
772-13 \\
643-12 \\
722-10 \\
005-10 \\
665-10 \\
901-10 \\
913-10 \\
945-11 \\
975-11 \\
993-11 \\
990-11 \\
990-11\end{array}$ & $\begin{array}{l}905-10 \\
913-10 \\
922-11 \\
961-11 \\
986-11 \\
996-11 \\
996-11 \\
992-11\end{array}$ & $\begin{array}{l}917-! 3 \\
837-13 \\
733-13 \\
670-10 \\
773-10 \\
951-10 \\
901-10 \\
923-10 \\
938-11 \\
973-11 \\
994-11 \\
949-11 \\
992-11 \\
970-11\end{array}$ & $\begin{array}{l}9 \\
915-10 \\
931-10 \\
949-11 \\
901-11 \\
997-11 \\
990-11 \\
904-11 \\
955-11\end{array}$ & $\begin{array}{l}901-13 \\
962-13 \\
995-13 \\
997-13 \\
969-13 \\
910-13 \\
924-13 \\
712-13 \\
701-10 \\
900-10 \\
932-10 \\
927-10 \\
939-10 \\
955-11 \\
985-11 \\
990-11 \\
994-11 \\
975-11 \\
937-11\end{array}$ \\
\hline
\end{tabular}

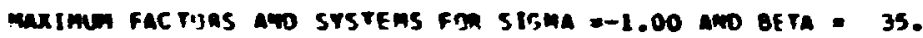

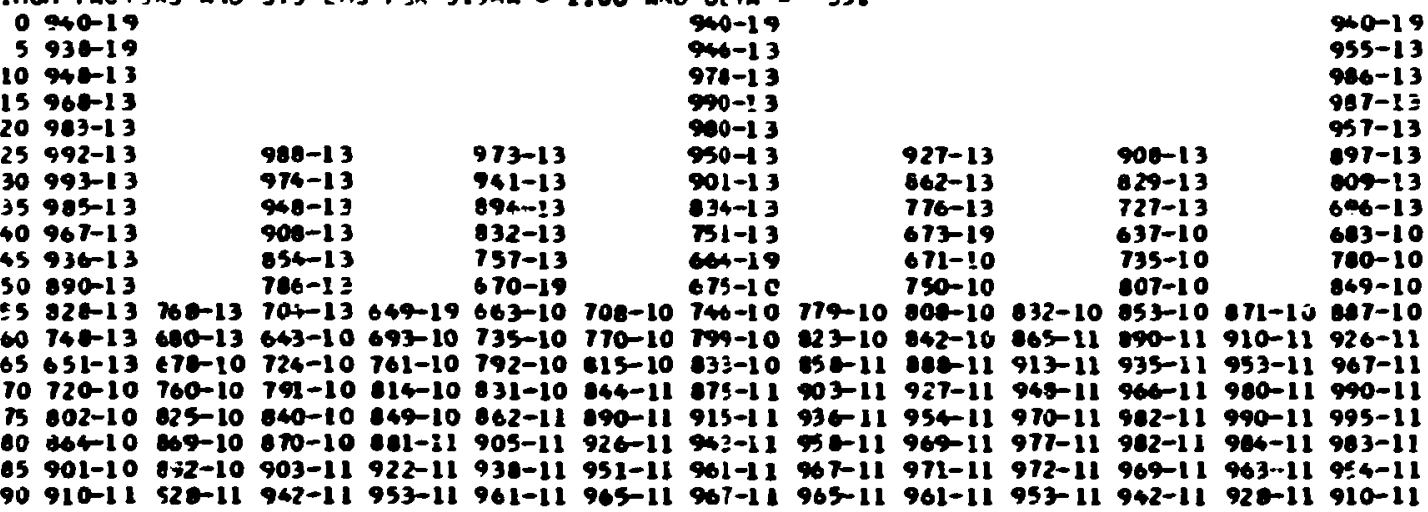

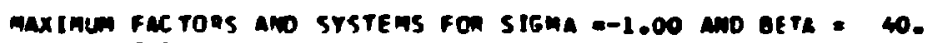

0 995-19

5 904-19

$905-19$

$981-19$

$970-19$

$10901-19$

964-13

$20967-19$

25 96-13

$30973-13$

$957-13$

$35974-13$

$\begin{array}{lll}961-13 & 954-13 & 932-13 \\ 960-13 & 930-13 & 939-13\end{array}$

809-13

$\begin{array}{lll}943-13 & 992-13 & 930-13 \\ 915-13 & 841-13 & 757-13\end{array}$

$10967-13$

$841-13$

$690-19$

$\begin{array}{rrr}074-13 & 770-13 & 696-19 \\ 021-13 & 710-19 & 633-19\end{array}$

So $920-13$

$021-13$ 710-19

633-19

-

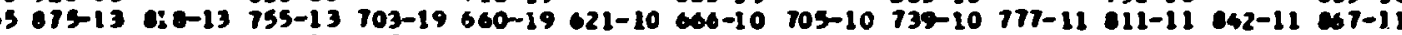

60 if 13 74:-13 692-19 647-19 342-10 604-10 719-10 756-11 797-11 834-11 866-11 695-11 919-11

65 735-13 470-19 634-19 664-10 701-10 731-10 772-11 e10-11 945-11 $076-11$ 907-11 932-11 954-11

$70653-19653$. 10 691-10 722-10 746-11 704-11 19-11 652-11 e02-11 900-11 932-11 953-11 970-11 75 697-10 727-10 750-10 765-10 799-11 629-11 57-11 693-11 905-11 925-11 942-11 956-11 967-11 60 773-10 705-10 791-10 010-11 043-11 005-11 655-11 902-11 917-11 926-11 537-11 944-11 947-11

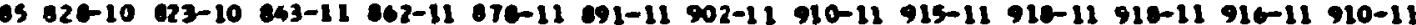

90 ast-11 874-11 886-11 896-11 902-11 906-11 907-11 906-11 902-11 e96-11 886-11 $874-11$ 858-11 


\begin{tabular}{|c|c|c|c|c|c|c|c|c|c|c|c|c|c|}
\hline $\begin{array}{l}170 \\
0 \\
5 \\
10 \\
15 \\
20 \\
25 \\
30 \\
35 \\
40 \\
45 \\
50 \\
55 \\
60 \\
65 \\
10 \\
15 \\
10 \\
65 \\
90\end{array}$ & 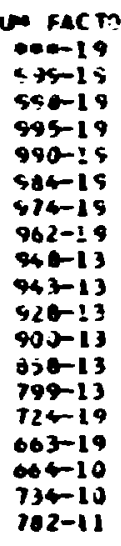 & 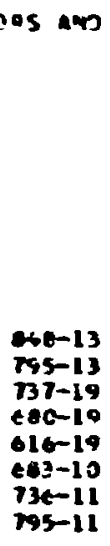 & $\begin{array}{l}964-19 \\
646-19 \\
975-19 \\
904-13 \\
671-13 \\
630-15 \\
709-19 \\
741-19 \\
687-19 \\
626-19 \\
644-10 \\
700-1 ! \\
757-11 \\
305-11\end{array}$ & $\begin{array}{l}7 \\
74-19 \\
741-19 \\
627-19 \\
617-16 \\
675-11 \\
729-11 \\
775-11 \\
612-11\end{array}$ & $\begin{array}{l}937-19 \\
909-19 \\
675-19 \\
837-19 \\
793-19 \\
763-19 \\
690-19 \\
628-19 \\
599-11 \\
658-11 \\
710-11 \\
754-11 \\
790-11 \\
617-11\end{array}$ & $\begin{array}{l}0 \\
632-19 \\
509-11 \\
647-11 \\
699-11 \\
743-11 \\
772-11 \\
604-11 \\
020-11\end{array}$ & 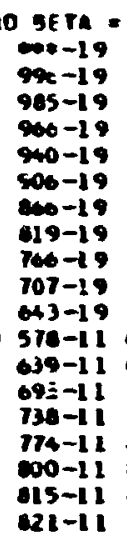 & $\begin{array}{l}45 . \\
030-11 \\
600-11 \\
720-11 \\
75-11 \\
603-11 \\
619-11 \\
625-11 \\
620-11\end{array}$ & $\begin{array}{l}676-19 \\
623-19 \\
7 \pm 3-19 \\
695-19 \\
622-19 \\
616-11 \\
670-11 \\
736-11 \\
770-11 \\
610-11 \\
629-11 \\
637-11 \\
833-11 \\
17-11\end{array}$ & $\begin{array}{l}0 \\
726-11 \\
770-11 \\
017-11 \\
042-11 \\
054-11 \\
653-11 \\
630-11 \\
312-11\end{array}$ & $\begin{array}{l}849-19 \\
764-19 \\
713-19 \\
633-19 \\
629-10 \\
705-11 \\
769-11 \\
619-11 \\
653-11 \\
673-11 \\
677-11 \\
606-11 \\
642-11 \\
005-11\end{array}$ & $\begin{array}{l}0 \\
00-11 \\
954-11 \\
907-11 \\
900-11 \\
997-11 \\
977-11 \\
243-11 \\
795-11\end{array}$ & $\begin{array}{l}000-19 \\
993-19 \\
972-19 \\
937-19 \\
691-19 \\
631-19 \\
758-19 \\
677-19 \\
017-10 \\
701-11 \\
700-11 \\
643-11 \\
690-11 \\
917-11 \\
925-11 \\
915-11 \\
906-11 \\
641-11 \\
702-11\end{array}$ \\
\hline $\begin{array}{l}x 10 \\
0 \\
5 \\
10 \\
15 \\
20 \\
25 \\
30 \\
35 \\
63 \\
45 \\
50 \\
55 \\
00 \\
05 \\
70 \\
75 \\
30 \\
65 \\
90\end{array}$ & $\begin{array}{l}\text { FAC m } \\
945-19 \\
905-19 \\
985-19 \\
905-19 \\
905-19 \\
903-19 \\
960-19 \\
914-15 \\
904-19 \\
950-19 \\
931-19 \\
906-19 \\
600-13 \\
640-13 \\
100-19 \\
129-19 \\
664-19 \\
620-10 \\
605-11\end{array}$ & $\begin{array}{l}0 \\
\\
\\
667-19 \\
630-19 \\
705-19 \\
734-19 \\
674-19 \\
697-19 \\
630-11 \\
693-11\end{array}$ & $\begin{array}{l}960-19 \\
647-19 \\
931-19 \\
910-19 \\
685-19 \\
654-19 \\
810-19 \\
775-19 \\
726-19 \\
670-19 \\
607-19 \\
599-21 \\
640-11 \\
699-1 !\end{array}$ & $\begin{array}{l} \\
\\
\\
162-19 \\
712-19 \\
657-19 \\
637-21 \\
631-21 \\
62 i-21 \\
604-11 \\
104-11\end{array}$ & $\begin{array}{l}520-19 \\
903-19 \\
873-19 \\
637-19 \\
791-19 \\
751-19 \\
760-19 \\
662-21 \\
650-21 \\
652-21 \\
641-21 \\
642-11 \\
679-11 \\
707-11\end{array}$ & $\begin{array}{l}0 \\
676-21 \\
670-21 \\
662-21 \\
651-21 \\
634-21 \\
666-11 \\
692-11 \\
704-11\end{array}$ & $\begin{array}{l}0 \text { OE TA } \\
905-19 \\
91-19 \\
970-19 \\
951-19 \\
925-19 \\
995-19 \\
553-19 \\
07-19 \\
754-19 \\
696-19 \\
677-21 \\
673-21 \\
665-21 \\
652-21 \\
635-21 \\
606-11 \\
690-11 \\
704-11 \\
709-11\end{array}$ & $\begin{array}{l}50 . \\
0621 \\
64-21 \\
640-11 \\
674-11 \\
690-11 \\
712-11 \\
715-11 \\
700-11\end{array}$ & $\begin{array}{l}457-19 \\
803-19 \\
741-19 \\
671-19 \\
605-21 \\
657-21 \\
643-21 \\
649-11 \\
679-1 \\
714-11 \\
729-11 \\
733-11 \\
725-11 \\
707-11\end{array}$ & $\begin{array}{l}0 \\
653-11 \\
699-11 \\
733-11 \\
753-11 \\
759-11 \\
752-11 \\
73-11 \\
704-11\end{array}$ & $\begin{array}{l}625-19 \\
759-19 \\
603-19 \\
644-21 \\
631-21 \\
446-11 \\
705-11 \\
749-11 \\
777-11 \\
790-11 \\
797-11 \\
771-11 \\
741-11 \\
699-11\end{array}$ & $\begin{array}{l}0 \\
0 \\
753-11 \\
794-11 \\
918-11 \\
625-11 \\
914-11 \\
797-11 \\
746-11 \\
693-11\end{array}$ & $\begin{array}{l}905-19 \\
977-19 \\
95-19 \\
917-19 \\
606-19 \\
602-19 \\
720-19 \\
640-19 \\
600-21 \\
606-11 \\
760-11 \\
790-11 \\
637-11 \\
657-11 \\
657-11 \\
838-11 \\
602-11 \\
750-11 \\
685-11\end{array}$ \\
\hline $\begin{array}{l}190 \\
0 \\
5 \\
10 \\
13 \\
20 \\
25 \\
30 \\
35 \\
40 \\
45 \\
50 \\
55 \\
80 \\
65 \\
70 \\
75 \\
80 \\
85 \\
90\end{array}$ & $\begin{array}{l}906-19 \\
674-19 \\
035-19 \\
785-19 \\
727-19 \\
659-19 \\
581-21\end{array}$ & $\begin{array}{l}0 \\
\\
\\
\\
284-19 \\
455-19 \\
819-19 \\
775-19 \\
723-19 \\
662-19 \\
637-21 \\
631-21\end{array}$ & $\begin{array}{l}929-19 \\
922-19 \\
\because 2-19 \\
690-19 \\
800-19 \\
857-19 \\
828-19 \\
793-19 \\
751-19 \\
702-19 \\
690-21 \\
683-21 \\
674-21 \\
661-21\end{array}$ & $\begin{array}{l}7 \\
764-19 \\
736-21 \\
731-21 \\
725-21 \\
116-21 \\
705-21 \\
690-21 \\
071-21\end{array}$ & $\begin{array}{l}893-19 \\
871-19 \\
845-19 \\
815-19 \\
779-19 \\
761-21 \\
750-21 \\
753-21 \\
746-21 \\
737-21 \\
724-21 \\
706-21 \\
697-16 \\
692-14\end{array}$ & $\begin{array}{l}7 \\
763-21 \\
763-21 \\
734-21 \\
745-21 \\
731-21 \\
722-14 \\
717-14 \\
713-14 \\
710-14\end{array}$ & $\begin{array}{l}940-19 \\
930-19 \\
925-19 \\
908-19 \\
803-19 \\
052-19 \\
14-19 \\
775-21 \\
774-21 \\
771-21 \\
765-21 \\
737-21 \\
745-21 \\
731-14 \\
727-14 \\
722-14 \\
719-14 \\
717-14 \\
717-14\end{array}$ & $\begin{array}{l}0 \\
740-21 \\
725-14 \\
719-14 \\
715-16 \\
711-14 \\
709-14 \\
709-14 \\
110-14\end{array}$ & $\begin{array}{l}611-19 \\
769-21 \\
765-21 \\
739-21 \\
746-21 \\
733-21 \\
713-21 \\
703-14 \\
656-14 \\
691-14 \\
600-14 \\
697-14 \\
609-14 \\
692-14\end{array}$ & $\begin{array}{l} \\
622-14 \\
671-14 \\
662-14 \\
656-14 \\
653-14 \\
653-14 \\
657-14 \\
671-20\end{array}$ & $\begin{array}{l}775-19 \\
751-21 \\
740-21 \\
724-21 \\
702-21 \\
674-21 \\
644-16 \\
658-11 \\
679-11 \\
685-11 \\
676-11 \\
654-11 \\
043-20 \\
661-20\end{array}$ & $\begin{array}{l} \\
079-11 \\
712-11 \\
729-11 \\
727-11 \\
710-11 \\
676-11 \\
630-11 \\
631-20\end{array}$ & 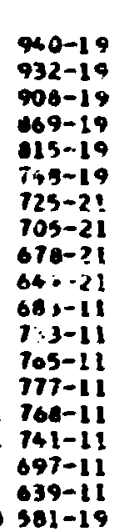 \\
\hline
\end{tabular}




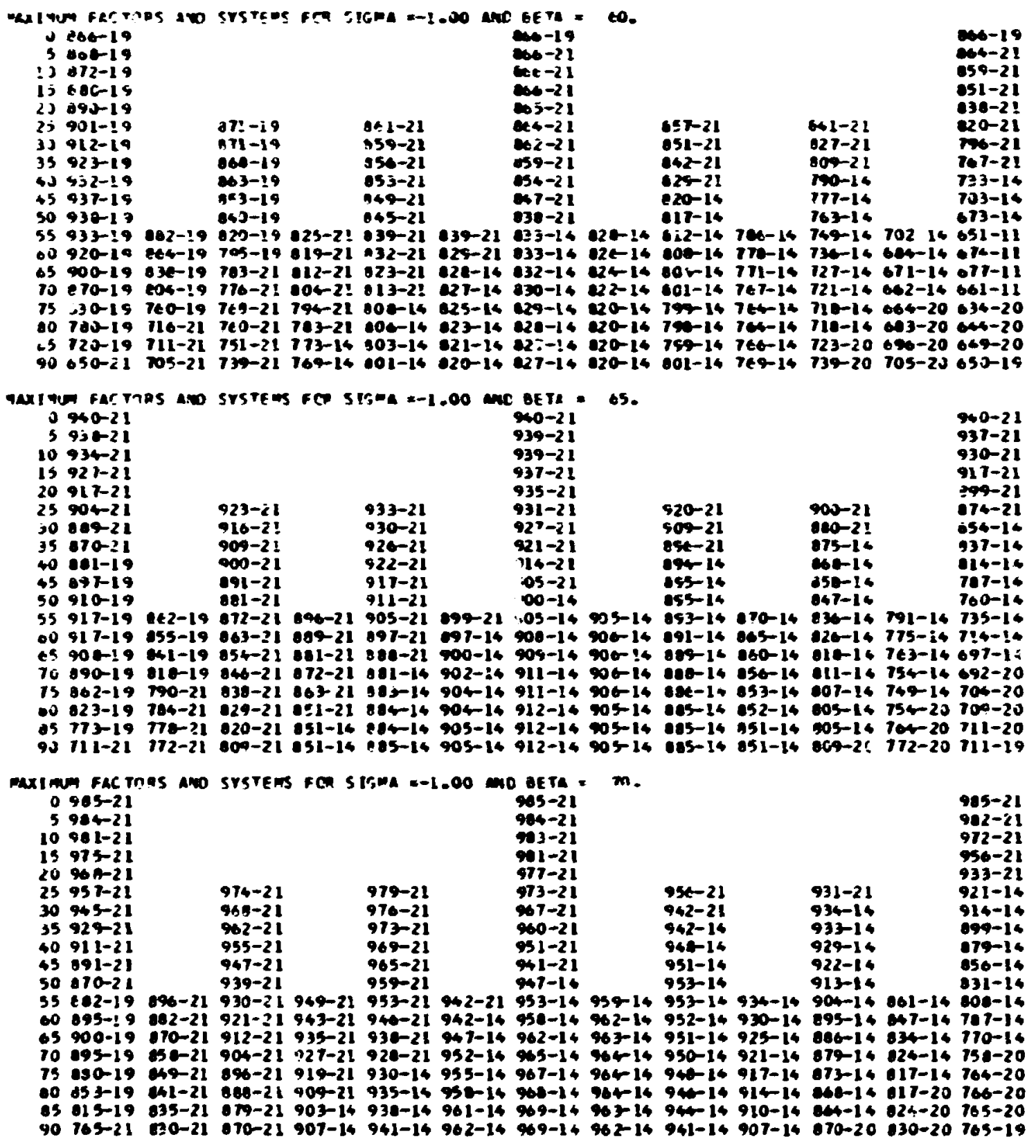




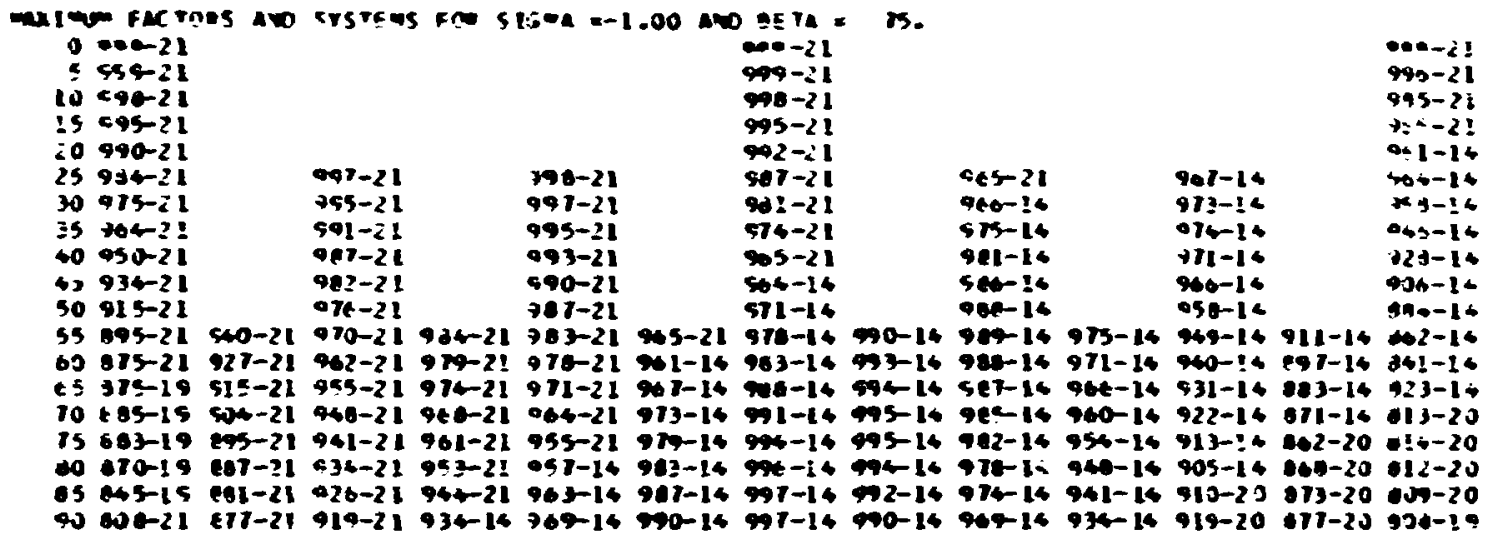

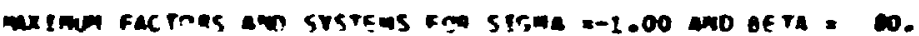

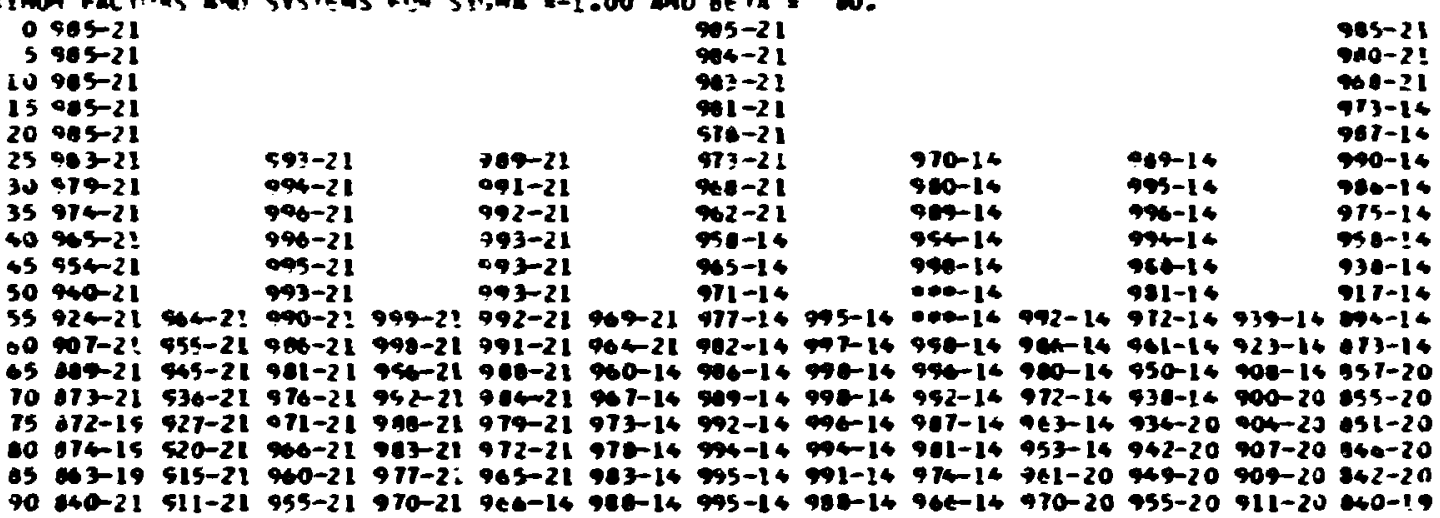

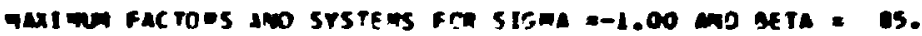

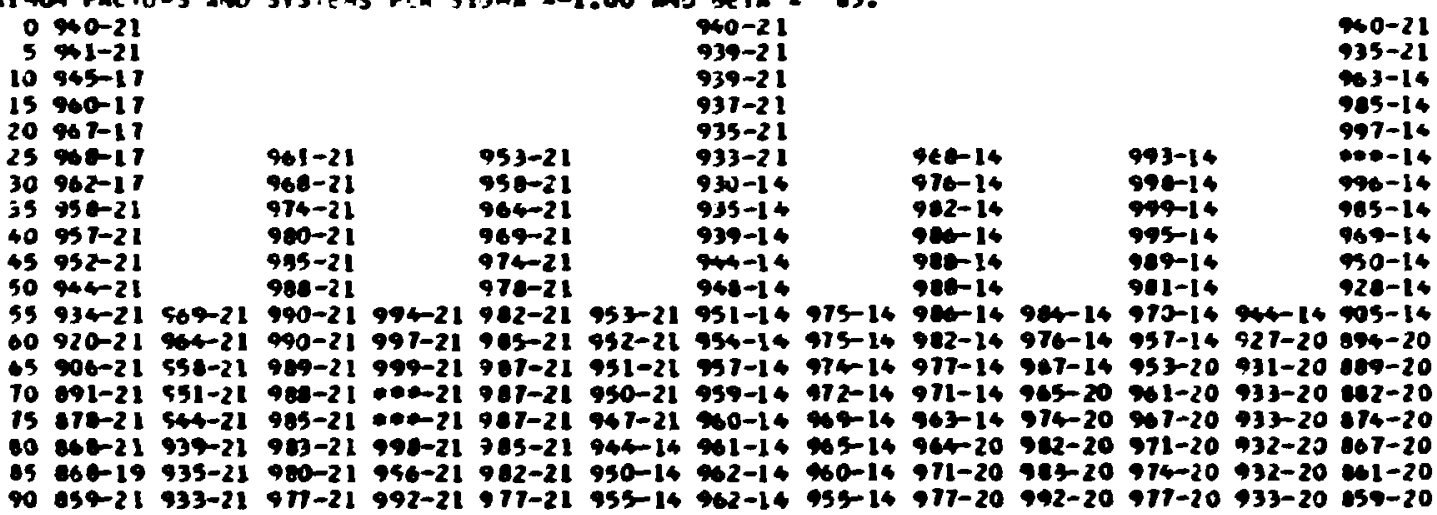




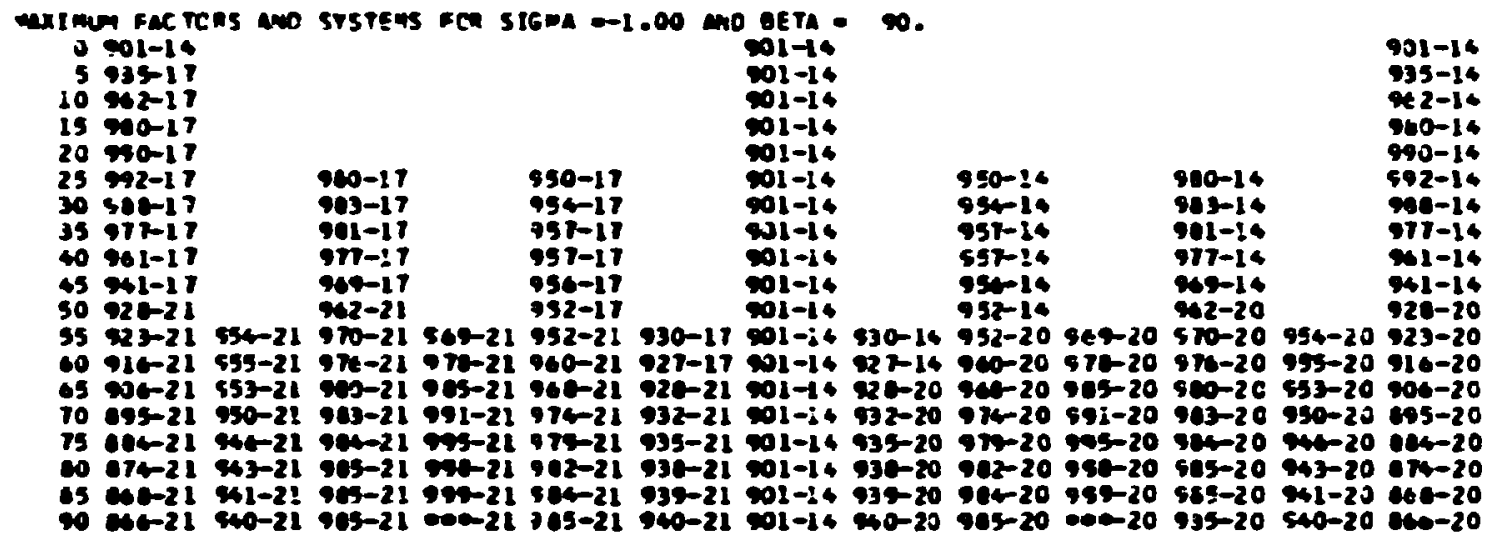

